NUREG/CR-6343

\title{
On-Line Testing of Calibration \\ of Process Instrumentation \\ Channels in Nuclear Power Plants
}

Phase II Final Report

Prepared by

H. M. Hashemian

Analysis and Measurement Services Corporation

Prepared for

U.S. Nuclear Regulatory Commission

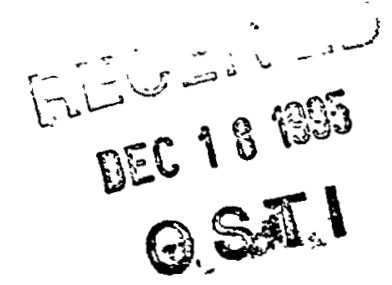




\section{AVAILABILITY NOTICE}

Availability of Reference Materials Cited in NRC Publications

Most documents cited in NRC publications will be available from one of the following sources:

1. The NRC Public Document Room, 2120 L Street, NW., Lower Level, Washington, DC 20555-0001

2. The Superintendent of Documents, U.S. Government Printing Office, P. O. Box 37082, Washington, DC 20402-9328

3. The National Technical Information Service, Springfield, VA 22161-0002

Although the listing that follows represents the majority of documents cited in NRC publications, it is not intended to be exhaustive.

Referenced documents available for inspection and copying for a fee from the NRC Public Document Room include NRC correspondence and internal NRC memoranda; NRC bulletins, circulars, information notices, inspection and investigation notices; licensee event reports; vendor reports and correspondence: Commission papers; and applicant and licensee documents and correspondence.

The following documents in the NUREG series are available for purchase from the Government Printing Office: formal NRC staff and contractor reports, NRC-sponsored conference proceedings, international agreement reports, grantee reports, and NRC booklets and brochures. Also available are regulatory guides, NRC regulatlons in the Code of Federal Regulations, and Nuclear Regulatory Commission Issuances.

Documents avallable from the National Technical Information Service include NUREG-series reports and technical reports prepared by other Federal agencies and reports prepared by the Atomic Energy Commission, forerunner agency to the Nuclear Regulatory Commission.

Documents available from public and special technical libraries include all open literature items, such as books, journal articles, and transactions. Federal Register notices. Federal and State legislation, and congressional reports can usually be obtained from these libraries.

Documents such as theses, dissertations, foreign reports and translations, and non-NRC conference proceedings are available for purchase from the organization sponsoring the publication cited.

Single coples of NRC draft reports are available free. to the extent of supply. upon written request to the Office of Administration, Distribution and Mail Services Section, U.S. Nuclear Regulatory Commission, Washington, DC 20555-0001.

Coples of industry codes and standards used in a substantive manner in the NRC regulatory process are maintained at the NRC Library. Two White Flint North, 11545 Rockville Pike. Rockville, MD 20852-2738, for use by the public. Codes and standards are usually copyrighted and may be purchased from the originating organization or, if they are American National Standards, from the American National Standards Institute. 1430 Broadway, New York, NY 10018-3308.

\section{DISCLAIMER NOTICE}

This report was prepared as an account of work sponsored by an agency of the United States Government. Neither the United States Government nor any agency thereof, nor any of their employees, makes any warranty, expressed or implied, or assumes any legal liability or responsibility for any third party's use, or the results of such use, of any information, apparatus, product, or process disclosed in this report, or represents that its use by such third party would not infringe privately owned rights. 


\section{On-Line Testing of Calibration of Process Instrumentation Channels in Nuclear Power Plants}

\section{Phase II Final Report}

Manuscript Completed: October 1995

Date Published: November 1995

Prepared by

H. M. Hashemian

Analysis and Measurement Services Corporation AMS 9111 Cross Park Drive

Knoxville, TN 37923

\section{E. Antonescu, NRC Project Manager}

\section{Prepared for}

Division of Systems Technology

Office of Nuclear Regulatory Research

U.S. Nuclear Regulatory Commission

Washington, DC 20555-0001

NRC Job Code L2562 
Copyright ${ }^{\odot}$ by Analysis and Measurement Services Corporation (AMS), 1995

ISBN: 1-882148-04-5

This manuscrip thas been authored by a contractor of the $\mathrm{S}$. Government under Contract No. NRC-0492-095. Accordingty, the U.S Government has a nonexclusive, royalty free focense to publishor reproduce the published form of this contribution, or atow ottiers to 0 so, for $\mathrm{U} S$, Government purposes. 


\section{DISCLAIMER}

Portions of this document may be illegible electronic image products. Images are produced from the best available original document. 


\begin{abstract}
Process instrumentation channels in nuclear power plants are usually calibrated at each refueling outage. The calibrations are currently performed manually and are time consuming, costly, and in some cases involve radiation exposure to test personnel. In addition, the hands-on calibrations can wear out the instruments and cause premature aging and failure. Therefore, the nuclear industry is interested in automating the calibration of the instruments and has sponsored a number of research projects to determine the validity of automated calibrations. This report provides the key results of one of these projects.
\end{abstract}

ABSTRACT

This project was conducted over a three-year period under a contract with the NRC. It involved both experimental and theoretical work. The experimental work included laboratory and in-plant validation tests on typical nuclear plant instrumentation systems. The theoretical work involved empirical and physical modeling and neural network fitting techniques to separate process effects from sensor effects, account for common mode problems, and improve the reliability of automated calibrations.

The conclusion of this project is that the normal outputs of instrument channels in nuclear plants can be monitored over a fuel cycle while the plant is operating to determine calibration drift in the field sensors and associated signal conversion and signal conditioning equipment. The plant computer or a dedicated on-line monitoring system can be used to obtain the test data. The advantage of automated calibrations is that they provide the opportunity to test the calibration of instrument channels on a continuous basis. This improves the safety and efficiency of the plant while reducing the cost of the calibrations and eliminating much of the personnel radiation exposure associated with conventional calibrations.

The procedure for on-line calibration tests involves calculating the deviation of each instrument channel from the best estimate of the process parameter that the instrument is measuring. Several methods are available and were evaluated in this project for determining the best estimate of the process. These methods are: (1) simple and weighted averaging of redundant signals, (2) empirical and physical modeling, (3) neural networks, and (4) a reference channel that is calibrated before and after each fuel cycle. The deviation of each signal from the best estimate of the process is updated frequently while the plant is operating and plotted as a function of time for the entire fuel cycle. This provides time history plots that can reveal channel drift and other anomalies. Any instrument channel that exceeds the allowable drift or the channel accuracy band is then scheduled for calibration during a refueling outage, or sooner if necessary.

The above procedure provides calibration test results at the process operating point. This is one of the most critical points of the channel operation, and should suffice for most narrow-range instruments. However, it is often necessary to verify the calibration of some instruments at other points throughout their calibrated range. This may be accomplished by sampling the channel outputs during plant startup and shutdown periods and evaluating the adequacy of the data for widerange calibration verification.

It should be pointed out that the calibration of some process signals such as the high pressure coolant injection flow in BWRs, which are normally off-scale during plant operation, can not be tested on-line. Therefore, the instrument channels for these signals must continue to be calibrated manually using the conventional procedures. However, the number of instrument channels that cannot be tested on-line is much smaller than the number of instrument channels that are testable during plant operation. 


\section{TABLE OF CONTENTS}

Chapter $\quad$ Page

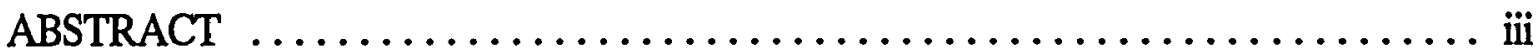

1. INTRODUCTION $\ldots \ldots \ldots \ldots \ldots \ldots \ldots \ldots \ldots \ldots \ldots \ldots \ldots \ldots \ldots \ldots \ldots$

2. HISTORICAL BACKGROUND .......................

$2.1 \quad$ Electric Power Research Institute $\ldots \ldots \ldots \ldots \ldots \ldots \ldots \ldots \ldots \ldots \ldots$

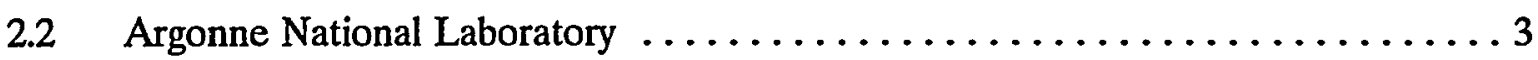

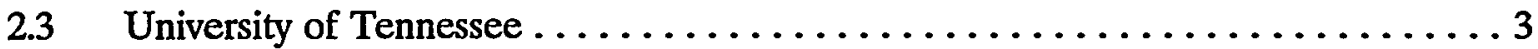

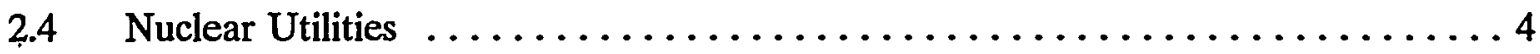

3. PROJECT PARTICIPANTS $\ldots \ldots \ldots \ldots \ldots \ldots \ldots \ldots \ldots \ldots \ldots \ldots \ldots \ldots$

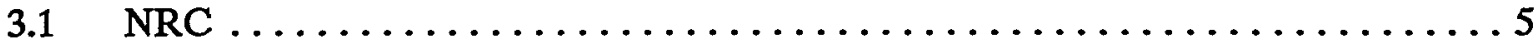

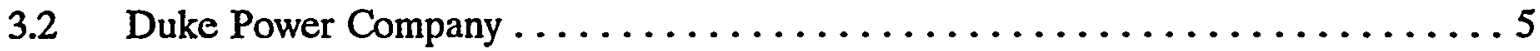

$3.3 \quad$ AMS Corporation $\ldots \ldots \ldots \ldots \ldots \ldots \ldots \ldots \ldots \ldots \ldots \ldots \ldots$

4. INSTRUMENT CHANNELS AND THEIR CALIBRATION REQUIREMENTS $\ldots \ldots 8$

$4.1 \quad$ Instrument Channel $\ldots \ldots \ldots \ldots \ldots \ldots \ldots \ldots \ldots \ldots \ldots \ldots \ldots \ldots$

4.2 Calibration Requirements $\ldots \ldots \ldots \ldots \ldots \ldots \ldots \ldots \ldots \ldots \ldots \ldots$

4.2.1 Calibration Requirements for Sensors . . . . . . . . . . . . . 8

4.2.2 Calibration Requirements for Remainder of Channel . . . . . . . . 10

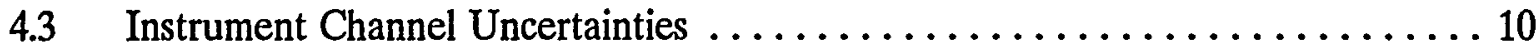

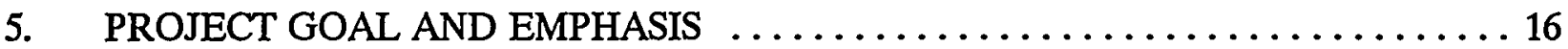

6. PRINCIPLE OF ON-LINE CALIBRATION TESTING . . . . . . . . . . . 19

7. IN-SITU CALIBRATION OF TEMPERATURE SENSORS . . . . . . . . . . . 24

8. ON-LINE CALIBRATION TESTS OF PRESSURE TRANSMITTERS . . . . . . 30

9. PROCESS ESTIMATION TECHNIQUES $\ldots \ldots \ldots \ldots \ldots \ldots \ldots \ldots \ldots \ldots \ldots$

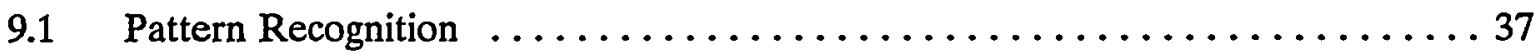

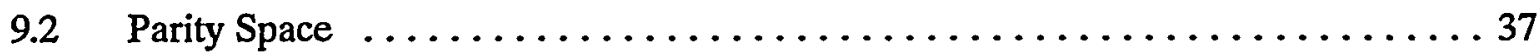

9.3 Generalized Consistency Checking . . . . . . . . . . . . . . . . . 39

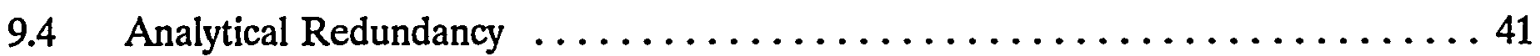

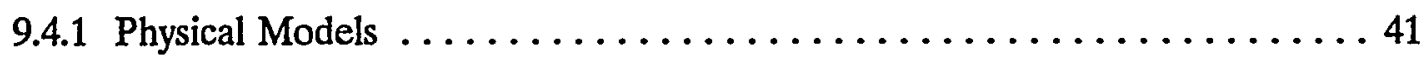

9.4 .2 Empirical Models . . . . . . . . . . . . . . . . . . 43 


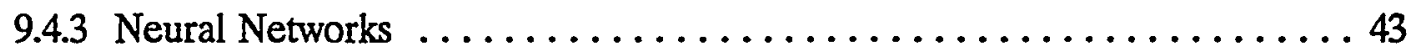

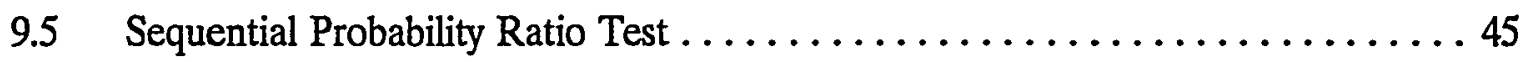

9.6 Process Hypercube Comparison $\ldots \ldots \ldots \ldots \ldots \ldots \ldots \ldots \ldots \ldots \ldots \ldots \ldots \ldots \ldots \ldots \ldots \ldots \ldots$

10. UNCERTAINTIES OF PROCESS ESTIMATION TECHNIQUES $\ldots \ldots \ldots \ldots \ldots 48$

11. ACCEPTANCE CRITERIA FOR ON-LINE MONITORING RESULTS . . . . . . 53

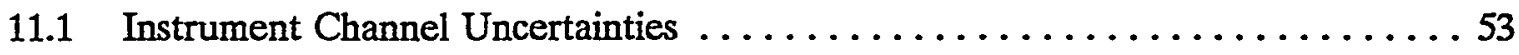

11.2 CSA Band and Drift Band ................................ 56

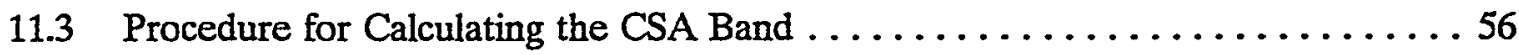

11.4 CSA and Drift Bands for McGuire Signals . . . . . . . . . . . . . . 63

12. TECHNOLOGY DEVELOPMENT AND VALIDATION $\ldots \ldots \ldots \ldots \ldots \ldots$

13. ON-LINE MONITORING SYSTEM DEVELOPMENT $\ldots \ldots \ldots \ldots \ldots \ldots \ldots$

14. VALIDATION OF ON-LINE MONITORING SYSTEM $\ldots \ldots \ldots \ldots \ldots \ldots \ldots$

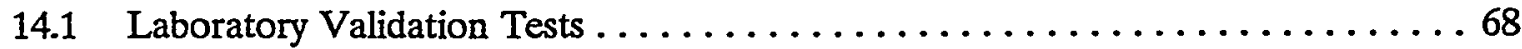

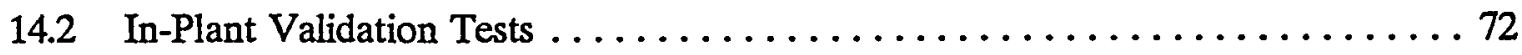

15. LABORATORY VALIDATION RESULTS $\ldots \ldots \ldots \ldots \ldots \ldots \ldots \ldots \ldots \ldots \ldots \ldots \ldots \ldots$

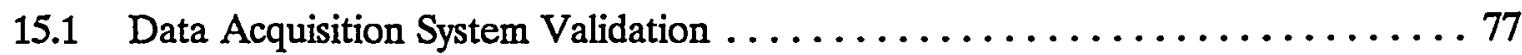

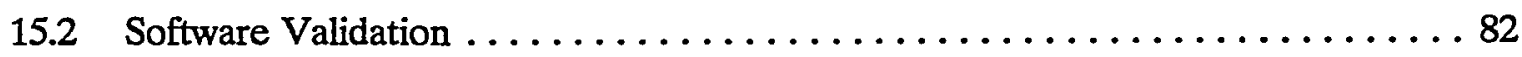

15.3 Validation of Analytical Techniques Using Differential Pressure Signals . . . . . . 86

15.4 Validation of Analytical Techniques Using Absolute Pressure Signals . . . . . . . 103

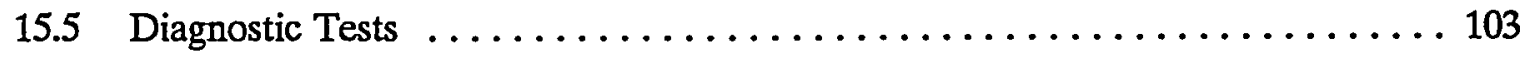

15.6 Effects of Damping on Transmitter Response $\ldots \ldots \ldots \ldots \ldots \ldots \ldots \ldots \ldots \ldots \ldots$

15.7 Test Results in Environment Chamber ...................... 111

16. IN-PLANT VALIDATION RESULTS $\ldots \ldots \ldots \ldots \ldots \ldots \ldots \ldots \ldots \ldots \ldots \ldots \ldots \ldots \ldots \ldots$

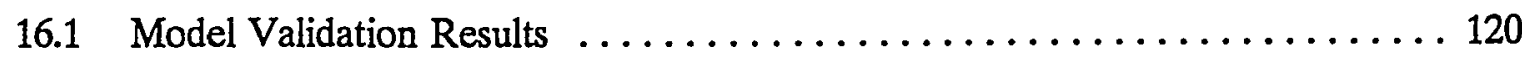

16.2 Comparison of Manual Calibration and On-line Monitoring Results ........ 120

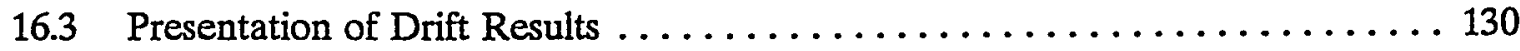

16.4 Presentation of Deviation Results . . . . . . . . . . . . . . . . . 149

16.5 Comparison of Averaging and Modeling Results $\ldots \ldots \ldots \ldots \ldots \ldots \ldots \ldots$

16.6 Success and Failure of Process Estimation Techniques ............... 166

16.7 Instrument Drift and Failure Problems at McGuire $\ldots \ldots \ldots \ldots \ldots \ldots \ldots \ldots \ldots$

16.8 Comparison of Deviation Plobts for Redundant Plant Equipment ........ 173 
17. TEMPERATURE STRATIFICATION AND ITS EFFECT ON

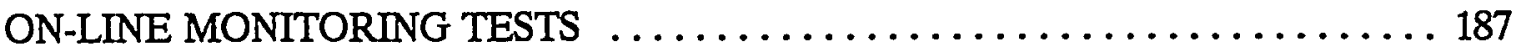

18. CALIBRATION OF ON-LINE MONITORING SYSTEM . .............. 194

19. BENEFITS AND COSTS OF ON-LINE CALIBRATION MONITORING $\ldots \ldots \ldots 196$

20. CONCERNS ABOUT ON-LINE CALIBRATION MONITORING .......... 204

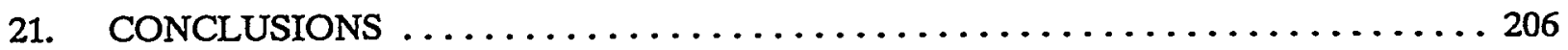

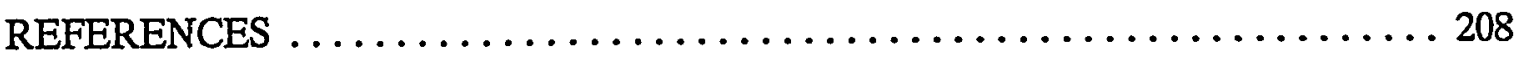

Appendices
A Neural Network Theory
B Empirical Modeling, Theory
C Generalized Consistency Checking Theory
D Sequential Probability Ratio Test
E Pattern Recognition Methods
F Raw Data Plots for Cycle 1
G Raw Data Plots for Cycle 2 


\section{LIST OF FIGURES}

Chapter

Page

4.1 Typical Components of a Safety-Related Instrument Channel in a PWR . . . . . . . . 9

4.2 A General View of Components of an Instrument Channel and Associated Uncertainties . . . . . . . . . . . . . . . . . . . . . . . . 11

4.3 Illustration of Activities to Verify the Performance of an Instrument Channel $\ldots \ldots \ldots 13$

4.4 Relative Location of Typical Operating Limits in Nuclear Power Plants . . . . . . . . 15

5.1 Summary of Search of LER Database $\ldots \ldots \ldots \ldots \ldots \ldots \ldots \ldots \ldots \ldots \ldots$

5.2 Summary of Search of NPRDS Database $\ldots \ldots \ldots \ldots \ldots \ldots \ldots \ldots \ldots \ldots$

6.1 Illustration of Potential Outcome of an On-Line Monitoring Test $\ldots \ldots \ldots \ldots$

6.2 Effect of Process Drift on Instrument Drift $\ldots \ldots \ldots \ldots \ldots \ldots \ldots \ldots \ldots \ldots \ldots$

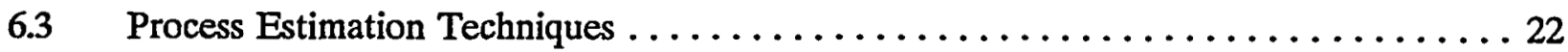

6.4 Process Estimation Techniques Used Together $\ldots \ldots \ldots \ldots \ldots \ldots \ldots \ldots \ldots \ldots \ldots$

8.1 Deviation Plot for McGuire Signals $\ldots \ldots \ldots \ldots \ldots \ldots \ldots \ldots \ldots \ldots \ldots \ldots \ldots \ldots \ldots$

8.2 Drift Plot for McGuire Signals $\ldots \ldots \ldots \ldots \ldots \ldots \ldots \ldots \ldots \ldots \ldots \ldots \ldots \ldots \ldots \ldots$

8.3 Use of Reference Sensor to Track the Process ................... 33

8.4 Process Estimating Using Empirical Modeling and a Neural Network to Identify Drift in a Single Instrument . . . . . . . . . . . . . . . . 34

8.5 Estimation of Steam Generator Level Process at McGuire Using Four

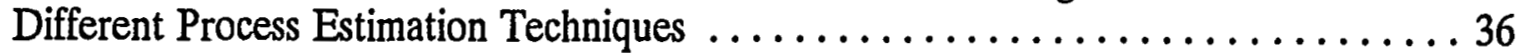

9.1 Analytical Estimation of EBR-II Temperature by Pattern Recognition . . . . . . . 38

9.2 Detection of Faulty Temperature Sensor at EBR-II Using Pattern Recognition . . . . . 39

9.3 Illustration of Principle of Parity Space Technique $\ldots \ldots \ldots \ldots \ldots \ldots \ldots \ldots$

9.4 Detection of Signal Inconsistencies Using the GCC Method . . . . . . . . . . 42

9.5 Example of an Empirical and Physical Modeling Results for a McGuire Pressure Signal . . . . . . . . . . . . . . . . . . . . . . . . . 44

9.6 Three Dimensional Cell for PHC Technique $\ldots \ldots \ldots \ldots \ldots \ldots \ldots \ldots \ldots \ldots$

10.1 Illustration of Effect of Process Estimation Uncertainties on the Results of On-Line Drift Monitoring . . . ....................... 49

10.2 Recovering Drift from a Noisy Plant Signal $\ldots \ldots \ldots \ldots \ldots \ldots \ldots \ldots \ldots \ldots$

10.3 Recovering a Sudden Jump in the Signal in Spite of Noise . . . . . . . . . . . 51

10.4 Raw Data and Deviation Plot Without Filtering the Signals . . . . . . . . . . 52

11.1 Deviation Plot and CSA Bands for McGuire Signals $\ldots \ldots \ldots \ldots \ldots \ldots \ldots$

11.2 Drift Plots and Drift Bands for McGuire Signals $\ldots \ldots \ldots \ldots \ldots \ldots \ldots \ldots$

$11.3 \quad$ CSA Band for a Single Signal . . . . . . . . . . . . . . . . . . . . 60 
11.4 Uncertainty of Simple Averaging for Process Estimation . . . . . . . . . . . 61

11.5 Illustration of How CSA Band is Established for Redundant Signals . . . . . . . . 62

11.6 Flow Signals and CSA Band Showing a Signal which Fails from the Start . . . . . . 65

14.1 Photograph of Laboratory Test Loop . . . . . . . . . . . . . . . . 69

14.2 Schematic of Laboratory Loop . . . . . . . . . . . . . . . . . . 70

14.3 Photograph of 7300 Instrumentation System and Loop Operations and Control Console ................................. 71

14.4 Simplified Schematic of One of the Four Coolant Loops of McGuire

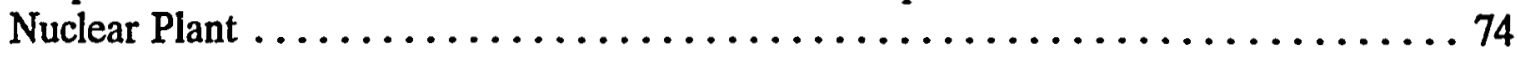

14.5 Block Diagram of On-Line Monitoring System Used at McGuire . . . . . . . . 75

14.6 Block Diagram of On-Line Monitoring System Connection to One of the

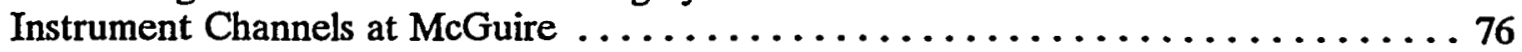

15.1 Laboratory Test Results when Sensors were Drifted and Process was Stable . . . . . . 78

15.2 Laboratory Test Results when Both the Test Sensors and the Loop Pressure

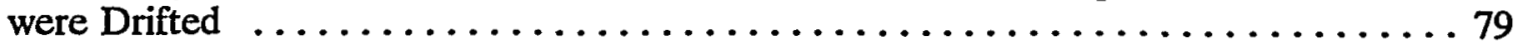

15.3 Laboratory Test Results with Loop Flow Manipulated to Simulate Plant-Type Data . . . . . . . . . . . . . . . . . . . . . . . . . 80

15.4 Test Results with Plant-Type Data Generated in the Laboratory $\ldots \ldots \ldots \ldots \ldots . . .81$

15.5 Raw Data and Deviation Plot for Three. Flow Transmitters $\ldots \ldots \ldots \ldots \ldots$

15.6 Software Validation Results for Flow Transmitters Induced with Linear Drift . . . . . . 84

15.7 Software Validation Results for Temperature and Flow Sensors Induced with a

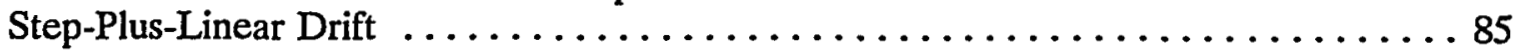

15.8 Software Validation Results for Rosemount Smart Transmitter . . . . . . . . . . . 87

15.9 Software Validation Results for a Foxboro Transmitter . . . . . . . . . . . . 88

15.10 Software Validation Results for a Statham Transmitter . . . . . . . . . . . . . . . . 89

15.11 Raw Data and Results of Process Estimation Techniques . . . . . . . . . . . . 90

15.12 Drift Detected by Three Process Estimation Techniques . . . . . . . . . . . 91

15.13 Quantitative Results of Process Estimation Techniques . . . . . . . . . . . . 92

15.14 Raw Data and Results of Process Estimate Results when Two of

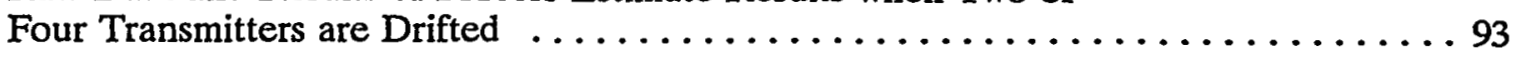

15.15 Drift Plots when Two of Four Transmitters are Drifted . . . . . . . . . . . . 94

15.16 Quantitative Results of Process Estimation Techniques when Two Transmitters are Drifted ............................. 95

15.17 Raw Data and Process Estimation Results when Three of Four

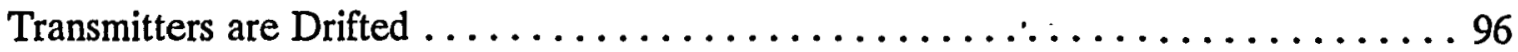

15.18 Drift Plots when Three of Four Transmitters are Drifted . . . . . . . . . . 97

15.19 Quantitative Results of Process Estimation Techniques when Three Transmitters are Drifted . . . . . . . . . . . . . . . . . . . 98 
15.20 Raw Data and Process Estimation Results when Four Transmitters are Drifted . . . . 99

15.21 Drift Plots when Four Transmitters are Drifted $\ldots \ldots \ldots \ldots \ldots \ldots \ldots \ldots \ldots$

15.22 Quantitative Results of Process Estimation Techniques when Four

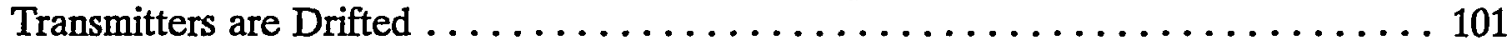

15.23 Raw Data Plot and Process Estimation Results when One Transmitter was Drifted .. 104

15.24 Deviation Plots when One Transmitter was Drifted . . . . . . . . . . . . . . . . 105

15.25 Comparison of Induced Drift Versus Detected Drift when One Transmitter

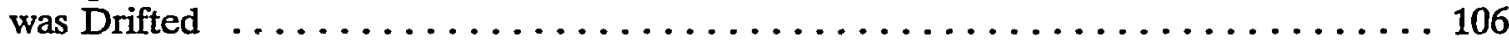

15.26 Raw Data Plot and Process Estimation Results when Both Transmitters

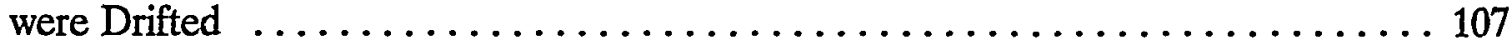

15.27 Deviation Plots for when Both Transmitters were Drifted . . . . . . . . . . . 108

15.28 Comparison of Induced Drift Versus Detected Drift . . . . . . . . . . . . . . . 109

15.29 On-Line Monitoring Results Revealing a Transmitter with a Loose Wire . . . . . . . 110

15.30 Response of a Transmitter with a Loose Wire . . . . . . . . . . . . . . 112

15.31 Response of a Failing Signal Conditioning Card in an Instrument Channel

Tested in the Laboratory . . . . . . . . . . . . . . . . . . . . . 113

15.32 RTD Output Signal when Extension Wires were Intentionally Loosened . . . . . . 114

15.33 RTD Output as a Function of Mechanical Stress $\ldots \ldots \ldots \ldots \ldots \ldots \ldots \ldots$

15.34 Decreasing Resistance of an RTD Subjected to Mechanical Stress . . . . . . . . 116

15.35 Responses of a Damped and an Undamped Pressure Transmitter . . . . . . . . . 117

15.36 Response of Pressure Transmitters Inside the Environmental Chamber

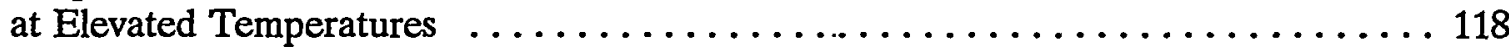

15.37 Comparison of Thermal Responses of Different Transmitters . . . . . . . . . . . 119

16.1 Reactor Power and Periods when On-Line Monitoring Data were Collected at the McGuire Nuclear Plant . . . . . . . . . . . . . . . . . . . . 121

16.2 Removal of Discontinuities and Gaps from On-Line Monitoring Data . . . . . . . 122

16.3 Raw and Filtered Signals . . . . . . . . . . . . . . . . . . . . 123

16.4 Model Validation Results for Two Feedwater Flow Signals . . . . . . . . . . . . . . . 124

16.5 Model Validation Results for Two Steam Flow Signals . . . . . . . . . . . . . . . 125

16.6 Model Validation Results for Four Steam Generator Level Signals . . . . . . . . . . 126

16.7 Model Validation Results for Three Steam Generator Pressure Signals . . . . . . . 127

16.8 Model Validation Results for Four Hot Leg RTD Signals . . . . . . . . . . . . . 128

16.9 Model Validation Results for Three Pressurizer Level Signals . . . . . . . . . . . . 129

16.10 Comparison of Drift from Manual Calibrations with Drift from On-Line Calibration for Three Pressurizer Level Transmitters at McGuire . . . . . . . . . . 131

16.11 Comparison of Manual Calibration Results and On-Line Monitoring Results . . . . . 132

16.12 Raw Data and Drift Plots for Steam Flow Signals . . . . . . . . . . . . . 133

16.13 Raw Data and Drift Plots for Steam Pressure Signals . . . . . . . . . . . . . . 134

16.14 Raw Data and Drift Plots for Steam Generator Level Signals . . . . . . . . . . . . . . 135 
16.15 Raw Data and Drift Plots for Feedwater Flow Signals $\ldots \ldots \ldots \ldots \ldots \ldots \ldots$

16.16 Raw Data and Drift Plots for Reactor Coolant Flow Signals ........ . . . . . 137

16.17 Raw Data and Drift Plots for Pressurizer Level Signals . . . . . . . . . . . . 138

16.18 Raw Data and Drift Plots for Pressurizer Pressure Signals . . . . . . . . . . . 139

16.19 Raw Data and Drift Plots for Wide-Range Pressure Signals . .............. 140

16.20 Raw Data and Drift Plots for Neutron Flux Signals ................. 141

16.21 Raw Data and Drift Plots for Hot Leg RTD Signals .................. 142

16.22 Raw Data and Drift Plots for Cold Leg RTD Signals ................. 143

16.23 Raw Data and Drift Plots for Core Exit Thermocouple Signals .............. 144

16.24 Raw Data and Drift Plots for Auxiliary Feedwater Flow Signals ............ 145

16.25 Raw Data and Drift Plots for Containment Pressure Signals ............... 146

16.26 Raw Data and Drift Plots for RVLIS Signals .................... 147

16.27 Raw Data and Drift Plots for Turbine Impulse Pressure Signals . . . . . . . . . 148

16.28 Raw Data and Deviation Plots for Steam Flow Signals ............... 150

16.29 Raw Data and Deviation Plots for Steam Pressure Signals . . . . . . . . . . . 151

16.30 Raw Data and Deviation Plots for Steam Generator Level Signals . . . . . . . . . 152

16.31 Raw Data and Deviation Plots for Feedwater Flow Signals . . . . . . . . . . . . 153

16.32 Raw Data and Deviation Plots for Reactor Coolant Flow Signals ........... . 154

16.33 Raw Data and Deviation Plots for Pressurizer Level Signals ............... 155

16.34 Raw Data and Deviation Plots for Pressurizer Pressure Signals .............. 156

16.35 Raw Data and Deviation Plots for Wide-Range Pressure Signals . . . . . . . . . . 157

16.36 Raw Data and Deviation Plots for Neutron Flux Signals . . . . . . . . . . . . 158

16.37 Raw Data and Deviation Plots for Hot Leg RTD Signals ................ 159

16.38 Raw Data and Deviation Plots for Cold Leg RTD Signals . . . . . . . . . . . . 160

16.39 Raw Data and Deviation Plots for Core Exit Thermocouple Signals ............ 161

16.40 Raw Data and Deviation Plots for Auxiliary Feedwater Flow Signals . . . . . . . . 162

16.41 Raw Data and Deviation Plots for Containment Pressure Signals . . . . . . . . . 163

16.42 Raw Data and Deviation Plots for RVLIS Signals . . . . . . . . . . . . . 164

16.43 Raw Data and Deviation Plots for Turbine Impulse Pressure Signals .......... 165

16.44 Comparison of Results of Physical Modeling with Averaging and Empirical

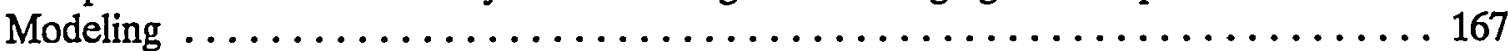

16.45 Comparison of Deviation Results from Three Process Estimation Techniques for McGuire Thermocouple Signals $\ldots \ldots \ldots \ldots \ldots \ldots \ldots \ldots \ldots \ldots \ldots . \ldots \ldots$

16.46 Empirical and Neural Network Results of Process Estimation Compared with Actual Plant Signals . ......................................... 169

16.47 Averaging and Empirical Modeling Results for McGuire Flow Transmitters . . . . . 170 
16.48 Drift Results Showing Good Agreement Between Process Estimation Techniques ... 171

16.49 Drift Results Showing the Failure of Empirical Modeling Technique .......... 172

16.50 Drift Results for McGuire Steam Pressure Transmitters Showing the Failure of Analytical Techniques . .................................. 174

16.51 Data for Three McGuire Steam Generator Level Transmitters of which One was Replaced ..................................... 175

16.52 Data for Three McGuire Reactor Coolant Flow Transmitters of which One was Replaced ............................ 176

16.53 On-Line Monitoring Results Showing Failed Core Exit Thermocouples . . . . . . . 177

16.54 Behavior of an Erratic Hot Leg RTD at McGuire ................. 178

16.55 Cycle 1 and Cycle 2 Signals from Redundant Steam Flow Loops ............ 179

16.56 Cycle 1 and Cycle 2 Signals from Redundant Steam Pressure Loops ............ 180

16.57 Cycle 1 and Cycle 2 Signals from Redundant Steam Generator Level Loops . . . . . 181

16.58 Cycle 1 and Cycle 2 Signals from Redundant Feedwater Flow Loops . . . . . . . . . 182

16.59 Cycle 1 and Cycle 2 Signals from Redundant Reactor Coolant Flow Loops . . . . . . 183

16.60 Cycle 1 and Cycle 2 Signals from Redundant Hot Leg RTD Loops . . . . . . . . . 184

16.61 Cycle 1 and Cycle 2 Signals from Redundant Cold Leg RTD Loops ............ 185

16.62 Cycle 1 and Cycle 2 Signals from Redundant Core Exit Thermocouple Loops ..... 186

17.1 Deviation of a Hot Leg RTD as a Function of Reactor Power Showing the Effect of

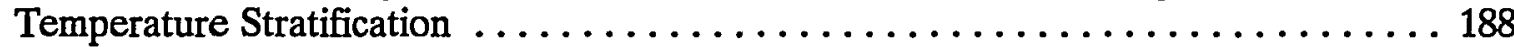

17.2 On-Line Monitoring Results for Six Hot Leg RTDs in One Loop of McGuire Plant ........................................... 189

17.3 On-Line Monitoring Results for Cold Leg RTDs at One Loop of McGuire Plant . . . 190

17.4 Deviation of Core Exit Thermocouples in One of the Four Quadrant of McGuire Plant ......................................... 191

17.5 Deviation of McGuire Hot Leg RTDs . ...................... 192

17.6 Deviation of McGuire Cold Leg RTDs ...................... 193

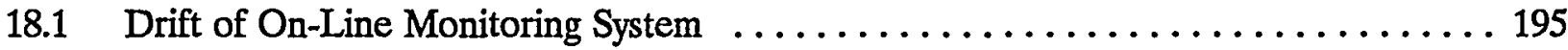

19.1 On-Line Monitoring Data Showing an Erratic RTD .................. 197

19.2 One-Line Monitoring Data Showing an Erratic Pressure Transmitter . . . . . . . . 198

19.3 Drift of a Temperature Channel Identified by Simple Averaging ........... 203

A.1 Schematic of a Four Layer Feedforward ANN $\ldots \ldots \ldots \ldots \ldots \ldots \ldots \ldots$ A7

A.2 Schematic of a Single Processing Element $\ldots \ldots \ldots \ldots \ldots \ldots \ldots \ldots$ A8

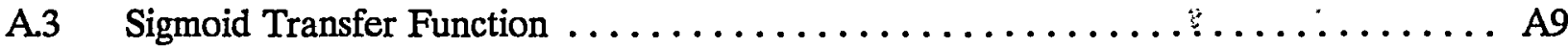

E.1 Two Dimensional Hypercube Nearest Neighbor Example $\ldots \ldots \ldots \ldots \ldots \ldots \ldots$ E3

F.1 McGuire Cycle 1 Data for Steam Flow Signals for Loop A $\ldots \ldots \ldots \ldots \ldots \ldots$ F1

F.2 McGuire Cycle 1 Data for Steam Flow Signals for Loop B . . . . . . . . . . . F2 
F.3 McGuire Cycle 1 Data for Steam Flow Signals for Loop C . . . . . . . . . . F 3

F.4 McGuire Cycle 1 Data for Steam Flow Signals for Loop D ............... F 4

- F.5 McGuire Cycle 1 Data for Steam Pressure Signals for Loop A ............. F5

F.6 McGuire Cycle 1 Data for Steam Pressure Signals for Loop B .............. F6

F.7 McGuire Cycle 1 Data for Steam Pressure Signals for Loop C . ............ F7

F.8 McGuire Cycle 1 Data for Steam Pressure Signals for Loop D . . . . . . . . . . . F8

F.9 McGuire Cycle 1 Data for Steam Generator Level Signals for Loop A . . . . . . . . . F9

F.10 McGuire Cycle 1 Data for Steam Generator Level Signals for Loop B . . . . . . . . F F10

F.11 McGuire Cycle 1 Data for Steam Generator Level Signals for Loop C . . . . . . . . . F11

F.12 McGuire Cycle 1 Data for Steam Generator Level Signals for Loop D .......... F12

F.13 McGuire Cycle 1 Data for Feedwater Flow Signals for Loop A . . . . . . . . . F F13

F.14 McGuire Cycle 1 Data for Feedwater Flow Signals for Loop B . . . . . . . . . . . F14

F.15 McGuire Cycle 1 Data for Feedwater Flow Signals for Loop C $\ldots \ldots \ldots \ldots \ldots$ F15

F.16 McGuire Cycle 1 Data for Feedwater Flow Signals for Loop D . . . . . . . . . . F16

F.17 McGuire Cycle 1 Data for Auxiliary Feedwater Flow Signals . . . . . . . . . . . F17

F.18 McGuire Cycle 1 Data for Reactor Coolant Flow Signals for Loop A . . . . . . . . . F18

F.19 McGuire Cycle 1 Data for Reactor Coolant Flow Signals for Loop B . . . . . . . . . F19

F.20 McGuire Cycle 1 Data for Reactor Coolant Flow Signals for Loop C . . . . . . . . F20

F.21 McGuire Cycle 1 Data for Reactor Coolant Flow Signals for Loop D . . . . . . . . F21

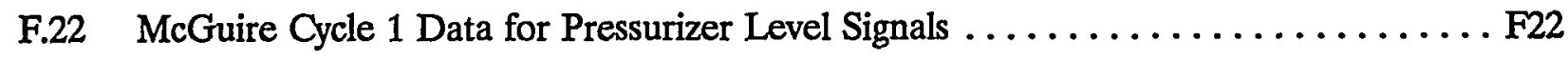

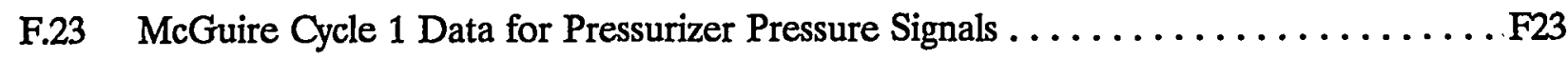

F.24 McGuire Cycle 1 Data for Wide-Range Pressure Signals $\ldots \ldots \ldots \ldots \ldots \ldots \ldots$ F24

F.25 McGuire Cycle 1 Data for Containment Pressure Signals . . . . . . . . . . . F F25

F.26 McGuire Cycle 1 Data for Reactor Vessel Level Indication System Signals . . . . . . . F26

F.27 McGuire Cycle 1 Data for Turbine Impulse Pressure Signals . . . . . . . . . . F F27

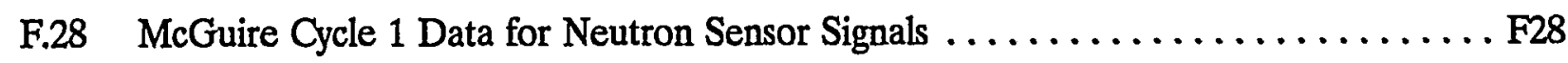

F.29 McGuire Cycle 1 Data for Hot Leg RTD Signals for Loop A ............... F29

F.30 McGuire Cycle 1 Data for Hot Leg RTD Signals for Loop B . . . . . . . . . . . F30

F.31 McGuire Cycle 1 Data for Hot Leg RTD Signals for Loop C . . . . . . . . . . F31

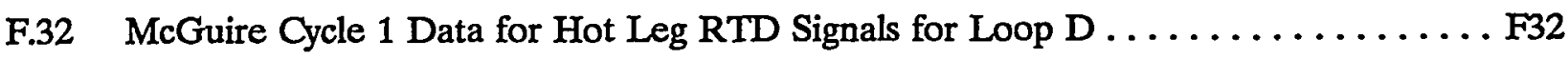

F.33 McGuire Cycle 1 Data for Cold Leg RTD Signals for Loop A $\ldots \ldots \ldots \ldots \ldots \ldots$ F33

F.34 McGuire Cycle 1 Data for Cold Leg RTD Signals for Loop B . . . . . . . . . . . F34

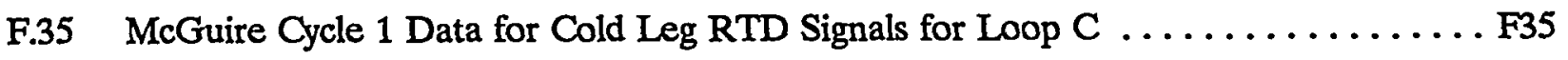

F.36 McGuire Cycle 1 Data for Cold Leg RTD Signals for Loop D . . . . . . . . . . . F36

F.37 McGuire Core Exit Thermocouple Signals for Quadrant $1 \ldots \ldots \ldots \ldots \ldots \ldots \ldots$ F37

F.38 McGuire Core Exit Thermocouple Signals for Quadrant $2 \ldots \ldots \ldots \ldots \ldots \ldots \ldots$ F38 
F.39 McGuire Core Exit Thermocouple Signals for Quadrant $3 \ldots \ldots \ldots \ldots \ldots$ F39

F.40 McGuire Core Exit Thermocouple Signals for Quadrant $4 \ldots \ldots \ldots \ldots \ldots \ldots$ F40

G.1 McGuire Cycle 2 Data for Steam Flow Signals for Loop A .............. G1

G.2 McGuire Cycle 2 Data for Steam Flow Signals for Loop B ............. G2

G.3 McGuire Cycle 2 Data for Steam Flow Signals for Loop C .............. G3

G.4 McGuire Cycle 2 Data for Steam Flow Signals for Loop D ............... G4

G.5 McGuire Cycle 2 Data for Steam Pressure Signals for Loop A ............. G5

G.6 McGuire Cycle 2 Data for Steam Pressure Signals for Loop B ............. G6

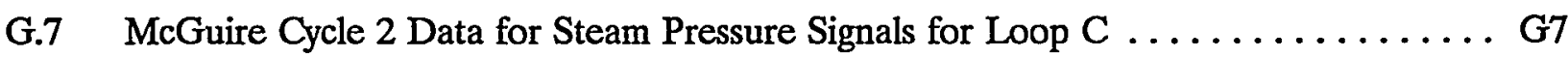

G.8 McGuire Cycle 2 Data for Steam Pressure Signals for Loop D .............. G8

G.9 McGuire Cycle 2 Data for Steam Generator Level Signals for Loop A .......... G9

G.10 McGuire Cycle 2 Data for Steam Generator Level Signals for Loop B ......... G10

G.11 McGuire Cycle 2 Data for Steam Generator Level Signals for Loop C . . . . . . . G11

G.12 McGuire Cycle 2 Data for Steam Generator Level Signals for Loop D . . . . . . . . G12

G.13 McGuire Cycle 2 Data for Feedwater Flow Signals for Loop A ............ G13

G.14 McGuire Cycle 2 Data for Feedwater Flow Signals for Loop B ............. G14

G.15 McGuire Cycle 2 Data for Feedwater Flow Signals for Loop C . . . . . . . . . G15

G.16 McGuire Cycle 2 Data for Feedwater Flow Signals for Loop D ............ G16

G.17 McGuire Cycle 2 Data for Auxiliary Feedwater Flow Signals . . . . . . . . . . . G17

G.18 McGuire Cycle 2 Data for Reactor Coolant Flow Signals for Loop A .......... G18

G.19 McGuire Cycle 2 Data for Reactor Coolant Flow Signals for Loop B . . . . . . . . G19

G.20 McGuire Cycle 2 Data for Reactor Coolant Flow Signals for Loop C . . . . . . . . G20

G.21 McGuire Cycle 2 Data for Reactor Coolant Flow Signals for Loop D . . . . . . . . G21

G.22 McGuire Cycle 2 Data for Pressurizer Level Signals ................ G22

G.23 McGuire Cycle 2 Data for Pressurizer Pressure Signals ................ G23

G.24 McGuire Cycle 2 Data for Wide-Range Pressure Signals ............... G24

G.25 McGuire Cycle 2 Data for Containment Pressure Signals ............... G25

G.26 McGuire Cycle 2 Data for Reactor Vessel Level Indication System Signals ....... G26

G.27 McGuire Cycle 2 Data for Turbine Impulse Pressure Signals $\ldots \ldots \ldots \ldots \ldots \ldots$ G27

G.28 McGuire Cycle 2 Data for Neutron Sensors .................... G28

G.29 McGuire Cycle 2 Data for Hot Leg RTD Signals for Loop A .............. G29

G.30 McGuire Cycle 2 Data for Hot Leg RTD Signals for Loop B ............. G30

G.31 McGuire Cycle 2 Data for Hot Leg RTD Signals for Loop C . ............ G31

G.32 McGuire Cycle 2 Data for Hot Leg RTD Signals for Loop D . . . . . . . . . . G32

G.33 McGuire Cycle 2 Data for Cold Leg RTD Signals for Loop A ............. G33

G.34 McGuire Cycle 2 Data for Cold Leg RTD Signals for Loop B ............. G34 
G.35 McGuire Cycle 2 Data for Cold Leg RTD Signals for Loop C ............ G35

G.36 McGuire Cycle 2 Data for Cold Leg RTD Signals for Loop D ............. G36

G.37 McGuire Cycle 2 Data for Core Exit Thermocouple Signals for Quadrant $1 \ldots \ldots$. G37

G.38 McGuire Cycle 2 Data for Core Exit Thermocouple Signals for Quadrant 2 ...... G38

G.39 McGuire Cycle 2 Data for Core Exit Thermocouple Signals for Quadrant 3 ...... G39

G.40 McGuire Cycle 2 Data for Core Exit Thermocouple Signals for Quadrant $4 \ldots \ldots$. G40 


\section{LIST OF TABLES}

\section{Chapter}

$\underline{\text { Page }}$

3.1 Listing of AMS Reports of R\&D Work for the NRC $\ldots \ldots \ldots \ldots \ldots \ldots \ldots$

4.1 Typical Uncertainties of an Instrument Channel in a Nuclear Power Plant . . . . . . 14

7.1 Typical Cross Calibration Results for a Group of Narrow-Range RTDs $\ldots \ldots \ldots \ldots 25$

7.2 Typical Cross Calibration Results for a Group of Wide-Range RTDs $\ldots \ldots \ldots \ldots 26$

7.3 Typical Cross Calibration Results for a Group of Core Exit Thermocouples in

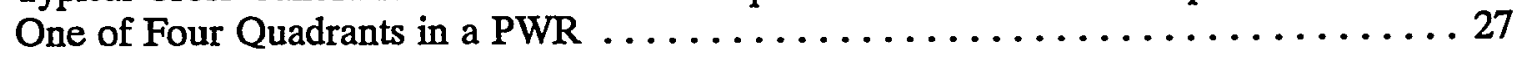

11.1 Typical Sources of Uncertainties for Process Instrumentation Channels in PWR Plants and Corresponding Values that Applies to the McGuire Plant .......... 54

11.2 Description of Sources of Instrument Channel Uncertainties $\ldots \ldots \ldots \ldots \ldots \ldots 5$

11.3 Representative CSA and Drift Bands for Representative Instrument Channels

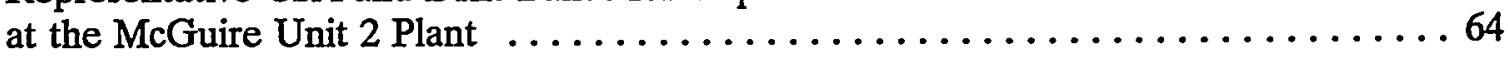

14.1 Listing of Signals Monitored at McGuire Unit $2 \ldots \ldots \ldots \ldots \ldots \ldots \ldots \ldots$

19.1 Comparison of Conventional Calibration Technique with On-Line Calibration Monitoring . . . . ................................ 199

19.2 Potential Benefits of On-Line Calibration Monitoring in Nuclear Power Plants . . . . 200

19.3 Costs of Implementation of On-Line Calibration Monitoring in Nuclear Power Plants . 


\section{INTRODUCTION}

Critical process sensors and associated instrumentation in nuclear power plants are usually calibrated at each refueling outage. The calibrations are performed manually and involve two steps; each of which requires essentially the same work. The two steps are:

1. DETERMINE IF CALIBRATION IS NEEDED. This step is performed by providing the instrument with a series of known inputs covering the operating range of the instrument. The output of the instrument is recorded for each input and compared with the acceptance criteria for the instrument.

2. CALIBRATE IF NEEDED. If the instrument does not meet its acceptance criteria, it is calibrated by providing the same series of input signals as in Step I while adjusting the output to meet the acceptance criteria.

The first step can be automated and performed while the plant is operating. This approach is therefore referred to as on-line calibration monitoring, on-line calibration testing, or on-line drift monitoring. It involves tracking the output of instrument channels over the fuel cycle to identify drift, bias errors, noise and other anomalies. The advantage of this approach is that it identifies calibration problems as they occur, accounts for installation and process condition effects on calibration, and prevents unnecessary calibration of instruments that have maintained their calibrations. Furthermore, it can include most components of an instrument channel in the calibration test as opposed to the conventional procedures which require some components to be calibrated individually. The method may be used for pressure, level, flow, temperature, neutron flux, and other process instrumentation channels including both safetyrelated and non-safety related channels in the primary and secondary systems of nuclear power plants.

This report presents the results of a research and development project to determine if on-line calibration monitoring is as effective as manual calibrations in identifying the instruments which have suffered a significant change in calibration. The project involved laboratory and in-plant tests and analysis. The laboratory tests were performed in a test loop instrumented with sensors and signal conditioning equipment of the types used in nuclear power plants. The loop served to prove the test principles and validate the data acquisition and data analysis software packages. The in-plant tests were performed at the McGuire Nuclear Power Station Unit 2. This is a pressurized water reactor (PWR) operated by Duke Power Company who served as the host utility for this project. A data acquisition system was installed at McGuire in March 1992 to monitor the outputs of nearly 170 instrument channels in the primary and secondary systems of the plant. These included temperature, pressure, level, flow, and neutron flux signals. To date, the system has collected data for two fuel cycles. At the end of each fuel cycle, the on-line monitoring results were compared with the results of the hands-on calibrations that are normally performed during refueling outages. In addition, the on-line monitoring results were evaluated against the allowable drift bands for each instrument channel and the channel statistical accuracy band. These efforts have successfully demonstrated the feasibility of the on-line monitoring approach for instrument calibration verification in nuclear power plants.

This project has led not only to the development of a system for automating 
testing of calibration of instrument channels in nuclear power plants, but also for on-line monitoring of performance of other plant equipment such as the emergency diesel generators, turbine generators, etc. For example, diesel generator parameters such as oil temperature and pressure, coolant water temperature, air pressure, and many other signals can be monitored to ensure that the engine will operate properly if it is needed for emergency power.

The on-line monitoring system can be fitted with transient data acquisition and data analysis modules to provide on-line capability to perform reactor diagnostics, measure core barrel vibration frequency and amplitude, estimate fluid flow rates and detect flowblockages and flow anomalies in the reactor coolant system, measure moderator temperature coefficient while the plant is operating, perform vibration and loose parts monitoring, sensor response time degradation testing, etc. It is envisioned that by the turn of the century, nuclear power plants and other power generation facilities and most process industries can be equipped or retrofitted with on-line monitoring systems to perform static and dynamic performance tests to aid in predictive maintenance, verify the performance of plant equipment, and automate the maintenance efforts currently performed by hands-on procedures.

In addition to nuclear power plants, the on-line monitoring system described in this report will have applications in chemical, petrochemical and other process industries, manufacturing facilities, aviation and aerospace, etc.

Copyright ${ }^{\odot}$ by Analysis and Measurement Services Corporation (AMS), 1995. 


\section{HISTORICAL BACKGROUND}

The interest in implementing on-line monitoring techniques for instrument calibration testing in nuclear power plants peaked in the late 1980s following nearly a decade of research and development efforts in the area of signal validation. These efforts were sponsored by EPRI, Department of Energy (DOE), and nuclear utilities. The EPRI efforts were carried out by various contractors, and the DOE efforts were carried out mostly by the Argonne National Laboratory (ANL) and the University of Tennessee. These efforts are summarized below.

\subsection{Electric Power Research Institute}

In the early 1980s, EPRI sponsored a number of projects in the signal validation area in support of the Safety Parameter Display System (SPDS), digital control systems, and other applications for nuclear power plants. ${ }^{(1,2)}$ These efforts not only helped SPDS, digital control, and other system developments, but also laid the foundation for on-line calibration testing in nuclear power plants and gave rise to the EPRI's instrument calibration reduction/extension program..$^{(3,4)}$ This program, which was initiated in the late 1980s, was originally referred to as the Instrument Calibration Reduction Program (ICRP) and subsequently renamed to Instrument Calibration and Monitoring Program (ICMP). The new name reflects the fact that on-line calibration monitoring amounts to an increase in the calibration frequency of instruments, rather than a decrease.

In addition to sponsoring $R \& D$ efforts in this area, EPRI has taken a leading role in seeking NRC approval for nuclear utilities to use online drift monitoring as a basis for determining which instrument channels should be manually calibrated. In a draft report released for NRC review in August 1995, EPRI has addressed the technical and regulatory issues for the implementation of on-line performance monitoring techniques in nuclear power plants..$^{(5)}$

\subsection{Argonne National Laboratory}

ANL in both Idaho (ANL-West) and in Illinois (ANL-East), have worked in the signal validation area and on the development of new methods for on-line calibration testing of instrument channels in nuclear power plants.

At ANL-West, an on-line monitoring system called the System State Analyzer (SSA) was developed in the early 1980s using the pattern recognition techniques described later in this report. ${ }^{(\bullet)}$ This system was successfully tested at the Experimental Breeder Reactor (EBR-II) in Idaho in the mid-1980s.

At ANL-East, the well-known Sequential Probability Ratio Test (SPRT) has been successfully implemented for determining instrument channel anomalies in nuclear power plants. ${ }^{(7,8)}$

\subsection{University of Tennessee}

In addition to ANL, DOE has sponsored research at the University of Tennessee (UT) on the signal validation and on-line calibration testing areas. The UT work, which was performed over a three-year period between 1986 and 1989, involved empirical, physical and neural network model development and testing. $\left.{ }^{(9,} 10\right)$ In particular, UT developed a number of advanced signal processing techniques that are useful for instrument fault detection and isolation. These techniques are 
described in numerous reports written by UT for DOE. AMS has taken advantage of UT's work in implementing the analytical techniques used in this project.

\subsection{Nuclear Utilities}

One of the first implementations of the on-line monitoring approach for instrument calibration verification in a commercial nuclear power plant occurred in 1987 at the Millstone Nuclear Power Station Unit 3. This is a fourloop Westinghouse PWR operated by Northeast Utilities. As a part of its Emergency Response Facility, which was established in the aftermath of the Three Mile Island accident, Millstone 3 used EPRI's signal validation technologies to develop their SPDS which included a module called the Off-site Facility Information System or OFIS. As its name implies, this system was used off-site at the engineering offices of Northeast Utilities and remotely interfaced with the Millstone plant computer to store operational data and plot them in a variety of formats for analysis of both normal and off-normal conditions. It was also used to monitor the outputs of redundant instrument channels for drift and other anomalies. In fact, it was the OFIS data which helped reveal the now well-known "oil-loss" problem in some models of Rosemount pressure transmitters in 1987. ${ }^{(11)}$

Since 1987, several utilities have tested a number of on-line monitoring systems for instrument calibration verification in nuclear power plants. These include V.C. Summer and South Texas Project which are Westinghouse PWRs and San Onofre Units 2 and 3 and Millstone Unit 2 which are Combustion Engineering PWRs. 


\section{PROJECT PARTICIPANTS}

The project reported here was a joint effort involving the Analysis and Measurement Services Corporation (AMS), the U.S. Nuclear Regulatory Commission (NRC), and Duke Power Company. AMS and the NRC provided the funding for the project and Duke Power Company served as the host utility for the project. The involvement of each of these three organizations is summarized below.

\subsection{NRC}

The project reported here was partially funded by the NRC under the Small Business Innovation Research (SBIR) program. This is a three-phase program that is aimed at meeting the government's research and development (R\&D) needs while stimulating innovative research in the private sector and commercialization of the $R \& D$ product by small businesses. The awards are made on a competitive basis to U.S. companies with less than 500 employees.

Under the SBIR program, a feasibility study is first performed over a six-month period in Phase I to demonstrate the merit of the R\&D and establish the qualification of the small business to conduct a comprehensive $R \& D$ effort over a two-year period in Phase II. The Phase II award is made upon successful completion of Phase I and demonstration of commercial viability of the $R \& D$ product. During or after the completion of the Phase II project, the small business is expected to use its own funds or secure other non-federal funds to launch a Phase III commercialization effort. The Phase III effort is expected to ensure that the government investments during Phase I and Phase II will result in commercial products in the form of equipment, services, training, or a combination of these for the benefit of both the private sector and the federal government.

AMS has been active in the SBIR program since 1984 and has completed $R \& D$ projects under this program for DOE, U.S. Department of Defense (DOD), National Aeronautics and Space Administration (NASA), and the NRC. The NRC projects have resulted in a number of NUREG/CR reports, a listing of which is presented in Table 3.1.

The project reported here began at AMS in 1992 with the award of a Phase I contract from the NRC. The Phase I effort was successfully completed in December 1992 and the results were documented in NUREG/CR5903 published by the NRC in January 1993. The successful completion of the Phase I project resulted in a Phase II award which began in early 1993.

The Phase III commercialization effort began during Phase I by formulating a plan to develop commercial equipment, training, and services for the nuclear power industry. In addition, the Phase III commercialization effort has involved the publication of several technical papers to disseminate the details of the project, participation in national and international exhibitions and trade shows, and demonstration of project results to interested utilities and nuclear plant owners groups.

\subsection{Duke Power Company}

As a part of the Phase I project, AMS negotiated a no-cost contract with Duke Power Company to use the McGuire Unit 2 facility as the test bed for the in-plant validation of the technologies described in this report. The McGuire plant is a two-unit site near Charlotte, North Carolina with two Westinghouse four-loop Pressurized Water 


\begin{tabular}{|c|c|c|c|}
\hline \multicolumn{4}{|c|}{ TABLE 3.1} \\
\hline Item & Report Number & Report Title & Description of Report/Project \\
\hline 1 & NUREG/CR-4928 & $\begin{array}{l}\text { Degradation of Nuclear } \\
\text { Plant Temperature Sensors }\end{array}$ & \multirow{2}{*}{$\begin{array}{l}\text { These reports concentrate on } \\
\text { calibration accuracy and stability } \\
\text { of Resistance Temperature } \\
\text { Detectors (RTDs) and describe } \\
\text { the cross calibration technique for } \\
\text { in-situ testing of accuracy of } \\
\text { nuclear plant RTDs. }\end{array}$} \\
\hline 2 & NUREG/CR-5560 & $\begin{array}{l}\text { Aging of Nuclear Plant } \\
\text { Resistance Temperature } \\
\text { Detectors }\end{array}$ & \\
\hline 3 & NUREG/CR-5383 & $\begin{array}{l}\text { Effect of Aging on } \\
\text { Response Time of Nuclear } \\
\text { Plant Pressure Sensors }\end{array}$ & \multirow{2}{*}{$\begin{array}{l}\text { These reports describe the } \\
\text { principle of operation of nuclear } \\
\text { plant pressure transmitters, and } \\
\text { their failure mechanisms and } \\
\text { failure modes. The reports } \\
\text { concentrate on the effects of } \\
\text { aging on transmitter calibration } \\
\text { and response time, describe } \\
\text { sensing line effects, and provide } \\
\text { data on the validation of new } \\
\text { methods for in-situ response time } \\
\text { testing of pressure transmitters } \\
\text { and detection of blockages and } \\
\text { voids in pressure sensing lines. }\end{array}$} \\
\hline 4 & NUREG/CR-5851 & $\begin{array}{l}\text { Long Term Performance } \\
\text { and Aging Characteristics of } \\
\text { Nuclear Plant Pressure } \\
\text { Transmitters }\end{array}$ & \\
\hline 5 & NUREG/CR-5903 & $\begin{array}{l}\text { Validation of Smart Sensor } \\
\text { Technologies for Instrument } \\
\text { Calibration Reduction in } \\
\text { Nuclear Power Plants } \\
\end{array}$ & \multirow{2}{*}{$\begin{array}{l}\text { This project has evaluated the } \\
\text { validity of on-line monitoring } \\
\text { techniques for instrument } \\
\text { calibration verification in nuclear } \\
\text { power plants. }\end{array}$} \\
\hline 6 & NUREG/CR-6343 & $\begin{array}{l}\text { On-Line Testing of } \\
\text { Calibration of Process } \\
\text { Instrument Channels in } \\
\text { Nuclear Power Plants } \\
\end{array}$ & \\
\hline 7 & NUREG/CR-6312 & $\begin{array}{l}\text { Assessment of Fiber Optic } \\
\text { Pressure Sensors }\end{array}$ & $\begin{array}{l}\text { The state-of-the-art in fiber optic } \\
\text { pressure sensing was established } \\
\text { in the Phase I project and the } \\
\text { potential of these sensors for use } \\
\text { in nuclear power plants was } \\
\text { investigated. }\end{array}$ \\
\hline 8 & NUREG/CR-6334 & $\begin{array}{l}\text { New Sensor for } \\
\text { Measurement of Low Air } \\
\text { Flow Velocity }\end{array}$ & $\begin{array}{l}\text { A new sensor for measurement of } \\
\text { very low air flow rates was } \\
\text { developed and tested. The } \\
\text { sensor can provide new capability } \\
\text { to establish air flow patterns to } \\
\text { determine where to locate air } \\
\text { samplers for more effective } \\
\text { personnel radiation protection in } \\
\text { nuclear facilities. }\end{array}$ \\
\hline
\end{tabular}


Reactors (PWRs). This plant was selected for the following reasons.

1. AMS has had a long term relationship with Duke Power Company which dates back to the mid-1970s when personnel now working for AMS were provided access to the Oconee nuclear power station for inplant validation of the Loop Current Step Response (LCSR) technology. This technology was developed under a contract with EPRI for in-situ response time testing of Resistance Temperature Detectors (RTDs) in nuclear power plants. Furthermore, in the late 1980s, Duke Power Company served as the host utility for in-plant validation of new methods that AMS had developed for on-line testing of response times of pressure transmitters and detection of blockages in pressure sensing lines in nuclear power plants. The validation work was performed at the Catawba Nuclear Power Station. The project was partially funded by the DOE.

2. A large number of isolated primary and secondary instrumentation signals were readily available from the McGuire plant for the on-line monitoring tests. These signals were used by McGuire in an earlier project. The signals were easily connected to an on-line monitoring system provided by AMS. The system was installed at McGuire in March 1992.

3. McGuire Unit 2 is a typical PWR plant. As such, the results of the in-plant validation work are equally applicable to a majority of PWRs. However, it should be pointed out that plant characteristics do not have a strong bearing on the on-line calibration results. Therefore, the validation work described in this report should be applicable not only to most PWRs, but also to Boiling Water Reactors (BWRs), Pressurized Heavy
Water Reactors (PHWRs), Russian PWRs (referred to in Russian as VVERs or WWERs), and other plants.

4. AMS provides testing services to Duke Power Company on a regular basis at the McGuire and Catawba plants and is therefore familiar with Duke Power Company's equipment, procedures and personnel. Also, many of the tests on the on-line monitoring project could be done in conjunction with other AMS service calls to McGuire.

\subsection{AMS Corporation}

In addition to performing the $R \& D, A M S$ has shared in the cost of performing the validation effort described in this report.

The cost of this project was approximately $\$ 600,000$, half of which was provided by the NRC, and the rest was funded by AMS. The motivation for AMS cost sharing has been the potential of the R\&D for commercialization in nuclear power plants and other industries. It is expected that on-line monitoring techniques will have wide applications not only for instrument calibration verification, but also for a variety of predictive maintenance and diagnostic tests in nuclear and non-nuclear facilities.

AMS is using the results of this project to provide commercial products for on-line monitoring of process instrumentation channels and other equipment in nuclear power plants, fossil power plants, process industries, manufacturing facilities, etc. These products will be provided in the form of hardware, software, training, technical support, or a combination of these as necessary to enable a customer to implement the technologies developed in this project. 


\section{INSTRUMENT CHANNELS AND THEIR CALIBRATION REQUIREMENTS}

\subsection{Instrument Channel}

An instrument channel consists of a sensor that is located in the field and signal conversion, signal conditioning, and logic and trip circuitry that are located in instrument cabinets in the control room or cable spreading room areas of most nuclear power plants. Figure 4.1 shows typical components of a safety-related instrument channel in a PWR plant. The sensor could be a pressure, level, or flow transmitter, a resistance temperature detector (RTD) that is used for measurement of reactor coolant hot leg or cold leg temperatures, a core exit thermocouple, a neutron sensor, etc. The signal conversion equipment could be a current-to-voltage convertor, a voltage-to-current convertor, a resistance bridge, etc. The signal conditioning equipment could be amplifiers and filters which are also referred to as lead and lag cards, summing and isolation amplifiers, signal comparators, etc. The safety systems of most nuclear power plants have up to four redundant instrument channels for critical measurements.

\subsection{Calibration Requirements}

The current calibration requirements or calibration practices in nuclear power plants call for the sensors and the rest of the instrument channels to be calibrated separately as described below.

\subsubsection{Calibration Requirements for Sensors}

- Pressure, level, and flow transmitters (hereafter referred to as pressure transmitters) are manually calibrated in the field once every fuel cycle usually during a refueling outage while the plant is at cold shutdown. The calibration involves a pressure signal generator and a calibrated pressure gauge. A number of constant pressures covering the operating range of the transmitter are applied to the transmitter and adjustments (if necessary) are made to the zero, span, and/or linearity of the transmitter electronics to produce the desired output.

- There are no rigid requirements for calibration of RTDs in nuclear power plants and no consensus on how many, how often, and by what means RTDs should be calibrated. As a result, RTD calibration practice in the nuclear industry is sporadic and ranges from no calibration of any RTDs to in-situ calibration of all primary coolant RTDs in some PWRs once every fuel cycle. The in-situ calibrations are performed using the cross calibration technique described later in thiș report. There are also plants which remove some or all of their primary coolant RTDs occasionally and calibrate them in a laboratory or replace them with newly calibrated RTDs.

The removal of RTDs from a plant for calibration in a laboratory is a time consuming and expensive practice. It involves personnel radiation exposure, and can cause response time problems in thermowell-mounted RTDs every time they are removed and reinstalled in thermowells. As such, removal and recalibration of RTDs is practiced in only a few plants on an occasional basis. New RTDs, RTDs which have been in storage for more than a year, reference RTDs that may be used to verify the RTD cross calibration results, 
AMS-DWG BLK054DS

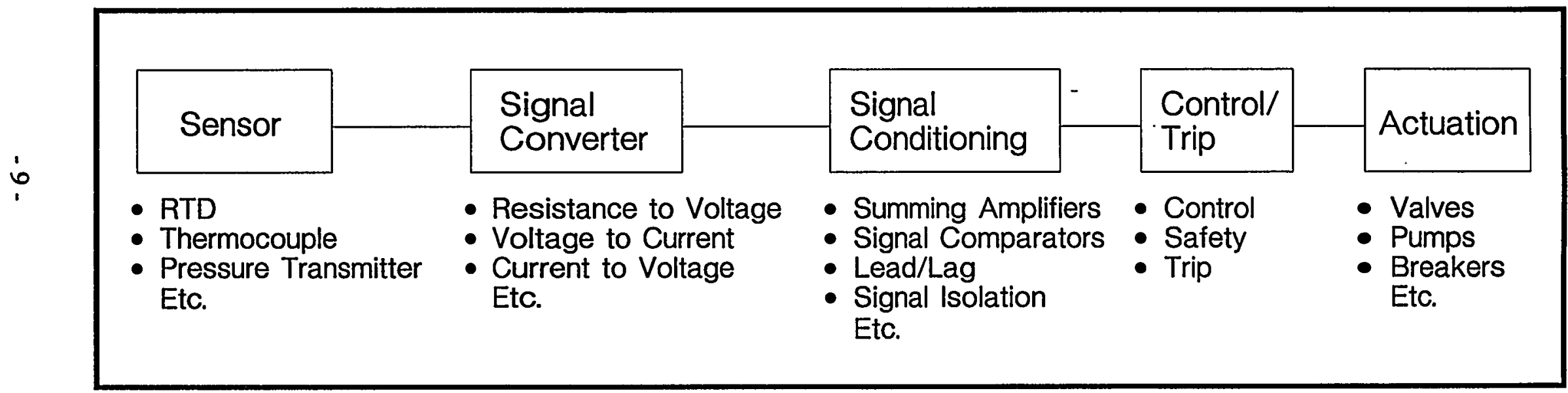

Figure 4.1 Typical Components of a Safety-Related Instrument Channel in a PWR 
and RTDs which are required to meet calibration requirements of better than one or two tenths of a degree must, however, be calibrated in a laboratory.

The laboratory calibration of RTDs for nuclear power plants is typically performed in the range of 0 to $400^{\circ} \mathrm{C}\left(32\right.$ to $\left.752^{\circ} \mathrm{F}\right)$ and involves an ice bath and an oil bath. The RTD is installed in the bath and its resistance is measured at three or more widely spaced temperatures covering the operating range of the RTD. The resistance-versus-temperature data are then used in a quadratic equation to produce a calibration table for the RTD. The calibration table gives the resistance of the RTD at any temperature within the calibrated range. The procedure for laboratory calibration of nuclear plant RTDs is described in detail in NUREG/CR-5560, including a discussion of the uncertainties of laboratory calibrations and how these uncertainties can be identified and combined to assess the accuracy of temperature measurements with RTDs. ${ }^{(12)}$

- As in the case of RTDs, there are no rigid requirements for calibration of thermocouples. However, in some PWRs where the RTD cross calibration method is used, core exit thermocouples are sometimes cross calibrated along with the primary coolant RTDs.

It should be pointed out that most plants use Type K (chromel/alumel) thermocouples. Unlike RTDs, Type K thermocouples will not yield reliable calibration results if they are removed from the plant and recalibrated in a laboratory. This is due to two inherent effects in Type $\mathrm{K}$ thermocouples. These effects are inhomogeneity and short-range ordering. ${ }^{(13)}$ Once a thermocouple is installed in a process and exposed to the high temperatures typical of nuclear power plants, their characteristics can change such that a meaningful recalibration would be difficult to perform. Therefore, the only reasonable method for verifying the calibration of installed thermocouples is to include them in the RTD cross calibration tests, if possible.

- Neutron detectors are not directly calibrated but they are sometimes tested to verify that they operate properly. For this purpose, a neutron source is introduced in the plant to verify that the sensors respond properly.

The calibrations of neutron sensors are checked against the reactor power that is calculated from other measurements. The neutron sensor outputs are then adjusted as necessary to match the calculated power.

\subsubsection{Calibration Requirements for Remainder of Channel}

The components of instrument channels beside the sensors are calibrated manually during each refueling outage. These components are calibrated individually or together depending on the instrument channel design and the plant requirements. A calibration test signal is injected into each component or a group of components and necessary adjustments are made to ensure that the component has the desired output.

In addition to full channel calibrations that are performed at each refueling outage, instrument channels (excluding the sensors) are surveillance tested once every month or once every quarter depending on the plant. The surveillance tests involve temporarily removing the channel from service while the plant is operating, and injecting calibration test signals to the channel to ensure that trip signals are initiated at the required levels.

\subsection{Instrument Channel Uncertainties}

Figure 4.2 provides a general view of an instrument channel and the uncertainties that 
AMS-DWG CRSO21A

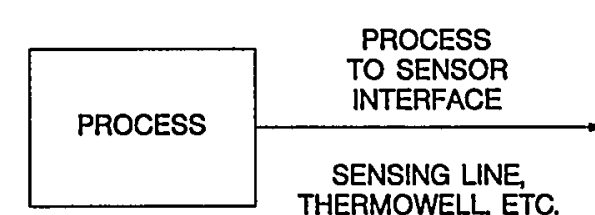
THERMOWELL, ETC.

Process Piping.

Tank

Etc.

\begin{tabular}{|c|c|}
\hline PROCESS & $\begin{array}{c}\text { SIGNAL } \\
\text { INTERFACE }\end{array}$ \\
\cline { 2 - 2 } & MEASUREMENT \\
& CABLES, ETC.
\end{tabular}

Primary Element

Sensor, Transmitter

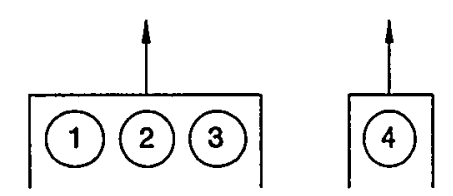

\begin{tabular}{|c|c|}
\hline $\begin{array}{c}\text { SIGNAL } \\
\text { CONDITIONING } \\
(\text { IN THE FIELD) }\end{array}$ & $\begin{array}{c}\text { SIGNAL } \\
\text { INTERFACE }\end{array}$ \\
\cline { 2 - 2 } & CABLES, ETC. \\
\hline
\end{tabular}

Signal Conversion,

Etc.

\begin{tabular}{c|c|}
\hline $\begin{array}{c}\text { SIGNAL } \\
\text { CONDITIONING } \\
\begin{array}{c}\text { (IN INSTRUMENT } \\
\text { CABINETS) }\end{array}\end{array}$ & $\begin{array}{c}\text { SIGNAL } \\
\text { INTERFACE }\end{array}$ \\
& CABLES, ETC.
\end{tabular}
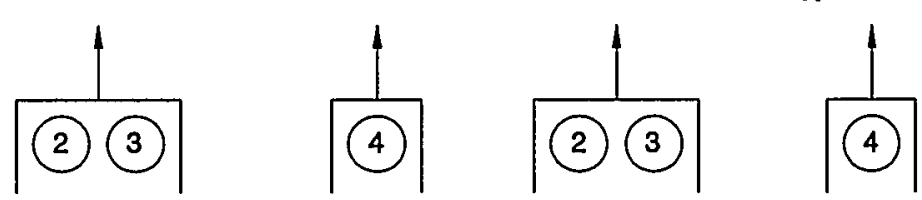

ACTUATION

Bistable

$\cdots$

SOURCES OF UNCERTAINTY
(1) Process Measurement Effects
(2) Instrument Uncertainty
(3) Calibration Uncertainty
(4) Other Uncertainties (IR, leadwire effects, etc.)

Reference: ISA-RP67.04, Part II, The International Society for Measurement and Control

(formerly Instrument Society of America ISA), Reaserch Triangle Park, North Carolina, Sept. 1994.

Figure 4.2 A General View of Components of an Instrument Channel and Associated Uncertainties. 
apply to each component of the channel. This is followed by Figure 4.3 which shows the activities that are practiced in nuclear power plants to verify the performance of instrument channels. The number of components that are covered by each activity is also shown in Figure 4.3. Besides surveillance or functional tests and full channel calibrations which were mentioned earlier, these activities include channel checks that are performed once every shift on the instrument channels which feed the Reactor Trip Systems and the Engineered Safety Feature Actuation Systems. A channel check is performed visually by comparing the outputs of redundant and related instruments on panel meters or other indicators to ensure that they are within reasonable agreement. A channel check covers the entire instrument channel (except for the actuation system) including the sensor.

The components of an instrument channel that can be included in on-line calibration monitoring are also shown in Figure 4.3. Note that virtually all components of an instrument channel can be monitored together for drift except for the actuation system whose performance is verified by functional testing.

Table 4.1 provides a listing of typical measurement uncertainties of an instrument channel in a nuclear power plant. ${ }^{(5)}$ Because of these and other uncertainties, conservative limits are assigned to critical process parameters to ensure safety. Figure 4.4 presents a relative view of these limits.

The "safety limit" shown in Figure 4.4 is an extreme that must not be exceeded to preserve the integrity of the plant. To ensure that a process parameter will not exceed the safety limit, a conservative limit called "analytical limit" as shown in Figure 4.4 is established by the plant safety analysis. To avoid exceeding the analytical limit, the plant is automatically tripped if certain safety-related process parameters exceed the trip setpoint shown in Figure 4.4. The trip setpoint is specified in the plant technical specifications based on the uncertainties associated with measurement of a critical process parameter. If the parameter can be measured with a small uncertainty, then the trip setpoint is set farther from the normal operating point. This leaves a larger operating margin and reduces the potential for spurious trips. If on the other hand, the process measurement uncertainty is large, then the trip setpoint is set closer to the normal operating point, leaving a smaller operating margin. 
AMS-DWG CRSOZOA

Full Channel Calibration (Performed Once Every Fuel Cycle at Each Refueling Outage)

Channel Chocks (Performed Daily)

On-Line Calibration Monitoring (To Be Performed Continuously While the Plant is On-Line and During Startups, Shutdowns, and Plant Trips) $\longrightarrow$

\begin{tabular}{|c|c|c|c|c|}
\hline \multirow{2}{*}{ PROCESS } & PROCESS & SIGNAL & $\begin{array}{c}\text { SIGNAL } \\
\text { CONDITIONING }\end{array}$ & \multirow{2}{*}{ ACTUATION } \\
\hline & MEASUREMENT & (IN THE FIELD) & $\begin{array}{c}\text { (IN INSTRUMENT } \\
\text { CABINETS) }\end{array}$ & \\
\hline
\end{tabular}

Figure 4.3 Illustration of Activities to Verify the Performance of an Instrument Channel 


\section{TABLE 4.1}

Typical Uncertainties of an Instrument Channel in a Nuclear Power Plant

\section{Calibration Uncertainties}

- Calibration standard

- Calibration equipment

- Calibration method

\section{Normal Operation Uncertainties}

- Reference accuracy

- linearity

- hysteresis

- dead band

- repeatability

- Power supply voltage changes

- Power supply frequency changes

- Temperature changes
- Humidity changes

- Pressure changes

- In-service vibration

- Radiation exposure

- Analog-to-digital (A-D) conversion

- Digital-to-analog (D-A) conversion

\section{Instrument Drift}

\section{Instrument Uncertainties Caused by Design Basis Events}

- Temperature effects

- Radiation effects

- Seismic/vibration effects

\section{Process Dependent Effects}

- Fluid stratification

- Process oscillations

- Fluid density effects

- etc.

\section{Other Effects}

- Calculation effects

- Dynamic effects

- Calibration and installation biases 
Safety Limit

Analytical Limit

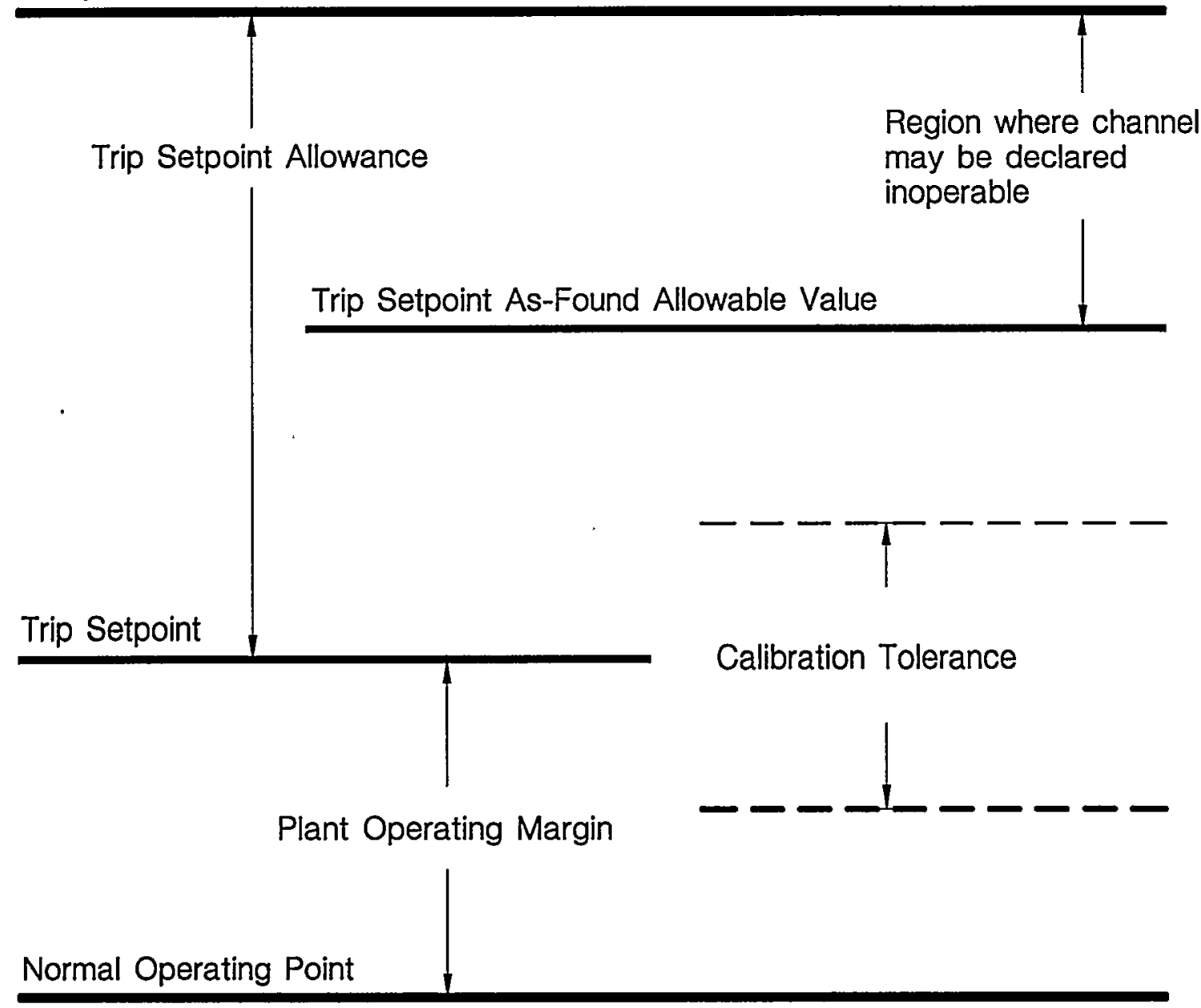

Figure 4.4 Relative Location of Typical Operating Limits in Nuclear Power Plants 


\section{PROJECT GOAL AND EMPHASIS}

The goal of this project was to develop and validate new equipment and techniques for online testing of calibration of safety-related instrumentation channels in nuclear power plants. Although these developments can be applied to any process instrumentation channel, the project emphasis was mainly on pressure transmitters that are located in the reactor containment or other radiation or hazardous areas of the plant. Other sensors such as RTDs, thermocouples, or neutron sensors are either not subject to regular calibration requirements or are in-situ calibrated by other established procedures such as the cross calibration method used for temperature sensors. Furthermore, although the on-line calibration techniques can include other components of instrument channels in addition to the sensors, the project emphasis was more on the sensors. This is because the balance of an instrument channel are usually located in instiument cabinets in mild environments of the plant, are easily accessible even while the plant is operating, and are not as difficult to calibrate as the sensors.

Searches of the License Event Report (LER) and Nuclear Plant Reliability Data System (NPRDS) databases as well as an informal survey of the nuclear power industry performed by AMS for the NRC under this and other projects have revealed that less than ten percent of pressure transmitters in nuclear power plants have been found in the past to drift out of tolerance. Figures 5.1 and 5.2 present the results of searches of the LER and NPRDS databases. The NPRDS showed 2089 reports of failures in about fifteen years or 139 per year, while the LER database showed 391 reports in 12 years or 33 per year. This amounts to about 2.8 failures per plant per fuel cycle from the NPRDS database and 0.66 failures per plant per fuel cycle from the LER database assuming 100 reactors and two-year fuel cycles. Of course, the actual number of failures is higher because LER and NPRDS databases do not include all failures.

Assuming that there are 50 safety-related pressure transmitters in a nuclear power plant, the LER database shows 1.32 percent failures per plant per fuel cycle while NPRDS shows 5.6 percent failures. The difference between the results of the LER and NPRDS is probably due to the differences in reporting requirements for the two databases. For example, since 1983 when the LER reporting requirements were changed by the NRC, single event failures are not reported in LERs. In any event, as indicated above, these results and other information gathered in the course of this and other research projects have indicated less than 10 percent failure for pressure transmitters per plant per fuel cycle. Therefore, by identifying the transmitters which have drifted out of tolerance, an on-line monitoring system has the potential to save a majority of the efforts that are currently spent on manual calibrations of pressure transmitters in nuclear power plants. This can provide substantial cost savings to utilities including direct reductions in labor, personnel radiation exposure, and material and indirect savings from increased instrument reliability and plant safety, simplified outage planning and scheduling, etc. 


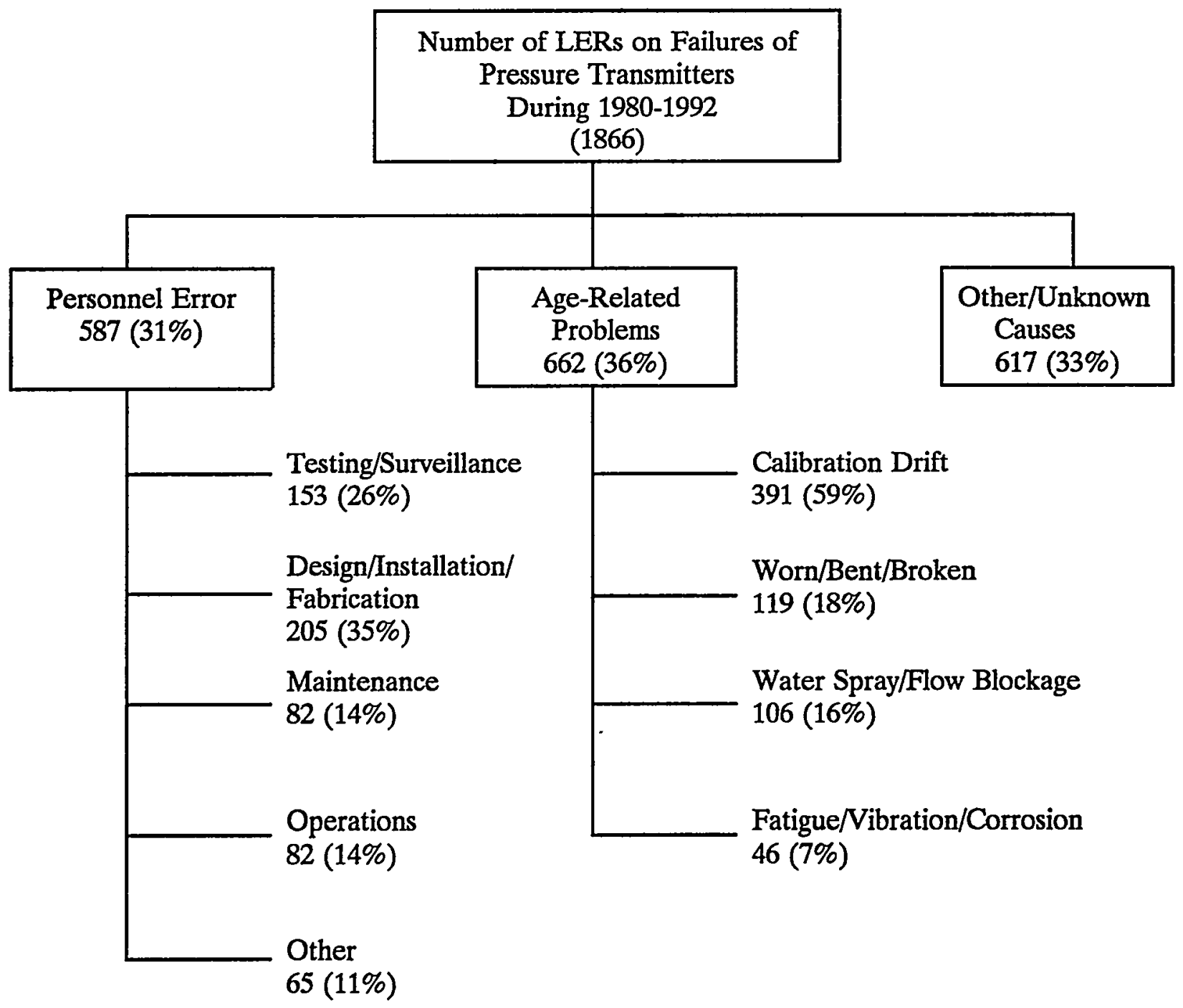

Figure 5.1 Summary of Search of LER Database 


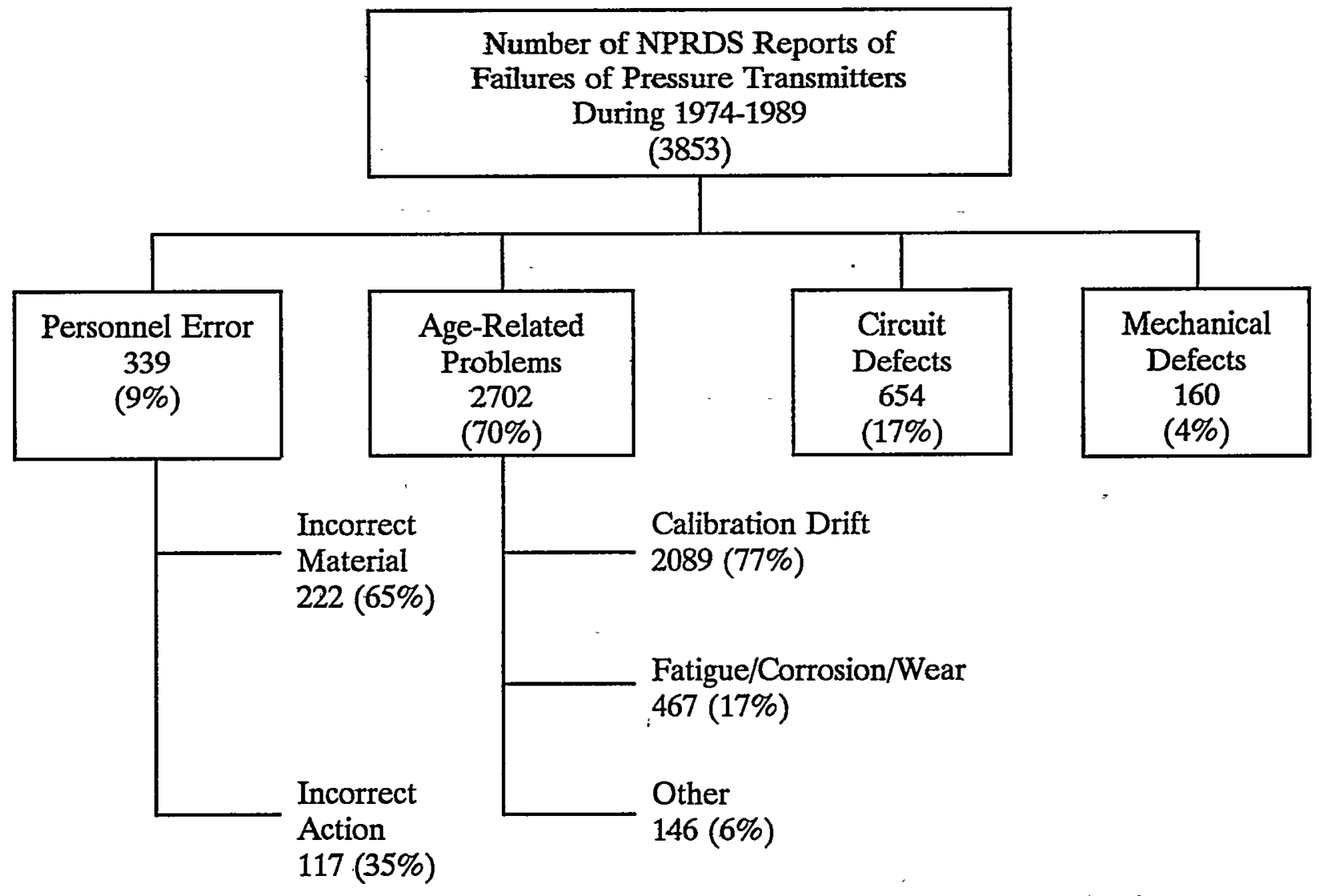

Figure 5.2 Summary of Search of NPRDS Database 


\section{PRINCIPLE OF ON-LINE CALIBRATION TESTING}

On-line testing of calibration of process instrumentation channels in nuclear power plants involves a simple procedure: monitor the steady-state output of each channel on a periodic or continuous basis throughout each fuel cycle and determine if the channel is drifting beyond an acceptable limit. Figure 6.1 illustrates the potential outcome of such a test. The channel may show no drift with respect to the process parameter being monitored, a bias error, or an upward or downward drift. There are other possibilities, but a simple view is presented here to help illustrate the principle of the test.

If the process parameter that is being monitored (e.g., pressurizer pressure, steam generator level, reactor coolant flow) does not remain reasonably stable throughout the on-line monitoring period, it can mask the instrument drift and invalidate the results of the on-line monitoring tests. Figure 6.2 illustrates a situation in which a process parameter is shown to drift upward and the instrument channel to drift downward at the same rate, thereby causing the channel output to show no drift. This simple example illustrates that a means must be established to track the process and help distinguish between process drift and instrument drift.

Several options are available for tracking a process parameter. These options are (Figure 6.3):

1. Averaging of redundant channels

2. Process analytical modeling

\section{Using a calibrated reference channel}

These options can be used individually or together to obtain a "best estimate" of a process parameter on an on-going basis (Figure 6.4). The data qualification module shown in Figure 6.4 screens the data for extraneous spikes, noise, discontinuities and other artifacts.

If a large number of redundant channels are available, then a simple average of the redundant readings can provide a reasonable estimate of the process to be used to verify the calibration of individual instruments. This is the case for the primary coolant RTDs and core exit thermocouples in PWRs. At isothermal conditions at the end of a refueling outage while the plant is heating up toward power operation, there are often 10 to 40 primary coolant RTD elements and about 40 to 70 core exit thermocouples in PWRs that are exposed to essentially the same temperature. Chapter 7 describes how these highly redundant temperature sensors are in-situ calibrated by a single scan of their readings and a simple averaging procedure.

If the number of redundant channels is limited, a single scan and a simple averaging of instrument outputs cannot provide a reasonable estimate of the process. This is the case for most nuclear plant pressure transmitters. Therefore, in addition to averaging, a calibrated reference channel or analytical techniques should be used to characterize the process as described in Chapter 8. 

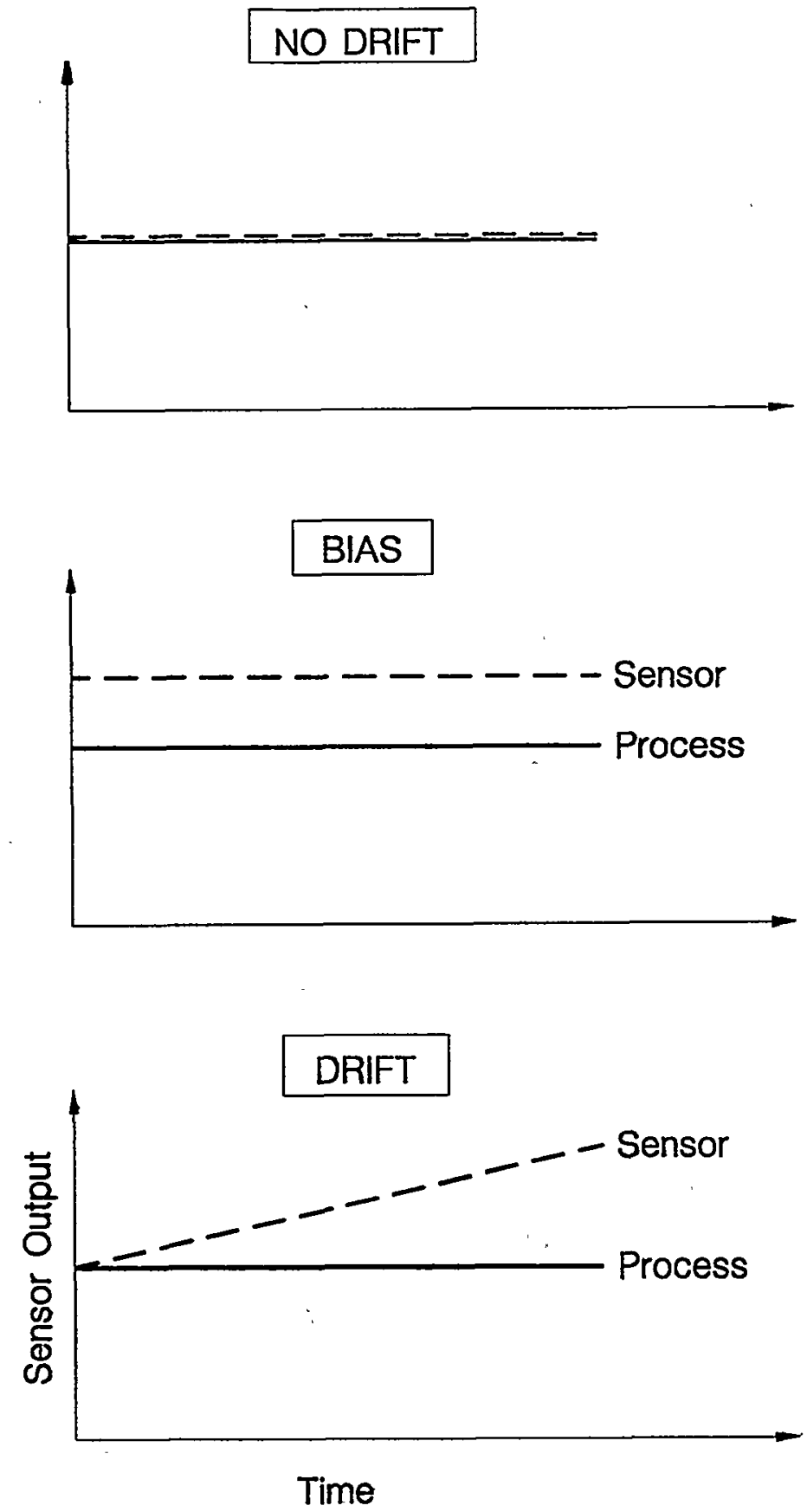

Figure 6.1 Illustration of Potential Outcome of an On-Line Monitoring Test 

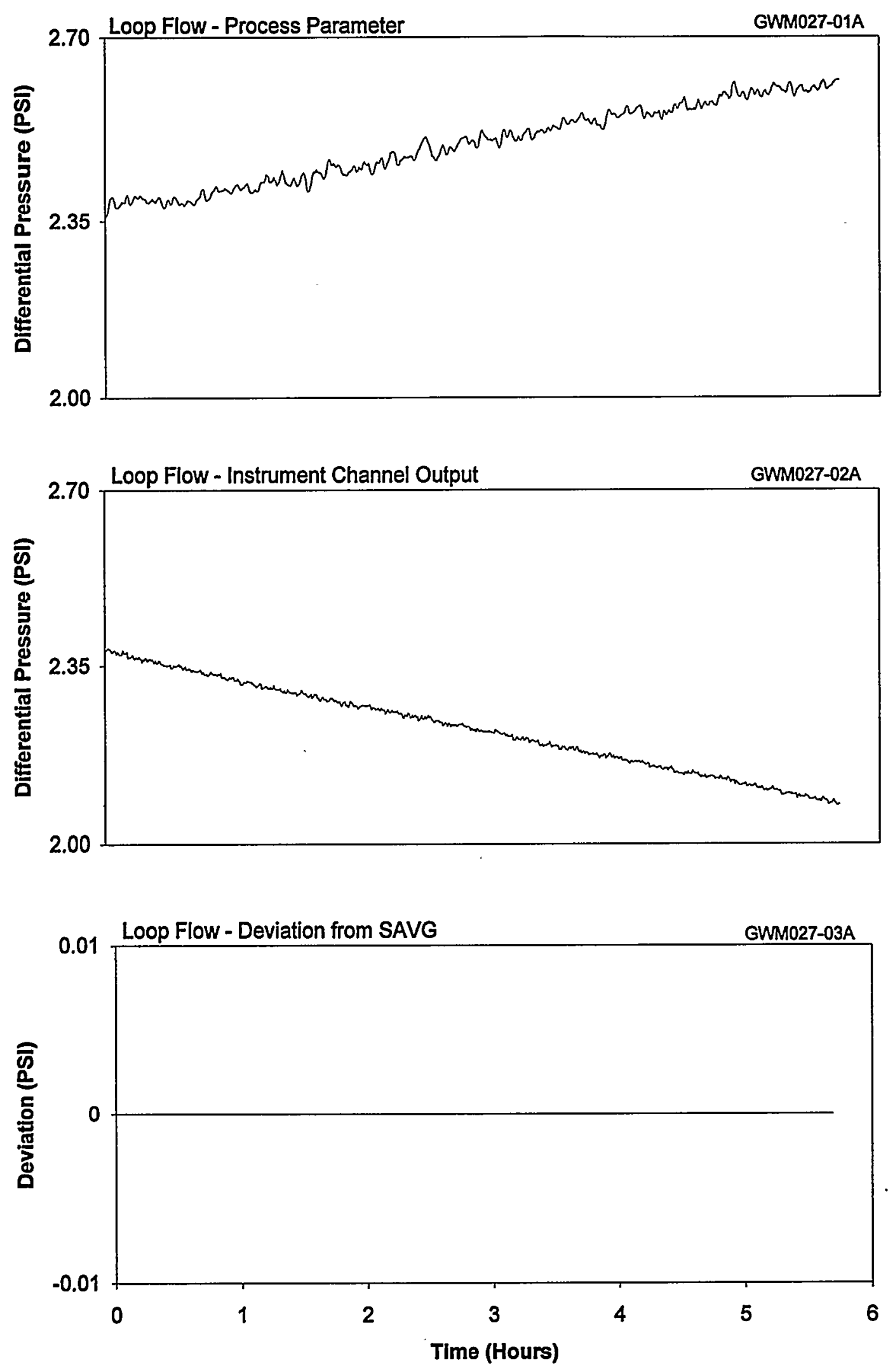

Figure 6.2 Effect of Process Drift on Instrument Drift 

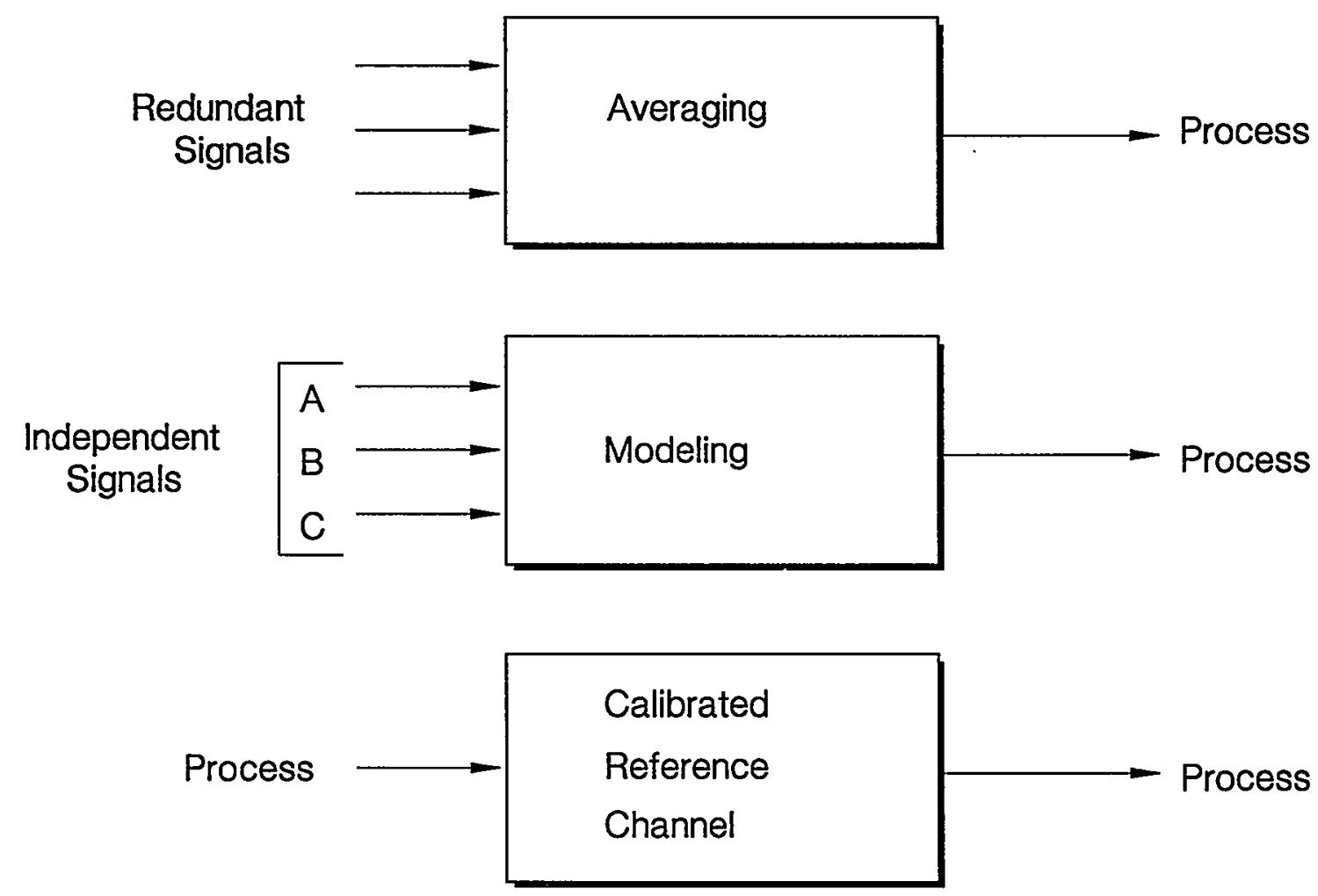

Figure 6.3 Process Estimation Techniques 


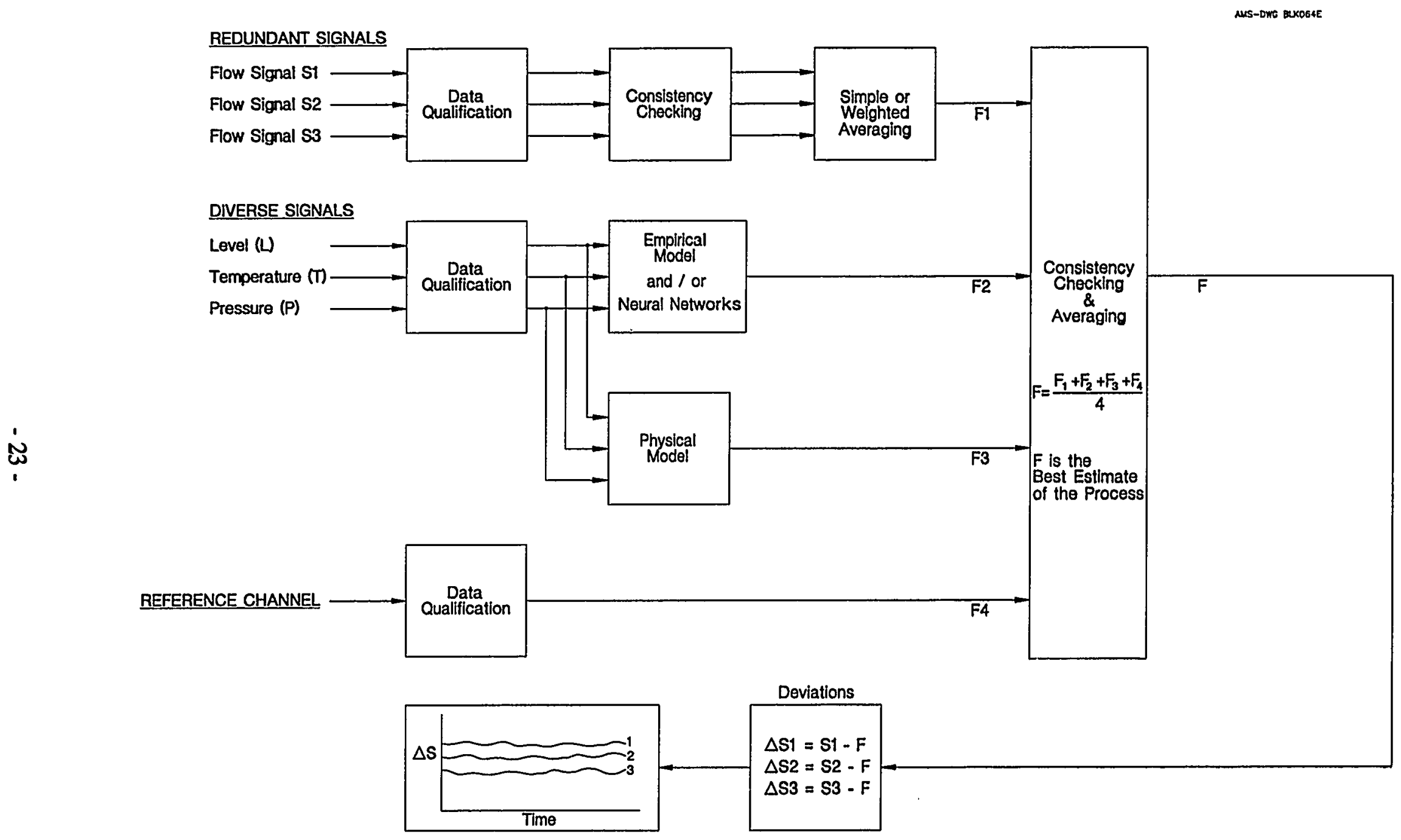

Figure 6.4 Process Estimation Techniques Used Together 


\section{IN-SITU CALIBRATION OF TEMPERATURE SENSORS}

On-line testing of calibration of process instrumentation channels in nuclear power plants requires a means to track the process to distinguish between process drift and instrument drift and account for commonmode effects. However, this effort may not be needed when a large number of redundant instruments are available. A simple average of redundant readings can usually provide a reasonable estimate of the process to serve as the basis for verifying the calibration of the individual instruments. Referred to as the cross calibration test, this approach is successfully used in PWRs for testing the calibration of primary coolant RTDs and core exit thermocouples (CETs) while they remain installed in the plant (in-situ testing).

Cross calibration tests are performed at isothermal conditions either at the end of a fuel cycle while the plant is proceeding toward cold shutdown or at the end of a refueling outage during plant heatup toward power operation. At isothermal conditions, all primary coolant RTDs and CETs can be assumed to be exposed to the same temperature. The temperature readings of the narrow-range RTDs are then averaged and the average temperature is assumed to represent the "true" temperature of the process. The deviation of each sensor from this average is then identified and compared against an acceptance criteria. A typical acceptance criteria for narrow-range RTDs in the primary coolant system of PWRs is $\pm 0.5^{\circ} \mathrm{F}$. The acceptance criteria for wide-range RTDs and CETs are normally less stringent than narrowrange RTDs. For example, wide-range RTDs are often expected to have deviations of less than $\pm 2^{\circ} \mathrm{F}$, and CETs are generally expected to meet $\mathrm{a} \pm 5^{\circ} \mathrm{F}$ requirement.

Table 7.1 shows typical results of a cross calibration test of twenty-four narrow-range
RTDs. The first column identifies the number of RTDs that were tested followed by four columns of repeated temperature measurements for each RTD. The temperature is obtained by measuring the RTD resistance and converting it to equivalent temperature using the most recent calibration table for the RTD. The four temperatures given in each row are averaged and listed in column six. The temperatures in column six are then averaged and this average is subtracted from the temperature indication of each RTD. The results are recorded in the last column as the RTD deviation. This column represents the results of the cross calibration test. The test can be repeated at several temperatures during plant heatup or cooldown to verify the calibration of the RTDs over a wide temperature range. Furthermore, the resistance-versus-temperature data at three or more widely spaced temperatures obtained during plant heatup can be used to generate new calibration tables for any outliers; provided that the number of outliers is small compared to the number of RTDs that are cross calibrated. This effort amounts to in-situ recalibration of those RTDs which do not meet the acceptance criteria.

The wide-range RTDs and CETs are typically calibrated using the average temperature indicated by the narrow-range RTDs. This is because narrow-range RTDs in nuclear power plants are usually subject to more stringent performance requirements than wide-range RTDs and CETs, and are therefore more accurate. Tables 7.2 and 7.3 show cross calibration test results for a number of widerange RTDs and CETs in a PWR.

These sensors were scanned along with the narrow-range RTDs, but were not used in obtaining a best estimate for the process temperature. The data for the wide-range 


\section{TABLE 7.1}

Typical Cross Calibration Results for a Group of Narrow-Range RTDs

\begin{tabular}{|c|c|c|c|c|c|c|}
\hline \multirow{2}{*}{$\begin{array}{l}\text { Item } \\
\text { Number }\end{array}$} & \multicolumn{4}{|c|}{ Temperature $(\%)$ T } & \multirow{2}{*}{$\begin{array}{l}\text { Average } \\
\text { Témp. ( } \mathrm{F})\end{array}$} & \multirow{2}{*}{$\begin{array}{l}\text { Devlation } \\
\text { AT (oF) }\end{array}$} \\
\hline & Pass 1 & Pass ? & Pass 3 & Pass 4 & & \\
\hline 1 & 540.5665 & 540.8777 & 540.8827 & 541.1903 & 540.879 & 0.153 \\
\hline 2 & 540.5785 & 540.8797 & 540.8983 & 541.1944 & 540.888 & 0.161 \\
\hline 3 & 540.4254 & 540.7135 & 540.7447 & 541.0252 & 540.727 & 0.001 \\
\hline 4 & 540.4356 & 540.7156 & 540.7589 & 541.0293 & 540.735 & 0.009 \\
\hline 5 & 540.4429 & 540.7162 & 540.7762 & 541.0425 & 540.744 & 0.018 \\
\hline 6 & 540.4592 & 540.7221 & 540.7901 & 541.0480 & 540.755 & 0.029 \\
\hline 7 & 540.3988 & 540.6256 & 540.6724 & 540.9132 & 540.653 & -0.074 \\
\hline 8 & 540.4016 & 540.6221 & 540.6684 & 540.9066 & 540.650 & -0.077 \\
\hline 9 & 540.3969 & 540.6180 & 540.7062 & 540.9279 & 540.662 & -0.064 \\
\hline 10 & 540.4097 & 540.6166 & 540.7113 & 540.9258 & 540.666 & -0.060 \\
\hline 11 & 540.3959 & 540.6010 & 540.7048 & 540.9118 & 540.653 & -0.073 \\
\hline 12 & 540.4047 & 540.5971 & 540.7149 & 540.9048 & 540.655 & -0.071 \\
\hline 13 & 540.4563 & 540.6306 & 540.7449 & 540.9327 & 540.691 & -0.035 \\
\hline 14 & 540.4992 & 540.6619 & 540.7843 & 540.9631 & 540.727 & 0.001 \\
\hline 15 & 540.5001 & 540.6587 & 540.7907 & 540.9912 & 540.735 & 0.009 \\
\hline 16 & 540.5072 & 540.6517 & 540.7943 & 540.9872 & 540.735 & 0.009 \\
\hline 17 & 540.4202 & 540.5598 & 540.7311 & 540.8808 & 540.648 & -0.078 \\
\hline 18 & 540.4475 & 540.5749 & 540.7536 & 540.8906 & 540.667 & -0.060 \\
\hline 19 & 540.7500 & 540.8649 & 541.0600 & 541.1860 & 540.965 & 0.239 \\
\hline 20 & 540.6176 & 540.7188 & 540.9238 & 541.0411 & 540.825 & 0.099 \\
\hline 21 & 540.4661 & 540.5607 & 540.7765 & 540.8786 & 540.670 & -0.056 \\
\hline 22 & 540.4544 & 540.5380 & 540.7601 & 540.8578 & 540.653 & -0.074 \\
\hline 23 & 540.5428 & 540.6097 & 540.8499 & 540.9133 & 540.729 & 0.003 \\
\hline 24 & 540.5397 & 540.5972 & 540.8421 & 540.8961 & 540.719 & -0.008 \\
\hline
\end{tabular}

Average Temperature Indicated by Narrow-Range RTDs: $540.726^{\circ} \mathrm{F}$ 


\section{TABLE 7.2}

Typical Cross Calibration Results for a Group of Wide-Range RTDs

\begin{tabular}{|c|c|c|c|c|c|c|}
\hline \% / lem & $3 ., 4$ & Temp & $\mathrm{res})$ & 1.2 .1 .2 & & Devlation \\
\hline Number & Pass & Pass 2 & Pass 3 & Pass 4 & Temp (of) & AT $(0 \mathrm{~F})$ \\
\hline 1 & 540.0240 & 540.0829 & 540.2940 & 540.3598 & 540.190 & -0.536 \\
\hline 2 & 539.9838 & 540.0231 & 540.2863 & 540.3384 & 540.158 & -0.568 \\
\hline 3 & 540.5137 & 540.5539 & 540.8250 & 540.8563 & 540.687 & -0.039 \\
\hline 4 & 540.2070 & 540.2316 & 540.5115 & 540.5371 & 540.372 & -0.355 \\
\hline 5 & 540.4871 & 540.5021 & 540.7723 & 540.7889 & 540.638 & -0.089 \\
\hline 6 & 540.5501 & 540.5601 & 540.8533 & 540.8639 & 540.707 & -0.019 \\
\hline 7 & 541.3847 & 541.4781 & 541.5195 & 541.5708 & 541.488 & 0.761 \\
\hline 8 & 539.5880 & 539.6675 & 539.7107 & 539.7680 & 539.683 & -1.044 \\
\hline 9 & 540.6260 & 540.6905 & 540.7257 & 540.7779 & 540.705 & -0.022 \\
\hline 10 & 540.1643 & 540.2307 & 540.2730 & 540.3213 & 540.248 & -0.479 \\
\hline 11 & 541.0316 & 541.1171 & 541.1603 & 541.2085 & 541.130 & 0.403 \\
\hline 12 & 541.2609 & 541.3354 & 541.3817 & 541.4330 & 541.353 & 0.626 \\
\hline 13 & 540.6287 & 540.6969 & 540.7425 & 540.7959 & 540.716 & -0.011 \\
\hline 14 & 540.5922 & 540.6750 & 540.7162 & 540.7535 & 540.684 & -0.043 \\
\hline
\end{tabular}

Average Temperature Indicated by Narrow Range RTDs $=540.726^{\circ} \mathrm{F}$ 
TABLE 7.3

Typical Cross Calibration Results for a Group of Core Exit Thermocouples in One of Four Quadrants in a PWR

\begin{tabular}{|c|c|c|c|c|c|c|}
\hline \multirow{2}{*}{ Number } & \multicolumn{3}{|c|}{ ২ Tempexature $(\mathrm{O})$} & अి & \multirow{2}{*}{$\begin{array}{l}\text { Average, } \\
\text { Temp }(\text { f })\end{array}$} & \multirow{2}{*}{$\begin{array}{l}\text { Deviation } \\
\text { AT (of) }\end{array}$} \\
\hline & Pass 1 & pass 2 & Pass 3 & $\mathrm{Pass} 4$ & & \\
\hline 1 & 541.5739 & 541.6448 & 541.7042 & 541.7541 & 541.668 & 0.941 \\
\hline 2 & 542.1380 & 542.1713 & 542.1791 & 542.1897 & 542.169 & 1.442 \\
\hline 3 & 540.8568 & 540.8908 & 540.9758 & 540.9767 & 540.925 & 0.198 \\
\hline 4 & 540.5961 & 540.6512 & 540.6224 & 540.6066 & 540.619 & -0.108 \\
\hline 5 & 540.9023 & 541.0177 & 541.0771 & 541.0526 & 541.013 & 0.286 \\
\hline 6 & 541.5014 & 541.5757 & 541.5861 & 541.5678 & 541.559 & 0.832 \\
\hline 7 & 541.5372 & 541.5915 & 541.6430 & 541.6833 & 541.614 & 0.887 \\
\hline 8 & 563.7292 & 564.8915 & 564.5894 & 564.3734 & 564.395 & 23.668 \\
\hline 9 & 541.6806 & 541.7366 & 541.8014 & 541.7978 & 541.755 & 1.028 \\
\hline 10 & 543.0238 & 543.0947 & 543.1725 & 543.2110 & 543.125 & 2.398 \\
\hline 11 & 542.1756 & 542.2044 & 542.2613 & 542.2998 & 542.235 & 1.508 \\
\hline 12 & 541.0404 & 541.0982 & 541.1462 & 541.2242 & 541.127 & 0.400 \\
\hline 13 & 540.8296 & 540.8507 & 540.9914 & 540.9801 & 540.912 & 0.185 \\
\hline
\end{tabular}

Average Temperature Indicated by Narrow Range RTDs $=540.726^{\circ} \mathrm{F}$ 
RTDs were analyzed using the same procedure as for the narrow-range RTDs. The data for thermocouples were converted from voltage readings to temperature using generic calibration tables available for Type $\mathrm{K}$ thermocouples.

A detailed description of the cross calibration technique is provided in the NUREG/CR-5560 report. ${ }^{(12)}$ This report also includes research results which show that a group of temperature sensors generally drift in a random fashion and common-mode drift is not usually a problem. Previous research had shown that thermal and vibration aging usually cause an RTD's resistance to increase. As such, RTDs have often been suspected of common-mode drift. Although this is generally true for Standard Platinum Resistance Thermometers (SPRTs), industrial RTDs of the types used in nuclear power plants are generally immune from this problem as. indicated by the results in NUREG/CR-5560. The sensing elements (a pure platinum wire) in SPRTs are designed to have unrestricted support as opposed to being secured to a support structure. This helps prevent interaction between the element and the support structure. Under vibration stress, however, the unrestricted element in the SPRT can experience cold working which can result in an increase in resistance. It is for this reason that SPRTs are annealed to relieve the mechanical stress before they are recalibrated. The sensing elements in industrial RTDs are not free to move as in SPRTs. Rather, they are secured to a support structure. As such, the common-mode drift that may occur in SPRTs is not prevalent in industrial RTDs.

The accuracy of the cross calibration results depends on the number of temperature sensors that are cross calibrated, the number of outliers, the accuracy of the sensor calibration tables used to convert the sensor output to equivalent temperature, the accuracy and stability of resistance and voltage measurement equipment, the stability and uniformity of the plant temperature during the tests, the measurement precision, etc. These uncertainties should be identified and statistically combined to assess the accuracy of the cross calibration results.

Another method called "Johnson Noise" has been studied for in-situ calibration of nuclear plant RTDs. ${ }^{(14)}$ This method is based on measurement of a small noise voltage (microvolt) or current (nanoamp) that is produced naturally in any passive resistor such as the sensing element of an RTD. This noise voltage or current is a function of the RTD's resistance and temperature by the following equations:

$$
\begin{gathered}
\bar{V}^{2}=4 k T R \Delta f \\
\bar{I}^{2}=\frac{4 k T \Delta f}{R}
\end{gathered}
$$

where $\mathrm{k}$ is the Boltzmann constant, $\quad \overline{\mathrm{V}}^{2}$ is the mean-squared open-circuit noise voltage in the bandwidth $\Delta \mathrm{f}$ (Hertz) across a resistor of $\mathrm{R}$ (Ohms) at an absolute temperature of $\mathrm{T}$ (Kelvin); and $I$ is the short-circuit noise current. The frequency band $(\Delta f)$ at which the Johnson noise voltage is measured is in the kilohertz $(\mathrm{KHz})$ range; typically about $50 \mathrm{KHz}$.

These equations can be used to identify the absolute resistance or absolute temperature of the RTD element as follows:

$$
T=\left(\sqrt{\bar{V}^{2} I^{2}}\right) / 4 k \Delta f
$$

$$
R=\sqrt{\bar{V}^{2} / \bar{I}^{2}}
$$

The Johnson Noise method requires sophisticated equipment, procedures and expertise to measure the small noise voltage or current at the end of several hundred feet of wire from the field to where the RTD 
extension leads are terminated. As such, the method is not currently used in nuclear power plants especially since the cross calibration method can provide the desired in-situ testing capability with a simple set of equipment and a straightforward procedure: 


\section{ON-LINE CALIBRATION TESTS OF PRESSURE TRANSMITTERS}

Chapter 7 described how redundant temperature sensors are cross calibrated by a single scan of the sensors and simple averaging. When a large number of redundant sensors is not available, a single scan of sensors is not sufficient for calibration testing. The instruments must be scanned repeatedly over a long period of time and their deviations from a best estimate of the process plotted to verify their calibrations based on whether or not they have exhibited a significant or consistent drift.

Except for primary coolant RTDs and core exit thermocouples (CETs) in PWRs, there are usually no more than four redundant sensors in nuclear power plants for most measurements. Therefore, the calibration of these instruments must be tested using a different procedure. The procedure calls for the cross calibration tests to be performed on the instruments on a periodic or continuous basis throughout each fuel cycle, and the deviation of the instruments to be plotted as a function of time and examined for evidence of drift. Figure 8.1 shows typical results of this procedure for four redundant signals from the McGuire nuclear plant. Note that the signals do not show a significant drift, but there are steady-state (bias) differences between the signals. These biases are generally due to normal calibration differences between instruments, different tap locations, etc. The deviation plots can be made to all begin at the same point on the vertical axis by removing the bias differences to show only the drift of the signals. Figure 8.2 shows the drift plot for four McGuire signals with the bias differences removed.

Figure 8.3 shows a raw data and a deviation plot for five signals from laboratory tests including a calibrated reference signal. Note in the raw data that although the five signals commonly drift, the deviation plot shows normal results. If the reference sensor was not included in this test, then it would have been difficult to determine whether the drift was due to the sensors or the process. The sensors could have all been drifting together at the same rate resulting in a deviation plot that only indicates that the sensors agree with each other, but no information to detect any common-mode drift. That is, the deviation plot alone cannot reveal common-mode drift. An independent means is needed to determine any significant drift. A calibrated reference instrument can provide the capability to distinguish between process drift and instrument drift. The reference instrument in the example of Figure 8.3 shows the same drift as the redundant instruments meaning that the common-mode drift is due to the process. For in-plant tests, the reference channel could be one of the redundant channels that is manually calibrated before and after each on-line monitoring period. A good practice would be to calibrate one of the redundant channels on a rotational basis at the end of each fuel cycle so that all channels are manually calibrated after a few fuel cycles whether or not they have drifted.

Analytical modeling of the process can be used in lieu of or in addition to a reference channel to characterize the behavior of the process. Both physical and empirical modeling techniques can be used; however, empirical models are more practical and advantageous than physical models and were therefore used in almost all of the analytical work performed in this project.

Neural networks, which are a form of empirical modeling, can also provide a means to obtain a best estimate of the process to be used for drift monitoring. Figure 8.4 shows empirical modeling and neural network results 


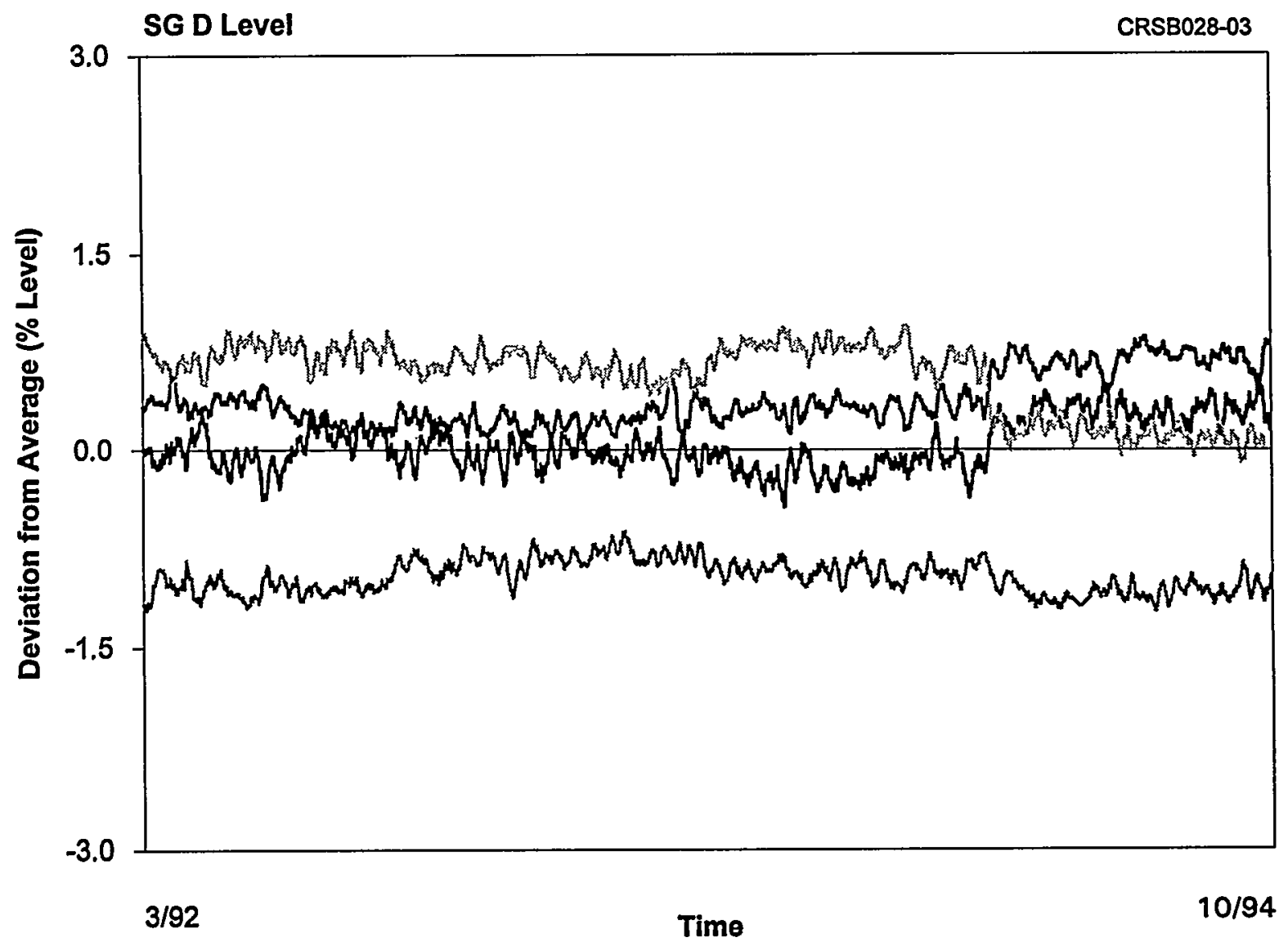

Figure 8.1 Deviation Plot for McGuire Signals 


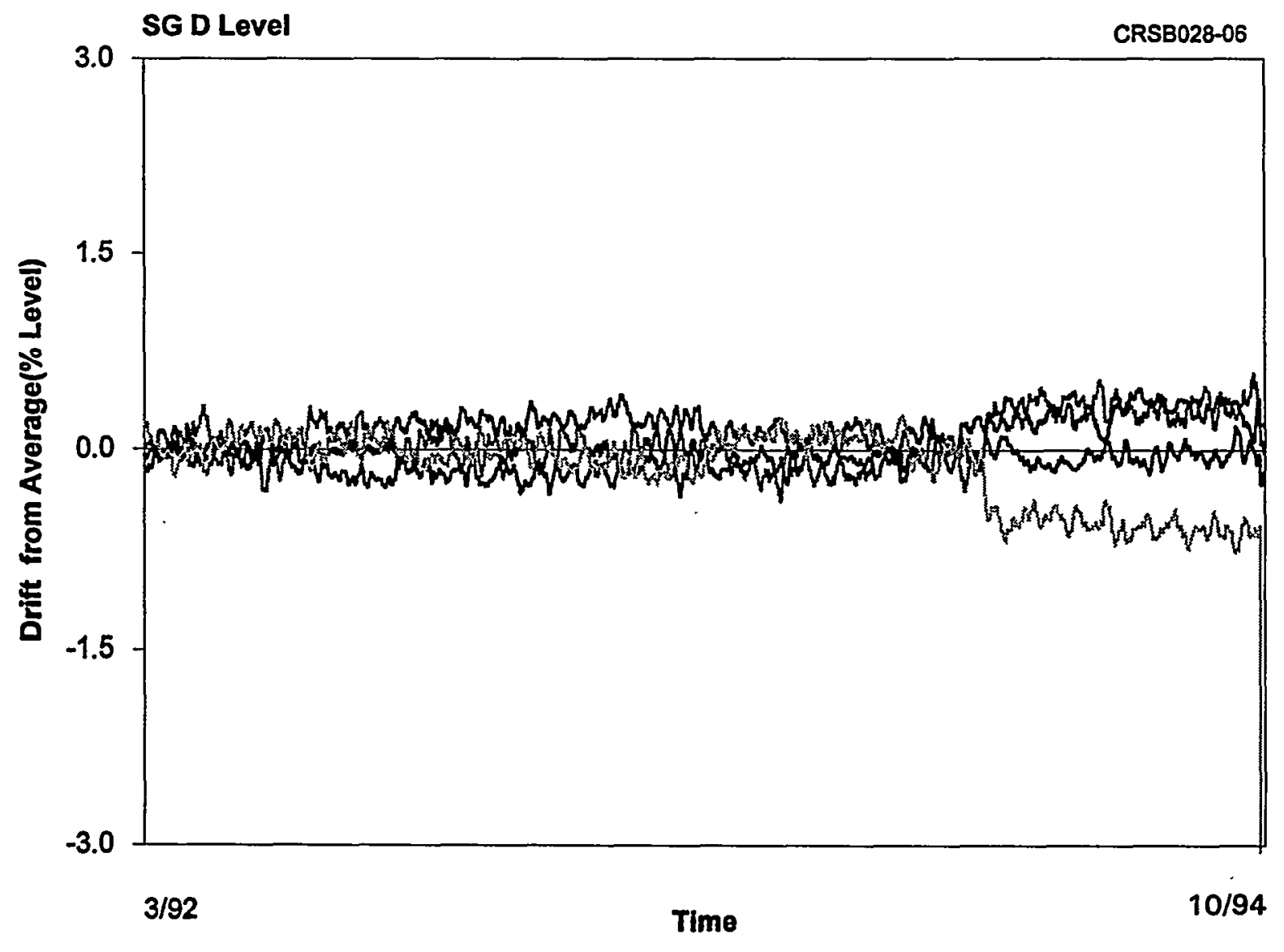

Figure 8.2 Drift Plot for McGuire Signals 

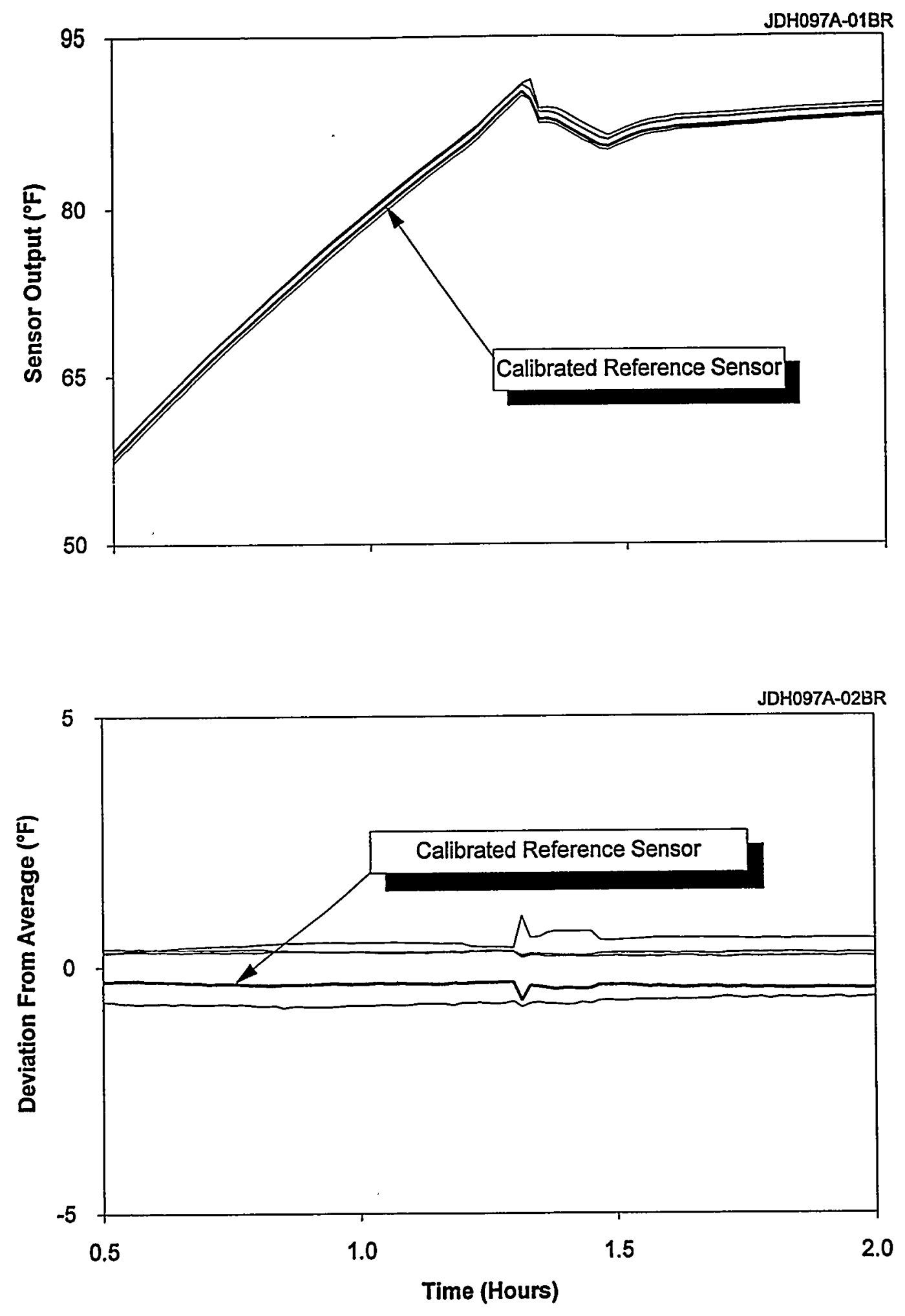

Figure 8.3 Use of Reference Sensor to Track the Process 


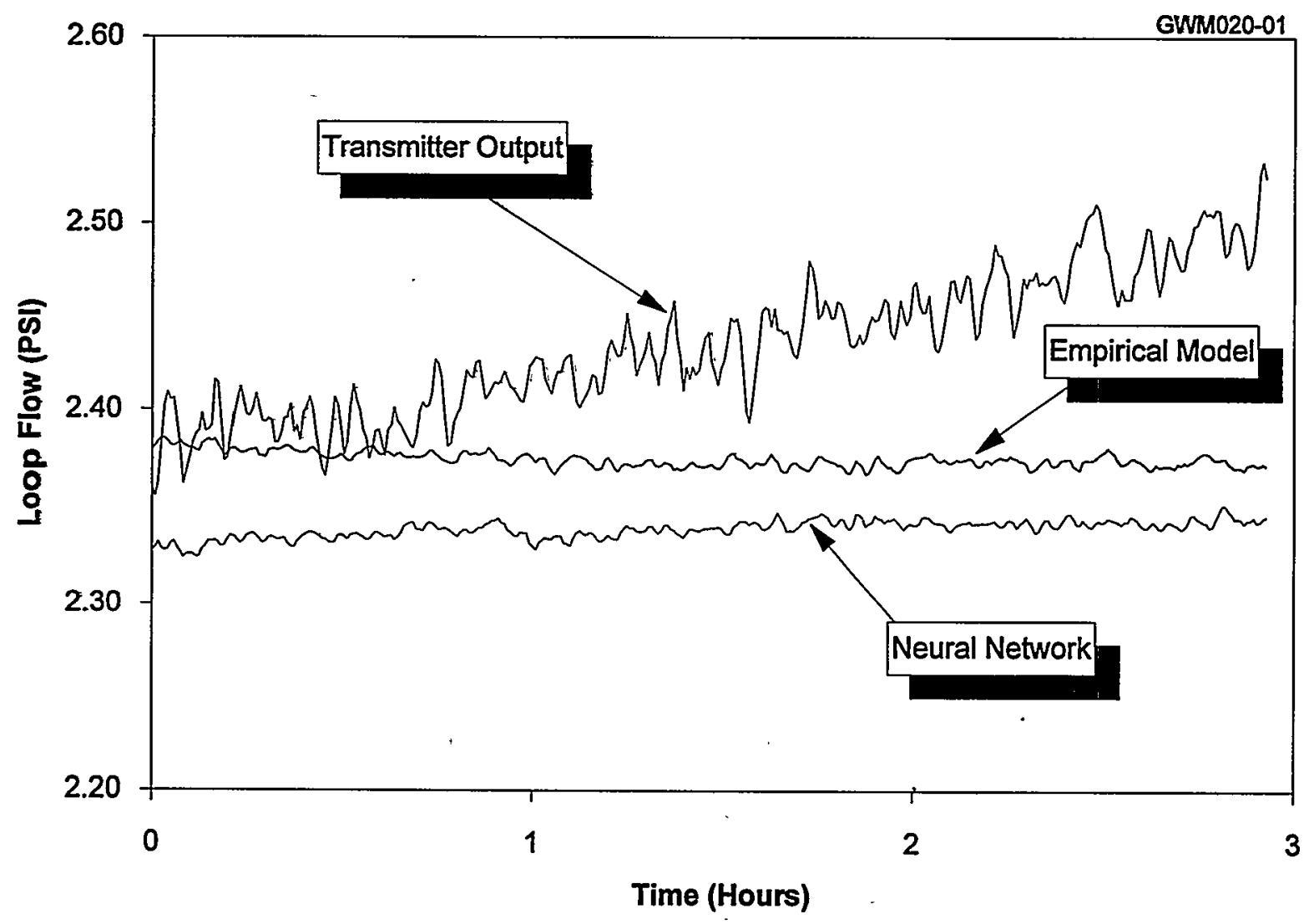

Figure 8.4 Process Estimating Using Empirical Modeling and a Neural Network to Identify Drift in a Single Instrument 
for laboratory data which involved a drifting sensor. As shown in this figure, the empirical model and neural network techniques estimated the process as being stable. Therefore, the drift observed here is due to the sensor and not the process. This example shows that analytical techniques such as empirical modeling and neural networks can help identify drift in even a single sensor or instrument channel. If redundant instruments are also available, a good approach is to estimate the process by averaging the redundant instruments, empirical modeling, neural networks, and other valid process estimation techniques. The results of these techniques can then be averaged together as was shown earlier in Chapter 6 to provide a "best estimate" for the process.

Figure 8.5 shows an example of process estimation results from simple and weighted averaging, empirical modeling, and neural networks. These results are from three years of on-line monitoring data collected at the McGuire nuclear plant over two consecutive fuel cycles. The process estimation results in Figure 8.5 are reasonably close to one another, and an average of the four traces could provide a "best estimate" for the steam generator level process.

A description of empirical models, neural networks, and other analytical techniques for process estimation and on-line drift monitoring is presented in Chapter 9. In Chapter 15, laboratory validation results are shown in which redundant signals were artificially drifted one at a time to demonstrate how simple and weighted averaging as well as empirical and neural network modeling can identify drift. 


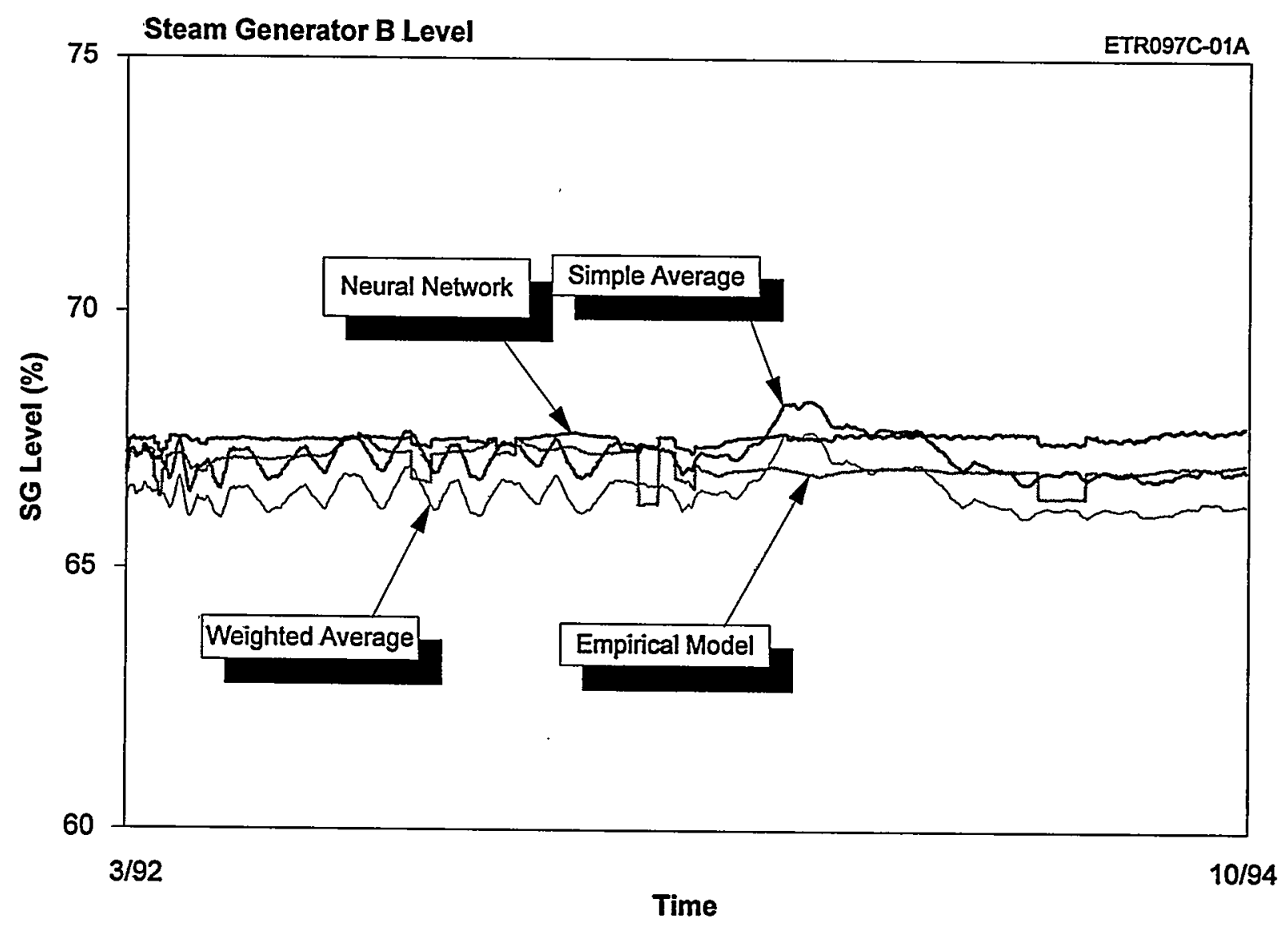

Figure 8.5 Estimation of Steam Generator Level Process at McGuire Using Four Different Process Estimation Techniques 


\section{PROCESS ESTIMATION TECHNIQUES}

During the last fifteen years, a number of advanced signal processing methods have been developed for a variety of applications including on-line monitoring for instrument calibration verification in nuclear power plants. The methods that may be used for on-line calibration monitoring are summarized in this chapter, and the details of some of these methods are presented in Appendices A through E.

\subsection{Pattern Recognition}

This method is used to identify the deviations of signals from their normal or historical behavior. It is a valuable tool in processes where there is a large database from which to develop the baseline behavior or normal pattern of the process. The method does not depend on analytical models. Rather, it uses matrix manipulation of a large number of signals to establish "patterns" representing best fits to previous operations. A list of signals which have a relationship to the signal of interest is used to establish the baseline patterns.

The first use of pattern recognition in a nuclear facility occurred at the Experimental Breeder Reactor (EBR-II) in Idaho to provide estimates of the reactor power level, reactor outlet temperature, etc. ${ }^{(15)}$ Once the parameters of interest are chosen, the next step is to select periods of reactor operation that are typical for the plant conditions of interest. These periods of operation are used to "teach" the system what to expect from each of the signals involved. For example, to estimate the power level at EBR-II, 129 signals were used in learning the necessary patterns. If any of the related signals exhibited unusual behavior relative to the others, its behavior was flagged. For example, when a reactor coolant outlet temperature sensor at EBR-II drifted, giving an incorrect reading, the pattern recognition method was able to predict the temperature with a reasonable accuracy, as shown in Figures 9.1 and 9.2.

\subsection{Parity Space}

The parity space technique is one of several methods that can be used to determine the consistency of redundant signals and identify weighing factors based on the consistency of the signals. References 1 and 2 provide detailed descriptions of the parity space technique and how the technique may be used to identify faulty signals.

\subsection{Generalized Consistency Checking}

Like the parity space method, the Generalized Consistency Checking (GCC) method is used to track redundant signals and identify inconsistencies. ${ }^{(9)}$ An inconsistency counter is employed in the GCC method to record the number of times that a signal is found to be inconsistent. After excluding the signals with maximum inconsistency indices, the best estimate of the process at any time is computed as a weighted average of the remaining signals. The procedure is illustrated in Figure 9.3. Three signals are compared two at a time to determine if their differences lie within a pre-determined acceptance criteria. If the acceptance criteria is satisfied, then the two signals are said to be consistent. Otherwise, the signals are inconsistent and must be compared with other signals to determine which of the two signals is inconsistent. Alternatively, analytical models, as described below, can be used to track the 


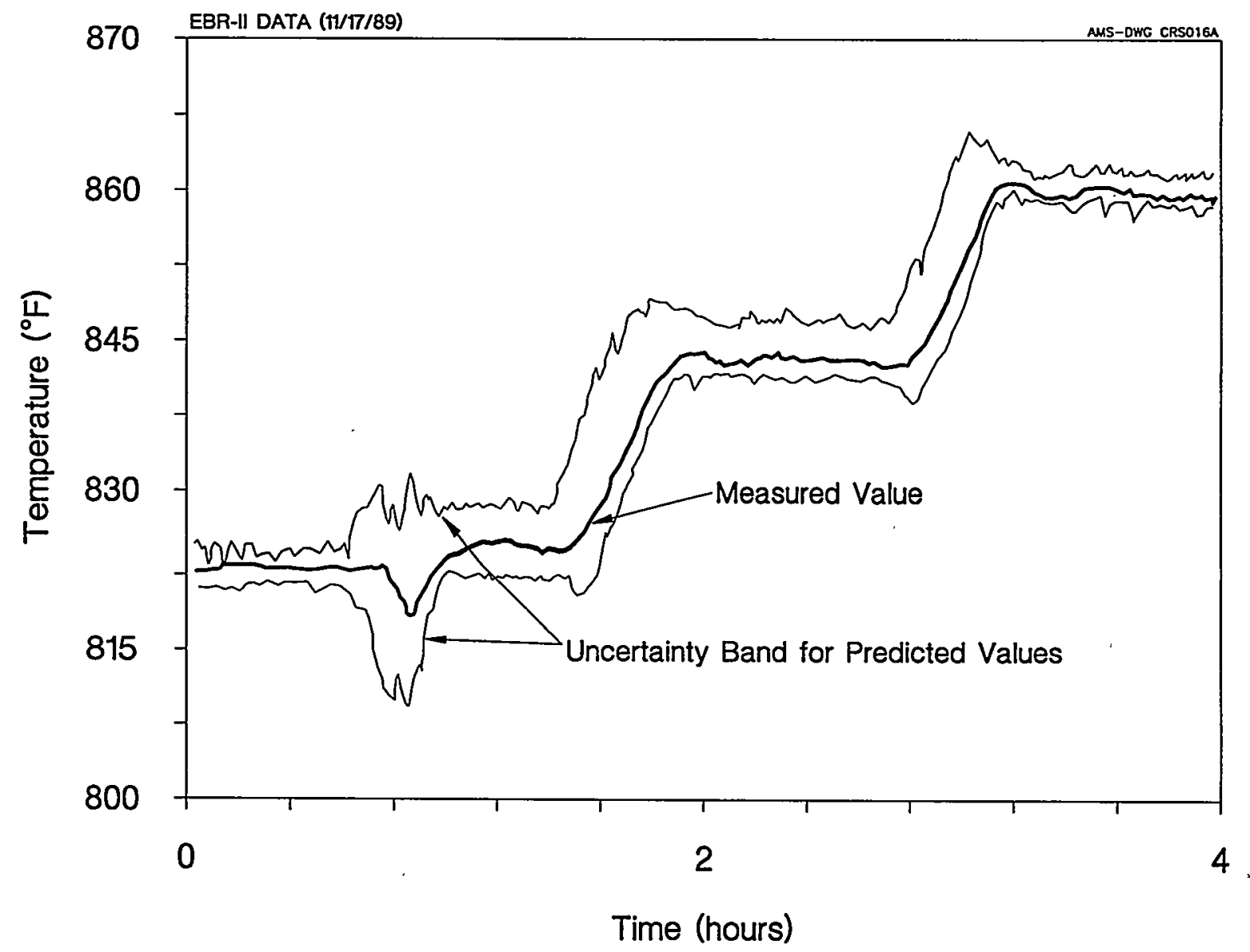

Figure 9.1 Analytical Estimation of EBR-II Temperature by Pattern Recognition (reproduced from a figure given in reference 15) 


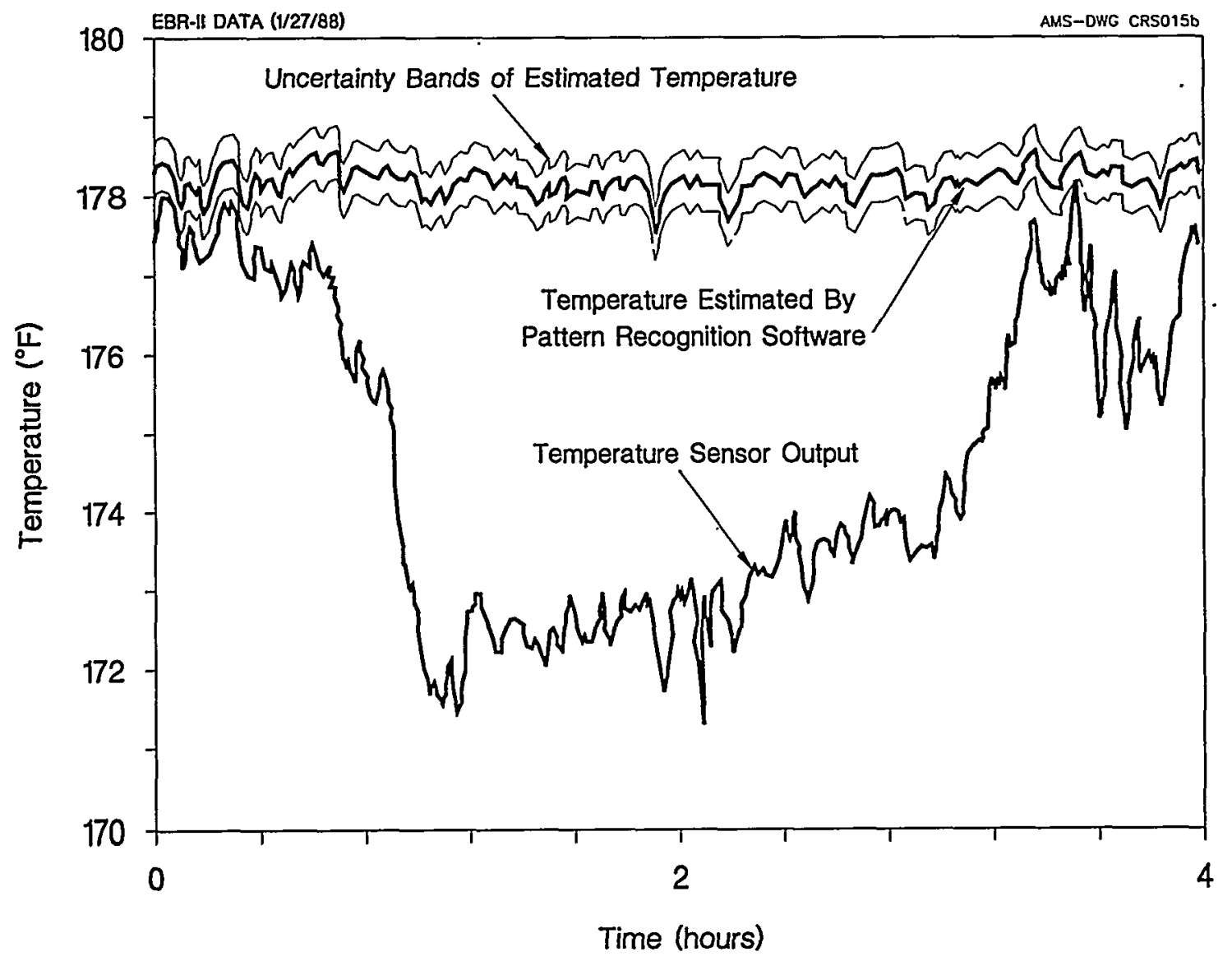

Figure 9.2 Detection of Faulty Temperature Sensor at EBR-II Using Pattern Recognition (reproduced from a figure given in reference 15) 

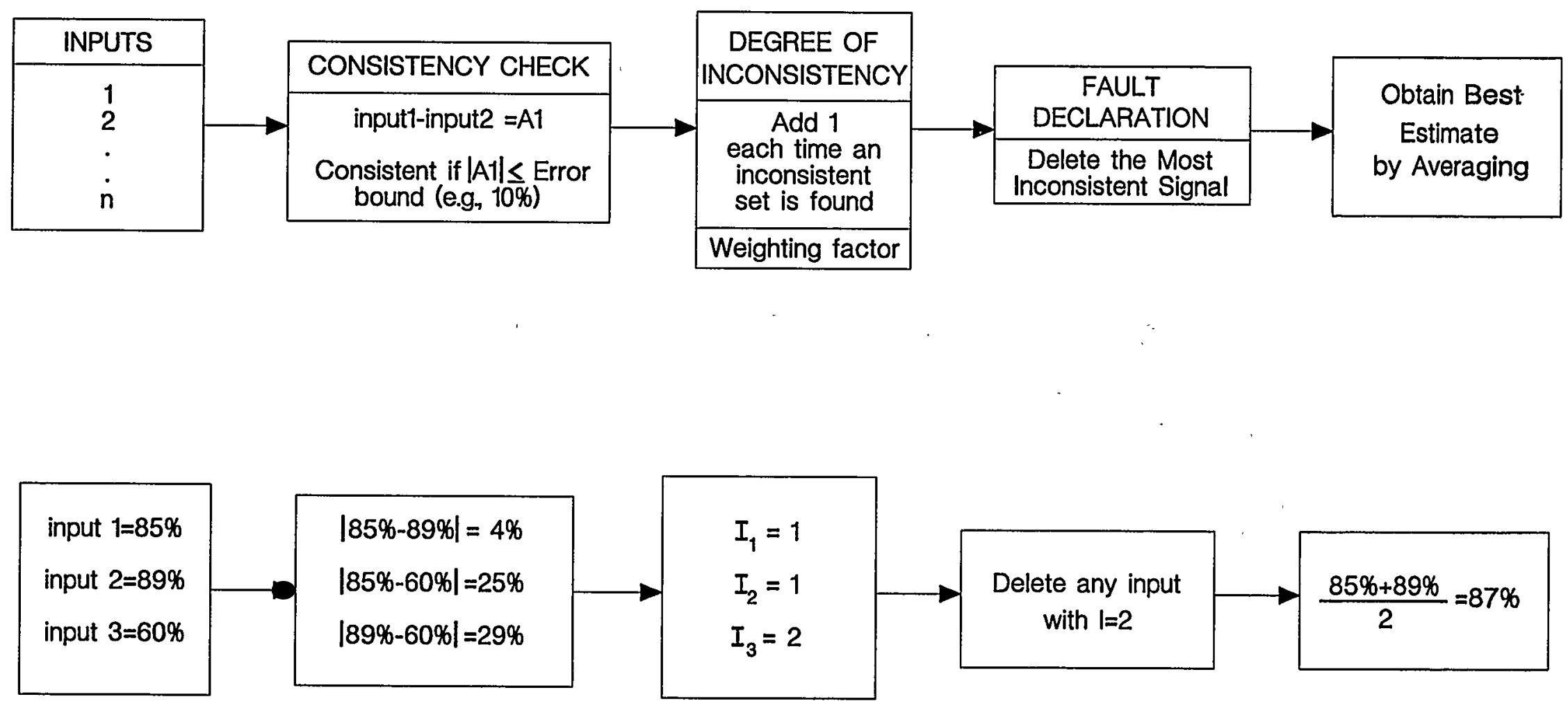

Figure 9.3 Illustration of Principle of Parity Space Technique 
process and compare the individual signals with the process estimate to identify the inconsistent signals.

Figure 9.4 shows two examples of inconsistent signals which were identified and flagged in laboratory demonstration tests of the GCC method.

\subsection{Analytical Redundancy}

Analytical redundancy is the general term for most techniques which combine equations and operating data to characterize the behavior of a process. As such, empirical and physical modeling, pattern recognition, neural networks, and other mathematical techniques are subsets of analytical redundancy.

Analytical redundancy is a term that is used as opposed to hardware redundancy. When a sufficient number of redundant channels are not available to be intercompared, hardware redundancy is said to be inadequate and analytical techniques are thus used to create redundant signals. The word "analytical" is used because the method creates new signals from results of calculations using data-driven physical or empirical models. A model is a set of mathematical equations that are developed based on the laws of physics (physical model) or experience with the process (empirical model). Models are used to calculate a process parameter from measurements of other parameters that have a relationship with the process parameter of interest.

In addition to instrument calibration verification in nuclear power plants, the analytical redundancy approach has been used in the development of "fault tolerant" systems so that signal loss due to an instrument failure cannot have a significant effect on the process operation. The lost signal is replaced by a calculated signal that is obtained from a model.

The constants in physical models are identified from material properties, dimensions, and other known characteristics of the plant. The constants of empirical models, on the other hand, are identified by fitting the operational plant data to the empirical model. As such, these and most other analytical modeling techniques are somewhat specific to the process for which they are developed. Also, the analytical techniques are generally dependent on the operating state of the process and the same model that is designed and optimized for an operating condition may not be suitable for other operating regimes. $A$ description of physical and empirical models and neural networks follows.

\subsubsection{Physical Models}

In closed loop systems such as nuclear power plants, many process parameters are related to one another. Therefore, each parameter can usually be estimated from measurement of other parameters. A simple example is from BWRs in which the reactor coolant temperature can be determined from a measurement of pressure and vice versa. Similarly, in a PWR, steam temperature can be estimated from measurement of steam pressure. In these examples, the physical model is a simple thermodynamic relationship between two parameters. However, most process parameters cannot be estimated from a simple relationship and a single signal. Two or more signals and several relationships are often needed to relate the parameter of interest to the measured signals. An example of a physical model that may be used for on-line calibration monitoring is shown by Equation 9.1 on the next page.

Equation 9.1 represents one of many differential equations that must be solved to model a process. For example, a physical model, attempted in this project, for a steam generator involved 24 differential equations and 30 process parameters. As such, physical modeling of nuclear plant parameters requires an extensive amount of analytical work, computing time, and computing power. Thus, 

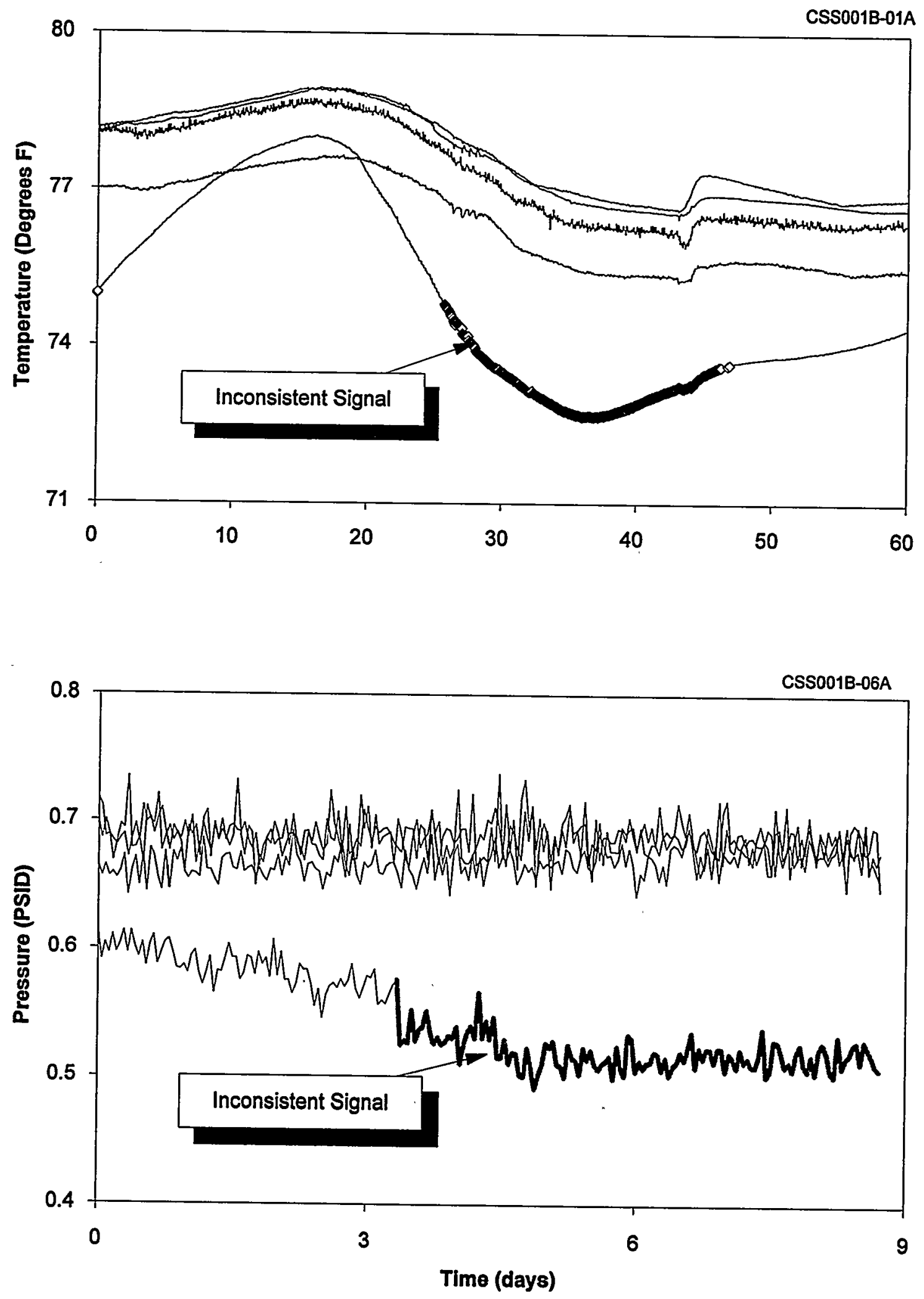

Figure 9.4 Detection of Signal Inconsistencies Using the GCC Method 


$$
\frac{d L_{W}}{d t}=\frac{1}{\rho_{s} A_{p I}}\left[\left(A_{p I}\left(L-L_{W}\right) \frac{\partial \rho_{W}}{\partial P_{p I}}-\frac{A_{p I}\left(L-L_{W}\right) \frac{\rho_{W}}{\rho_{s}} \frac{\partial \rho_{W}}{\partial P_{p I}}+A_{p Y} L_{w} \frac{\partial \rho_{s}}{\partial P_{p I}}}{\frac{\rho_{W}}{\rho_{S}}-1}\right) \frac{d P_{p I}}{d t}+\frac{W_{s I}+W_{c o}}{\frac{\rho_{W}}{\rho_{S}}-1}\right]
$$

where:

$$
\begin{array}{ll}
\mathrm{L}_{w w} & =\text { water level in the pressurizer } \\
\rho_{\mathrm{s}} & =\text { vapor density in the pressurizer } \\
\mathrm{A}_{\mathrm{pr}} & =\text { cross-sectional area of the pressurizer } \\
\mathrm{L} & =\text { effective pressurizer length } \\
\rho_{\mathrm{w}} & =\text { water density in the pressurizer } \\
\mathrm{P}_{\mathrm{pr}} & =\text { pressurizer pressure } \\
\mathrm{W}_{\mathrm{sc}} & =\text { in/out surge flow rate } \\
\mathrm{W}_{\mathrm{co}} & =\text { condensation/evaporation flow rate in } \\
& \text { the pressurizer }
\end{array}
$$

physical models are not generally as convenient as other analytical techniques for estimating a process parameter, and we are therefore not including much test data in this report involving physical models.

\subsubsection{Empirical Models}

Empirical models are linear or nonlinear equations that are written based on assumptions made regarding the form of relationships between the variables of the modeled process. An example of an empirical model that may be used in process estimation for drift monitoring is given below:

$\mathrm{y}=\mathrm{Ax}_{1}^{2}+\mathrm{Bx}_{1}+\mathrm{Cx}_{1} \mathrm{x}_{3}+\mathrm{Dx}_{2}^{2}+\mathrm{Ex}_{2}+\mathrm{F}$

where:

$$
\begin{aligned}
& y=\text { Pressurizer Level } \\
& x_{1}=\text { Pressurizer Pressure } \\
& x_{2}=\text { Hot Leg Temperature } \\
& x_{3}=\text { Reactor Coolant System Pressure }
\end{aligned}
$$

The following are the constants of this equation which were obtained by fitting operational data from the McGuire nuclear plant to Equation 9.2.
$\mathrm{A}=2.81 \times 10^{-4}$
$\mathrm{B}=-1.25$
$\mathrm{C}=-1.34 \times 10^{-6}$
$D=5.03 \times 10^{-4}$
$\mathrm{E}=4.56 \times 10^{-2}$
$\mathrm{F}=1.23 \times 10^{3}$

Figure 9.5 provides a comparison of empirical and physical modeling results for a McGuire pressure signal. The measured pressure signal is also shown in this example indicating that the modeling results are in reasonable agreement with the measurement.

\subsubsection{Neural Networks}

The advantage of empirical models over physical models is that empirical models do not require a knowledge of material properties, geometry, and other characteristics of the plant. They are identified by fitting plant operational data to an equation that represents a plant subsystem and determining the constants of the equation. This fitting process is often referred to as "learning." Neural networks can help facilitate and expedite the learning process involved in empirical modeling.

Neural networks are generic empiriçal models which use iterative fitting techniques to establish the nominal relationships between 


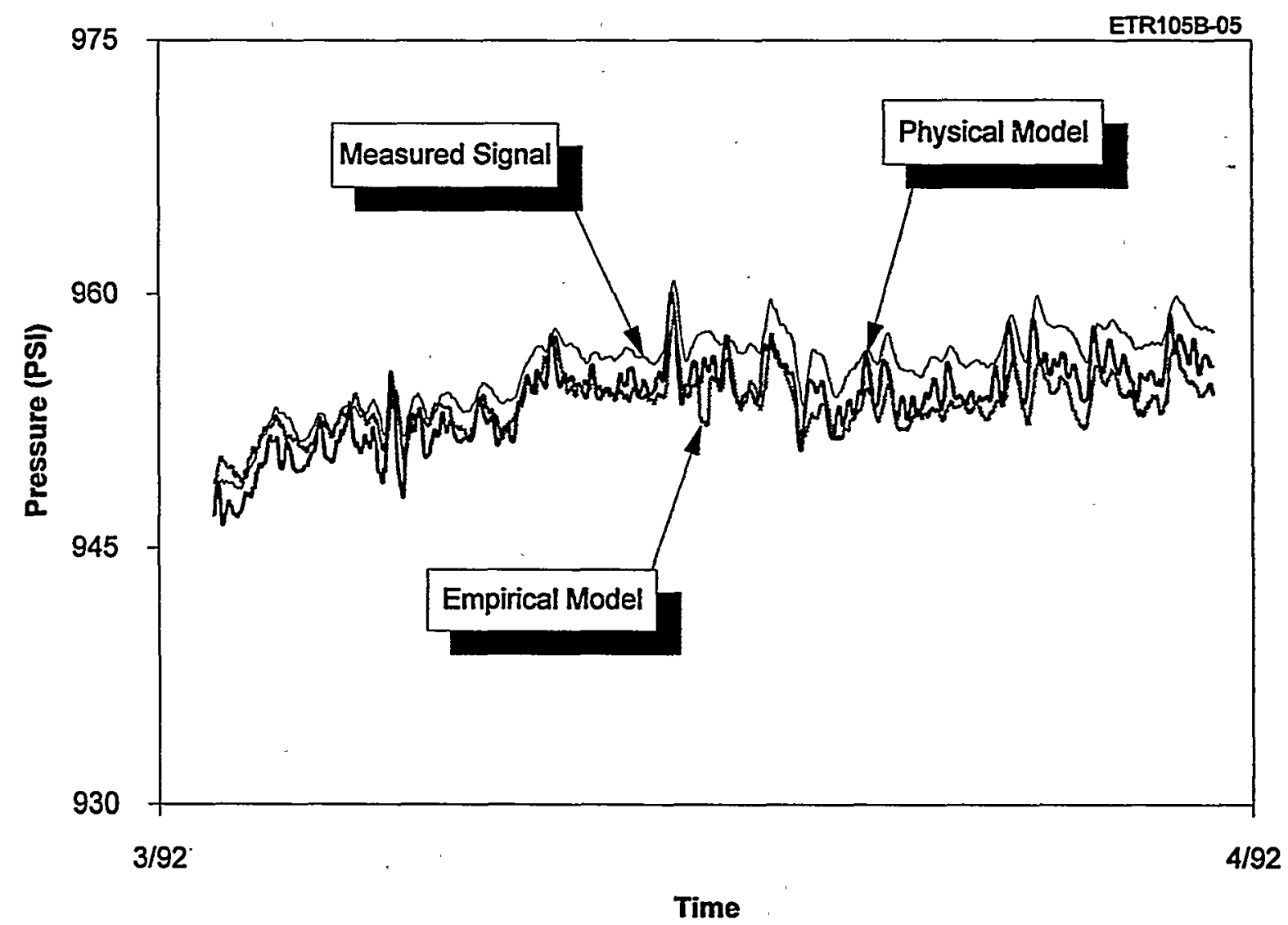

Figure 9.5 Example of Empirical and Physical Modeling Results for a McGuire Pressure Signal 
inputs and outputs of a system at a given operating condition. Once the nominal relationship is identified, the neural network can track outputs of the system and reveal if they are deviating from the nominal condition. The advantage of neural networks is that they do not require any knowledge of the system being monitored, nor do they require the user to define the specific form of the empirical model.

Neural networks can take the place of empirical modeling and pattern recognition in applications such as on-line drift monitoring discussed in this report. They are easy to employ and can be used to identify the drift of most process parameters under a variety of operating conditions; provided that the neural network is sufficiently trained with appropriate input/output data. The training involves feeding the input(s) and output(s) of the system to a neural network to establish the input/output relationship. If at a later time, an input to the system drifts, or experiences other anomalies, neural networks can identify the problem by revealing a change in the input/output relationship that was established during training.

\subsection{Sequential Probability Ratio Test}

SPRT was developed by Wald in the 1940s and has been adopted in the recent years for analysis of on-line monitoring data for detecting drift and other anomalies in nuclear plant instruments. ${ }^{(16)}$ The method is applied to a random signal that is derived from measurements. The following procedure illustrates the principle of the SPRT method using pairs of redundant signals. One of the two signals is first subtracted from the other. This usually leaves a steady-state difference or bias between the two signals plus random fluctuations (noise) which normally exist at the output of process instruments when the plant is operating. The bias is then subtracted out leaving only the noise. The noise should have a Gaussian distribution given by:

$$
p(x)=\frac{1}{\sqrt{2 \pi \sigma^{2}}} \exp \left[-\frac{(x-\mu)^{2}}{2 \sigma^{2}}\right]
$$

Where $\mathrm{p}(\mathrm{x})$ is the probability density function of the noise signal $\{x\}$, and $\mu$ and $\sigma$ are the mean and the standard deviation of the noise signal, respectively.

The term "sequential probability" or SP in the SPRT is derived from the fact that the probability of obtaining the sequence of measurements $x_{1}, x_{2}, x_{3}, \ldots, x_{n}$ is equal to the product of probabilities of attaining each measurement. That is:

$\mathrm{p}\left(\mathrm{x}_{1}, \mathrm{x}_{2}, \mathrm{x}_{3}, \ldots \mathrm{x}_{2}\right)=\mathrm{p}\left(\mathrm{x}_{1}\right) \cdot \mathrm{p}\left(\mathrm{x}_{2}\right) \cdot \mathrm{p}\left(\mathrm{x}_{3}\right) \ldots \cdot \mathrm{p}\left(\mathrm{x}_{\mathrm{n}}\right)$

The term "ratio test" or RT in the SPRT comes from the ratio of two probabilities: the probability that something is wrong divided by the probability that the signal is normal. Something is said to be wrong if $\mu$ or $\sigma$ exceed an acceptance criteria defined by the user. The signal is said to be normal if $\mu$ is near zero and $\sigma$ is within an acceptable range during the monitoring process.

If $\mu_{\text {good }}$ and $\sigma_{\text {good }}$ are the acceptable limits for the mean and standard deviations and $\mu_{\mathrm{bad}}$ and $\sigma_{\text {bad }}$ represent values that deviate from the acceptance criteria, the probability ratio mentioned above may be written as:

$$
\mathrm{RT}=\frac{\begin{array}{c}
\text { probability that the measured noise has } \\
\text { a bad distribution characterized by } \mu_{\mathrm{bad}} \text { and } \sigma_{\mathrm{bad}}
\end{array}}{\begin{array}{c}
\text { probability that the measured noise has a } \\
\text { good distribution characterized by } \mu_{\mathrm{good}} \text { and } \sigma_{\mathrm{good}}
\end{array}}
$$

The above ratio is calculated and compared against an acceptance criteria to determine if the results are normal, abnormal, or in between. The acceptance criteria for the 
SPRT are selected so that the results of the SPRT applications are not so sensitive to produce "false alarms" or so weak to result in "missed alarms."

\subsection{Process Hypercube Comparison}

This method is similar to pattern recognition with an improved data storage capability. ${ }^{(17,18)}$ As with pattern recognition, the state of the process is "learned" using plant operational data. All possible states within the normal operating region of the process are stored. If in the future, a state is identified that has not existed before, the responsible signal(s) are searched for and identified.

The Process Hypercube Comparison (PHC) technique derives its name from the fact that the learned process states are stored in a hypercube data structure. The structure contains a history of valid process conditions.

Consider a system in which only three variables $(x, y, z)$ are monitored. Suppose that the range of each variable is divided into five intervals. Also, suppose that all possible states of the process lie on the diagonal of the threedimensional cube that is formed by the three variables (Figure 9.6). The cells shown in Figure 9.6 should be numbered $(1,1,1),(2,2,2)$, ..., $(5,5,5)$. Now while monitoring the process, suppose that a new state is seen at $(1,1,5)$ as shown in Figure 9.6. It is obvious that both the $\mathrm{x}$ and $\mathrm{y}$ have been observed together before in this combination but that $\mathrm{z}$ was observed in a different state (i.e., $z=1$ instead of $\mathrm{z}=5$ ). Thus, the true state is probably $(1,1,1)$ and the variable $z$ is in error. 


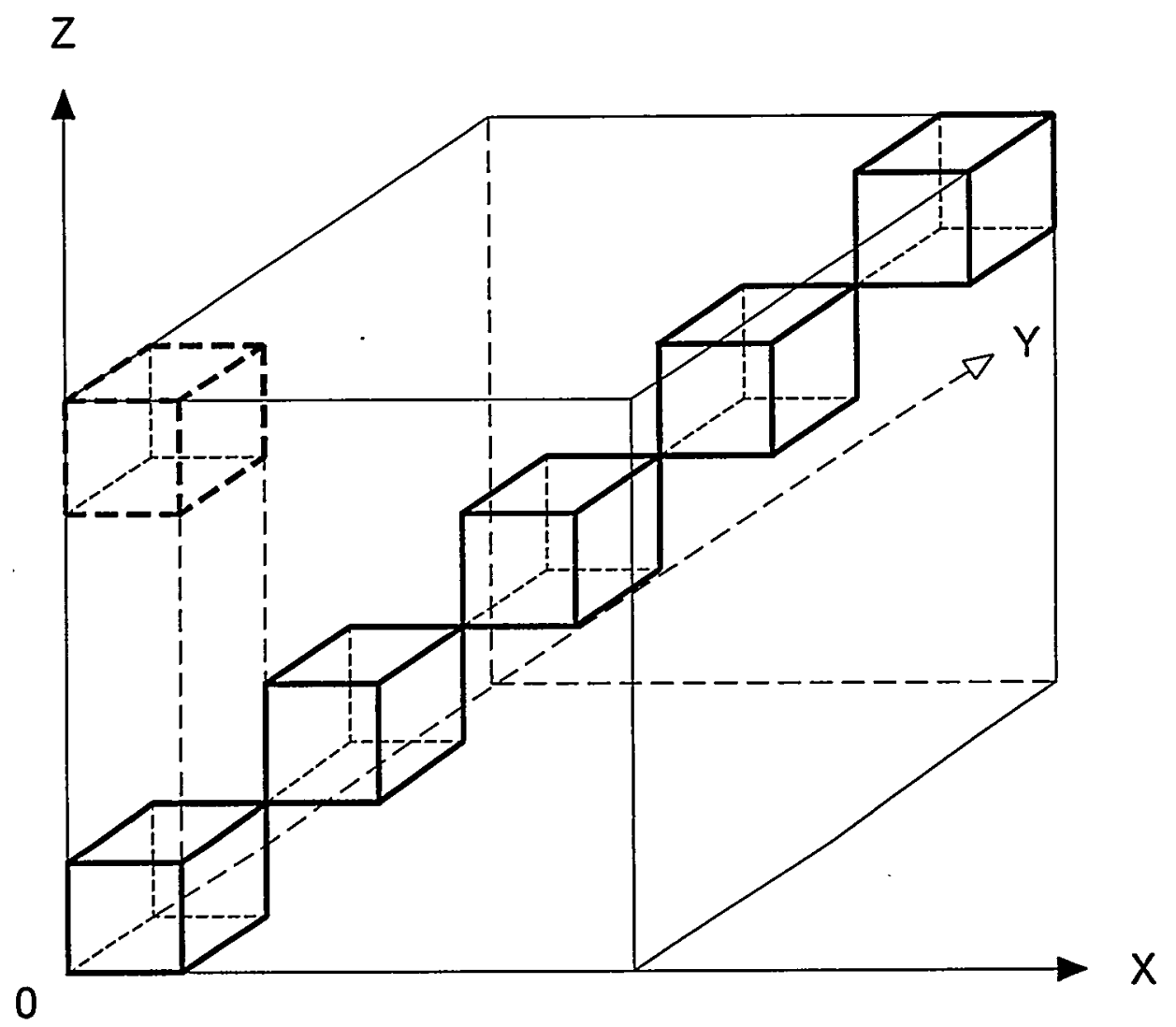

Figure 9.6 Three Dimensional Cell for PHC Technique 


\section{UNCERTAINTIES OF PROCESS ESTIMATION TECHNIQUES}

Process estimation techniques such as simple and weighted averaging, empirical modeling, pattern recognition, and neural networks have uncertainties which depend on many factors such as the number of redundant signals, frequency and length of data collection, measurement uncertainties, model order and convergence criteria for data fitting, reliability of input signals, etc. These factors can make it difficult to determine the overall uncertainties of process estimation results. However, process estimation uncertainties may or may not play an important role in determining the drift of instrument channels. Figure 10.1 illustrates two columns of plots, each with three plots showing the following cases: an instrument channel or sensor that is drifting, an estimate of the process parameter that is being measured, and the channel deviation from the process estimate. The column on the left shows the situation assuming that the process estimation result has no uncertainties. In this case, of course, the instrument drift which is the slope of the deviation plot is identified exactly.

The column on the right shows the situation assuming that the process estimation result has an uncertainty denoted as $\pm x$. If the uncertainty is constant throughout the on-line monitoring process, then the drift (i.e., the slope of the deviation plot) can be identified correctly in spite of the uncertainty. However, if the uncertainty is not constant, then the drift results may be affected by the process estimation uncertainty.

Although process estimation uncertainties may be difficult to quantify, it is reasonable to assume that their values, whatever they may be, remain constant throughout the on-line monitoring process and that drift results from on-line monitoring of instruments are not generally affected by the uncertainties of process estimation techniques. The process estimation uncertainty does, however, affect the ability to determine when the deviation of an instrument channel is unacceptable.

Another concern with the use of on-line drift monitoring is often expressed as follows. If the process is noisy and the noise level is larger than the instrument drift, then the noise could hide the drift. Figures 10.2 and 10.3 show two series of McGuire plant data which illustrate that signal drift and even sudden changes in the signals can be recovered from the noise. In Figure 10.2, a three-month record of three flow signals is shown. It is apparent that the spikes and noise in the raw data are larger than the slow changes in the signal levels. Nevertheless, as shown in the filtered data, the average changes in the signal can be readily extracted from the noise. The drift is a low frequency phenomenon that can be extracted from noise using a low-pass filter. A window-averaging algorithm was developed in this project and successfully tested for removing the extraneous spikes and noise from the raw data. The algorithm functions as a digital low-pass filter which is implemented during the data qualification process.

Figure 10.3 shows another example in which the raw data experiences a sudden shift. The shift is not much larger in amplitude than the noise of the signal. Nevertheless, it is easily recovered from the noise. Figure 10.3 also shows the deviation plot for the four signals.

In Figure 10.4, the same signals of Figure 10.3 are shown without filtering. Although the deviation plot indicates a smaller level of noise than the raw data, it is not as desirable as the clean deviation plot shown in Figure 10.3 for which the data was first filtered. This illustrates the benefit of the filtering and points out that the noise in the raw data is not cancelled out in the deviation plot. 
Uncertainty $=0$
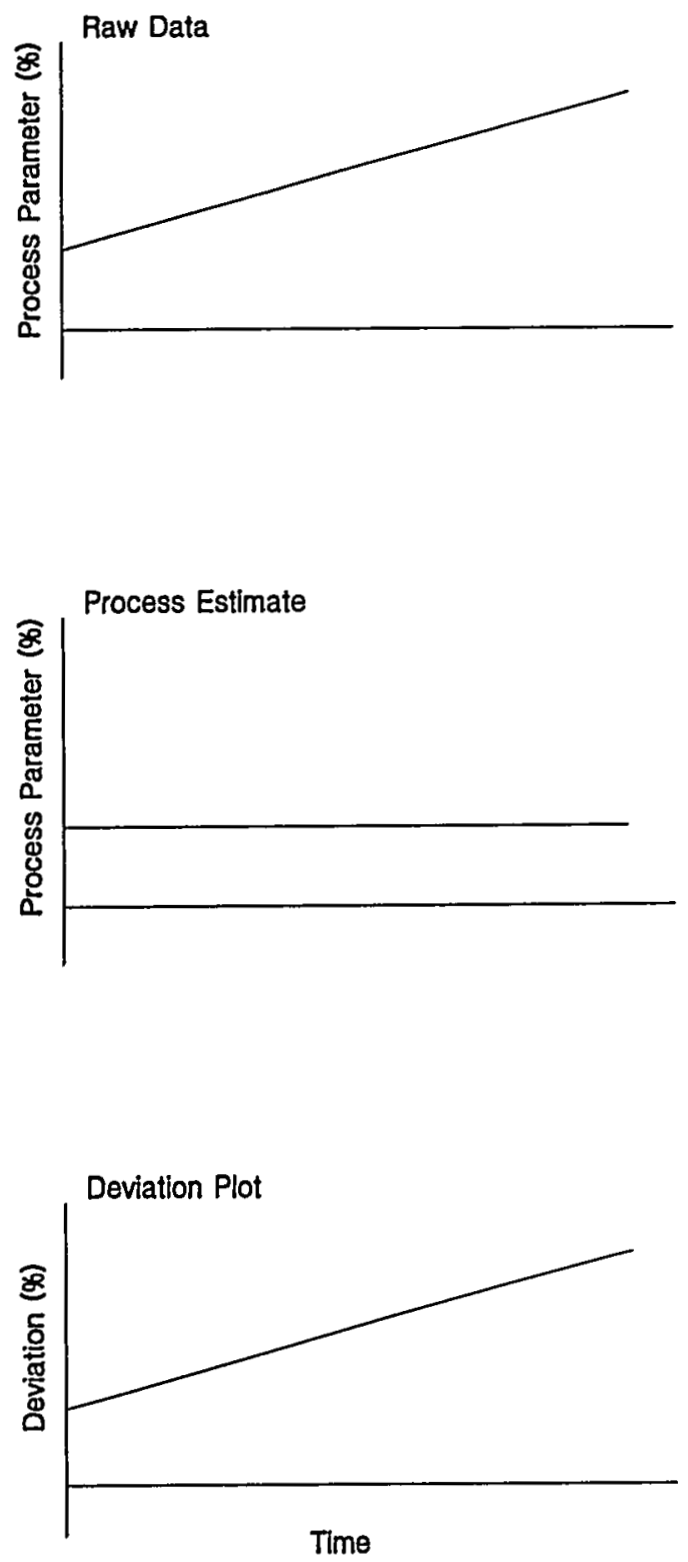

Uncertainty $=x$
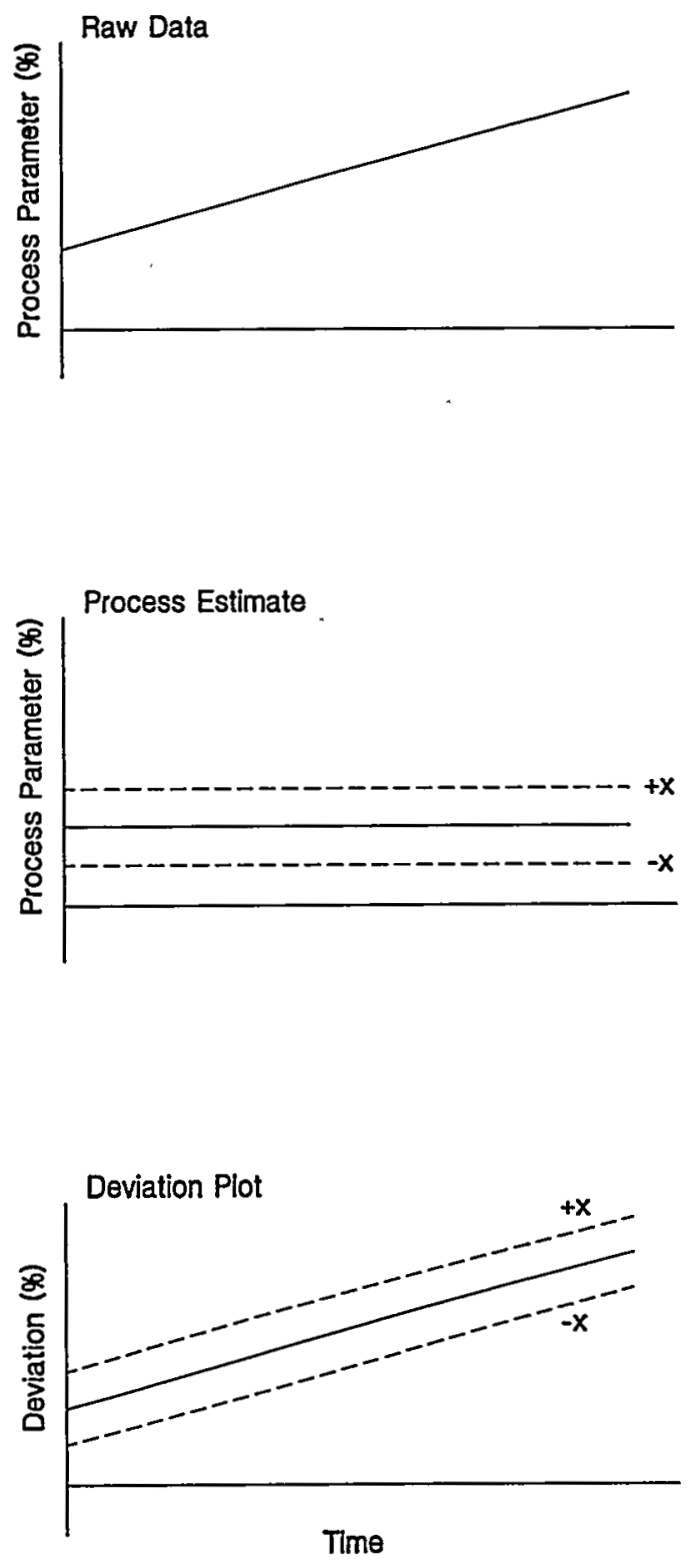

Figure 10.1 Illustration of Effect of Process Estimation Uncertainties on the Results of On-Line Drift Monitoring 

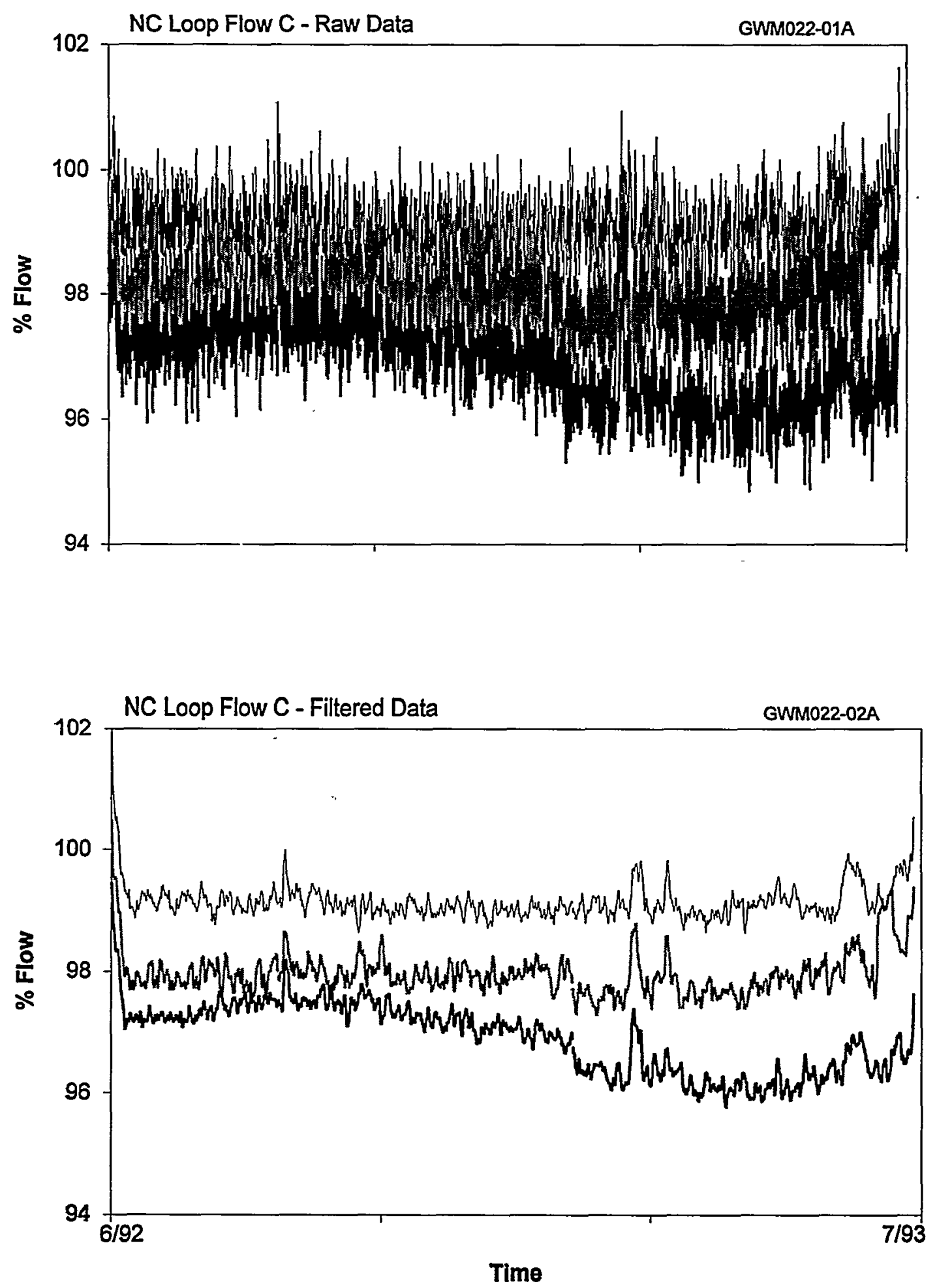

Figure 10.2 Recovering Drift from a Noisy Plant Signal 
- IS -

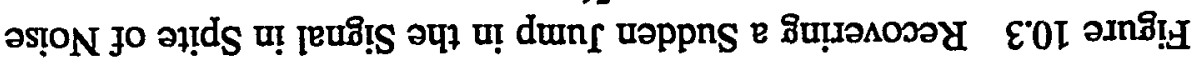
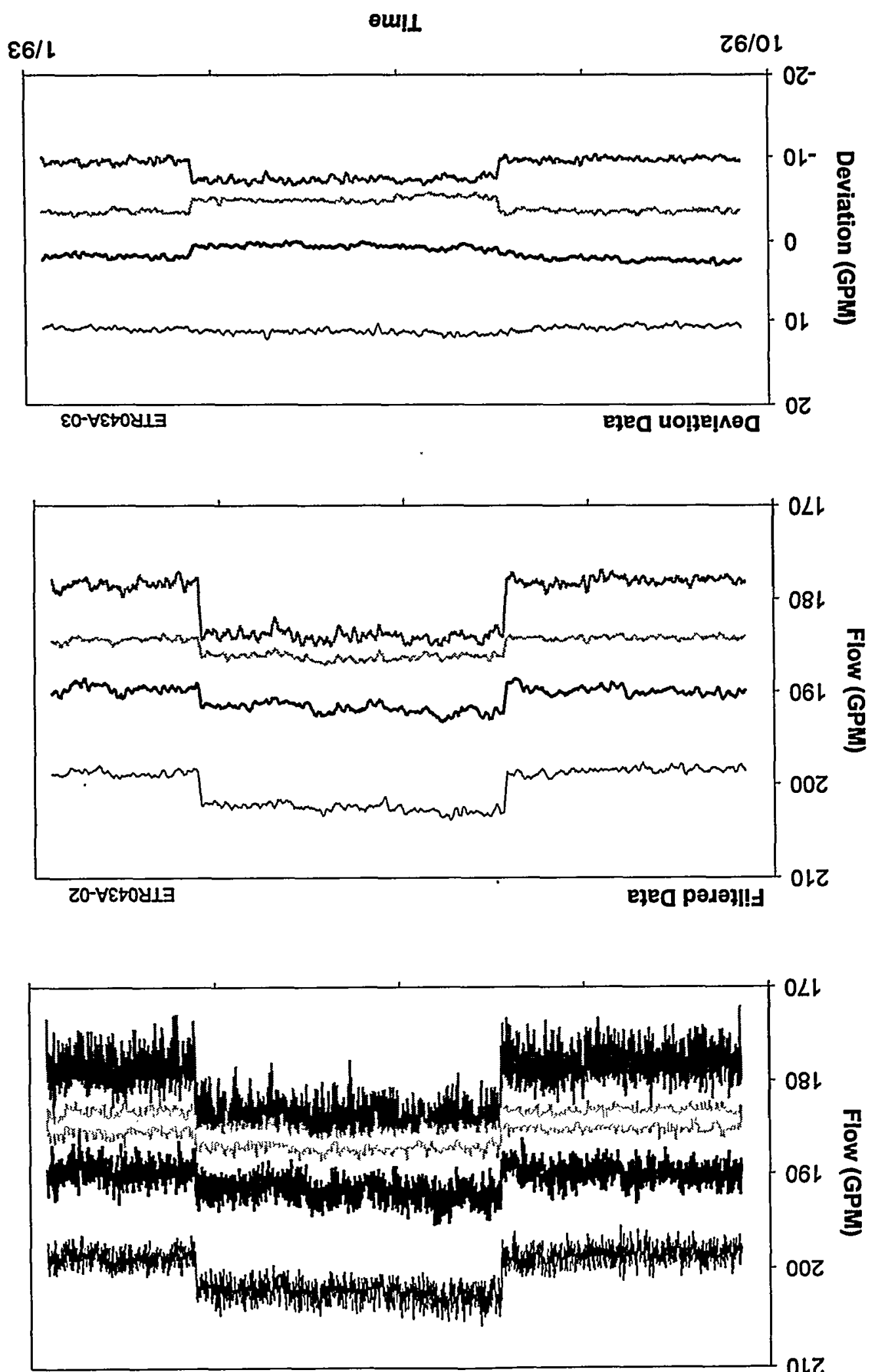

OLL

081

$061 \stackrel{\frac{\pi}{0}}{\frac{9}{0}}$

002

10- $\forall$ Etoy

ejeq MEY - MOJ $\exists$ porg XnY 

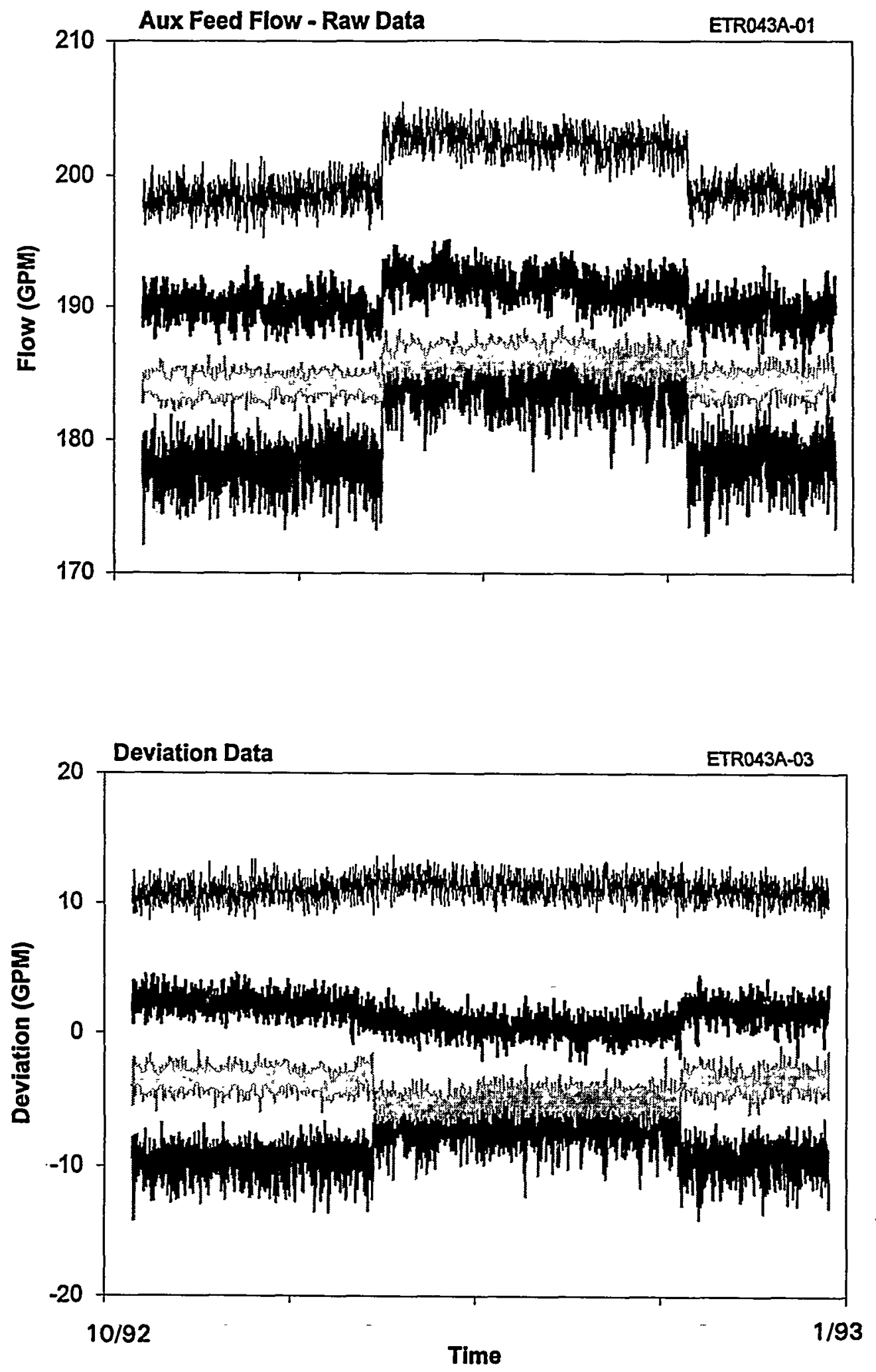

Figure 10.4 Raw Data and Deviation Plot Without Filtering the Signals. 


\section{ACCEPTANCE CRITERIA FOR ON-LINE MONITORING RESULTS}

On-line monitoring can identify drift in a sensor such as a pressure transmitter or an entire instrument channel (except for the actuation system) depending on where the online monitoring system is connected to the instrument channel. The next step after identifying the drift is to determine if the drift is acceptable and to set alert and alarm limits for corrective action as necessary to remedy the drift problem or mitigate its consequences. An alert limit is a conservative band that may be used to identify the onset of a potential drift problem, and an alarm limit is a band that may be used to identify the point at which corrective action should be initiated to prevent a channel from exceeding its drift allowance. also referred to as bias errors. The words uncertainty, error, and accuracy are used interchangeably.)

If the uncertainties are random, then they are squared and the square root of the sum of the squares (called RSS error) is calculated and added to the sum of the biases to yield the total uncertainty for the channel as shown in Equation 11.1 below. Note, in this equation that the errors that are dependent are first added together and then squared in calculating the RSS error. ${ }^{(19)}$

The total channel uncertainty calculated from Equation 11.1 is often referred to as the

$$
C S A=\sqrt{P M A^{2}+P E A^{2}+(S C A+S M T E+S D)^{2}+S P E^{2}+S T E^{2}+(R C A+R M T E+R C S A+R D)^{2}+R T E^{2}}+E A+B I A S
$$

\subsection{Instrument Channel Uncertainties}

Table 11.1 shows typical sources of uncertainties and their corresponding values for most of the services that were monitored at the McGuire plant. Some columns in Table 11.1 are left blank because they do not apply to the McGuire signals monitored in this project. They were, nevertheless, included in the table because they generally play a role in arriving at instrument channel uncertainties in nuclear power plants.

The uncertainties listed in Table 11.1 are defined in Table 11.2. These uncertainties are combined in a manner which depends on whether they are random or systematic, dependent or independent. (The random uncertainties are also referred to as accidental errors, and the systematic uncertainties are channel statistical accuracy or channel statistical allowance (CSA). The CSA is a parameter of interest in determining the trip setpoints for the plant. A larger CSA means that there is more room for instrument drift, but a smaller margin for trips, and vice-versa. Therefore, a plant would normally desire as small a CSA as possible to allow operation with as much margin as possible.

For a more detailed description of setpoints and CSA methodologies for nuclear power plants, the reader may consult references 19 through 21 listed at the end of this report. In this report, a general view of setpoint information is provided using some of the McGuire instrument specifications as an example to illustrate how the uncertainties of individual components of a channel may be combined to determine the acceptance criteria for on-line monitoring results. 


\begin{tabular}{|c|c|c|c|c|c|c|c|c|c|c|c|c|c|c|}
\hline \multicolumn{15}{|c|}{$\begin{array}{l}\text { Typical Sources of Uncertainties for Process Instrumentation Channels in PWR Plants } \\
\text { and Corresponding Values that Apply to the McGuire Plant } \\
\text { (\% of Span) }\end{array}$} \\
\hline Service & PMA & PEA & SCA & SMTE & SD & SPE & STE & RCA & RMTE & RCSA & RD & RTE & $E A$ & BIAS \\
\hline Feedwater Flow & 0.00 & $0.25^{(1)}$ & 0.10 & & 0.30 & 0.56 & 0.10 & 1.50 & & 0.00 & 1.00 & 0.50 & & 0.00 \\
\hline $\begin{array}{l}\text { Steam Generator } \\
\text { Level }\end{array}$ & $2.00^{(2)}$ & & 0.50 & & 1.00 & 0.30 & 0.50 & 0.50 & & 0.48 & 1.00 & 0.50 & & 0.00 \\
\hline $\begin{array}{l}\text { Reactor Coolant } \\
\text { Flow } \\
\end{array}$ & $1.40^{(3)}$ & & 0.00 & & 0.60 & 0.00 & 0.00 & 0.30 & & 0.17 & 0.60 & 0.30 & & $0.05^{(4)}$ \\
\hline Pressurizer Level & $2.00^{(2)}$ & & 0.50 & & 1.00 & 0.50 & 0.50 & 0.50 & & 0.35 & 1.00 & 0.50 & & 0.00 \\
\hline $\begin{array}{l}\text { Wide Range } \\
\text { Pressure }\end{array}$ & 0.00 & & 0.50 & & 1.50 & 0.00 & 0.50 & 0.50 & & 0.35 & 1.00 & 0,50 & & 0.00 \\
\hline $\begin{array}{l}\text { Pressurizer } \\
\text { Pressure }\end{array}$ & 0.00 & & 0.50 & & 1.00 & 0.00 & 0.50 & 0.50 & & 0.35 & 1.00 & 0.50 & & $1.50^{(5)}$ \\
\hline $\begin{array}{l}\text { Containment } \\
\text { Pressure } \\
\end{array}$ & 0.00 & & 0.50 & & 1.00 & 0.00 & 0.80 & 0.50 & & 0.35 & 1.00 & 0.50 & & 0.00 \\
\hline Steam Pressure & $0.20^{(6)}$ & & 0.50 & & 1.73 & 0.00 & 1.12 & 0.50 & & 0.35 & 1.50 & 0.50 & & 0.00 \\
\hline $\begin{array}{l}\text { Turbine Impulse } \\
\text { Pressure }\end{array}$ & 0.00 & & 0.50 & & 0.63 & 0.00 & 0.72 & 0.50 & & 0.00 & 1.00 & 0.50 & & 0.00 \\
\hline Power Range & 4.17 & & 4.17 & & 0.00 & 0.00 & 0.00 & 0.50 & & 0.25 & 1.00 & 0.50 & & 0.00 \\
\hline $\begin{array}{l}\text { Incore } \\
\text { Thermocouples }\end{array}$ & 0.00 & & 7.20 & & 10.00 & 0.00 & 2.30 & 6.90 & & 0.00 & 2.30 & 11.50 & & 0.00 \\
\hline
\end{tabular}

Footnotes: 1. $0.25 \%$ represents uncertainty in flow measurements due to flow orifice

2. $2.00 \%$ represents uncertainty in level measurements due to the density of water

3. 0.33 of this 1.4 is uncertainty in flow measurements due to the density of water

4. $0.05 \%$ is bias due to tap location

5. $1.50 \%$ bias represents thermal non-repeatability

6. $0.20 \%$ is due to water leg compensation

Above are bias terms that are common to redundant sensors. Thus, they were not included in calculating the process estimation uncertainties presented in this chapter for the McGuire instruments. 


\begin{tabular}{|c|c|}
\hline \multicolumn{2}{|r|}{ Description of Sources of Instrument Channel Uncertainties } \\
\hline PMA & $\begin{array}{l}\text { Process measurement accuracy. Inherent noise in the process. PMA sources are listed as Water Leg Correction, Elbow Tap } \\
\text { Error, Streaming \& Thermal Mismatch (Power Range Detectors). For the RC Flow Channel, PMA is a RSS combination of } 0.33 \\
\text { for Density, } 0.30 \text { for noise, and } 1.33 \text { for calorimetric uncertainties. This RSS combination equals to } 1.4 \% \text {. }\end{array}$ \\
\hline PEA & Primary Element Accuracy. Represents the error due to the use of a metering device like a flow orifice, etc. \\
\hline SCA & Sensor Calibration Accuracy. Inherent accuracy of the sensor at reference conditions; typically vendor supplied. \\
\hline SMTE & $\begin{array}{l}\text { Sensor Measurement \& Test Equipment. McGuire calculation assumes } 0.0 \text { for SMTE because equipment used in this plant } \\
\text { meets 4:1 accuracy ratio. }\end{array}$ \\
\hline SD & Sensor Drift. Observed change in sensor accuracy as a function of time; typically supplied by the vendor. \\
\hline SPE & Sensor Pressure Effects \\
\hline STE & Sensor Temperature Effects \\
\hline RCA & Rack Calibration Accuracy \\
\hline RMTE & $\begin{array}{l}\text { Rack Measurement \& Test Equipment. McGuire calculation assumes } 0.0 \text { for SMTE because equipment used meets } 4: 1 \\
\text { accuracy ratio. }\end{array}$ \\
\hline RCSA & Rack Comparator Setting Accuracy \\
\hline RD & Rack Drift \\
\hline RTE & Rack Temperature Effects \\
\hline EA & $\begin{array}{l}\text { Environmental Allowance. Represents the change in the instrument channel's response due to accident environmental } \\
\text { conditions. McGuire calculation uses } 0.0 \text { for EA because these are normal CSAs rather than accident CSAs. }\end{array}$ \\
\hline BIAS & For the RC Flow channel, this represents the flow measurement error for the elbow taps, \\
\hline
\end{tabular}




\subsection{CSA Band and Drift Band}

An instrument channel is said to be in calibration if the difference between its input and output is less than the CSA or if its drift is contained within the drift allowance for the channel. For on-line calibration monitoring, the channel output is subtracted from the best estimate of the process input and the results are plotted over the entire fuel cycle to check for drift and other problems. Figure 11.1 shows the results of this exercise for two services at the McGuire plant. The results in this figure are shown in terms of the deviation of each signal from the average of the redundant signals. Also shown in Figure 11.1 are the CSA bands. A discussion on how the CSA band may be determined is given later in this chapter.

Figure 11.2 shows the same data as in Figure 11.1 except that in this case, the signals are biased as necessary to start them at zero on the vertical axis. Appropriate drift bands for these signals are also shown on the figure. The drift band is calculated by squaring the sensor drift (SD) and rack drift (RD) terms, adding the two results, and calculating the square root of the sum. The result is then multiplied by $(n-1) / n$ to account for the number of redundant signals that are intercompared on the same plot. Following is the equation for the drift band:

$$
D R I F T B A N D=\sqrt{S D^{2}+R D^{2}} \frac{(n-1)}{n}
$$

where $\mathrm{n}$ is the number of redundant signals that are intercompared on the same plot. If analytical methods are used for process estimation instead of averaging methods, then the above equation for drift band does not need the $(n-1) / n$ term.

Drift band is a simpler means than the CSA band for determining the calibration acceptability of instrument channels. In using the drift band, one assumes that all channels agree with each other when the plant starts from a refueling outage. This assumption is valid because redundant instrument channels are all manually calibrated with the same procedure during a refueling outage and they should thus agree with each other. If they do not agree, however, it could be due to inherent uncertainties involved in the calibrations. These uncertainties only affect where the signals may start on the on-line monitoring plot, but have no bearing on how much the signals may drift from the beginning to the end of a fuel cycle. Ideally, after a full channel calibration which is performed during a refueling outage, and when the plant is at full power, there should be no deviation between a process estimate and the corresponding instrument channel output. If there is, the difference represents a bias. This bias can be subtracted from the deviation plot and the residual deviation attributed to drift. With this approach, the acceptance criteria can be defined in terms of an allowable drift.

Furthermore, drift is the only component of CSA which drives the requirement for manual calibrations. As such, it is reasonable to monitor for drift to determine if an instrument channel needs a manual calibration.

\subsection{Procedure for Calculating the CSA Band}

A conservative procedure is outlined below for determining the CSA band for four steam generator level signals using the information given in Tables 11.1 and 11.2.

1. Combine the accuracies of the components that were included in the on-line monitoring of the channel. At McGuire, all components listed in Table 11.1 for the steam generator level channel were included in the on-line monitoring except for the comparator 

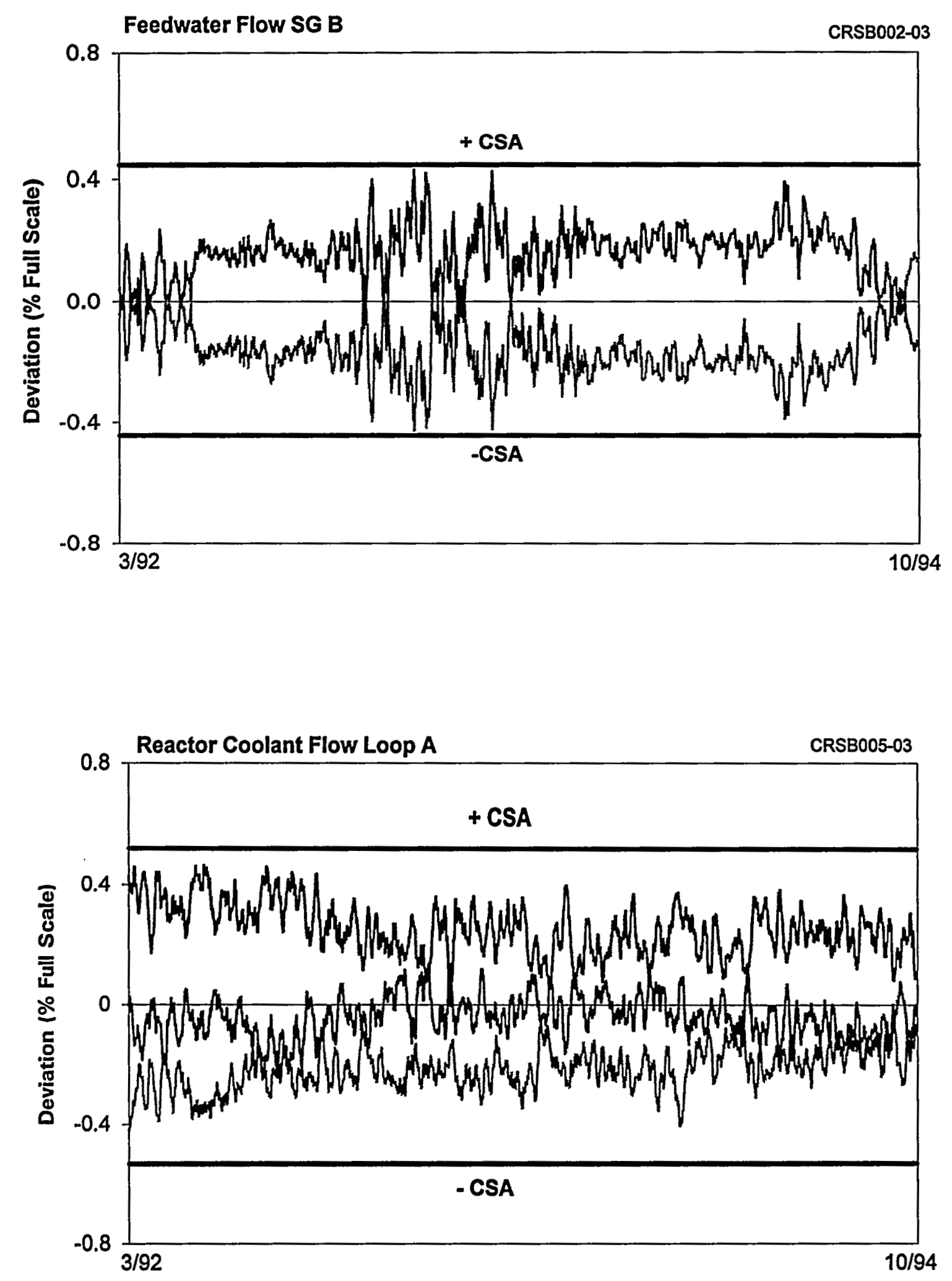

Figure 11.1 Deviation Plot and CSA Bands for McGuire Signals 

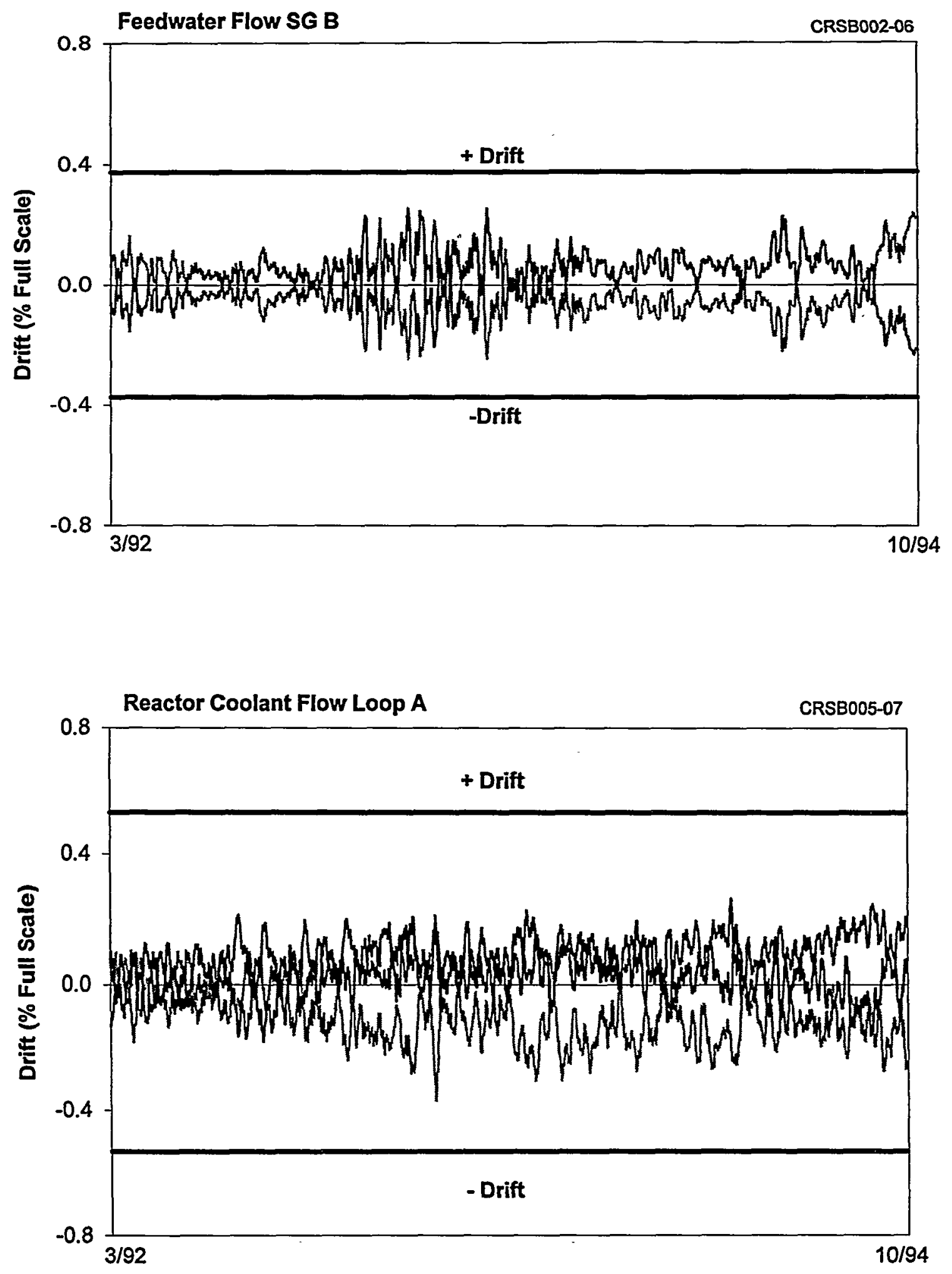

Figure 11.2 Drift Plots and Drift Bands for McGuire Signals 
whose error is designated in the table under the column marked RCSA. Therefore, the CSA is calculated as follows: that are not included in the on-line monitoring (e.g., RCSA). For steam generator level transmitters, the common bias is $2.0 \%$ due to uncertainty of water

$$
\begin{aligned}
& C S A=\sqrt{(P M A)^{2}+(S C A+S D)^{2}+(S P E)^{2}+(S T E)^{2}+(R C A+R D)^{2}+(R T E)^{2}} \\
& C S A=\sqrt{(2.0)^{2}+(1.5)^{2}+(0.3)^{2}+(0.5)^{2}+(1.5)^{2}+(0.5)^{2}}= \pm 3.01 \text { of of span }
\end{aligned}
$$

Figure 11.3 illustrates how the CSA band would have been used if only one signal was involved in the on-line monitoring. However for four signals to be intercompared on the same plot, the CSA band is reduced by $(n-1) / n$, where $n$ is the number of signals that are intercompared on the same plot.

$$
\sigma_{i}=\sqrt{(S C A+S D)^{2}+(S P E)^{2}+(S T E)^{2}+(R C A+R D)^{2}+(R T E)^{2}}
$$

2. The uncertainty of process estimation must be subtracted from the CSA calculated in step 1 . This uncertainty may be calculated as shown in Figure 11.4 and is described below assuming that simple averaging was used as the process estimation technique.

For a conservative treatment of the uncertainties, we assumed that each of the four steam generator level signals have an uncertainty $\left(\sigma_{i}\right)$ which is calculated using Equation 11.1, but excluding the following terms: (1) the effect of common biases (if any) given in the footnotes of Table 11.1, and (2) error terms for the components density. Therefore, the uncertainty of each steam generator level signal is calculated using Equation 11.1 but excluding not only the RCSA, but also the PMA term as shown in Equation 11.4 below.

3. This uncertainty must be subtracted for the CSA band calculated in step 1 to account for the uncertainty of the process estimation results. Figure 11.5 shows (by dashed lines) the CSA band that is used for intercomparing four signals.

As shown in Figure 11.4, the uncertainty of the process estimation using the average of the four redundant steam generator level sensors is:

$$
\sigma=\frac{\sqrt{(4)\left(\sigma_{i}\right)^{2}}}{4}=1.13 \% \text { of span }
$$




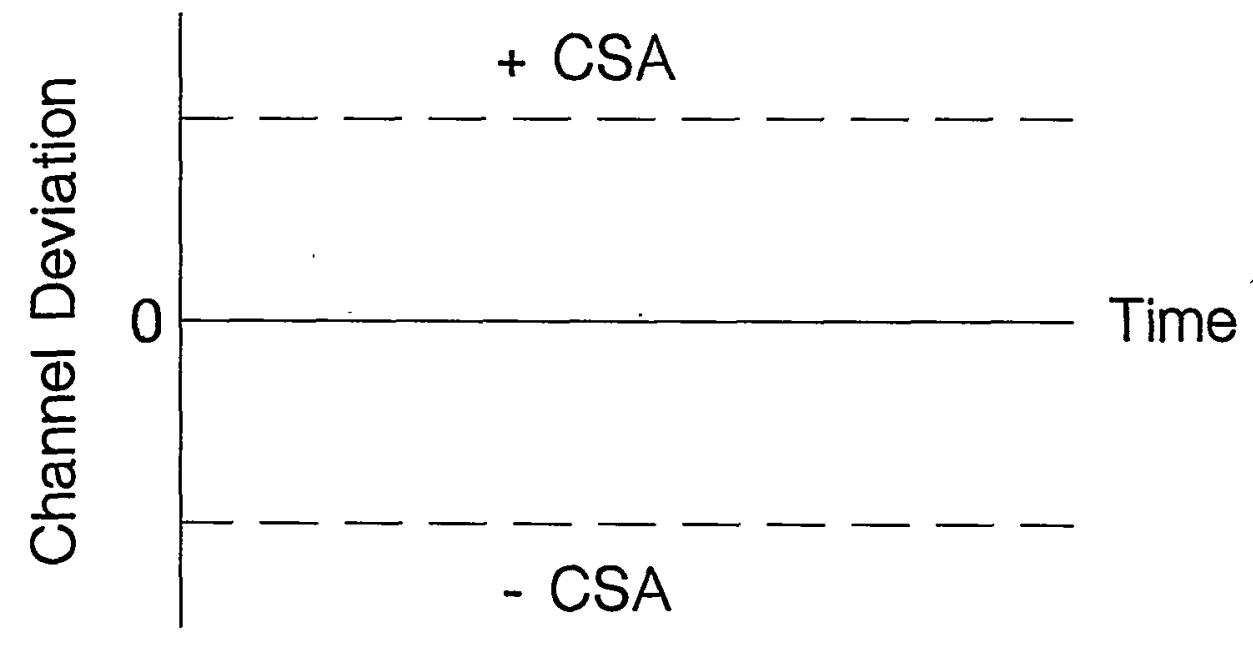

Figure 11.3 CSA Band for a Single Signal 


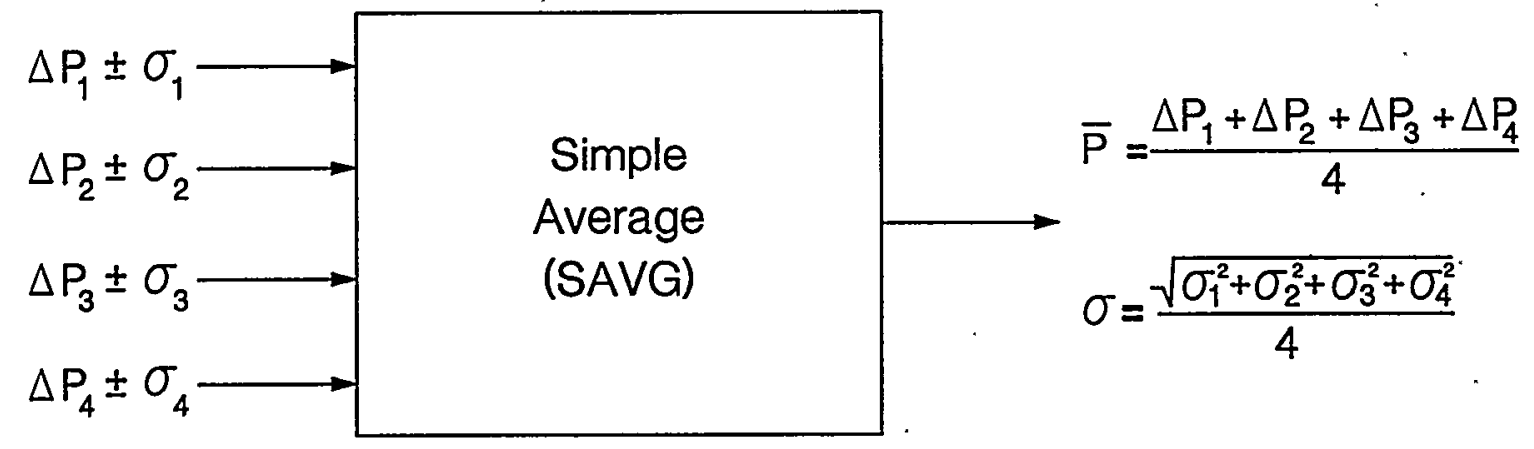

$(\bar{P}$ is the average value of the process and $\sigma$ is the uncertainty of this average. Thus, the best estimate of the process is $\bar{P} \pm \sigma$ )

Figure 11.4 Uncertainty of Simple Averaging for Process Estimation 


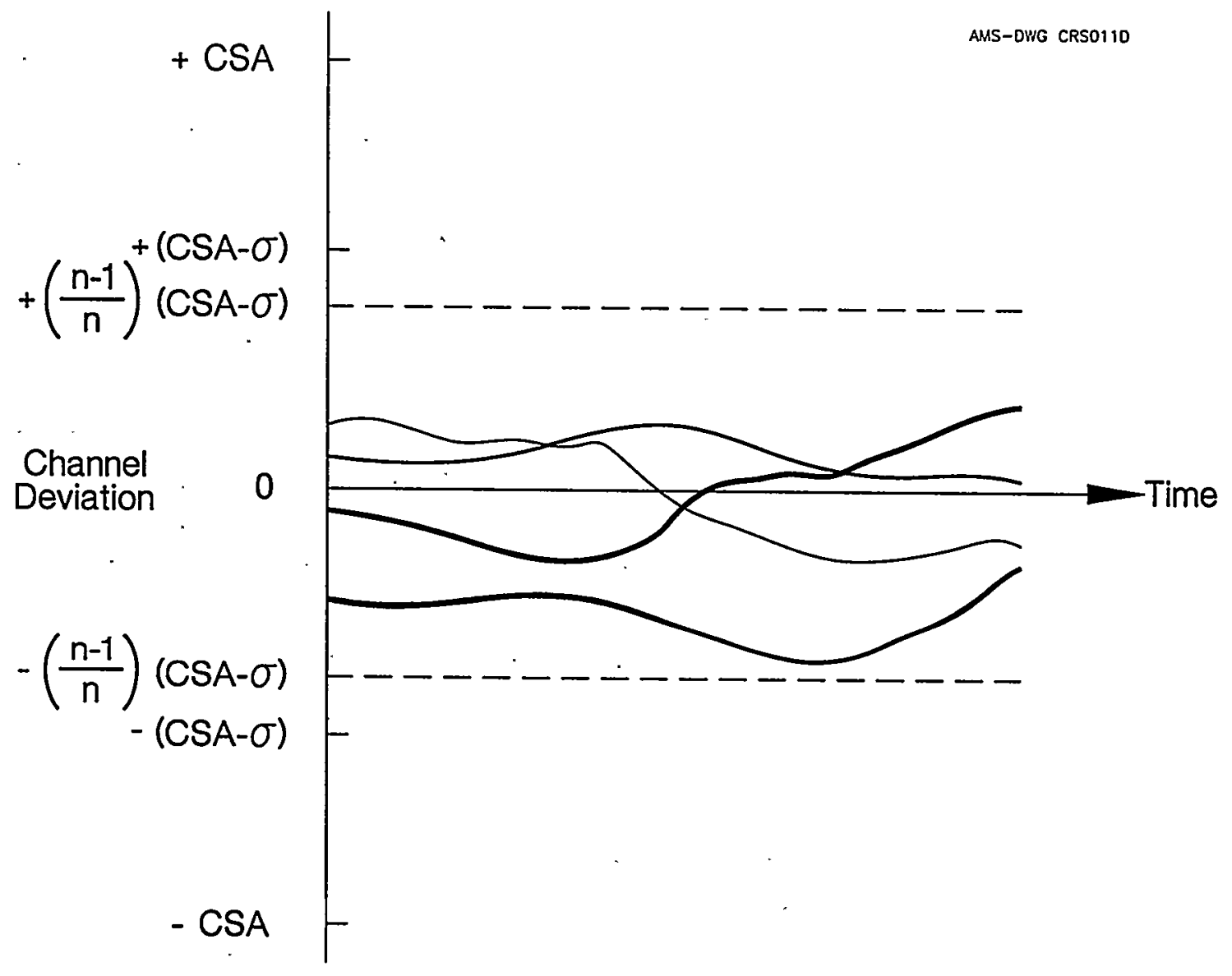

Figure 11.5 Illustration of How CSA Band is Established for Redundant Signals 


\subsection{CSA and Drift Bands for McGuire Signals}

Table 11.3 shows drift and CSA bands calculated as outlined above for nine of the McGuire instrument channels. The CSA bands in this table are arrived at as follows:

$\operatorname{CSA}$ Band $=(\operatorname{CSA}-\sigma) \cdot(n-1 / n)-0.05$

The 0.05 percent subtracted in the equation above is to account for the measurement uncertainties associated with the on-line monitoring system. This value was obtained from the specifications of the components in the on-line monitoring system that was developed in this project and used at the McGuire plant.

If analytical techniques such as empirical modeling or neural networks are used for process estimation, then the term $(n-1) / n$ is not needed in Equation 11.6.

Although the drift and CSA bands described in this chapter are for use with the results of averaging techniques, they can also be used with the results of analytical techniques. The drift and CSA bands for averaging techniques should be more conservative than those that would be obtained for analytical techniques. This is because the uncertainties of averaging techniques are generally larger than analytical techniques, thus, their drift and CSA bands are larger.

There are some arguments against subtracting the $\sigma$ from CSA (see Figure 11.5) to arrive at the CSA band given in Equation 11.6, and there is no consensus as to what constitutes a reasonably conservative CSA band to be used with the results of on-line calibration tests. Table 11.3 includes a column listing CSA bands without the effect of process estimation uncertainties. The CSA bands that were used in this report, however, are those which include the effect of process estimation uncertainties.

As indicated before, a drift band is less ambiguous and more straightforward than CSA for determining the calibration status of instrument channels. Nevertheless, discussions of the CSA band were presented in this chapter in addition to drift band to provide a better picture of the options that are available. The disadvantage of a CSA band is that it can produce false alarms depending on where a signal starts on the deviation plot. Figure 11.6 shows three signals and the corresponding CSA band. Note that one of the signals exceeds the CSA band from the beginning of the cycle even though the instrument channel for this signal was manually calibrated during the preceding refueling outage. This is because redundant signals cannot be expected to all start on the same point at the beginning of a cycle even if they are all manually calibrated prior to starting the cycle. The normal uncertainties in the manual calibrations can cause bias differences between the redundant signals.

It should be pointed out that the drift bands and CSA bands used with the results presented here are especially conservative and should not be used to draw conclusions about the acceptability of instrument channel calibrations at the McGuire plant. 


\section{TABLE 11.3}

Representative CSA and Drift Bands for Representative Instrument Channels at the McGuire Unit 2 Plant

(\% of Span)

\begin{tabular}{|c|c|c|c|c|c|}
\hline Item & Channel & $\begin{array}{c}\text { Number of } \\
\text { Signals }\end{array}$ & Drift Band & $\begin{array}{l}\text { CSA Without } \\
\text { Uncertainty }\end{array}$ & $\begin{array}{c}\text { CSA } \\
\text { With Uncertainty }{ }^{(2)}\end{array}$ \\
\hline 1 & Feedwater Flow & 2 & 0.52 & 1.33 & 0.34 \\
\hline 2 & $\begin{array}{l}\text { Steam Generator } \\
\text { Level }\end{array}$ & 4 & 1.06 & 2.26 & 1.36 \\
\hline 3 & $\begin{array}{l}\text { Reactor Coolant } \\
\text { Flow }\end{array}$ & 3 & 0.57 & 1.23 & 0.58 \\
\hline 4 & Pressurizer Level & 3 & 0.94 & 2.03 & 1.10 \\
\hline 5 & $\begin{array}{l}\text { Wide-Range } \\
\text { Pressure }\end{array}$ & 2 & 0.90 & 1.30 & 0.33 \\
\hline 6 & $\begin{array}{l}\text { Pressurizer } \\
\text { Pressure }\end{array}$ & 4 & 1.06 & 2.81 & 1.92 \\
\hline 7 & $\begin{array}{l}\text { Containment } \\
\text { Pressure }\end{array}$ & 3 & 0.94 & 1.55 & 0.60 \\
\hline 8 & Steam Pressure & 3 & 1.53 & 2.16 & 0.86 \\
\hline 9 & $\begin{array}{l}\text { Turbine Impulse } \\
\text { Pressure }\end{array}$ & 2 & 0.59 & 1.04 & 0.25 \\
\hline
\end{tabular}

(1) CSA band without uncertainty is calculated from Equation 11.3 and then multiplied by $(n-1) / n$, where $n$ is the number of redundant signals that are intercompared on the same deviation plot.

(2) CSA band with uncertainty is calculated from this equation: CSA Band $= \pm[(n-1) / n][C S A-\sigma]-0.05$, where $\sigma$ is the uncertainty of process estimation technique. 


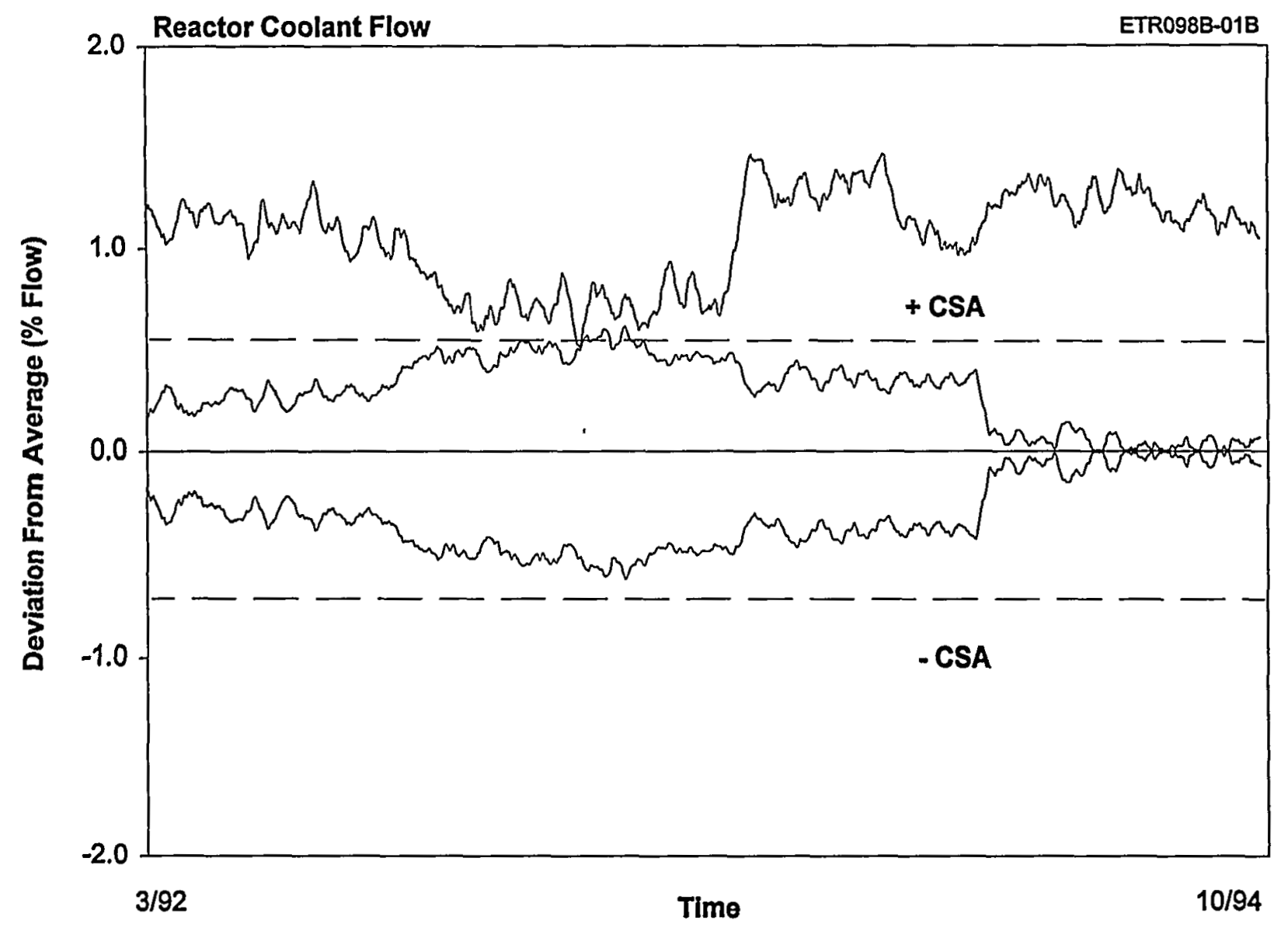

Figure 11.6 Flow Signals and CSA Band Showing a Signal which Fails from the Start 


\section{TECHNOLOGY DEVELOPMENT AND VALIDATION}

The on-line monitoring system development and validation conducted in this project involved two steps as follows:

1. On-line monitoringsystem development to include the following:

- Data acquisition hardware and software development

- Development of data analysis algorithms and software packages

- Preparation of procedure for in-plant tests and interpretation of test results

- Development of procedures for quality assurance tésting and calibration of on-line monitoring system and documentation of test results

2. Validation of on-line monitoring techniques to include the following:

- Laboratory demonstration tests

- Software verification and validation

- In-plant demonstration tests

- Documentation of validation results

The above steps are described separately in the next two chapters of this report.

It should be pointed out that on-line drift monitoring is a simple task which does not require sophisticated equipment, algorithms, or procedures. A variety of data acquisition equipment and software packages are available from a number of suppliers that can be used for on-line monitoring tests.

The analytical techniques that are needed for process estimations associated with on-line monitoring tests have been developed by a number of organizations and most of the information is available in open literature. In particular, neural networks, which are shown in this report to be very useful for on-line drift monitoring, are described in numerous publications not only in the field of nuclear engineering, but also in other engineering, scientific, and industrial reference publications. The references listed at the end of this report identify a few of the publications which contain most of the necessary information on analytical techniques for on-line calibration tests in nuclear power plants.

The interpretation of results is probably the only area of an on-line drift monitoring program that may need special expertise and in-depth training. Whether the data is analyzed by simple or weighted averaging techniques or analytical methods such as empirical modeling, neural networks, or pattern recognition, there are peculiarities that would require experience to understand to interpret the results.

Automated drift monitoring equipment incorporating expert systems that can analyze the data and interpret the results will eventually be deployed in the nuclear industry, but adequate experience does not currently exist to develop an expert system. On-line drift monitoring as a replacement for manual calibrations has not been in routine use in nuclear power plants. Upon NRC approval, it is expected that utilities will adopt the method and the necessary experience will begin to accumulate in order to develop an expert system for this application.

In addition to on-line calibration testing in nuclear power plants, an on-line monitoring system can be used for predictive maintenance tests and performance monitoring of equipment and systems in a variety of nuclear and non-nuclear applications. 


\section{ON-LINE MONITORING SYSTEM DEVELOPMENT}

On-line drift monitoring requires a multichannel data acquisition system and a data analysis and plotting software. The requirements for the data acquisition system depend on the source of the data. The sources from which the data are available in nuclear power plants often varies from one plant to another and the same on-line monitoring system will not be suitable for all plants. For example, in some plants, the data may simply be acquired from the plant computer, and in others, a dedicated data acquisition system may have to be installed to interface with the plant instrumentation cabinets through isolation devices. Other sources of process instrumentation data include SPDS, patch panels, and plant-wide information systems. Also, plants that are retrofitted with new computers may include provisions to allow on-line drift monitoring without a need for additional hardware.

To acquire data from the plant computer, a number of commercial software packages are available. These packages can be used to obtain the data and process them as they are collected, or store them on computer disks, or other storage media for subsequent analysis. A small computer such as a desktop or laptop personal computer may be used to transfer the data from the plant computer and store them on computer disks. The advantage of using data from the plant computer is that no additional equipment such as signal isolation devices and analog-to-digital converters are required. However, the plant computer may not have all the plant signals in a format that is suitable for on-line drift monitoring.

The advantage of using SPDS, patch panels, and the like is that signals from these sources are usually isolated and can be readily accessed, digitized, and used for on-line monitoring with no disturbance to plant operation. However, these sources do not usually provide all the redundant signals that are needed for on-line drift monitoring. These and other existing sources of plant data were explored during this project to determine what must be developed to meet the on-line monitoring needs of most nuclear power plants. This effort concluded that a dedicated data acquisition, storage, and analysis system would be needed in most nuclear power plants to perform on-line drift monitoring. Although in some plants, data can be accessed and recorded from existing sources, most plants are not configured to allow all the necessary signals to be obtained from one convenient location.

The challenge in implementing a dedicated data acquisition system in a plant is that, in most cases, plant data are available only from the plant protection cabinets in the control room or cable spreading room area. Furthermore, the signals are not usually available through isolated outputs. Therefore, for independent on-line monitoring, qualified isolation devices may have to be installed in some plants to allow access to the data.

The data sampling frequency is arbitrary as long as enough data points are collected at reasonable intervals throughout the fuel cycle to reveal any significant drift in the instrument channels. There are trade-offs in using high or low sampling frequencies. The advantage of high sampling rates (such as one sample per second) is that channel transients, fluctuations, and spikes will be captured which can provide diagnostics information about the channel in addition to drift information. The disadvantage of high sampling rates is that they increase the data storage requirements, and can lead to false alarms.

If the on-line monitoring system is only intended to identify drift, then continuous data acquisition may not be needed as long as reasonable amounts of data are collected at the beginning, middle, and the end of a fuel cycle. 


\section{VALIDATION OF ON-LINE MONITORING SYSTEM}

A comprehensive validation effort was carried out in this project to examine the suitability of on-line monitoring techniques for instrument calibration verification in nuclear power plants. This effort involved both laboratory and inplant tests. The equipment setup and other arrangements for the laboratory and in-plant tests are described in this chapter. Test results are presented in chapter 15 for the laboratory validation tests, and in chapter 16 for the inplant validation tests.

The term "validation tests" used in this and the remaining chapters of this report refers to tests that were conducted here to demonstrate the feasibility of on-line calibration testing techniques for individual instruments and sensors, or groups of instruments and sensors. The laboratory tests were designed to test the algorithms and associated software packages which were developed for the project and to gain experience on the interpretation of on-line monitoring results. The in-plant tests were designed to determine if on-line monitoring tests can be performed without interfering with the plant operation, and to verify that on-line monitoring results can provide adequate and useful information about the calibration stability of sensors and associated components which constitute an instrument channel.

\subsection{Laboratory Validation Tests}

This work was conducted at the AMS laboratory using a test loop developed during Phase I. The loop contains a 500 GPM pump and a 50,000 BTU/hr heat exchanger and is made of transparent PVC pipes. A photograph of the loop is given in Figure 14.1 followed by a schematic of the loop in
Figure 14.2. The loop can be operated at temperatures up to $120^{\circ} \mathrm{F}$, pressures up to 100 PSI, and flow rates up to 20 feet per second. While these conditions are not close to nuclear plant operating conditions, they are sufficient for testing the on-line monitoring equipment, algorithms, and software packages developed in this project.

The laboratory loop is instrumented with temperature, pressure and flow sensors and associated instrumentation of the types used in nuclear power plants. In particular, the loop contains nuclear grade pressure sensors and RTDs, and Westinghouse Model 7300 instrumentation racks acquired from nuclear power plants. A photograph of the 7300 system and the loop operations and control console is given in Figure 14.3. From the control console, the temperature, pressure, flow, and other parameters in the loop could be manipulated as necessary to provide data for a variety of demonstration and validation tests.

In addition to the laboratory loop that was constructed specifically for this project, existing equipment available at AMS from previous $\mathrm{R} \& \mathrm{D}$ projects for the NRC and others, as well as AMS' own equipment were used in the project. This included high temperature laboratory furnaces for testing of temperature sensors, an environmental chamber for testing of pressure sensors, oil and ice baths for calibration of temperature sensors, standard platinum resistance thermometers for calibration of RTDs and thermocouples, voltage, resistance, and other calibration standards, measurement and test equipment, personal computers, and digital data acquisition systems and associated software packages and interface equipment. 


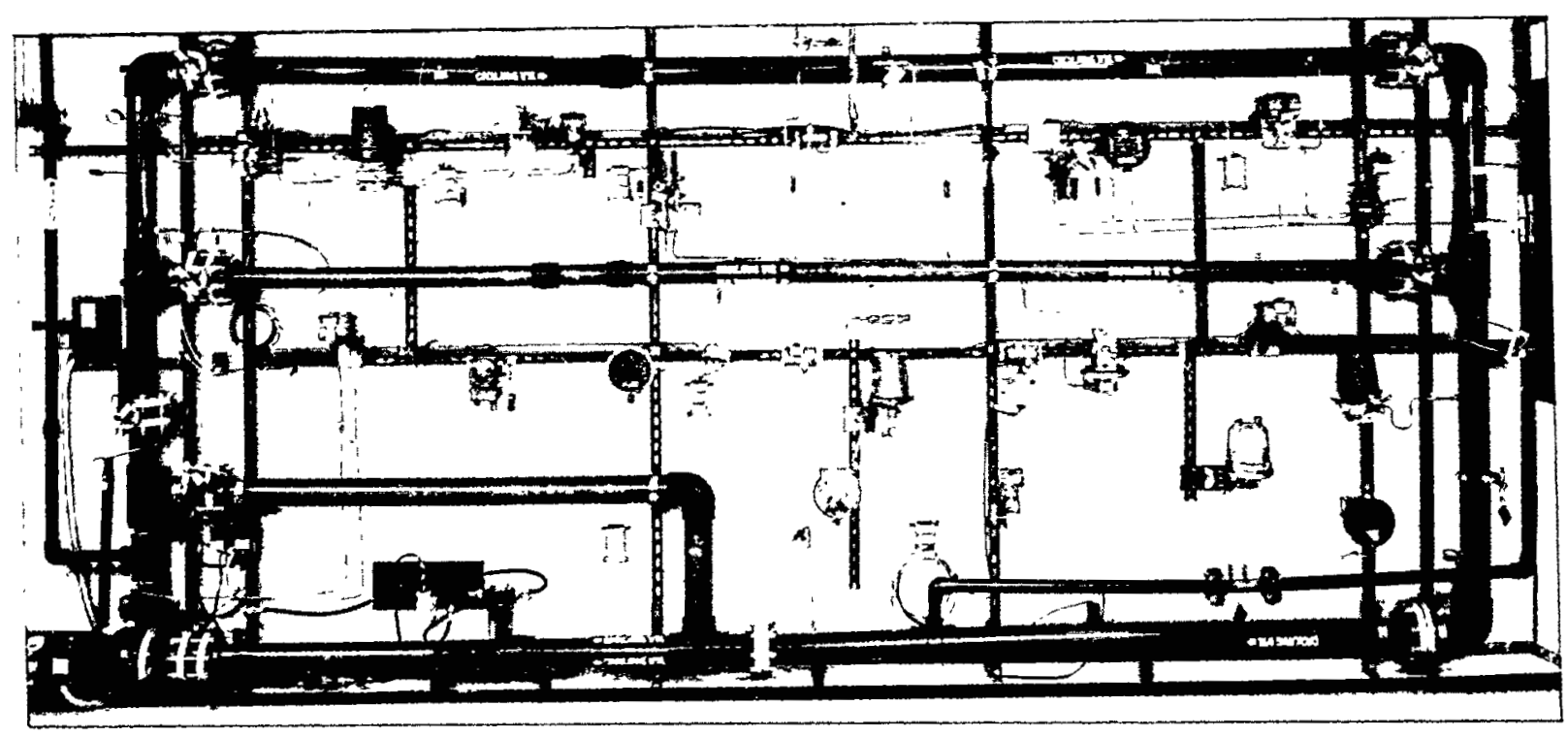

Figure 14.1 Photograph of Laboratory Test Loop 


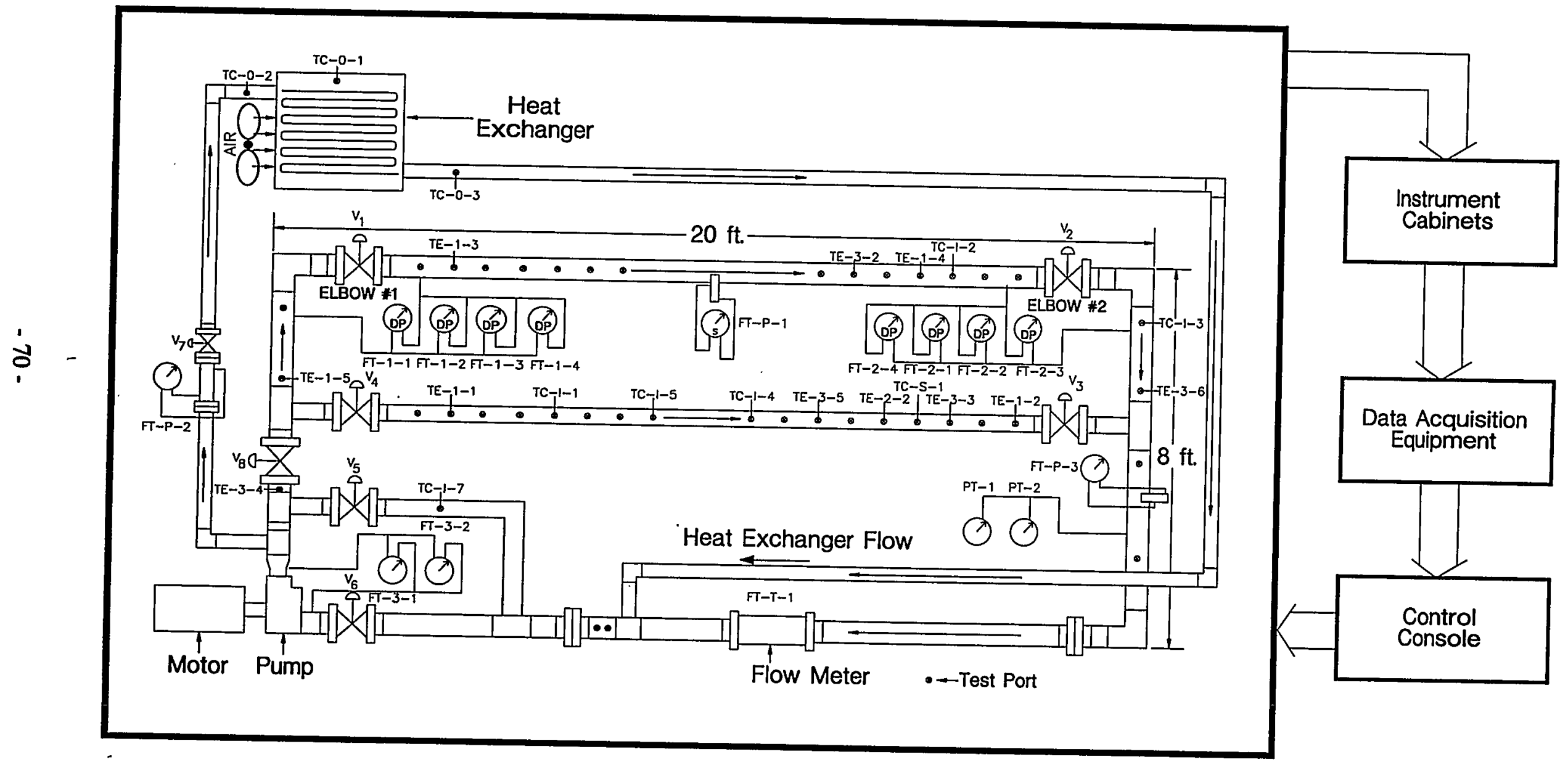

Figure 14.2 Schematic of Laboratory Loop 


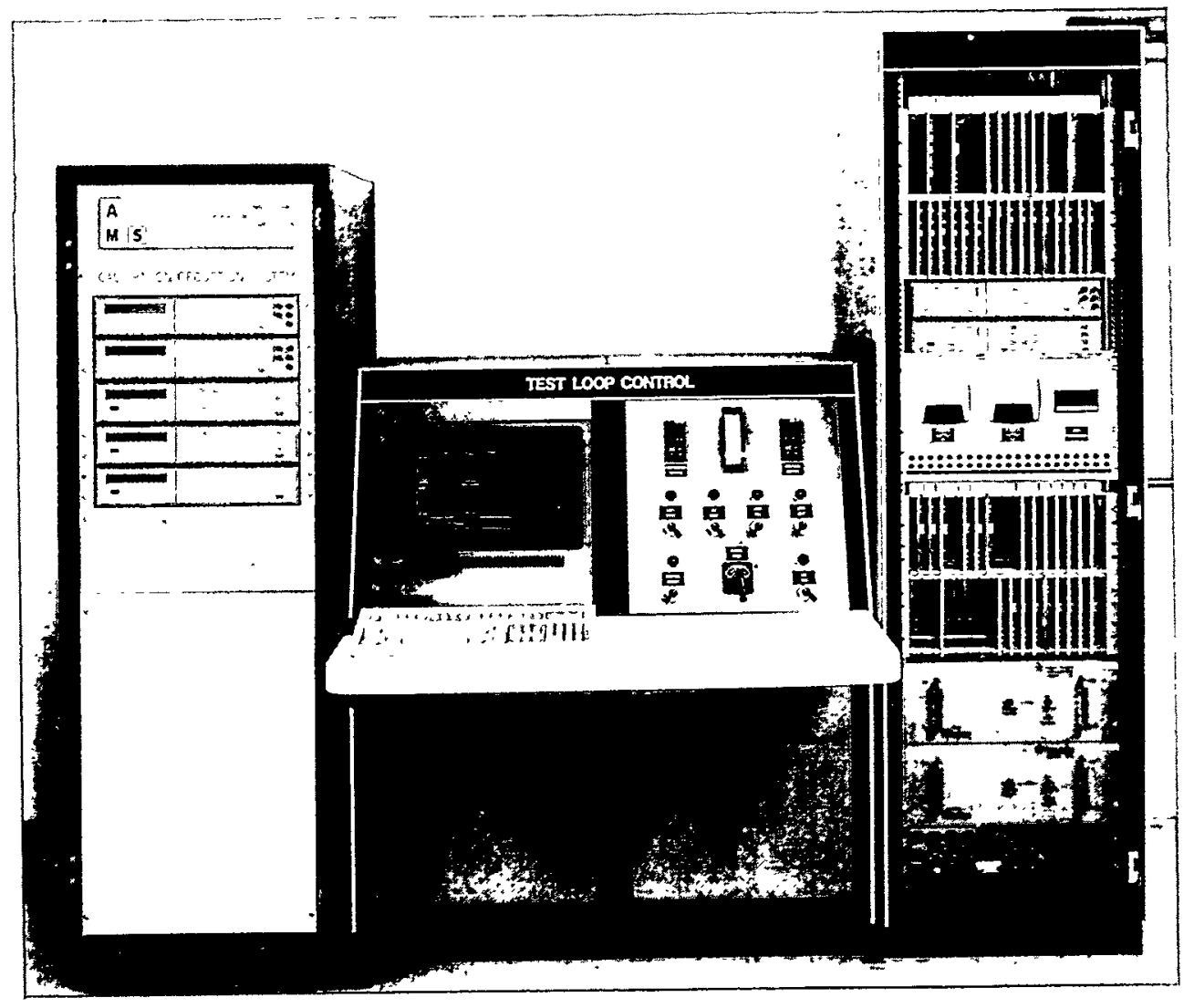

Figure 14.3 Photograph of 7300 Instrumentation System and Loop Operations and Control Console 


\subsection{In-Plant Validation Tests}

As mentioned before, the in-plant demonstration of on-line drift monitoring techniques was carried out at the McGuire Nuclear Power Station Unit 2. Data were recorded for two fuel cycles from approximately 170 signals involving eighteen categories of services in the primary and secondary systems of the plant. Table 14.1 gives a listing of these signals followed by Figure 14.4 which is a simplified drawing of one of the four coolant loops of the McGuire plant showing the typical locations of the sensors that were monitored for this project.

Both steady-state (DC) and transient (AC) data were sampled at McGuire, stored on computer disks, and subsequently analyzed at AMS. The DC data were used for validation of on-line calibration testing techniques, and the $\mathrm{AC}$ data were used to validate reactor diagnostic techniques and assess the validity of other dynamic tests. This report, however, is concerned only with the use of the McGuire DC data for instrument calibration verification. As such, no discussions are included in this report on the analysis of the $\mathrm{AC}$ data from the McGuire plant.

Figure 14.5 shows a block diagram of the on-line monitoring system that was installed at
McGuire, and Figure 14.6 shows how this system was connected to one of McGuire's instrument channels. Attempts were made in installing the on-line monitoring system to include as many components of the instrument channels in the on-line monitoring tests as possible. Note that signals from the McGuire plant were already isolated before connection to the multiplexer unit in the on-line monitoring system. As such, the isolation unit shown in Figure 14.5 is redundant to the plant's isolation devices.

The on-line monitoring system installed at McGuire consisted of a data acquisition cabinet and a data storage computer. The data acquisition cabinet included a number of Hewlett Packard multiplexers, and a digital multimeter to acquire and digitize the data. The data were then sent to the computer via an IEEE-488 bus where they were stored on hard disks and magnetic tapes. The data were then sent to AMS for analysis.

The on-line monitoring system also included a constant voltage power supply with a 5 volt DC output which was monitored along with the plant signals to detect any drift in the online monitoring system itself. The calibration of the on-line monitoring system is discussed further in Chapter 18. 


\section{TABLE 14.1}

\section{Listing of Signals Monitored at McGuire Unit 2}

Item

Description of Signals

Number of

Signals

\section{Pressure Signals}

Steam Flow

8

2

Steam Pressure

12

3

Steam Generator Level

20

4

Feedwater Flow

8

Auxiliary Feedwater Flow

4

6

Reactor Coolant Flow

12

Pressurizer Level

3

Pressurizer Pressure

4

Wide Range Reactor Coolant Pressure

2

Containment Pressure

3

Reactor Vessel Level Indicating System (RVLIS)

6

Turbine Impulse Pressure

Temperature Signals

13

14

Narrow-Range RTDs

16

Wide Range RTDs

8

15

Core Exit Thermocouples

40

\section{Other Signals}

16

Neutron Flux Detectors (NI Channels)

12

17

$\Delta T$ signals

$\mathrm{T}_{\text {ave }}$ signals

4

4

\section{Calibration Signals}


RVLIS - Reactor Vessel Level Indicating System

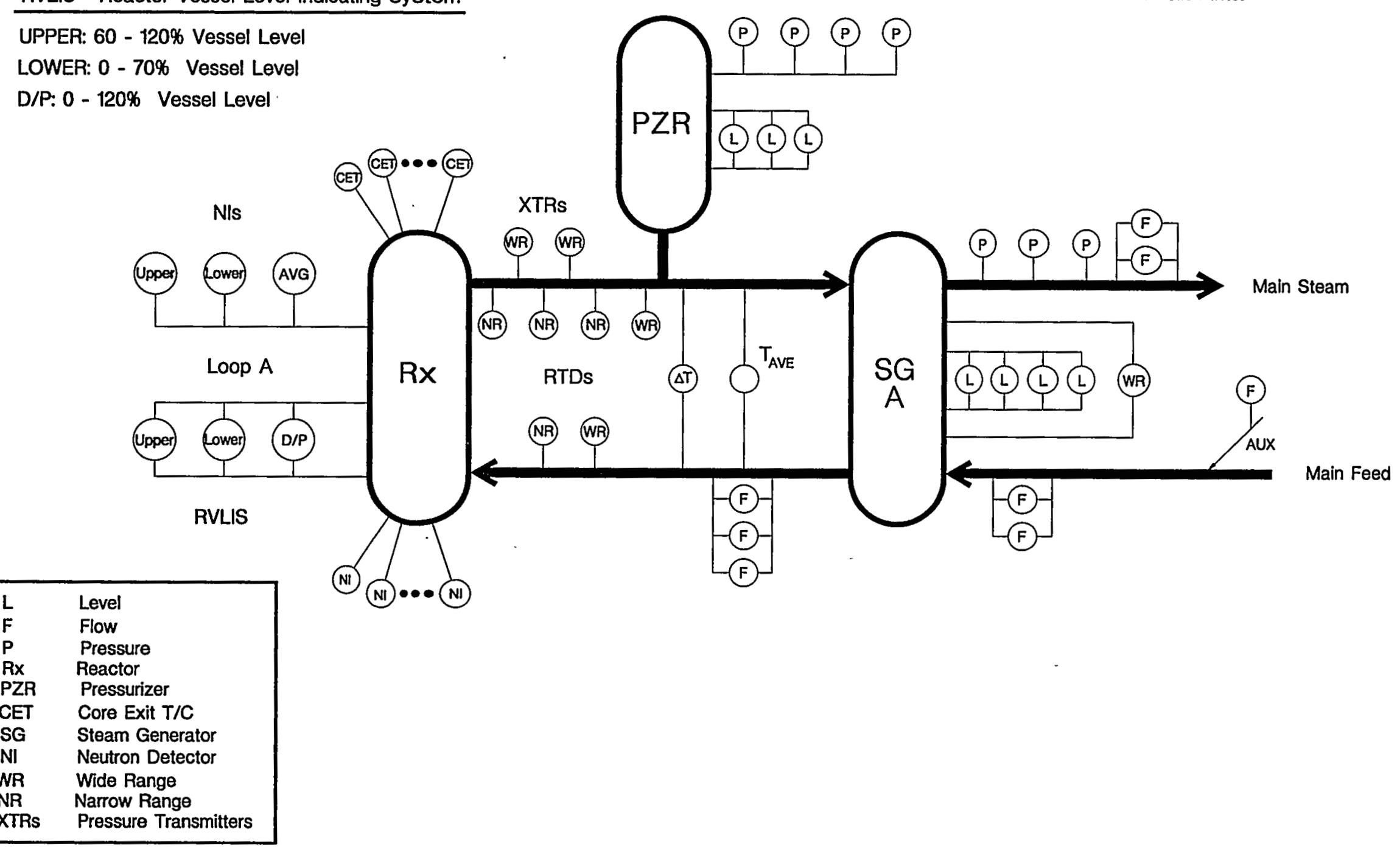

Figure 14.4 Simplified Schematic of One of the Four Coolant Loops of McGuire Nuclear Plant 


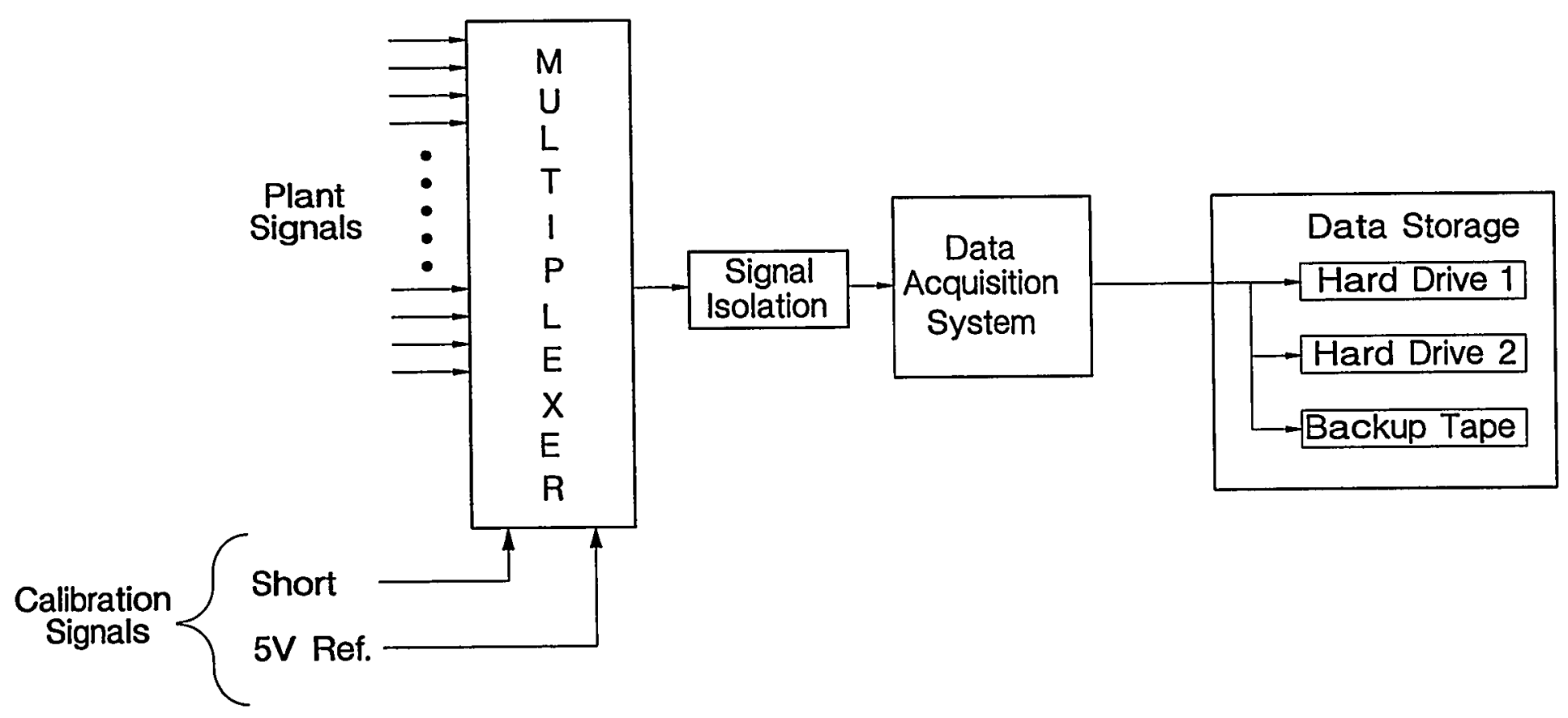

Figure 14.5 Block Diagram of On-Line Monitoring System Used at McGuire 


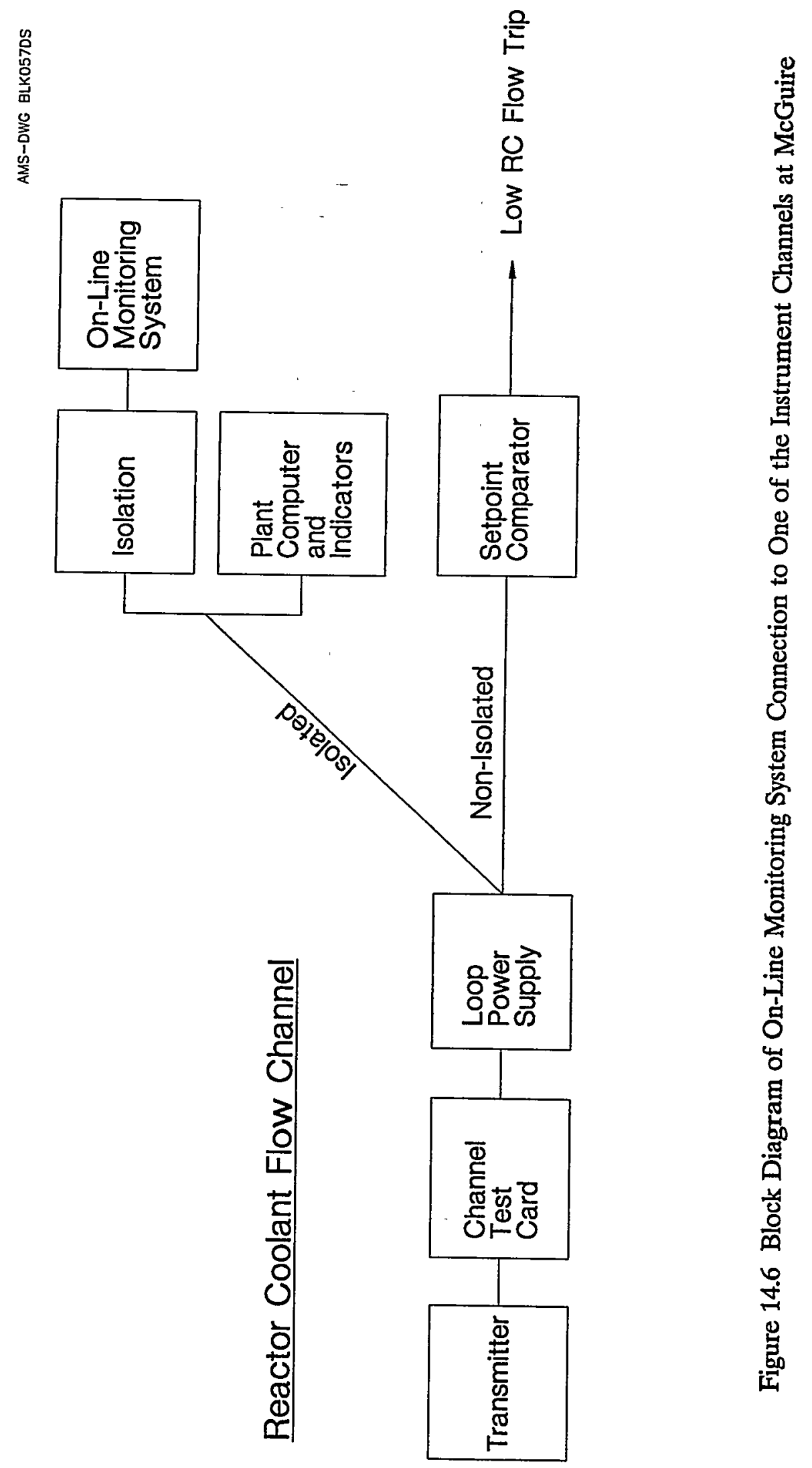




\section{LABORATORY VALIDATION RESULTS}

The purpose of the laboratory tests was to demonstrate that the data acquisition, data analyses, and the interpretation of results of on-line monitoring tests for the sensors in the laboratory test loop all occur as expected and thereby validate the data acquisition and data analysis algorithms, software packages, and the interpretation procedures.

The laboratory validation tests involved numerous series of simple experiments. In most of these experiments, drift was induced in the sensors or the process (i.e., the test loop) to verify that: (1) the drift identified by on-line monitoring system compares well with the induced drift, and (2) the data processing algorithms and interpretation procedures can distinguish between process drift and instrument drift. The results of these experiments are discussed below in terms of equipment validation, software validation, validation of empirical models and neural networks, and other tests. In most of the laboratory tests, pressure or differential pressure transmitters were used as these are the sensors of main interest in this project.

Validation of physical models was also attempted during the laboratory tests, but useful results to be included here were not obtained. As indicated earlier, it was concluded, through the efforts spent in this project, that physical models are not as practical and useful as empirical models and neural networks for on-line calibration tests. As such, very few results are provided in this report involving physical models.

\subsection{Data Acquisition System Validation}

Figure 15.1 shows typical results involving four pressure transmitters that were used in testing the data acquisition system developed in this project. Results are shown in this figure in terms of time history plots and bar charts.

The time history plots in Figure 15.1 show the drift that was induced in the output of each sensor. The drift was induced by adjusting the zero, span, or gain of the sensor electronics or the signal conditioning equipment. The bar charts show the comparison between induced drift as identified by manual calibrations and detected drift as identified by the on-line monitoring system. The small differences between the two results verifies that the on-line monitoring system performs properly. Note that the process pressure during the experiments shown in Figure 15.1 was stable as indicated by the reference transmitter.

Figure 15.2 shows results from a similar laboratory experiment in which the process was drifted in addition to the pressure transmitters. Again, the bar charts show that the differences between the induced drift and the detected drift are small thus verifying the proper operation of the data acquisition system. Experiments of this type were repeated numerous times with various combinations of sensors to verify the validity of the algorithms, equipment, and software packages developed in this project. Figures 15.3 and 15.4 show results for two cases where the loop flow was manipulated during data collection to generate plant-type data and verify the equipment operation. Data are shown in Figure 15.3 for two Foxboro and one Statham pressure transmitters and a reference sensor. The reference sensor is a Rosemount smart transmitter which provided information regarding the input to the transmitters under test. The bar charts in this figure again verify that the equipment and techniques are working as desired. Figure 15.4 provides similar data for a Barton, a Honeywell, and a 

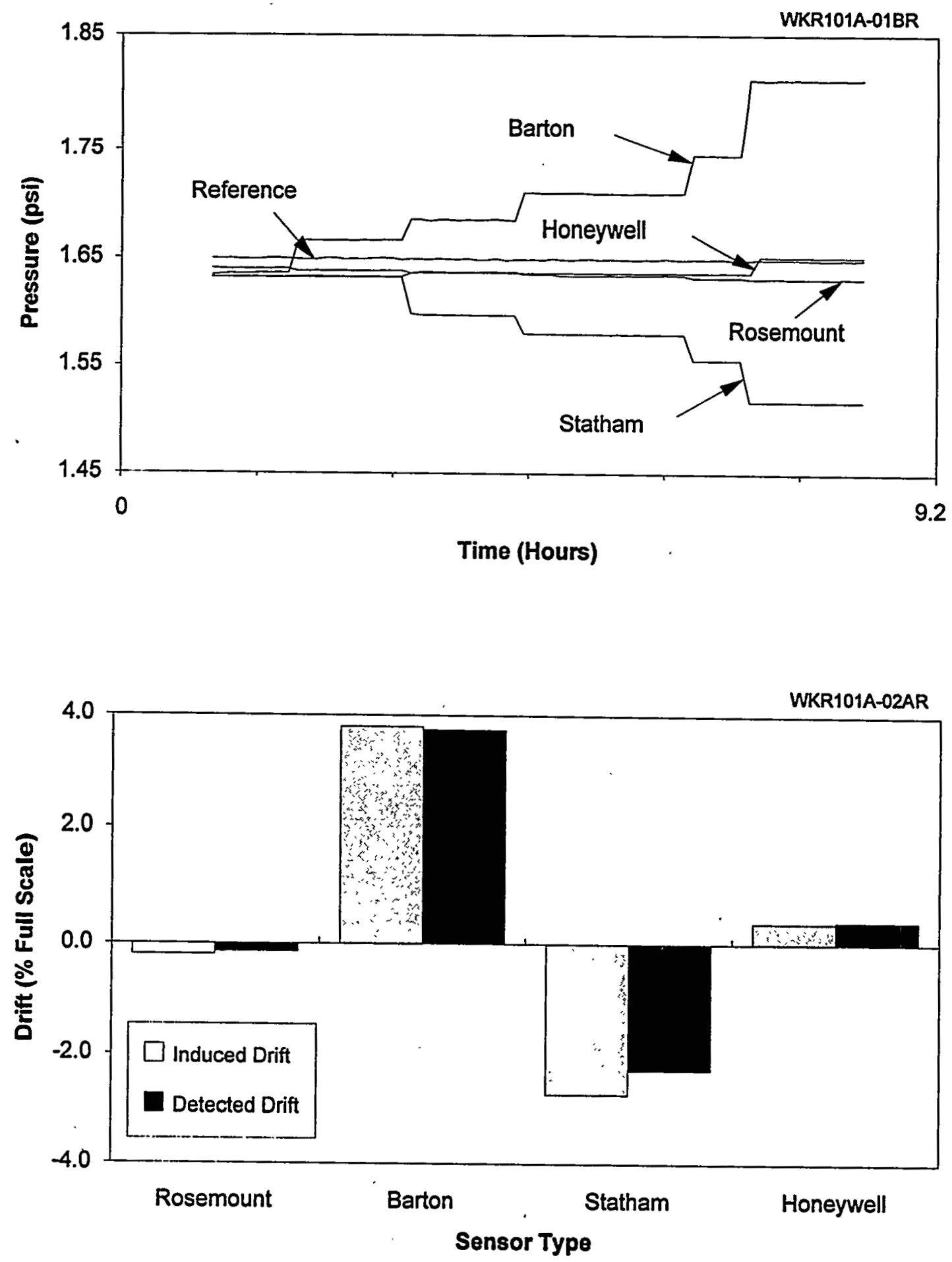

Figure 15.1 Laboratory Test Results when Sensors were Drifted and Process was Stable 

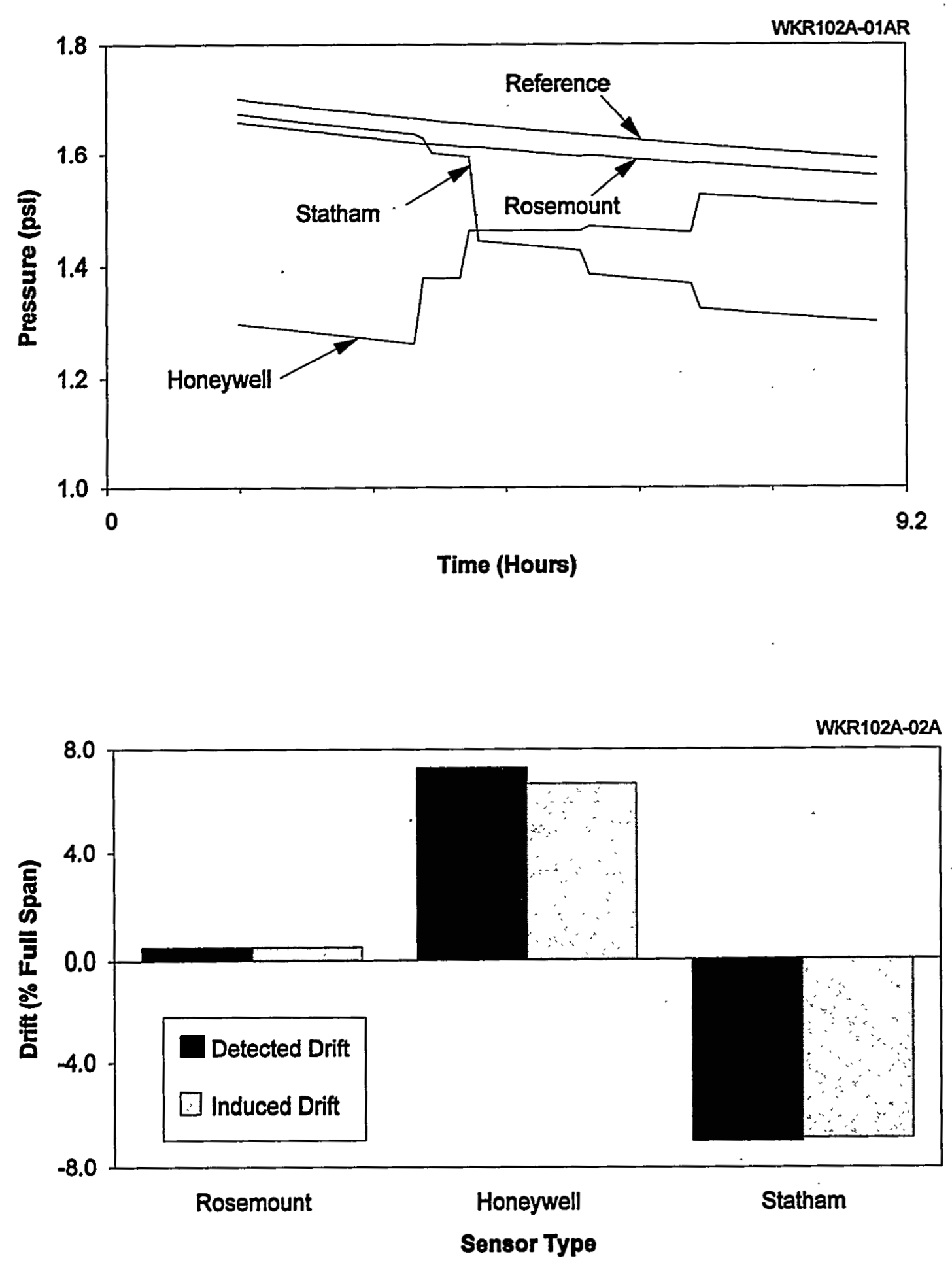

Figure 15.2 Laboratory Test Results when Both the Test Sensors and the Loop Pressure were Drifted 

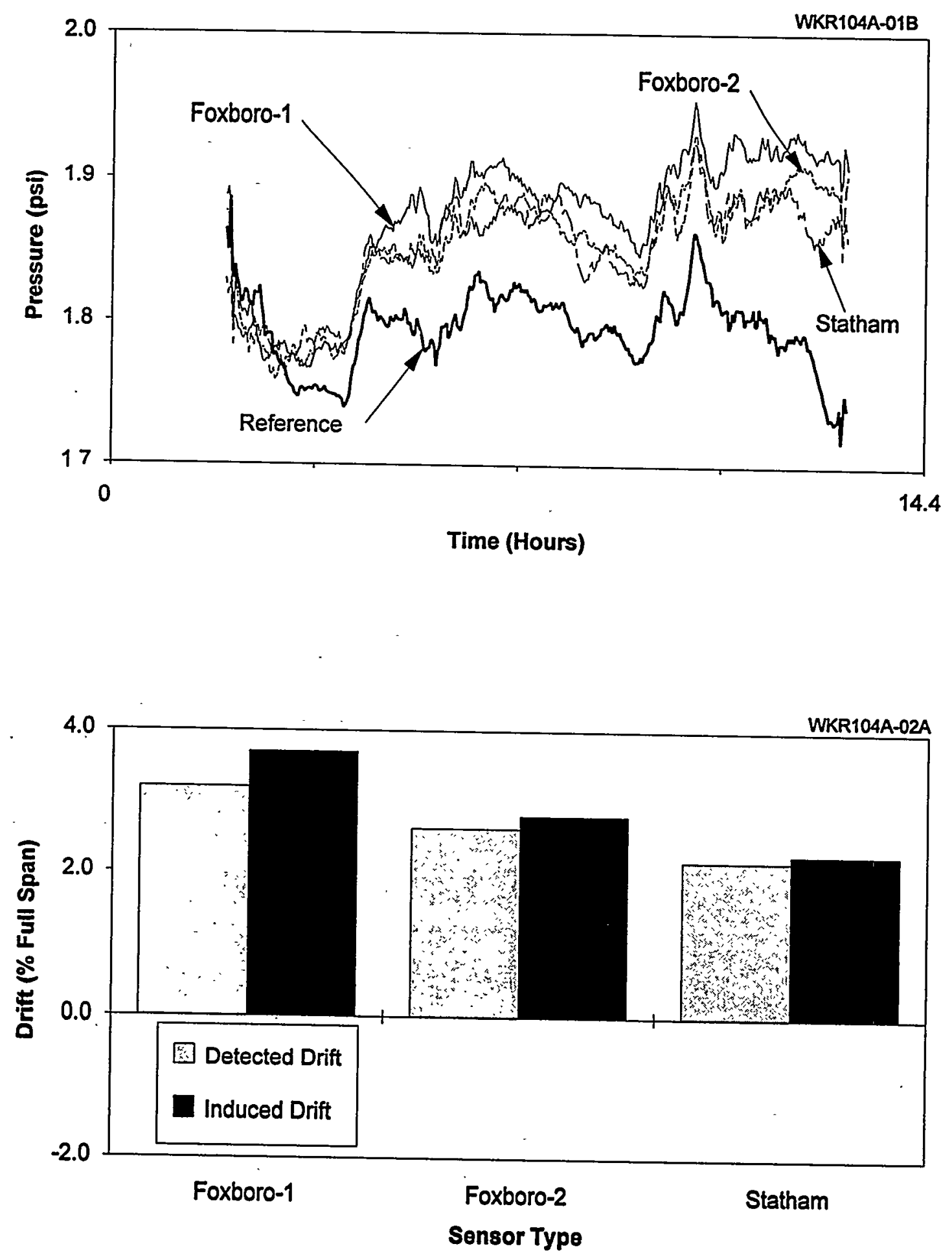

Figure 15.3 Laboratory Test Results with Loop Flow Manipulated to Simulate Plant-Type Data 

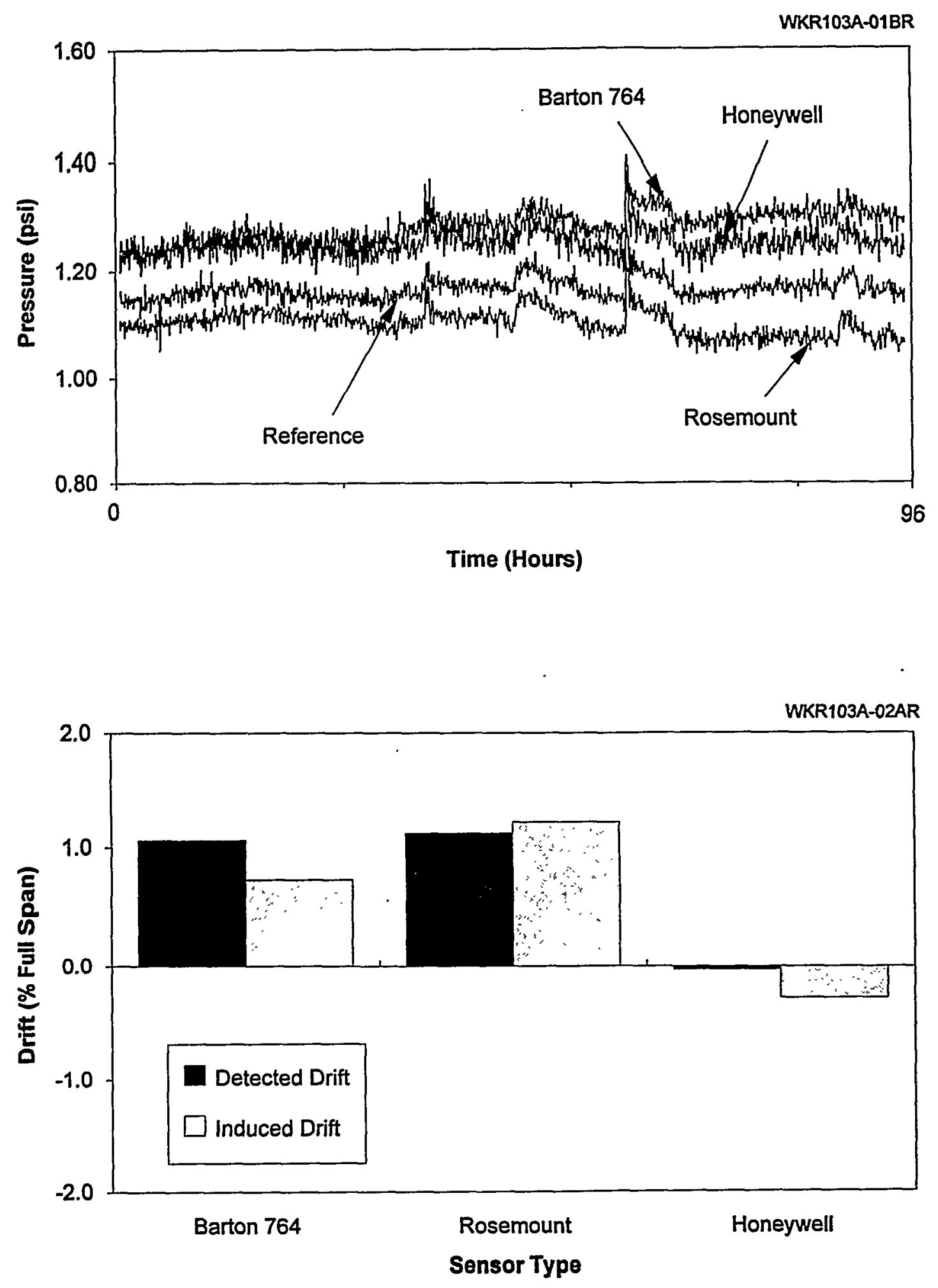

Figure 15.4 Test Results with Plant-Type Data Generated in the Laboratory 
Rosemount transmitter. The test results are good except for the Honeywell transmitter. This is a non-nuclear grade transmitter that was included in the project for comparison with nuclear-grade transmitters.

Figure 15.5 shows raw data for three flow transmitters as drift was being induced in.one of the transmitters. Data are shown in terms of both measured differential pressure and the deviation of individual pressure signals from the average of the three signals. The deviation plot shows that the drift in one of the three sensors causes the other two sensors to appear as if they are drifting as well. The drift appears until after the failed transmitter has been identified by a consistency checking algorithm and removed from the average. The criteria for determining when to exclude a sensor from the average depends on the application and must be specified by the user. It will be seen later in this chapter that the use of empirical models and neural networks can facilitate the identification of drifting instruments.

In presenting laboratory test results in the remaining parts of this chapter, consistency checking or parity space techniques were not used. These techniques are normally used during the analysis of on-line monitoring data to exclude the signals which have a large drift. They were not used in analyzing the laboratory test data to allow us to demonstrate the behavior of drifting signals.

\subsection{Software Validation}

An important aspect of the laboratory tests was software validation and Quality Assurance (QA) testing. Software validation work at AMS is usually performed according to formal software $Q A$ procedures which involve documentation of every step of the software development process and testing of the final software with induced (known) input data. Following is a partial listing of software QA guides and standards used by AMS in the development of software products for the nuclear power industry.

- NUREG/CR-4640, "Handbook of Software Quality Assurance Techniques Applicable to the Nuclear Industry," U.S. Nuclear Regulatory Commission, Washington, DC, August 1987.

- ANSI/IEEE Standard 730.1-1989, "IEEE Standard for Software Quality Assurance Plans," American National Standard Institute/Institute of Electrical and Electronics Engineers, Inc., New York, NY.

- IEEE Standard 983, "IEEE Guide for Software Quality Assurance Planning," Institute of Electrical and Electronics Engineers, Inc., New York, NY, February 1986.

- IEEE Standard 1012, "IEEE Standard for Software Verification and Validation Plans," Institute of Electrical and Electronics Engineers, Inc., New York, NY, February 1987.

Being a research and development project, the software QA work performed in this project concentrated mainly on testing the final software products using synthetic analog and digital data. The results of this work are discussed in the following paragraphs.

Figures 15.6 and 15.7 show typical software validation results for two types of simulated drift in pressure transmitters tested in the laboratory: (1) linear drift, and (2) linear drift plus a step change. The results are shown in terms of known drift that was induced in the instruments and drift that was detected by the on-line monitoring software.

Figure 15.6 shows results for three types of drift that were induced in pressure transmitters; small drift, medium drift, and large drift. In all three cases, the differences 

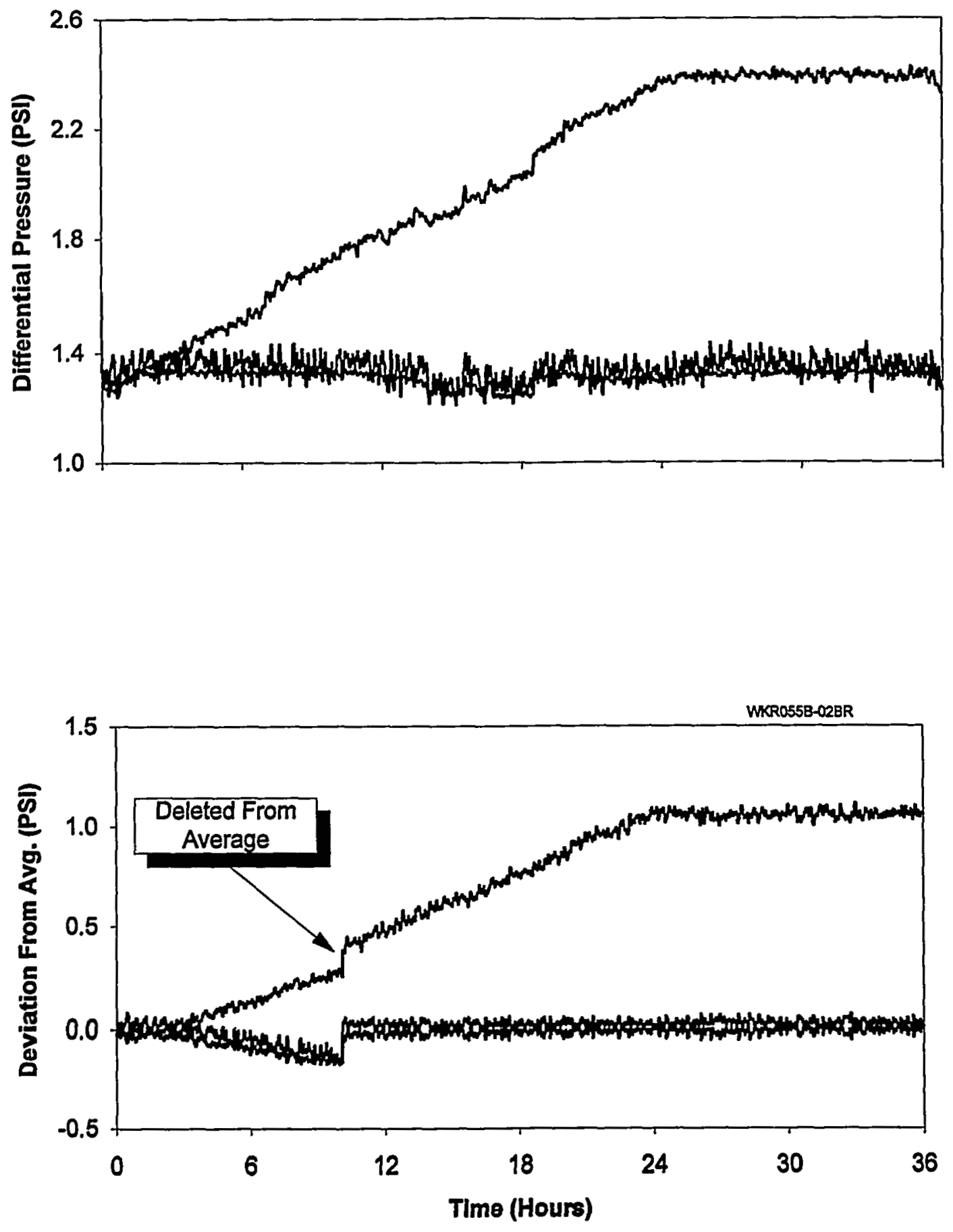

Figure 15.5 Raw Data and Deviation Plot for Three Flow Transmitters 


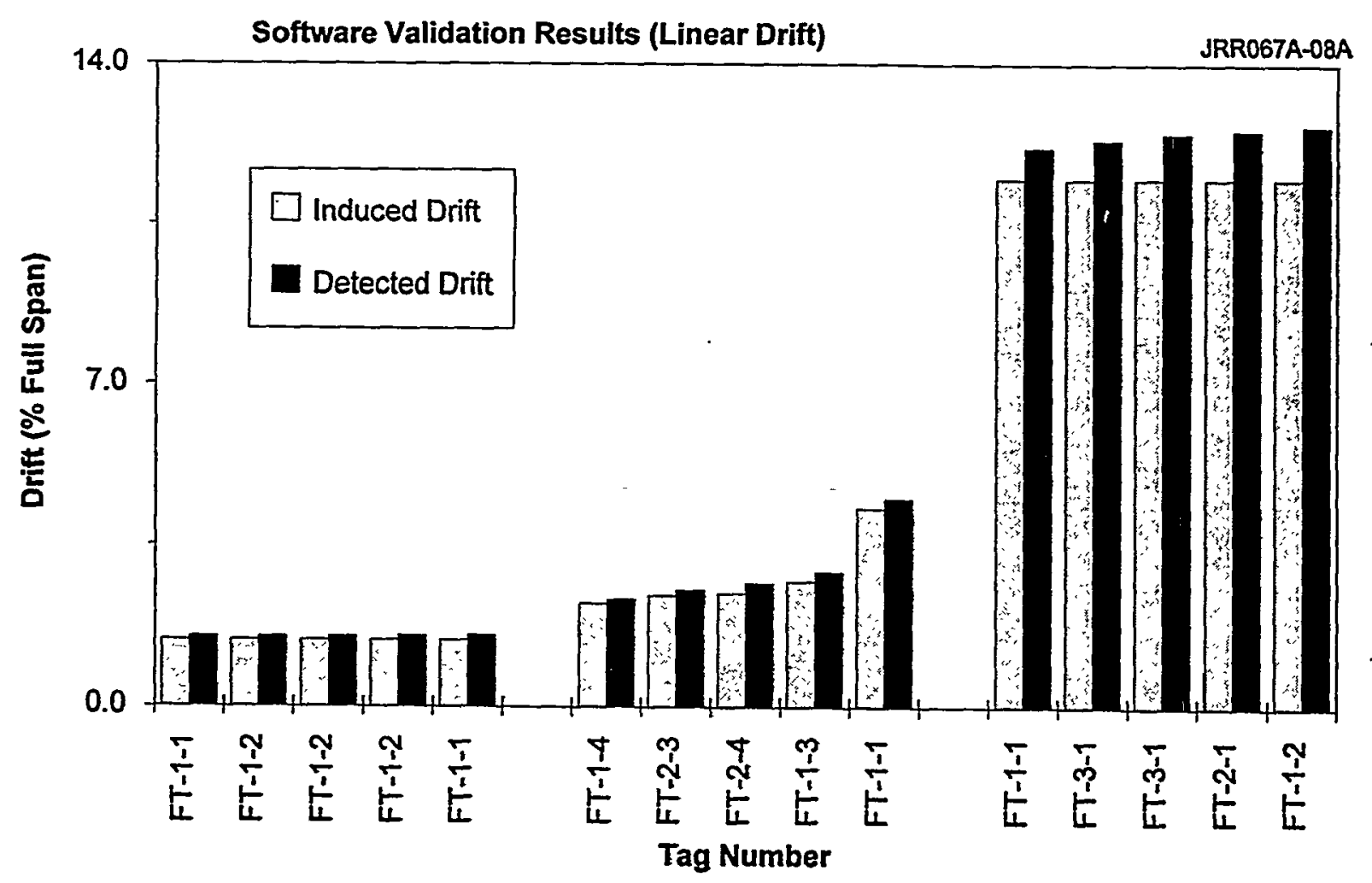

Figure 15.6 Software Validation Results for Flow Transmitters Induced with Linear Drift 


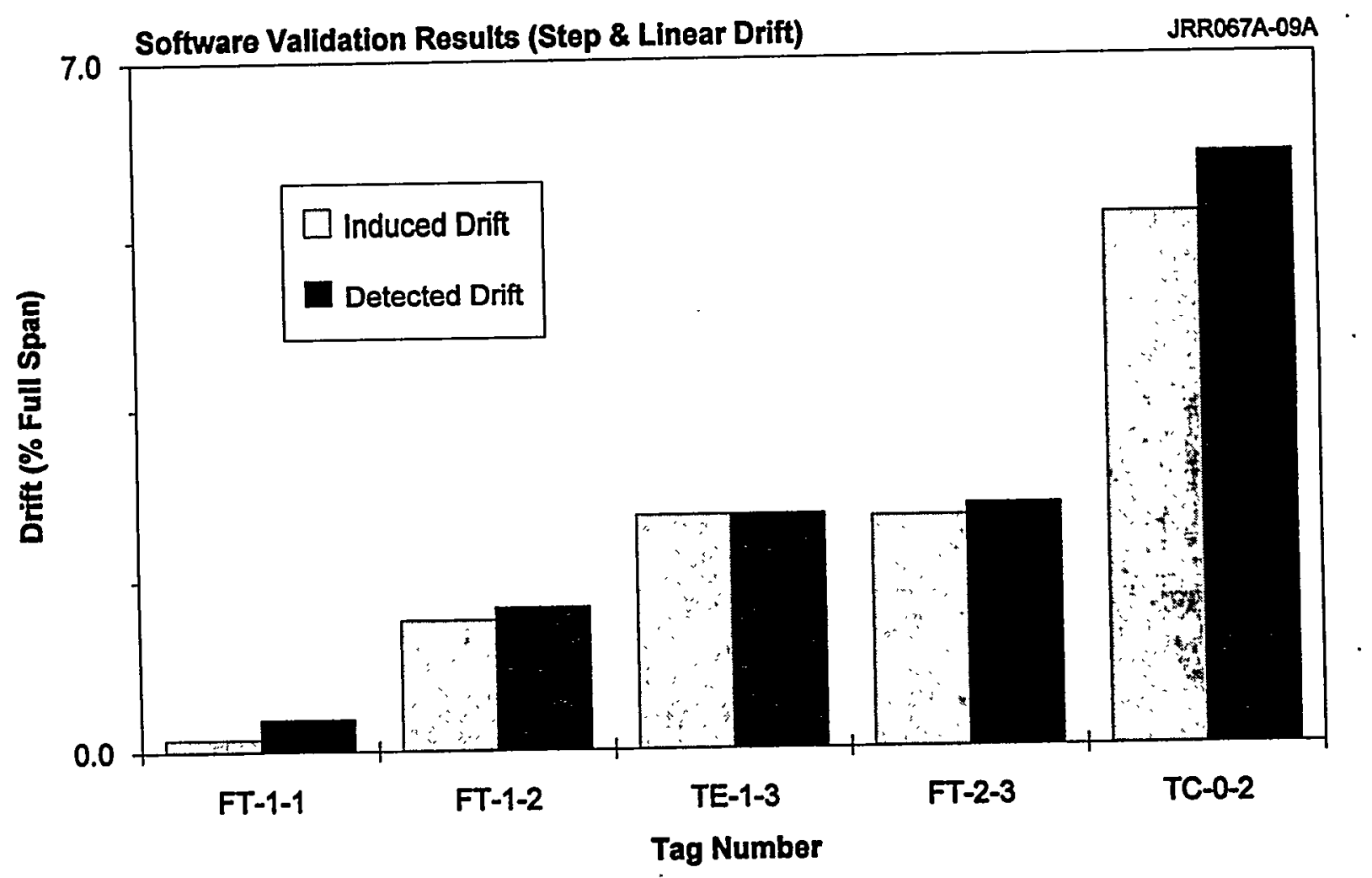

Figure 15.7 Software Validation Results for Temperature and Flow Sensors Induced with a Step-Plus-Linear Drift 
between the induced drift and detected drift is small indicating that the software is performing its function properly. Note that this validation includes the data acquisition, data analysis, and data display software packages as well as the data acquisition hardware. Note also that the results in Figure 15.6 includes repeated tests on the same sensors to validate various aspects of the software or analysis techniques. This is the reason why the tag numbers on the horizontal axis of Figure 15.6 are repeated for some of the sensors.

Figure 15.7 shows software validation results for a combination of flow transmitters and temperature sensors. Each instrument was subjected to a step change followed by a linear input drift. The results show that the induced anomaly is detected reasonably well indicating that the system is functioning as desired.

Figures 15.8 through 15.10 present representative results for three transmitters that were tested individually. Each transmitter was given various amounts of drift through a sequence of test runs as shown in the three figures to demonstrate that the induced drift can be effectively detected independent of its magnitude and direction. One of the three transmitters was a smart transmitter made by Rosemount. This and other smart transmitters tested in this project often yielded excellent agreement between induced drift that was measured by manual calibrations and drift identified by the on-line monitoring system. Note in Figures 15.8 through 15.10, that the run numbers on the horizontal axis of these figures are not in sequence. These numbers correspond to internal tracking numbers that were used during the research to identify the tests. As such, the run numbers do not have any significance.

\subsection{Validation of Analytical Techniques Using Differential Pressure Signals}

Laboratory validation of analytical techniques was performed by inducing drift in one or more redundant sensors, as installed in the laboratory test loop, and plotting the raw data and the results in terms of deviations from the averaging and modeling techniques. The plots are shown in Figures 15.11 through 15.22 for a set of four differential pressure signals obtained across an elbow in the laboratory test loop. These plots are intended to show how the results of averaging, empirical modeling, and neural networks are affected by the number of redundant signals that are monitored together. The discussions that follow describe the results in terms of the number of signals that were intentionally drifted.

\section{One Signal Drifted}

Figures 15.11 through 15.13 show results for when one of four redundant laboratory signals were intentionally drifted to determine how the following three process estimation techniques reveal the problem:

1. Simple averaging of redundant
signals

2. Empirical modeling of the process using temperature, pressure, and flow signals

3. Neural networks trained with temperature, pressure, and flow signals.

Figure 15.11 shows two plots: the raw data and the process estimation results. The raw data shows the three transmitters which follow each other and the one that has a constant drift. This experiment was conducted over a six-hour period. The process estimation results show that the simple average (SAVG) of the four signals is influenced by the drifting transmitter and therefore shows a drift, while the empirical model (EM) and neural network (NN) results correctly estimate the process as being stable. 


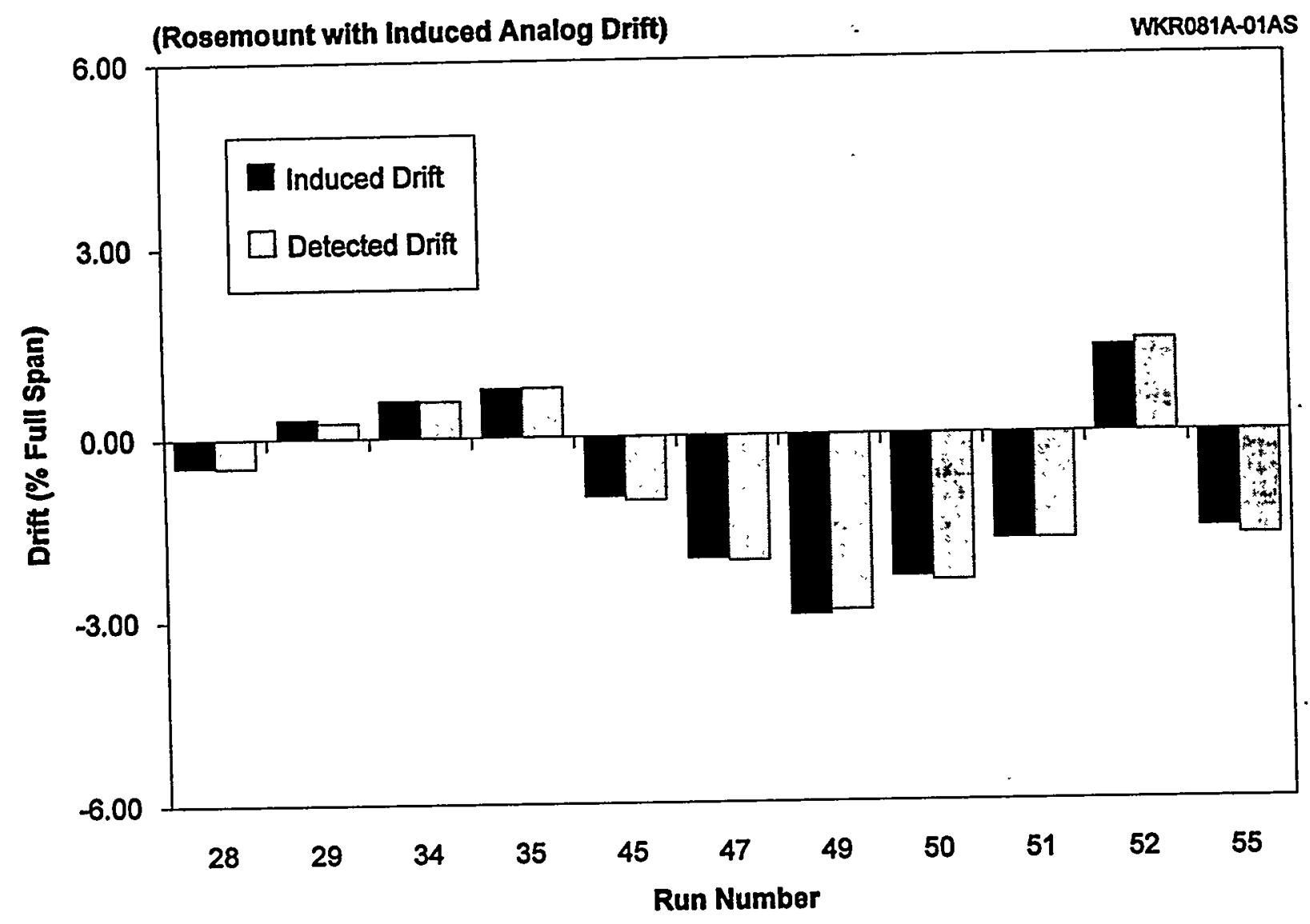

Figure 15.8 Software Validation Results for a Rosemount Smart Transmitter 


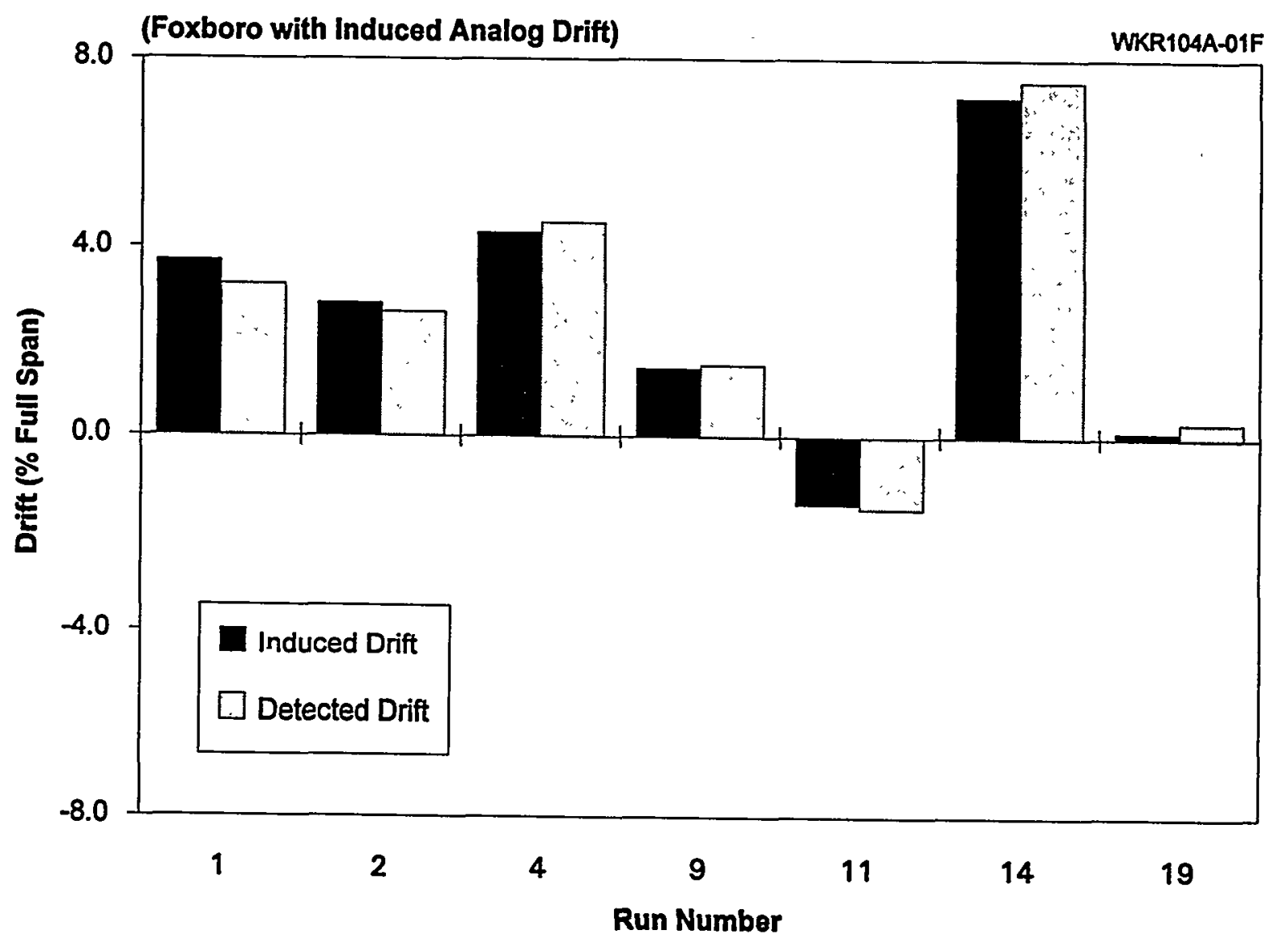

Figure 15.9 Software Validation Results for a Foxboro Transmitter 


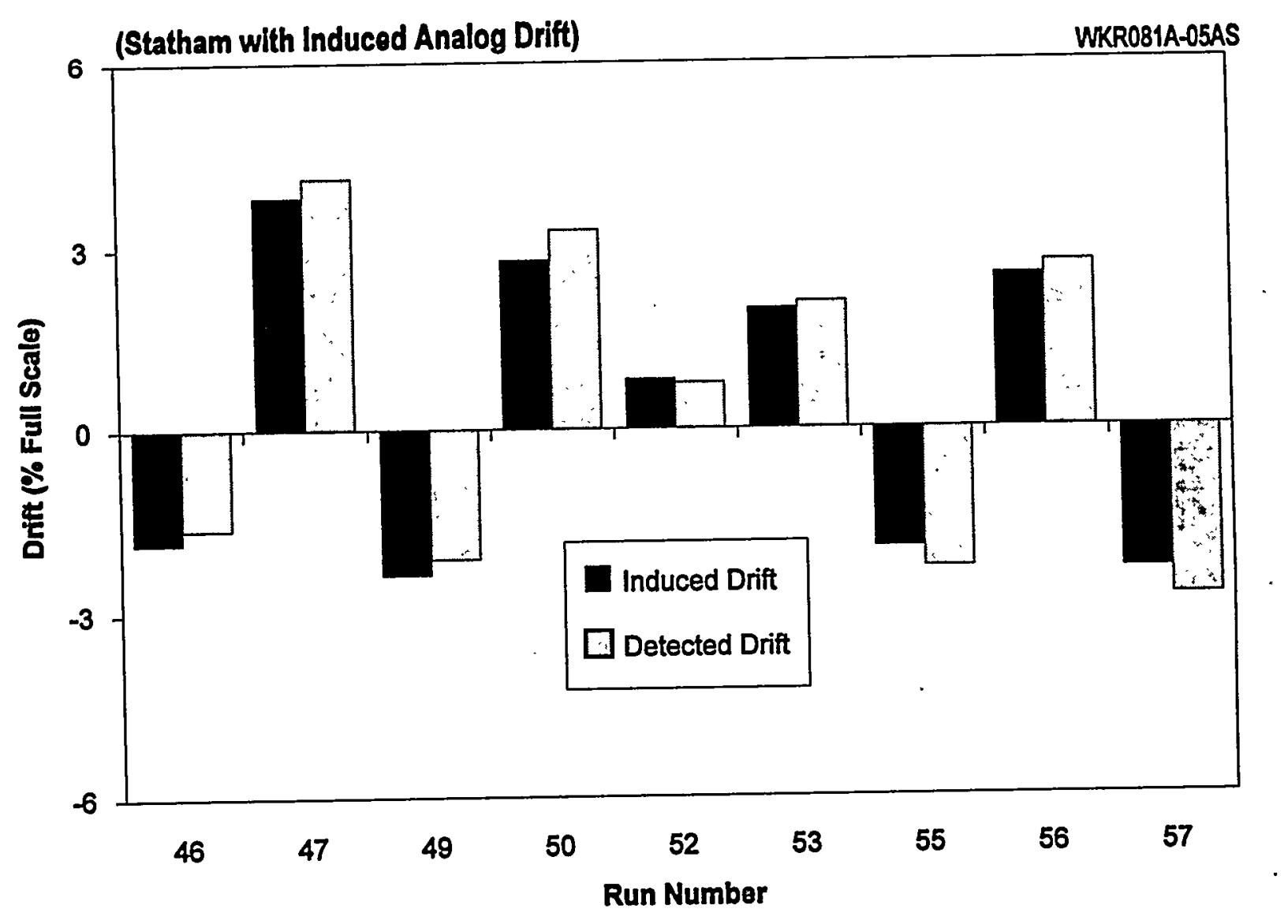

Figure 15.10 Software Validation Results for a Statham Transmitter 

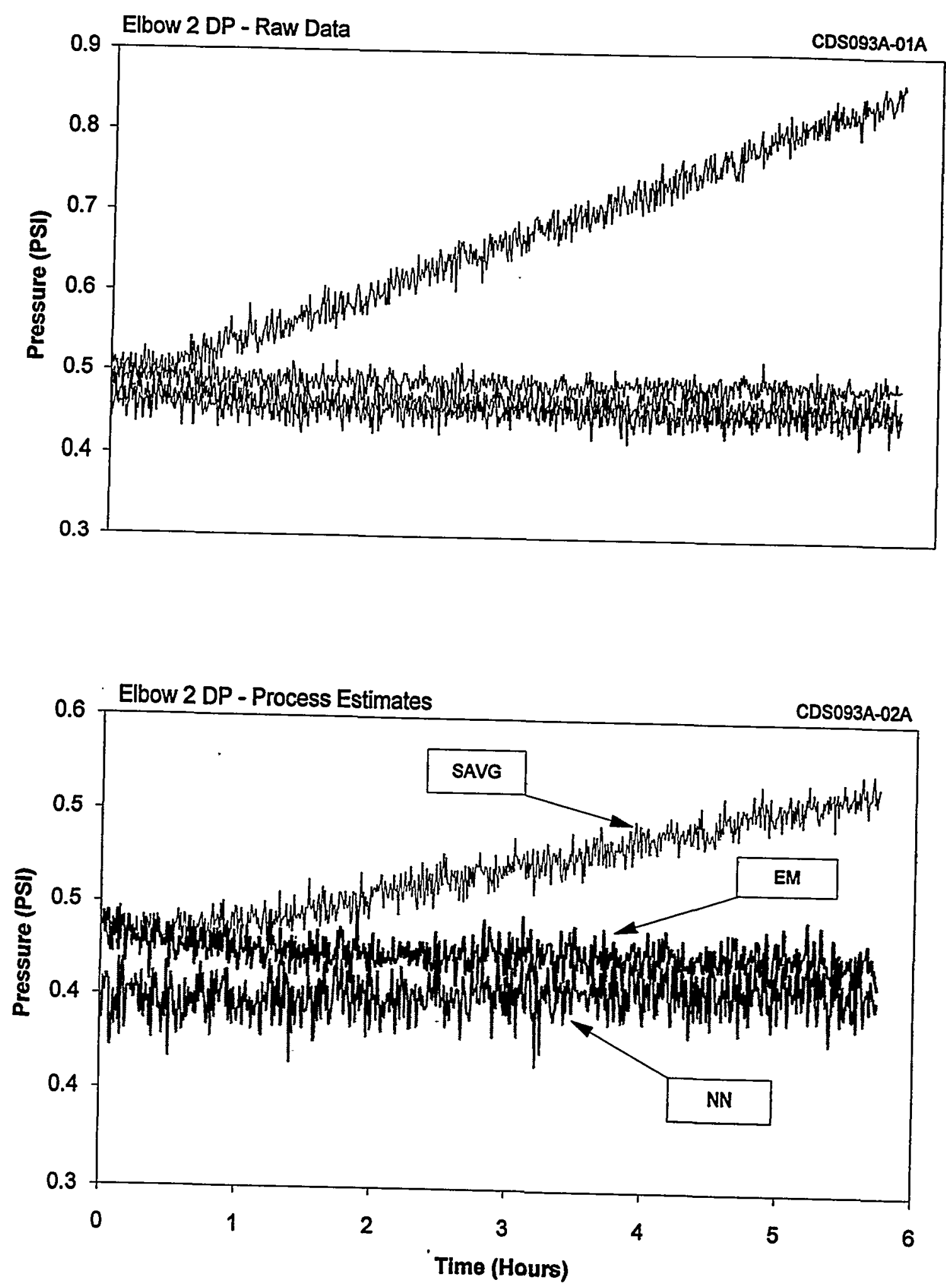

Figure 15.11 Raw Data and Results of Process Estimation Techniques 

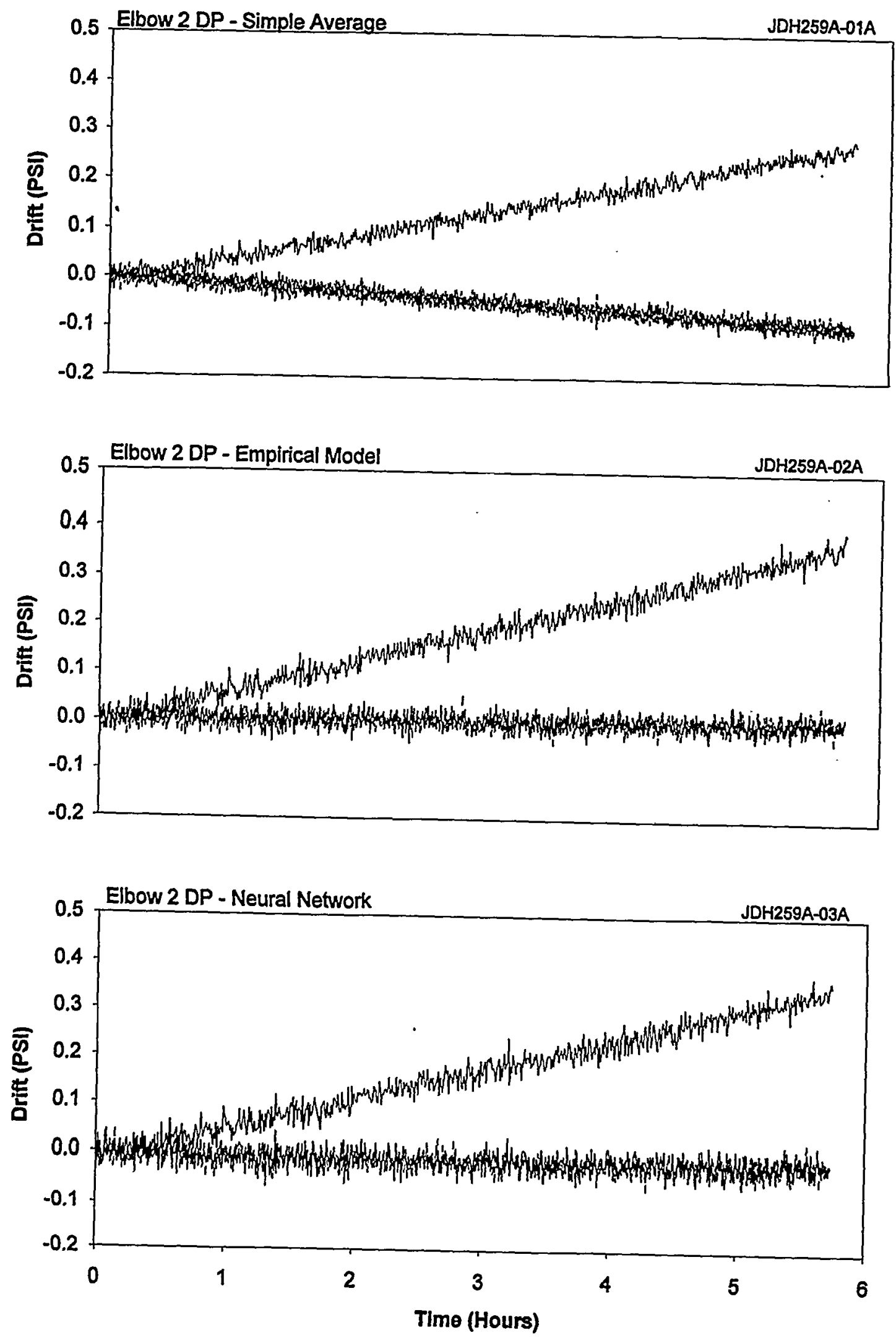

Figure 15.12 Drift Detected by Three Process Estimation Techniques 

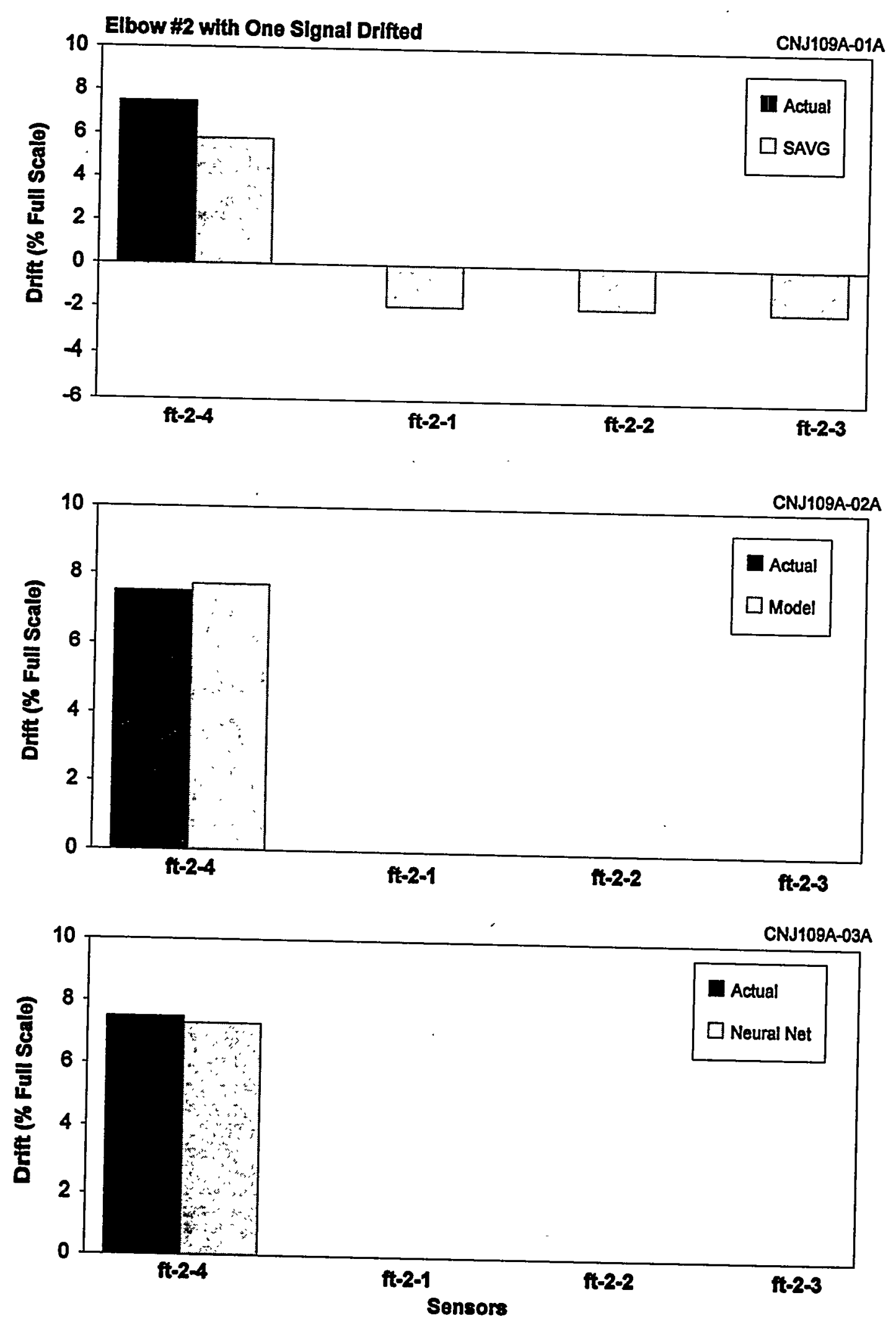

Figure 15.13 Quantitative Results of Process Estimation Techniques 

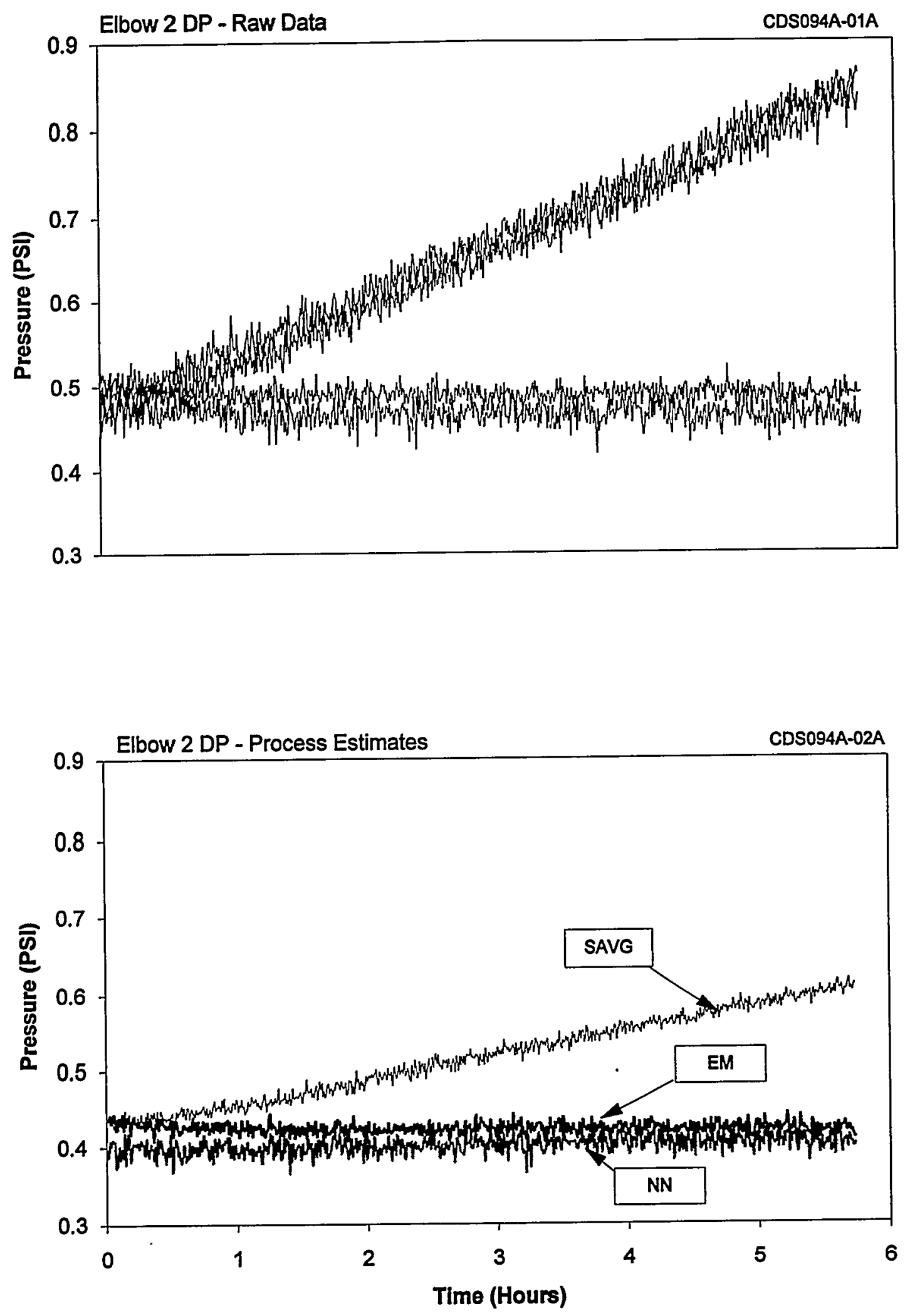

Figure 15.14 Raw Data and Results of Process Estimate Results when Two of Four Transmitters are Drifted 

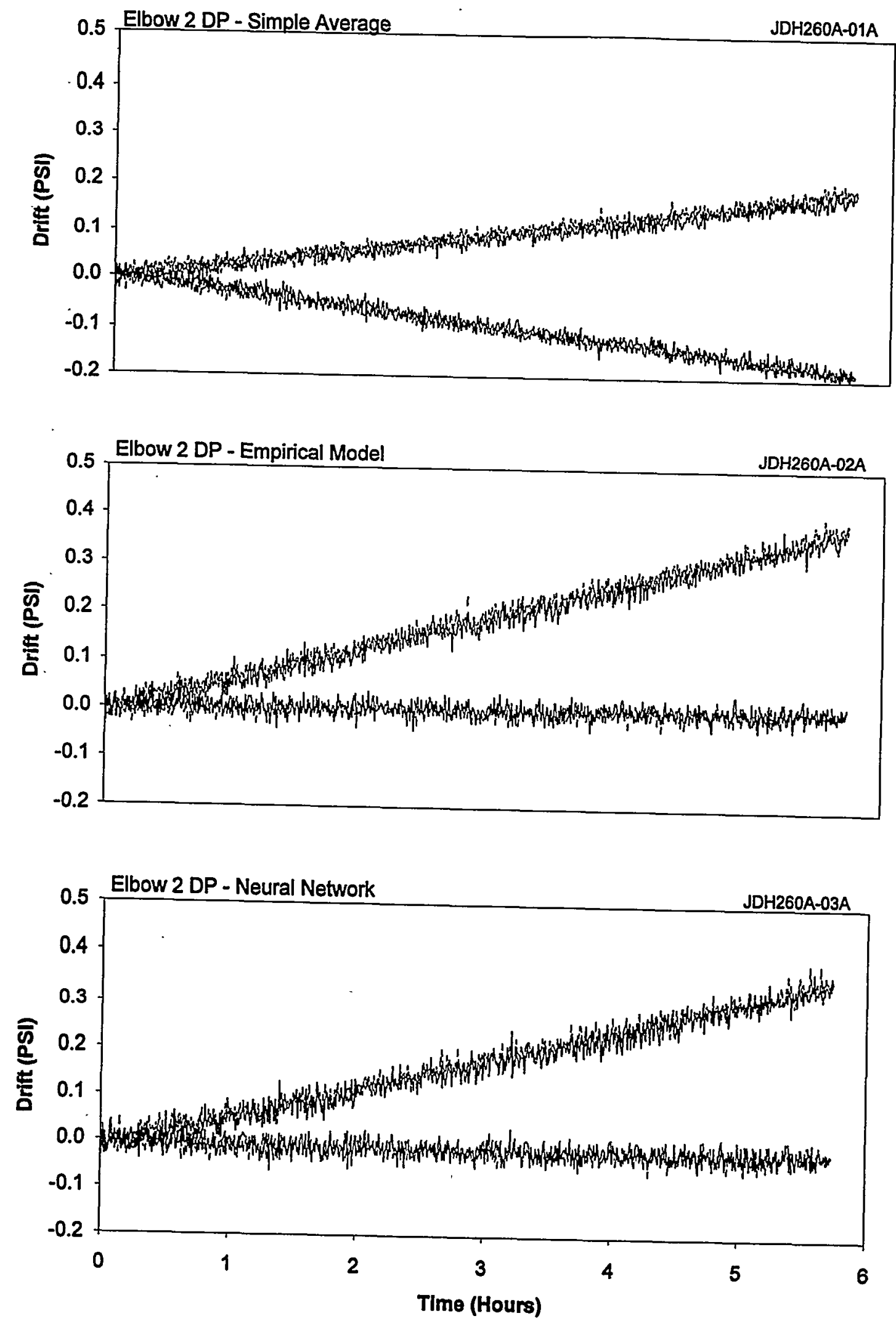

Figure 15.15 Drift Plots when Two of Four Transmitters are Drifted 

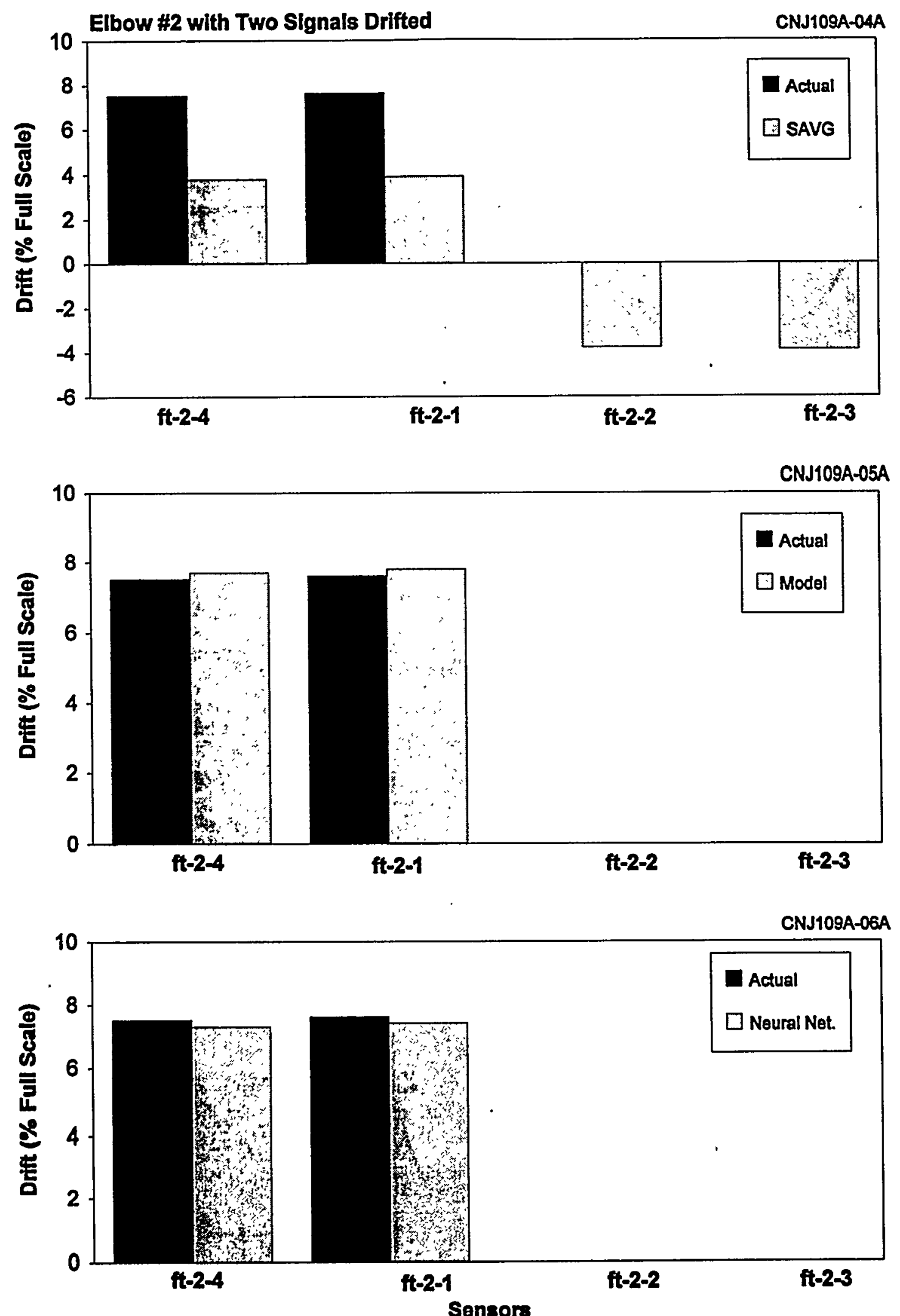

Figure 15.16 Quantitative Results of Process Estimation Techniques when Two Transmitters are Drifted 

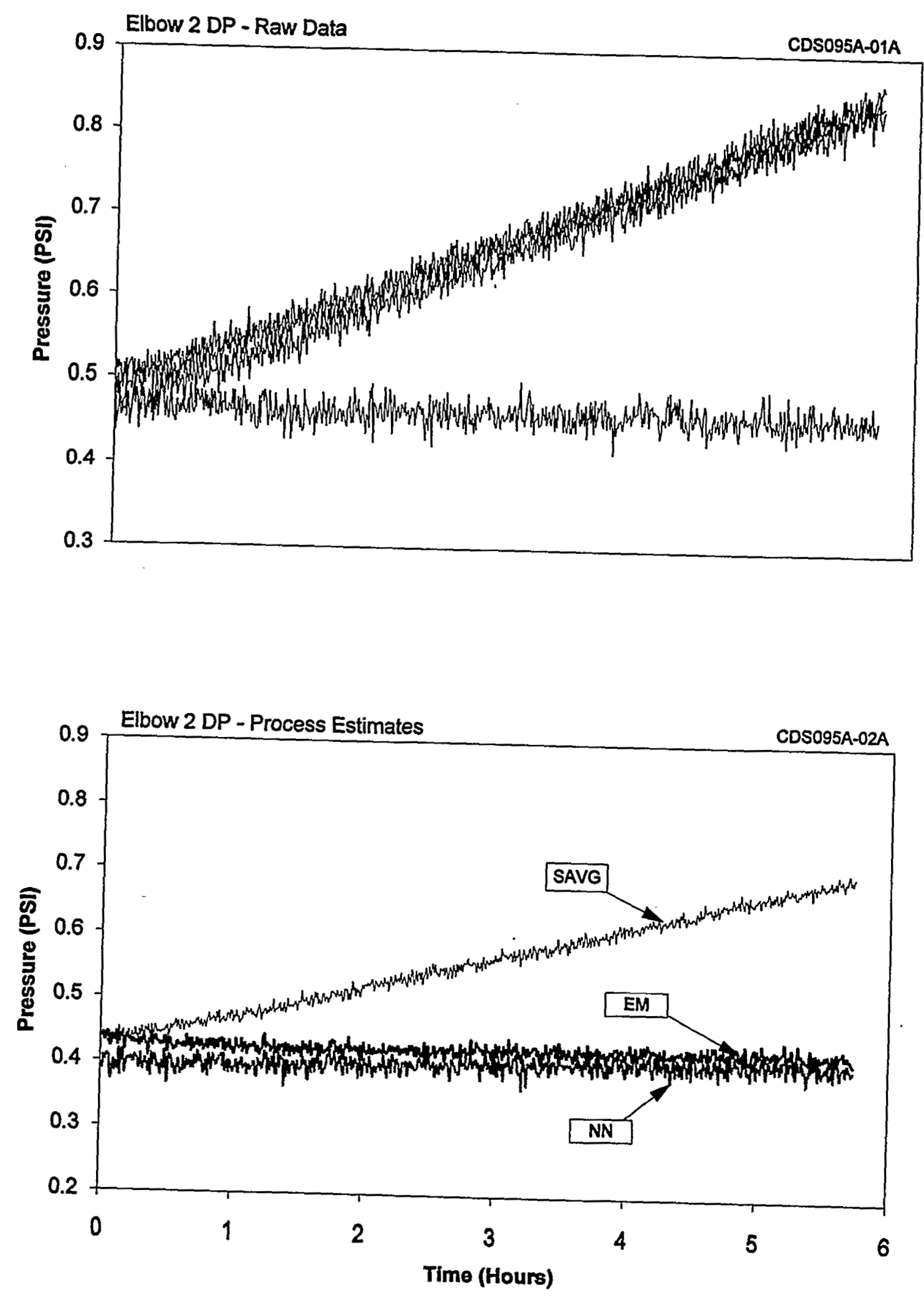

Figure 15.17 Raw Data and Process Estimation Results when Three of Four Transmitters are Drifted 

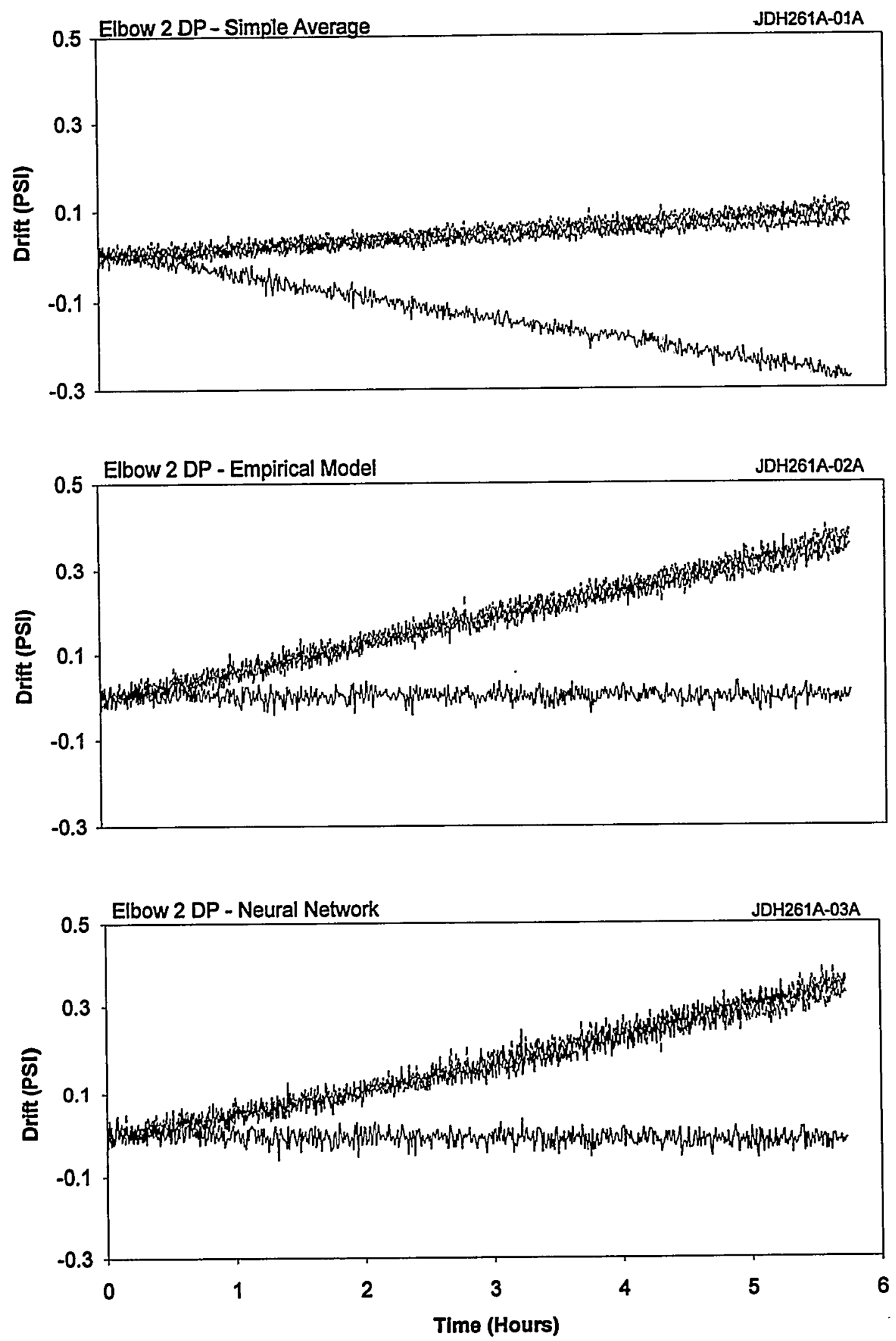

Figure 15.18 Drift Plots when Three of Four Transmitters are Drifted 

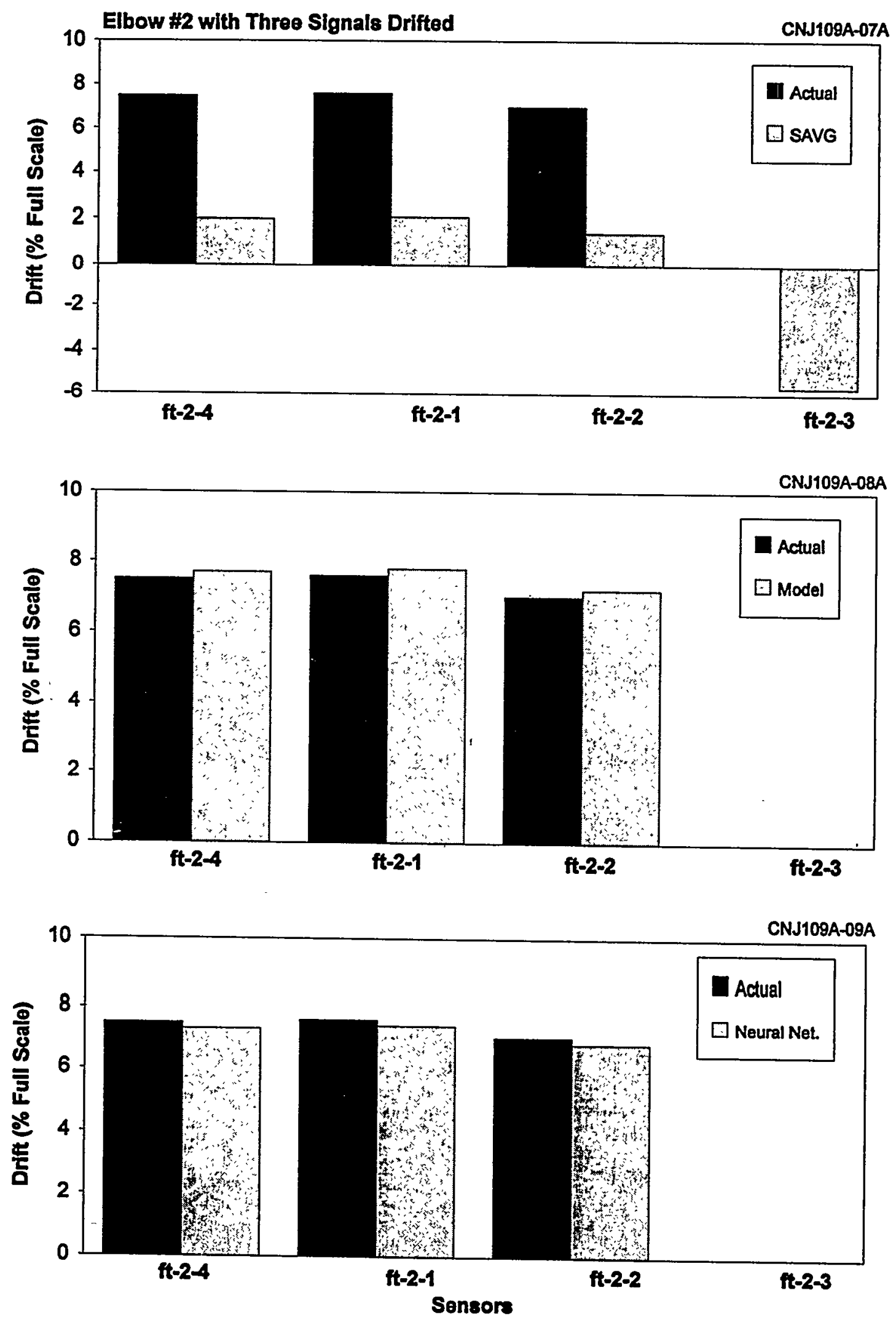

Figure 15.19 Quantitative Results of Process Estimation Techniques when Three Transmitters are Drifted 

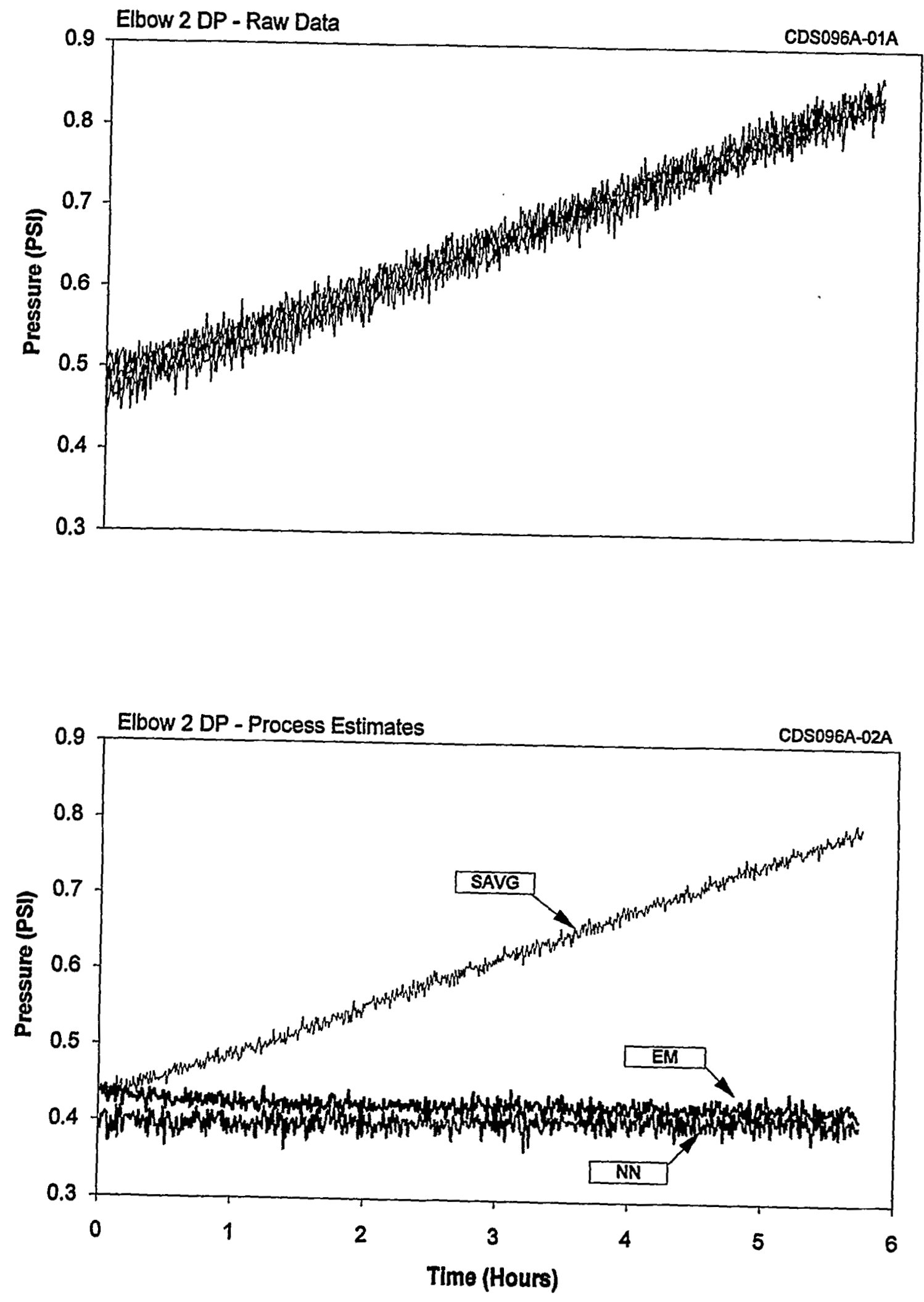

Figure 15.20 Raw Data and Process Estimation Results when Four Transmitters are Drifted 

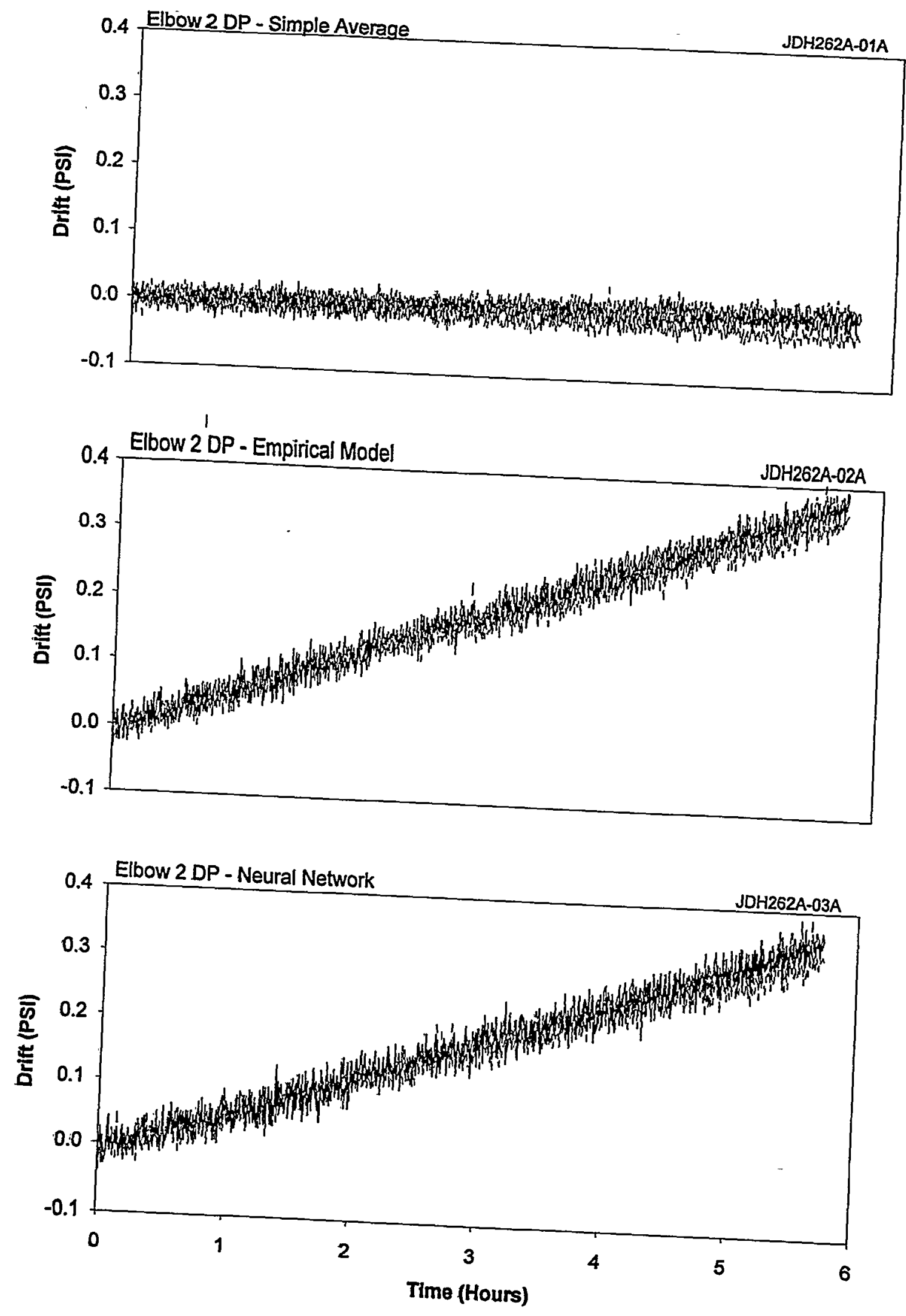

Figure 15.21 Drift Plots when Four Transmitters are Drifted 

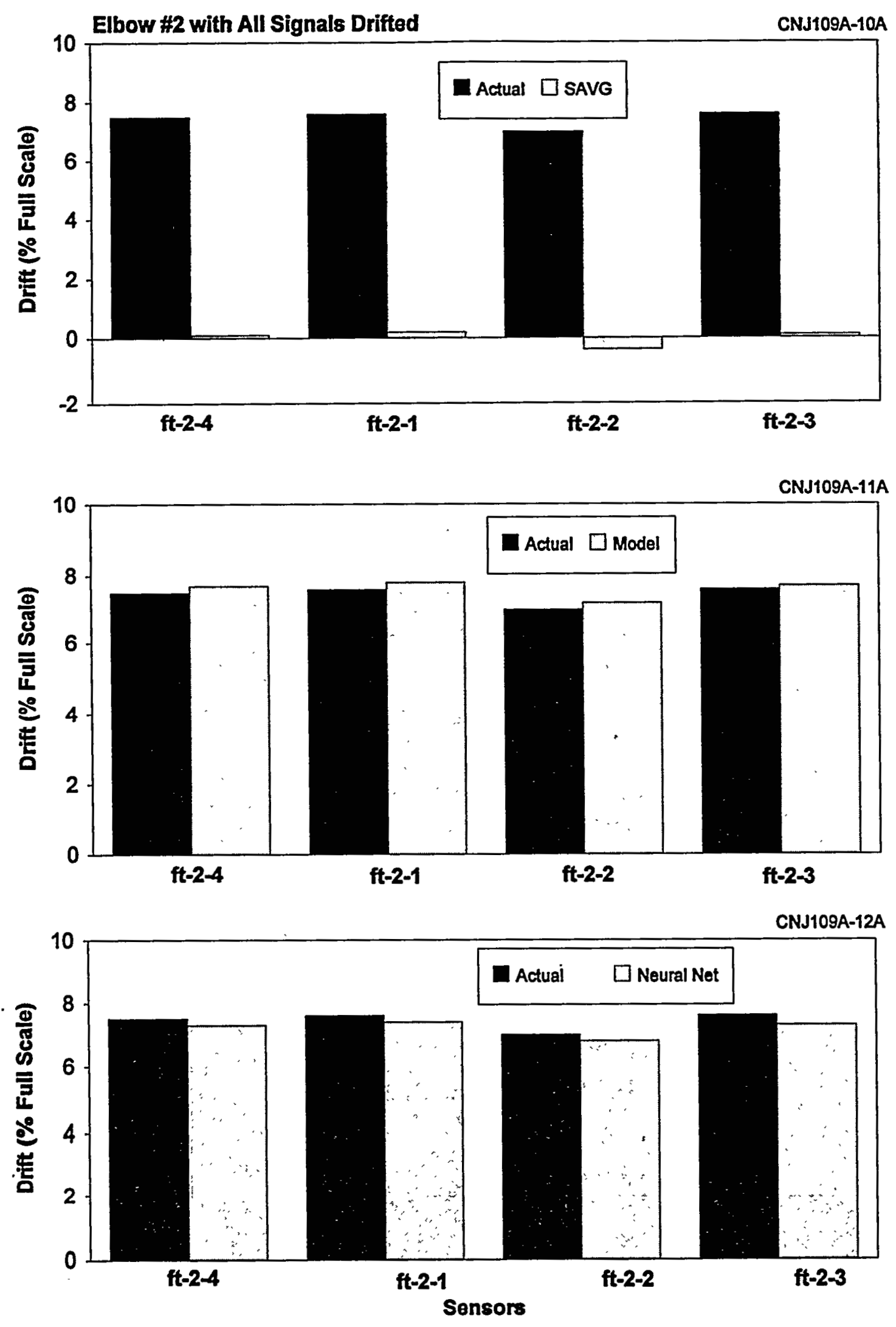

Figure 15.22 Quantitative Results of Process Estimation Techniques when Four Transmitters are Drifted 
Figure 15.12 shows three drift plots each providing the deviation of the signals from the best estimate of the process. The three plots correspond to the three process estimation techniques. Drift plots were used instead of deviation plots because there were no significant differences between the calibrations of the transmitters, thus, the drift plots and deviation plots would be almost identical.

Figure 15.13 shows the results of the tests in bar-chart format. Three plots are given in this figure for the three process estimation techniques: simple averaging, empirical modeling, and neural network. Each plot shows the actual drift induced in the transmitter and the drift identified by the process estimation technique. The figure shows that the empirical model and neural network techniques identify the drift very accurately while the simple averaging technique does not perform as well, and even shows incorrectly that the stable sensors have some drift.

\section{Two Signals Drifted}

Figures 15.14 through 15.16 show test results for when two of the four transmitters were drifted.

Figure 15.14 shows the raw data and the process estimation results, indicating that the averaging technique is not as successful as the analytical techniques.

Figure 15.15 shows drift plots that correspond to the three process estimation techniques. It is apparent that the results of the averaging technique do not correctly represent the situation while the empirical modeling and neural networks provide accurate assessment of the problem.

Figure 15.16 presents the test results in terms of bar charts of induced drift versus detected drift. Note that the averaging technique is not as successful as the other process estimation techniques.

\section{Three Signals Drifted}

Figures 15.17 through 15.19 present test results for when three of the four differential pressure transmitters were drifted.

Figure 15.17 shows the raw data and the process estimation results for the three techniques. As expected, the averaging technique incorrectly shows the process as drifting while the analytical techniques correctly show that the process is stable.

Figure 15.18 shows drift plots in which results are shown to point out that the three drifting signals are correctly identified by the empirical modeling and neural networks while the averaging technique fails as expected.

Figure 15.19 shows the induced drift versus the drift as detected by the three process estimation techniques. A review of this figure along with the two bar charts provided earlier in Figures 15.13 and 15.16 show that averaging results become less accurate as more sensors in the group are drifted.

\section{Four Signals ,Drifted}

Figures 15.20 through 15.22 present the results of laboratory tests for the case in which all four differential pressure transmitters were drifted.

Figure 15.20 shows the raw data and plots of process behavior from the three process estimation techniques.

In Figure 15.21, the averaging technique incorrectly shows no drift in any of the signals, while the empirical modeling and neural networks correctly reveal that all four signals are drifting together at the same rate.

The failure of the simple averaging techniques is obvious in Figure 15.22. 
In summary, the analytical techniques (i.e., empirical modeling and neural networks) provide accurate results if they are properly trained for the particular process and provided with adequate input. The success of the averaging technique depends on the number of redundant signals that are intercompared and the number of signals which are suffering a drift problem. Although the averaging technique was shown to be unsuccessful in the examples discussed above, it was proven to be useful in the analysis of in-plant data. This is because the in-plant data that were analyzed in this project for the McGuire plant contained very few cases where signals had any significant drift. Also, when parity space and GCC methods are used with the averaging technique to identify the inconsistent signals, reduce their weighting, or remove them from the average, the results of the averaging technique improve significantly.

\subsection{Validation of Analytical Techniques Using Absolute Pressure Signals}

A series of experiments similar to those discussed above were also performed using two absolute pressure transmitters. Test results are shown in Figures 15.23 through 15.28 for two cases. In the first case, one of the two pressure transmitters was drifted, and in the second case, both transmitters were drifted. The results are discussed below. The experiments discussed here and those described in the section above provide a database of potential cases involving either two or four signals.

\section{One Transmitter Drifted}

Figure 15.23 shows a raw data plot and best estimates of the process from three methods: simple averaging, empirical modeling, and neural networks. In this case, only one of the two transmitters is drifted.

Figure 15.24 shows three deviation plots: one shows the deviation of each signal from the average of the two signals, and the other two plots show the deviation of each signal from empirical modeling and neural network estimates of the process.

Figure 15.25 presents the test results in terms of bar charts. Note that the empirical modeling and neural network results show a small drift in the transmitter that was stable. That is, although these methods are more successful than the simple averaging technique, they do not provide the results as accurately as they did for the four pressure transmitters case discussed earlier.

\section{Both Transmitters Drifted}

Figure 15.26 through 15.28 show test results for two pressure transmitters that were both drifted.

Figure 15.26 shows the raw data as well as a plot of best estimates of the process from three techniques. Note that the two signals are superimposed in the raw data plot.

Figure 15.27 shows deviation plots for three process estimation techniques. The results of the averaging technique do not show the drift while the other two techniques identify the problem accurately. The validation results are quantified in Figure 15.28.

\subsection{Diagnostic Tests}

On-line monitoring data can provide diagnostic capabilities in addition to revealing calibration problems. Figure 15.29 shows on-line monitoring data for five pressure transmitters one of which exhibited erratic behavior during the tests. Troubleshooting revealed the problem to be due to a loose wire in the 

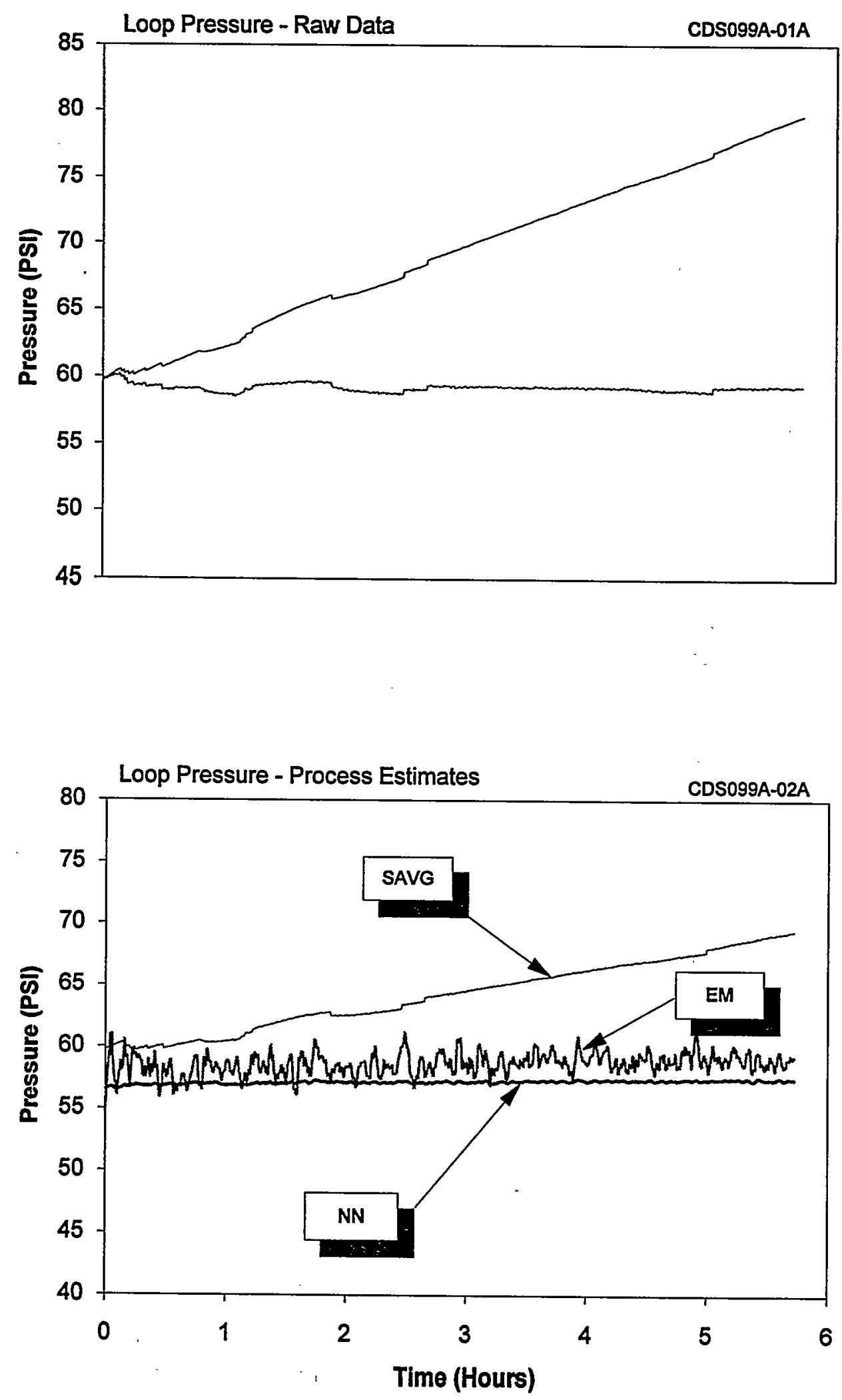

Figure 15.23 Raw Data Plot and Process Estimation Results when One Transmitter was Drifted 

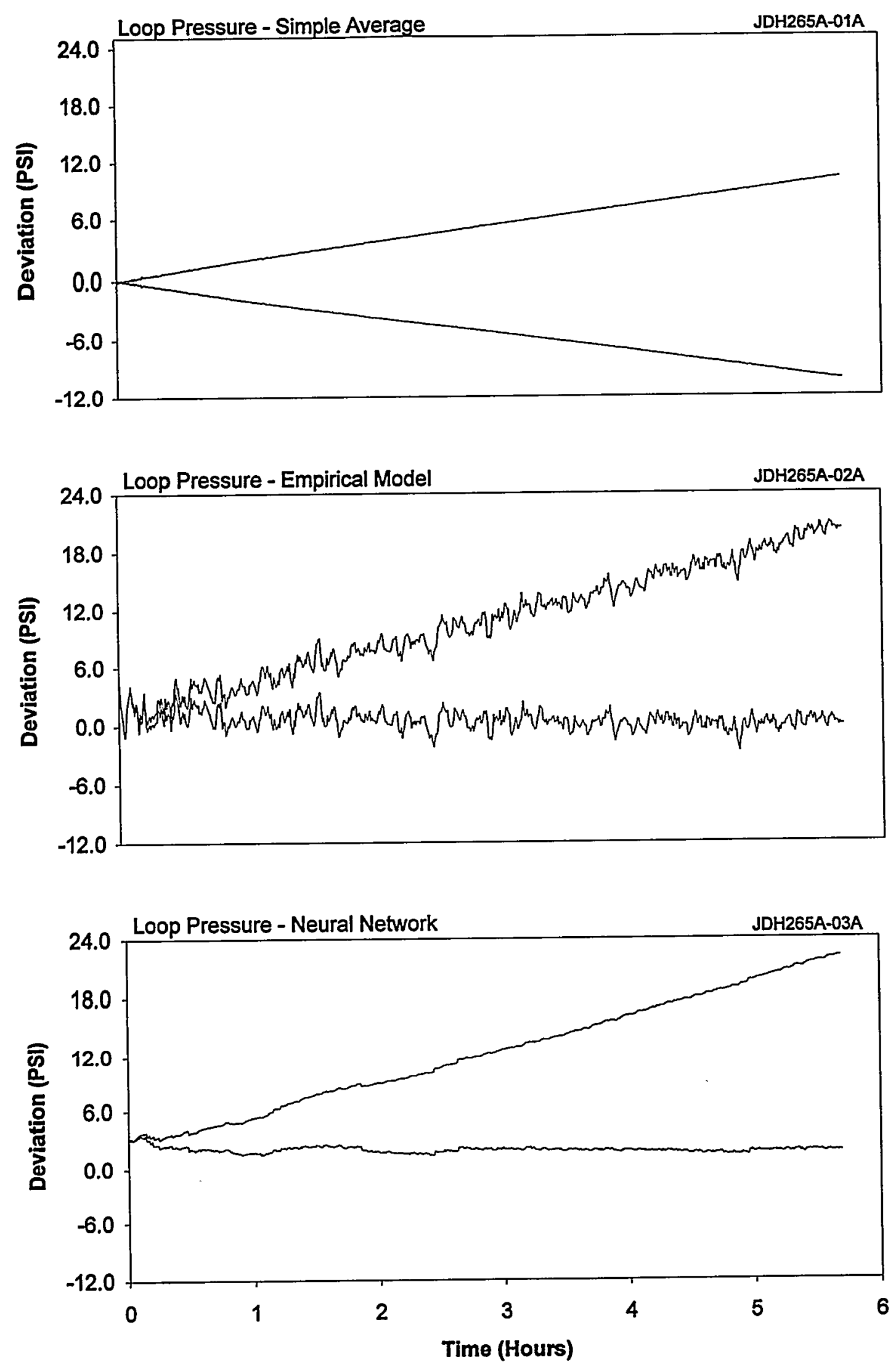

Figure 15.24 Deviation Plots when One Transmitter was Drifted 

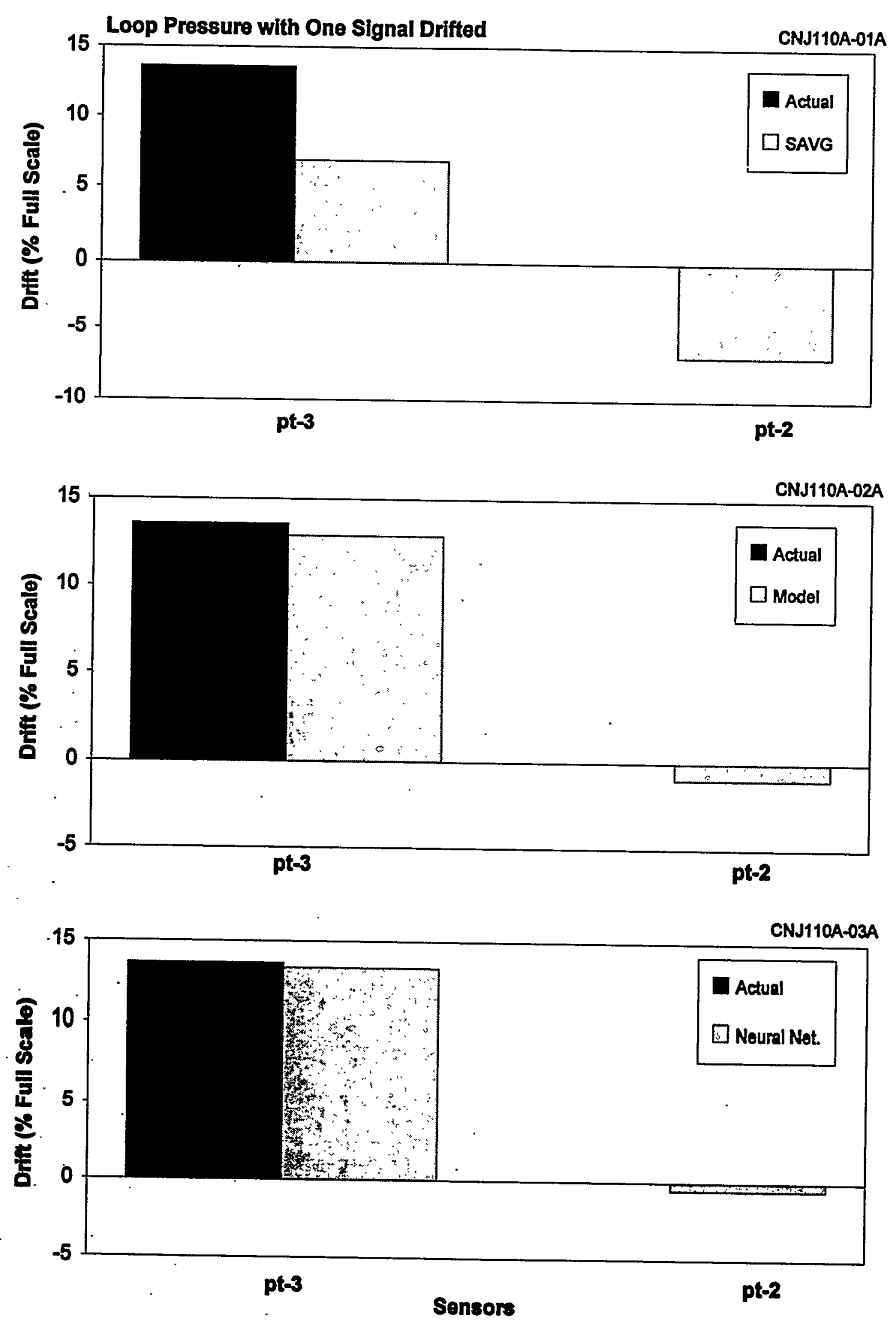

Figure 15.25 Comparison of Induced Drift Versus Detected Drift when One Transmitter was Drifted 

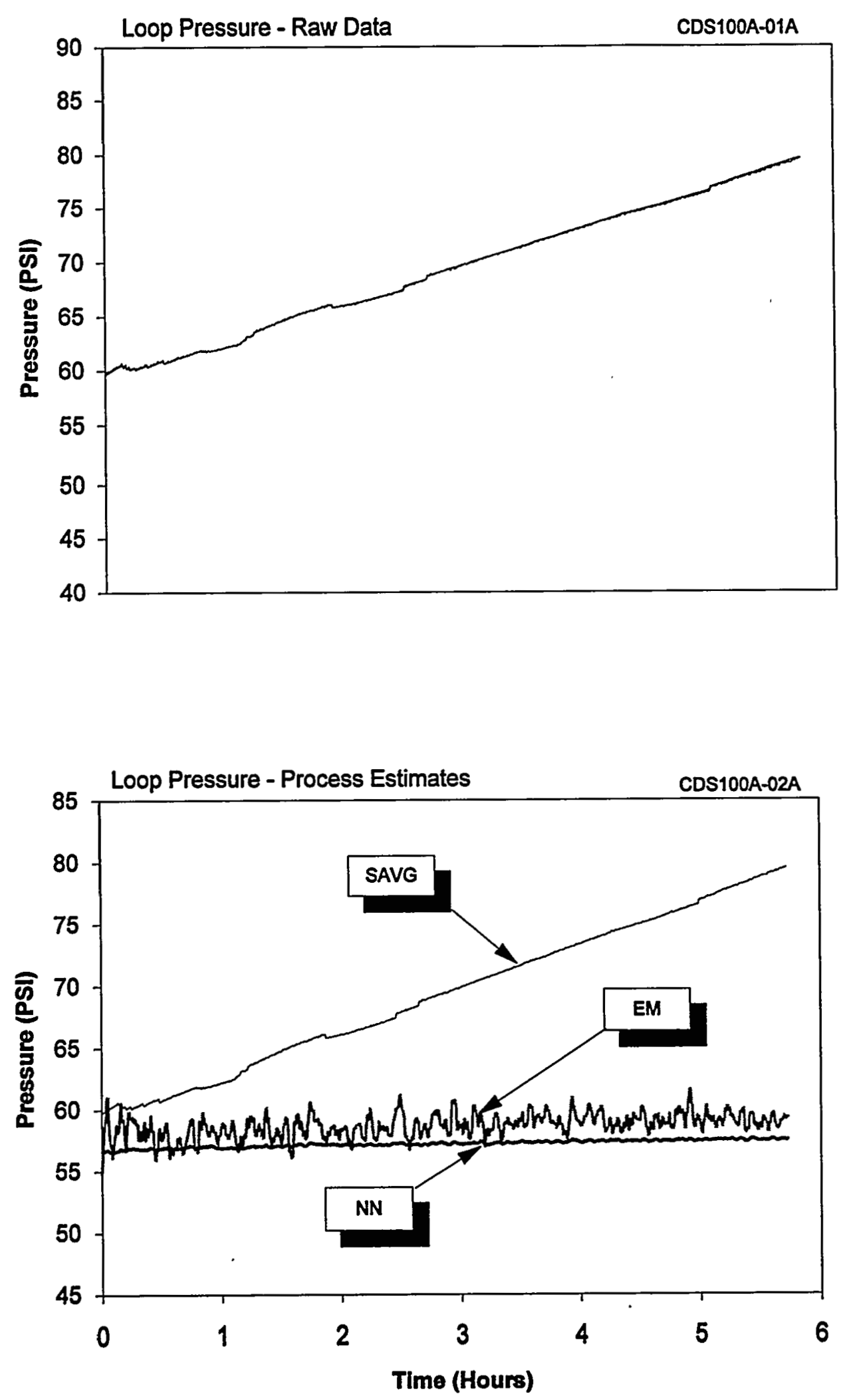

Figure 15.26 Raw Data Plot and Process Estimation Results when Both Transmitters were Drifted 

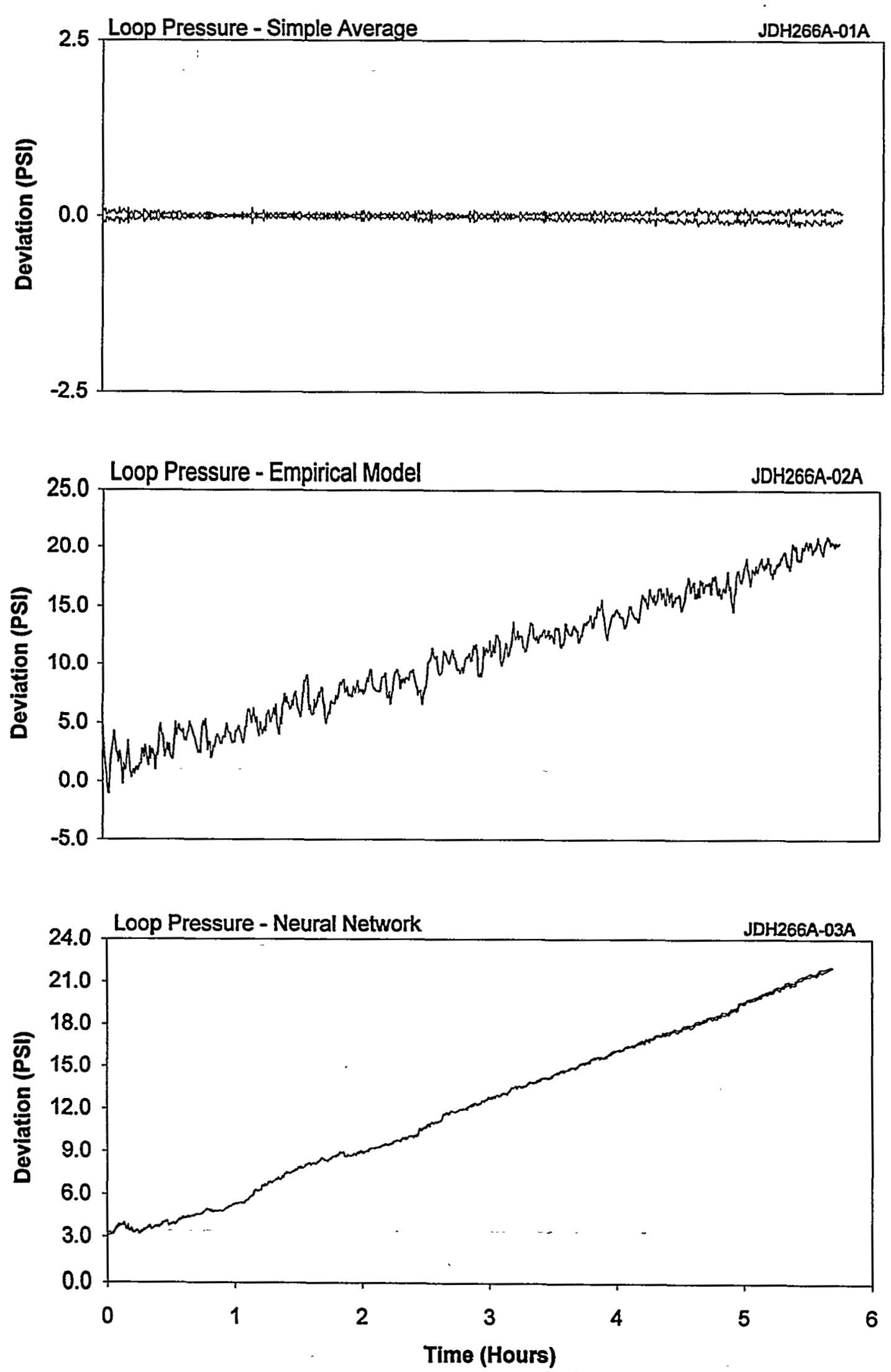

Figure'15.27 Deviation Plots for when Both Transmitters were Drifted 

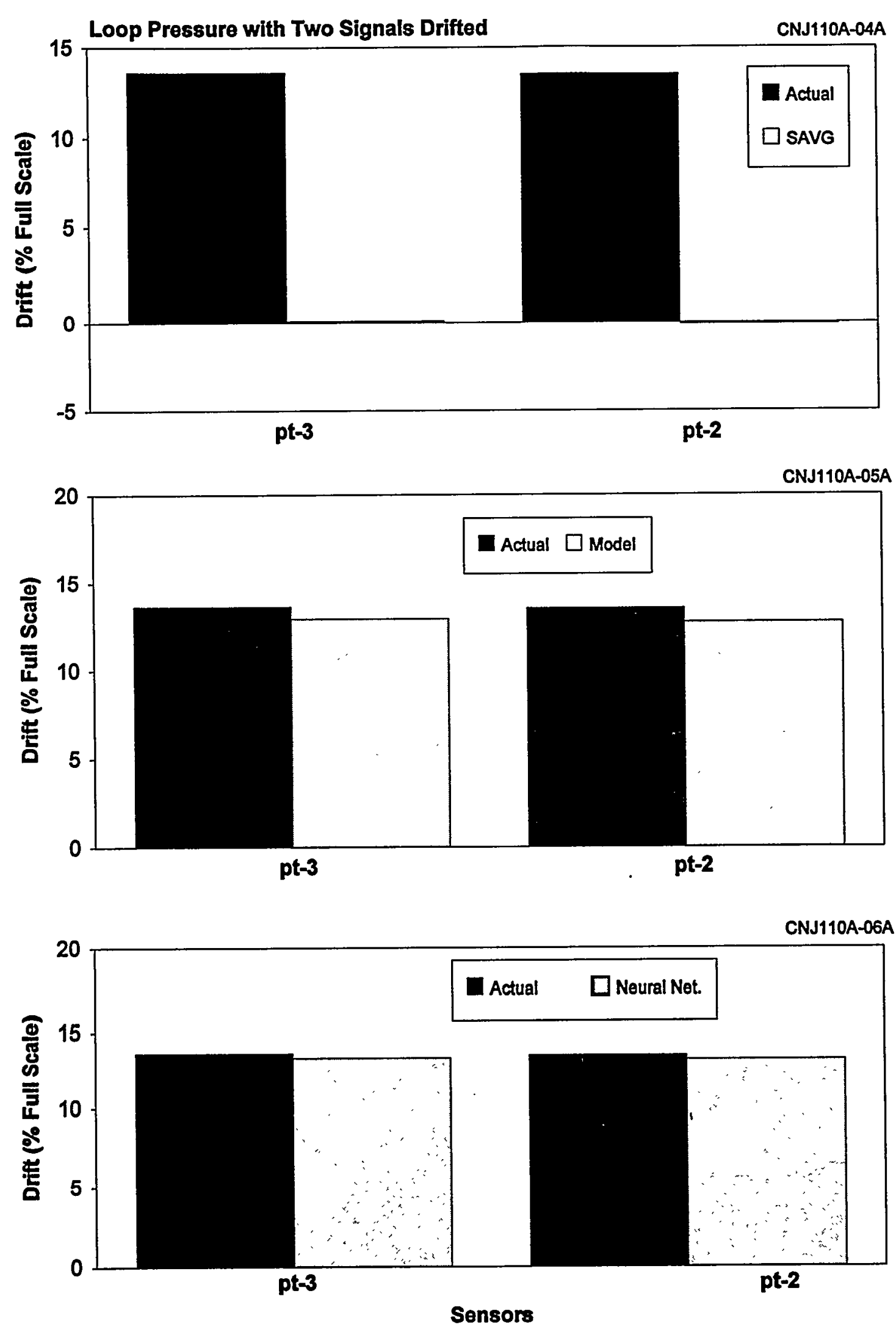

Figure 15.28 Comparison of Induced Drift Versus Detected Drift 


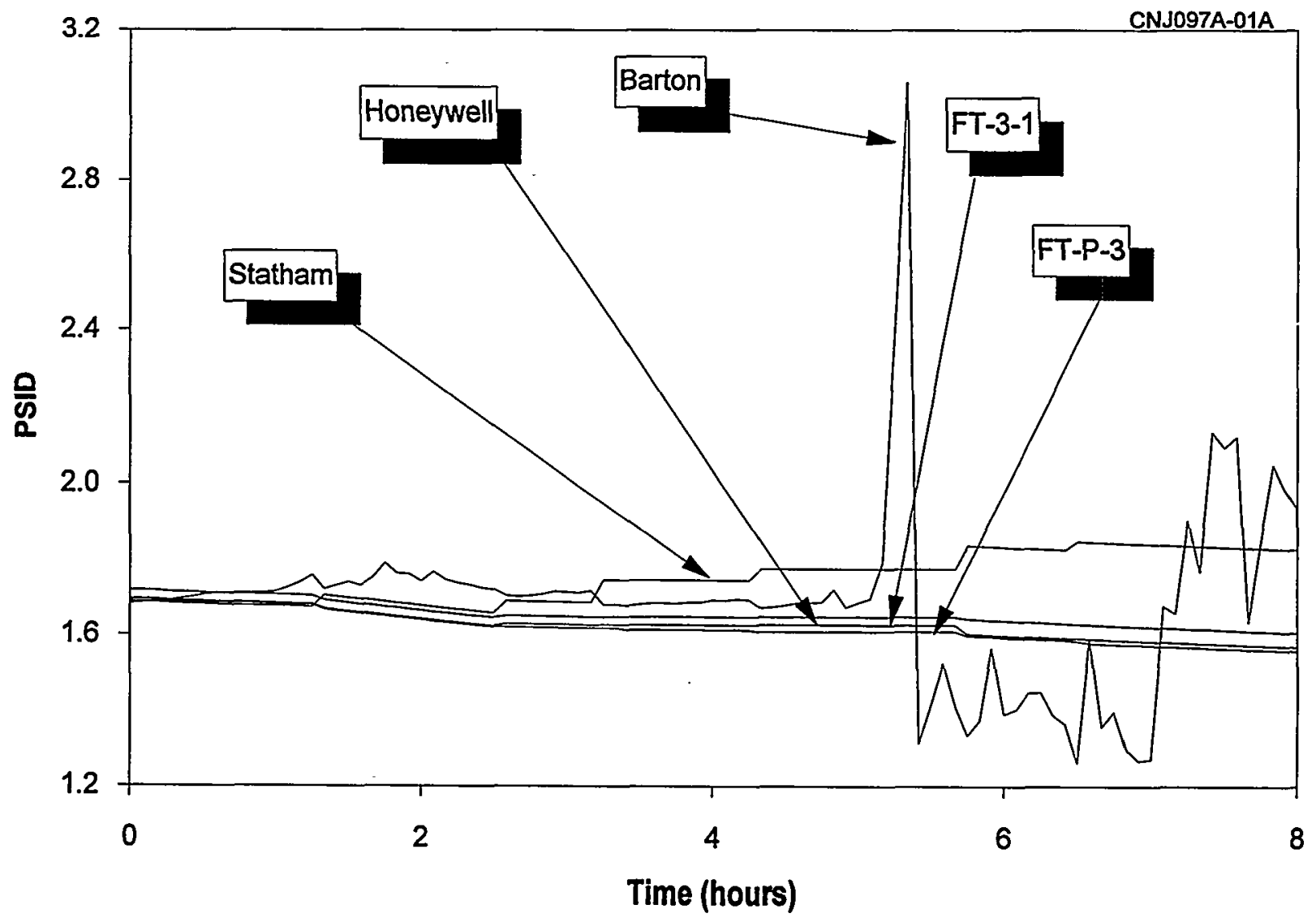

Figure 15.29 On-Line Monitoring Results Revealing a Transmitter with a Loose Wire 
transmitter. The behavior of another transmitter with a loose lead is shown in Figure 15.30. Note that in this case, in addition to an erratic response, the transmitter shows a large calibration error compared with the other transmitters that were monitored along with this transmitter.

Other interesting observations during the laboratory tests included a signal conditioning card which failed during laboratory tests as shown in Figure 15.31. Two views of the same data are shown. The important point here is that the card began to drift about two hours before it failed. An on-line monitoring system could detect the onset of instrument failures and provide an opportunity to repair or replace the instrument before it fails.

In addition to accidental failures and problems which were identified during the laboratory tests, failures and degradations were intentionally induced in sensors and their effects were recorded to determine how problems in sensors manifest themselves in the results of on-line monitoring tests. Figures 15.32 through 15.34 show typical results of such tests.

In Figure 15.32 results are shown for when the extension wires of an RTD were loosened. This caused the RTD to shift and indicate a higher temperature probably due to an increase in circuit resistance.

In Figure 15.33, results are shown for an RTD that was placed under mechanical stress while its output was monitored. The stress was irduced on the RTD sheath at two locations (A and $B$ ) as shown in Figure 15.33. The results show that these stresses have caused the RTD resistance to increase and result in a higher temperature indication. In a similar example, contrary to expectation, the RTD resistance dropped when it was mechanically stressed. Consequently, the RTD indicated a lower temperature as shown in Figure 15.34. The decrease in the RTD resistance could be due to a failure of insulation resistance from mechanical shock.

\subsection{Effects of Damping on Transmitter Response}

Some pressure transmitters that are used in nuclear power plants are equipped with a damping adjustment to help reduce any extraneous noise at the output of the transmitter. Figure 15.35 shows responses of two transmitters, one with a one-second damping and another without damping. Note that the undamped transmitter is much faster than the damped transmitter. The interesting point here is that the response time of the damped transmitter can be estimated from this data. Also, this example shows that in-plant monitoring data, if sampled frequently enough, could reveal sluggish response due to pressure transmitter damping, sensing line blockages, aging degradation, etc.

\subsection{Test Results in Environment Chamber}

Figures 15.36 and 15.37 show the outputs of several pressure transmitters while they were installed in an environmental chamber. Results are compared with the output of a reference sensor that was installed outside the environmental chamber. The results show that although these transmitters are temperature compensated, a significant amount of time is required for their temperature compensation to take effect. This indicates that if pressure sensors are exposed to temperature cycling, their steady-state output may have large errors depending on the magnitude and frequency of the temperature changes. Figure 15.36 shows the output of the transmitters when the temperature in the environmental chamber was increased by about $120^{\circ} \mathrm{F}$. Figure 15.37 shows that the temperature response of transmitters from three different manufacturers are essentially identical. 


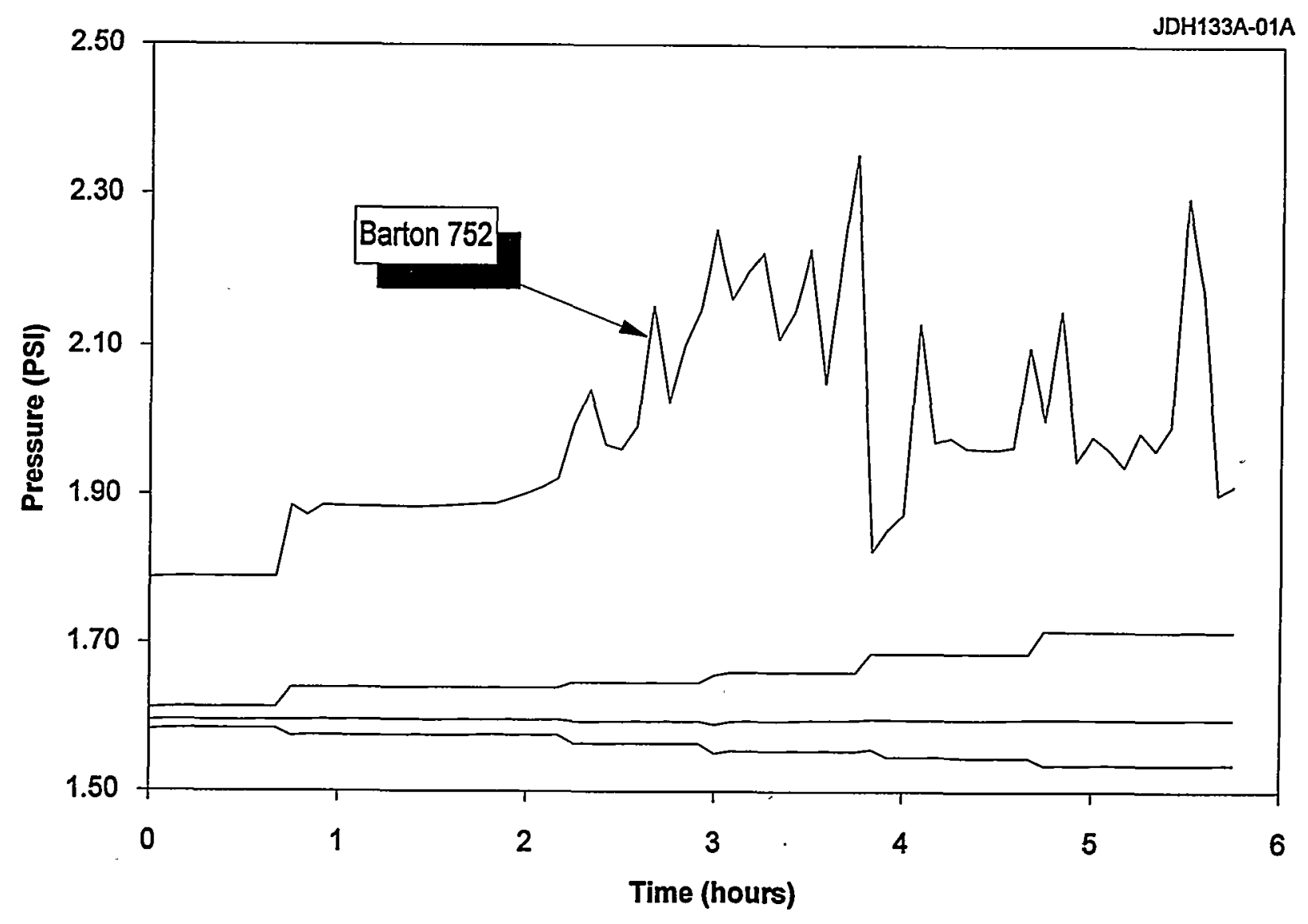

Figure 15.30 Response of a Transmitter With a Loose Wire 

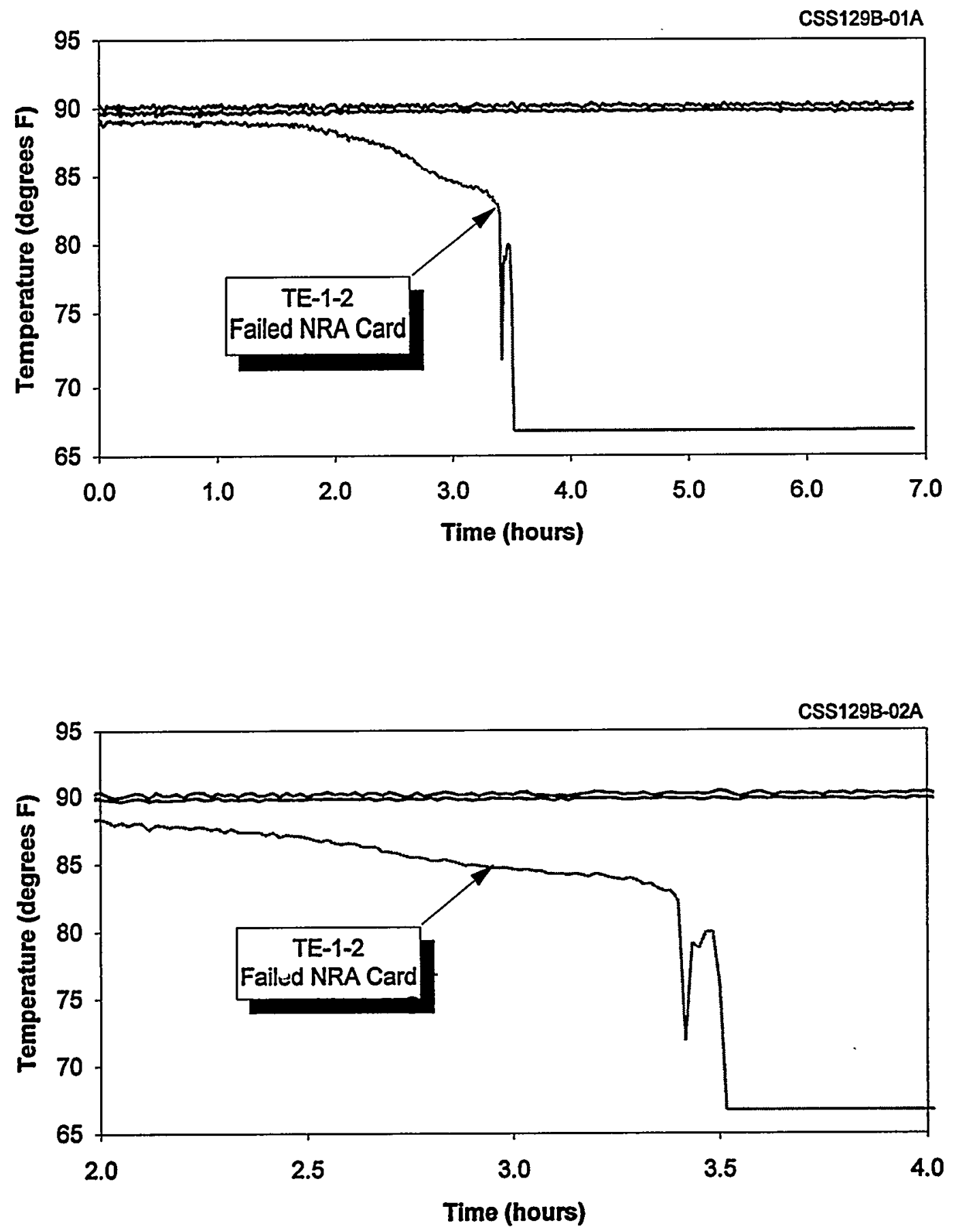

Figure 15.31 Response of a Failing Signal Conditioning Card in an Instrument Channel Tested in the Laboratory 


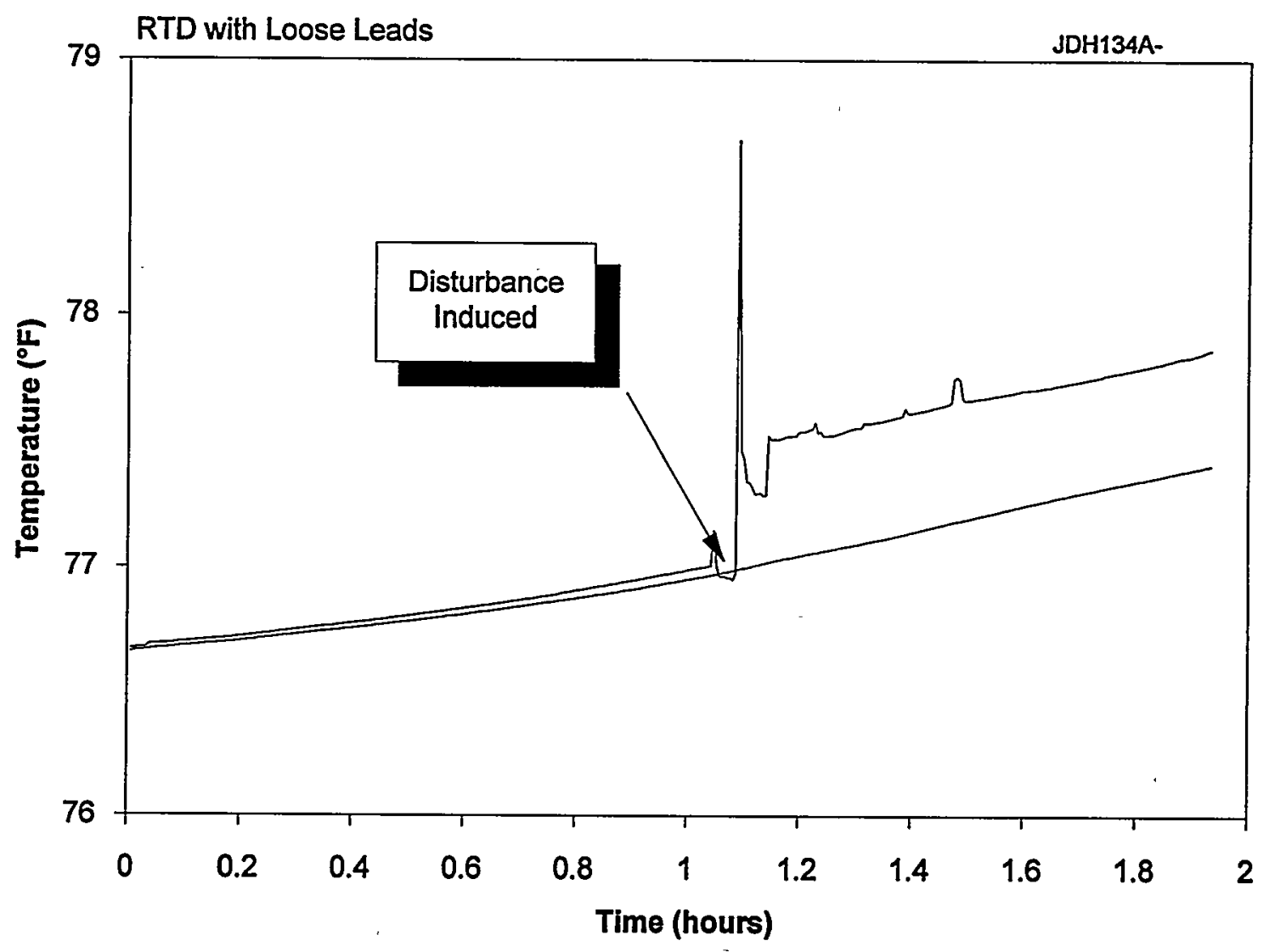

Figure 15.32 RTD Output Signal when Extension

Wires were Intentionally Loosened 

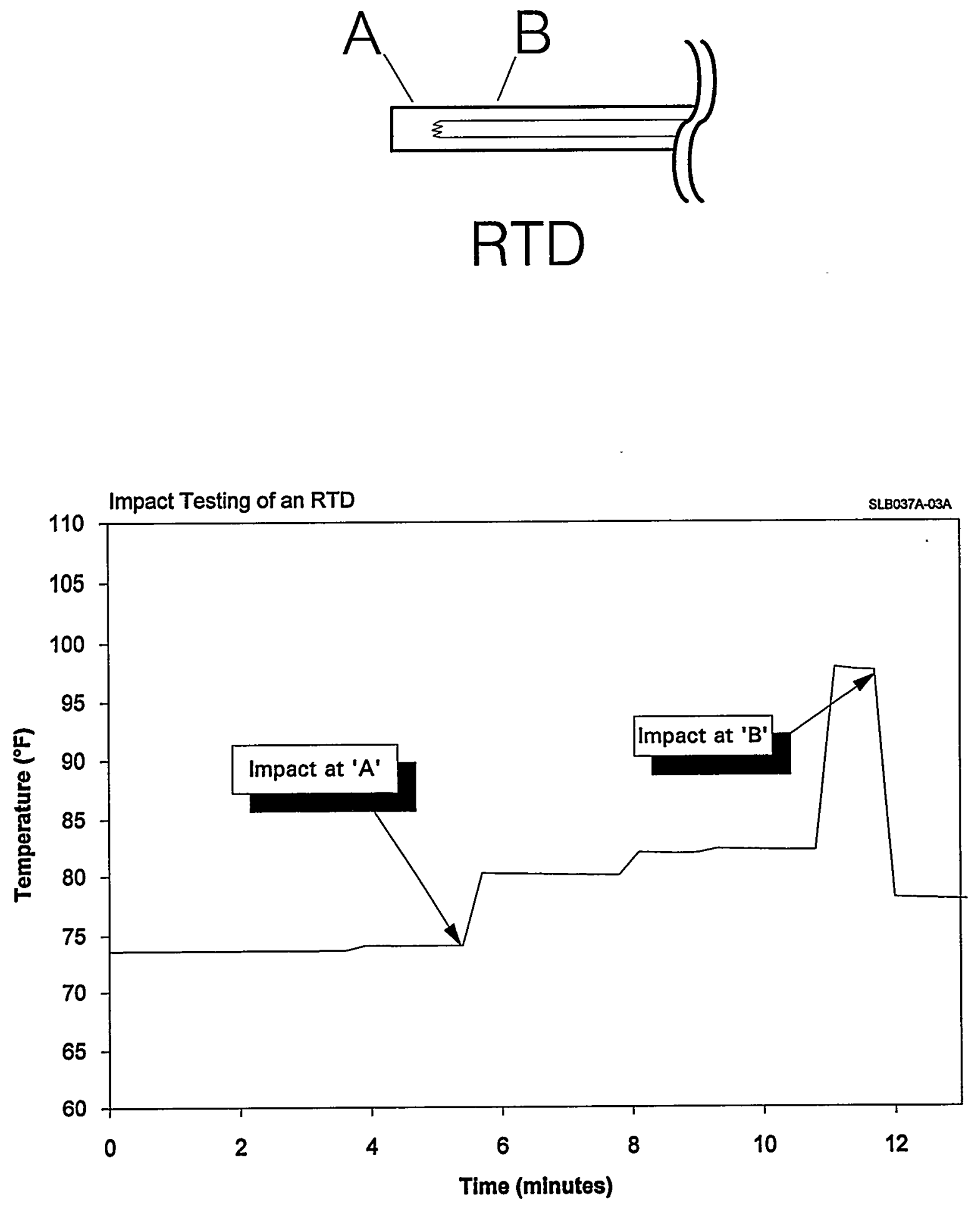

Figure 15.33 RTD Output as a Function of Mechanical Stress 


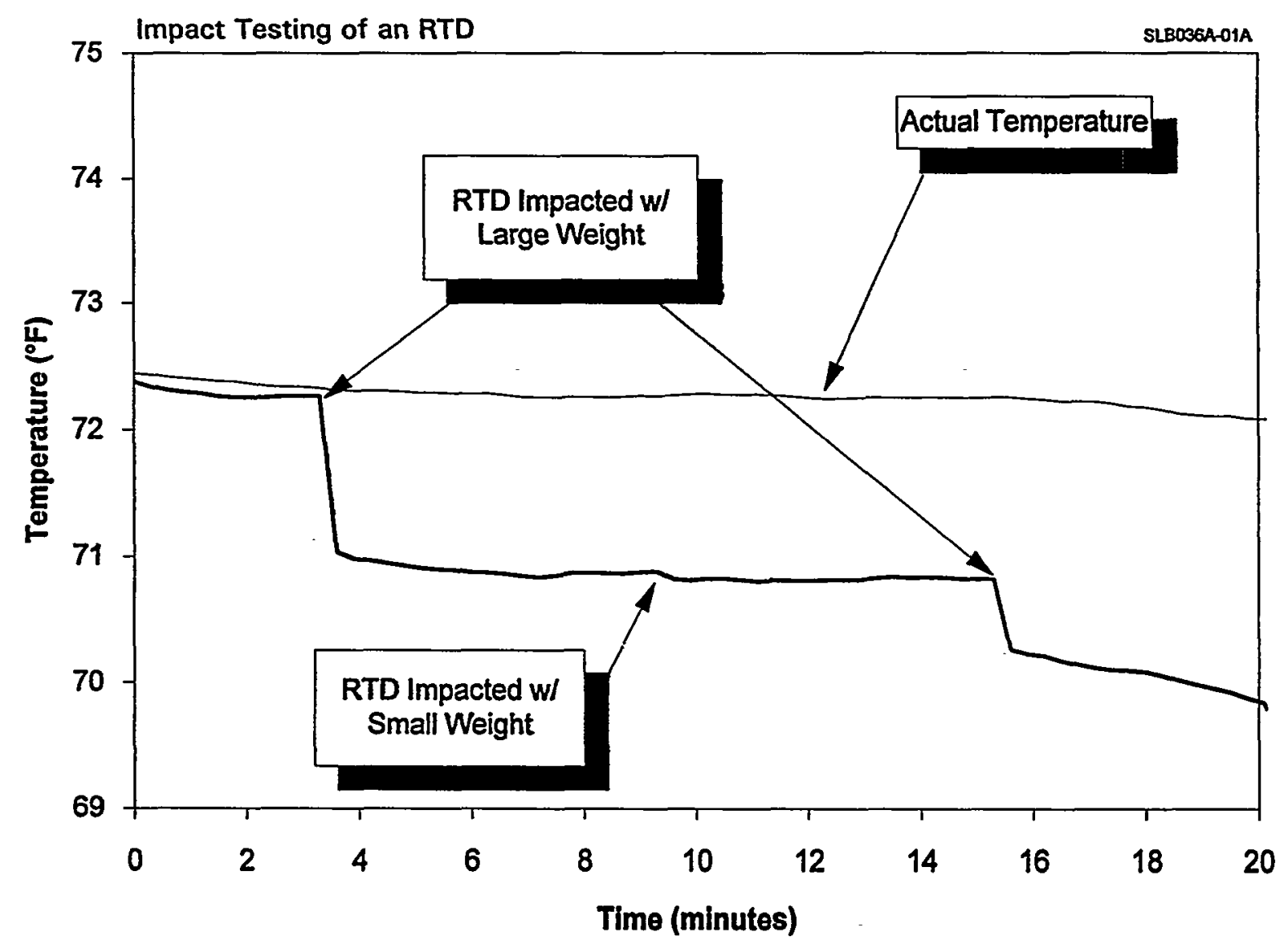

Figure 15.34 Decreasing Resistance of an RTD Subjected to Mechanical Stress 

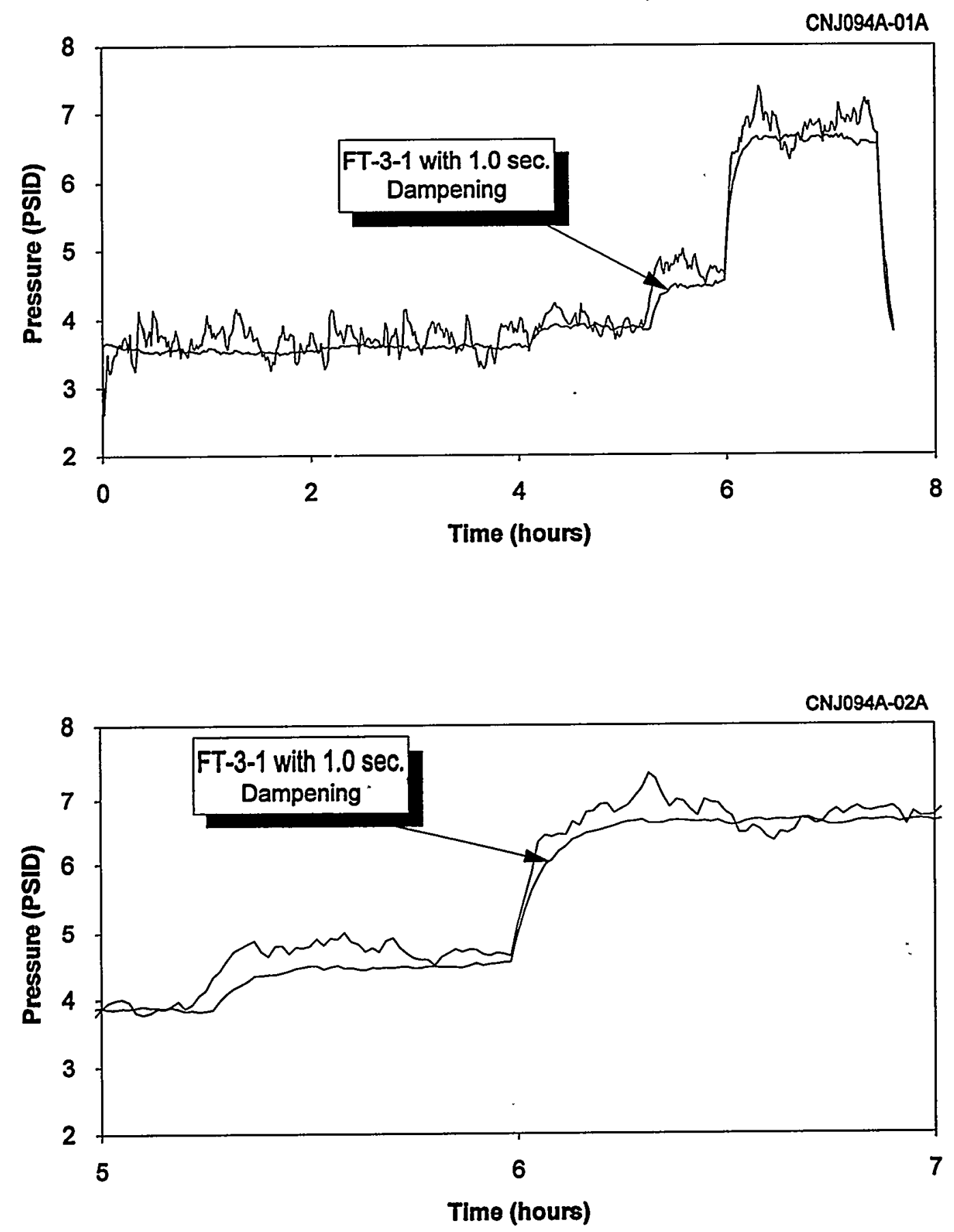

Figure 15.35 Responses of a Damped and an Undamped Pressure Transmitter 


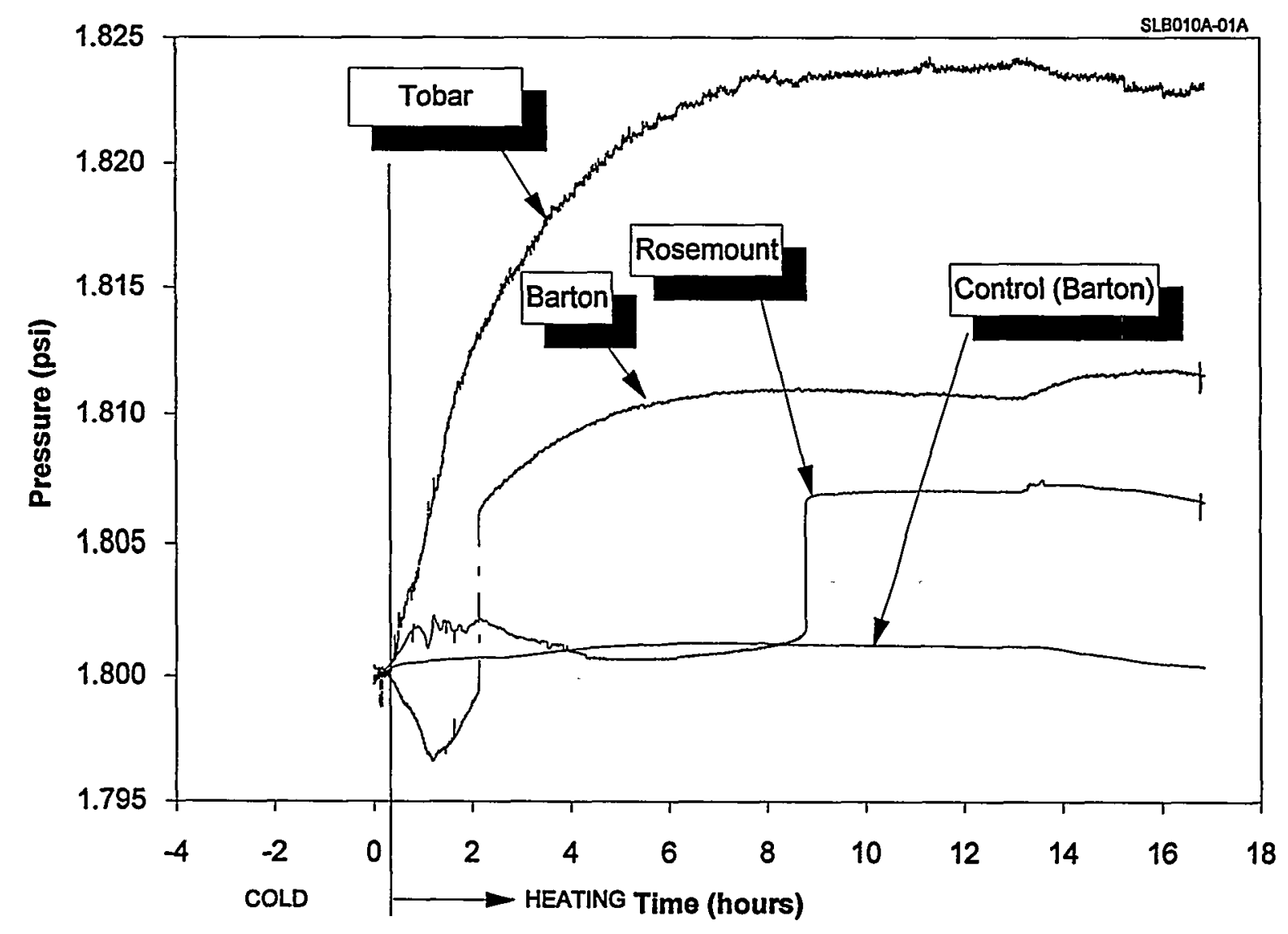

Figure 15.36 Response of Pressure Transmitters Inside the Environmental Chamber at Elevated Temperatures 


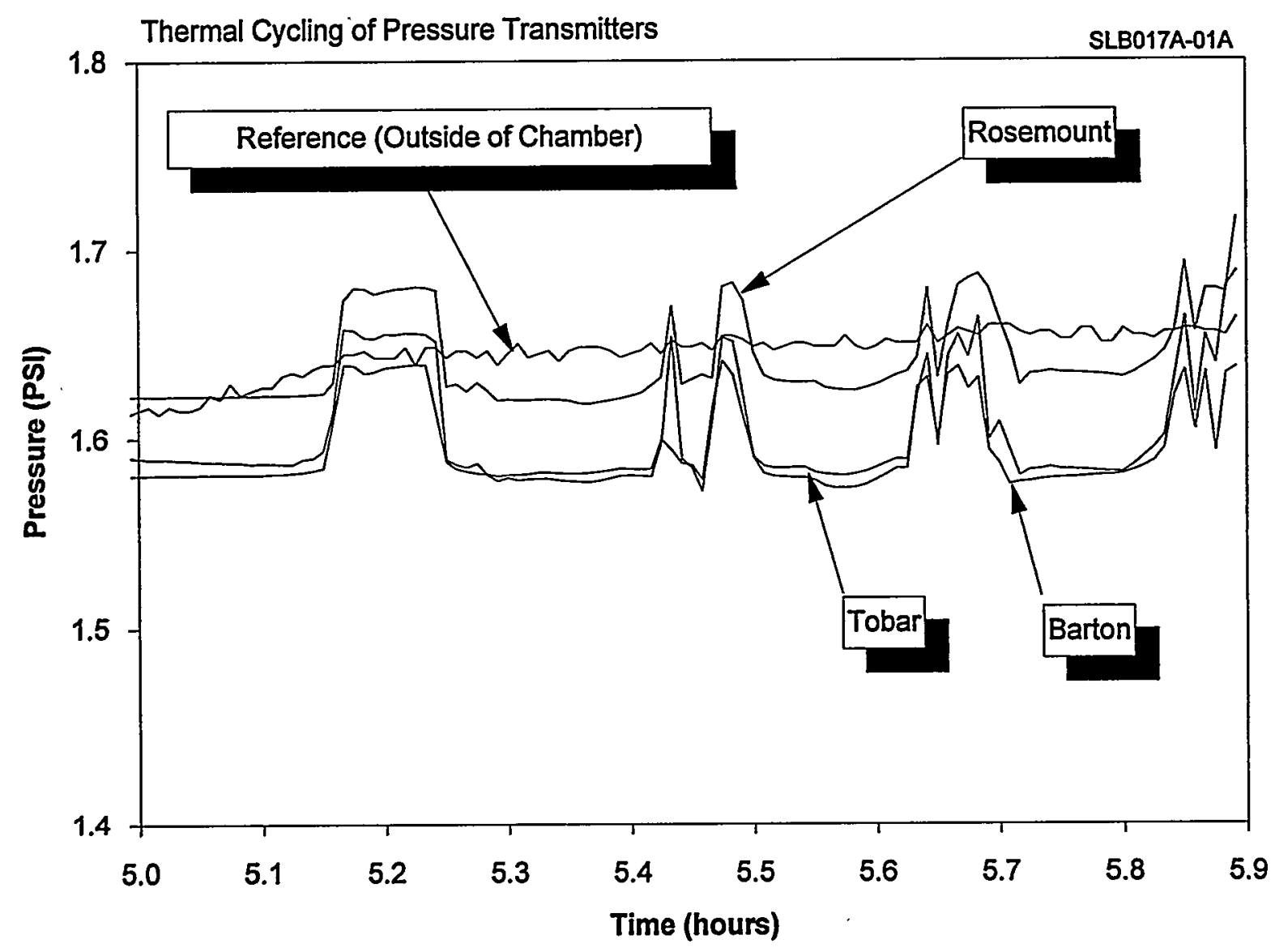

Figure 15.37 Comparison of Thermal Responses of Different Transmitters 


\section{IN-PLANT VALIDATION RESULTS}

The in-plant validation of the on-line monitoring technique was performed using data from two consecutive fuel cycles from the McGuire Nuclear Power Station Unit 2. The data covered the period of March 1992 to December 1994. The average length of a McGuire fuel cycle is about 14 months.

During the nearly thirty-three months of data collection at McGuire, there were plant trips and scheduled outages which resulted in discontinuities in the data as well as periods where on-line monitoring data could not be collected leaving gaps in the data. Figure 16.1 shows a plot of the reactor power during the two fuel cycles that on-line monitoring data were collected at McGuire. The figure shows the periods of reactor operation and shutdown as well as the gaps in the data. Figure 16.2 shows how discontinuities and gaps were removed in preparing the data for analysis.

In addition to discontinuities and gaps which had to be removed, some of the McGuire signals were noisy and had to be filtered. Filtering was performed by moving an averaging window through the digitized data. Figure 16.3 shows a plot of three redundant signals before and after filtering. Appendices $F$ and $G$ include plots of raw data with and without filtering for sixteen McGuire services tested during cycle 1 (March 1992 to June 1993) and cycle 2 (October 1993 to October 1994).

\subsection{Model Validation Results}

Although reactor trips and refueling outages interrupted the on-line monitoring process as shown in Figure 16.1, they provided an opportunity to examine the calibration of instrument channels over a wide range from normal operating conditions to shutdown and from shutdown to normal operating conditions.
In particular, the startup and shutdown data were used to validate the empirical models and the neural networks.

Figures 16.4 through 16.9 present typical results of validation of analytical estimates of the process during the plant startup and shutdown periods. The figures represent the following six services: feedwater flow, steam flow, steam generator level, steam generator pressure, hot leg RTDs, and pressurizer level. Two plots are given in each figure, one plot shows the results of the validation of the empirical model, and the other plot shows the results of validation of neural networks.

The empirical models and neural networks for the examples given here were trained with on-line monitoring data during a startup including a period of stable reactor operation after the startup. The models were then used to estimate the process during a subsequent plant transient.

The plots in Figures 16.4 through 16.9 show that there is. reasonable agreement between the empirical and neural network estimates of the process and the sensor measurements. For the hot leg RTDs, however, only two of the four redundant measurements agreed with the empirical model and neural network estimates. This is probably due to the temperature stratification phenomenon inherent in the hot leg loops of PWRs. The temperature stratification problem is discussed in Chapter 17.

\subsection{Comparison of Manual Calibration and On-line Monitoring Results}

The in-plant validation tests were originally planned to be conducted according to the 


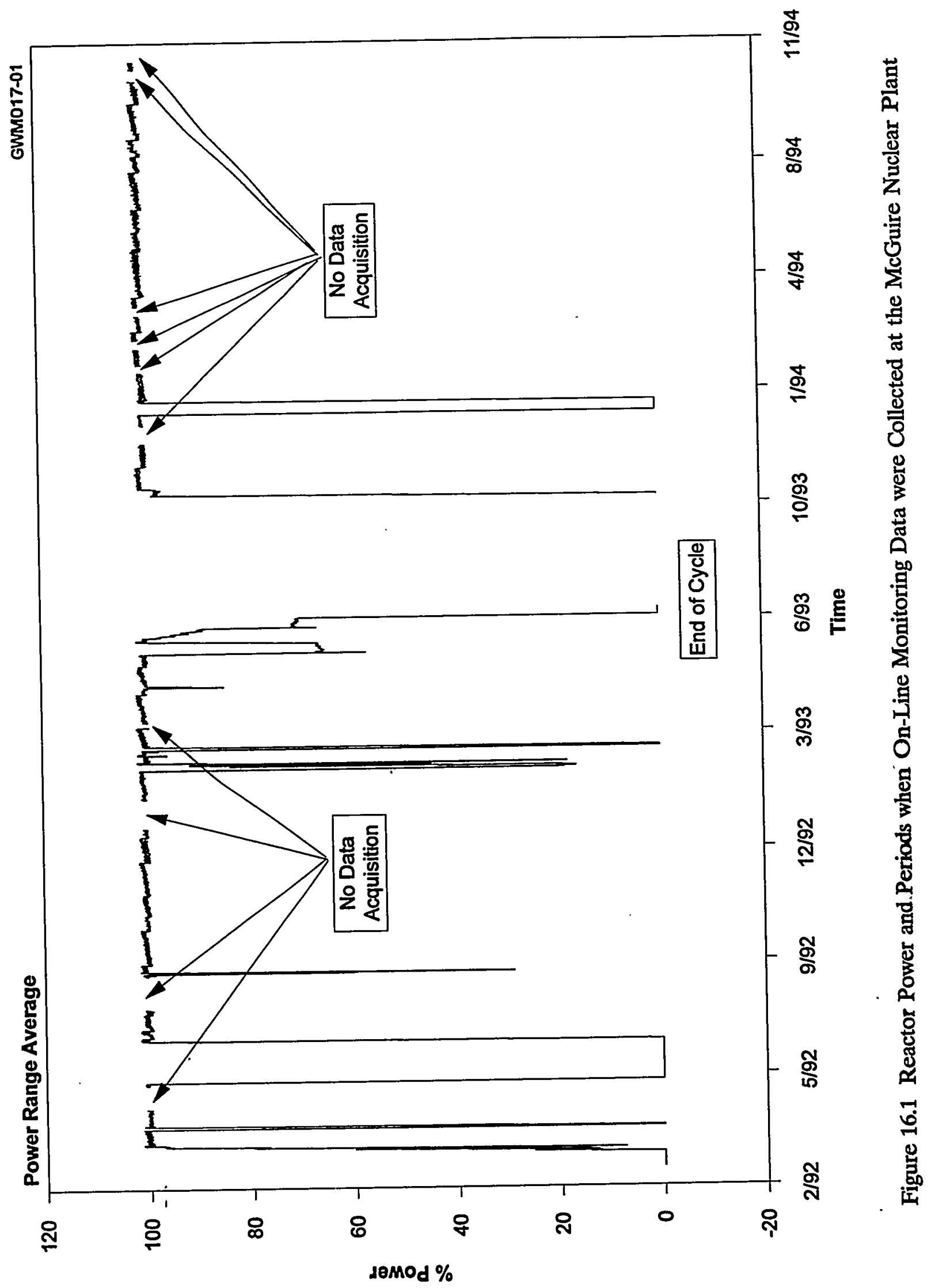



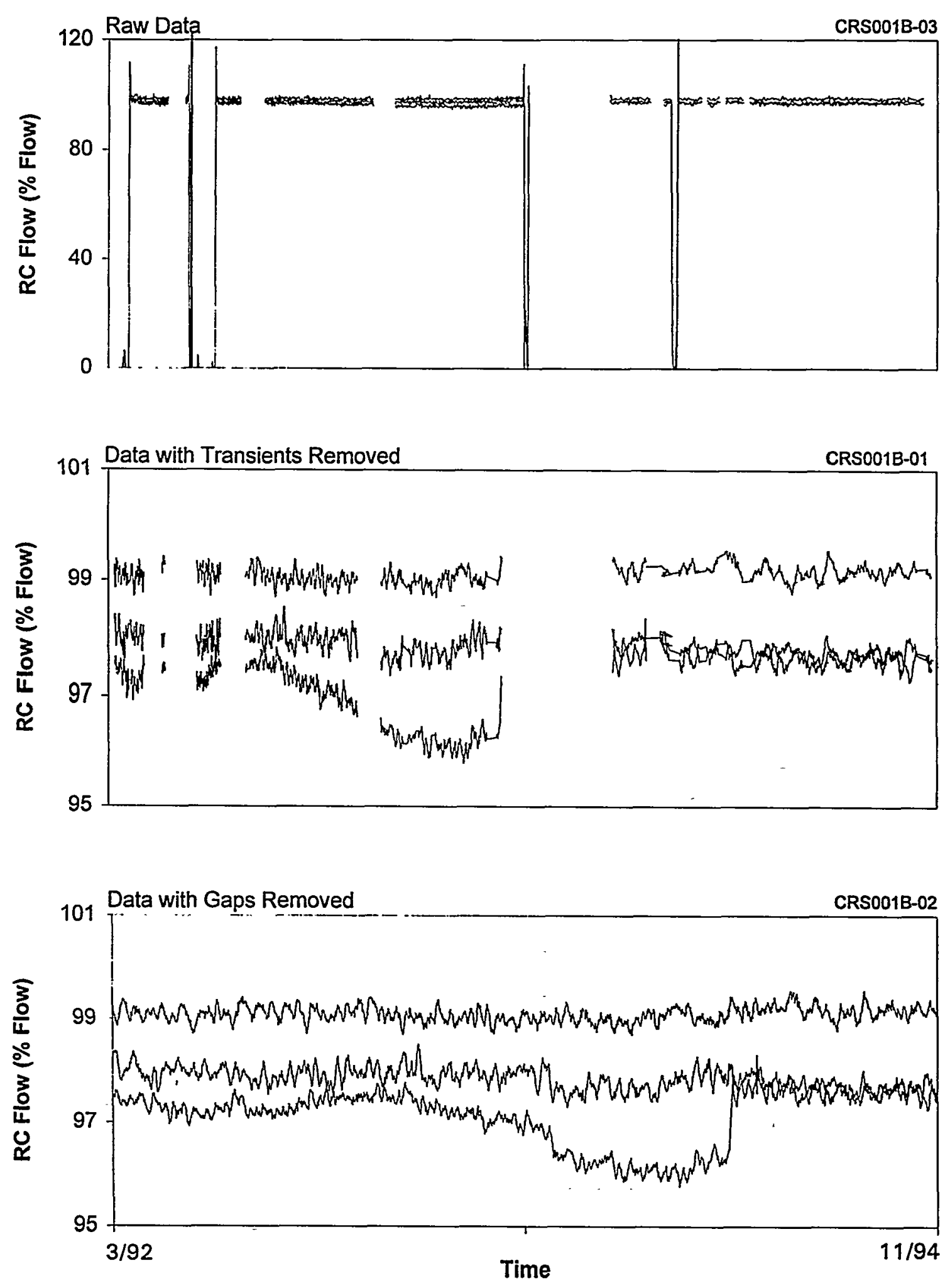

Figure 16.2 Removal of Discontinuities and Gaps from On-Line Monitoring Data 

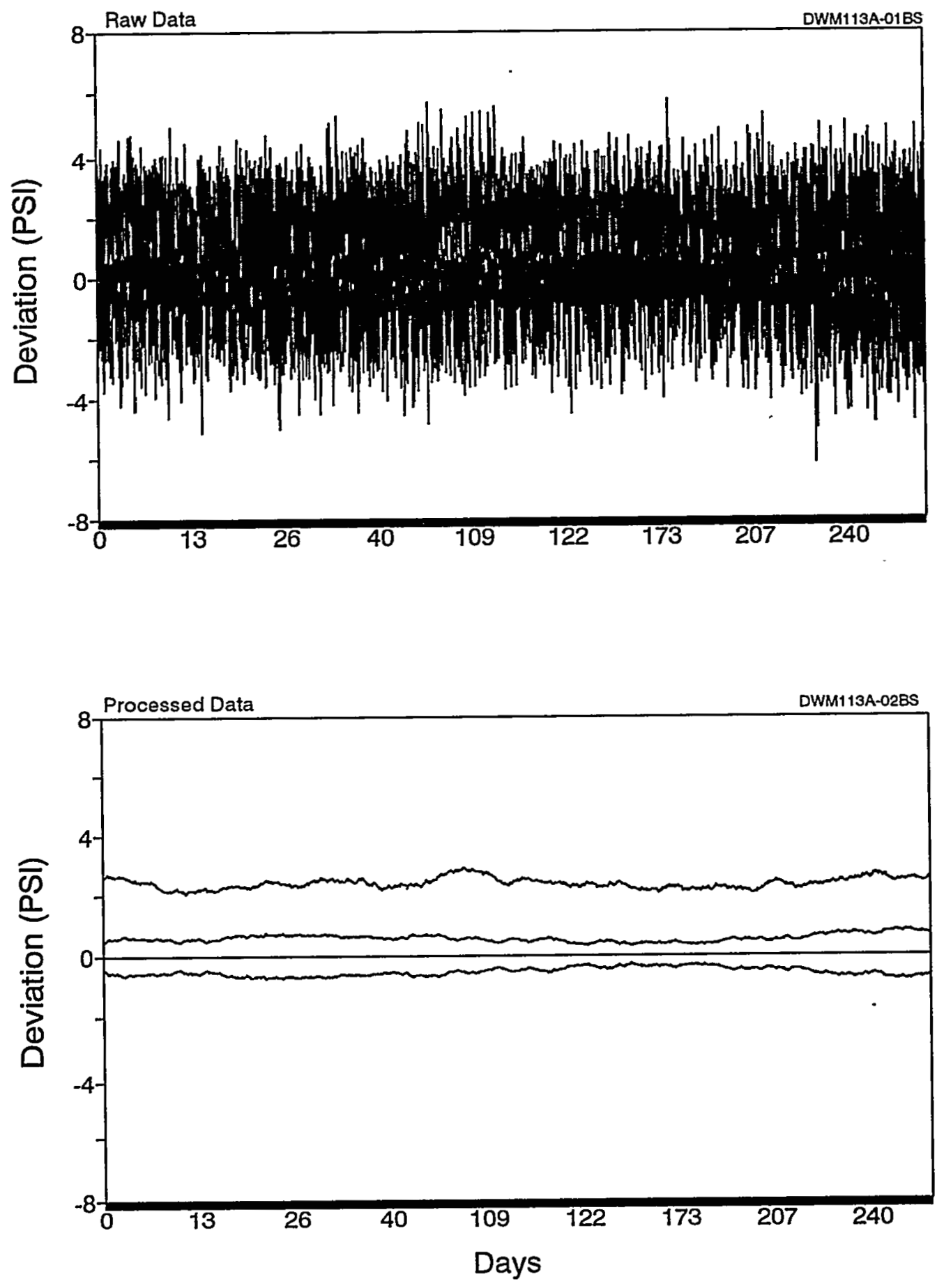

Figure 16.3 Raw and Filtered Signals 


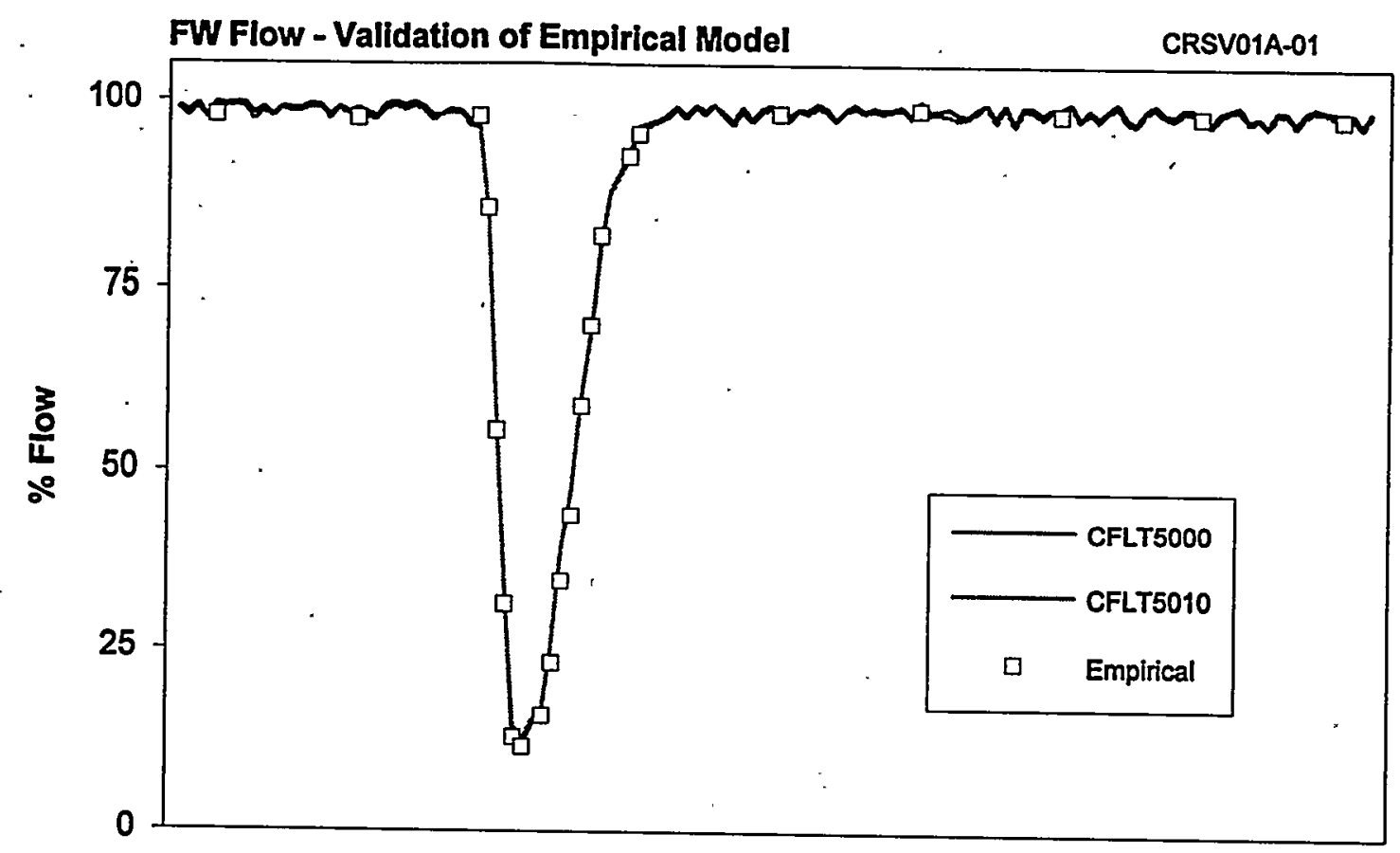

FW Flow - Validation of Neural Networks

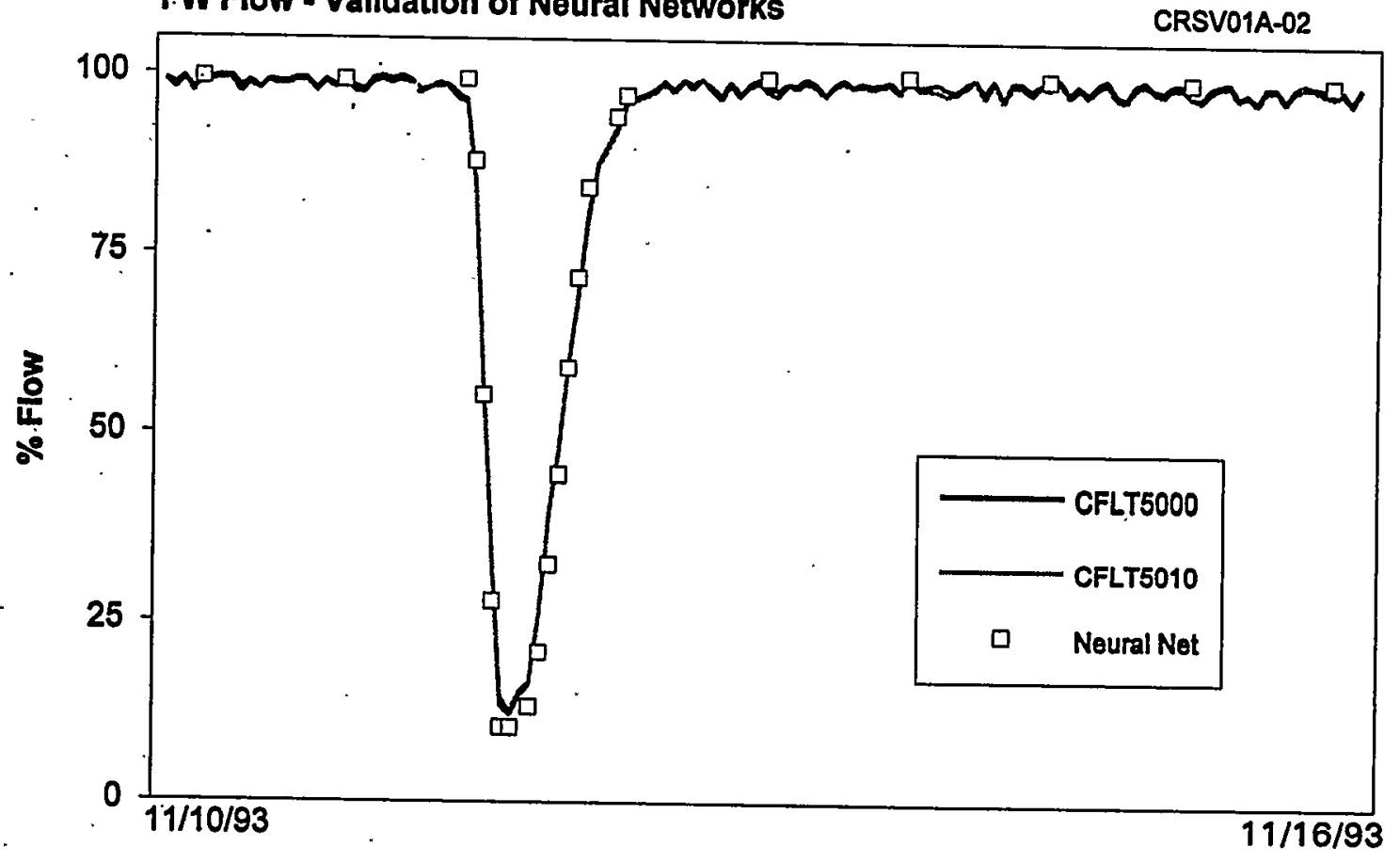

Figure 16.4 Model Validation Results for Two Feedwater Flow Signals 

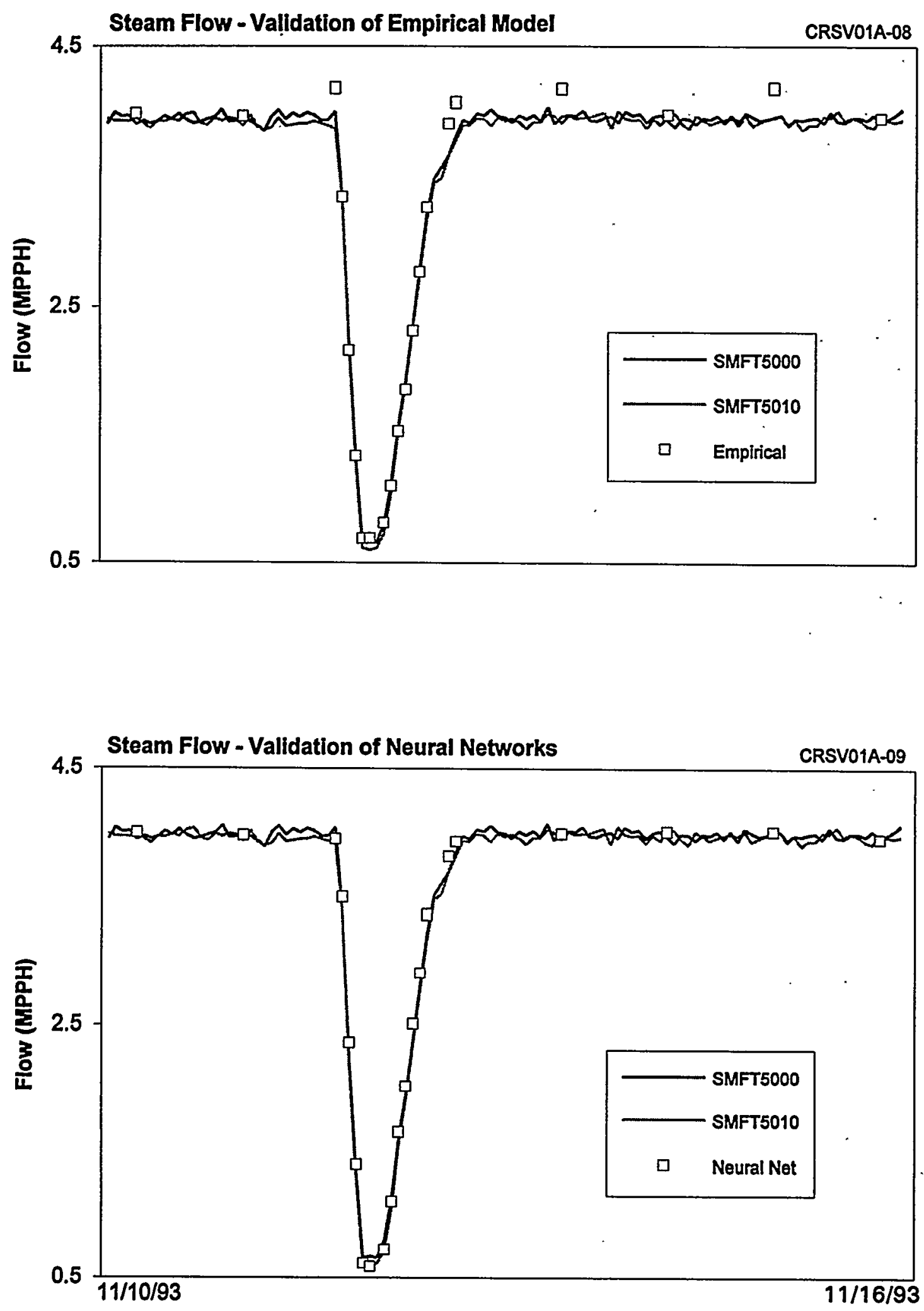

Figure 16.5 Model Validation Results for Two Steam Flow Signals 

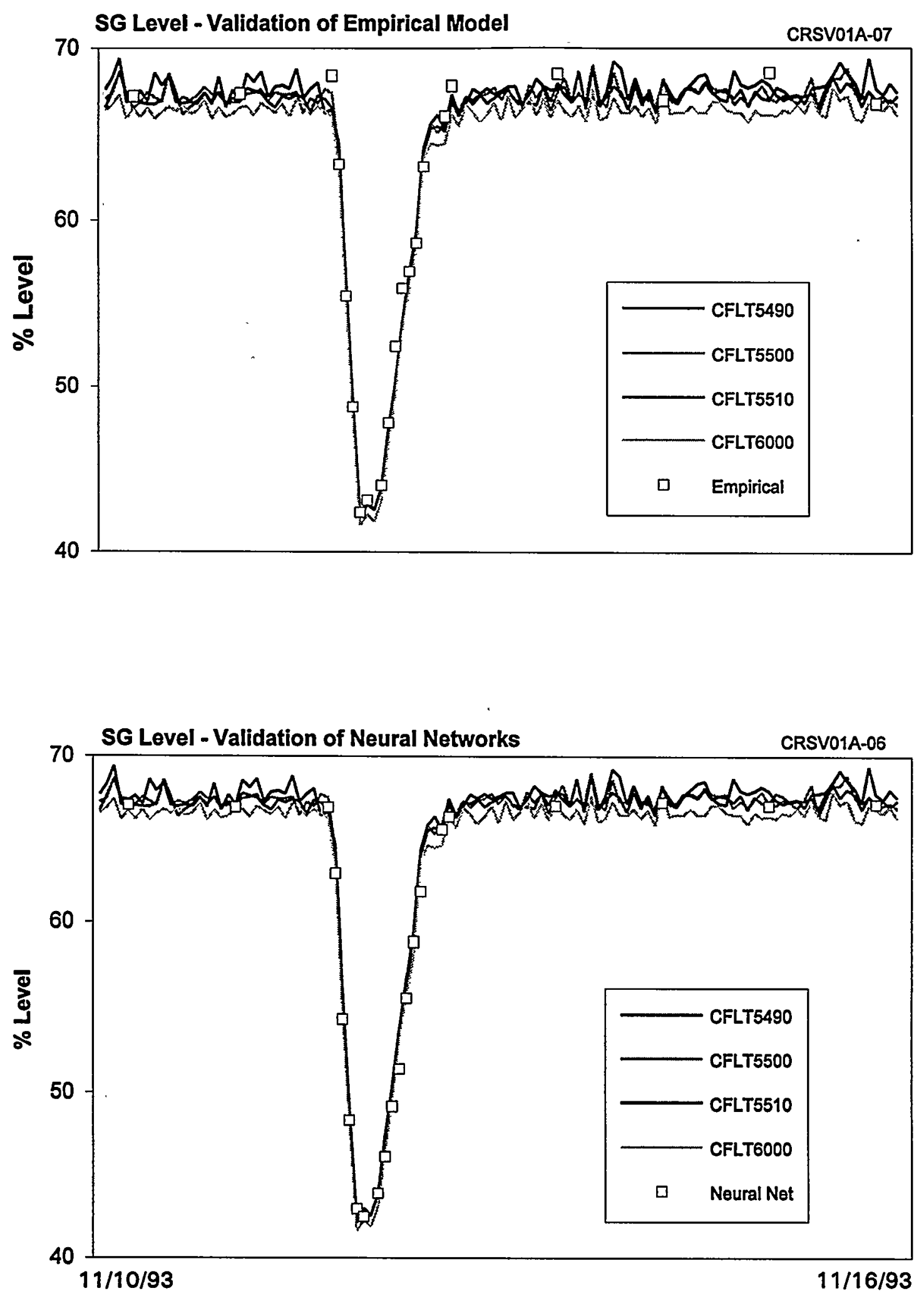

Figure 16.6 Model Validation Results for Four Steam Generator Level Signals 

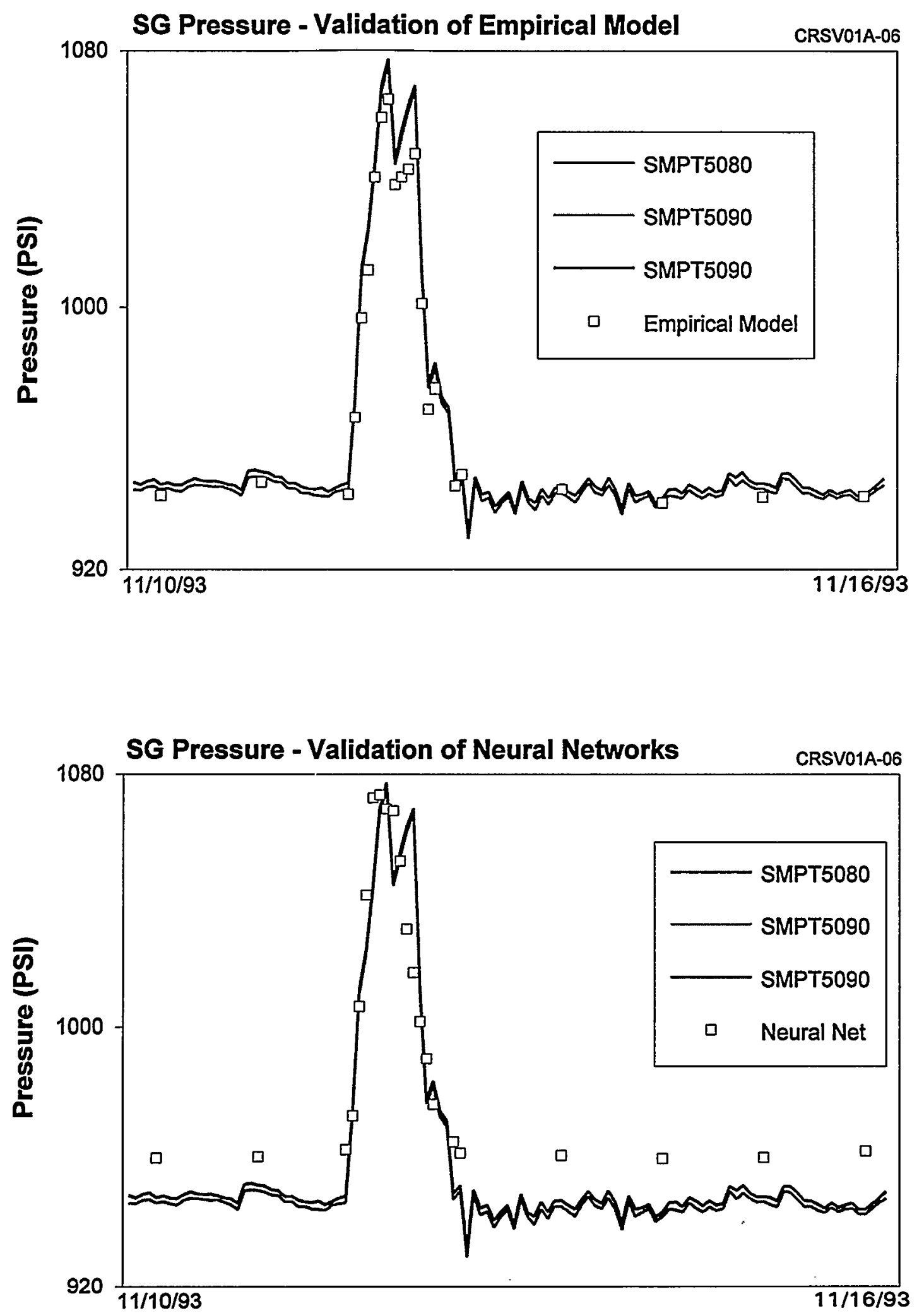

Figure 16.7 Model Validation Results for Three Steam Generator Pressure Signals 

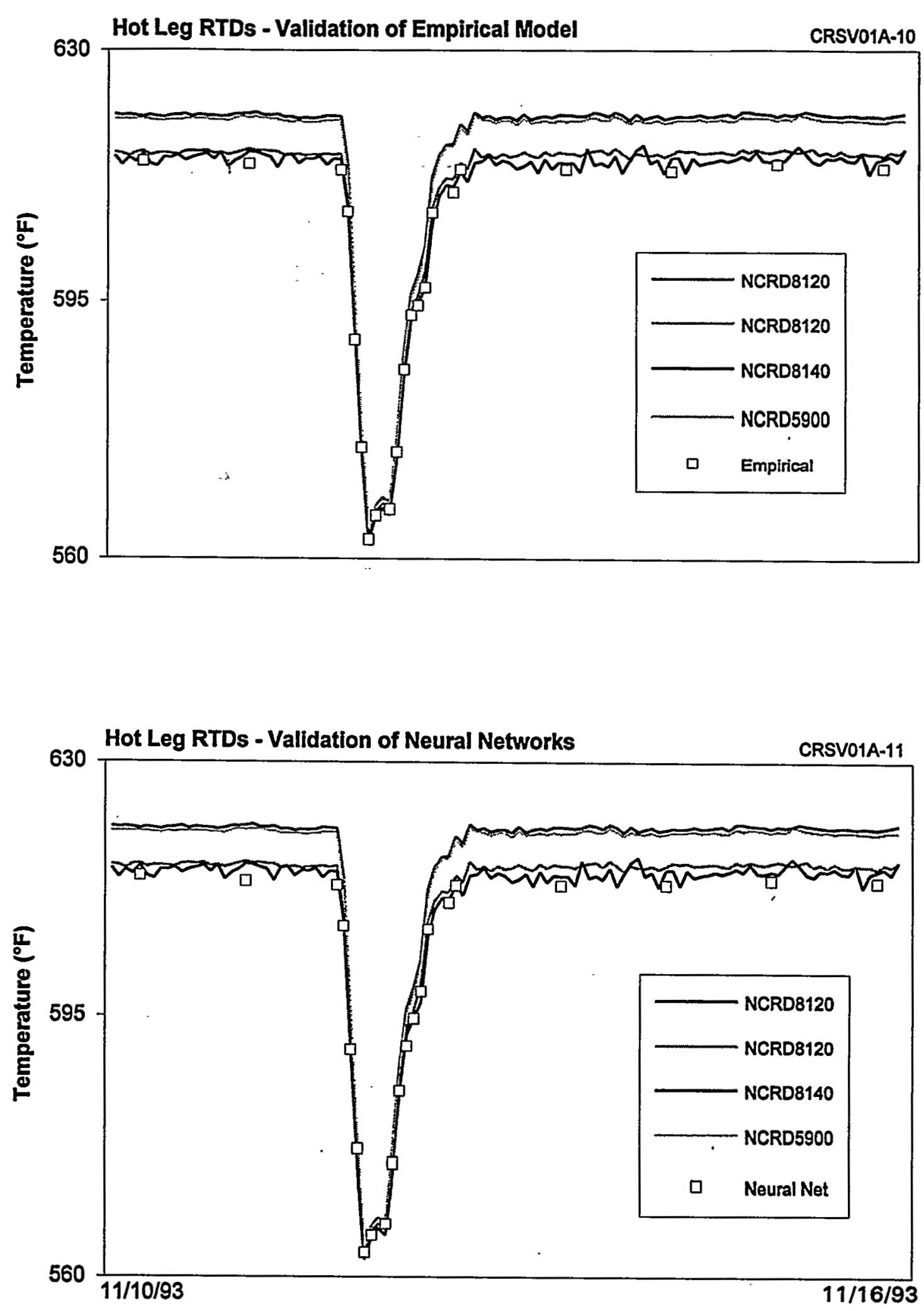

Figure 16.8 Model Validation Results for Four Hot Leg RTD Signals 

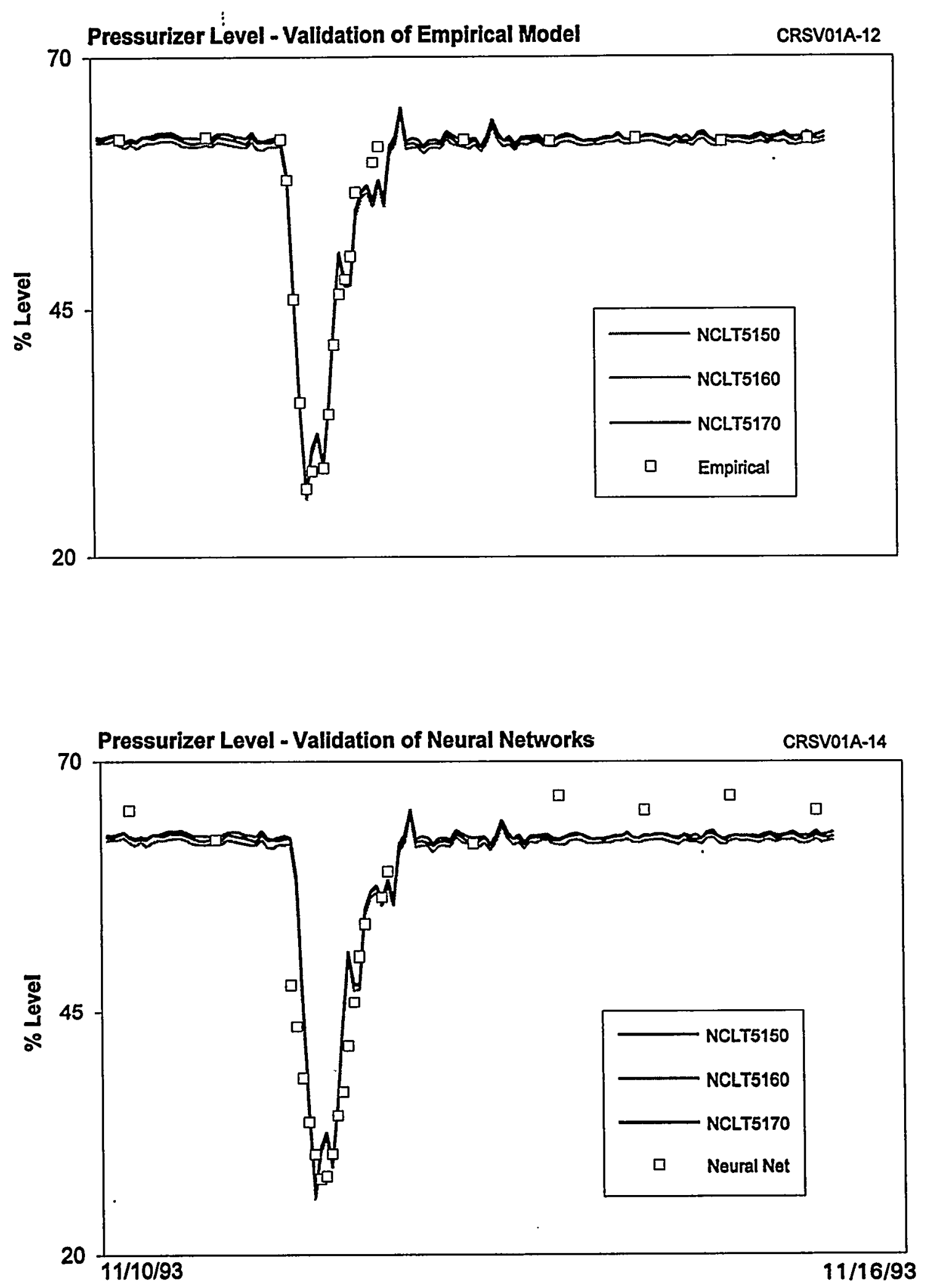

Figure 16.9 Model Validation Results for Three Pressurizer Level Signals 
following procedure:

1. Perform on-line drift monitoring on as many instrument channels at the McGuire nuclear plant as possible and identify those channels which have drifted.

2. Calculate the drift of each instrument using the on-line monitoring results.

3. Calculate the drift of each instrument using the manual calibration data.

4. Compare the drift from on-line monitoring with drift from manual calibrations.

Figure 16.10 shows the results of the above procedure for the McGuire pressurizer level transmitters. These results are from data obtained during the first of the two operating cycles during which this project was conducted at McGuire. The good agreement between the results of manual calibrations and on-line calibration tests is apparent in Figure 16.10. However, good agreements such as this were identified in only a few cases. In most cases, there were larger differences between the results of manual calibrations and on-line monitoring tests. Figure 16.11 shows the distribution of these results from both the averaging and analytical techniques. The averaging results in this figure are the average of both simple and weighted averaging techniques and the modeling results are the average of the empirical modeling and neural network results. These results show that differences between drift from manual and on-line calibration tests were less than 0.5 percent in about 80 percent of the cases and that 100 percent agreement was achieved when the band was increased to 1.5 percent. These results did not appear at first to support the validity of the on-line calibration tests. It was later concluded that a comparison of drift from manual calibrations with that of on-line monitoring is not a valid comparison since the two tests are not performed under the same environmental conditions. For example, manual calibrations are performed during refueling outages when the plant is at cold shutdown, while on-line calibrations are performed when the plant is at operating conditions. As a result, in-plant validation of on-line monitoring techniques cannot be based on the agreement between the drift identified from manual and on-line calibrations. The preferred method for evaluating the results of the on-line monitoring tests is to plot them within drift bands or CSA bands and identify channels that fall out of either of the two bands.

\subsection{Presentation of Drift Results}

Figure 16.12 through 16.27 provide raw data and drift plots for the sixteen services tested at McGuire. Drift bands are also shown on the drift plots for nine services for which allowable drift information was available (see Chapter 11). There are eight plots in each of the first twelve figures (Figures 16.12 through 16.23) and six plots in each of the last four figures (Figures 16.24 through 16.27).

The eight plots on a page include two columns of plots each with four plots for cycle 1 and four plots for cycle 2. The four plots on each column include the raw data as were sampled in the plant, filtered data to remove the extraneous noise, and two drift plots. One drift plot is based on the averaging techniques and the other drift plot is based on the analytical techniques. The plot that is based on the averaging techniques is the average of simple and weighted averages if both methods were used; and the plot that is based on the analytical techniques is the average of results from empirical modeling and neural networks.

For the four services shown in Figures 16.24 through 16.27, there are no analytical techniques because independent inputs for 


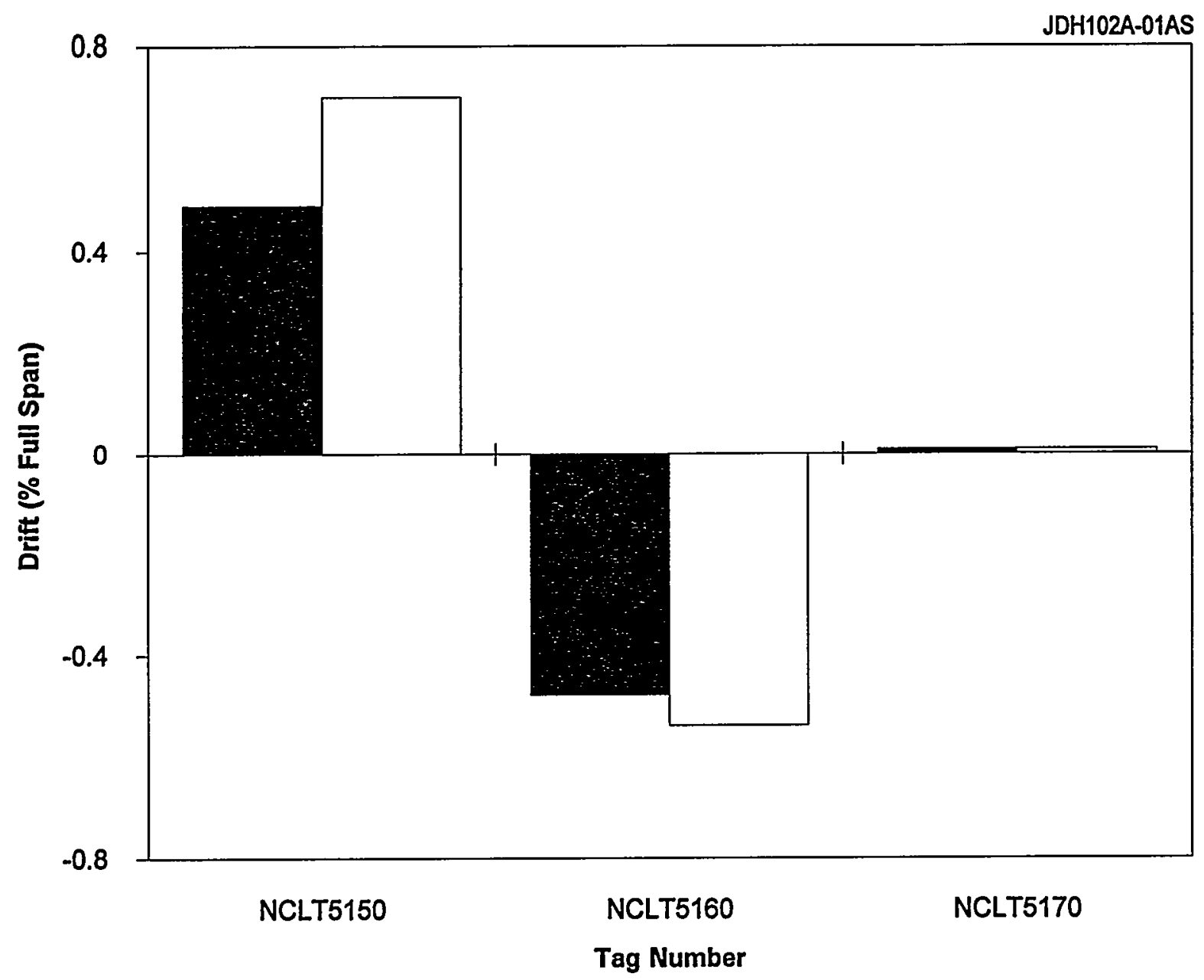

On-line Monitoring Results $\square$ Manual Calibrations

Figure 16.10 Comparison of Drift from Manual Calibrations with Drift from On-Line Calibration for Three Pressurizer Level Transmitters at McGuire 


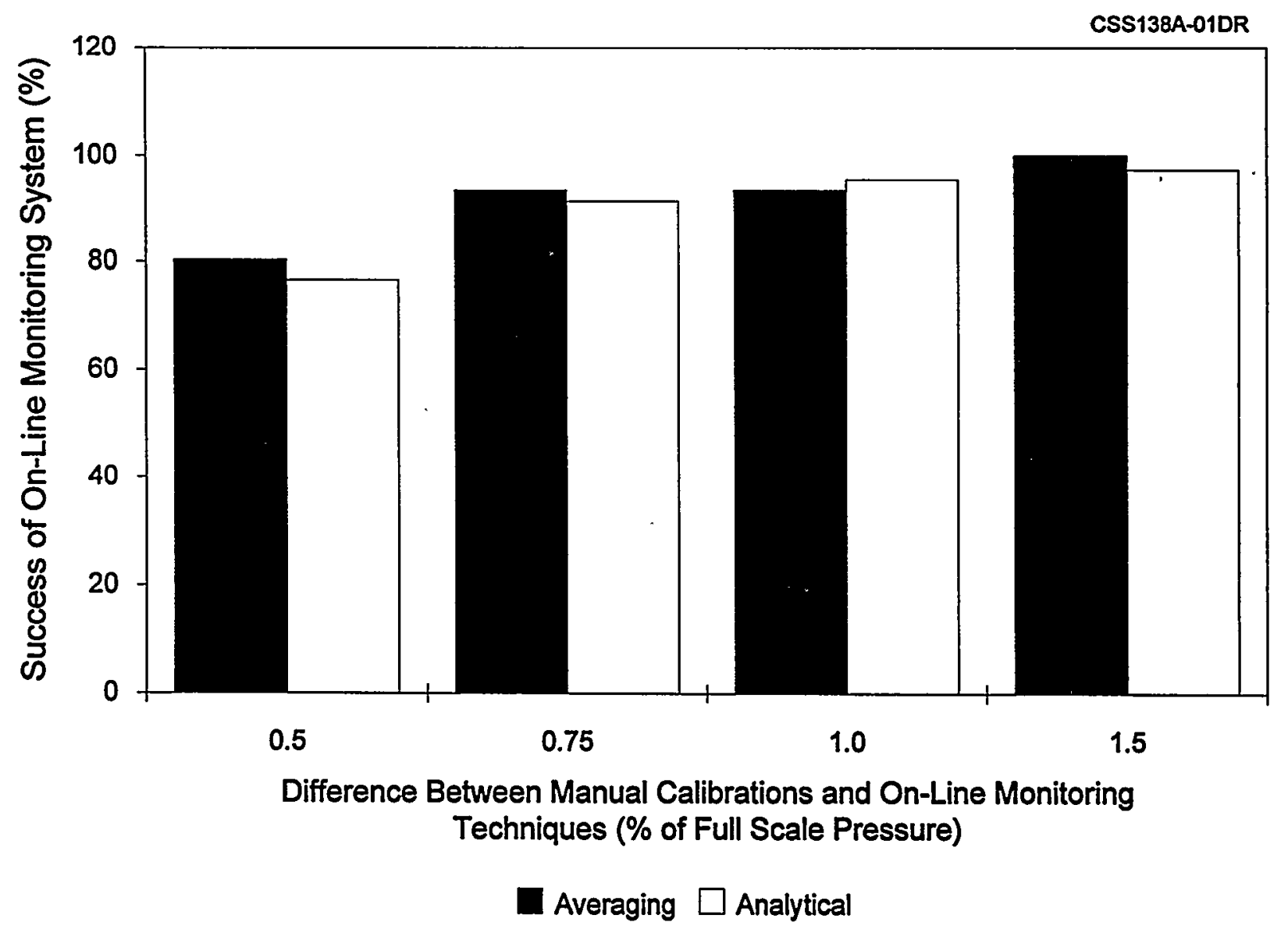

Figure 16.11 Comparison of Manual Calibration Results and On-Line Monitoring Results 


\section{Cycle 1}
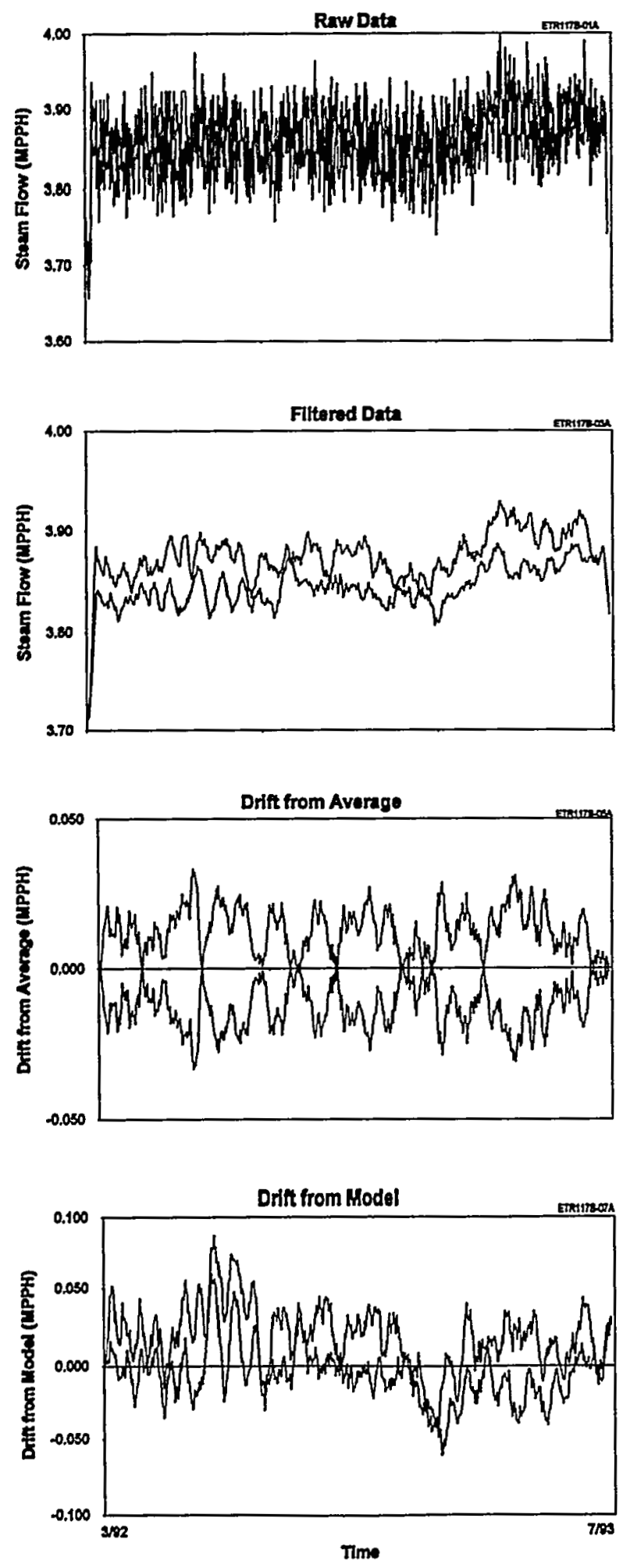

Cycle 2
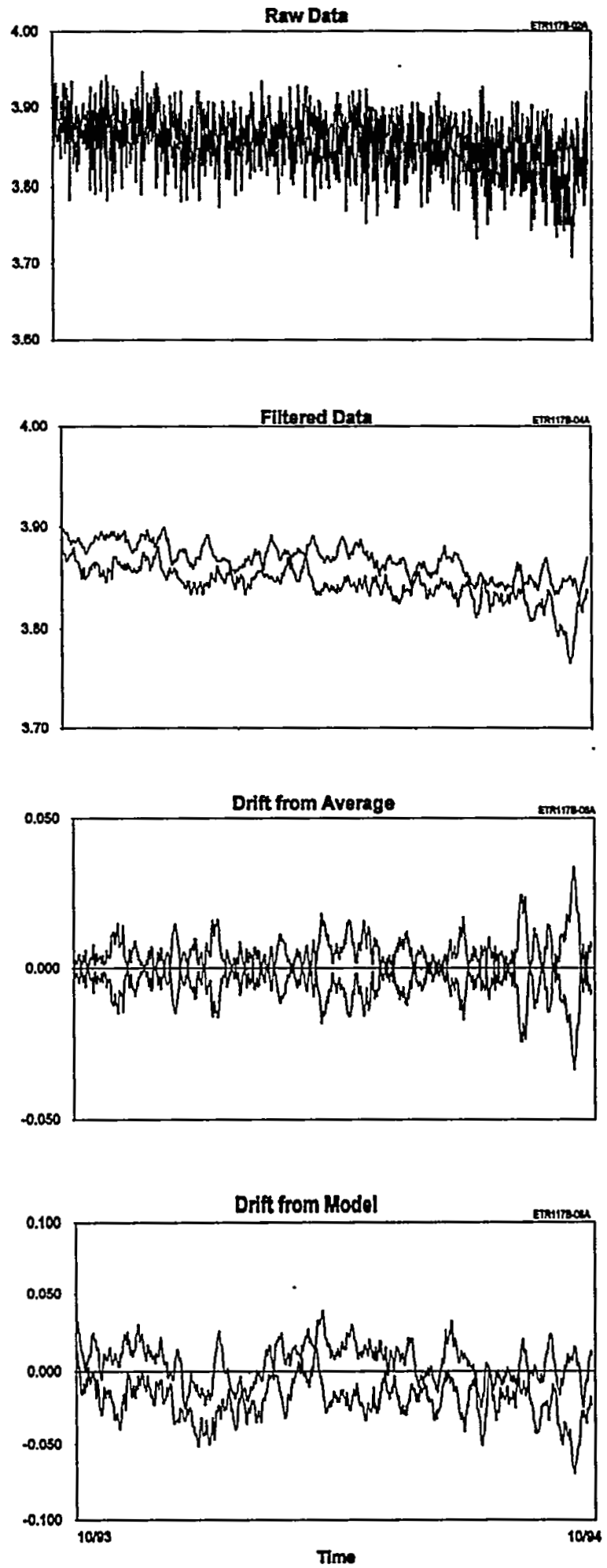

Figure 16.12 Raw Data and Drift Plots for Steam Flow Signals 


\section{Cycle 1}
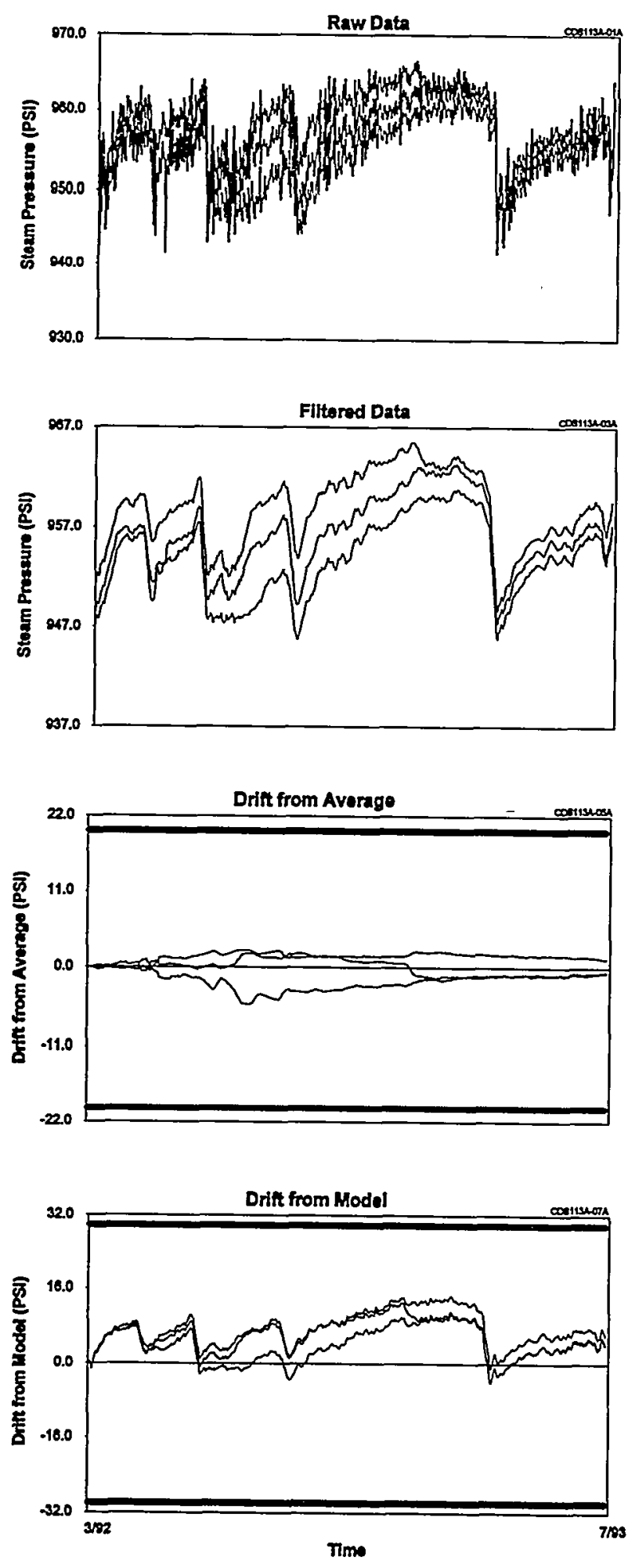

\section{Cycle 2}
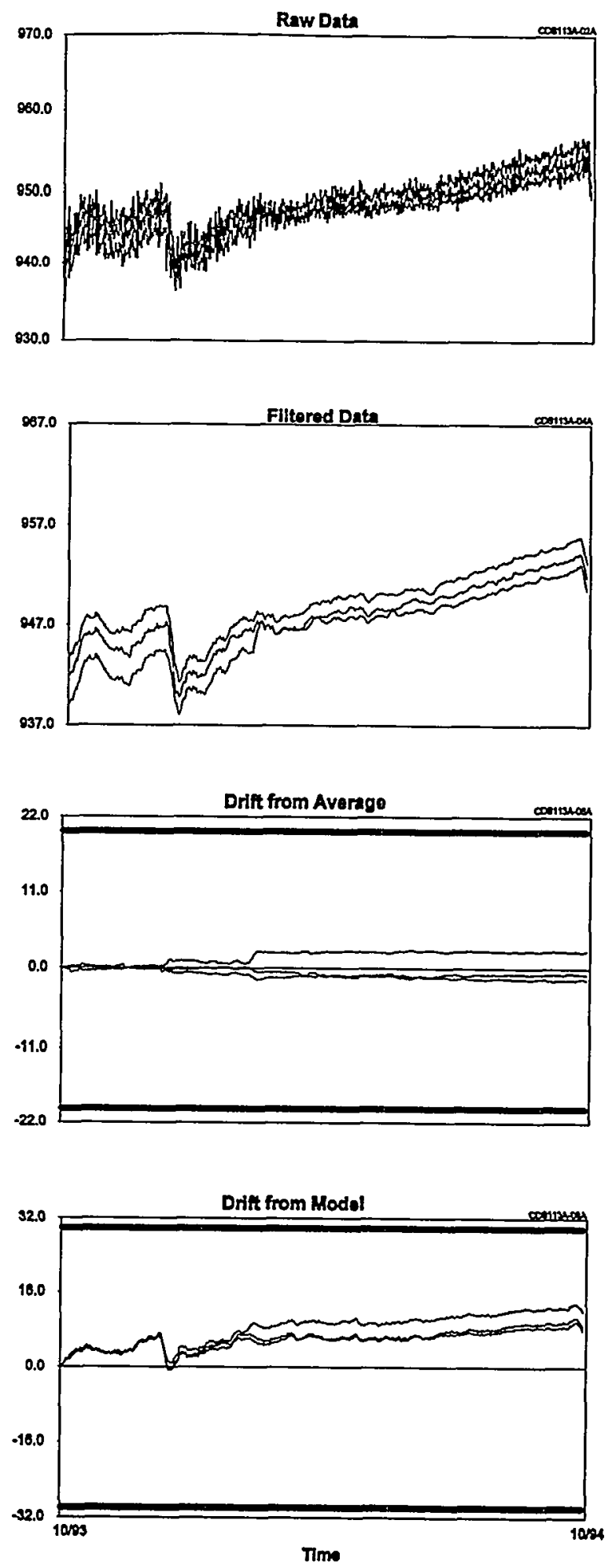

Figure 16.13 Raw Data and Drift Plots for Steam Pressure Signals 


\section{Cycle 1}
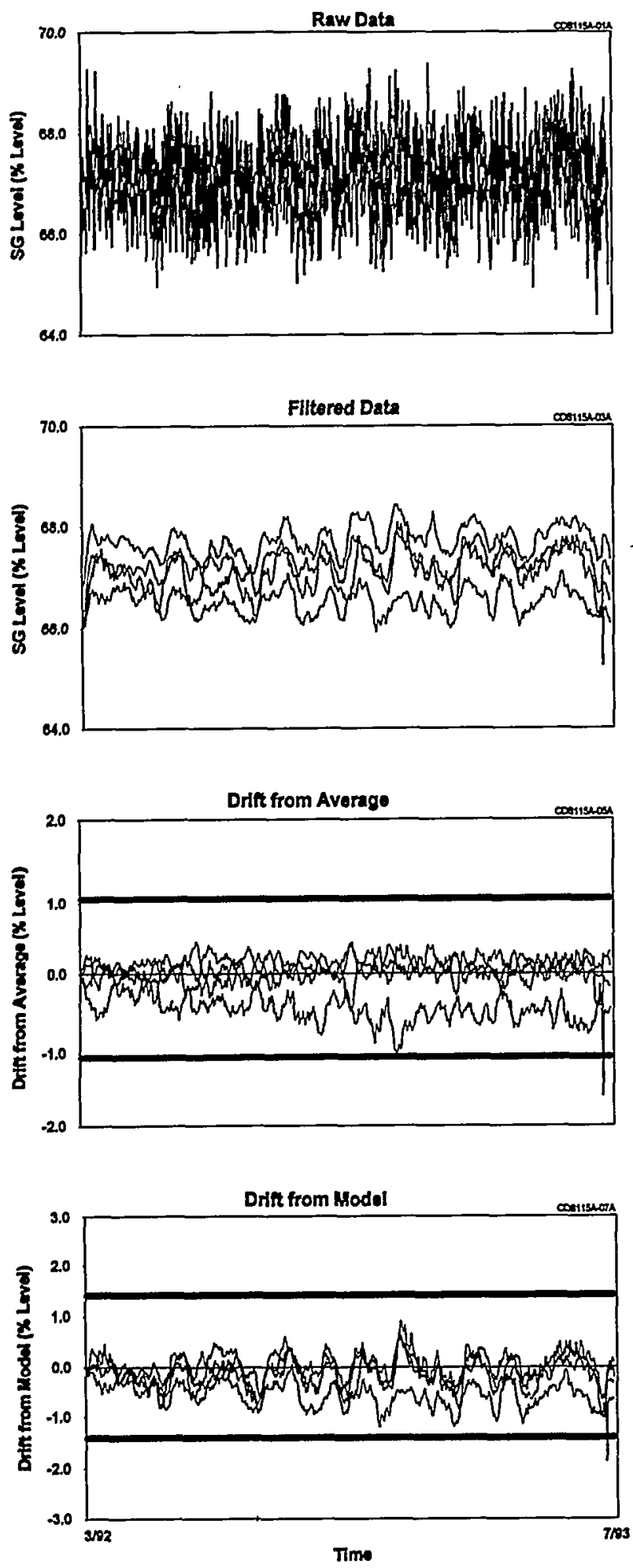

\section{Cycle 2}
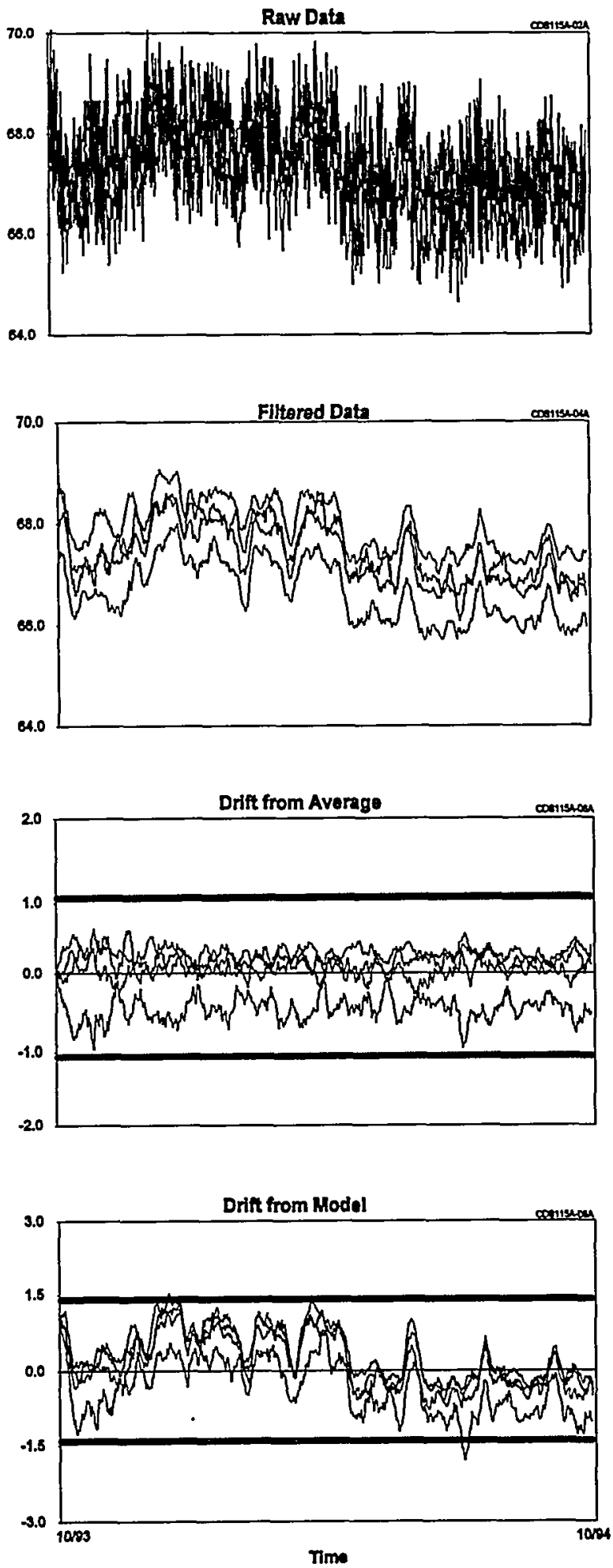

Figure 16.14 Raw Data and Drift Plots for Steam Generator Level Signals 


\section{Cycle 1}
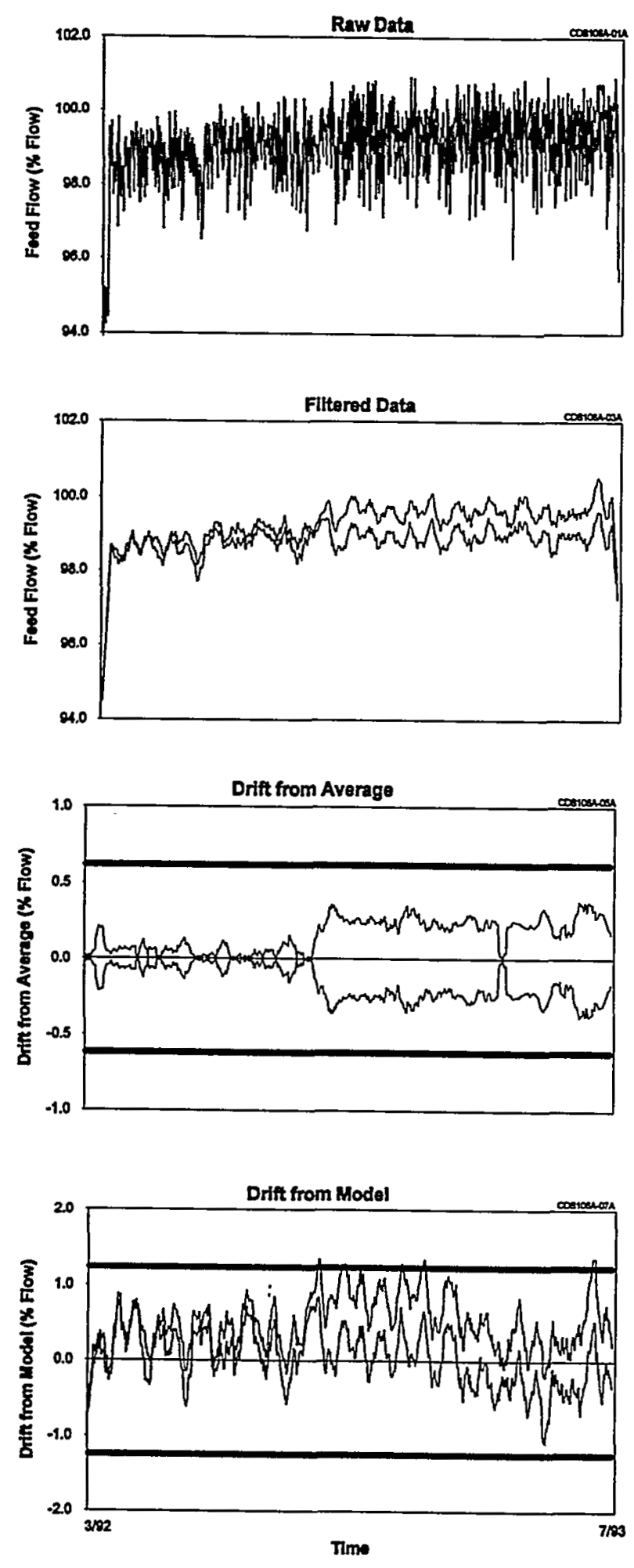

\section{Cycle 2}
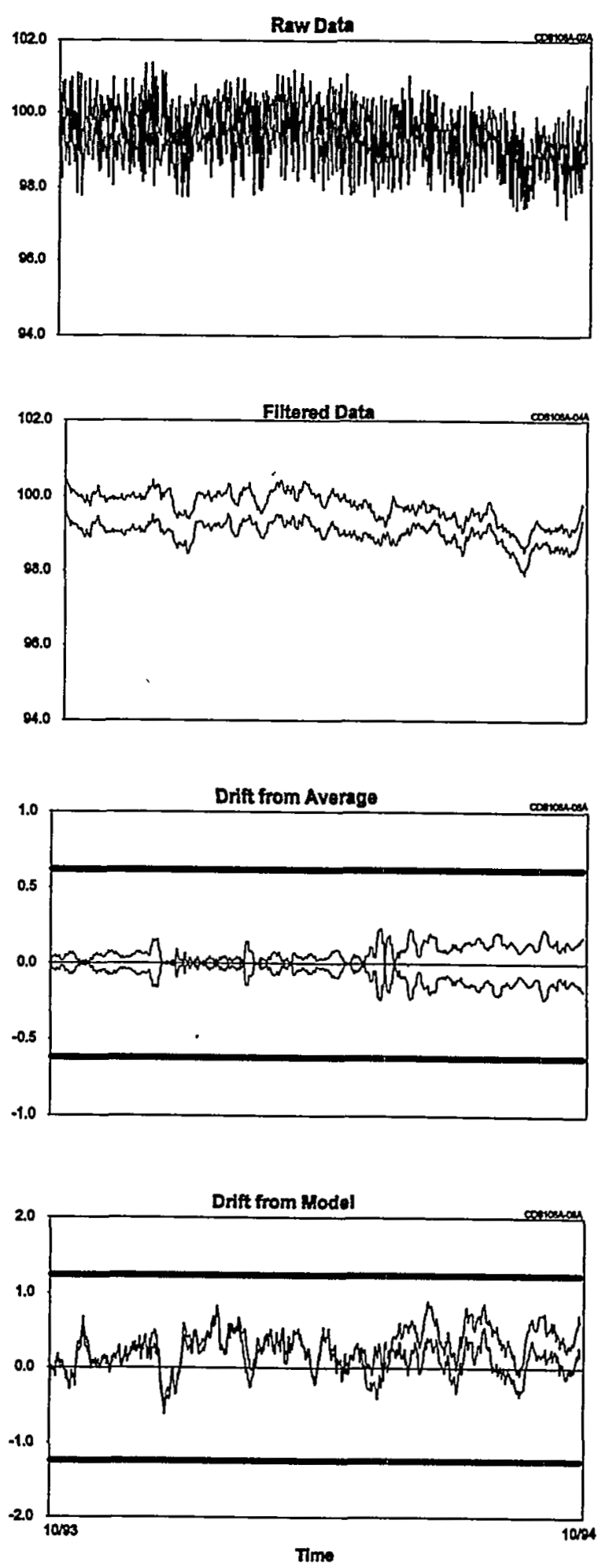

Figure 16.15 Raw Data and Drift Plots for Feedwater Flow Signals 
Cycle 1
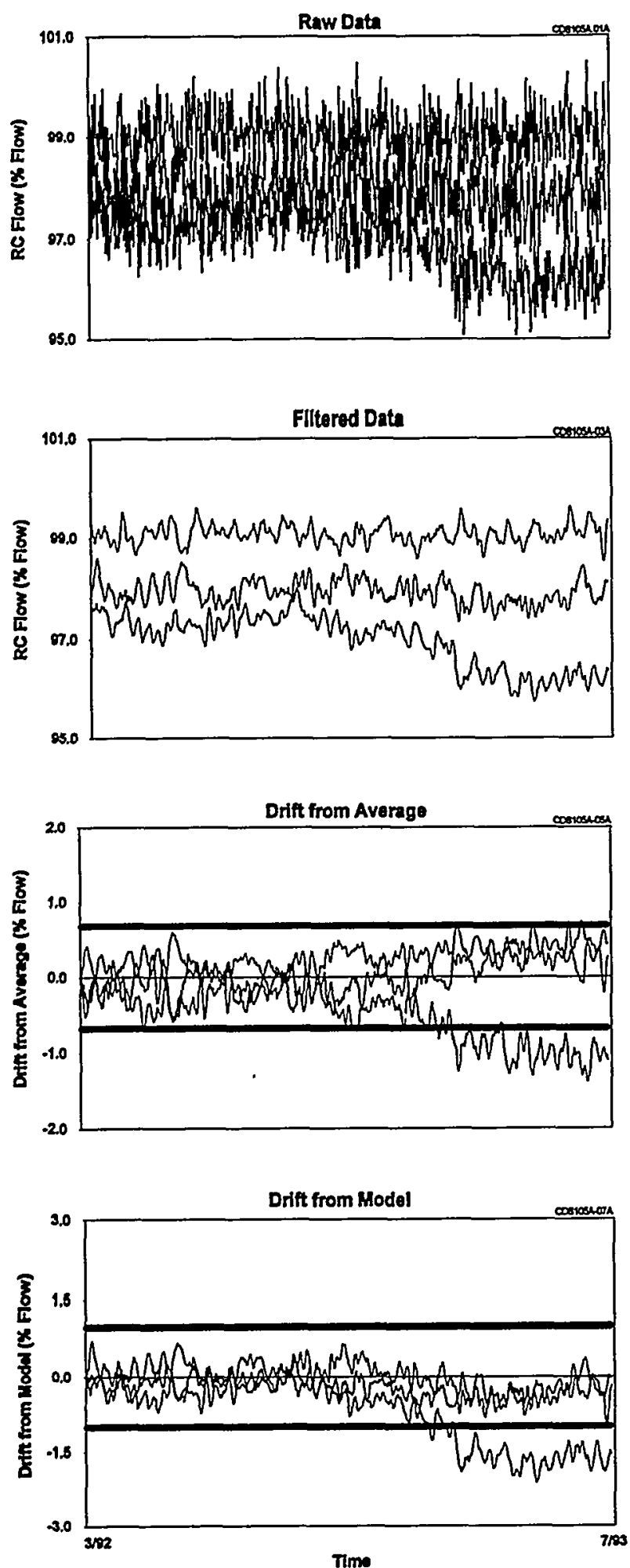

Cycle 2
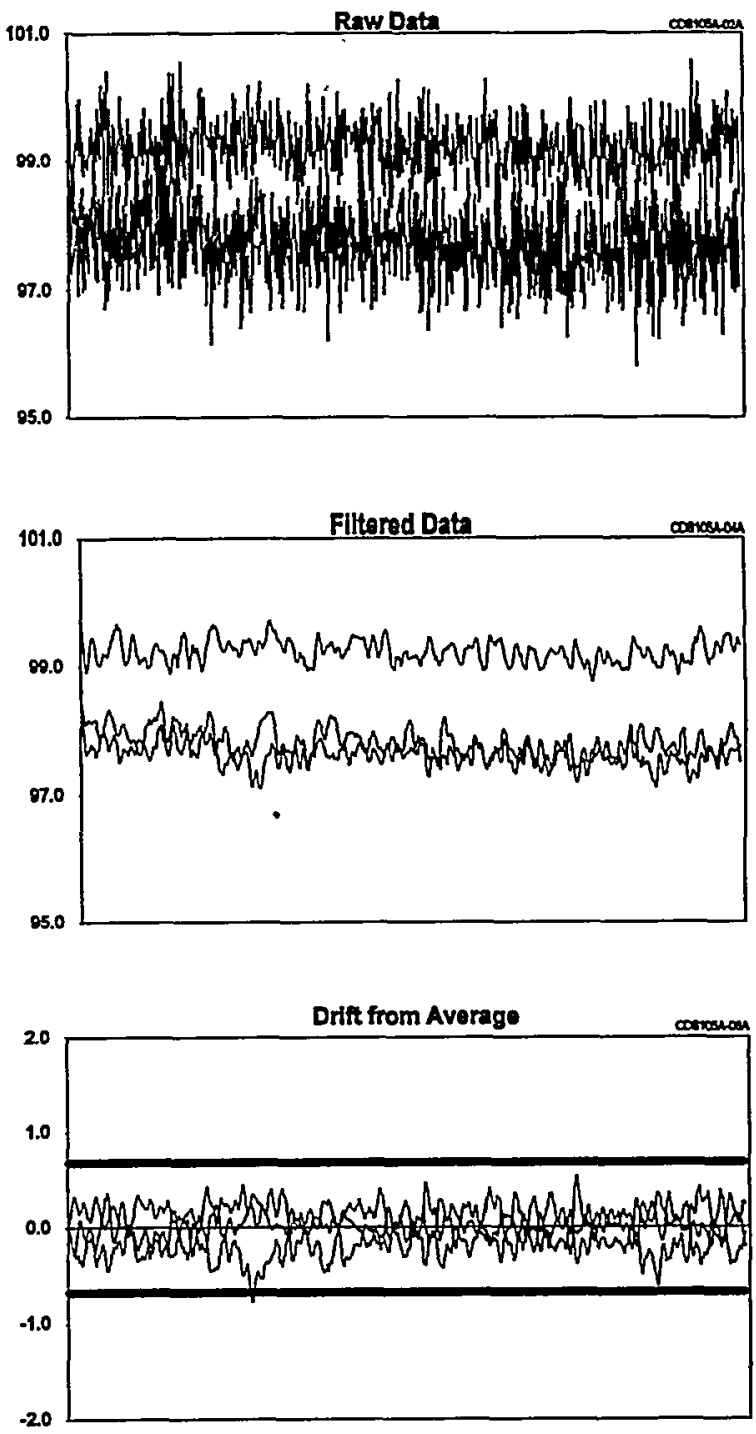

Drift from Hodel

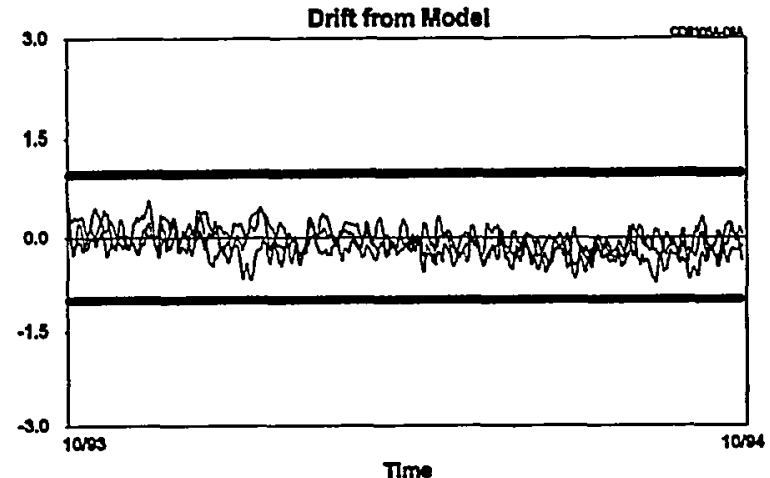

Figure 16.16 Raw Data and Drift Plots for Reactor Coolant Flow Signals 
Cycle 1
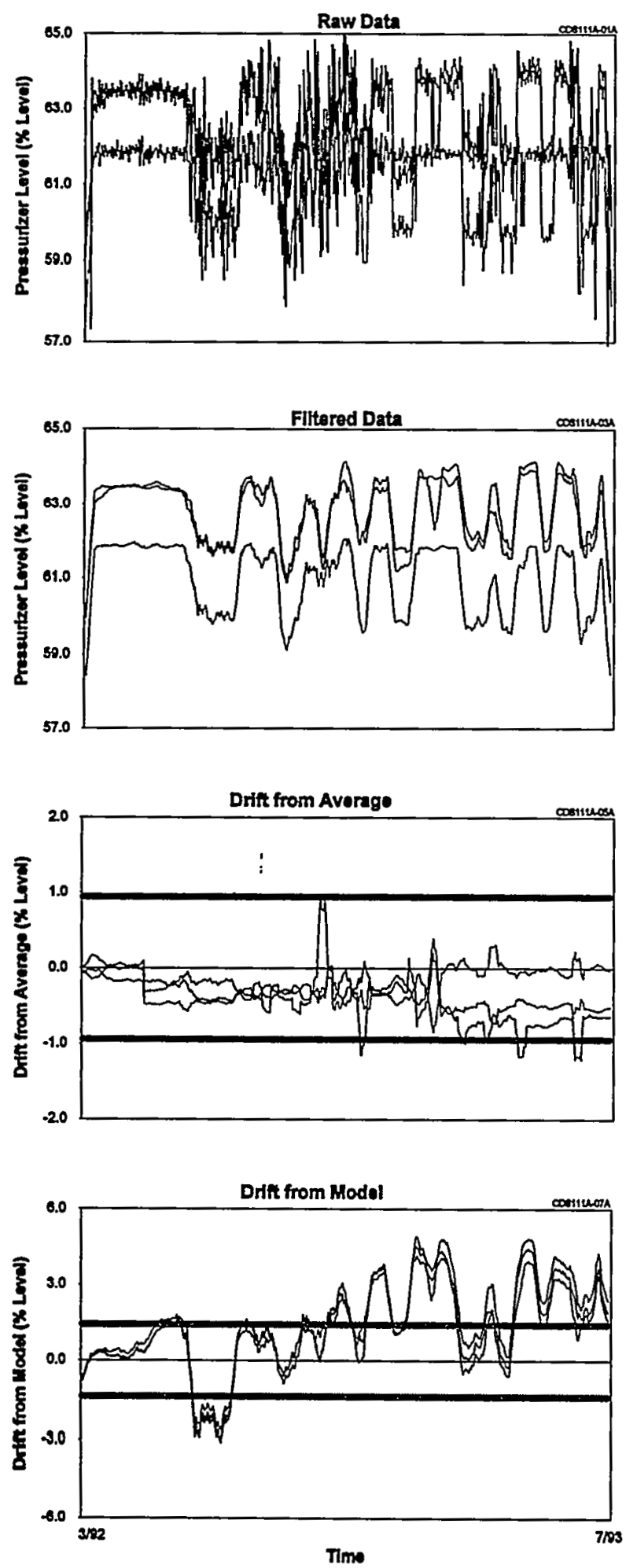

Cycle 2
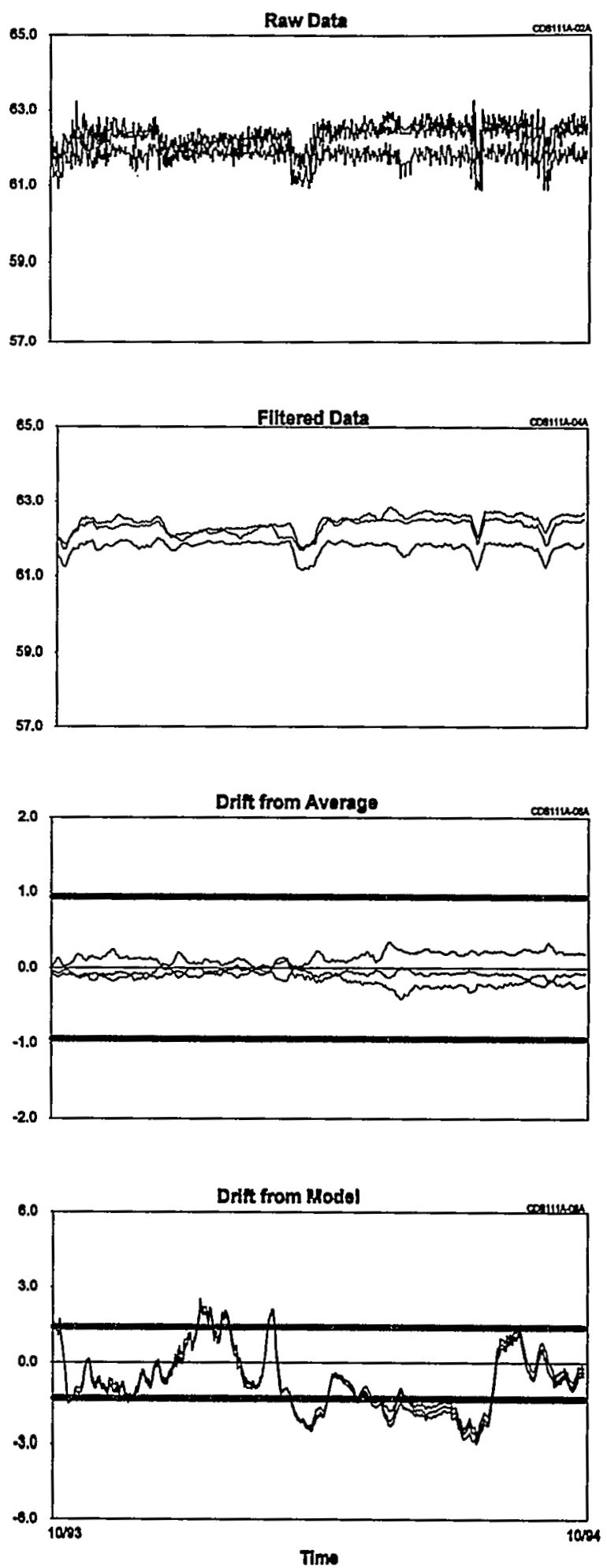

Figure 16.17 Raw Data and Drift Plots for Pressurizer Level Signals 
Cycle 1
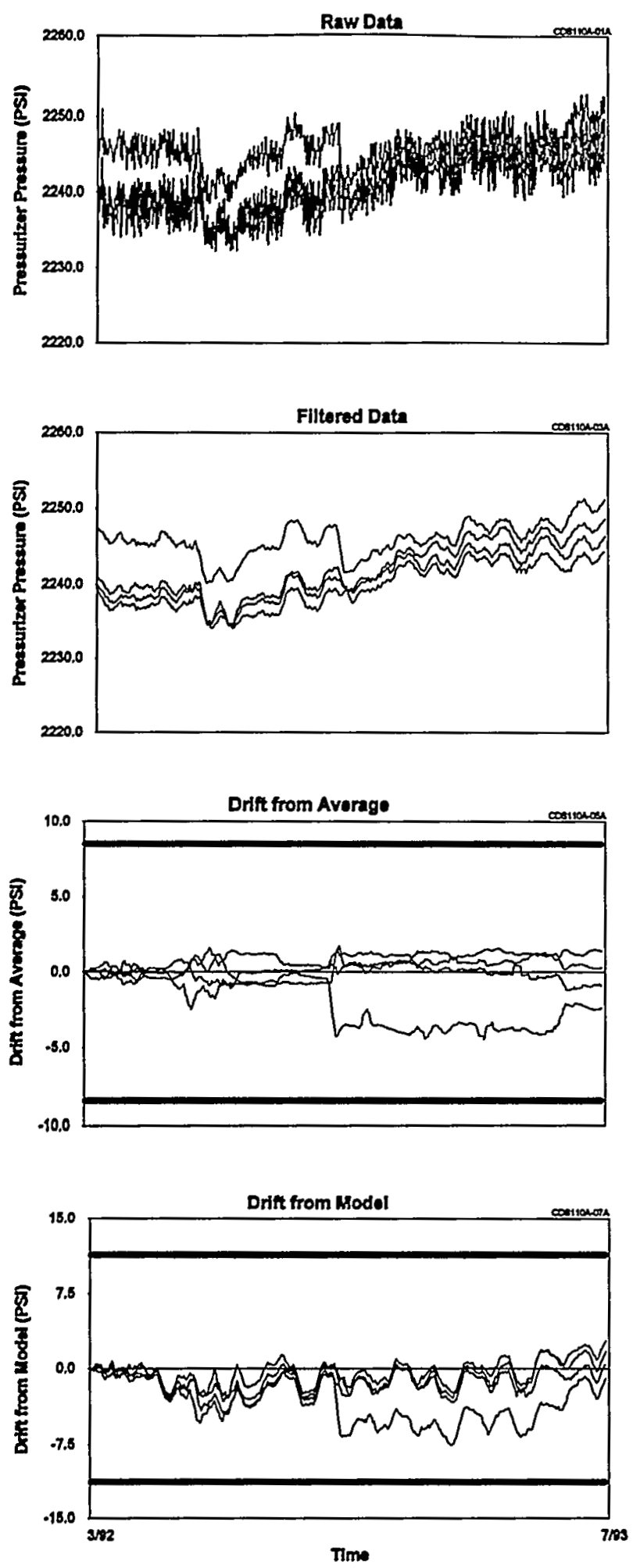

\section{Cycle 2}
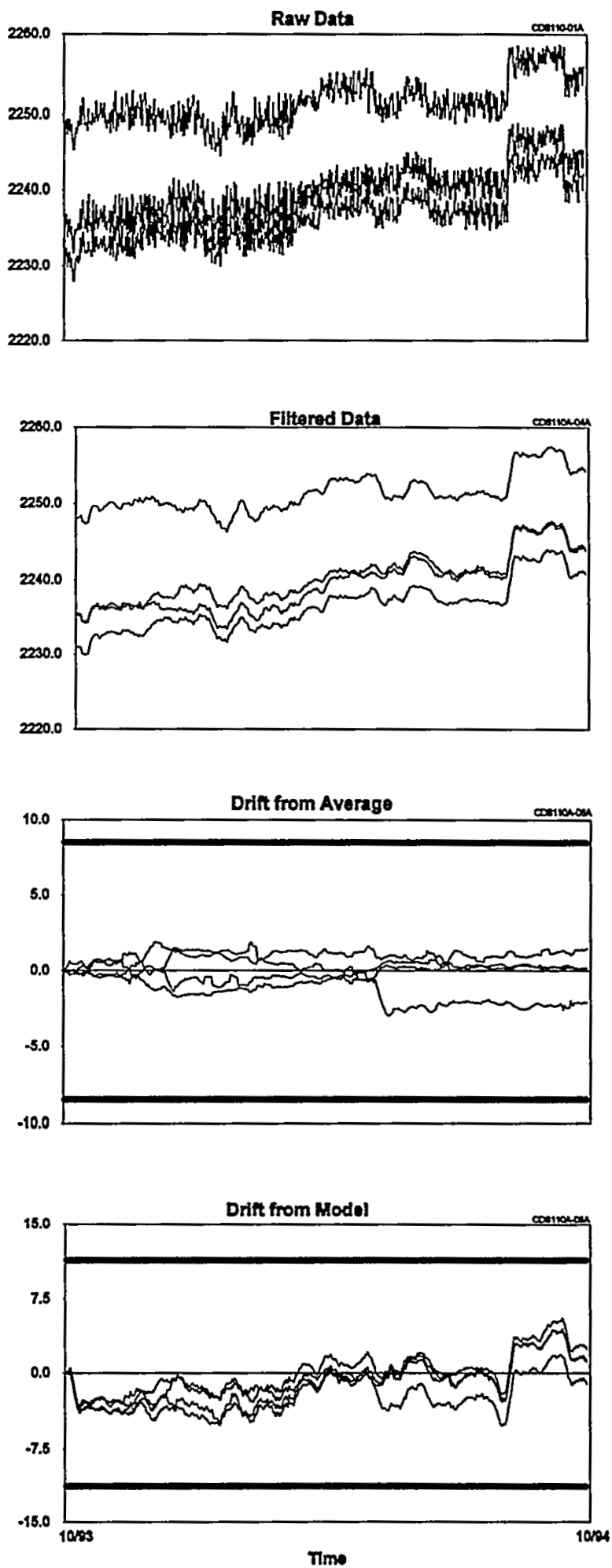

Figure 16.18 Raw Data and Drift Plots for Pressurizer Pressure Signals 
Cycle 1
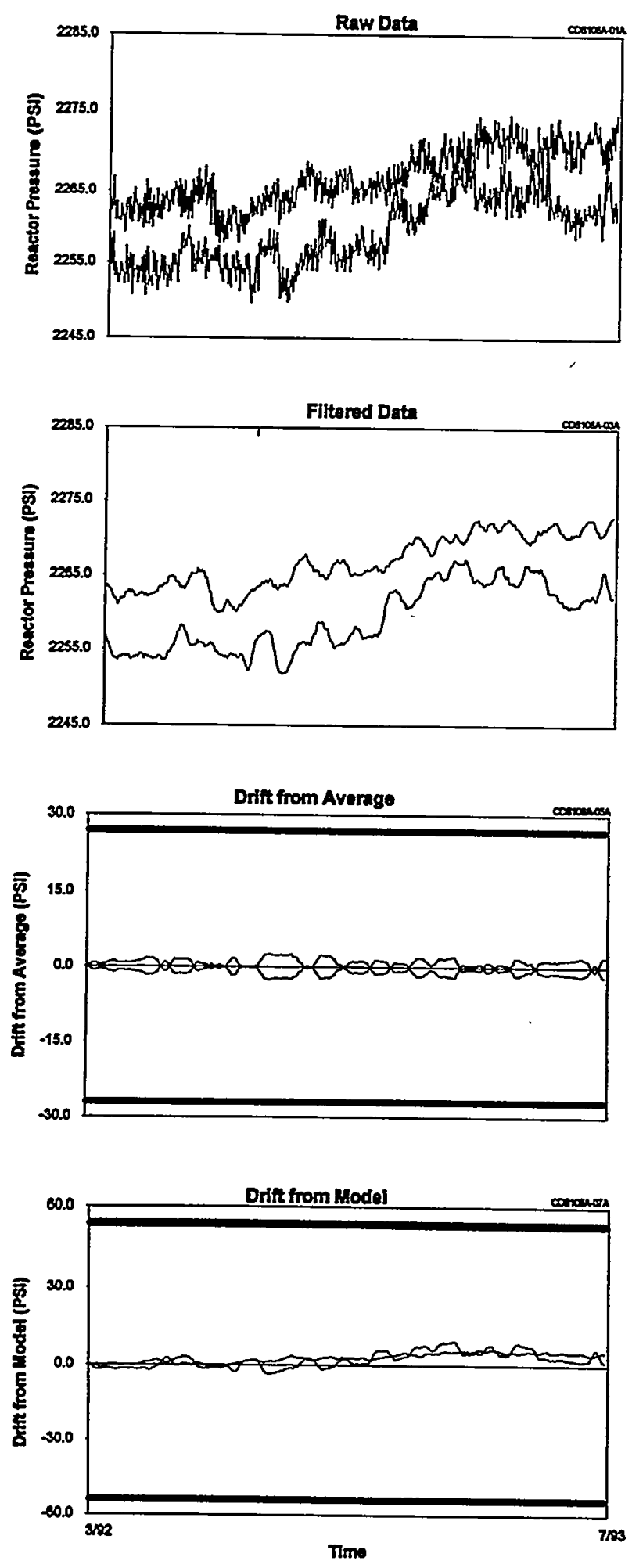

\section{Cycle 2}
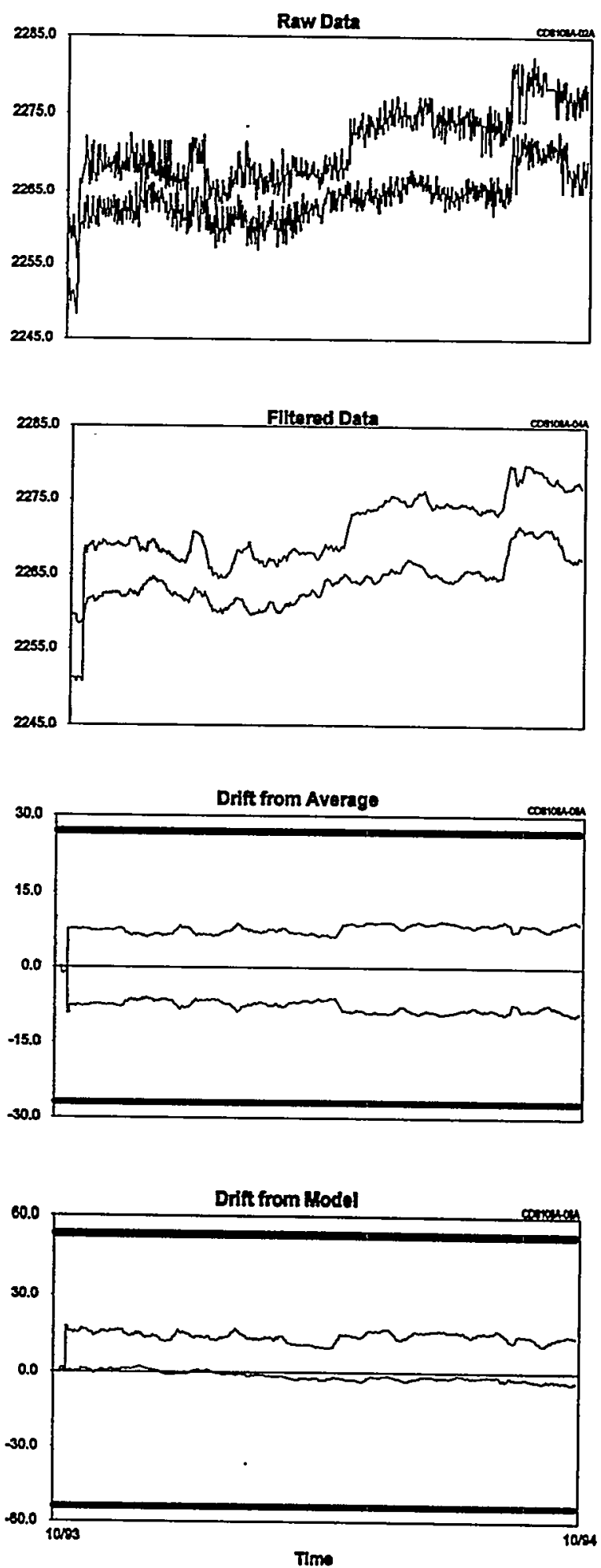

Figure 16.19 Raw Data and Drift Plots for Wide-Range Pressure Signals 
Cycle 1
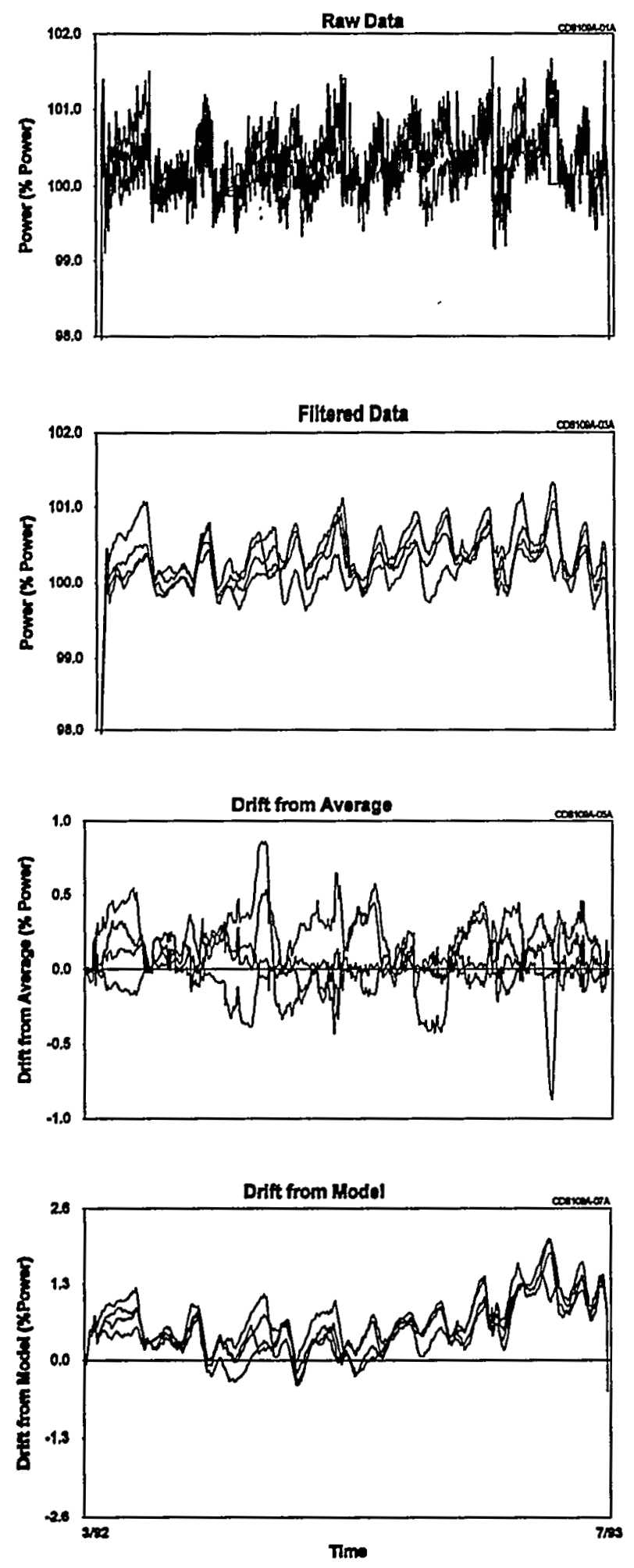

Cycle2
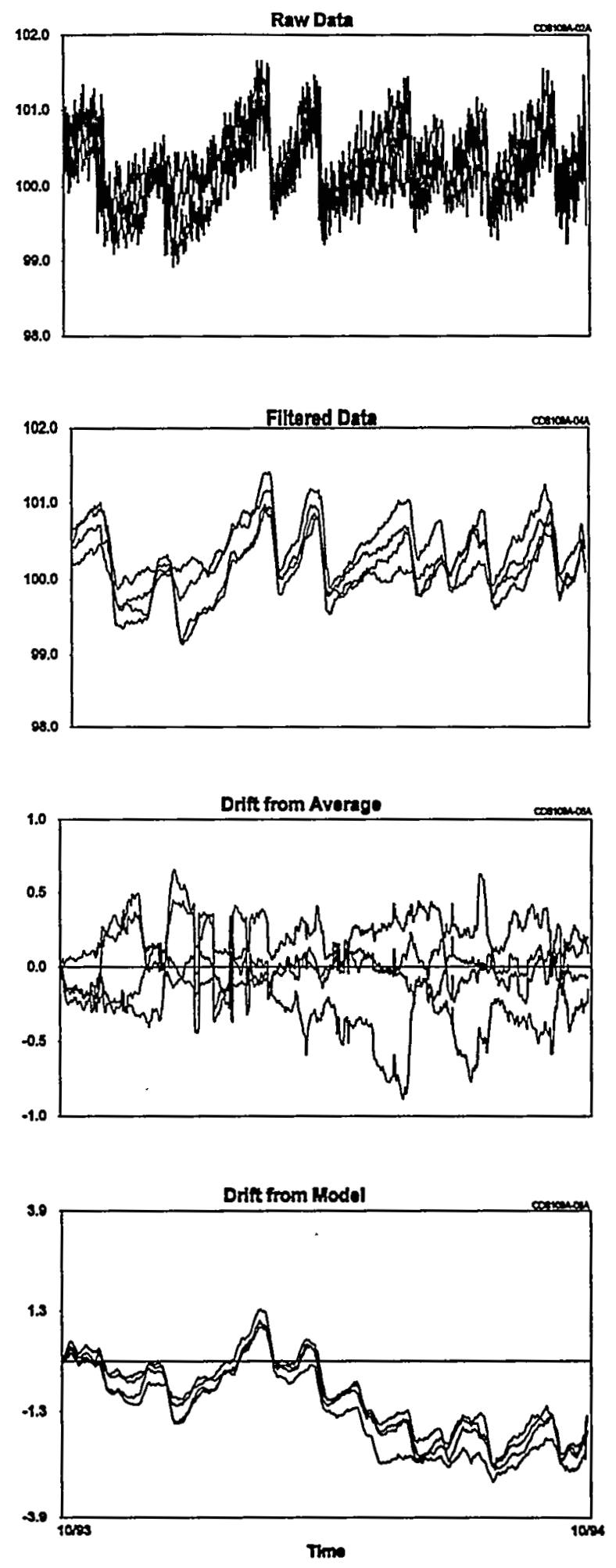

Figure 16.20 Raw Data and Drift Plots for Neutron Flux Signals 


\section{Cycle 1}
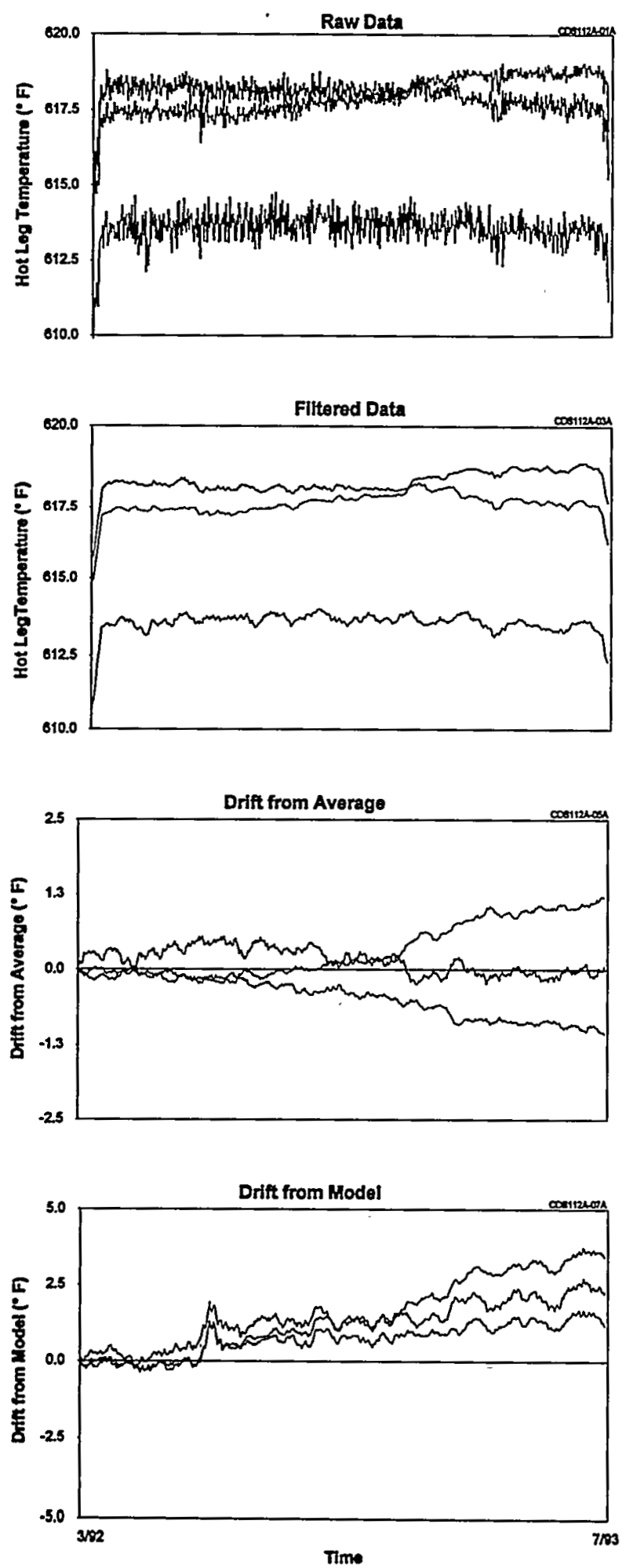

Cycle 2
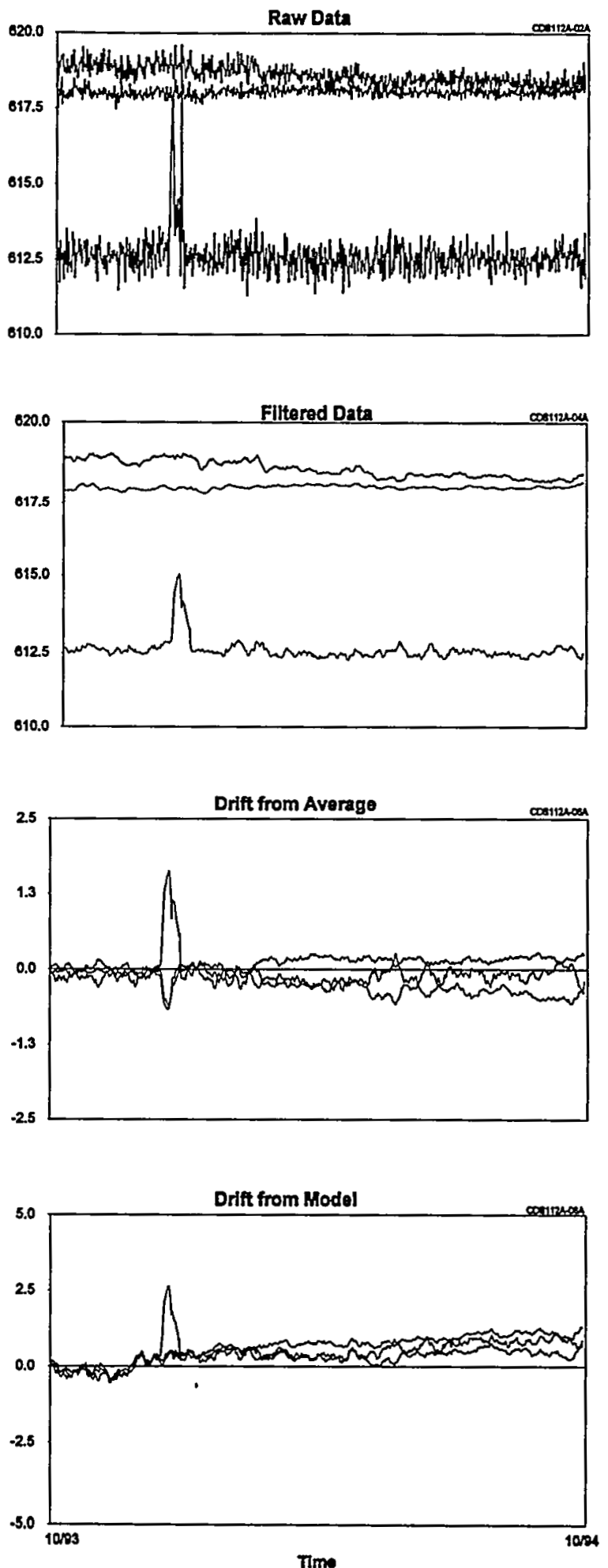

Figure 16.21 Raw Data and Drift Plots for Hot Leg RTD Signals 
Cycle 1
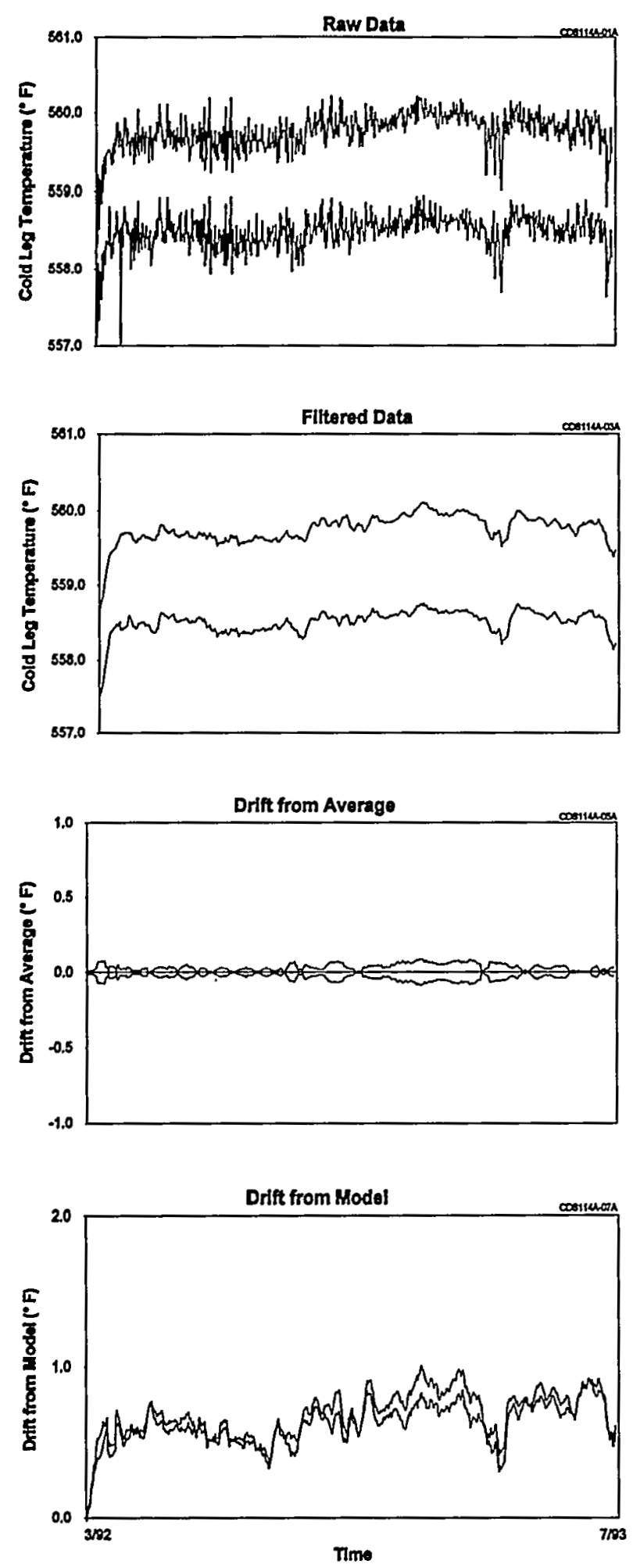

\section{Cycle 2}
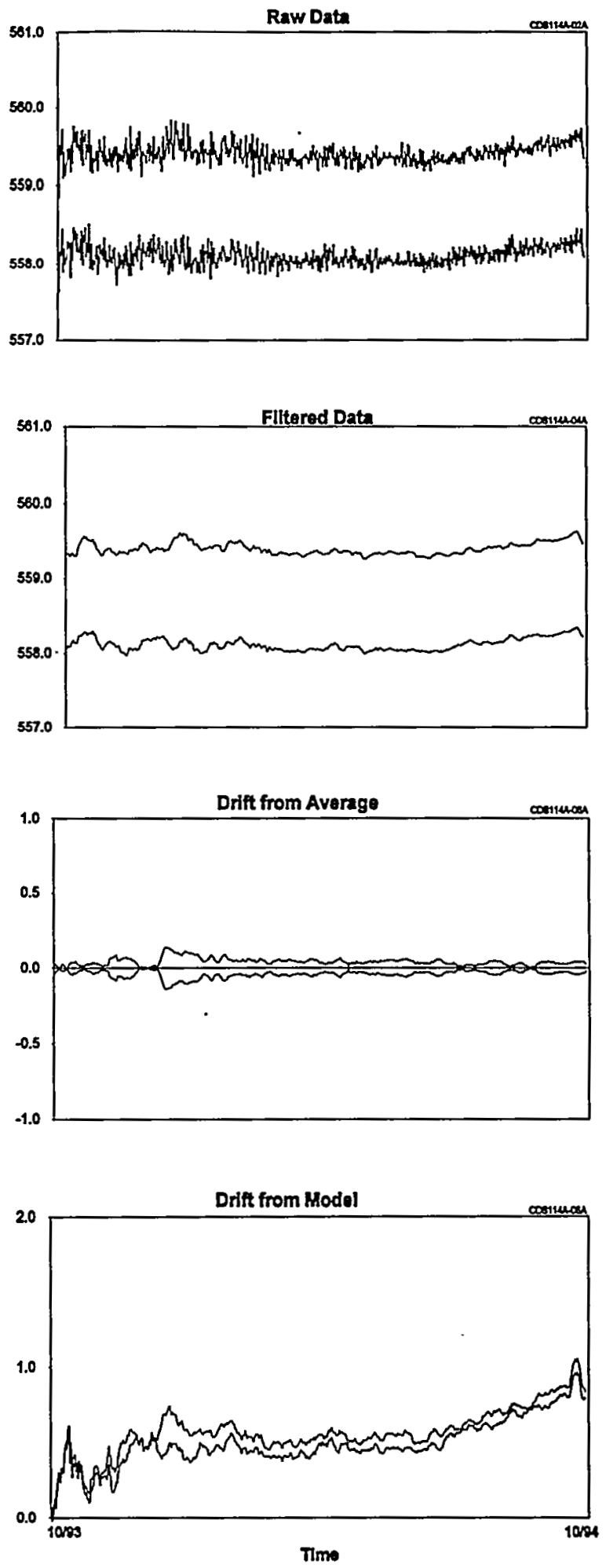

Figure 16.22 Raw Data and Drift Plots for Cold Leg RTD Signals 


\section{Cycle 1}
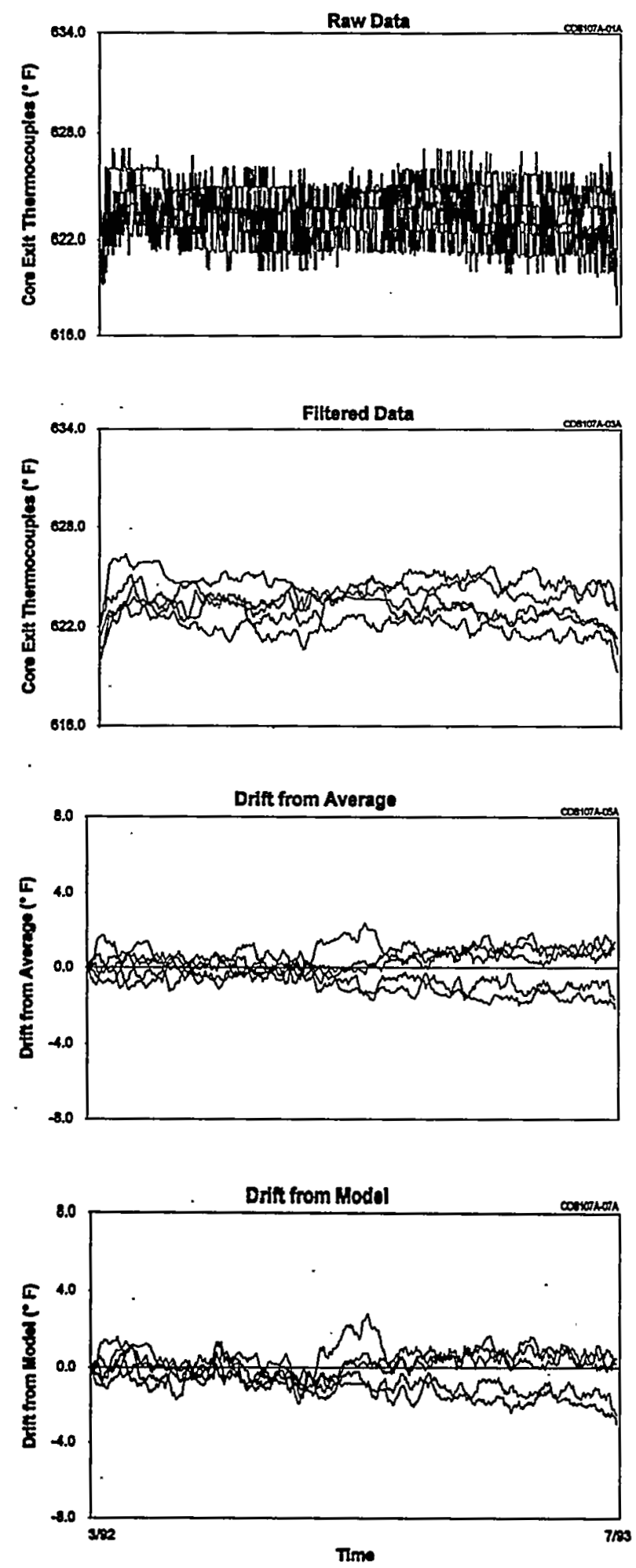

\section{Cycle 2}
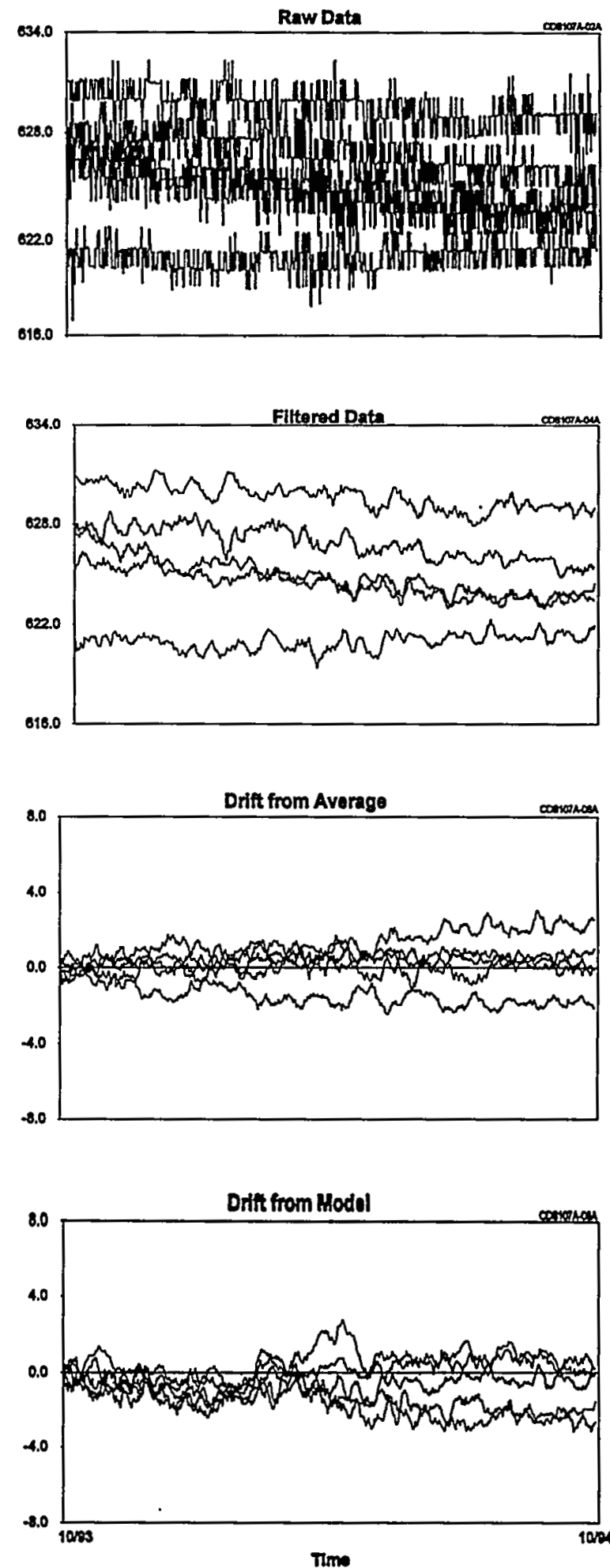

Figure 16.23 Raw Data and Drift Plots for Core Exit Thermocouple Signals 


\section{Cycle 1}
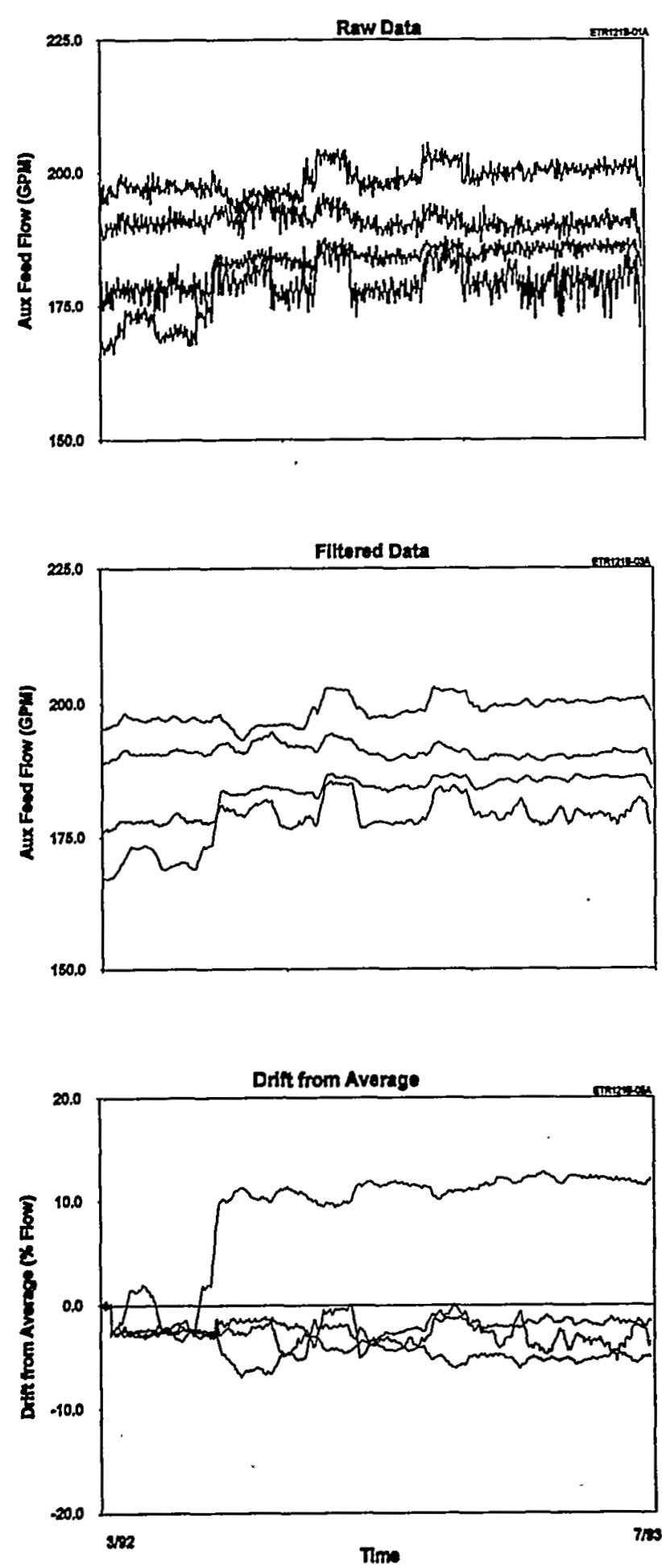

\section{Cycle 2}
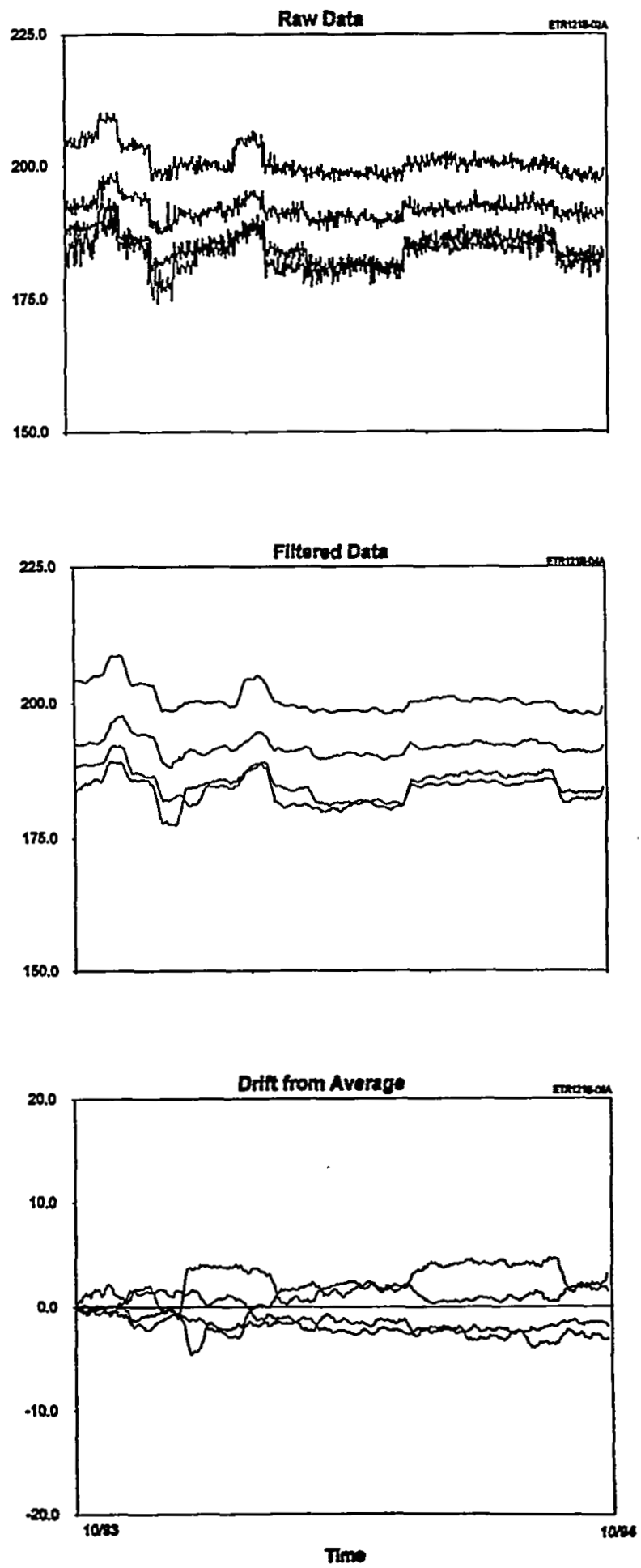

Figure 16.24 Raw Data and Drift Plots for Auxiliary Feedwater Flow Signals 


\section{Cycle 1}
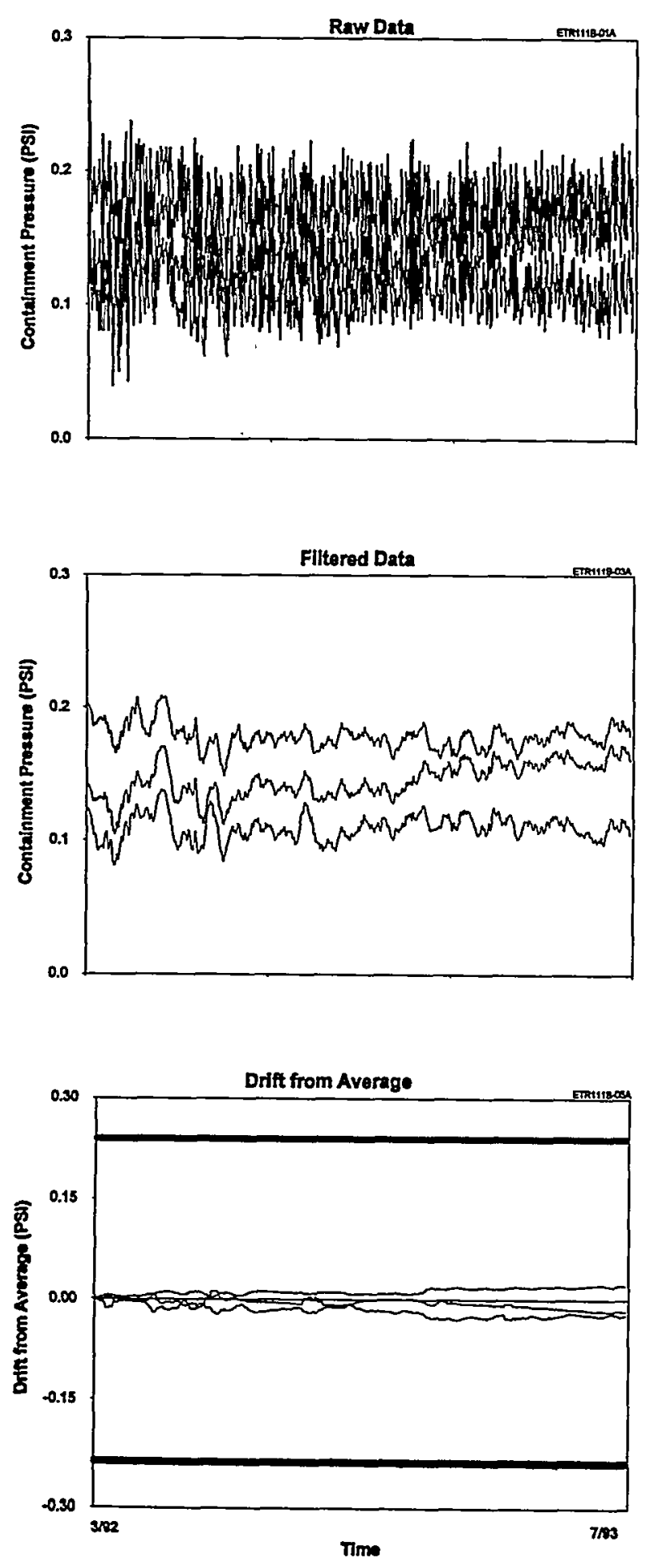

Cycle 2
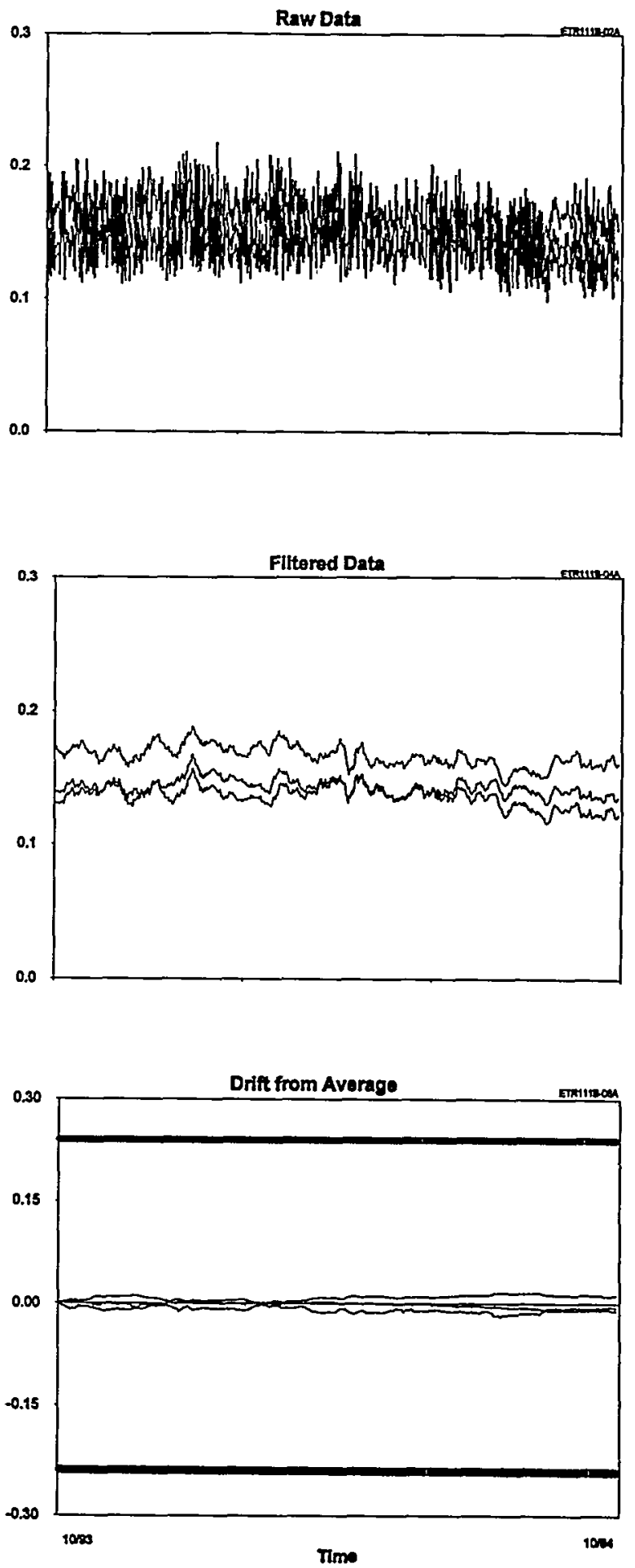

Figure 16.25 Raw Data and Drift Plots for Containment Pressure Signals 
Cycle 1
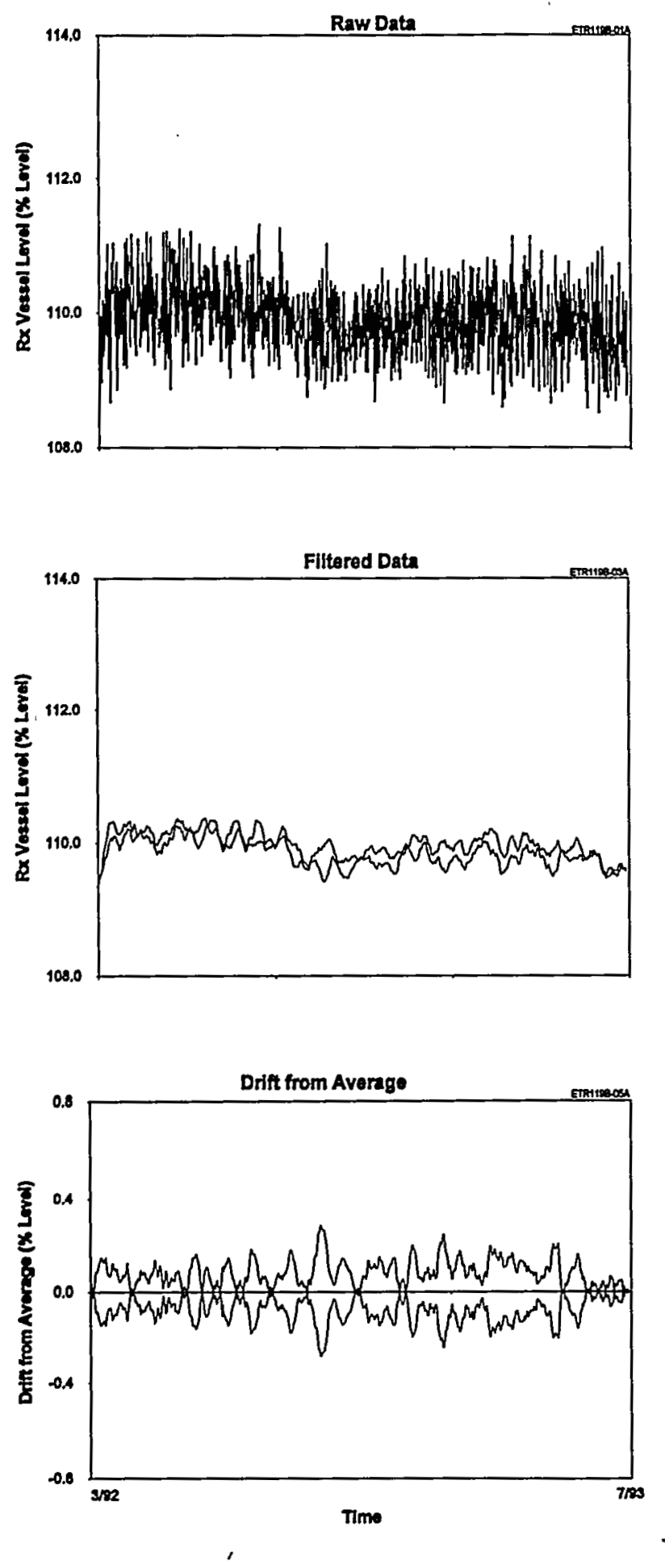

Cycle 2
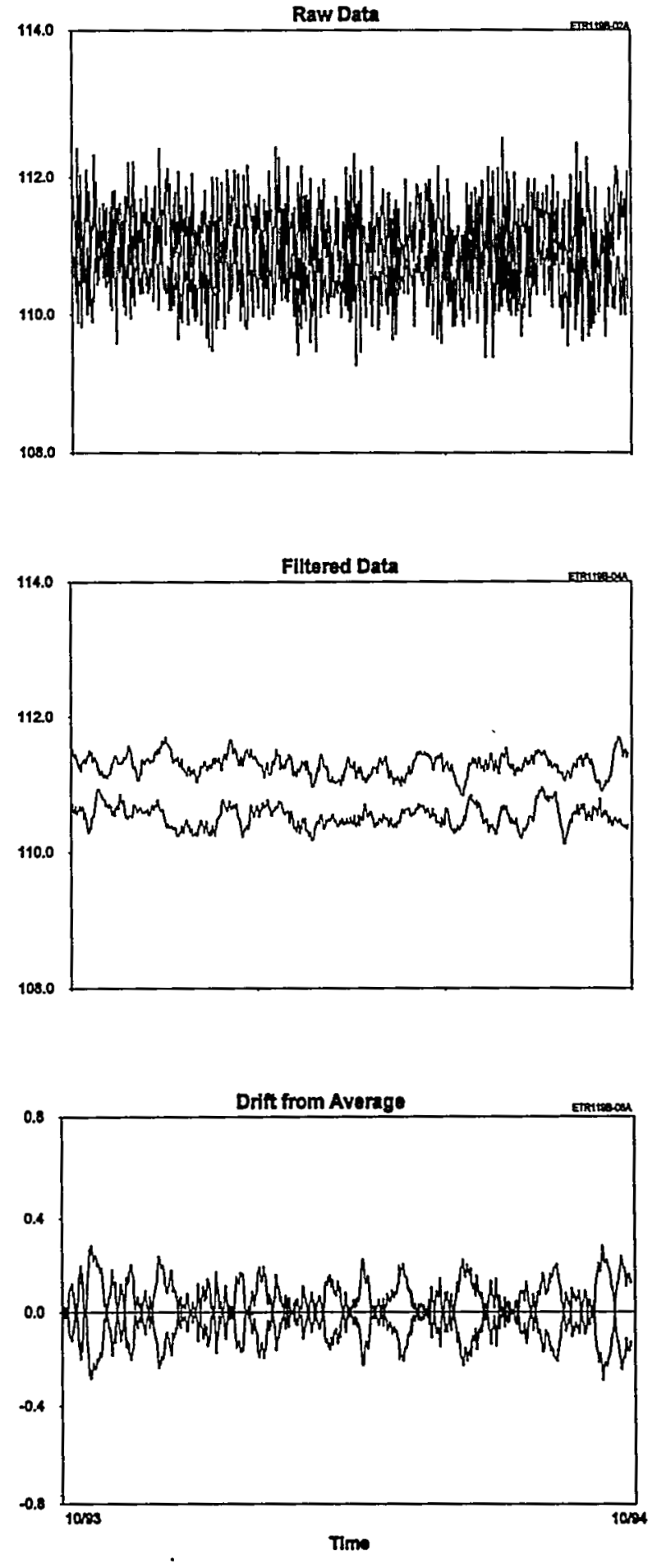

Figure 16.26 Raw Data and Drift Plots for RVLIS Signals 
Cycle 1
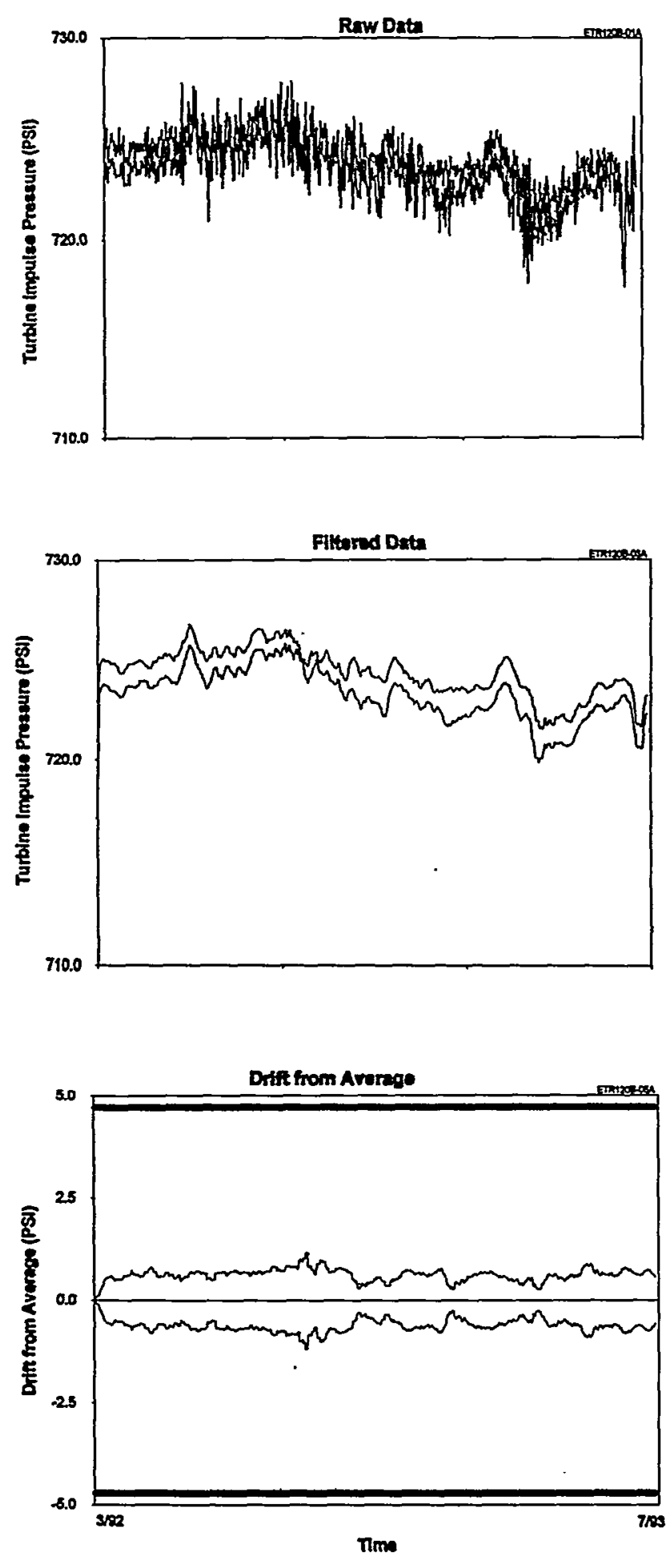

\section{Cycle 2}
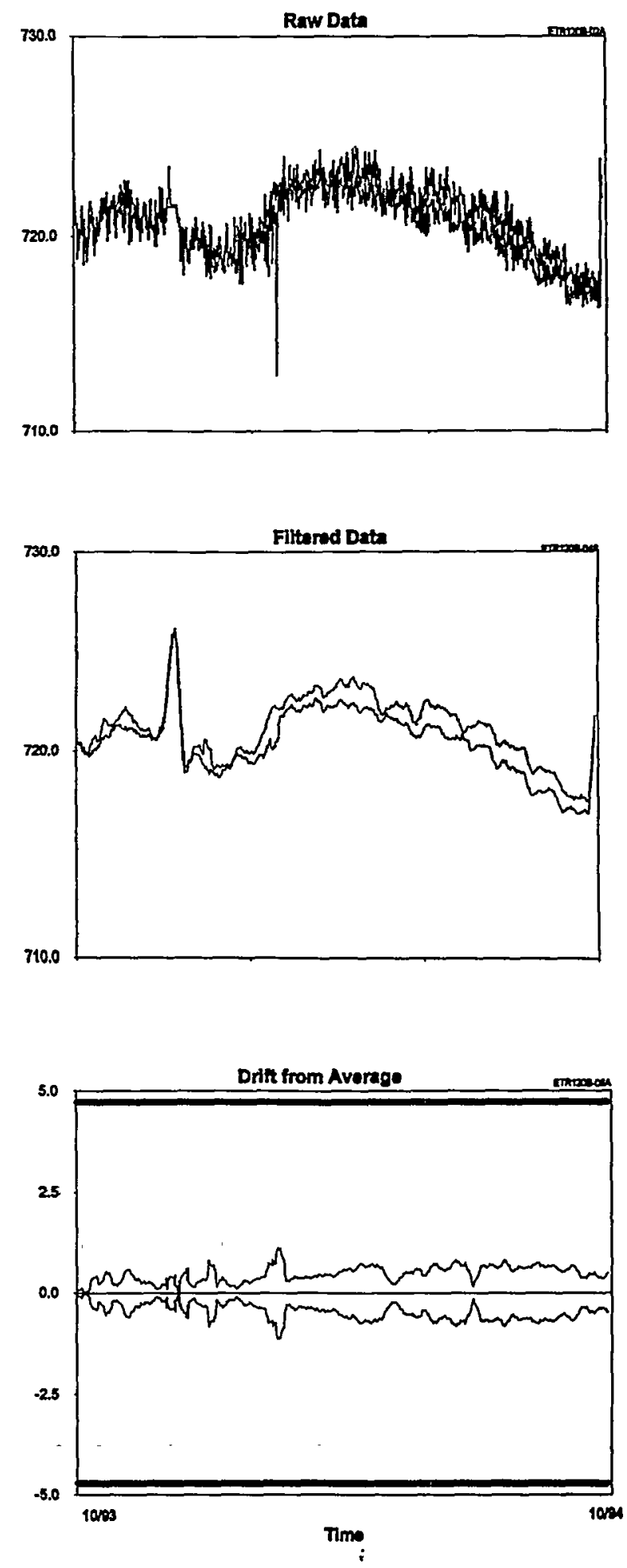

Figure 16.27 Raw Data and Drift Plots for Turbine Impulse Pressure Signals 
modeling were not available for these services.

The following points are pertinent to some of the information presented in Figures 16.12 through 16.27:

1. In Figure 16.17, which presents drift results for pressurizer level transmitters, the analytical techniques failed to provide a reasonable estimate of the process. As a result, the drift from modeling techniques incorrectly show the channels as exceeding the allowable drift. The results from averaging techniques, however, are correct and show that the deviation of pressurizer level signals are within the allowable drift band except in a few places in cycle 1 where the results exceed the band for short periods to time. Pressurizer level signals are generally more difficult to model due to plant perturbations which affect the inputs to the model.

2. In Figure 16.19 where test results are presented for two reactor pressure signals, one of the signals experiences a sudden shift in the second cycle. The modeling results correctly identifies the signal that shifted while the averaging results can only show the fact that a shift occurred, but cannot identify which one of the two signals was responsible for the shift.

\subsection{Presentation of Deviation Results}

Figures 16.28 through 16.43 provide raw data and deviation plots for sixteen services tested at McGuire. CSA bands are also shown on the deviation plots for the cases for which CSA bands were available. There are eight plots in each of the first sixteen figures (Figures 16.28 through 16.39) and six plots in each of the last four figures (Figures 16.40 through 16.43).

The eight plots on a page include two columns, each with four plots for each cycle. The four plots in each column include the raw data as was sampled in the plant, filtered data to remove the extraneous noise, and two deviation plots. One deviation plot is based on the averaging techniques and the other is based on the analytical techniques. The plot that is based on the averaging technique is the average of simple and weighted averages if both methods were used, and the plot that is based on the analytical techniques is the average of results from empirical modeling and neural networks.

For the four services shown in Figures 16.40 through 16.43, there are no analytical techniques because independent inputs for modeling were not available for these services.

The following points are pertinent to some of the plots shown in Figures 16.28 through 16.43:

1. In Figure 16.31 which presents results for two feedwater flow signals, the signal deviations increase near the middle of the first cycle and almost reach the CSA bands plotted on the figure. Both averaging and modeling results show the problem although averaging results are more clear than the modeling results. The deviations remain near the bands and continue to do so in the second fuel cycle.

2. In Figure 16.32, three signals are shown for the reactor coolant flow. One of the transmitters began to drift near the middle of cycle 1 as shown in both averaging and modeling results. This transmitter was replaced at the end of cycle 1 . Note also in cycle 1 that another one of the three transmitters exceeds the deviation band in the averaging results, but is on the borderline in the modeling results.

In cycle 2 , the averaging results show one transmitter as exceeding the band, but the modeling results show the 
Cycle 1
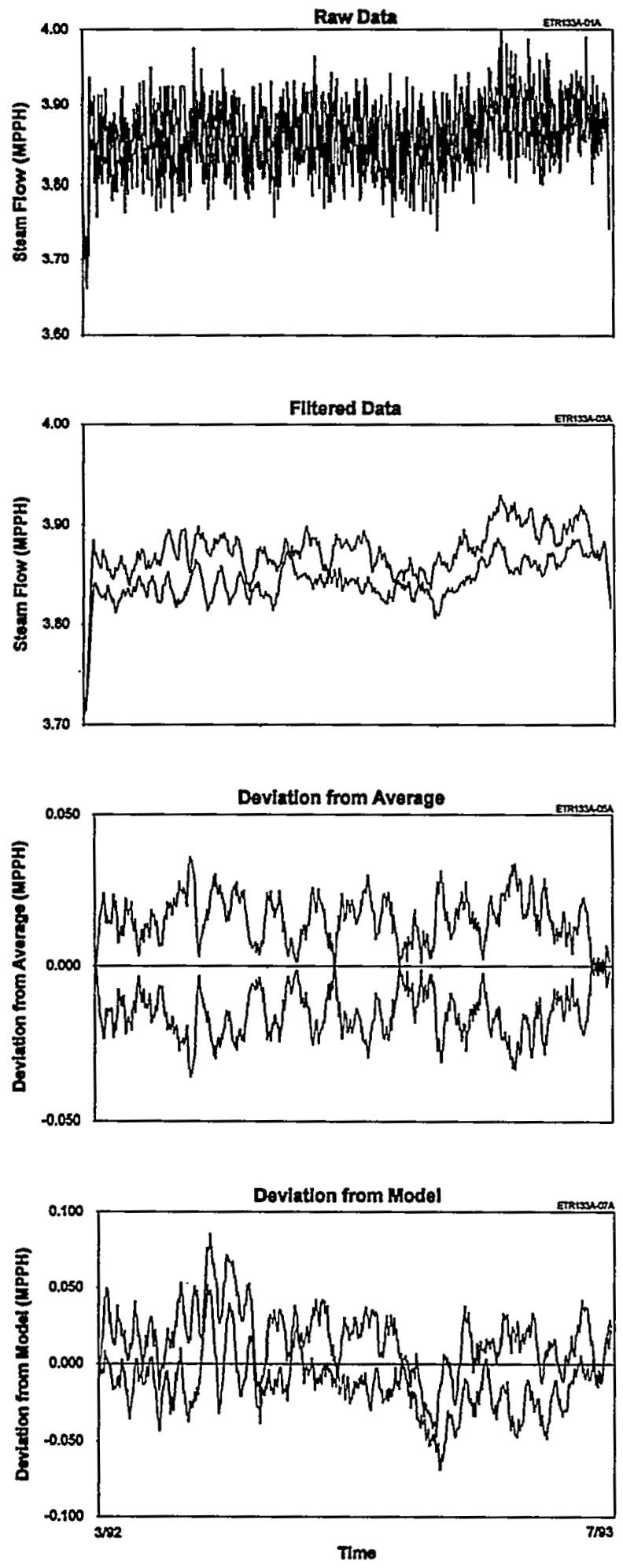

Cycle 2
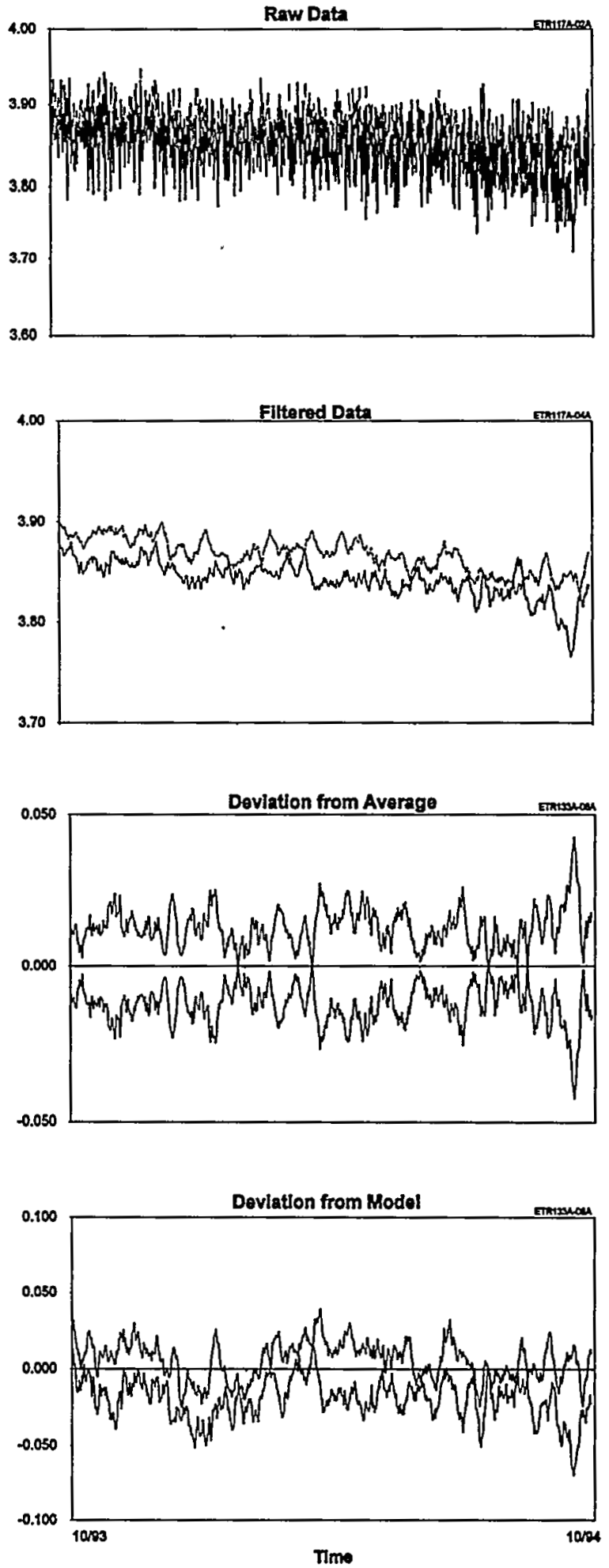

Figure 16.28 Raw Data and Deviation Plots for Steam Flow Signals 


\section{Cycle 1}
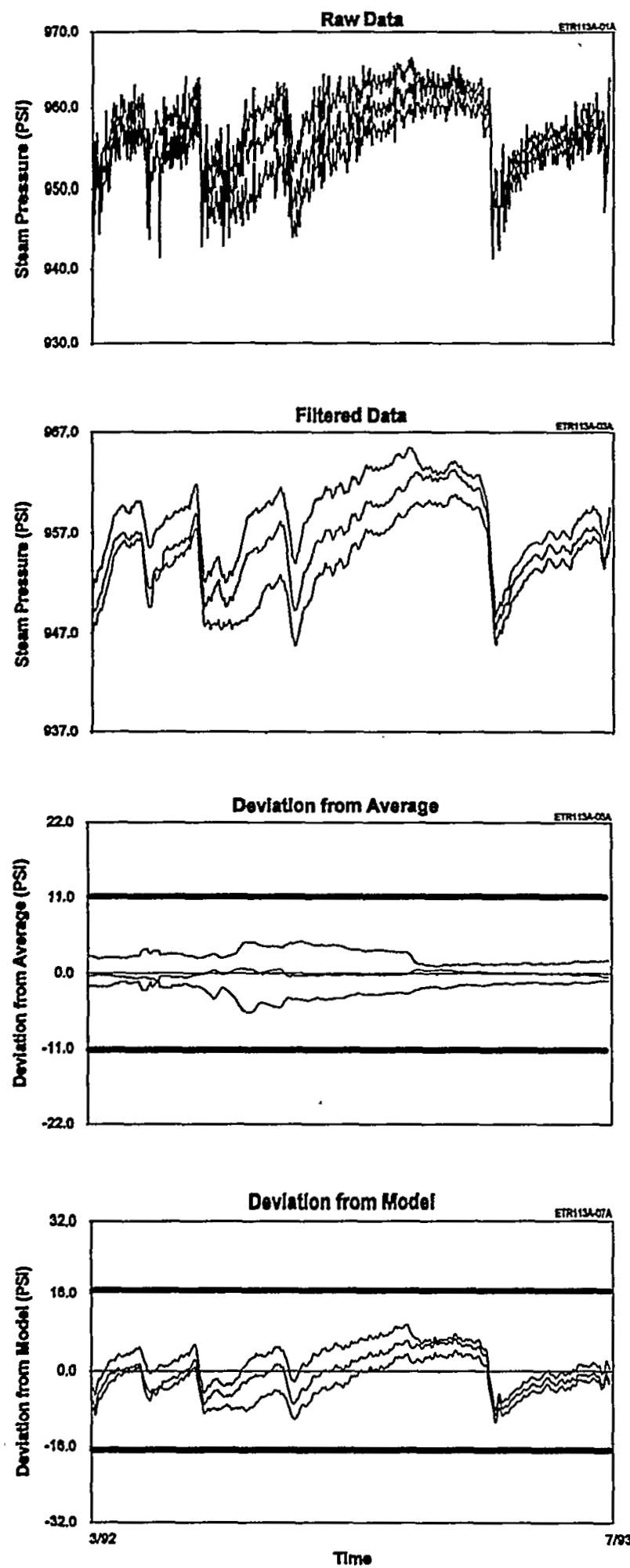

Cycle 2
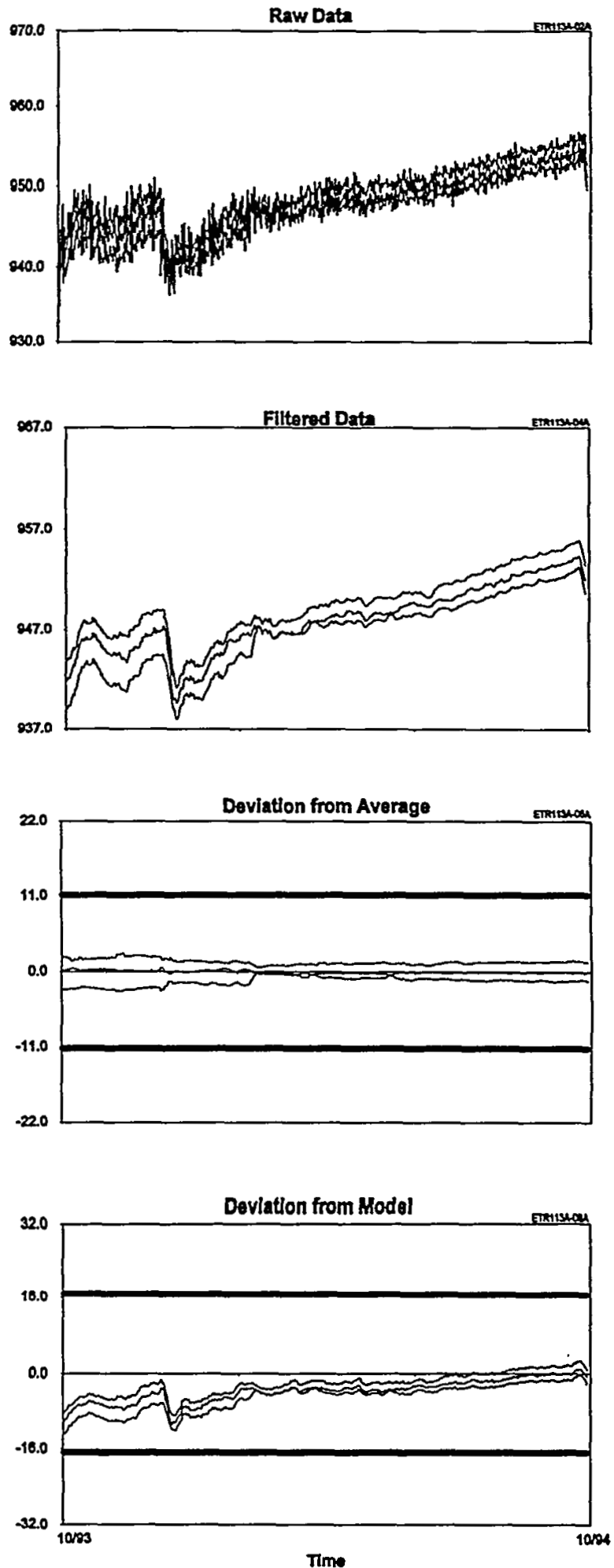

Figure 16.29 Raw Data and Deviation Plots for Steam Pressure Signals 


\section{Cycle 1}
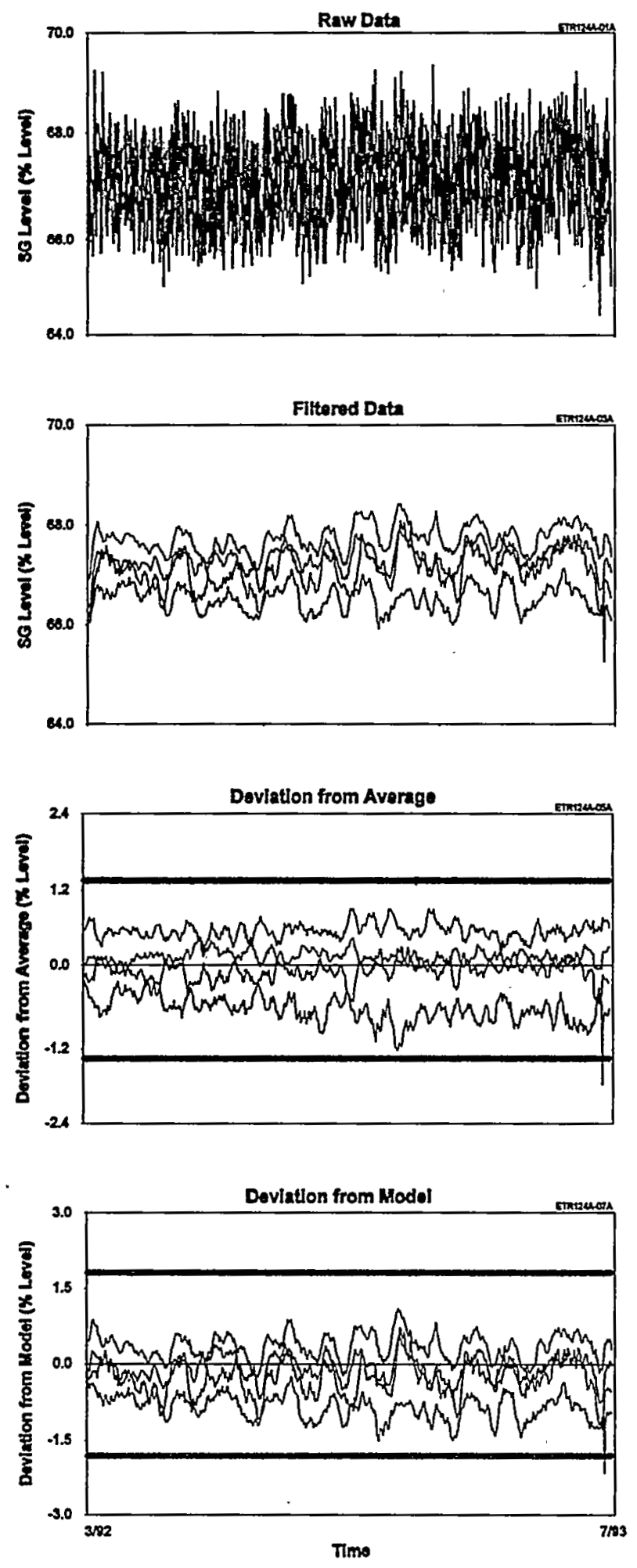

\section{Cycle 2}
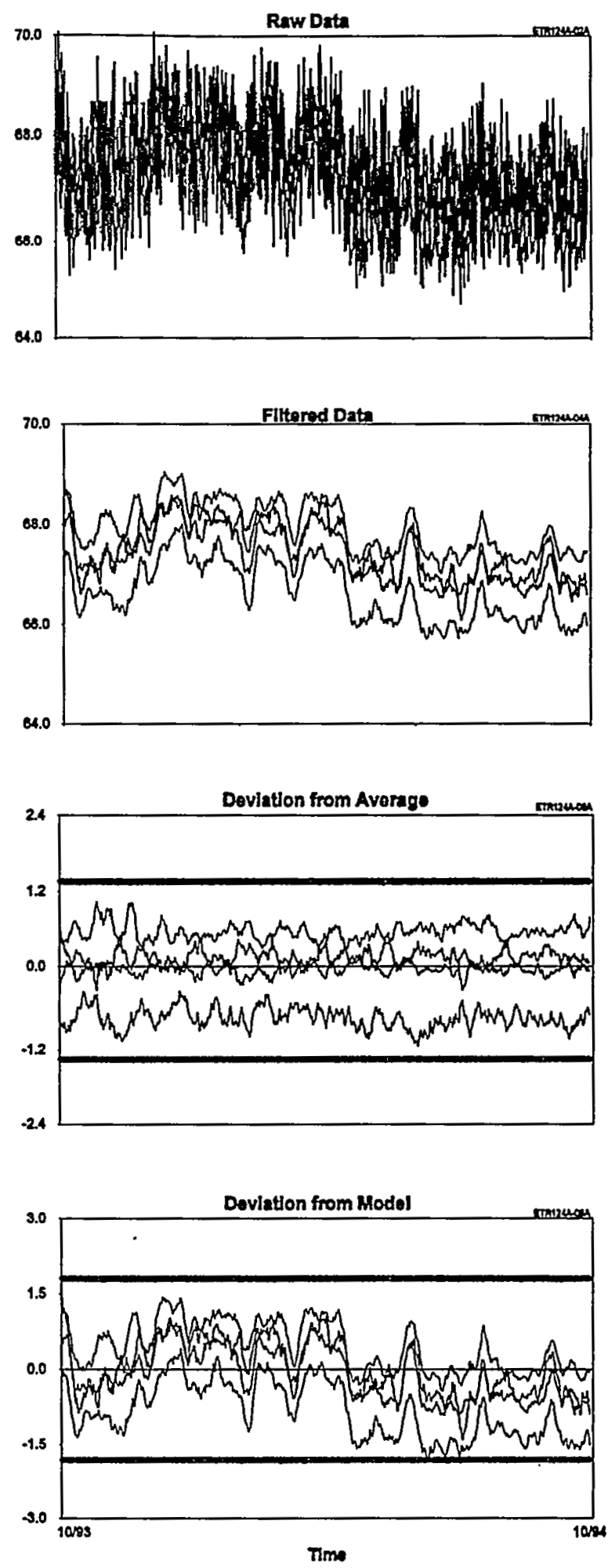

Figure 16.30 Raw Data and Deviation Plots for Steam Generator Level Signals 
Exucle 1
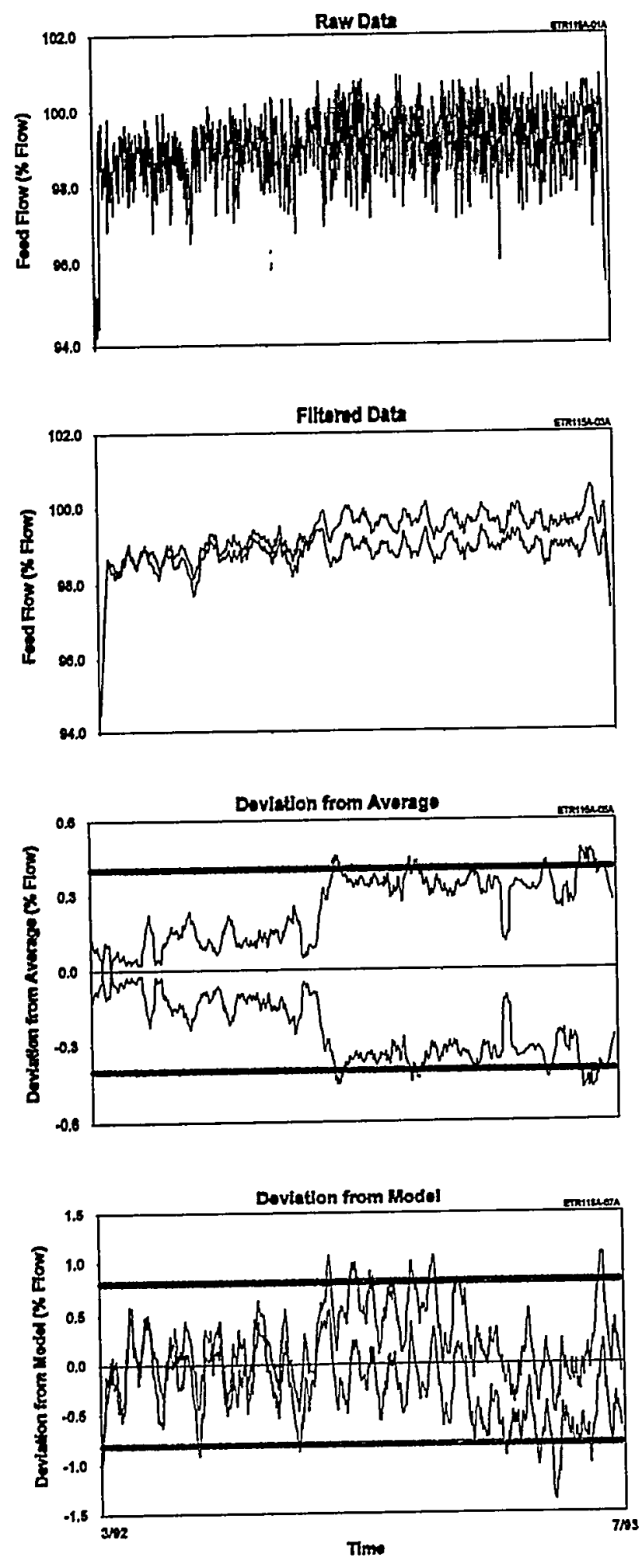

Cycle 2
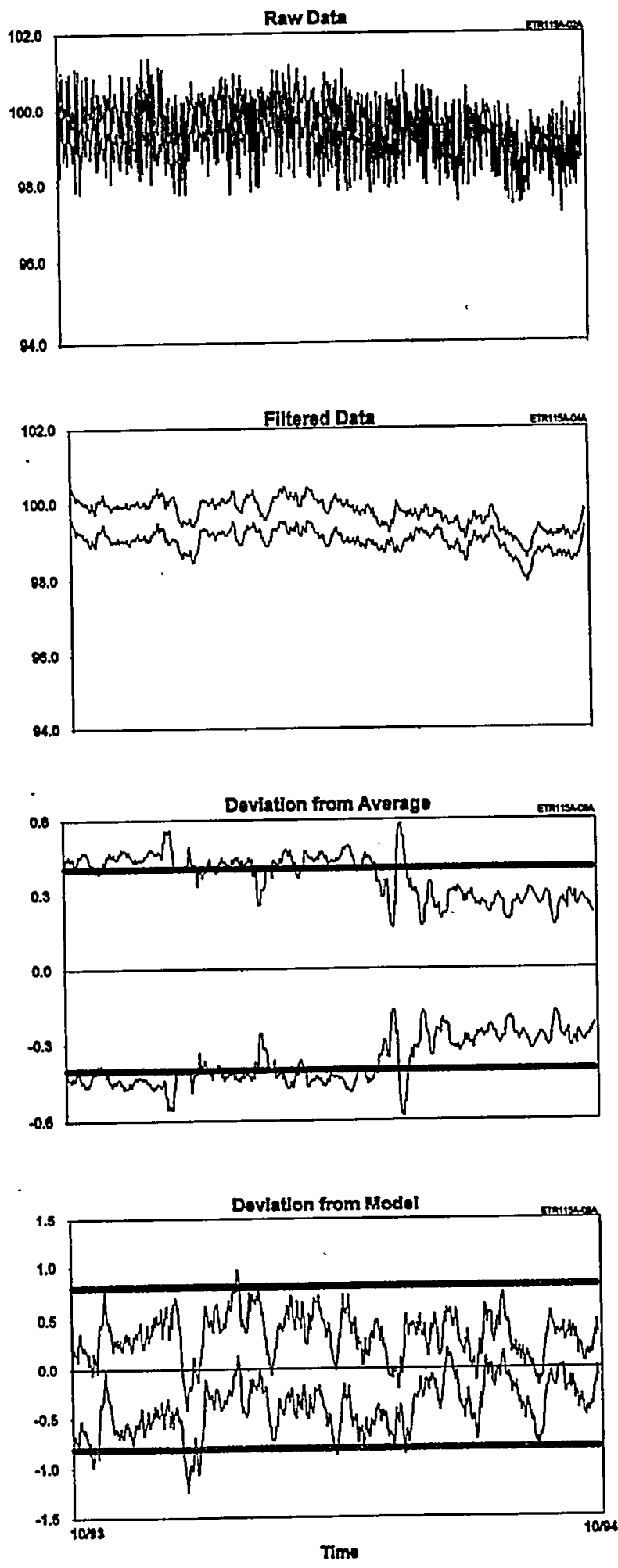

Figure 16.31 Raw Data and Deviation Plots for Feedwater Flow Signals 


\section{Cycle 1}
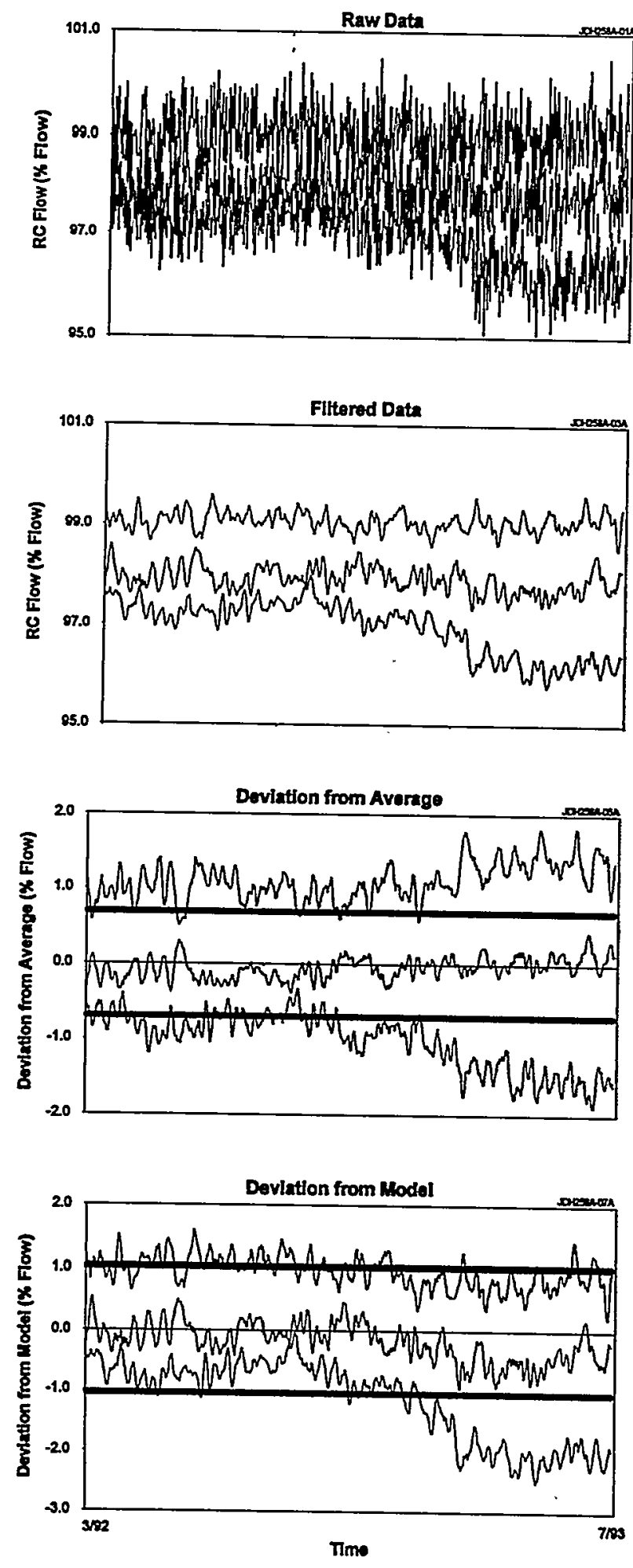

Cycle 2
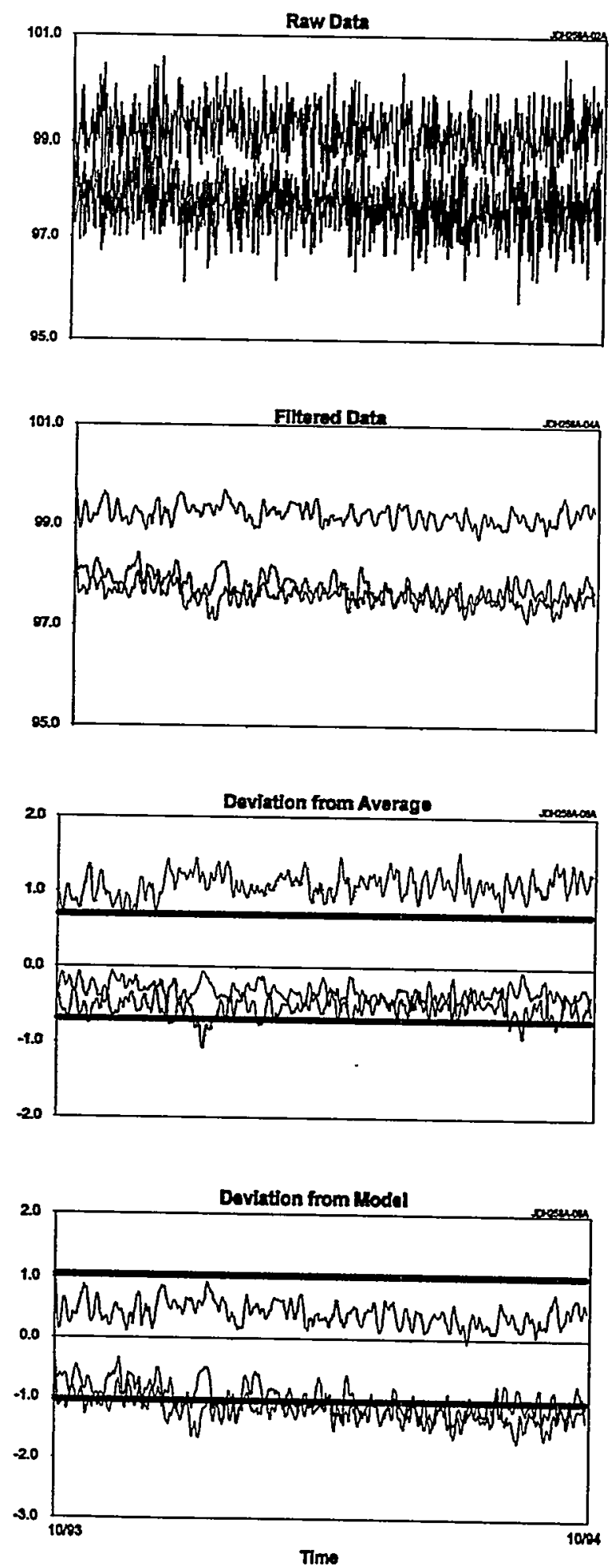

Figure 16.32 Raw Data and Deviation Plots for Reactor Coolant Flow Signals 
Cycle 1
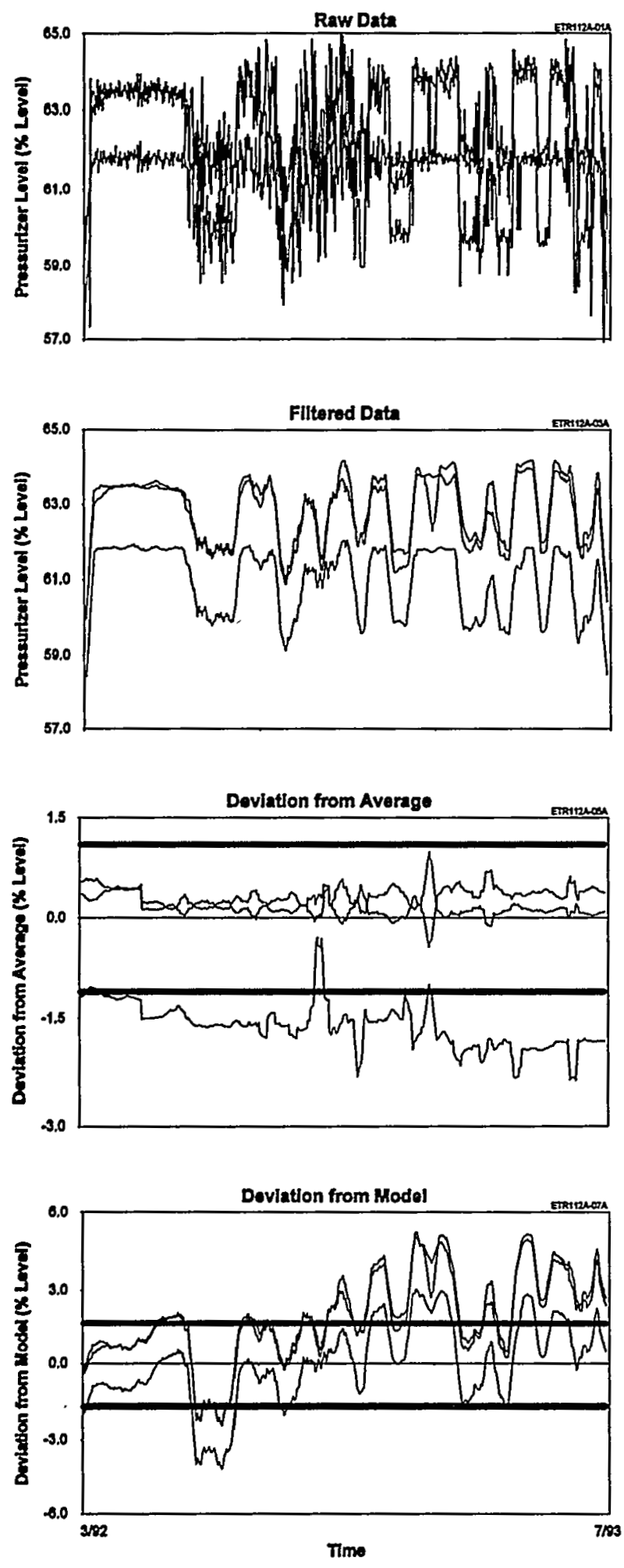

Cycle 2
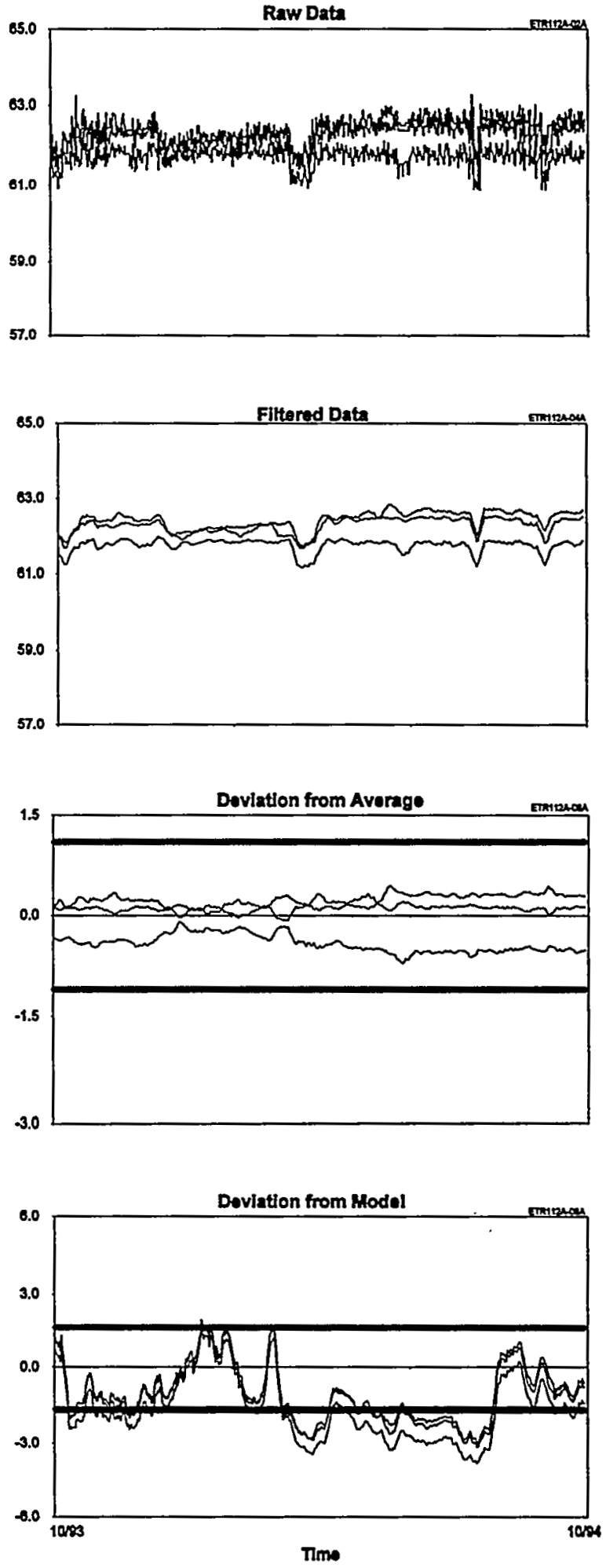

Figure 16.33 Raw Data and Deviation Plots for Pressurizer Level Signals 


\section{Cycle 1}
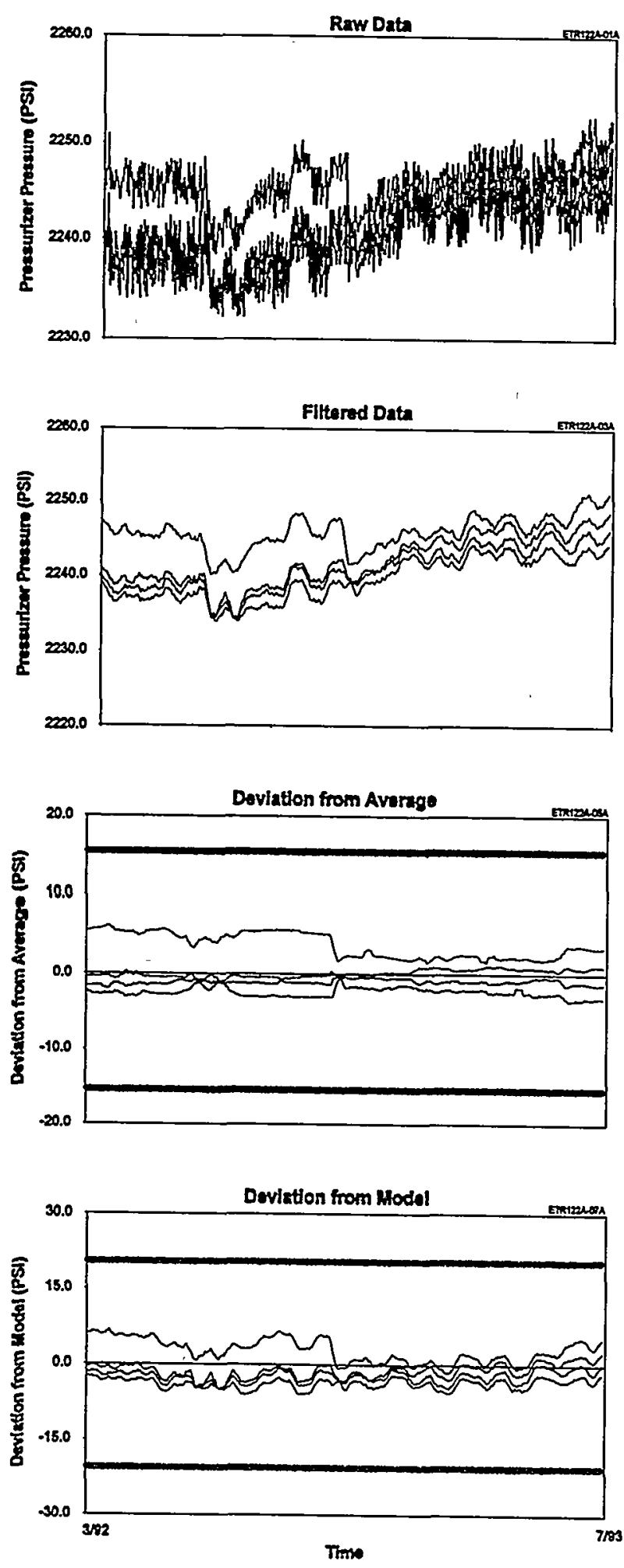

\section{Cycle?}
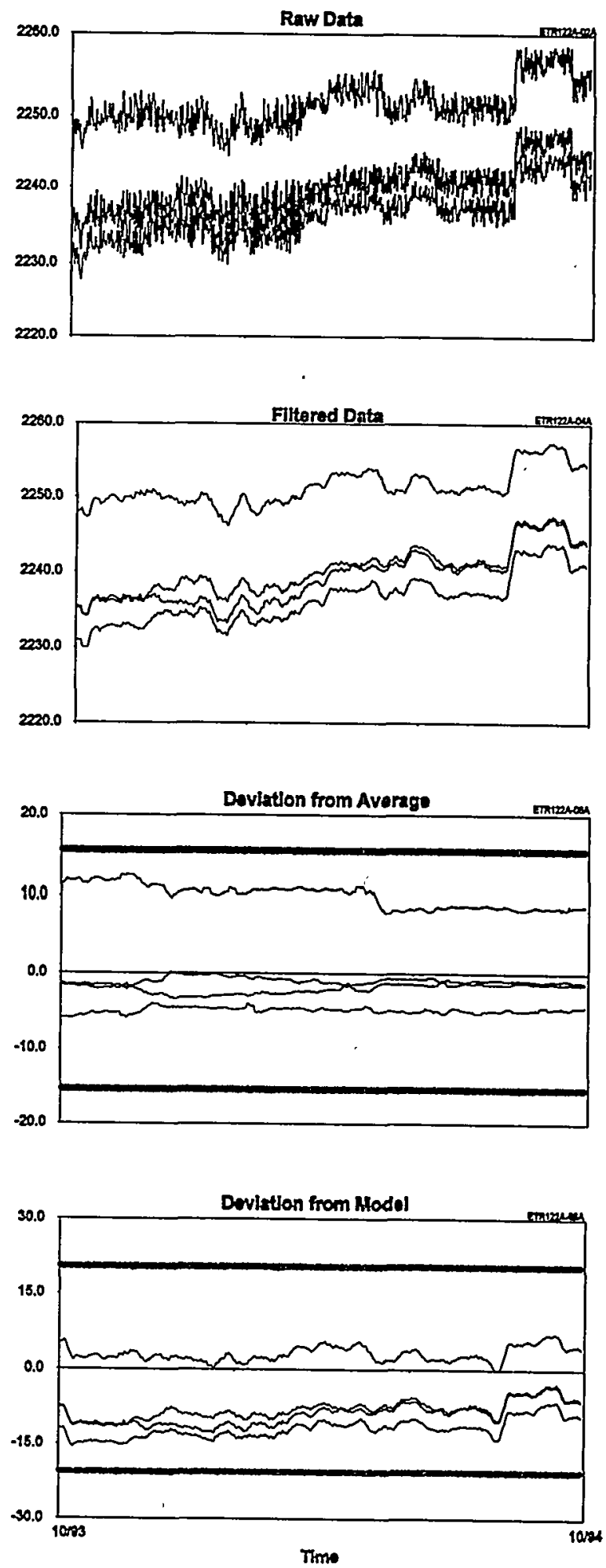

Figure 16.34 Raw Data and Deviation Plots for Pressurizer Pressure Signals 


\section{Cycle 1}
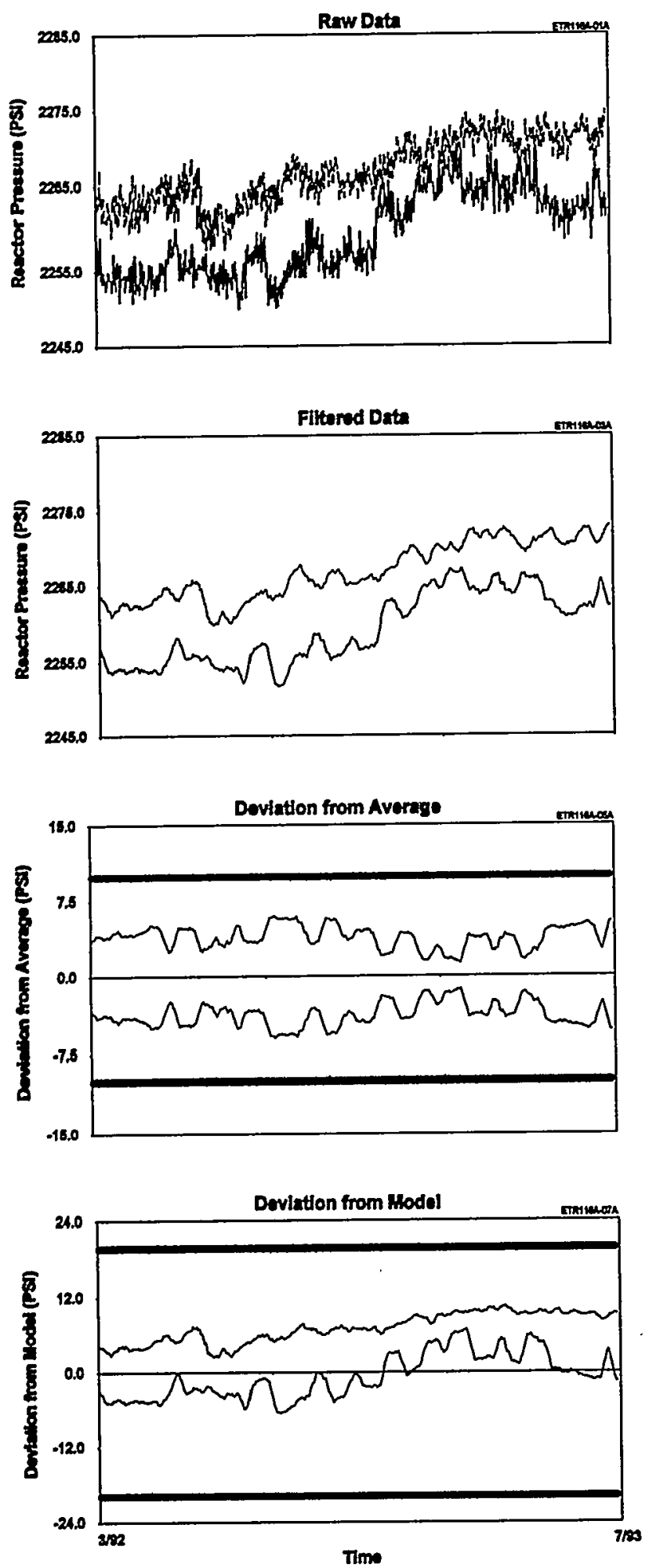

Cycle 2
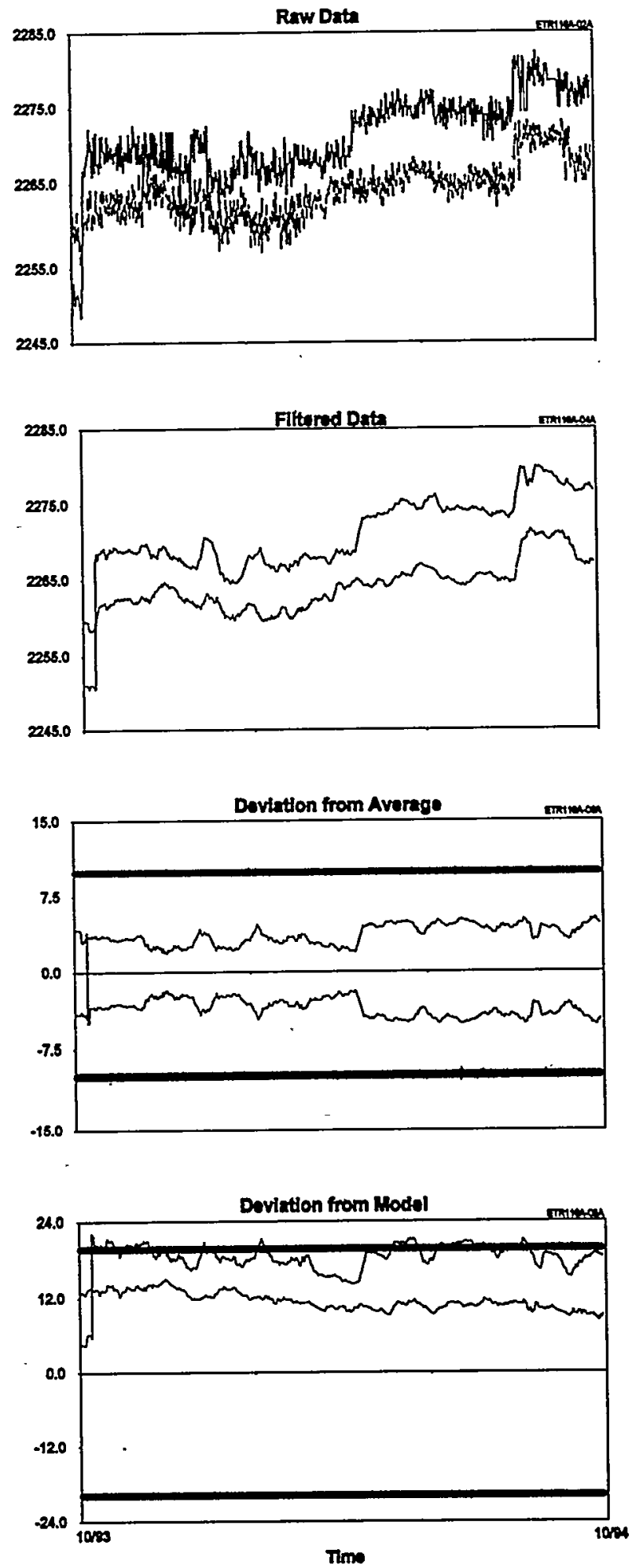

Figure 16.35 Raw Data and Deviation Plots for Wide-Range Pressure Signals 


\section{Cycle 1}
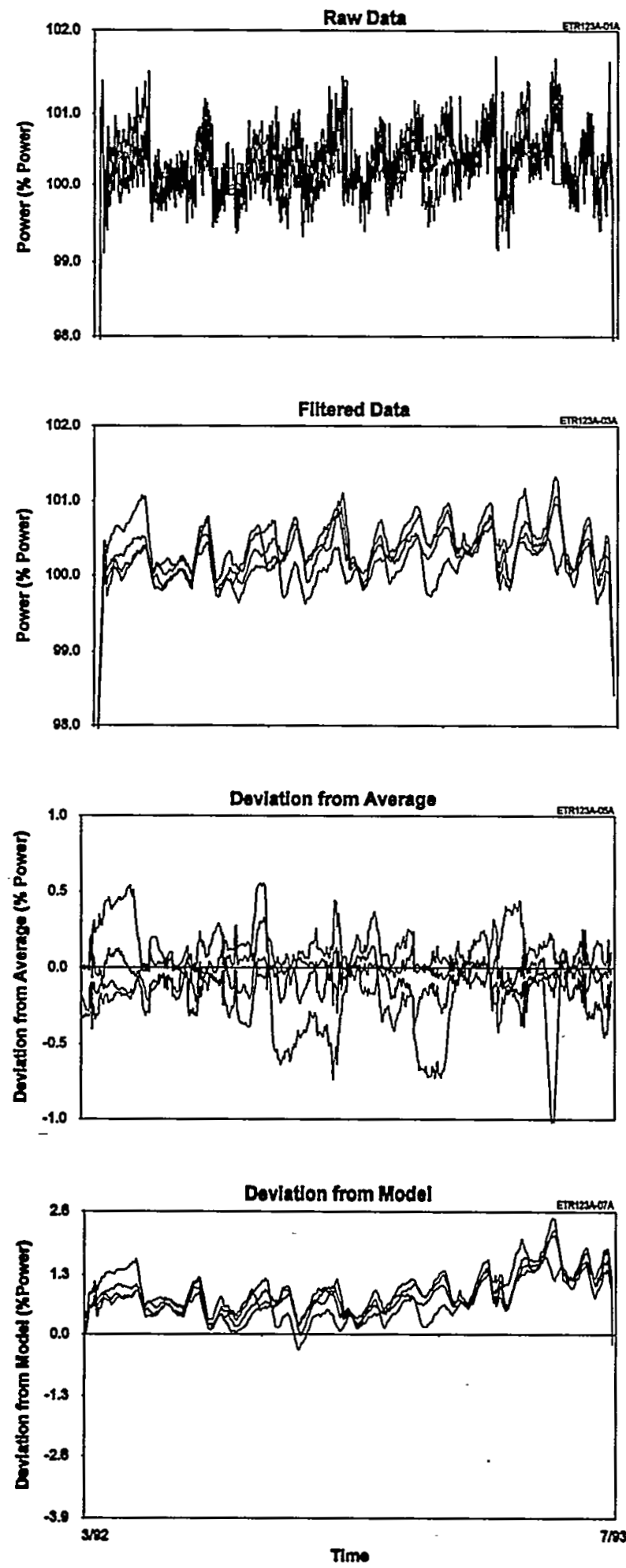

Cycle 2
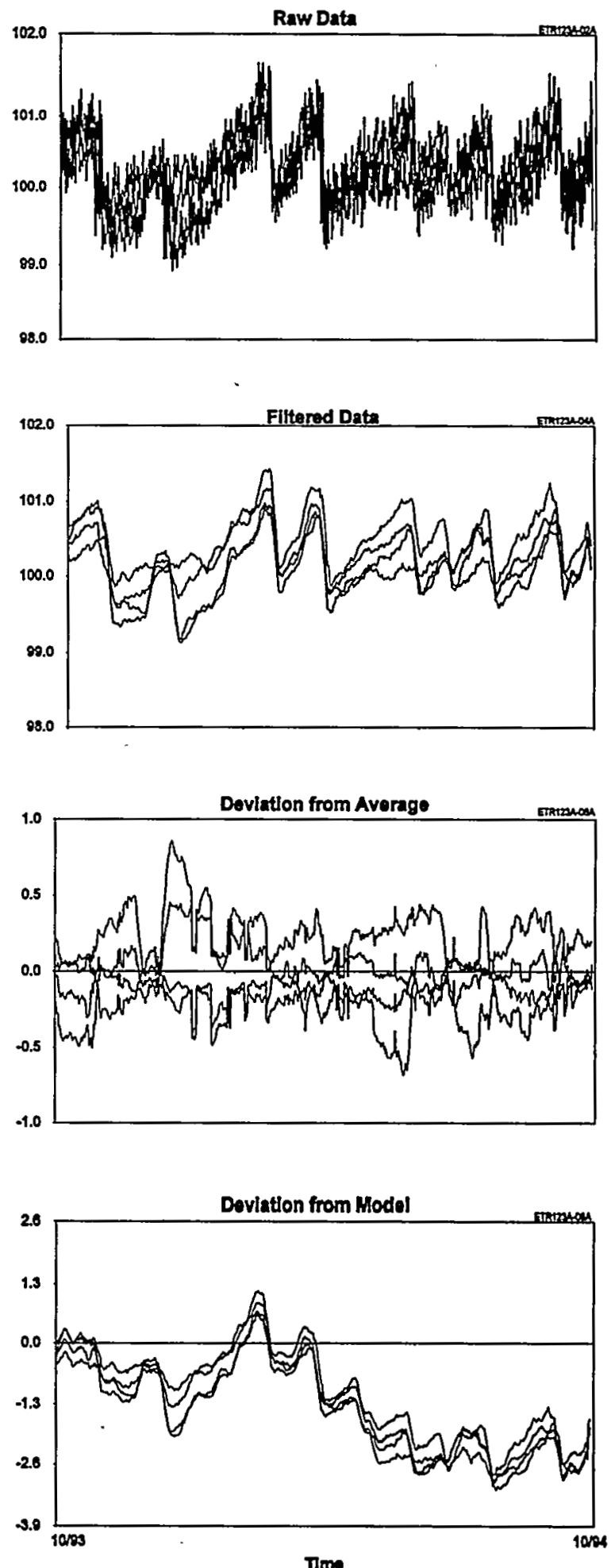

Figure 16.36 Raw Data and Deviation Plots for Neutron Flux Signals 
Cycle 1
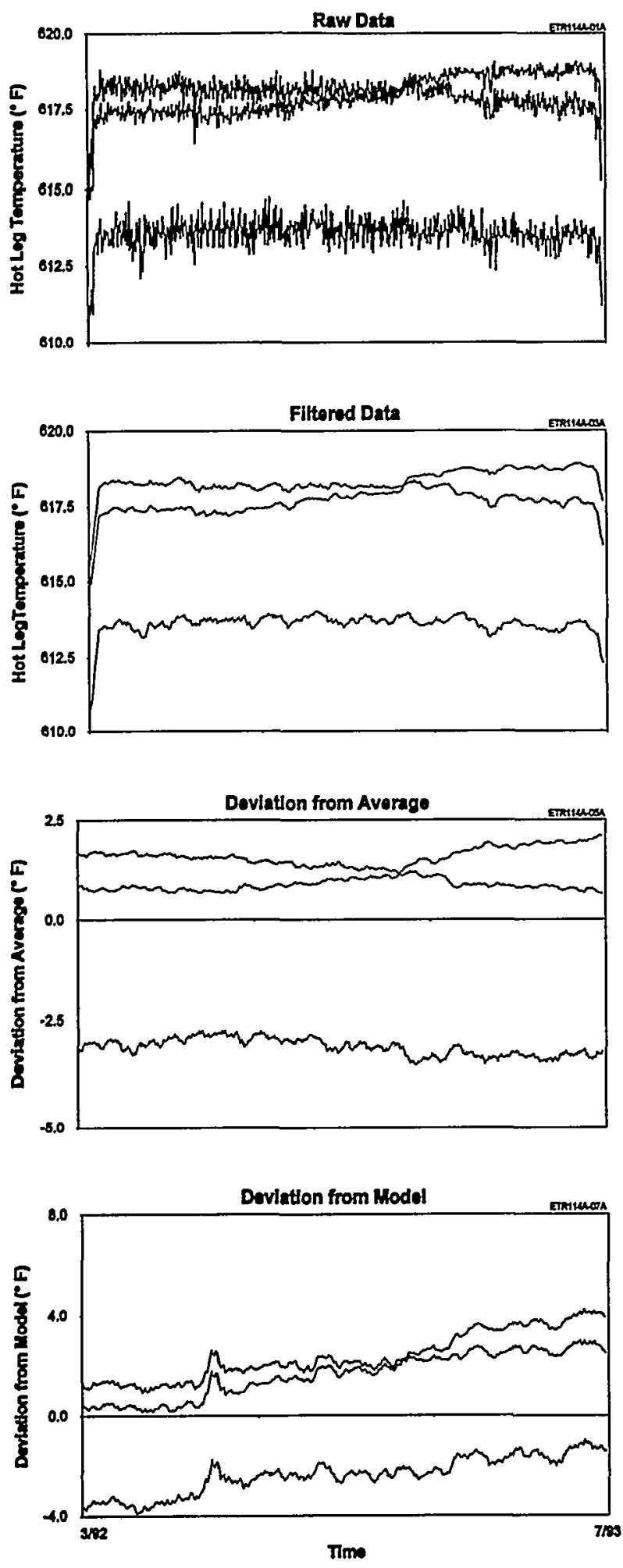

\section{Cycle 2}
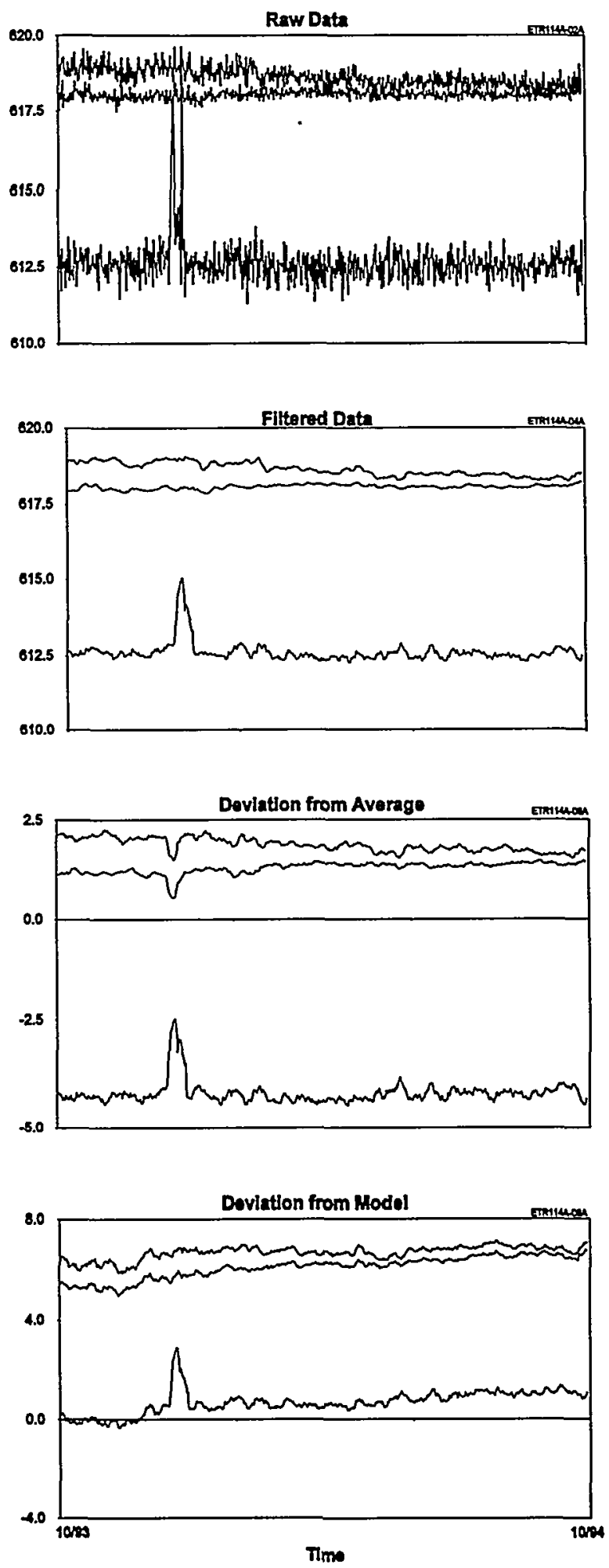

Figure 16.37 Raw Data and Deviation Plots for Hot Leg RTD Signals 


\section{Excle 1}
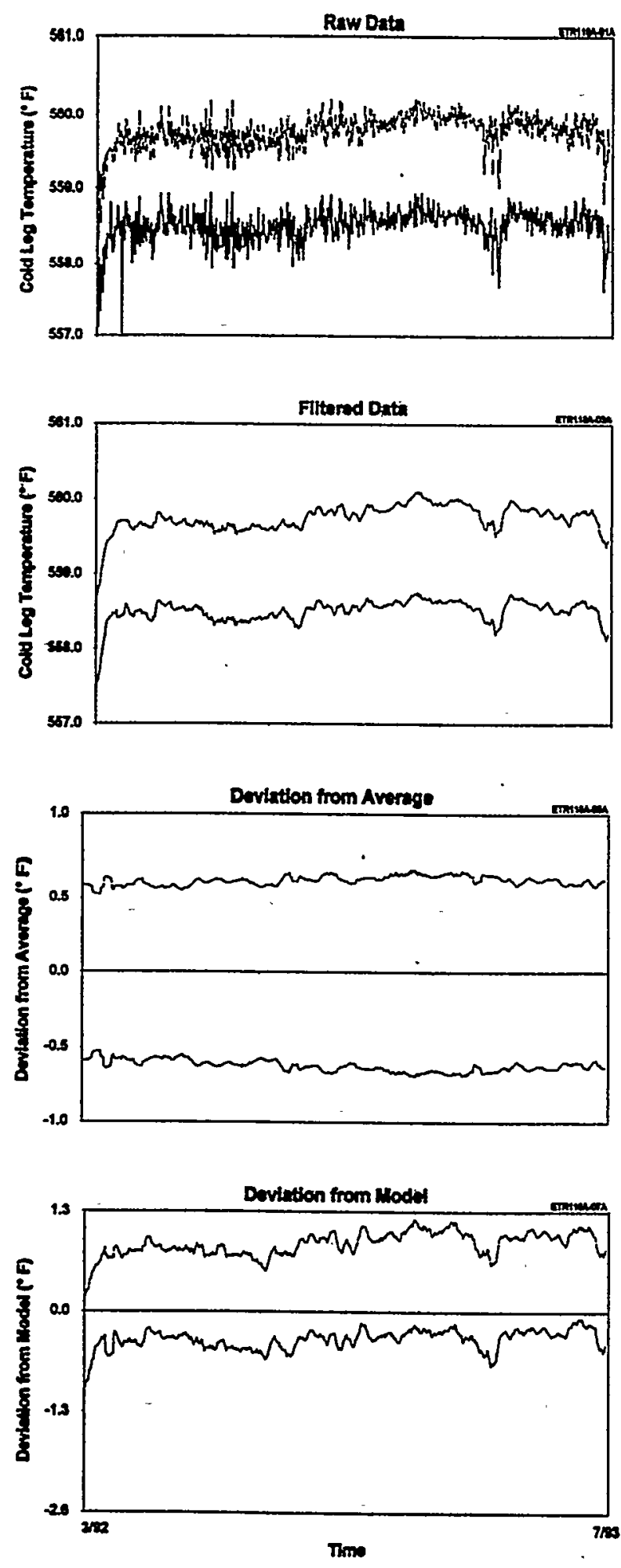

\section{Cycle 2}
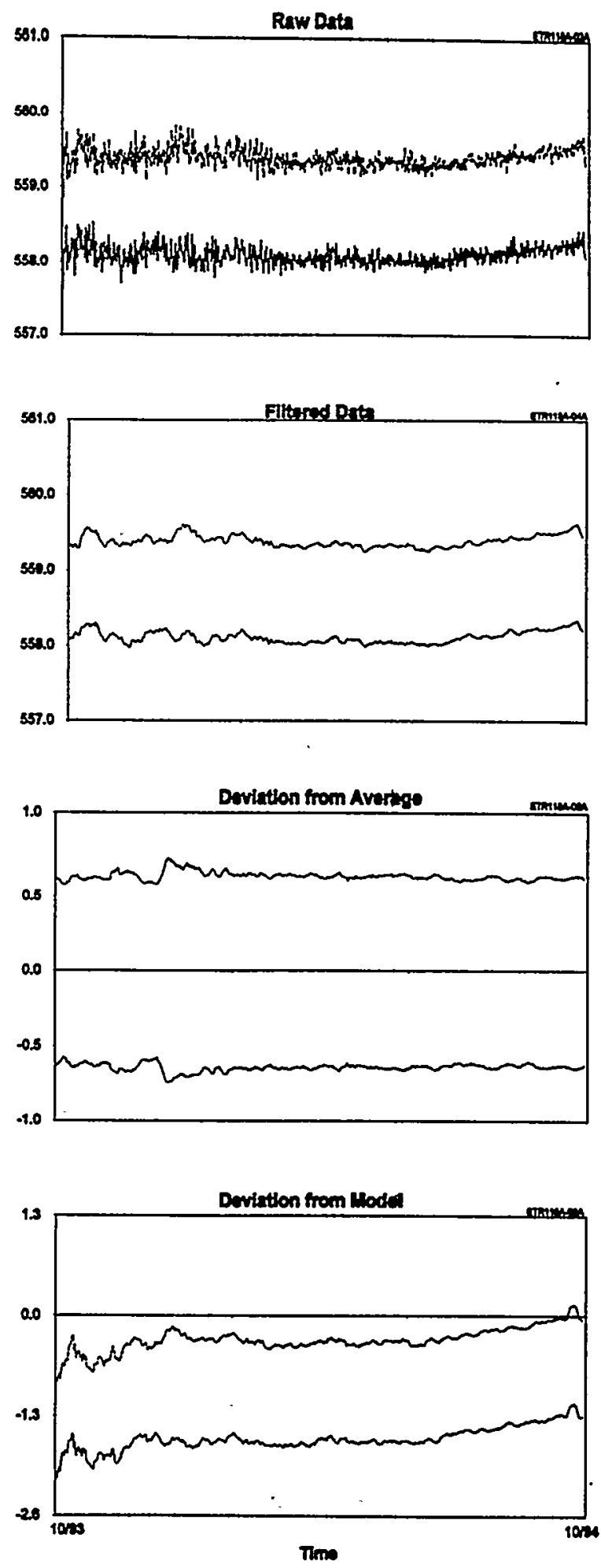

Figure 16.38 Raw Data and Deviation Plots for Cold Leg RTD Signals 


\section{Cycle 1}
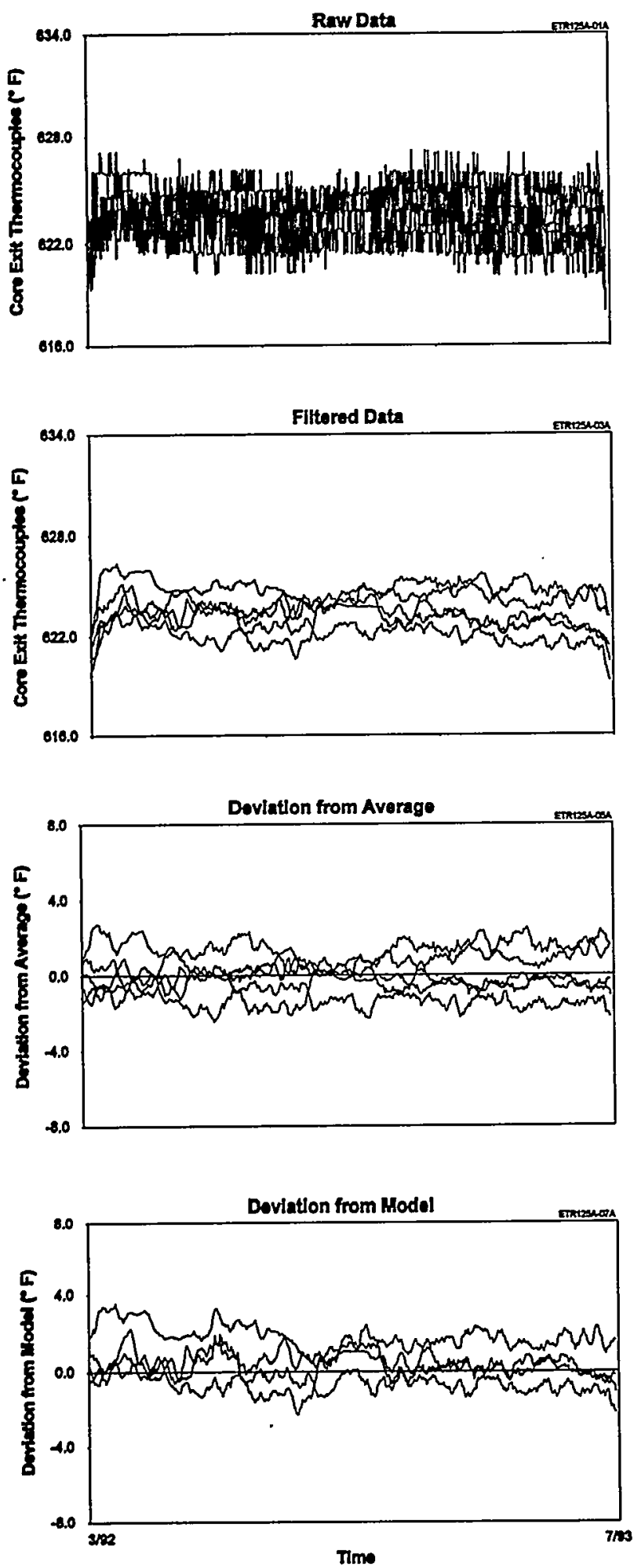

Cycle 2
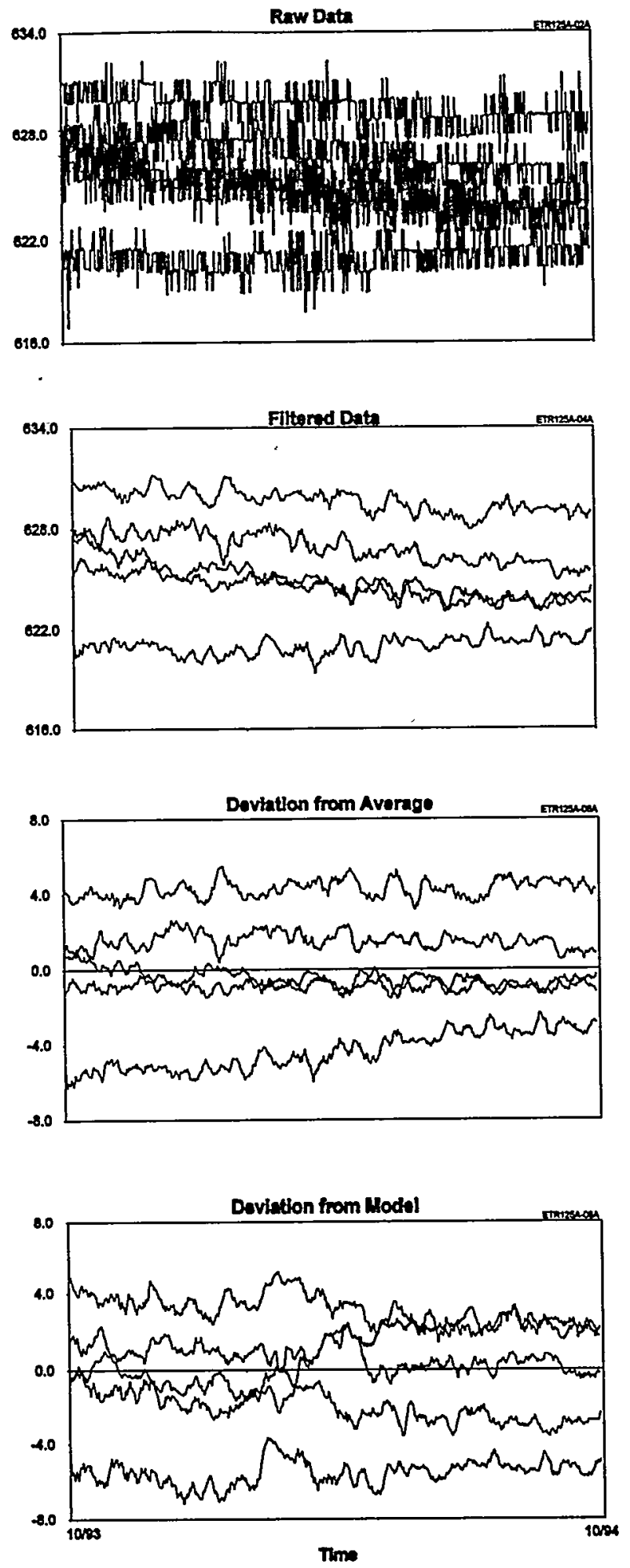

Figure 16.39 Raw Data and Deviation Plots for Core Exit Thermocouple Signals 


\section{Cycle 1}
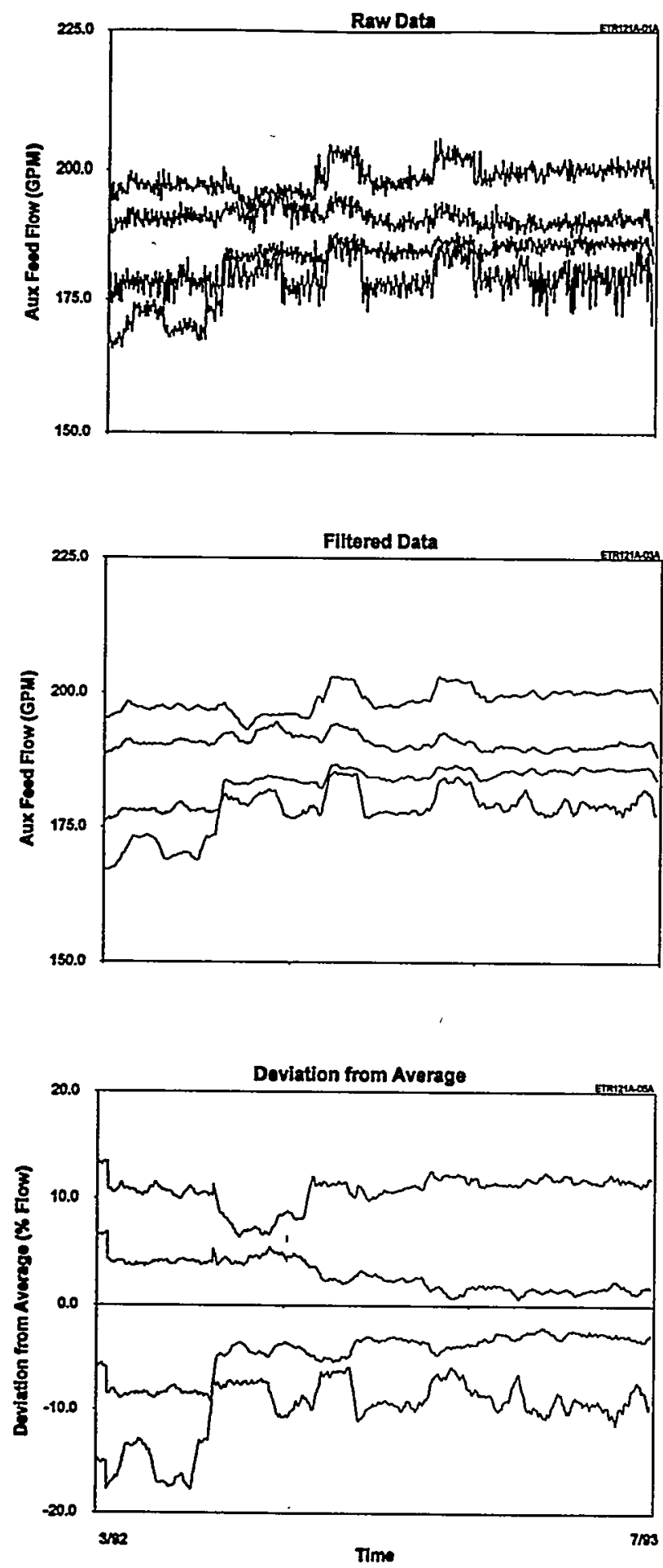

Cycle 2
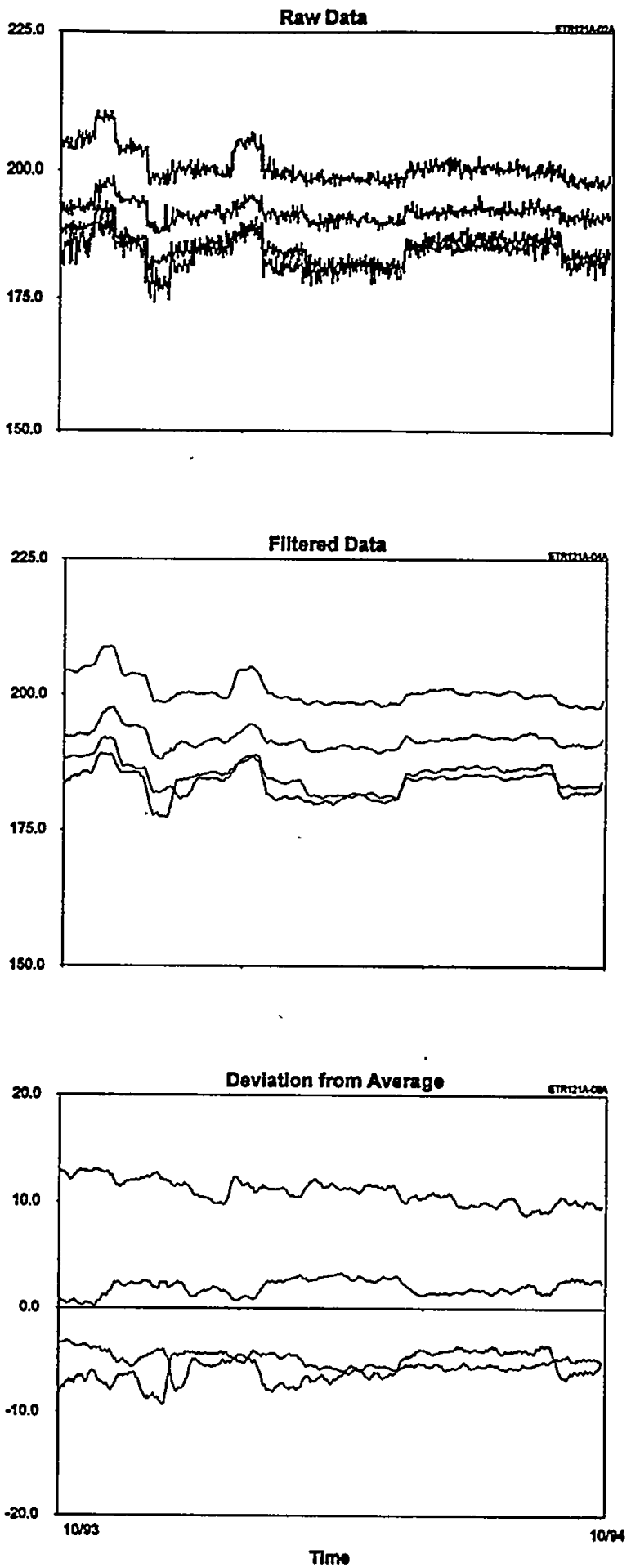

Figure 16.40 Raw Data and Deviation Plots for Auxiliary Feedwater Flow Signals 
Cycle 1
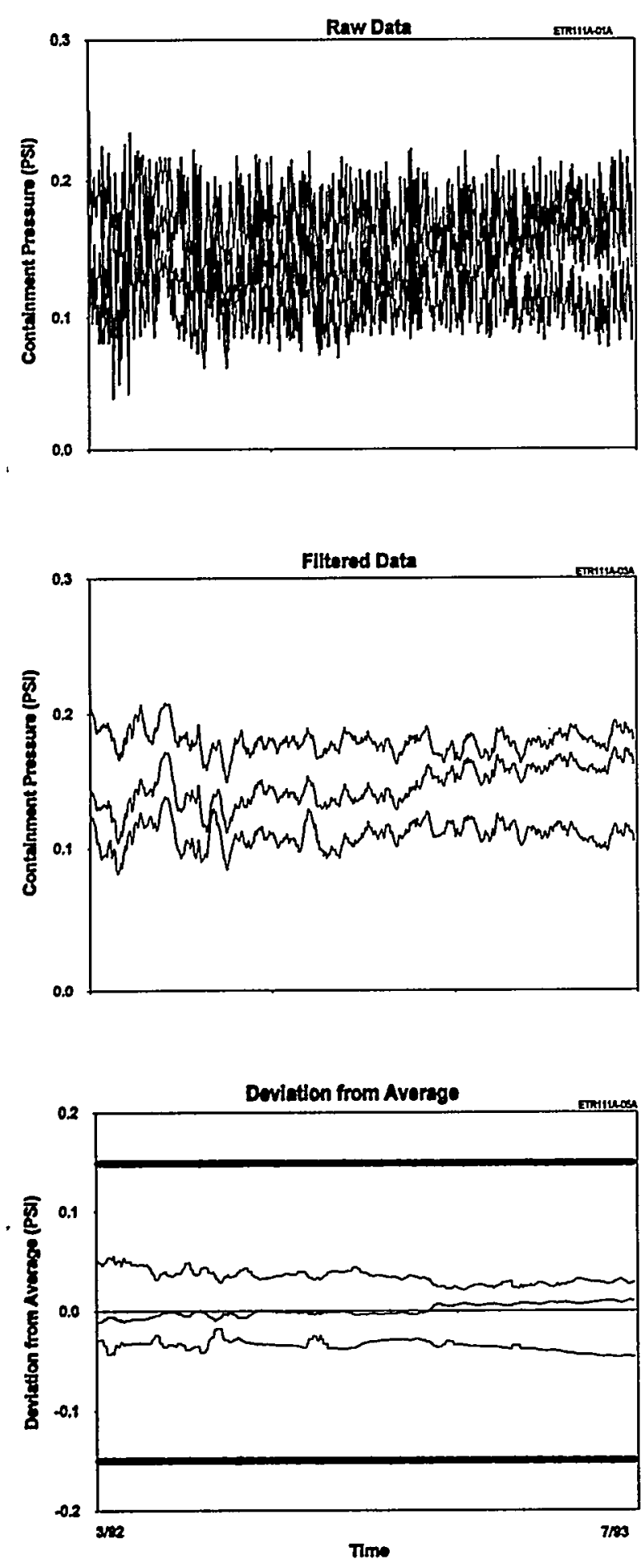

Cycle 2
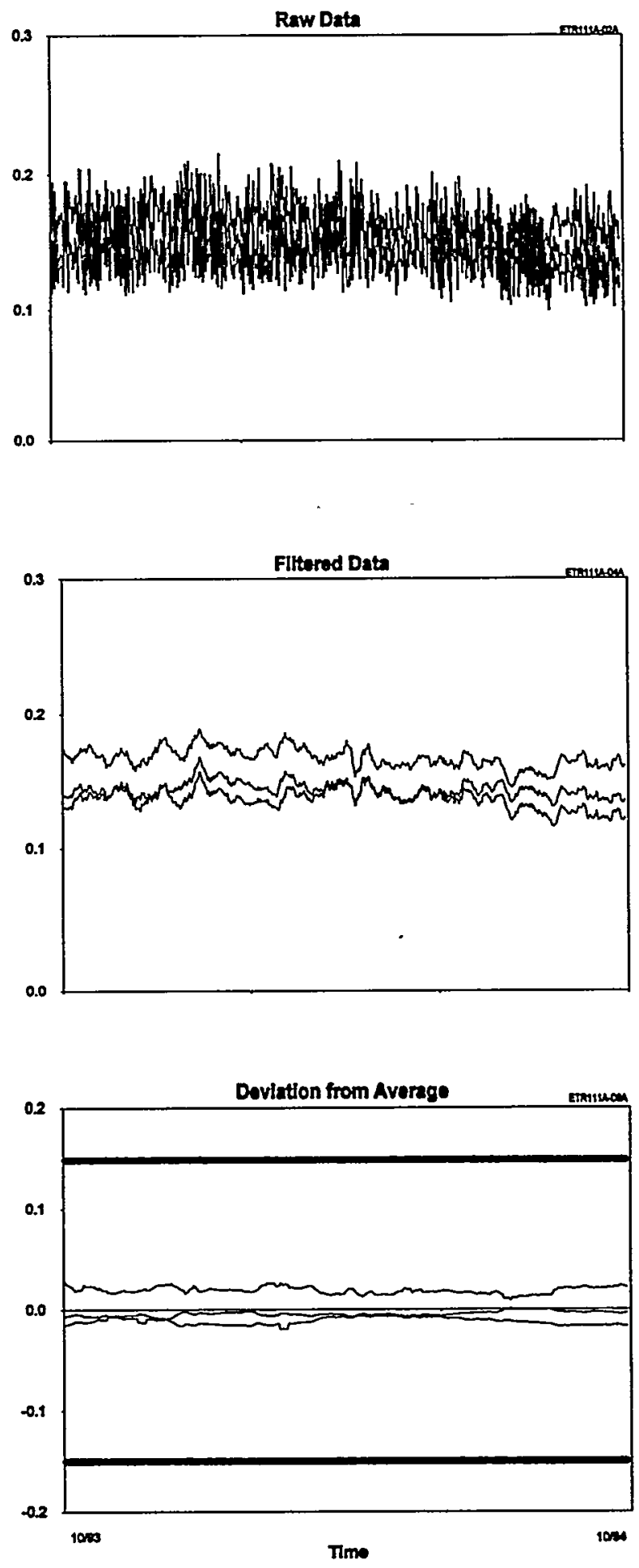

Figure 16.41 Raw Data and Deviation Plots for Containment Pressure Signals 
Cycle 1
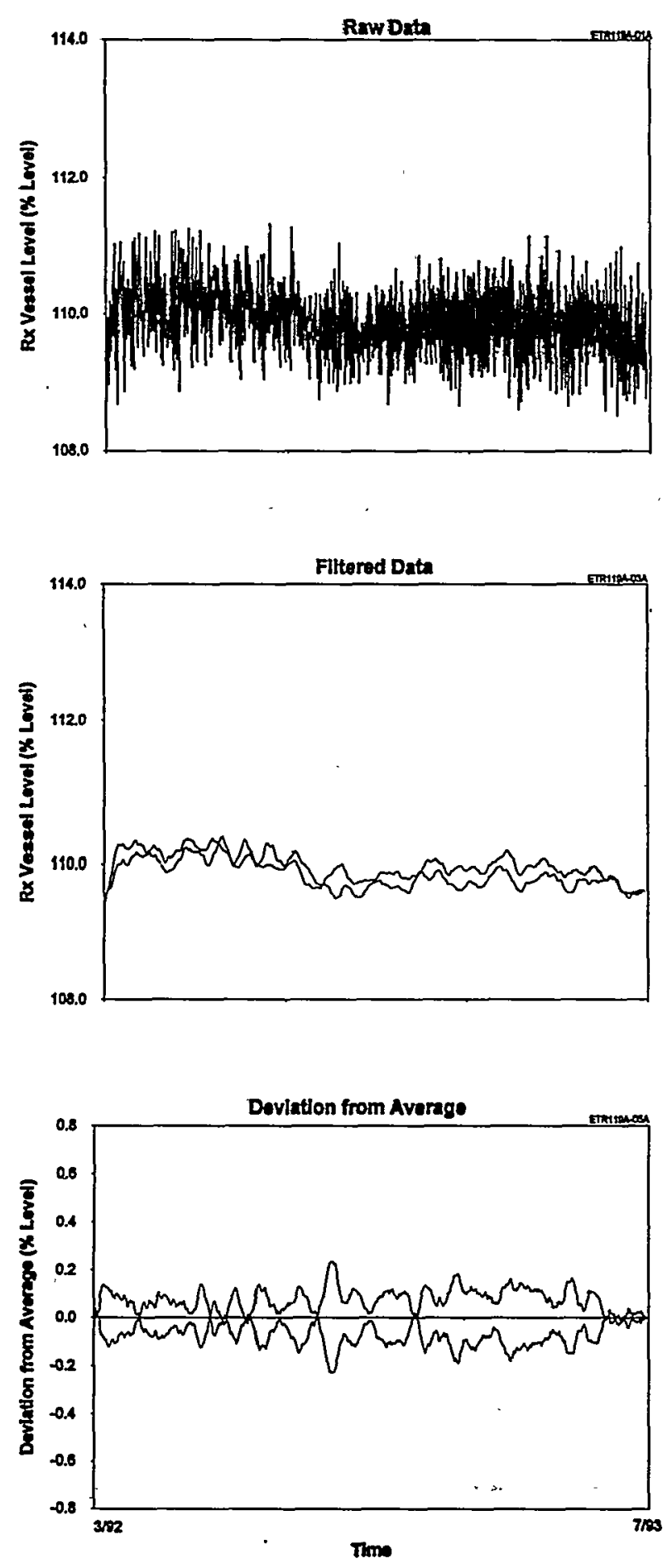

Cycle 2
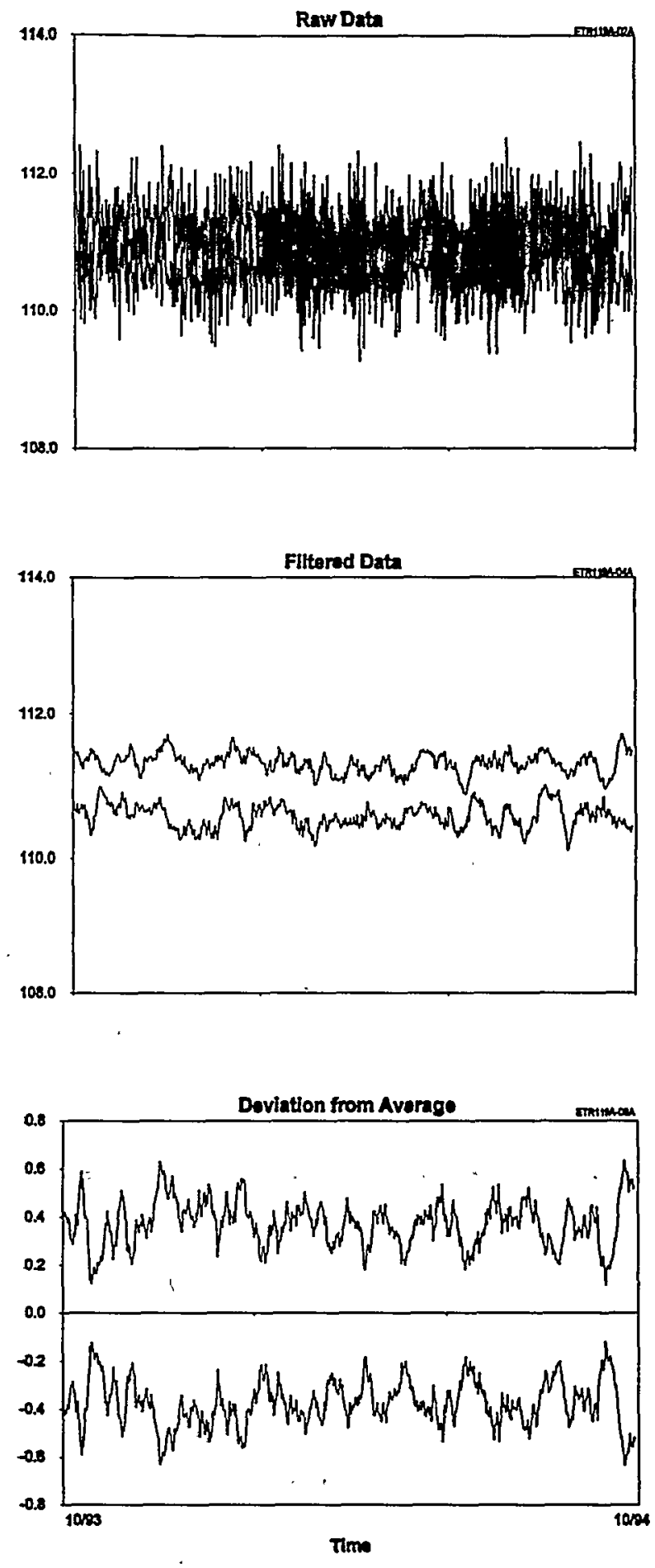

Figure 16.42 Raw Data and Deviation Plots for RVLIS Signals 
Cycle 1
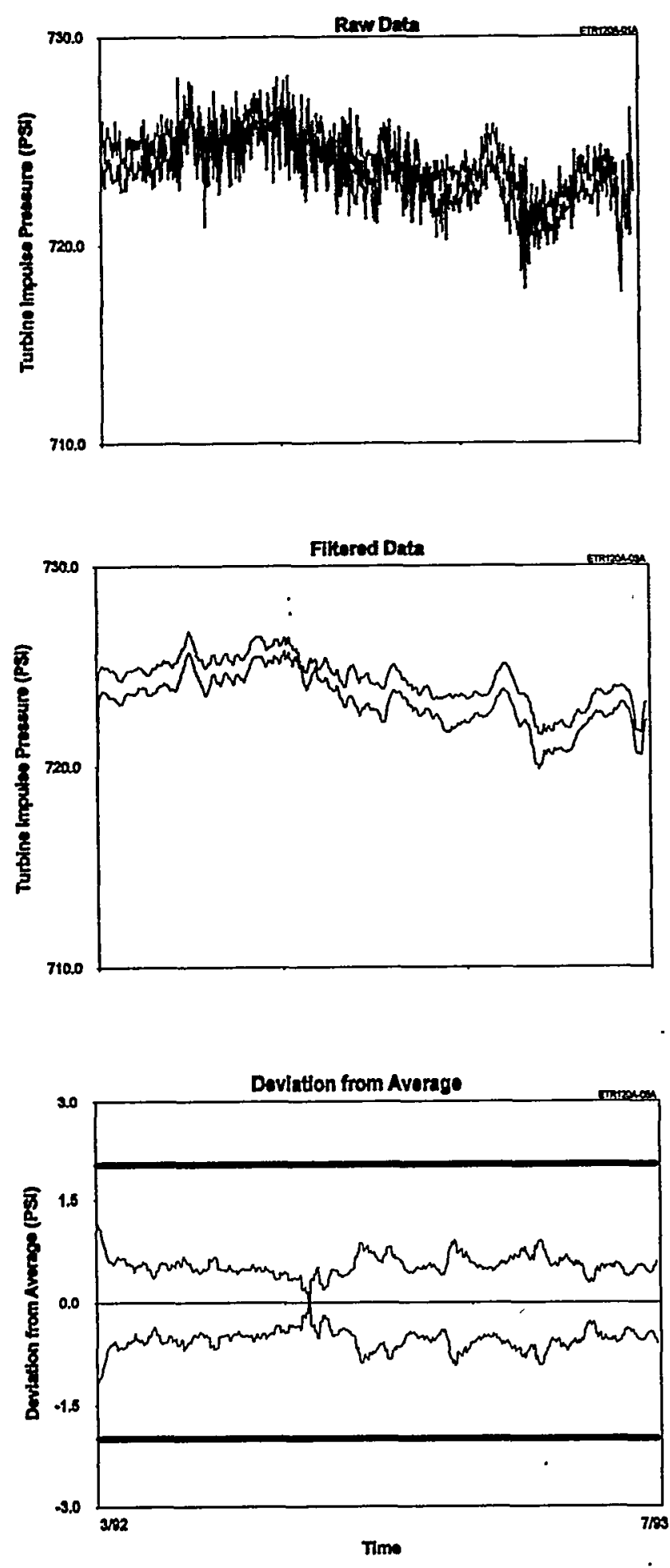

\section{Cycle 2}
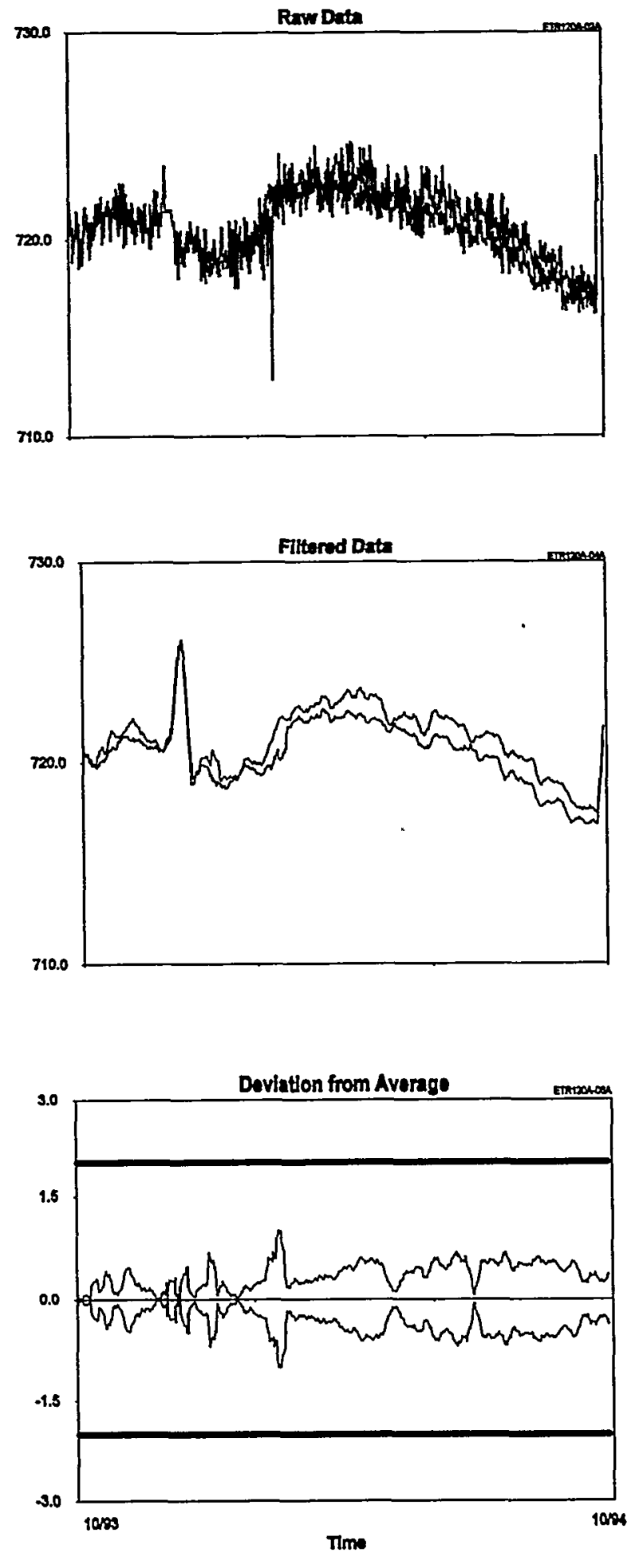

Figure 16.43 Raw Data and Deviation Plots for Turbine Impulse Pressure Signals 
transmitter as being well within the band.

3. In Figure 16.33, results are shown for three pressurizer level transmitters. The modeling results fail as was discussed earlier in describing the drift plot for the pressurizer level. The averaging results show a channel that exceeds the CSA band in cycle 1 . The channel was calibrated at the end of cycle 1 as indicated by the cycle 2 results.

\subsection{Comparison of Averaging and Modeling Results}

Results comparing the averaging, physical modeling, and empirical modeling are given in Figure 16.44 for a steam pressure transmitter. The models were trained with startup data and used to estimate the process behavior during a subsequent plant transient. The data in this figure shows that the empirical and physical modeling results agree well with the average of redundant steam pressure channels. Although physical modeling has provided reasonable results in this case, as indicated before, empirical modeling, neural networks, and simple and weighted averaging techniques have proven to be more efficient and more suitable than physical modeling. As such, physical modeling was used in very few cases in this project.

Figure 16.45 compares quantitative results of on-line drift monitoring for eight thermocouples. The results are shown from simple averaging, empirical modeling, and neural networks. Note that the three methods agree reasonably well for most of the eight cases shown. Thermocouples were the only sensors that showed enough drift to allow this type of comparison in this project.

Empirical modeling and neural network results are given in Figure 16.46 for a steam generator level and a pressurizer pressure signal and compared with actual measurements. The good performance of the models is apparent in these data.

\subsection{Success and Failure of Process Estimation Techniques}

Figure 16.47 shows drift plots for three flow signals. One plot shows the drift data obtained using the averaging technique and the other plot shows the drift data from using the empirical modeling technique. The drift bands are also shown on the figure. Note that the drift band for the modeling result is larger than the drift band for averaging results because the band for averaging results is multiplied by $(n-1) / n$ (where $n$ is the number of signals). One of the three signals is exceeding the allowable drift band. The empirical modeling results show the problem earlier than the averaging results. This is because the averaging estimate is biased toward the drifting signal which diminishes the amount of drift compared to the model estimate.

Figures 16.48 and 16.49 show drift results for three reactor coolant flow transmitters based on neural networks, empirical modeling and simple averaging of data for the two cycles during which on-line monitoring was performed at McGuire. Figure 16.48 shows that the three process estimation techniques agree reasonably well. However, the empirical modeling results in Figure 16.49 do not agree with the neural networks or simple averaging results. In this case, the empirical model has failed because of inadequate inputs.

The reliability of empirical modeling and neural networks depend on the adequacy of their training and the quality of the input signals that are given to the model. If the model is not trained properly for the 


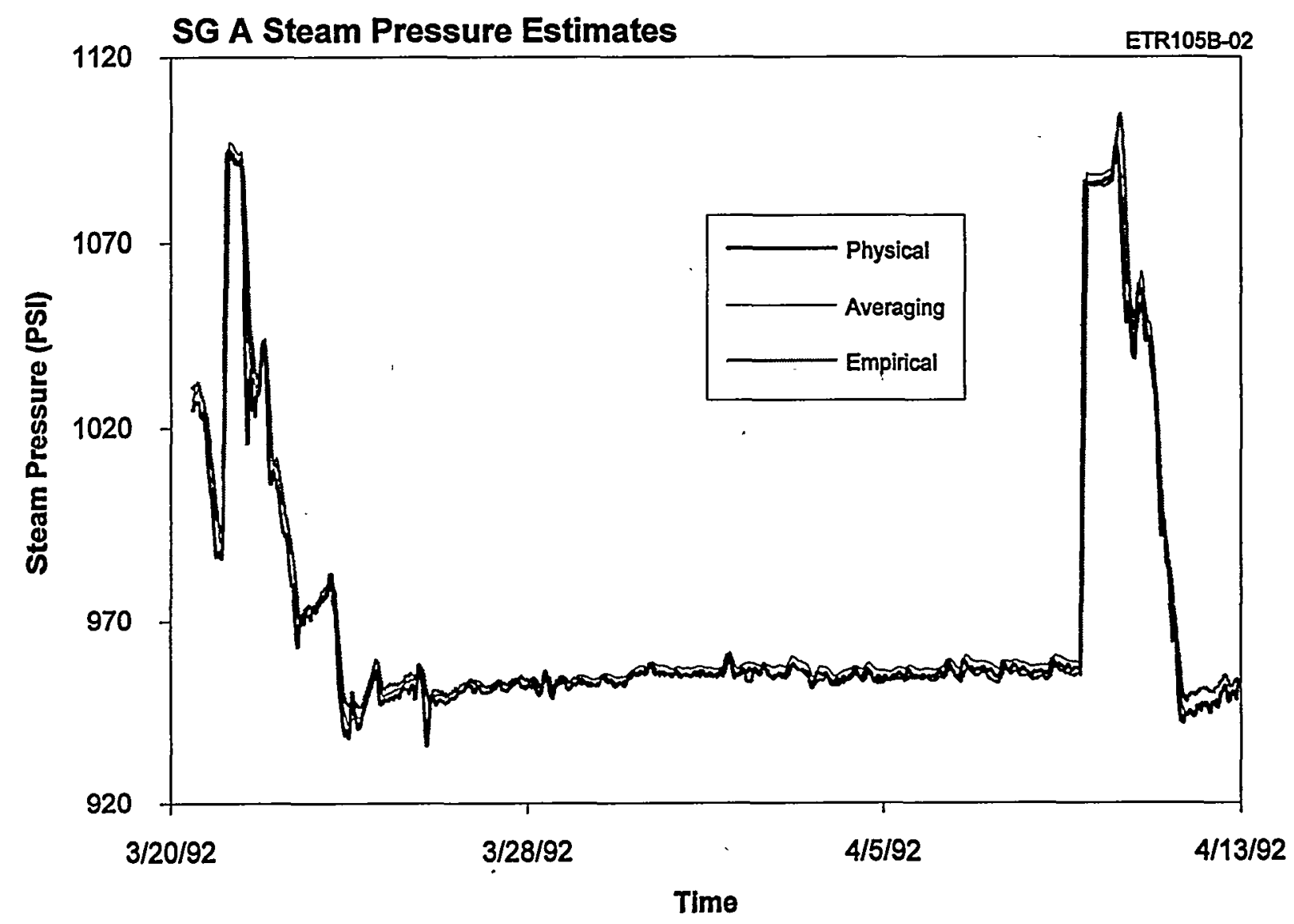

Figure 16.44 Comparison of Results of Physical Modeling with Averaging and Empirical Modeling 


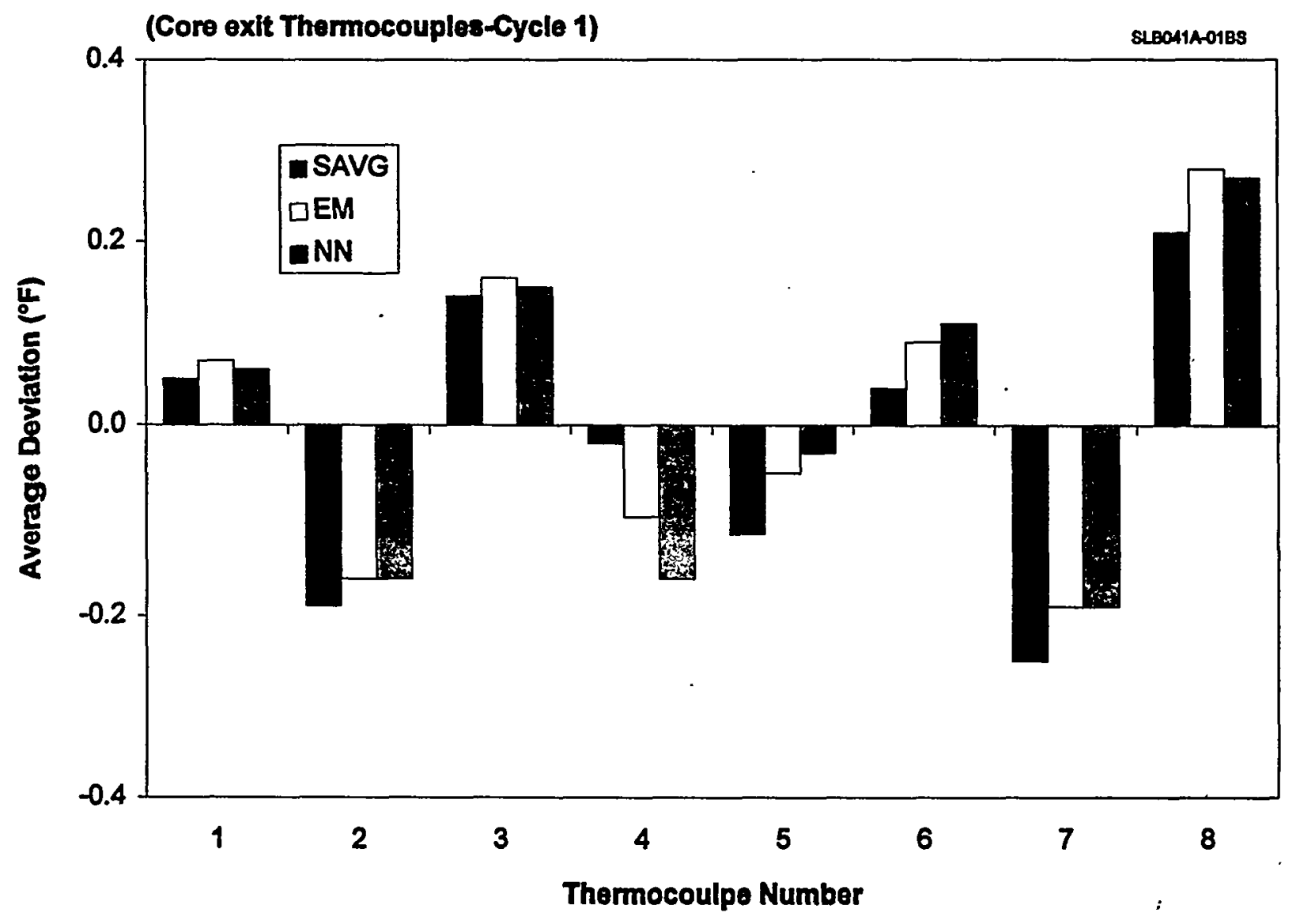

Figure 16.45 Comparison of Deviation Results From Three Process Estimation Techniques for McGuire Thermocouple Signals 

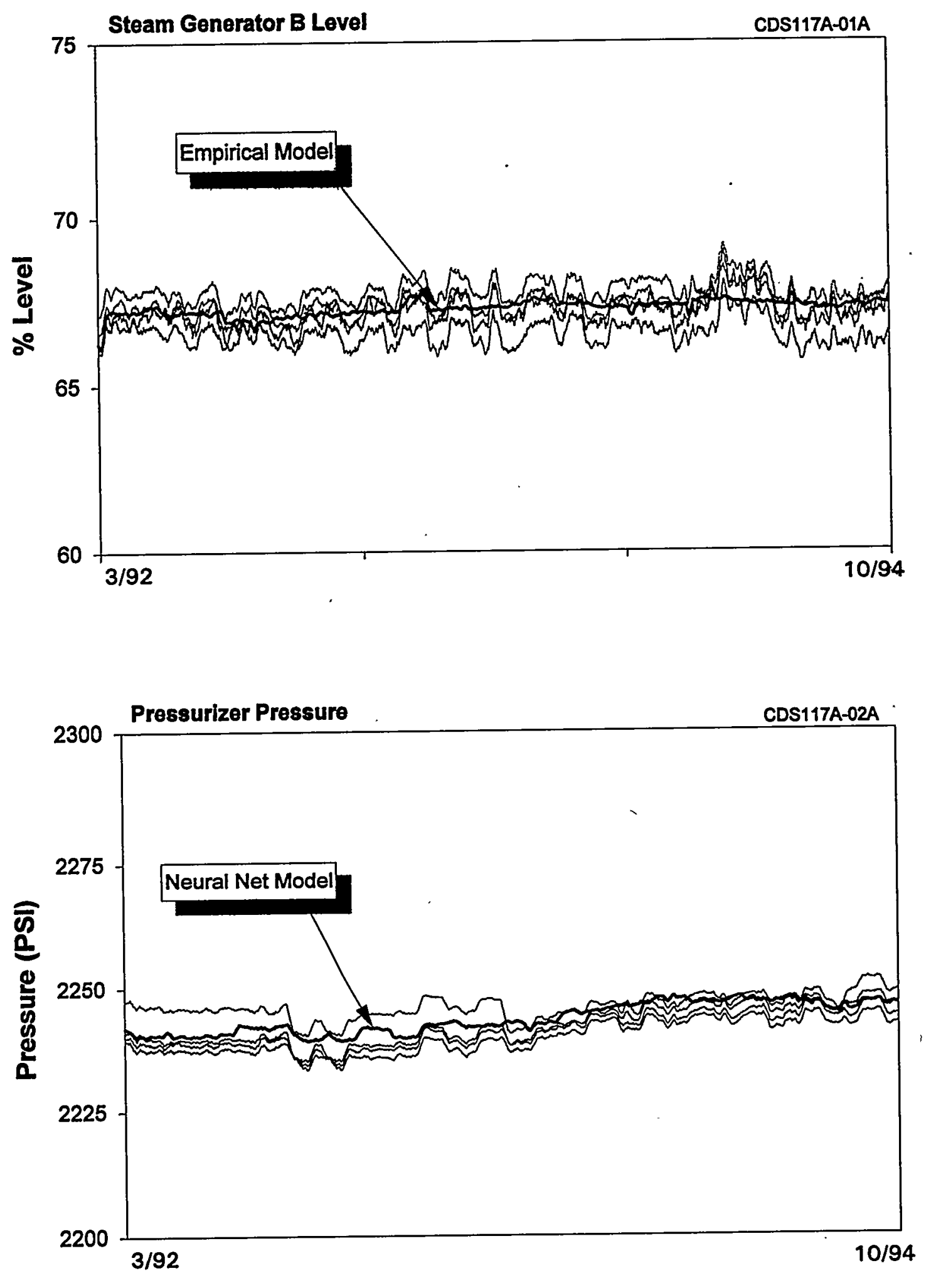

Figure 16.46 Empirical and Neural Network Results of Process Estimation Compared with Actual Plant Signals 

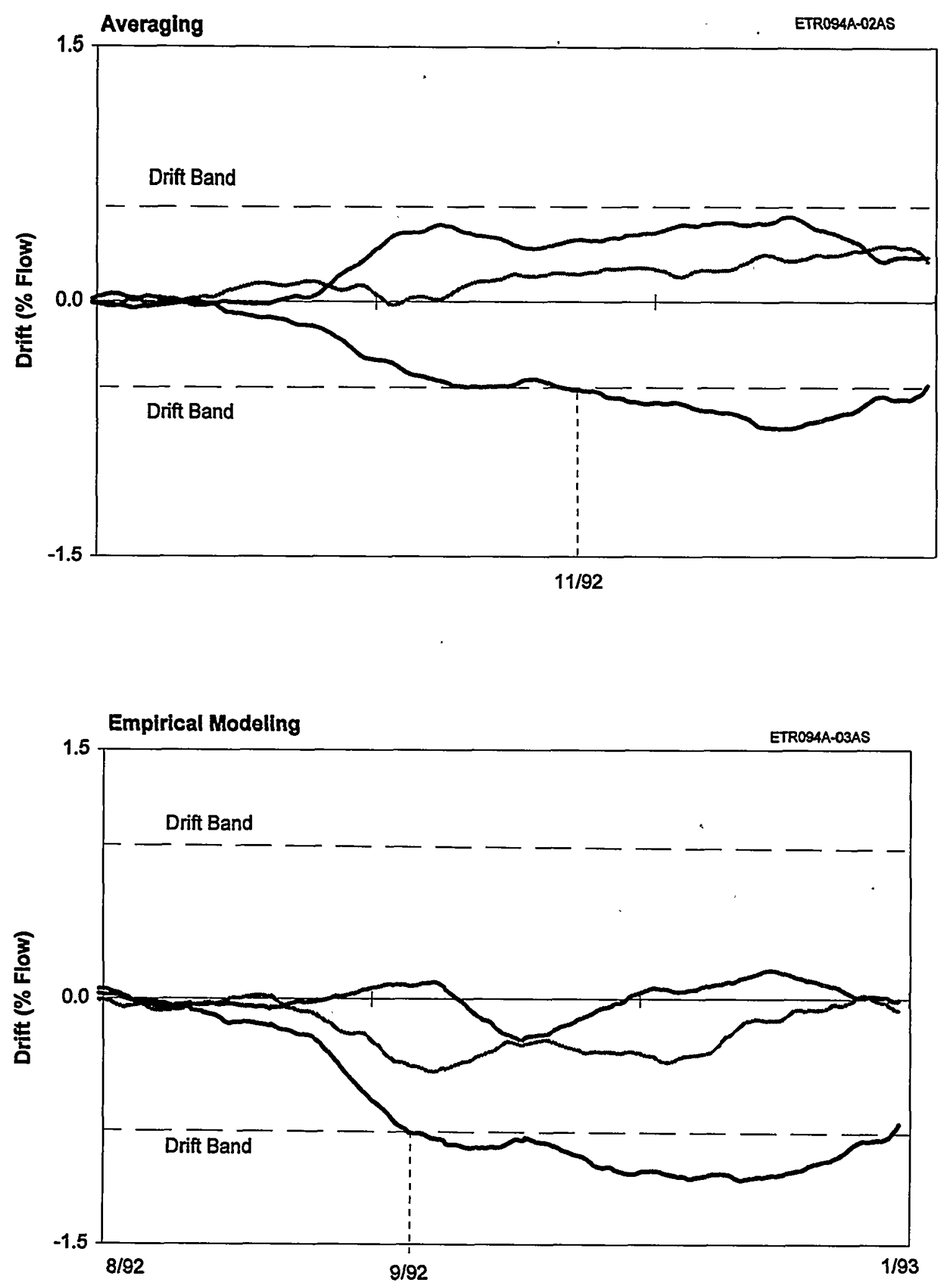

Figure 16.47 Averaging and Empirical Modeling Results for McGuire Flow Transmitters 

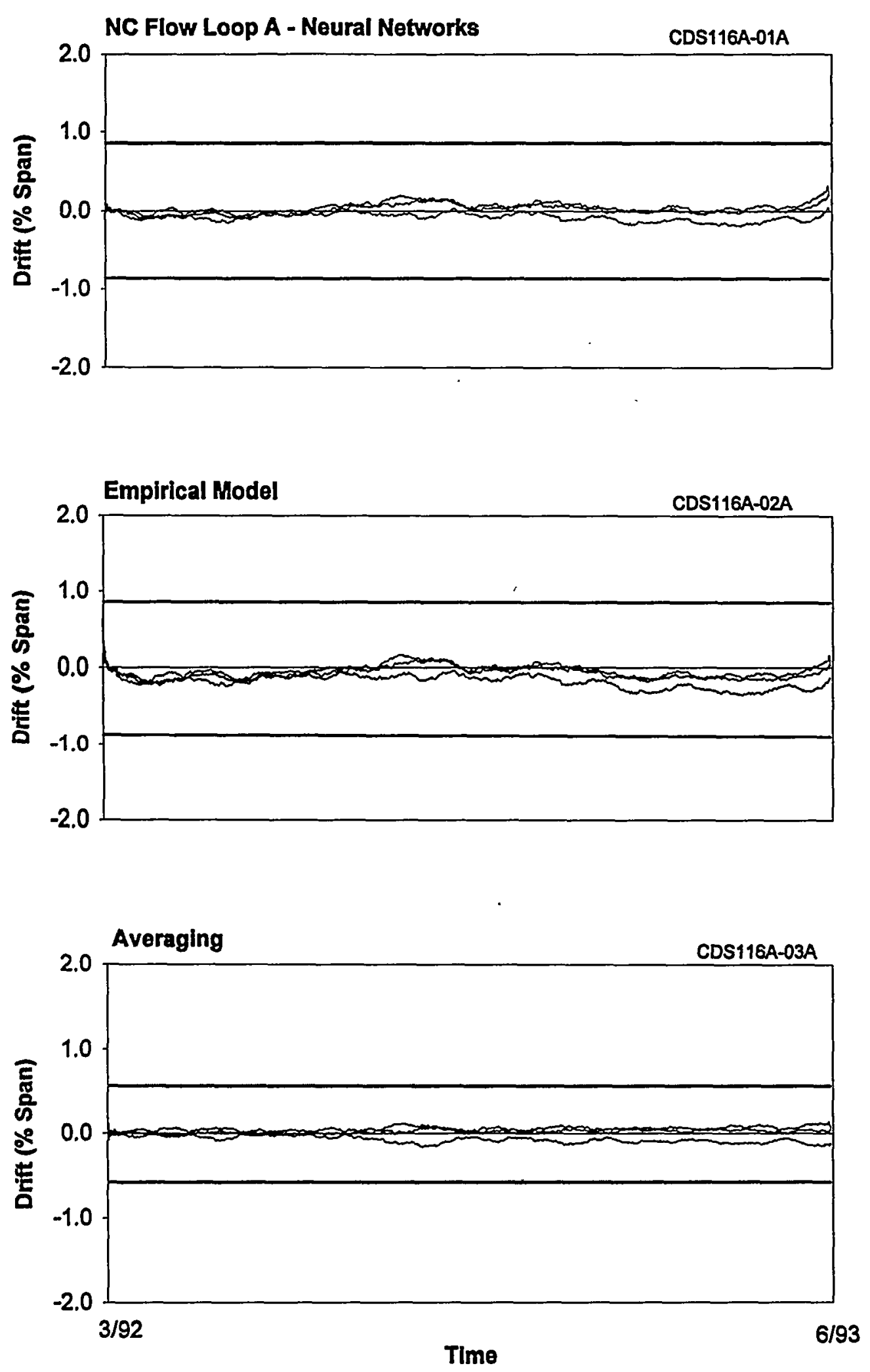

Figure 16.48 Drift Results Showing Good Agreement Between Process Estimation Technqiues 

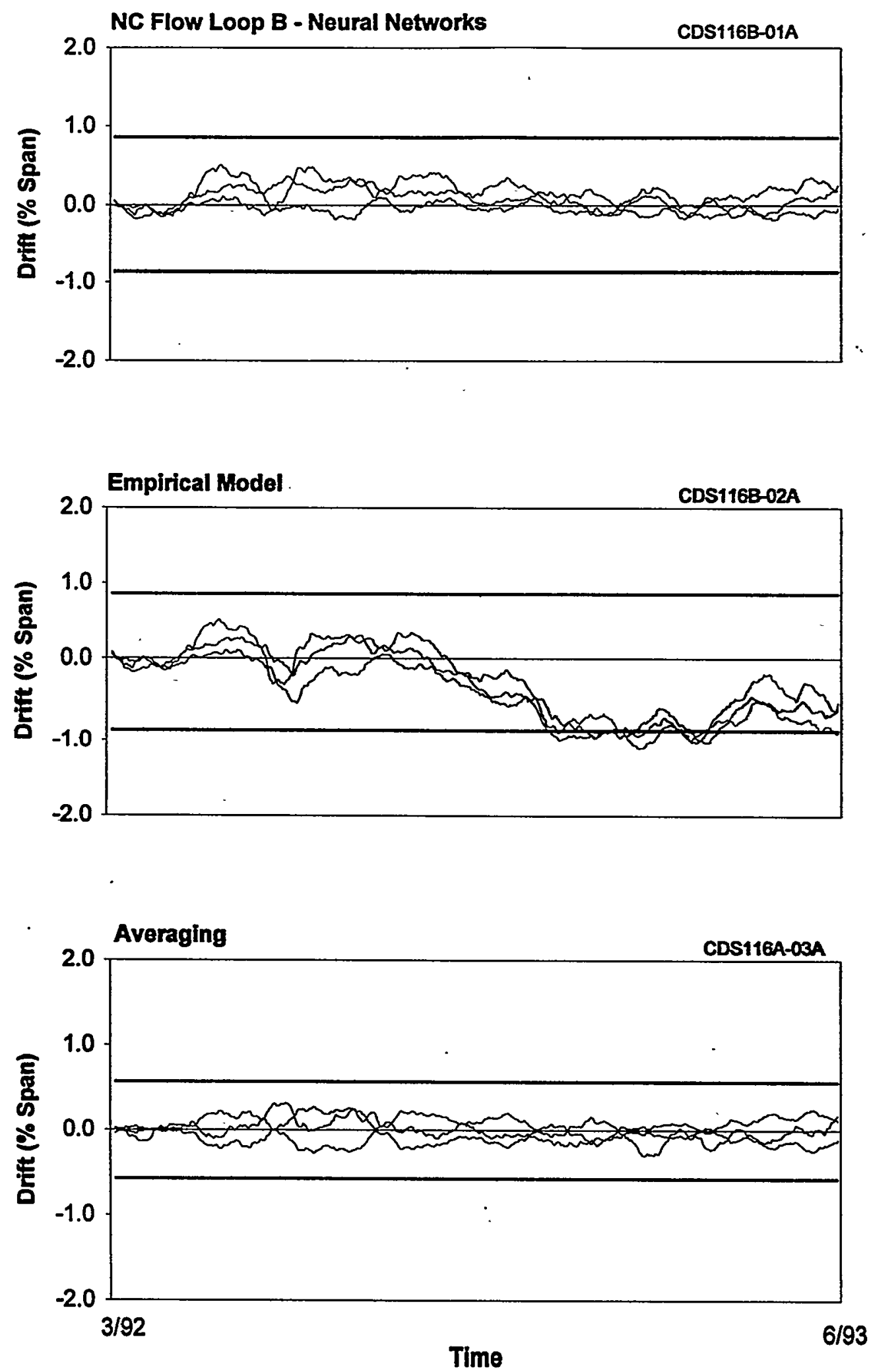

Figure 16.49 Drift Results Showing the Failure of Empirical Modeling Technique 
conditions at hand, then its results will not be reliable. Figure 16.50 shows analytical and averaging results for three steam pressure transmitters for which the empirical model and neural network have apparently failed due to improper inputs.

\subsection{Instrument Drift and Failure Problems at McGuire}

In the three years of on-line monitoring of nearly 170 signals at McGuire, there were less than five percent failures per fuel cycle in the pressure or temperature instrumentation channels.

Figure 16.51 shows signals from three steam generator level transmitters, one of which was replaced in the time frame shown on the figure. Note, in the middle of the figure that two of the three transmitters rise while the third one remains flat. The transmitter with the flat response was the one that was replaced. The data for another transmitter which was replaced is shown in Figure 16.52. The transmitter which shows the most drift in this plot was replaced.

Several non-safety related thermocouples at McGuire were found to have large errors. However, this is typical for any large group of thermocouples that are not normally calibrated or replaced. Figure 16.53 shows on-line monitoring traces for a group of McGuire thermocouples which include a failed thermocouple. Another temperature sensor which failed was a hot leg RTD in Loop D.
Figure 16.54 shows on-line monitoring results in terms of raw data and a drift plot. The RTD which failed began to drift a little after the beginning of the cycle. It then experienced a sudden shift which lasted until near the end of the cycle when the RTD shifted significantly. This RTD was replaced. This case illustrates the incipient failure detection capability of the on-line monitoring system. The RTD showed erratic behavior for a long period of time before it failed.

\subsection{Comparison of Deviation Plots for Redundant Plant Equipment}

Figure 16.55 through 16.62 show eight deviation plots for redundant plant equipment. Two columns of four plots are shown in each figure. One column is for cycle 1 and the other is for cycle 2. Each plot shows about two months of data. The data are presented in terms of deviation of each signal from the simple average of redundant signals. The purpose of these plots is to illustrate how redundant signals from redundant equipment such as steam flow signals from the four steam generators (A through D) compare with one another. Also, these plots are provided here to show how the signals from cycle 1 compare with corresponding signals from cycle 2 .

The figures show that the signals from redundant plant equipment have similar characteristics and that cycle 1 and cycle 2 signals look much alike. 


\section{Steam Generator B Pressure}

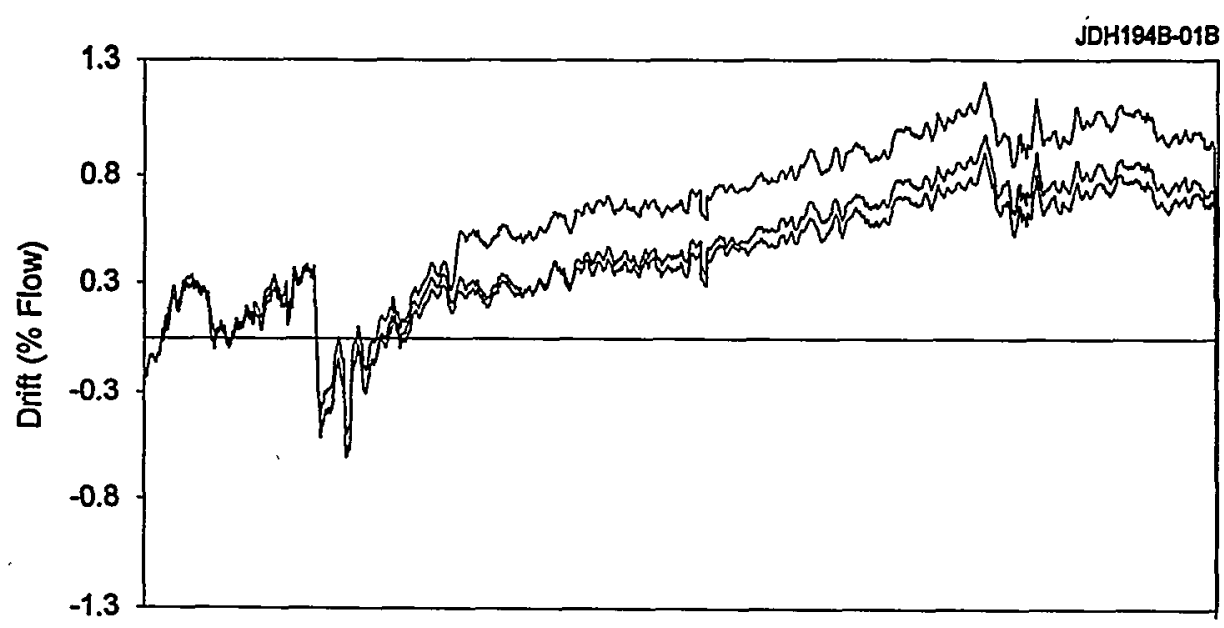

Neural Net

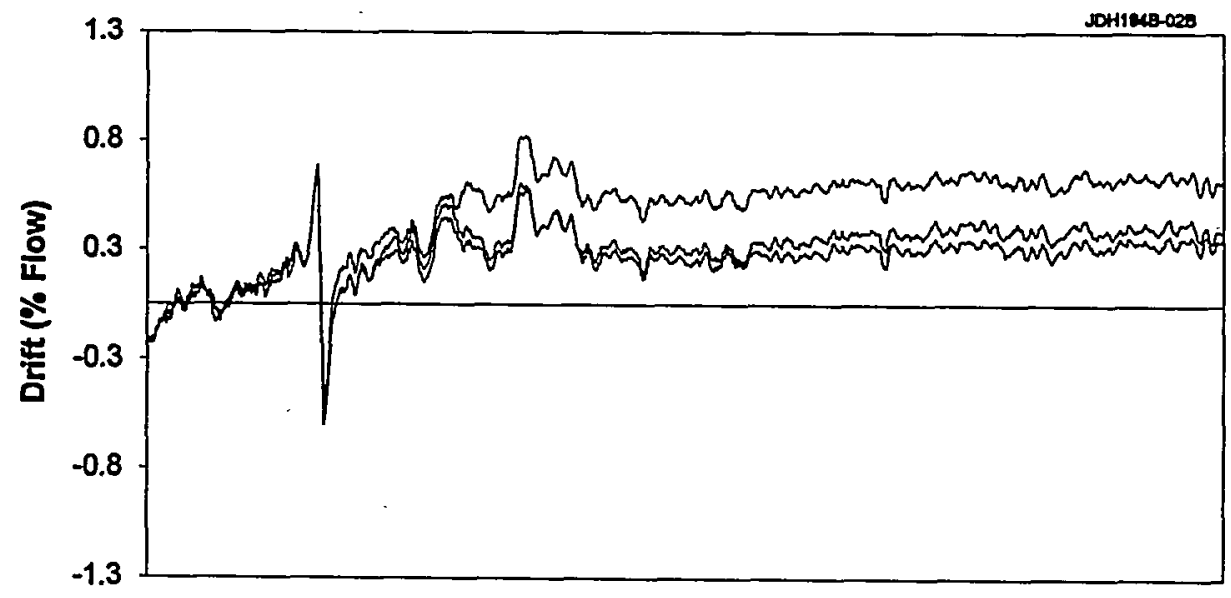

Empirical

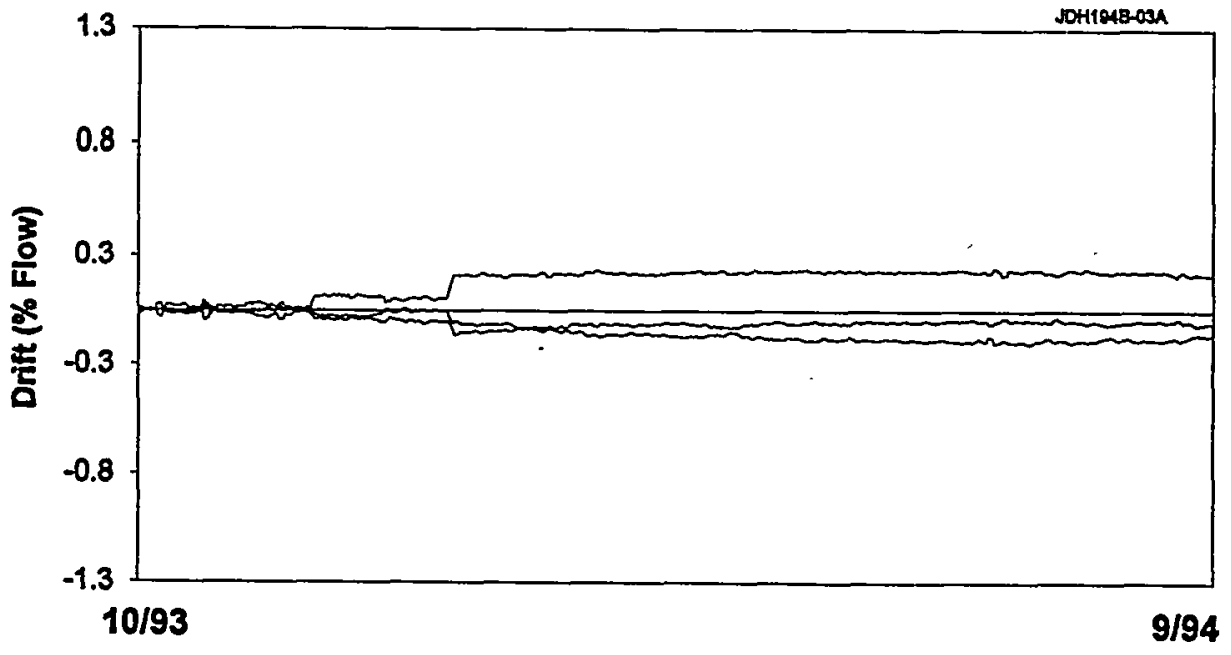

Averaging

Figure 16.50 Drift Results for McGuire Steam Pressure Transmitters Showing the Failure of Analytical Techniques 


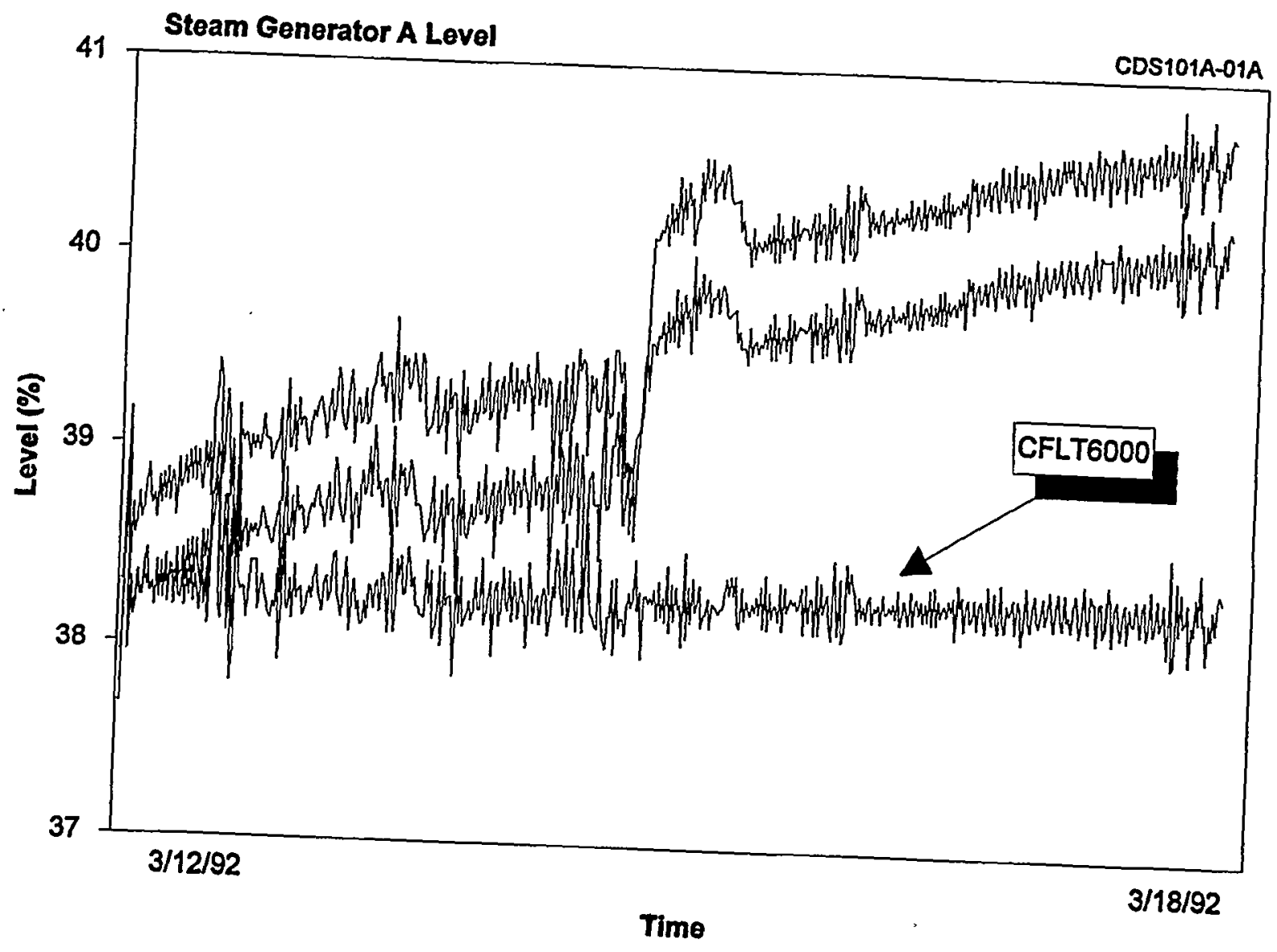
Figure 16.51 Data for Three McGuire Steam Generator Level Transmitters
of which One was Replaced 


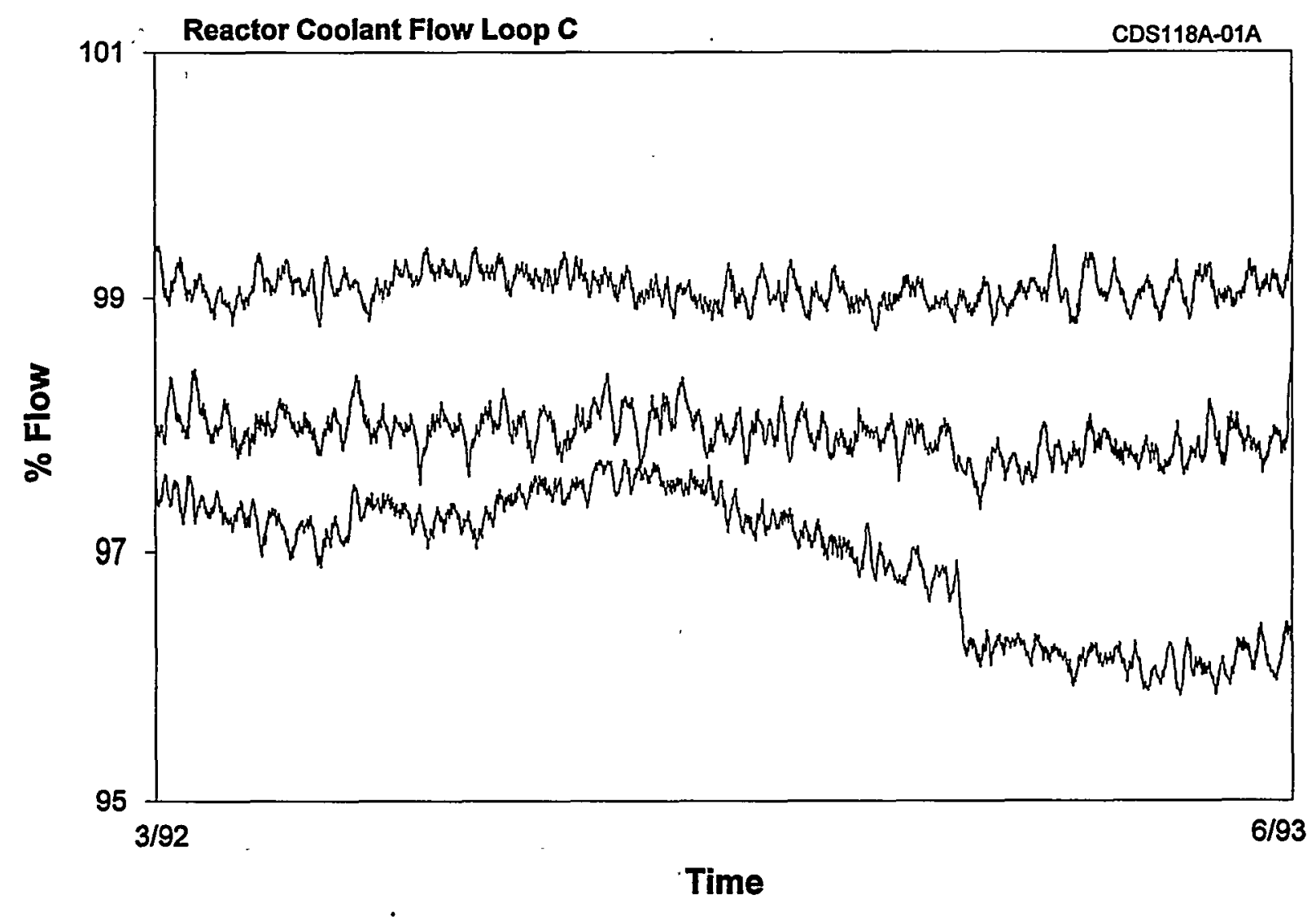

Figure 16.52 Data for Three McGuire Reactor Coolant Flow Transmitters of which One was Replaced 

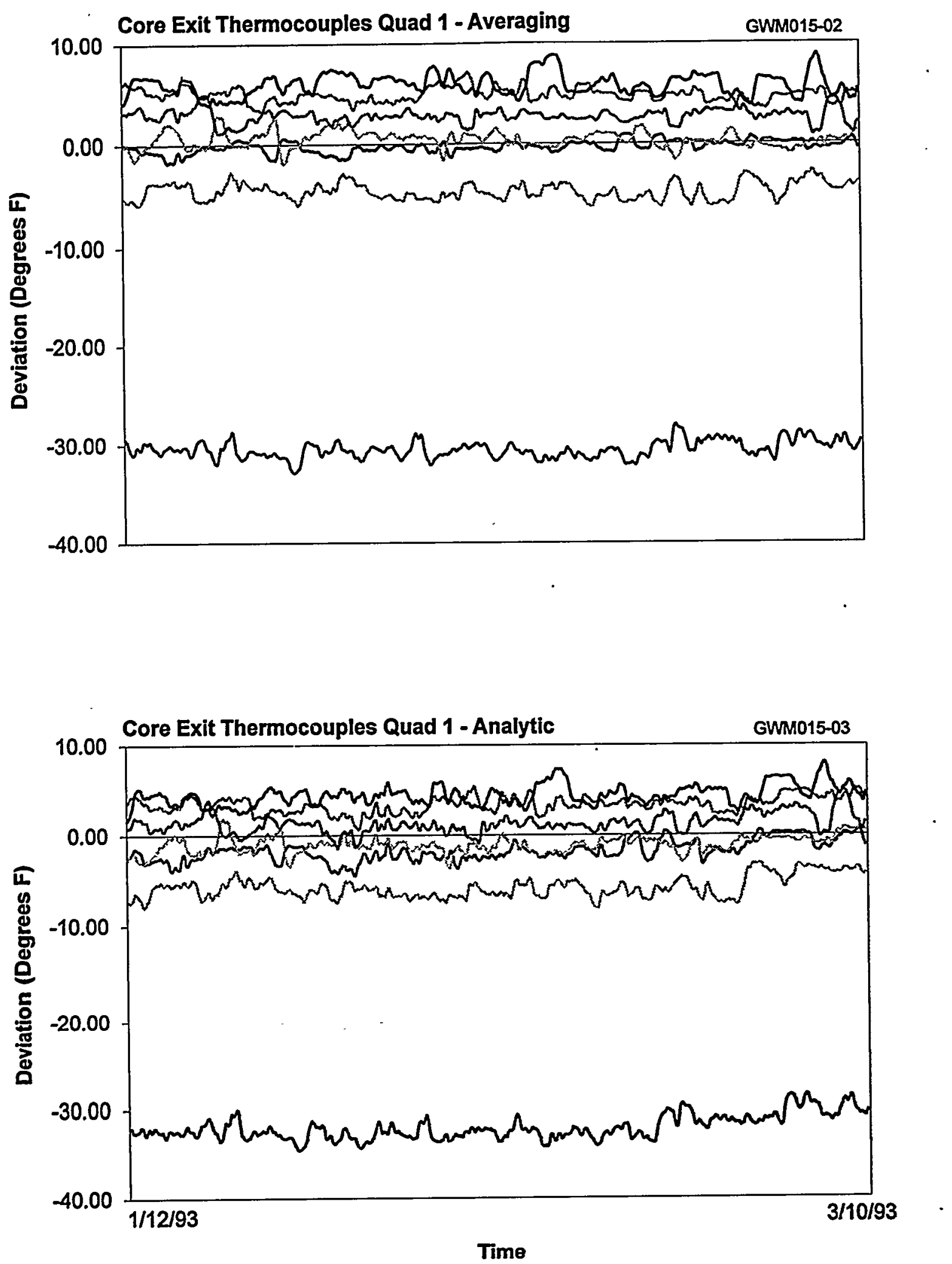

Figure 16.53 On-Line Monitoring Results Showing Failed Core Exit Thermocouples 

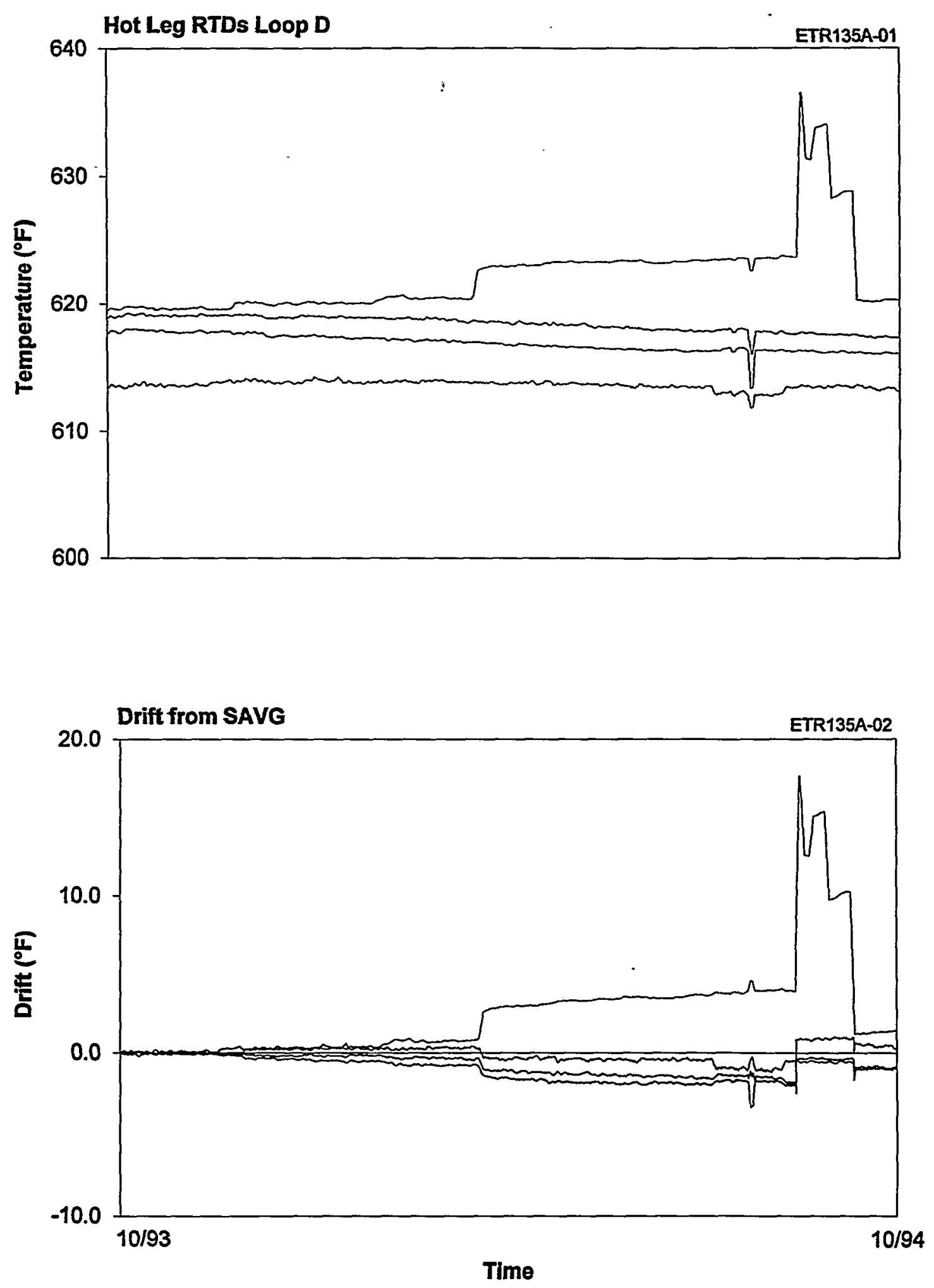

Figure 16.54 Behavior of an Erratic Hot Leg RTD at McGuire 
Cycle 1
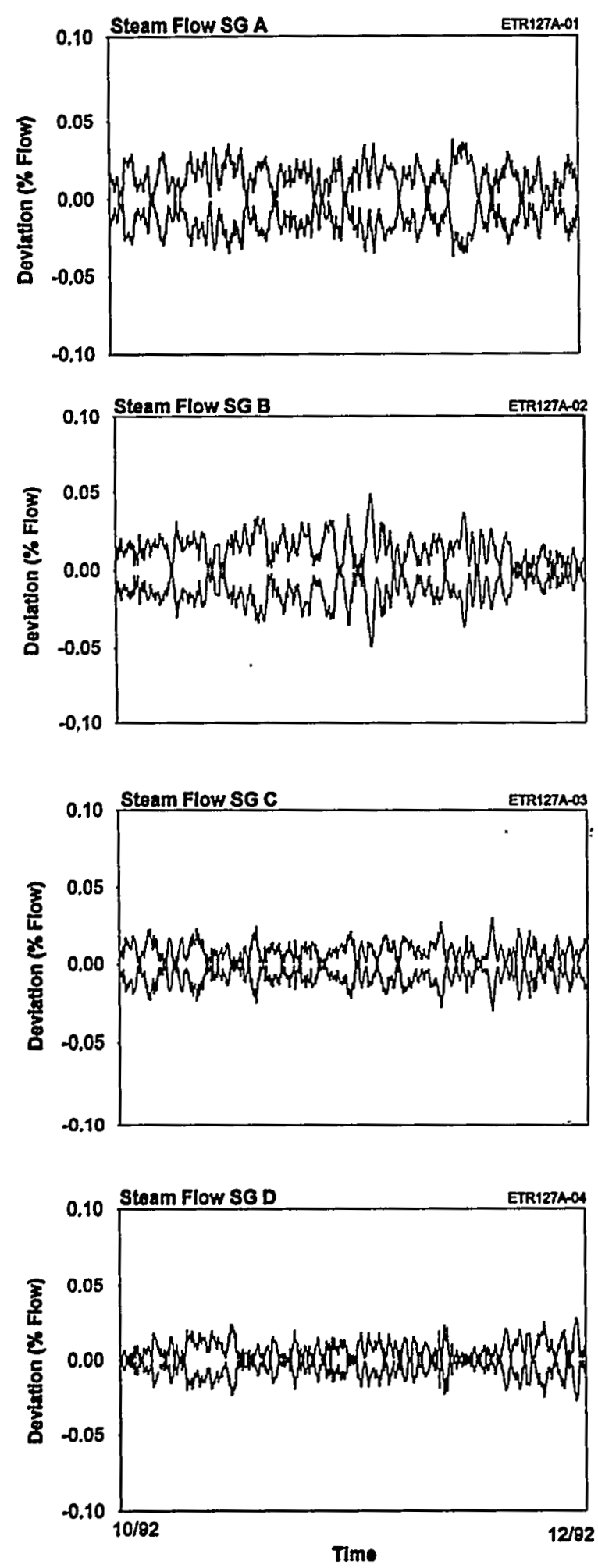

Cycle 2
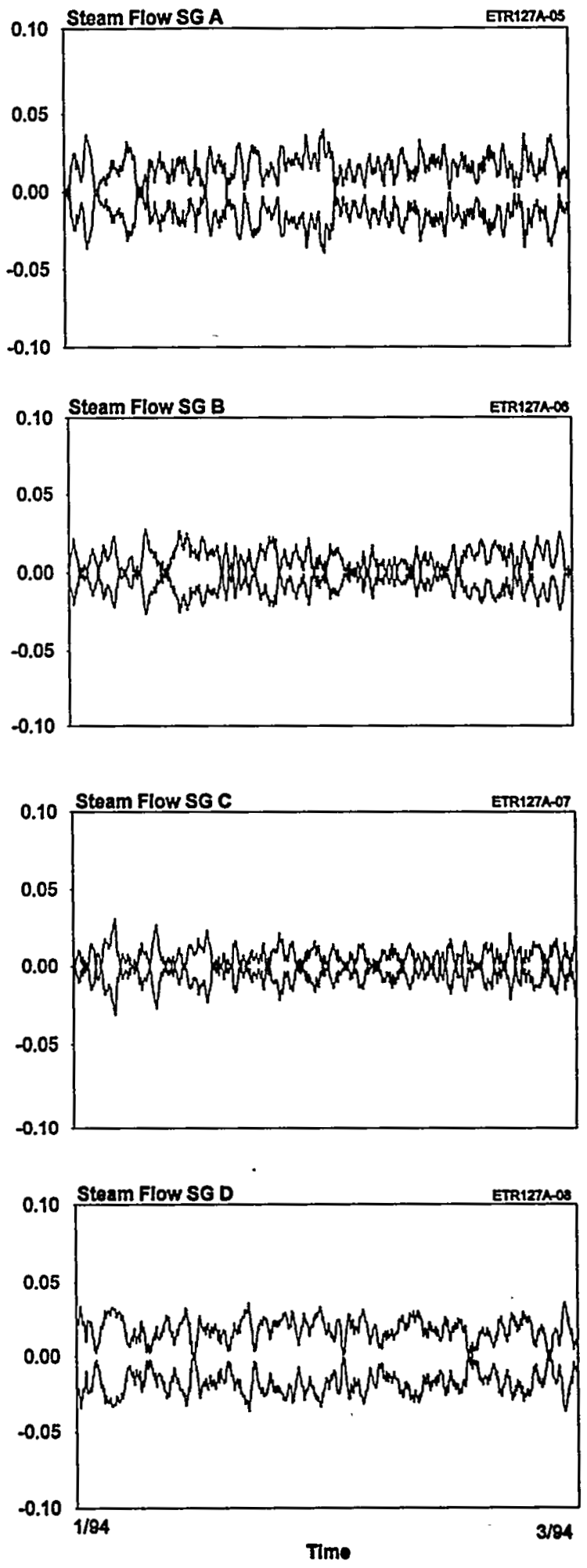

Figure 16.55 Cycle 1 and Cycle 2 Signals from Redundant Steam Flow Loops 


\section{Cycle 1}
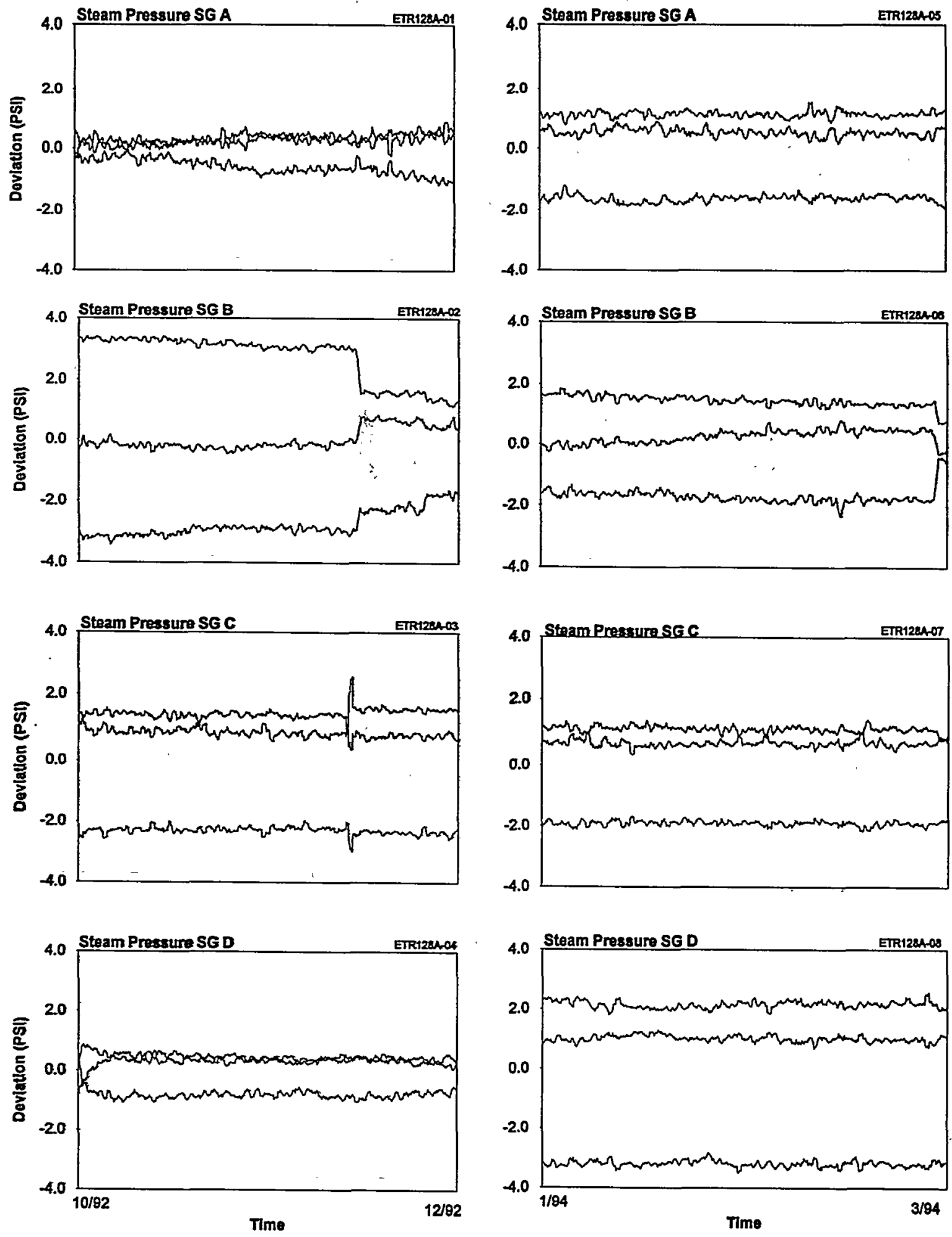

Figure 16.56 Cycle 1 And Cycle 2 Signals From Redundant Steam Pressure Loops 
Cycle 1
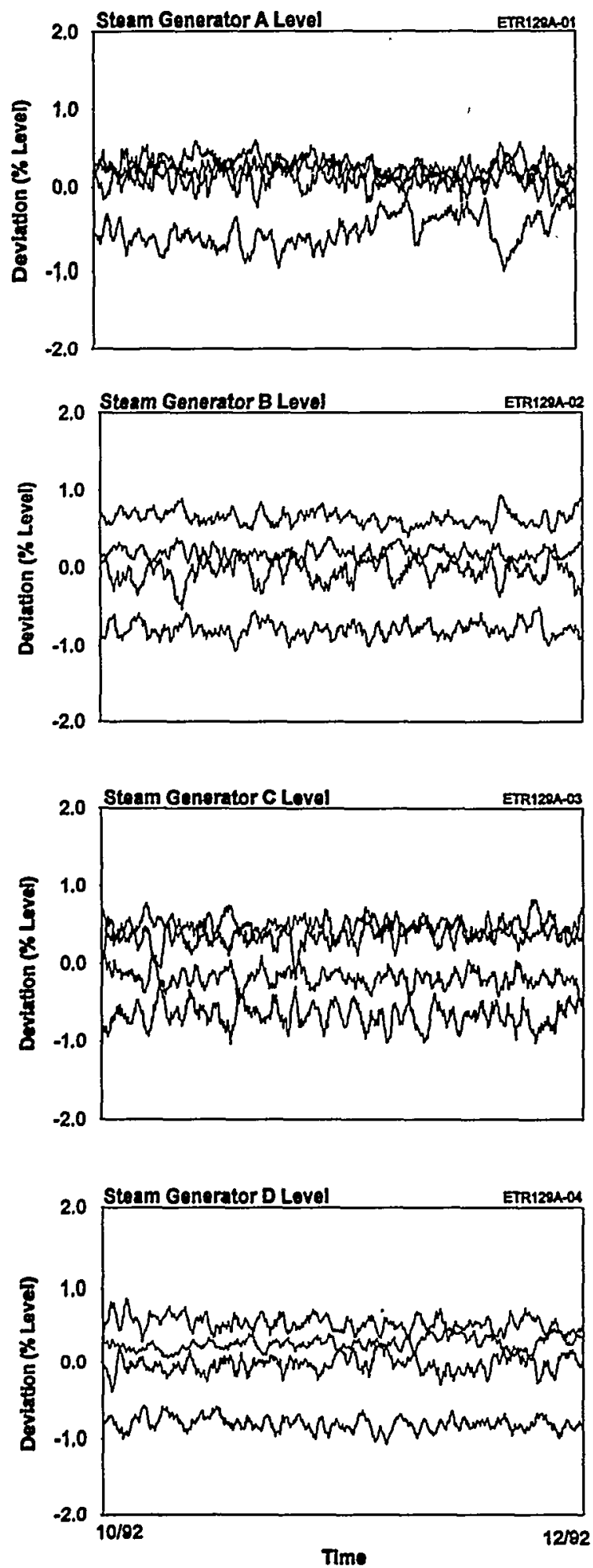

\section{Cycle 2}
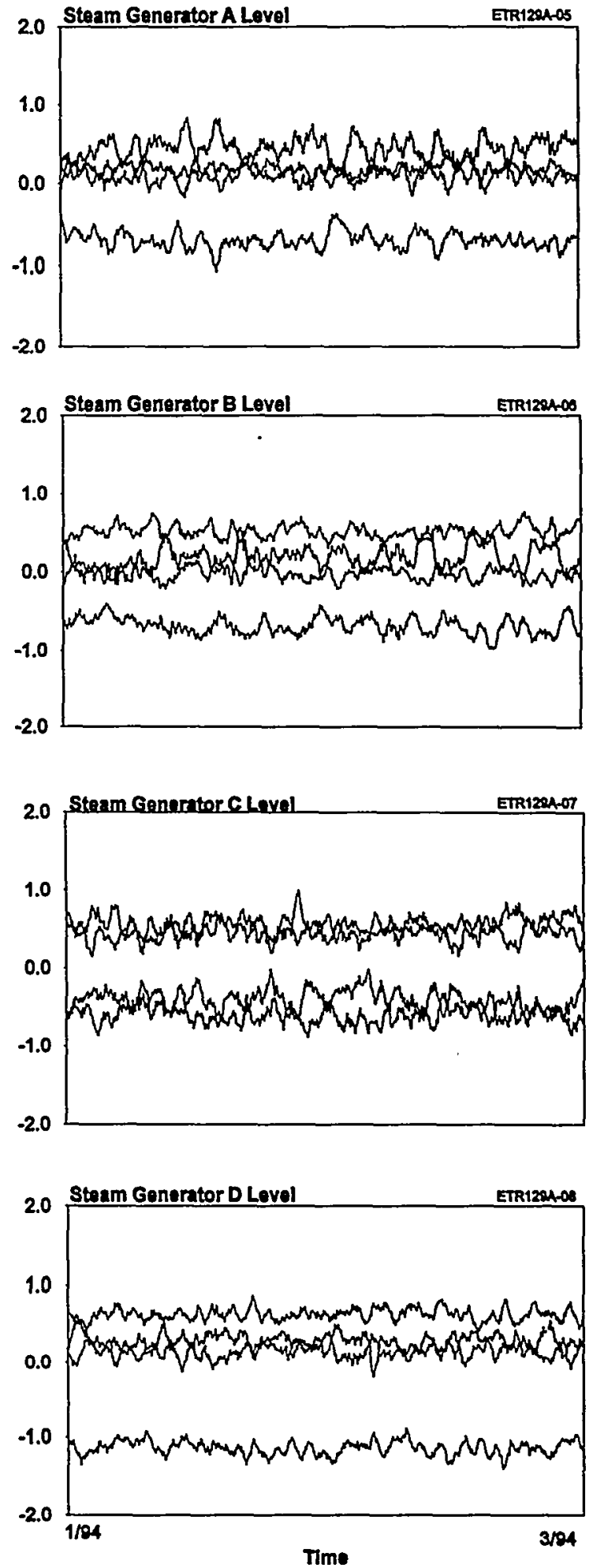

Figure 16.57 Cycle 1 and Cycle 2 Signals from Redundant Steam Generator Level Loops 


\section{Cycle 1}
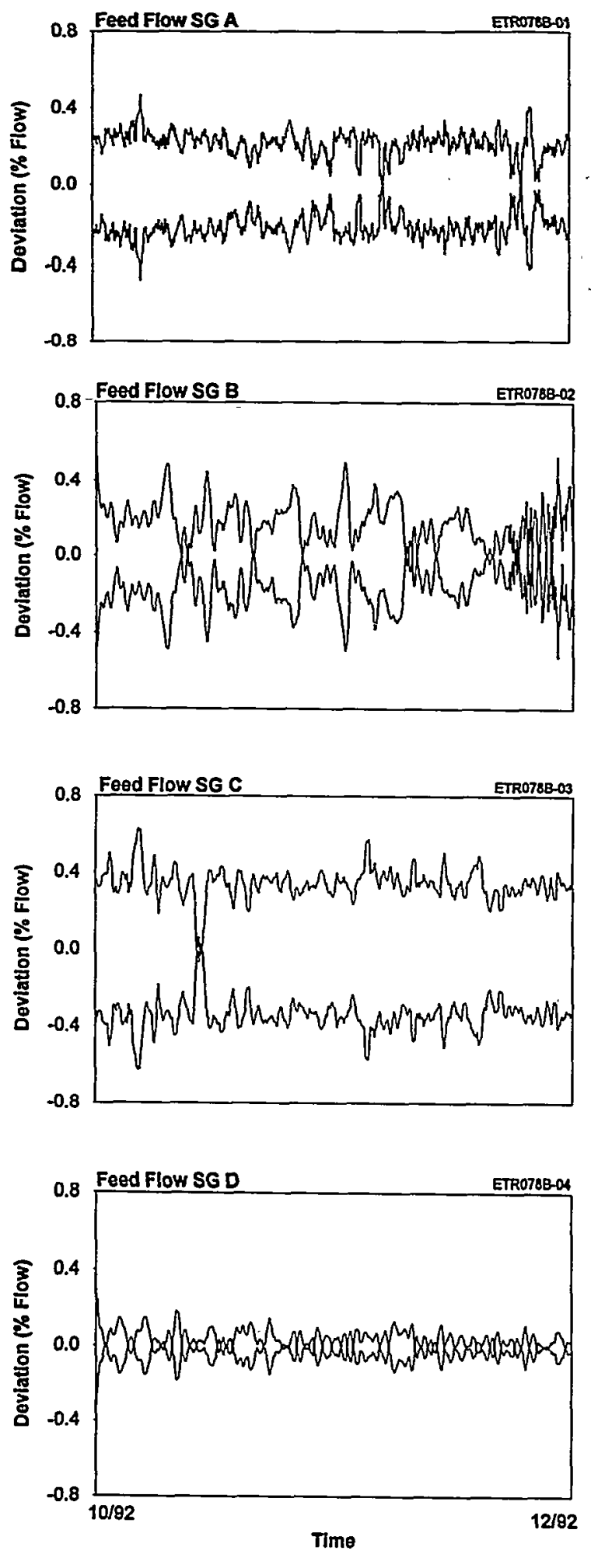

\section{Cycle 2}
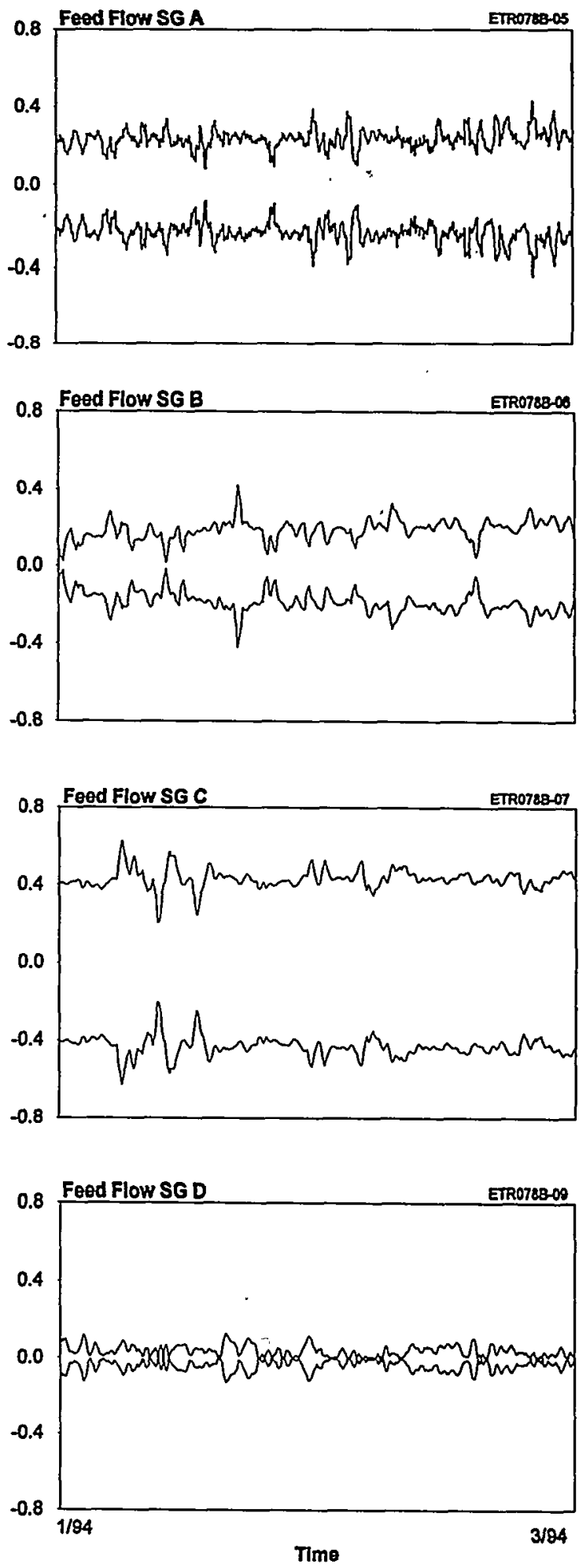

Figure 16.58 Cycle 1 and Cycle 2 Signals from Redundant Feedwater Flow Loops 
Cycle 1
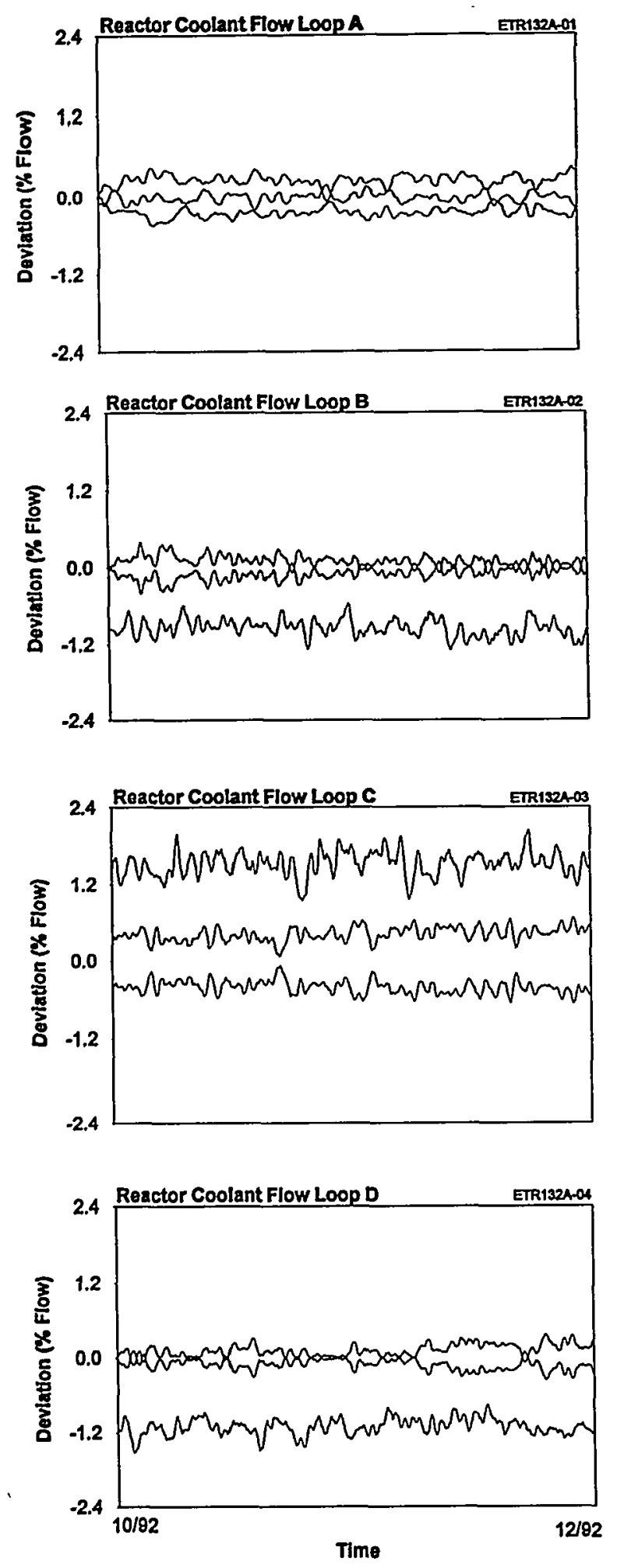

Cycle 2
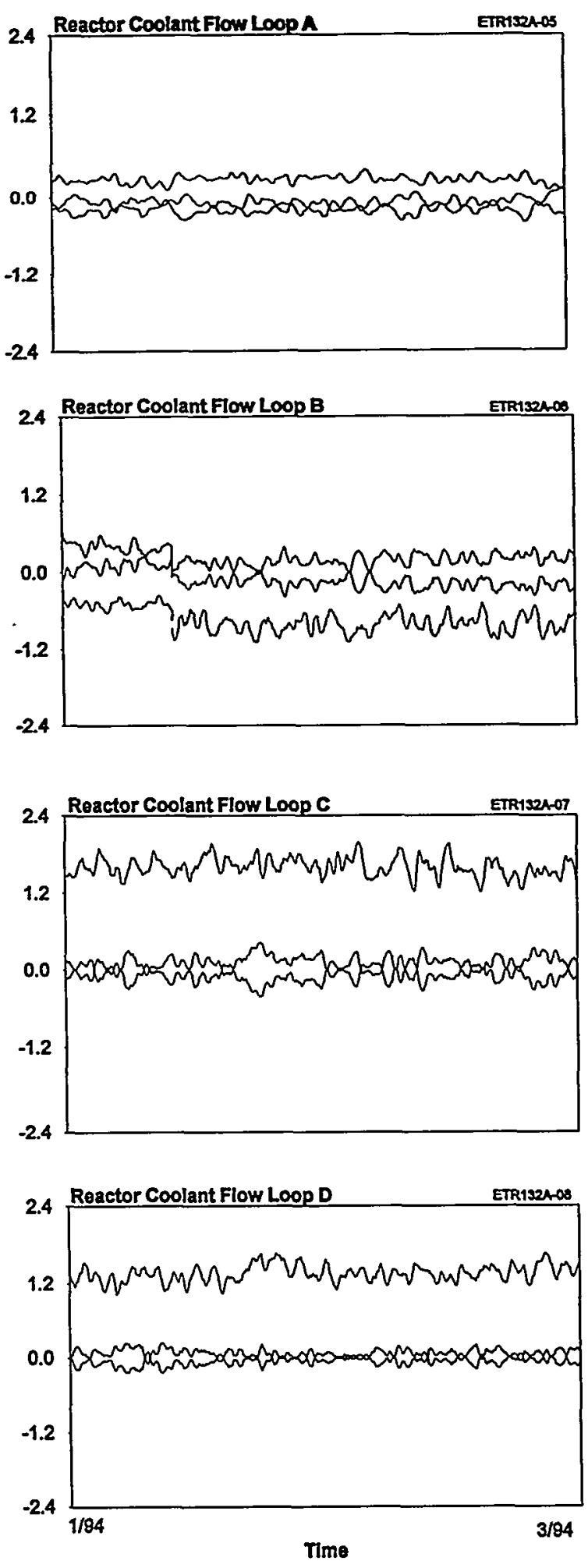

Figure 16.59 Cycle 1 and Cycle 2 Signals from Redundant Reactor Coolant Flow Loops 
Cycle 1
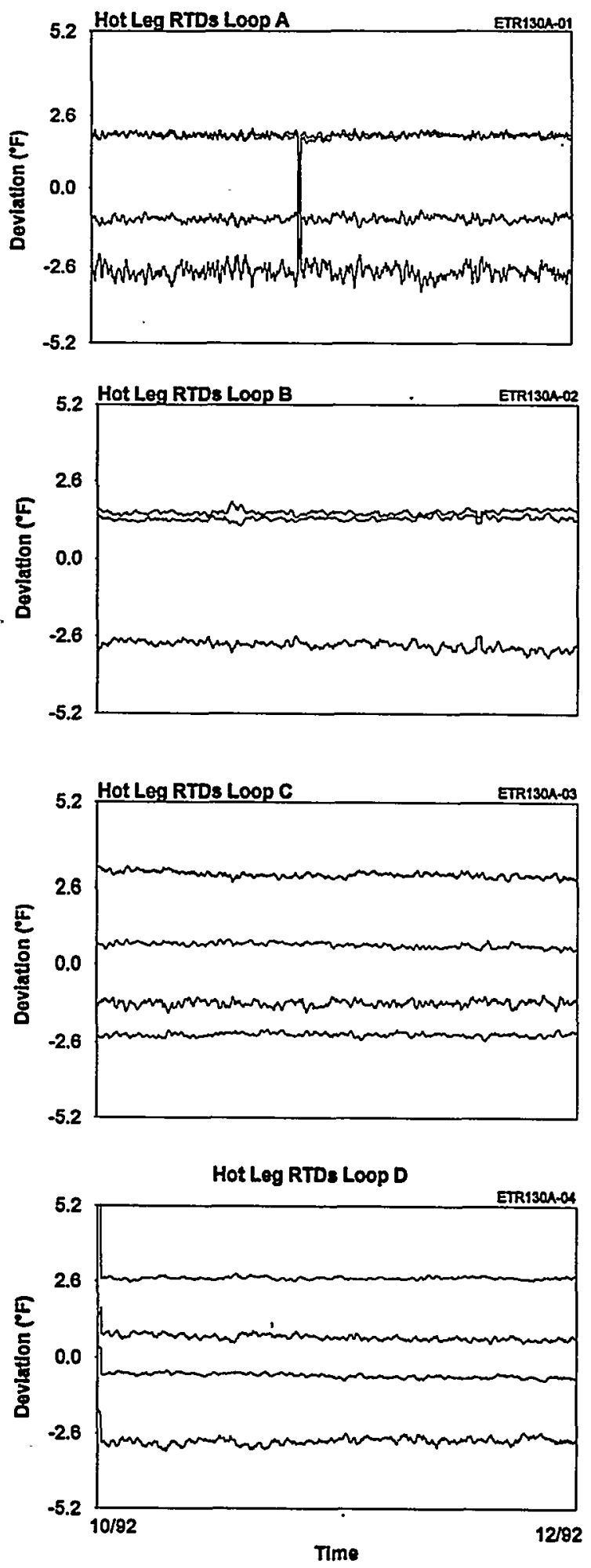

Cycle 2
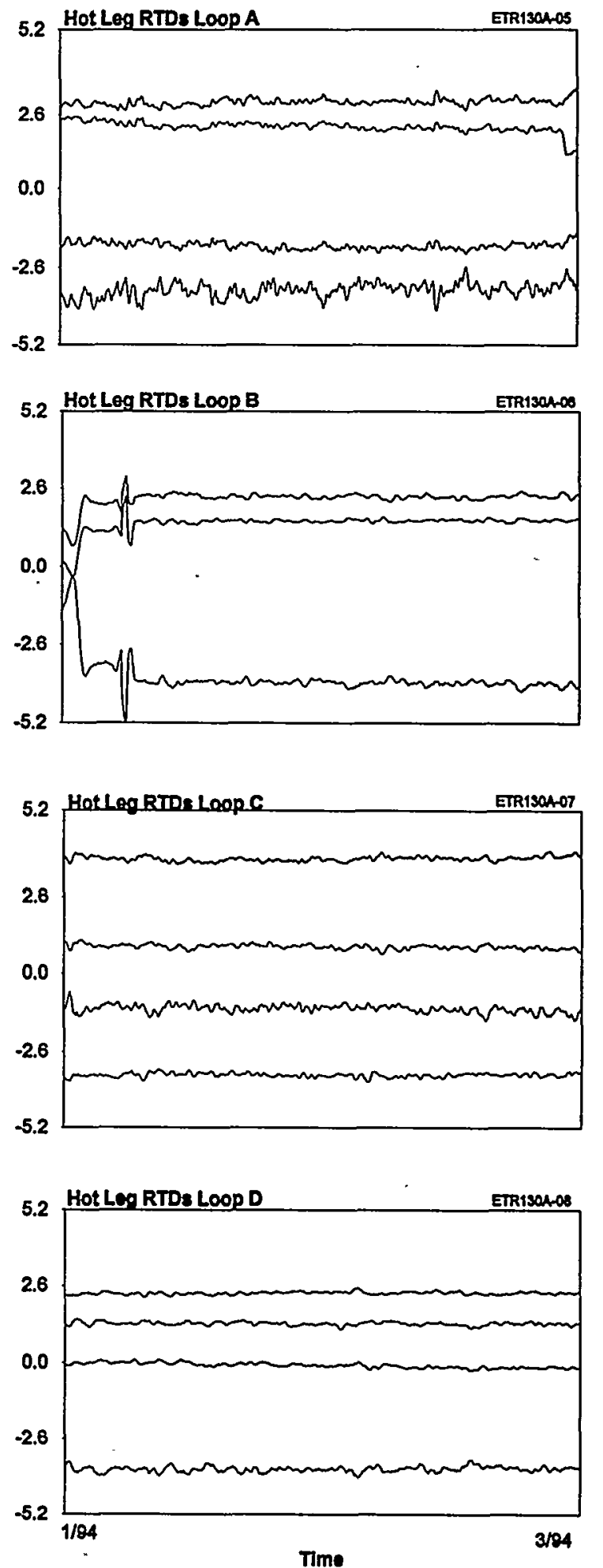

Figure 16.60 Cycle 1 and Cycle 2 Signals from Redundant Hot Leg RTD Loops 


\section{Cycle 1}
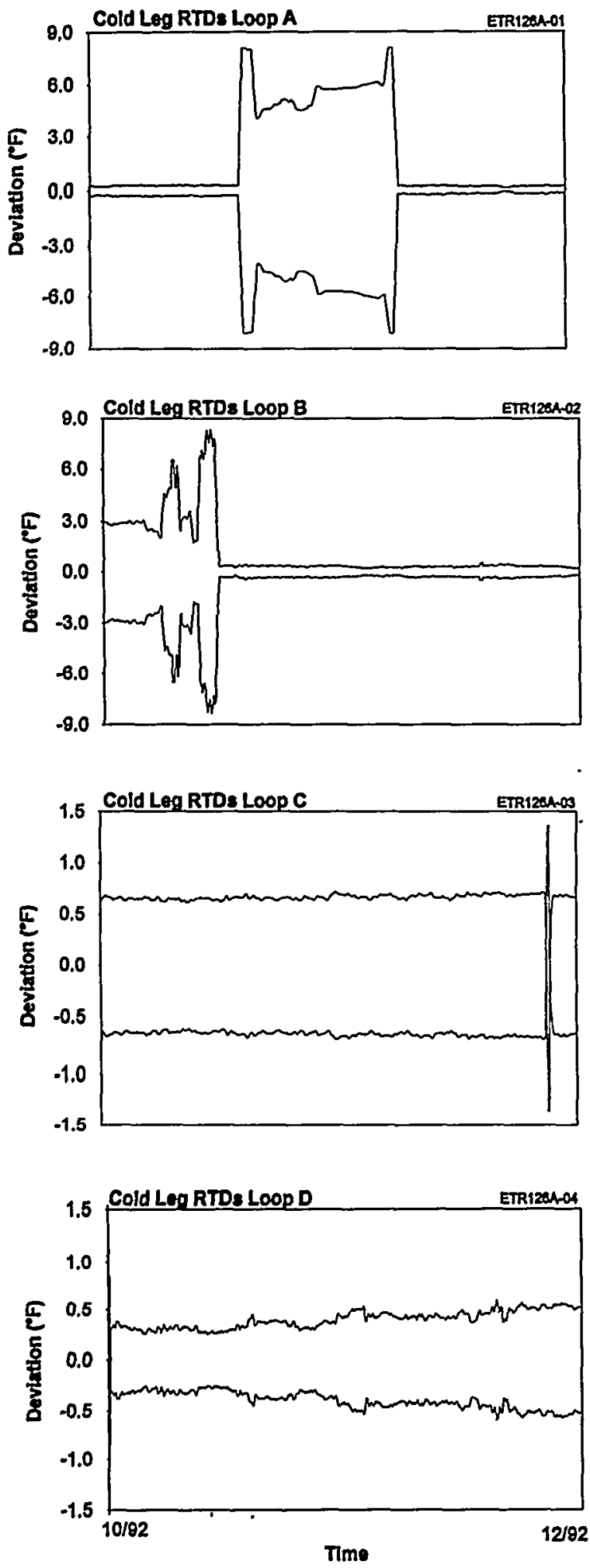

\section{Cycle 2}
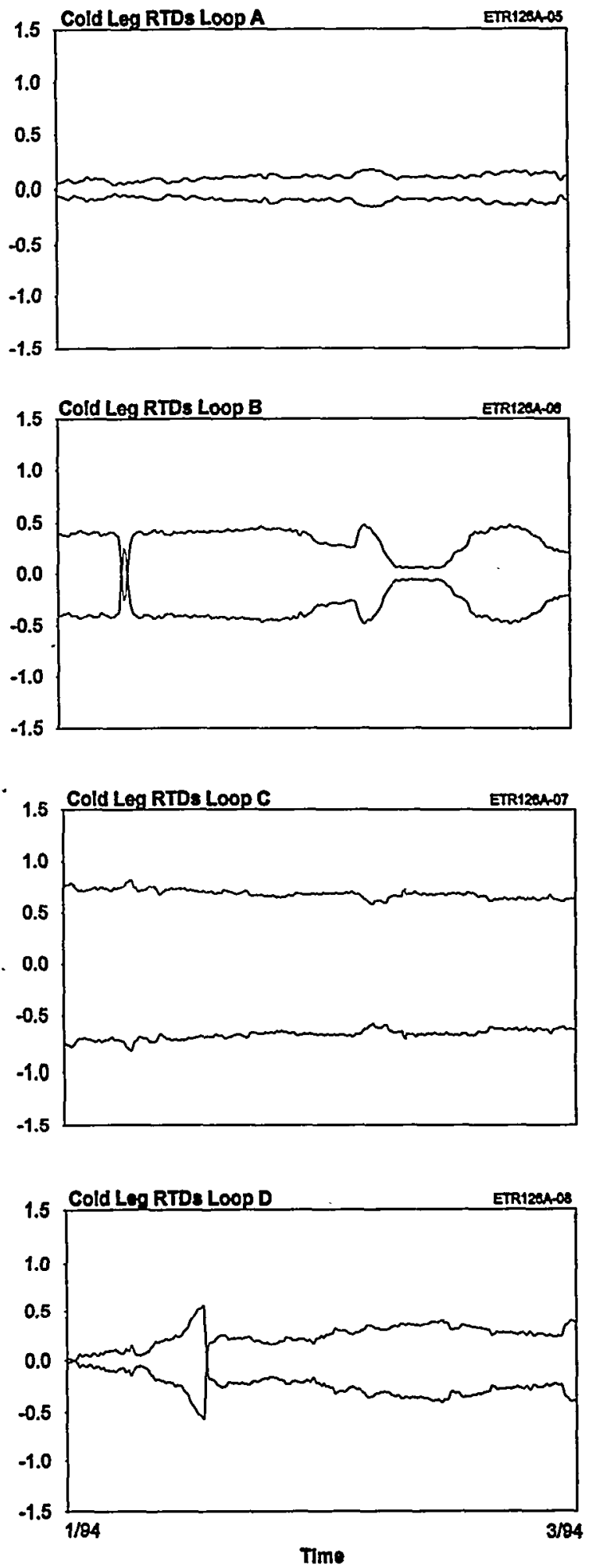

Figure 16.61 Cycle 1 and Cycle 2 Signals from Redundant Cold Leg RTD Loops 


\section{Cycle 1}
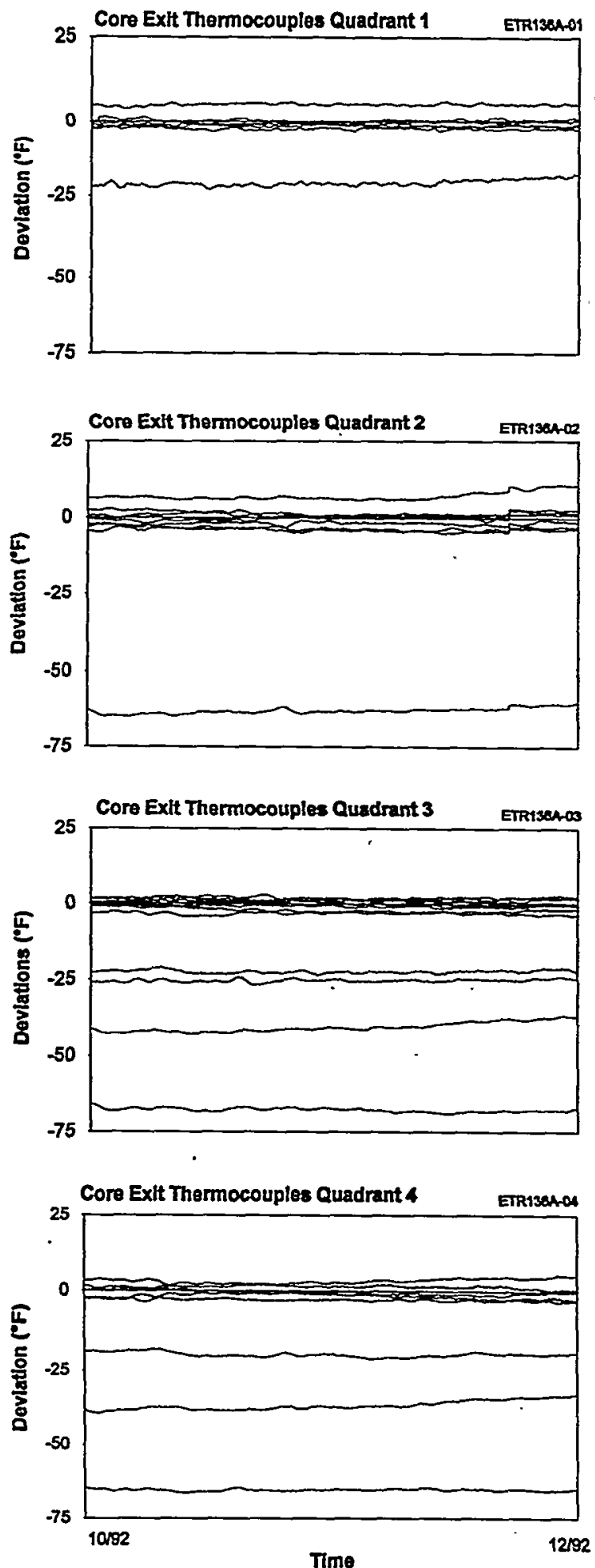

\section{Cycle 2}
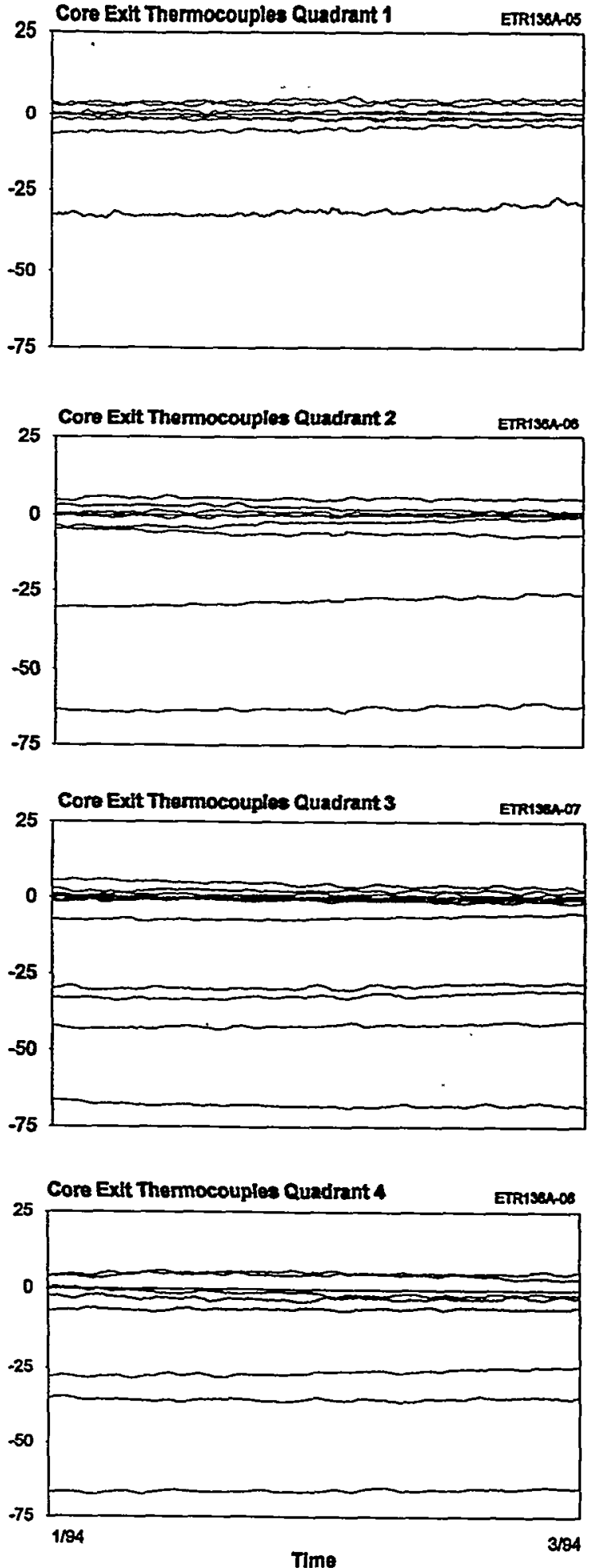

Figure 16.62 Cycle 1 and Cycle 2 Signals from Redundant Core Exit Thermocouple Loops 


\section{TEMPERATURE STRATIFICATION AND ITS EFFECT ON ON-LINE MONITORING TESTS}

Temperature stratification or temperature streaming occurs in PWRs during reactor operation because different streams of water that exit the core are normally at different temperatures due to uneven heating in the core. As a result, the hot leg RTD readings are affected by the location and depth of the RTD sensing elements in the hot leg pipes because: (1) the hot leg RTDs are relatively close to the reactor, and (2) the water is not well mixed when it reaches the hot leg RTDs. Consequently, temperature differences in the range of about 3 to $15^{\circ} \mathrm{F}$ may be encountered between redundant hot leg RTDs in the same loop of a PWR. This error is dependant on the reactor power and is not present at the hot standby conditions. Figure 17.1 shows a deviation plot for one of the hot leg RTDs at McGuire as a function of reactor power. The on-line monitoring system collected this data during a plant heatup that was followed by a plant cool down. The deviation plot shows data for both the startup and the cooldown period. Note that the RTD shows almost the same deviation during the startup and the shutdown of the plant.

Figure 17.2 shows on-line monitoring results for six hot leg RTDs at McGuire. The RTDs do not show a significant drift, but there are steady-state (bias) differences due to the temperature stratification phenomenon. In contrast, the cold leg RTDs do not show a significant bias as shown in Figure 17.3. This is because temperature stratification is not significant in the cold legs because the reactor coolant is well mixed by the time it reaches the cold leg RTDs.

The temperature stratification phenomena can also affect the core exit thermocouples, however, there were core exit thermocouples at McGuire that had large errors in addition to the temperature stratification effect. Figure 17.4 shows the deviations of eight core exit thermocouples at McGuire. One of the eight thermocouples agrees with the other seven when the plant is cold, but its deviation increases significantly with temperature. This indicates that thermocouples may have normal outputs at the shutdown temperature and significant differences at higher temperatures.

Figures 17.5 and 17.6 show representative online monitoring results for hot leg and cold leg RTDs over the two fuel cycles during which data were collected at McGuire. The results show the following: (1) the hot leg and cold leg RTDs shown here have not experienced a significant drift, and (2) the hot leg RTD deviations are within a band of about $\pm 4.5^{\circ} \mathrm{F}$, while the cold leg RTD deviations are within a band of about $\pm 1.5^{\circ} \mathrm{F}$; a factor of three difference. 


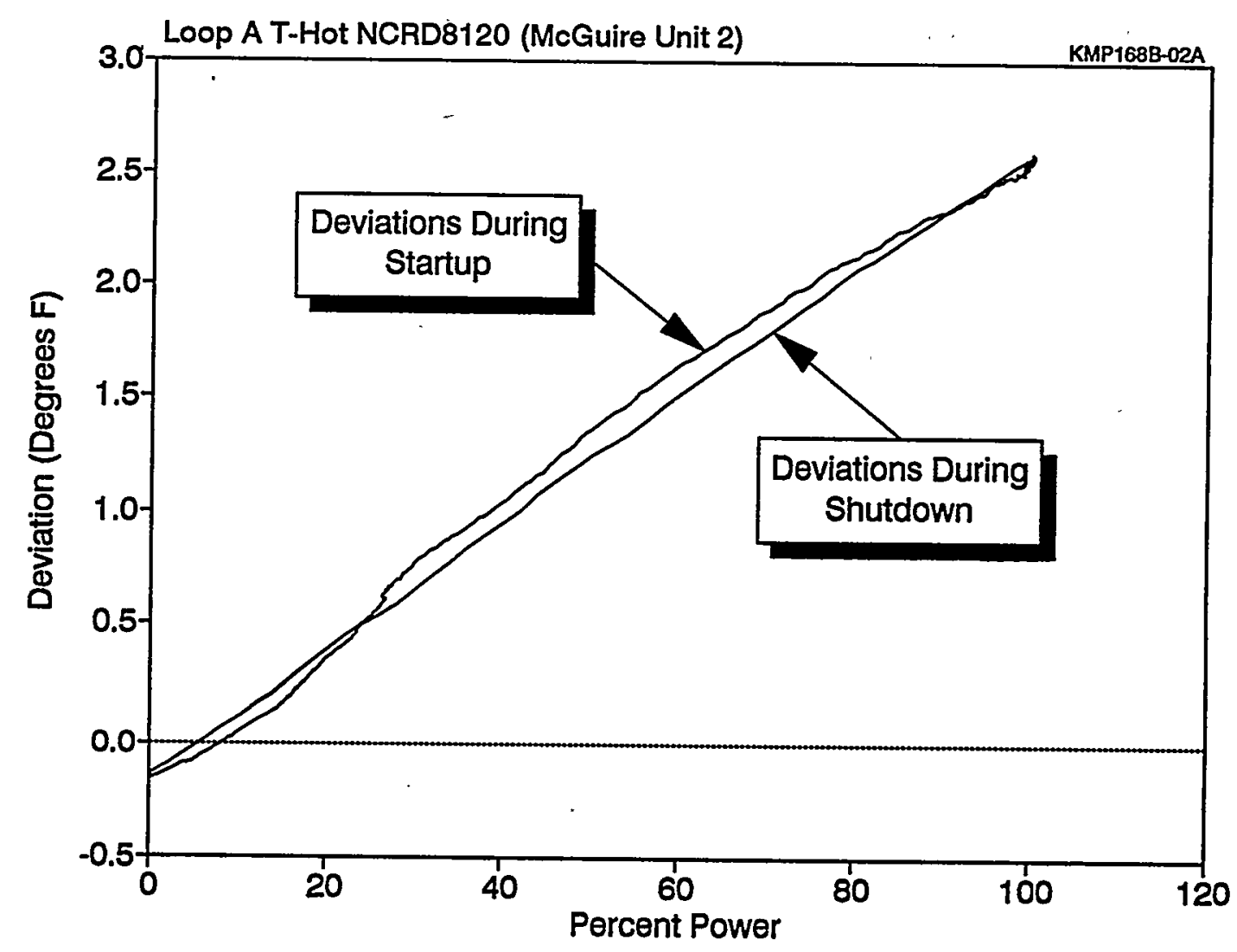

Figure 17.1 Deviation of a Hot Leg RTD as a Function of Reactor Power Showing the Effect of Temperature Stratification 


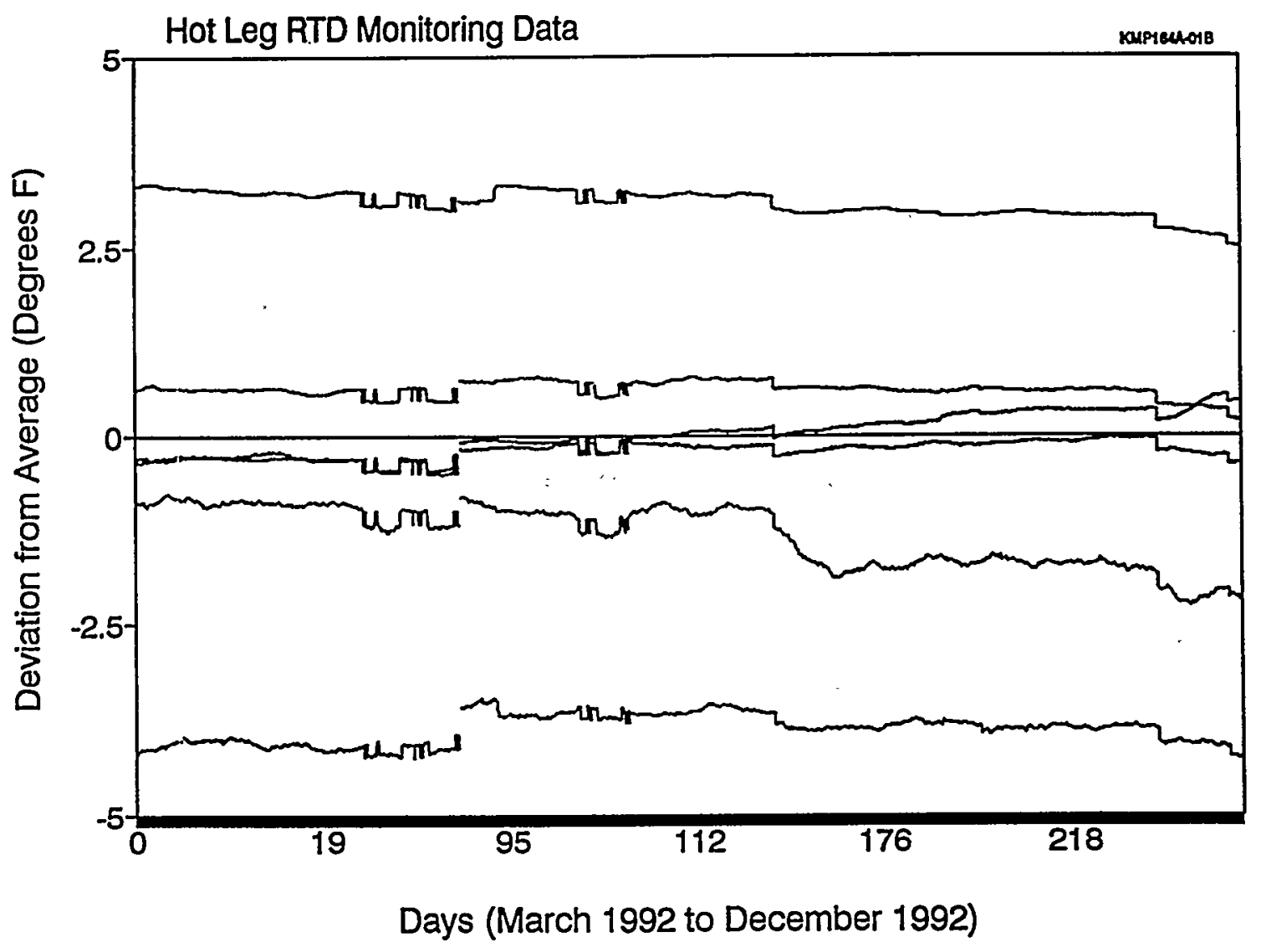

Figure 17.2 On-Line Monitoring Results for Six Hot Leg RTDs in One Loop of McGuire Plant 


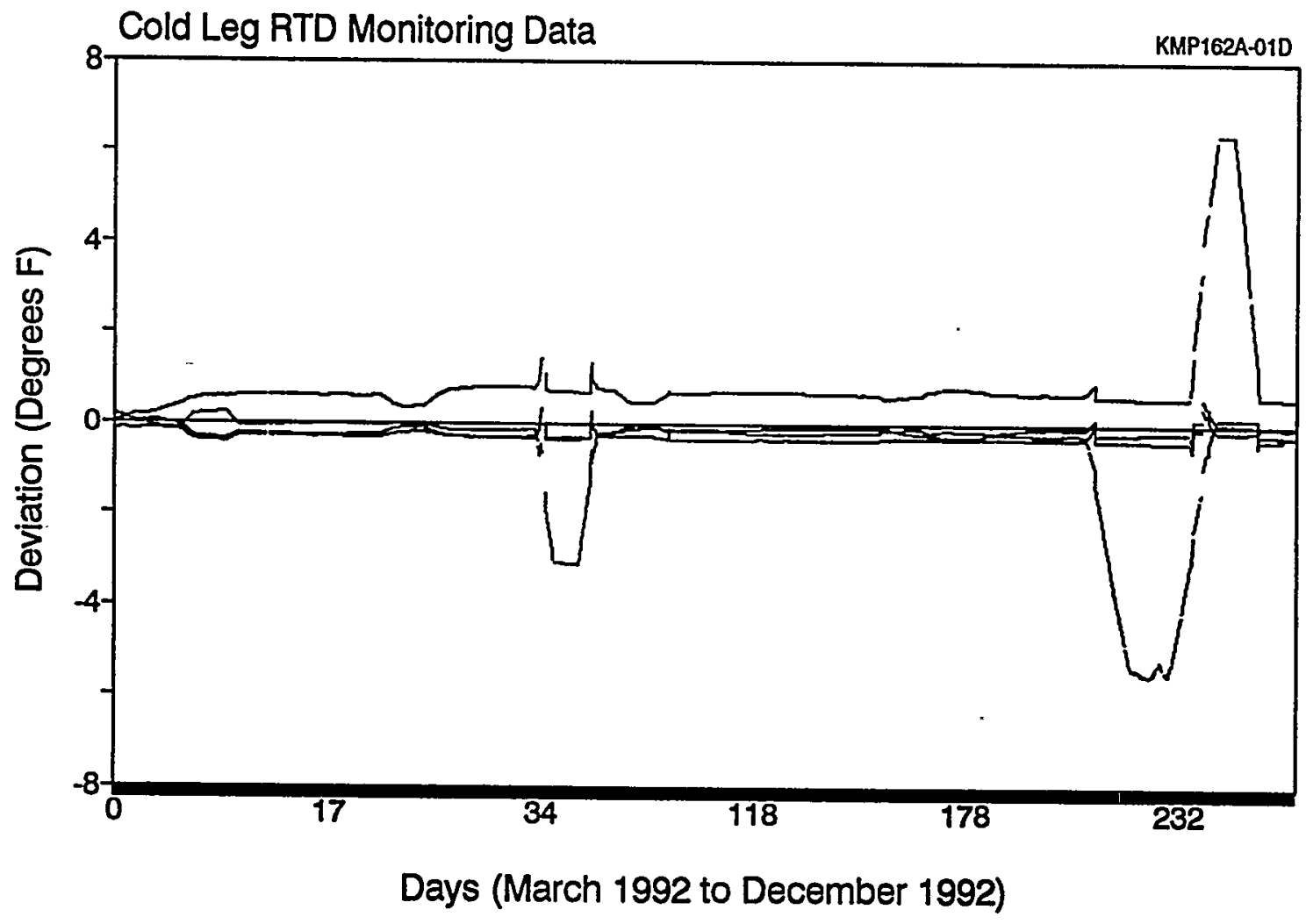

Figure 17.3 On-Line Monitoring Results for Cold

Leg RTDs at One Loop of McGuire Plant 


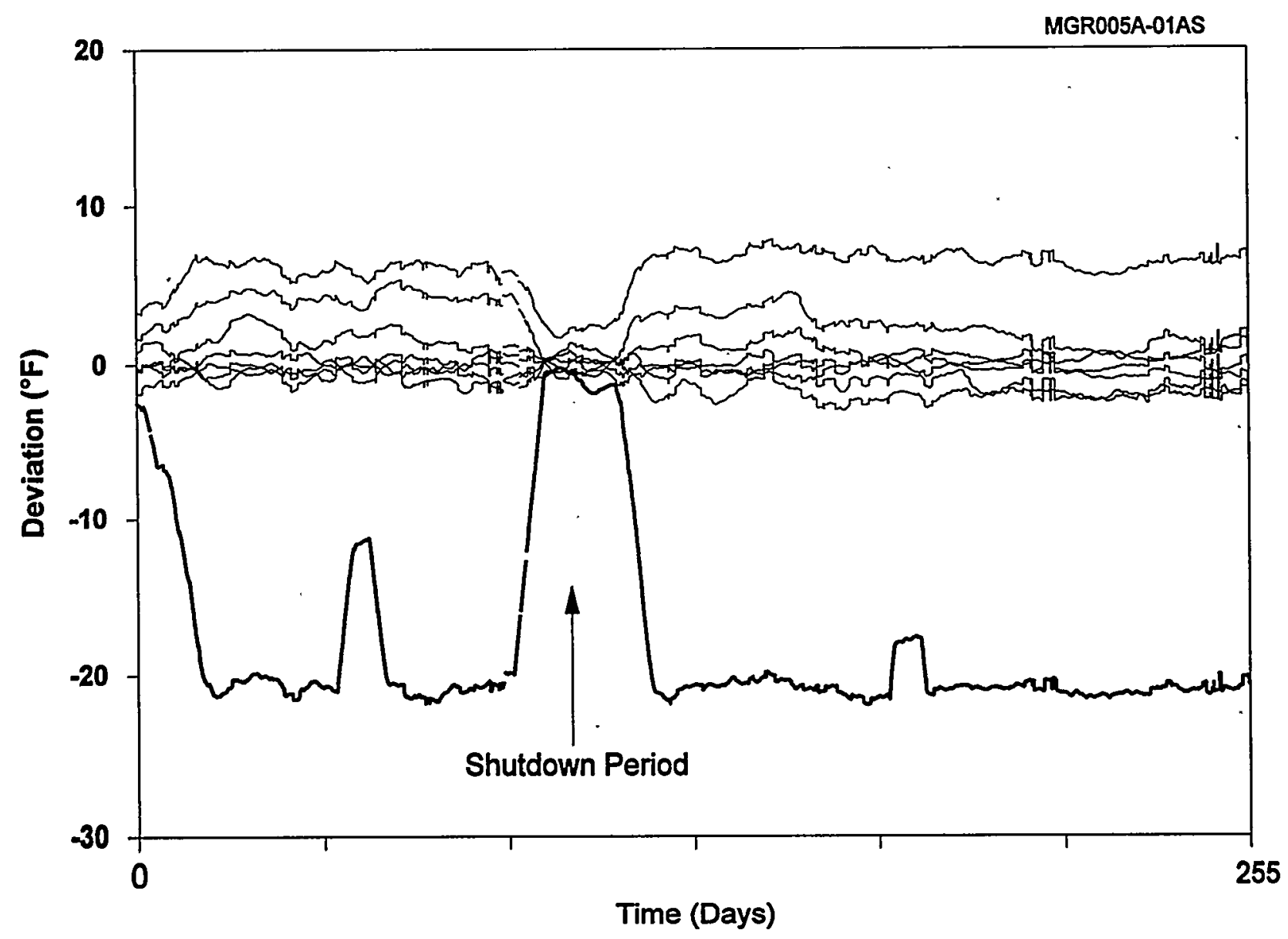

Figure 17.4 Deviation of Core Exit Thermocouples in One of the Four Quadrant of McGuire Plant 

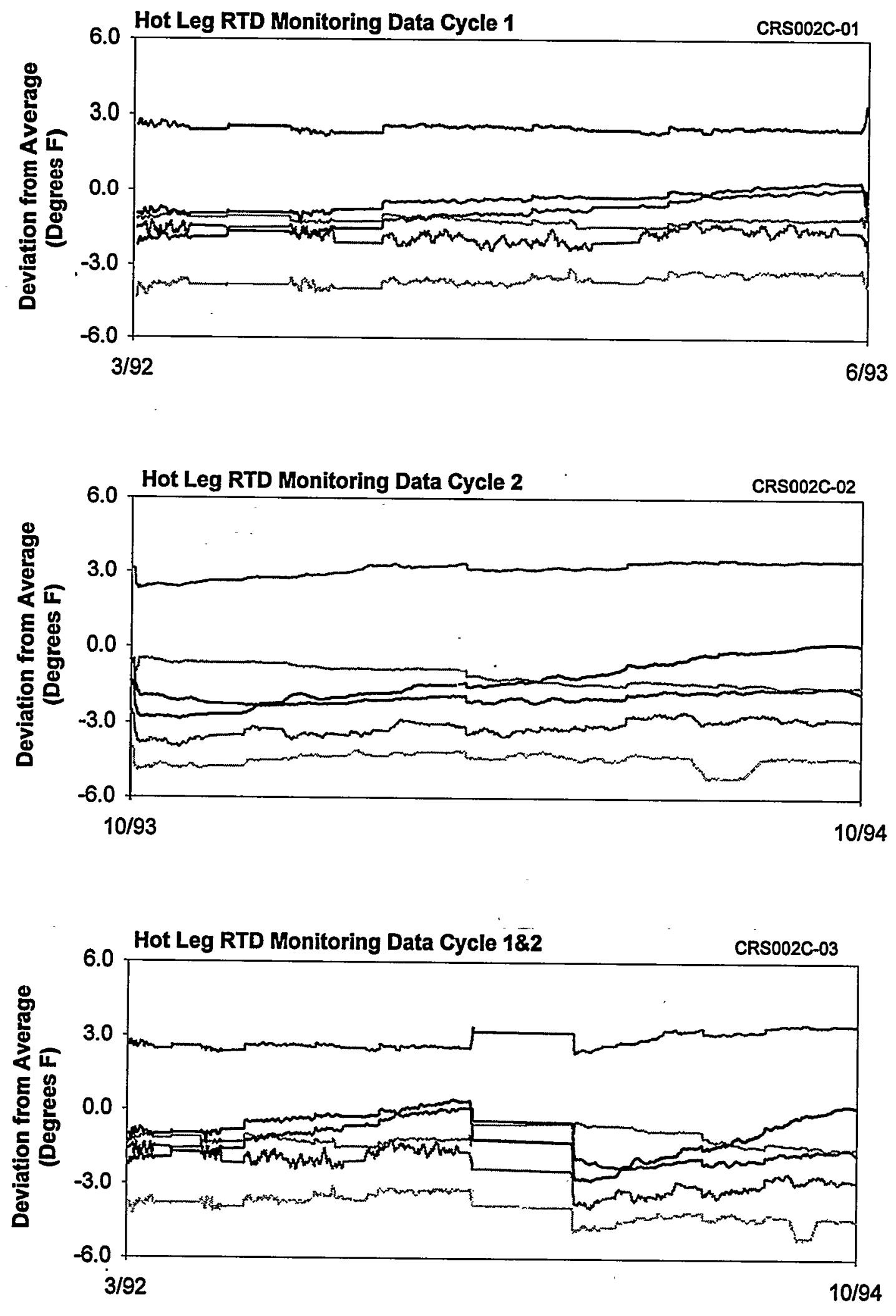

Time

Figure 17.5 Deviation of McGuire Hot Leg RTDs 

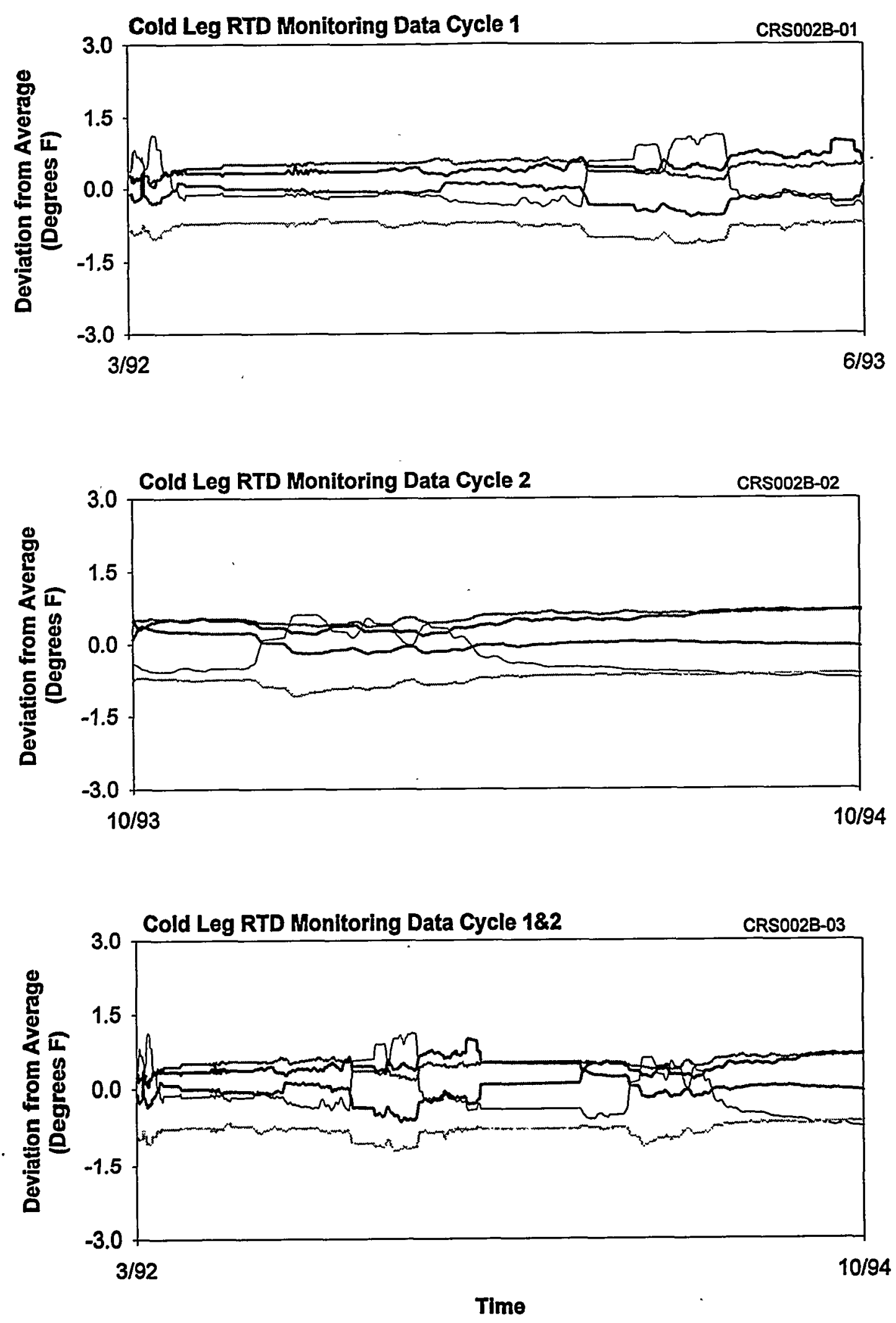

Figure 17.6 Deviation of McGuire Cold Leg RTDs 


\section{CALIBRATION OF ON-LINE MONITORING SYSTEM}

The on-line monitoring system must be calibrated periodically to establish traceability to national standards and to verify that the system is not drifting. Since this system is used mostly for relative measurements to identify deviations as opposed to measuring absolute values, its accuracy requirements do not have to be as stringent as a standard calibration system.

The calibration requirements for an on-line drift monitoring system would normally depend on how it is used. For example, if the system is used to extract digitized data from the plant computer, then it may not need a calibration. If, however, the on-line monitoring system is comprised of its own data acquisition modules, then these modules must be calibrated at least once a year or at the same frequency that other measurement and test equipment are calibrated in the plant.
The on-line monitoring system that was used at the McGuire plant was equipped with a calibrated reference source ( +5 VDC) and a "short." The outputs from the source and the short were monitored continuously to detect any significant drift in the system. Figure 18.1 shows the results of this monitoring. Two plots are shown in the figure for the 5 volt source. One represents the normal output of the source, and the other shows the drift of the source with respect to a straight line. It is apparent that although the system drifted, this drift has been very small compared to potential drift in instrument channels in a plant.

If the on-line monitoring system is found to have drifted significantly, this drift must be accounted for in analyzing the on-line monitoring data to ensure that the in-plant test results are conservative. 

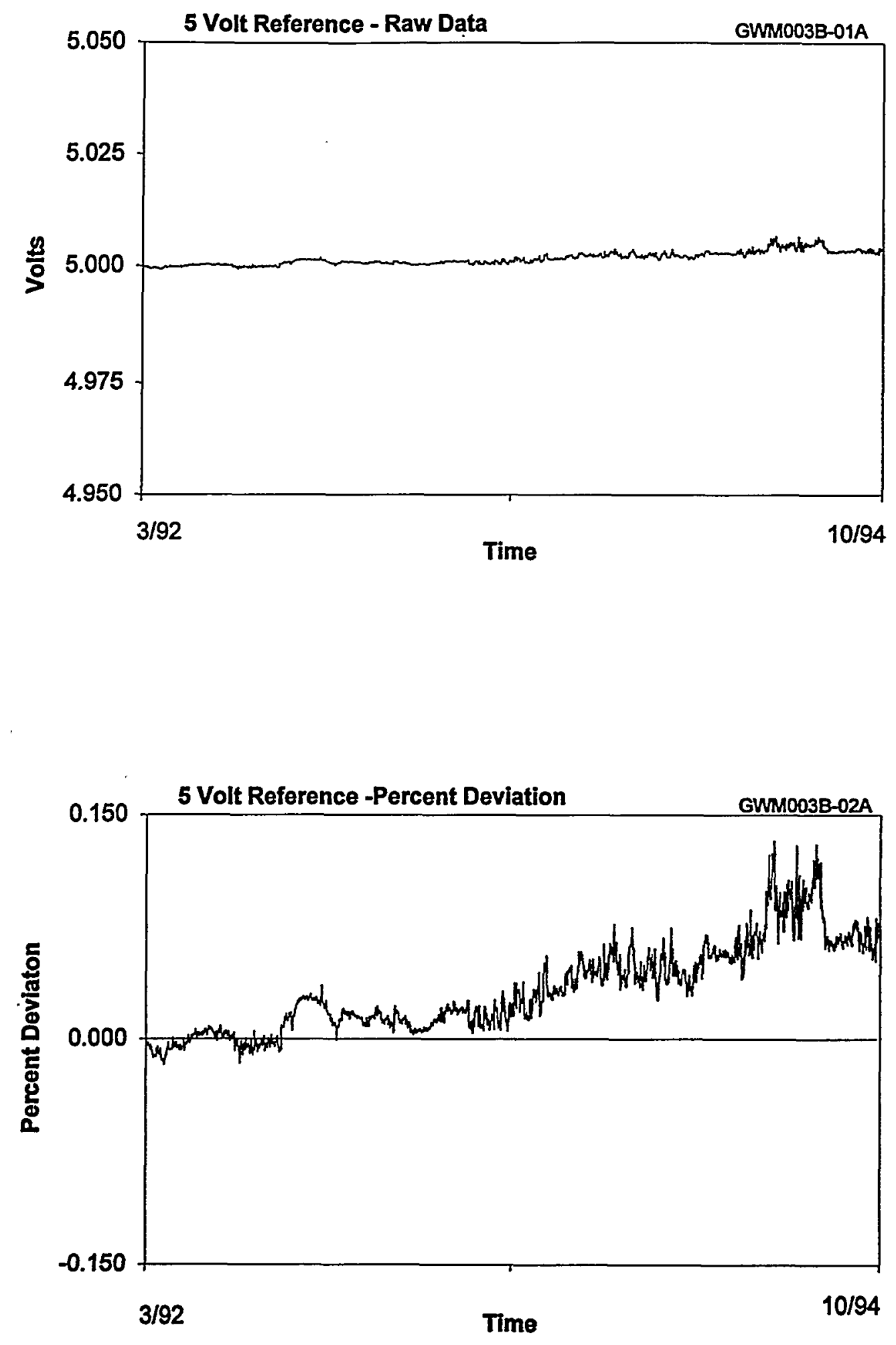

Figure 18.1 Drift of On-Line Monitoring System 


\section{BENEFITS AND COSTS OF ON-LINE CALIBRATION MONITORING}

On-line calibration monitoring has the potential to provide substantial saving in the cost of instrument calibrations and associated personnel radiation exposure while enhancing the plant safety. The safety of the plant is enhanced because on-line calibration tests will reveal calibration problems as they occur and will provide additional diagnostics. Also, personnel error during manual calibrations will be reduced once an on-line calibration system is implemented in the plant.

Figure 19.1 shows on-line monitoring results for four RTD signals from the McGuire plant. One of the RTDs had an erratic behavior as seen in the figure. However, the readings from this RTD are almost always in reasonable agreement with one or more of the other three redundant RTDs. As a result, such a sensor will not normally be identified by the plant personnel as an erratic sensor until after it fails or until its behavior becomes so erratic as to cause instrumentation alarms. With online monitoring data, an erratic sensor such as this is readily identified during plant operation and scheduled for repair or replacement at a convenient opportunity.

Figure 19.2 shows another example of an erratic sensor at McGuire. This one is a steam generator level transmitter. The sensor was replaced after the plant personnel had enough information to conclude that the sensor was indeed erratic. With an on-line monitoring system, the problem would have manifested itself in the first few weeks of operation.

Table 19.1 provides a listing of the advantages of on-line calibration monitoring over the conventional calibrations that are performed manually. This is followed by Table $\mathbf{1 9 . 2}$ outlining the cost benefits of on-line calibration tests. The direct cost savings have been estimated by AMS, EPRI, the nuclear industry and others to range from an average of about $\$ 50,000$ to $\$ 500,000$ per plant per fuel cycle depending on the number of instruments calibrated, frequency of calibrations, the instrument design and principle of operation, location, layout, etc.

The initial and recurring costs of implementing an on-line monitoring system in a nuclear power plant are summarized in Table 19.3. The initial cost is estimated to have a range of about $\$ 250,000$ to $\$ 500,000$ for a typical installation depending on the number of instruments to be monitored, the complexity of the installation and wiring of the plant instruments to the on-line monitoring system, etc. In some plants, the necessary data may be available from the plant computer. In this case, the cost of implementing an on-line calibration monitoring system will be closer to the lower end of the range of the estimated costs mentioned earlier.

Based on the estimated costs and benefits discussed in the above paragraph, it is reasonable to conclude that an on-line calibration monitoring system can pay for itself in a relatively short period of time.

It should be pointed out that after implementing an on-line monitoring system, manual calibrations may still be required for reference channels to account for common-mode drift and to help establish calibration traceability. This is especially true if analytical modeling is not used. As such, in the recurring cost section in Table 19.3, a line item is included for manual calibration of reference channels.

The use of analytical modeling in an on-line monitoring system is not mandatory. However, 


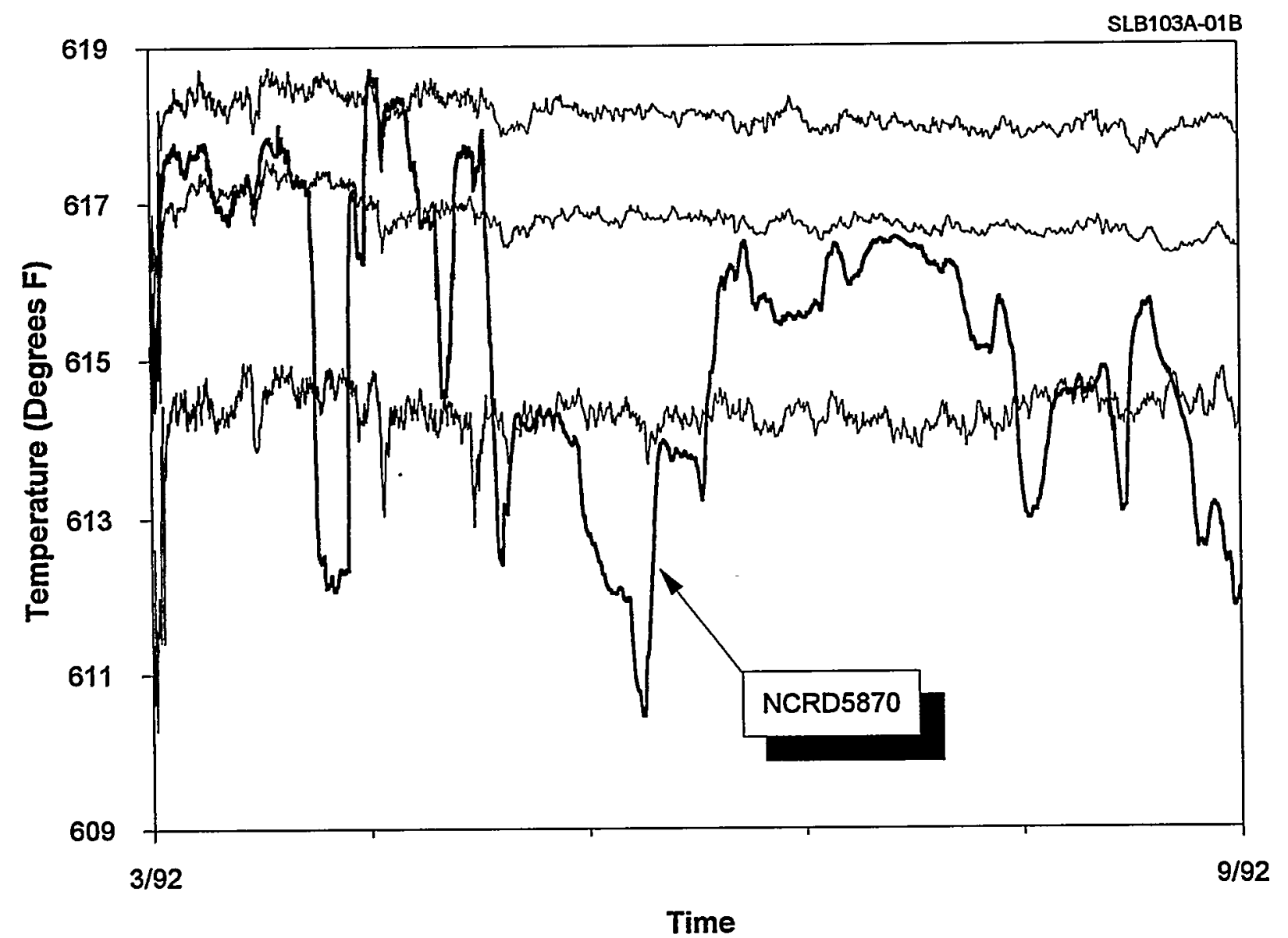

Figure 19.1 On-Line Monitoring Data Showing Erratic RTD 


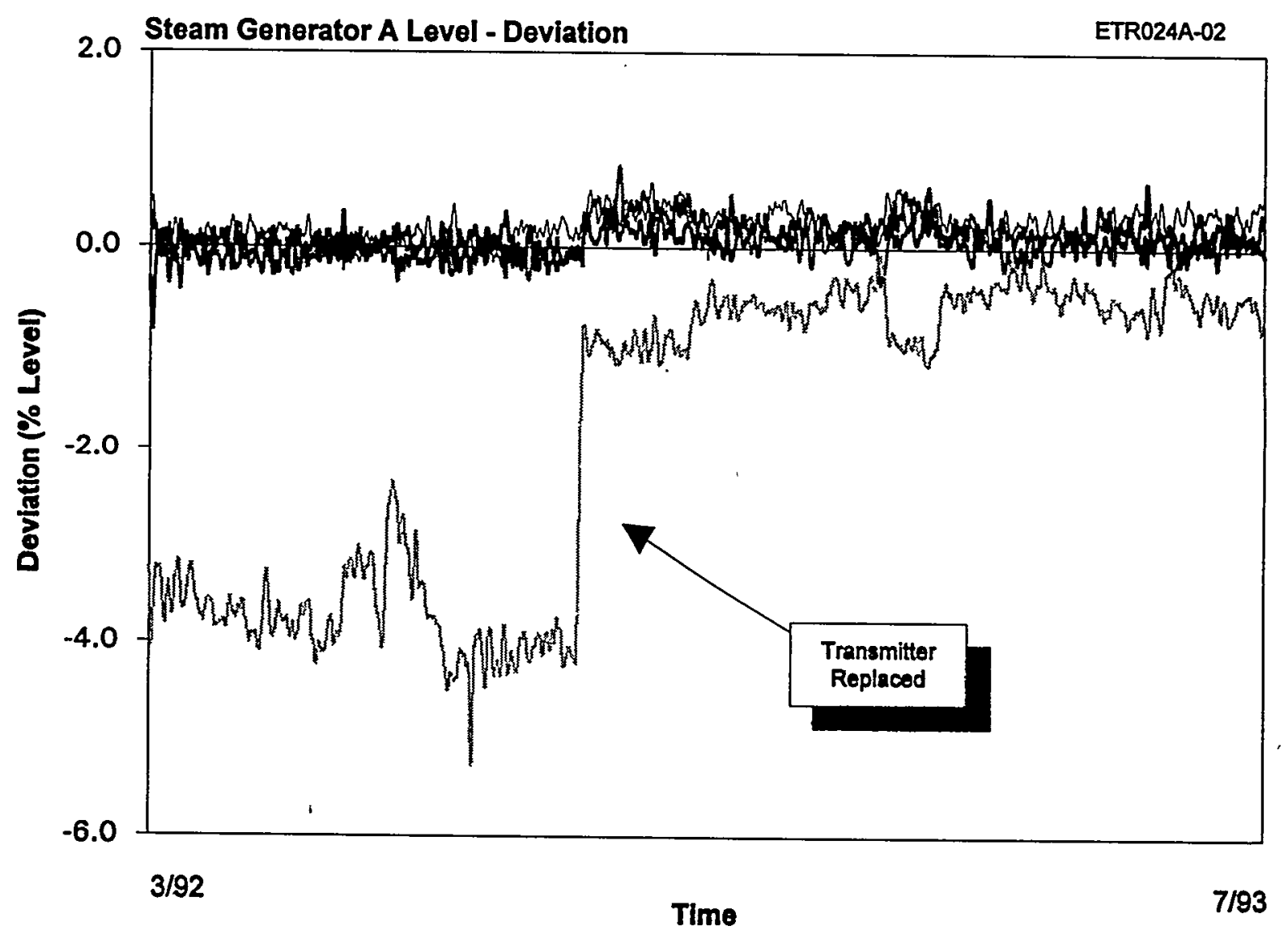

Figure 19.2 On-Line Monitoring Data Showing Erratic Pressure Transmitter 


\begin{tabular}{|l|l|}
\hline \multicolumn{2}{|c|}{ TABLE 19.1 } \\
$\begin{array}{l}\text { Comparison of Conventional Calibration Technique } \\
\text { with On-line Calibration Monitoring }\end{array}$ \\
\hline \multicolumn{1}{|c|}{ Conventional } & On-line \\
\hline $\begin{array}{l}\text { Performed manually and requires physical } \\
\text { access to each instrument }\end{array}$ & $\begin{array}{l}\text { Automated (computer-aided) and remote } \\
\text { calibration monitoring }\end{array}$ \\
\hline Performed once a fuel cycle & Performed almost continuously \\
\hline Performed at refueling outages & Performed while the plant is on-line \\
\hline Identifies calibration drift only & $\begin{array}{l}\text { Identifies calibration drift and other } \\
\text { instrument anomalies }\end{array}$ \\
\hline $\begin{array}{l}\text { Identifies calibration problems over the } \\
\text { entire operating range of an instrument }\end{array}$ & $\begin{array}{l}\text { Identifies calibration problems at the normal } \\
\text { operating point with the potential to reveal } \\
\text { calibration problems over the entire } \\
\text { operating range of instruments }\end{array}$ \\
\hline $\begin{array}{l}\text { Typically performed on one component of } \\
\text { an instrument channel }\end{array}$ & $\begin{array}{l}\text { Can cover multiple components or the } \\
\text { entire instrument channel (excluding the } \\
\text { actuation system) }\end{array}$ \\
\hline $\begin{array}{l}\text { Performed at cold shutdown; thus, } \\
\text { environmental effects such as temperature } \\
\text { that may have an influence on calibration } \\
\text { are not included. }\end{array}$ & $\begin{array}{l}\text { Performed at normal operating conditions; } \\
\text { thus, environmental effects are included in } \\
\text { the results. }\end{array}$ \\
\hline $\begin{array}{l}\text { Detects calibration problems after they } \\
\text { have occurred }\end{array}$ & \begin{tabular}{l} 
Detects calibration problems as they occur \\
\hline
\end{tabular} \\
\hline
\end{tabular}




\section{TABLE 19.2}

\section{Potential Benefits of On-line Calibration Monitoring}

in Nuclear Power Plants

\section{Direct Cost Savings}

- Labor

- Saves 500 to 1,000 hours per outage

- Relieves personnel

Instrumentation and Control (I\&C) Personnel

Quality Assurance and Quality Control (QA/QC) Personnel

Health Physics (HP) Personnel

Administrative Personnel

etc.

- Radiation Exposure

- Direct man-rem savings

- Cost of dress-out to enter radiation controlled zone

- etc.

\section{Indirect Cost Savings}

- Reduced Outage Length and Outage Risk

- Simplified Outage Planning and Outage Scheduling

- Increased instrument reliability and plant safety

- Reduced human errors and plant trips associated with instrument calibrations

- Reduced potential to damage plant equipment

- Improved performance trending, incipient failure detection, and troubleshooting

- Post trip review, troubleshooting, engineering analysis, etc.

- .Consistent with the "Maintenance Rule" 


\section{TABLE 19.3}

Costs of Implementation of On-Line Calibration Monitoring in Nuclear Power Plants

\section{Initial Costs}

- On-line Monitoring System Costs

- hardware

- software

- training

- technical support

- etc.

- Installation Costs

- Licensing Costs

- Procedure Preparation and QA Costs

\section{Recurring Costs}

- Cost to Operate and Maintain the On-Line Monitoring System

- Cost of data analysis, interpretation, data storage, trending, etc.

- Cost of manual calibration of any reference channels 
if analytical modeling is used, then a manual calibration of reference channels may not be required. Figure 19.3 shows the drift of a temperature channel as identified from simple averaging of four redundant signals. In this case, analytical modeling can help verify that the process was not drifting and thereby

- conclude that three of the four channels show no drift. If analytical modeling is not used in this case, one of the stable channels may have to be calibrated in addition to the drifting channel to ensure that there is no commonmode drift. 

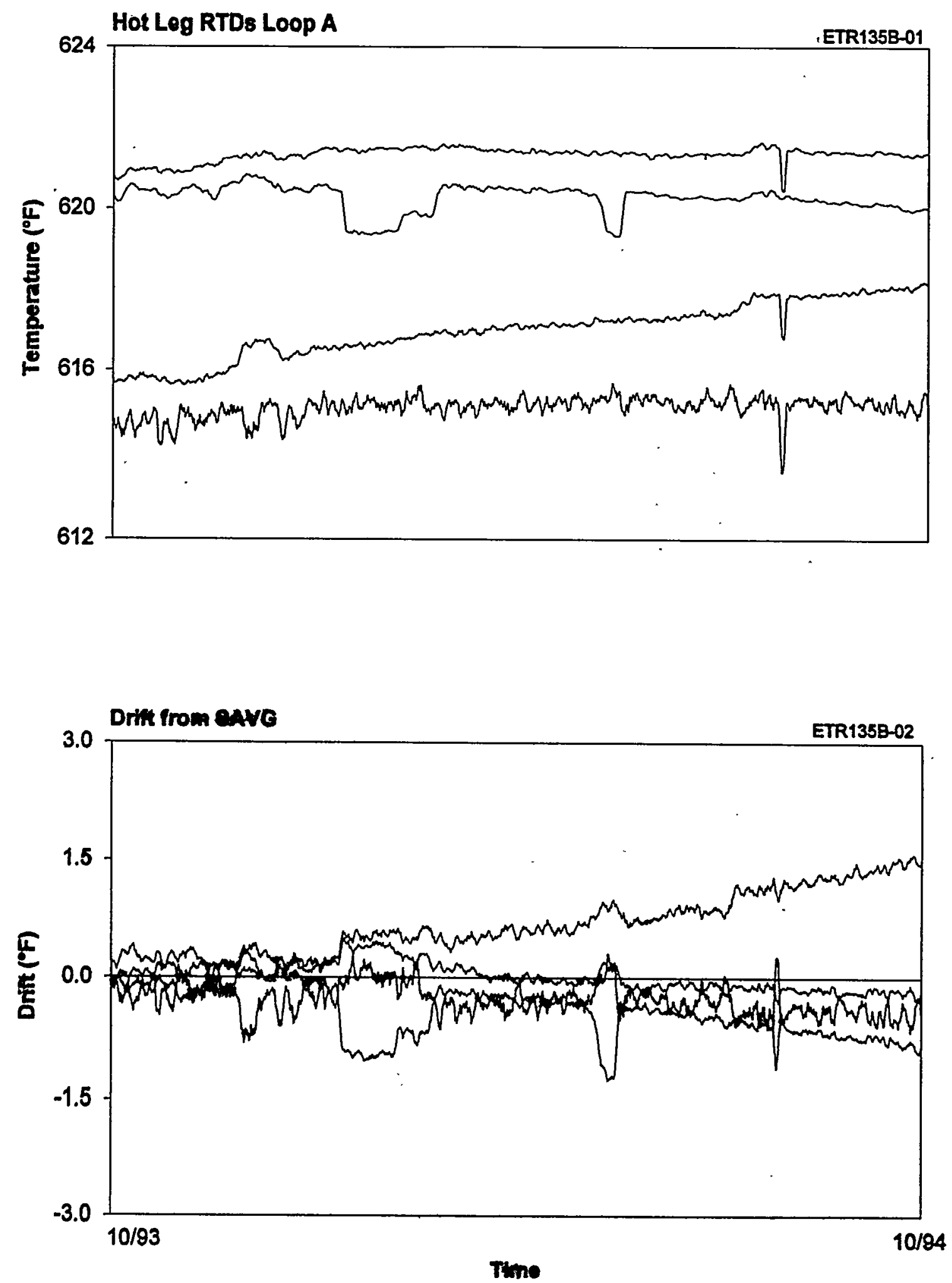

Figure 19.3 Drift of a Temperature Channel Identified by Simple Avaraging 


\section{CONCERNS ABOUT ON-LINE CALIBRATION MONITORING}

On-line calibration monitoring is mostly performed at normal operating conditions. Although, the plant operating point is usually the most important point of a channel operation, this method is often questioned as being a one-point calibration check. This question is addressed by programming the on-line monitoring system to sample data not only during normal operation, but also during startups, shutdowns, and plant trips. The data should then be evaluated to determine if they can be used to verify the performance of the instruments over a wide-range. This, of course, only partially addresses the question because data collected during startups, shutdowns, and plant trips do not provide for long term monitoring that may be needed to resolve subtle calibration differences and small calibration drifts. Furthermore, problems such as differences between dynamic responses of redundant instruments may interfere with the ability to detect calibration problems during fast process transients.

EPRI has also addressed the above question and indicated that changes in zero, span, hysteresis, and linearity of instruments generally manifest themselves at the normal operating point. ${ }^{(3,4,5)}$ That is, according to EPRI, on-line monitoring performed at normal operating conditions can reveal calibration problems at other points within the range of the instrument.

Another concern with the use of on-line calibration monitoring relates to common-mode drift. If redundant instruments experience unidirectional drift, then their deviation from their average may not adequately show the drift. To address this concern, analytical modeling and/or calibrated reference channels must be used as discussed earlier to separate process drift from instrument drift. If analytical models are used, the uncertainties of process estimation using the model must be evaluated and accounted for in determining which channels need a manual calibration. These uncertainties would depend on the number and diversity of the inputs used, the reliability of the inputs, the adequacy of the model training, the validity of the model for the process that is being monitored, etc. Due to these and other factors, the overall uncertainties of modeling techniques depend on each particular process monitored. However, as was shown earlier, modeling uncertainties may not be very important if it can be shown that the uncertainties are time-invariant.

Noise in the output of process instrumentation channels in nuclear power plants is another problem that may interfere with the ability of an on-line monitoring system to recover a small drift. Filtering and averaging was found in this project to help with this problem and in-plant data was presented here earlier in which the drift of a noisy signal could easily be identified in spite of the noise.

There are other questions, concerns, and challenges involved in implementing an on-line calibration monitoring system in a nuclear power plant. For example, determining when to declare a channel as needing a manual calibration and what actions to take if the problem is identified during an operating cycle. These and other questions and concerns may have to be addressed on a case by case basis while experience is being accumulated with on-line calibration monitoring technology. This technology has not been used in the nuclear or non-nuclear industries for an appreciable period of time to offer much information on the relevant questions and answers. However, the technology is ready for in-plant use with the understanding that some 
tuning may be necessary as experience is accumulated in actual practice. The technology is said to be ready for implementation in nuclear power plants because it has been independently examined by a number of organizations and consistent results have been obtained. 


\section{CONCLUSIONS}

A comprehensive R\&D project was successfully carried out to provide new technology for on-line monitoring of calibration of process instrumentation channels in nuclear power plants. The work involved hardware and software development for data acquisition and data processing, analytical modeling including physical and empirical models and neural networks for independent process estimation, laboratory and in-plant validation tests, searches of LER and NPRDS databases to examine failure rates due to calibration drift, development of interpretation techniques, review of related $R \& D$, presentation of project results to the nuclear power industry and the NRC, publication of a preliminary report (NUREG/CR-5903), and preparation of this final report.

A major task in this project was the adaptation of existing analytical modeling techniques for instrument calibration verification. More specifically, analytical techniques were used in laboratory and in-plant tests to obtain an independent estimate for each process parameter by measurement of other process parameters. This is important in determining whether a drift at the output of an instrument channel is from the process or the instruments comprising the channel. Both physical and empirical modeling techniques were examined in this project for process estimation. Empirical models, especially neural networks, were found to be much easier to develop and implement than physical models. As such, very little work was done in the project on experimental validation of physical models. A majority of the effort in the area of analytical modeling was spent on verification and validation of empirical models and neural networks. These efforts showed that the accuracy and reliability of these models depend on the inputs used in training and using the models. If a model is sufficiently trained with adequate inputs at a given process operating condition, then it will produce accurate results for the particular operating condition at which it was trained. However, if the model is trained in a given process condition and used in another process condition, then the results will not be as accurate.

Simple and weighted averaging techniques were found to be effective when an adequate number of reliable signals are available to be averaged and intercompared. The averaging techniques may be supplemented with analytical models to improve the accuracy and reliability of the on-line calibration monitoring results. Whether averaging or modeling is used, it is important to verify the validity and consistency of individual signals before they are averaged together or used as inputs to empirical models or neural networks. To accomplish this, data qualification procedures and algorithms must be used to screen the data. A variety of data qualification algorithms are available for this purpose. These algorithms can identify and remove any significant noise, spike, or other anomalies in the data, check the data for consistency, and reject or reduce the weighting of any signal or any portion of a signal that is contaminated with extraneous effects. Two methods called parity space and Generalized Consistency Checking (GCC) were mentioned in this project that could be used for both data qualification and interpretation of the results to identify the inconsistent signals. There are other methods that are as effective as parity space and GCC for data qualification. These methods are described in the literature in the areas of advanced signal processing, on-line monitoring, and predictive maintenance. Also described in literature are details of analytical methods such as pattern recognition and Sequential Probability Ratio Test (SPRT) that were only briefly reviewed in this project. 
These methods have been independently developed and are commercially available for on-line calibration monitoring and other applications in nuclear power plants and other industries.

The technology that was developed and validated in this project is commercially available through AMS in terms of hardware, software, training, technical support, and installation services. These products have applications not only in the nuclear industry, but also in the fossil power industry, chemical, petrochemical and other process industries, aviation and aerospace industries, defense industry, etc. In addition to AMS, on-line calibration monitoring equipment and techniques have been independently developed and successfully tested in nuclear power plants by other R\&D organizations, vendors, EPRI, and nuclear utilities.

Besides instrument calibration verification, on-line monitoring technology can be used in a variety of predictive maintenance applications in nuclear and non-nuclear industries. For example, AMS has successfully adapted the technology that was developed in this project for monitoring the performance of emergency diesel generators in nuclear power plants and other processes. The performance of turbine generators and other equipment in nuclear power plants can also be monitored with the products developed in this project.

Since on-line calibration monitoring in nuclear power plants is a relatively new application, a large database is not yet available to objectively characterize the benefits and drawbacks of this technology, nor has this technology been used in other industries to help produce the database. Nevertheless, it is obvious that on-line calibration monitoring can enhance the reliability of process instrumentation channels and contribute to plant safety and availability. The technology has the potential to replace much of the manual calibrations that are currently performed in nuclear power plants with more frequent or continuous and fully automated monitoring of drift and other instrument anomalies. As such, on-line calibration monitoring not only contributes to plant safety but also helps with plant economy. More specifically, utilities can realize substantial cost saving through reduced manpower to perform manual calibrations and the related activities, shorter plant outages due to reduced number of manual calibrations, reduction in maintenance-related plant trips and damage to the plant equipment, and substantial reduction in personnel radiation exposure and related HP work, QA/QC work, administrative work, etc. 


\section{REFERENCES}

1. Meijer, C.H., et al., "On-Line Power Plant Signal Validation Technique Utilizing Parity-Space Representation and Analytic Redundancy," EPRI NP-2110, November 1981.

2. Swisher, V.I., "User's Guide for Signal Validation Software," EPRI NP-5389, September 1987.

3. Electric Power Research Institute, "Instrument Calibration and Monitoring Program - Volume 1: Basis for the Method," EPRI TR-103436-V1, December 1993.

4. Electric Power Research Institute, "Instrument Calibration and Monitoring Program - Volume 2: Failure Modes and Effects Analysis," EPRI TR-103436-V2, December 1993.

5. Electric Power Research Institute, "Calibration Through On-line Performance Monitoring of Instrument Channels," EPRI TR-104965, Palo Alto, California, August 1995.

6. Mott, J.E., Radtke, W.H., and King, R.W., "EBR-II System Surveillance Using PatternRecognition Software," Proceedings of the ANS/ENS Topical Meeting on Operability of Nuclear Power Systems in Normal and Adverse Environments, Albuquerque, NM, September 29 October 3, 1986.

7. Humenik, KE. and Gross, K.C., "Sequential Probability Ratio Tests for Reactor Signal, Validation and Sensor Surveillance Applications," Nucl. Sci. and Eng. (105), pp. 383-390, August 1990.

8. Gross, K.C., Humenik, K.E., "Sequential Probability Ratio Test for Nuclear Plant Component Surveillance," Nuclear Technology, Volume 93, pp. 131-137, 1991.

9. Upadhyaya, B. R., Holbert, K.E., and Kerlin, T.W., "Development of an Integrated Signal Validation System and Application to Operating Power Plants," Proceedings of the 7th Power Plant Dynamics, Control \& Testing Symposium, Volume 2, Knoxville, Tennessee, May 1989.

10. Uhrig, R.E., "Artificial Neural Networks in Nuclear Power Plants," Nuclear News, Volume 39, pp. 38-40, July 1994.

11. Hashemian, H.M., et al., "Long Term Performance and Aging Characteristics of Nuclear Plant Pressure Transmitters,"NUREG/CR-5851, U.S. Nuclear Regulatory Commission, Washington, DC, March 1993.

12. Hashemian, H.M., et al., "Aging of Nuclear Plant Resistance Temperature Detectors," NUREG/CR-5560, U.S. Nuclear Regulatory Commission, Washington, DC, June 1990.

13. Hashemian, H.M., "New Technology for Remote Testing of Response Time of Installed Thermocouples," AEDC-TR-91-26, Volume 1, U.S. Airforce, Arnold Engineering Development Center, January 1992.

14. Electric Power Research Institute "Remote Calibration of Resistance Temperature Devices 
(RTDs)," EPRI NP-553, Palo Alto, California, February 1988.

15. Sackett, J. I., "Application of AI Technology to Nuclear Plant Operations," ASEE Annual Conference, CONF-8806146--2, Portland, Oregon, June 1988.

16. Wald, A., "Sequential Tests of Statistical Hypotheses," The Annals of Mathematical Statistics, Volume 16, No. 2, pp. 117-186, June 1945.

17. Holbert, K.E., "Process Hypercube Comparison for Signal Validation," IEEE Transactions on Nuclear Science, Volume 38, No. 2, pp. 803-811, April 1991.

18. Holbert, K.E. and Hashemian, H.M., "Instrument Calibration Reduction Using Signal Validation," Proceedings of the American Nuclear Society Winter Meeting, San Francisco, California, November 1993.

19. Tuley, C.R., "The Significance of the Nominal Trip Setpoint in the Westinghouse Setpoint Methodology," Proceedings of the 34th Power Instrumentation Symposium, Volume 34, Paper \#91-709, Instrument Society of America, June 1991.

20. Tuley, C.R., Williams, T.P., "The Significance of Verifying the SAMA PMC 20.1-1973 Defined Reference Accuracy for the Westinghouse Setpoint Methodology," Proceedings of the 35th Power Instrumentation Symposium, Volume 35, Paper \#92-0639, Instrument Society of America, June 1992.

21. Instrument Society of America, "Methodologies for Determination of Setpoints for Nuclear Safety-Related Instrumentation," ISA RP67.04, Part II, September 1994. 
APPENDIX A

NEURAL NETWORK THEORY 


\section{APPENDIX A}

\section{NEURAL NETWORK THEORY}

\section{INTRODUCTION}

This appendix provides a basic discussion on the algorithms and theory associated with the application of neural networks to process modeling. Specifically, the topics of feedforward networks and backpropagation training are explored to characterize the neural network modeling techniques used in this research. For an in-depth study of neural networks and their applications in nuclear power plants, the reader may consult the following references:

- $\quad$ Bartlett, E.B., Uhrig, R.E., "Nuclear Power Plant Diagnostics Using an Artificial Neural Network," Nuclear Technology, Volume 97, pp. 272-281, March 1992.

- $\quad$ Uhrig, R.E., "Potential Application of Neural Networks to the Operating of Nuclear Power Plants, Nuclear Safety, Volume 32, No. 1, January-March 1991.

- Wasserman, P.D., "Neural Computing: Theory and Practice," Van Nostrand Reinholt, New York, 1989.

- Caudill, M. and Butler, C., "Understanding Neural Networks: Computer Explorations," Volumes 1 and 2, MTI Press, Cambridge, MA, 1992.

- $\quad$ Aly, M.N., Nagy, M.E., Shaat, M.K., Abou Bakr, A.A., "Application of Nuclear Networks to Signal Validation in Nuclear Power Plants," Proceedings of the 9th Power Plant Dynamics, Control and Testing Symposium, University of Tennessee, Knoxville, Tennessee, May 1995.

- Ugolini, D., Saiki, A., Yoshikawa, S., Endou, A., "Enhancing Nuclear Power Plant Operations with the Artificial Neural Network Technique," Proceedings of the 3rd JSME/ASME Joint International Conference on Nuclear Engineering (ICONE-3), Kyoto, Japan, April 1995, Published by the Japan Society of Mechanical Engineers, Tokyo, Japan.

- $\quad$ Maren, A.J., Harston, C.T. and Pap, R.M., "Handbook of Neural Computing Applications," Academic Press, New York, NY, 1990.

- Nelson, M.M. and Illingsworth, W.T., "A Practical Guide to Neural Networks," Addison Wesley Publishing Co., Reading, MA, 1990.

- Proceedings of the Joint IEEE-INNS Neural Networks Conferences, Published by the IEEE Neural Network Council, Ann Arbor, MI, 1992. 


\section{PROCESS MODELING}

Mathematical models consist of relationships which describe how a set of input variables affect a set of output variables. Modeling techniques may be classified as being either physical or empirical. Empirical modeling has inherent advantages in simplicity of development over physical modeling. Neural network modeling is an empirical modeling technique which overcomes one of the limitations of other empirical modeling techniques by allowing complex relationships to be expressed and manipulated using generic algorithms.

Physical modèls, or first principle based models, are derived from first principle balance equations. The models are difficult and time consuming to develop, requiring intensive analysis of the system being modeled. The relationships between the system parameters are typically expressed in the form of differential equations. These differential equations describe all of the physical interactions of mass, energy, and momentum which comprise the physical system. Physical models often have large uncertainties due to linearized relationships, simplifications in geometry, and other assumptions. Another constraint on the accuracy of physical models is that some of the coefficients in the original balance equations may not be known precisely. If the system is sensitive to any of these approximations, then the model may be ineffective.

Empirical modeling consists of assuming the form of relationships (e.g., polynomial form) between input and output variables, and then determining the best parameters and coefficients of these relationships based on numerical regression of the measurements of the system. These measurements which are used to find the best model parameter coefficients are referred to as "training patterns" or example data. The main limitation of data-driven approaches, including neural networks, is that the training patterns must contain enough information to completely represent the physical system. All parameters which are variant and independent, and which also affect the system, must be supplied as inputs to the model. Additionally, all characteristics of the system must be demonstrated in the training data.

Neural networks provide an alternative empirical modeling technique. They provide a very general and powerful modeling tool, while requiring no prior knowledge of functional forms or probability density functions, and involving no complicated problem-specific algorithms. Empirical models, in general, require some prior knowledge of or assumptions regarding the functional form of the relationship between the system variables (e.g., assumptions regarding a polynomial-form 
relationship between the model output and input signals). The unique advantage of neural networks for complex system modeling is that they achieve an internal nonlinear representation of a modeled system upon merely being presented with proper input and output example data from the process.

\subsection{Feedforward Networks}

A schematic of a general four layer fully connected feedforward Artificial Neural Network (ANN), showing the direction and path of information flow, is presented in Figure A.1. The circles represent processing elements (PEs), also known as neurodes or nodes. The lines leading to and from each PE are referred to as connections. All the connections of a feedforward network are unidirectional, transmitting PE activations from the input layer towards the output layer. In a fully connected feedforward ANN, each PE receives inputs from every PE in the previous layer. More complex non-feedforward ANN architectures exist which permit flow of information or feedback from a layer to either a previous layer or to itself. A physical analogy for the structure of a feedforward ANN is the cerebral cortex of the brain. Each PE in the feedforward ANN represents a single artificial neuron and the connections represent the synapses between neurons.

The input layer of a feedforward network functions as a buffer with each processing element's activation corresponding to one of the network's input variables. The range of each input variable is typically linearly scaled to an appropriate range such as $[0.1,0.9]$ to limit the resulting activations in the first hidden layer. The intermediate layers of the network, known as hidden layers, contain an internal representation of the modeled system. The activations of the PEs in the intermediate layer(s) between the input and output layers are normally only utilized to obtain the activation at the output layer. The output layer of the network functions as a buffer for the output variables. The activation of each particular PE in the output layer is proportional to one of the output variables. The output PE activations, which are scaled similarly to the input layer activations to the region $(0,1)$, must be de-scaled to provide the output variables in the desired units.

The ANN given in Figure A.1 is a four layer example. This illustration may be generalized to represent any multi-layer feedforward network by reducing or increasing the number of hidden layers. Four (or more) layer networks as shown in Figure A.1 are not always required. Kolmogorov's mapping theorem states that any continuous function mapping $\mathrm{n}$ inputs onto $\mathrm{m}$ outputs may be implemented exactly by a three layer feedforward neural network with dimensions determined by the number of inputs and outputs. Kolmogorov's theorem guarantees the existence of such a 
perfect network, but with unspecified parameters. Three layer networks are usually found to be practical for most modeling applications.

Figure A.2 provides a more detailed diagram of a single processing element. The calculation of $x_{j}^{l}$, the activation of the $\mathrm{j}$-th PE in layer $l$, is given below in terms of the activations of each of $x_{i}^{l-1}$ and the $n$ PEs of the previous layer.

$$
x_{j}^{1}=\Phi\left(x_{0}^{1-1} w_{0 j}{ }^{1}+\sum_{i=1}^{n} x_{i}^{1-1} w_{i j}{ }^{2}\right),
$$

where $w_{i j}^{l}$ is the weight corresponding to the connection between the $x_{j}^{l} P E$ and the $j$-th PE of the previous level, and $\Phi$ is the transfer function. $x_{0}^{l-1}$ is a constant activation of 1.0 which is multiplied by the weight $\mathrm{w}_{0 \mathrm{j}}^{l}$ to provide a bias to the $\mathrm{PE}$.

The primary purpose of a transfer function is to limit the activation of a PE. This prevents any one PE from overwhelming the others in the same layer with respect to influencing PEs in the next layer. The transfer function forces the ANN to form very distributed input-output relationships and use all of the processing elements. Additionally, when nonlinear transfer functions are used, the resultant ANNs are able to model non-linear behavior. For this project, the transfer function utilized was the sigmoid function, which may be expressed as follows:

$$
\Phi(I)=\frac{1}{1+e^{-\gamma I}}
$$

where $\gamma$ is the sigmoidal slope, and corresponds to the slope of the sigmoid at $I=0$. The sigmoid transfer function which is shown in Figure A.3 limits the activation range of a PE to $(0,1)$.

A constant bias of 1.0 multiplied by a variable weight is introduced to create a neural threshold. The bias shifts the activation of a PE toward one of the asymptotes of the transfer function. A large bias can effectively force the activation of a PE to either zero or one, until the sum of the other inputs to that $\mathrm{PE}$ is large enough to cancel that bias.

\subsection{Backpropagation Training}

Once the initial architectural details have been decided about an ANN (e.g., type of ANN, type of transfer function, number of layers, number of PEs in each layer), the network has to be 
optimized to perform the desired function. This is done by adjusting the connection weights to appropriate values using training data.

A majority of all neural networks applications involve backpropagation training. This is a form of supervised learning which involves assigning numerical "responsibility" to each individual ANN weight for the error in output prediction. Supervised learning techniques are distinguished from unsupervised learning techniques in that the desired outputs are given to the network in the training data.

This "responsibility" for prediction error is obtained for each weight by taking the partial derivative of an error function in terms of the appropriate weight. The weights are all adjusted an increment in the opposite direction of the resultant error gradient. The prediction error of the ANN is reduced iteratively in this manner either until a minimum has been reached in the error "surface," and no further reduction is possible, or until the prediction error has been reduced to a predefined acceptance limit. This generally involves presenting all the patterns in the training data several times.

The algorithm which is used to adjust the weights is known as a training rule. The general delta rule (GDR) and the cumulative delta rule (CDR), the two most commonly used training rules for backpropagation, are described below.

The prediction error function used in GDR training is expressed as follows:

$$
E=\sum_{j=1}^{n} \frac{\left(t_{j}-x_{j}\right)^{2}}{2}
$$

where $t_{j}$ is the desired network output for component $j$, and $x_{j}$ is the actual activation of the $j$-th PE of the output layer.

The weight change $\Delta \mathrm{w}_{\mathrm{ij}}{ }^{1}$ is calculated as follows, at each presentation of a training pattern:

$$
\Delta w_{i j}^{1}=-\eta \frac{\partial E}{\partial w_{i j}^{2}}=\eta \delta_{j}^{1} x_{i}^{1-1},
$$

where $\eta$ is known as the learning coefficient and is set to a value contained in the interval $[0,1] . \delta$ may be expressed as follows for a PE belonging to the output layer: 


$$
\delta_{j}^{1}=\gamma x_{j}^{1}\left(1-x_{j}^{1}\right)\left(t_{j}-x_{j}^{1}\right)
$$

For PEs in the hidden layer(s), $\delta_{\mathrm{j}}{ }^{1}$ is expressed in terms of the $\delta_{\mathrm{k}}{ }^{1+1}$ of the PEs in the succeeding layer.

$$
\delta_{j}^{1}=\gamma x_{j}^{l}\left(1-x_{j}^{3}\right) \sum_{k} w_{j k}^{1+1} \delta_{k}^{1+1} .
$$

In order to avoid oscillatory weight changes which may occur with some training data sets, the cumulative delta rule (CDR) may be used. The CDR is distinguished from the GDR in that all weight changes are not implemented as they are evaluated, but are instead summed over the entire training data set. After all of the training examples have been presented to the network once, the summed (or cumulative) weight change is then implemented. Each presentation of all of the training examples over which the weight changes are summed is termed an epoch. The network is trained over many epochs to reduce the prediction error. The CDR is expressed as follows, summing the weight changes due to each of the individual training patterns (given above by the GDR, and indexed with the variable $\mu$ ) over the entire training epoch.

$$
\Delta w_{i j}^{1}=\eta \sum_{\mu}\left[\delta_{j}^{I} x_{j}^{1-1}\right](\mu) .
$$

Occasionally, the error gradient will lure the ANN's weights into a local minimum in the error surface. The global minimum may be nearby in the weight-space, but the intervening error surface may be a steep "hill." The concept of "momentum" is introduced to allow the network to "roll" out of "shallow" local minima while searching for the global error surface minimum. The CDR incorporating a momentum term representing a fraction of the last weight change may be expressed as follows:

$$
\Delta w_{i j}^{I}(t)=\beta \Delta w_{i j}^{I}(t-1)+\eta \sum_{\mu}\left[\delta_{j}^{l} x_{j}^{I-1}\right](\mu) .
$$

where $\beta$, the momentum coefficient, is selected from the interval $[0,1]$. 


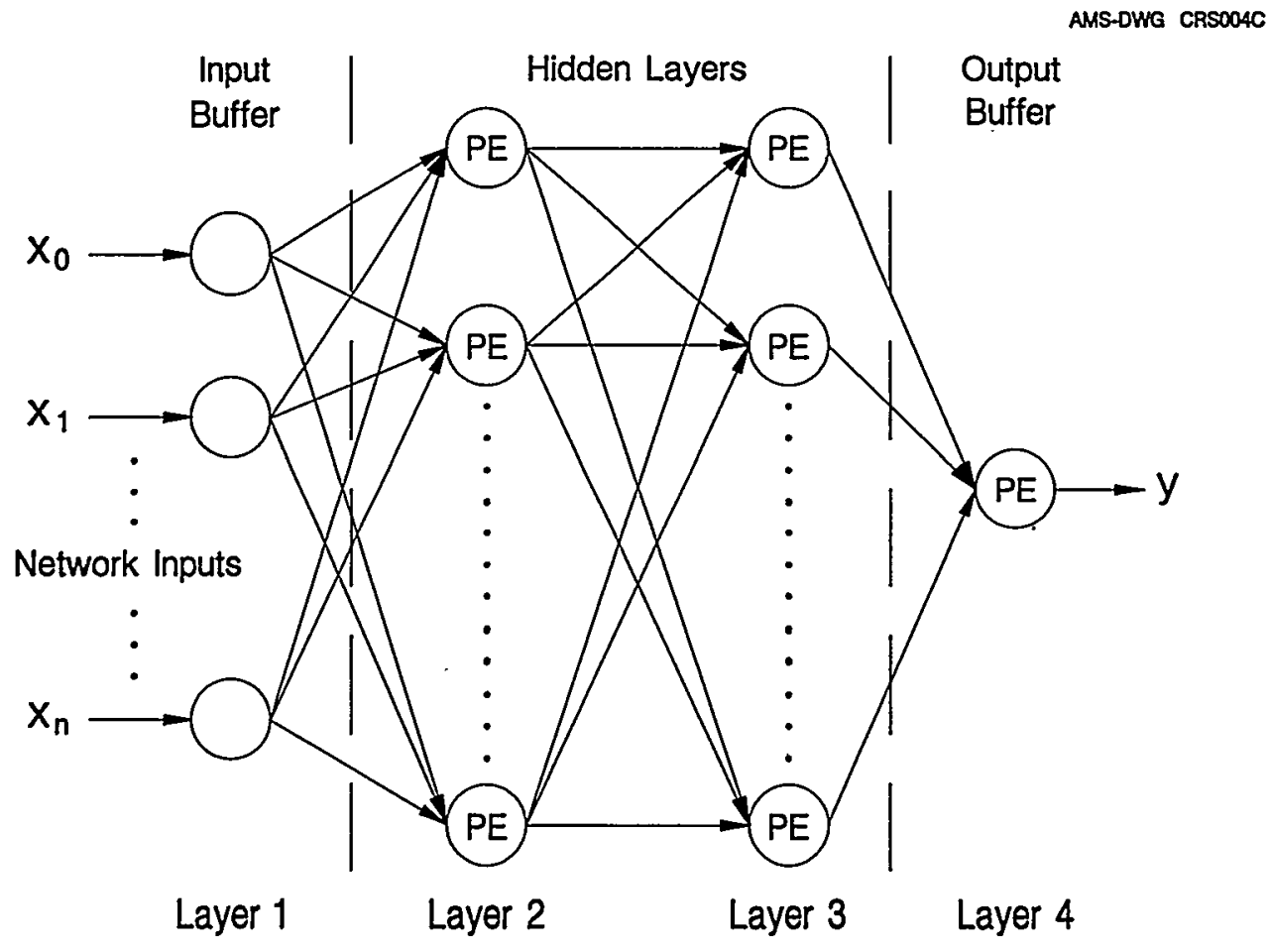

Figure A.1 Schematic of a Four Layer Feedforward ANN 


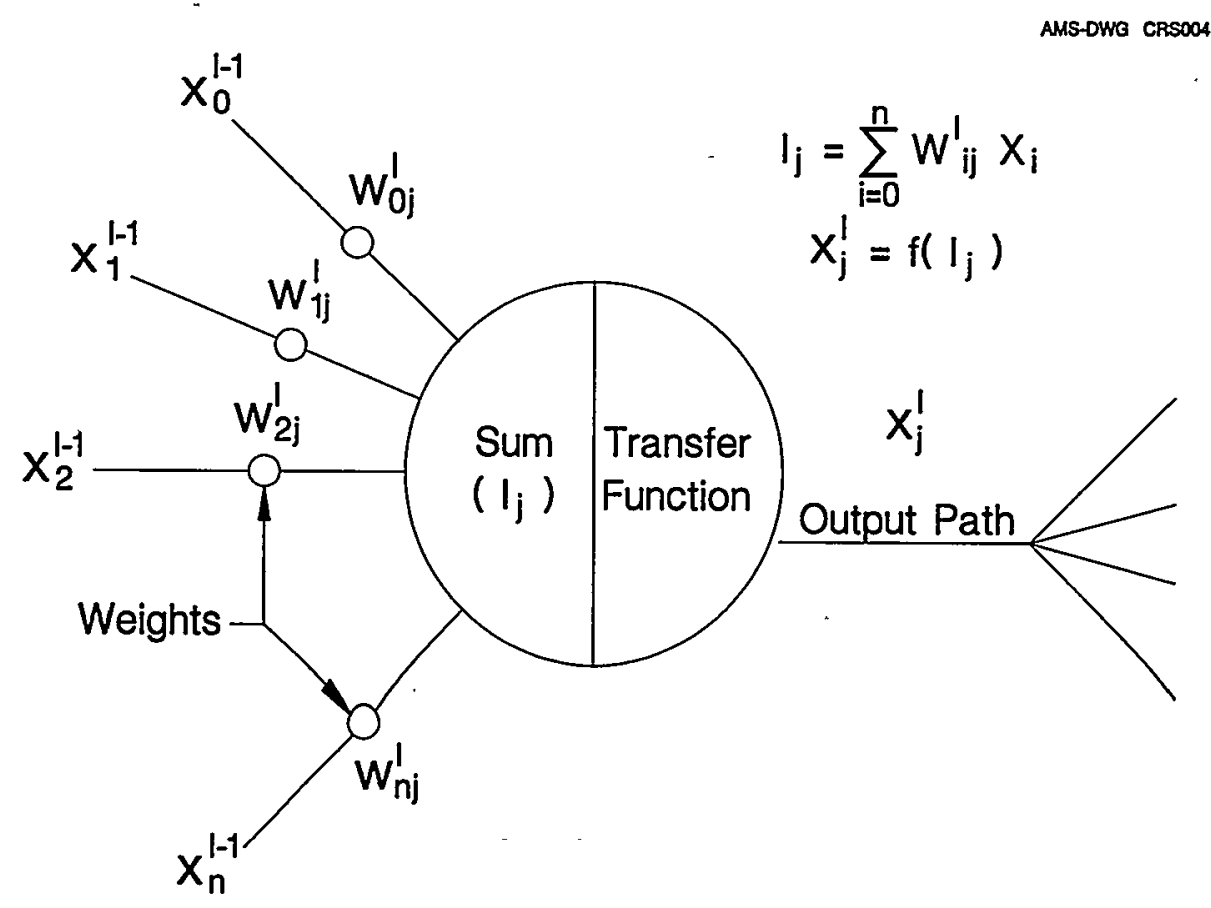

Figure A.2 Schematic of a Single Processing Element 


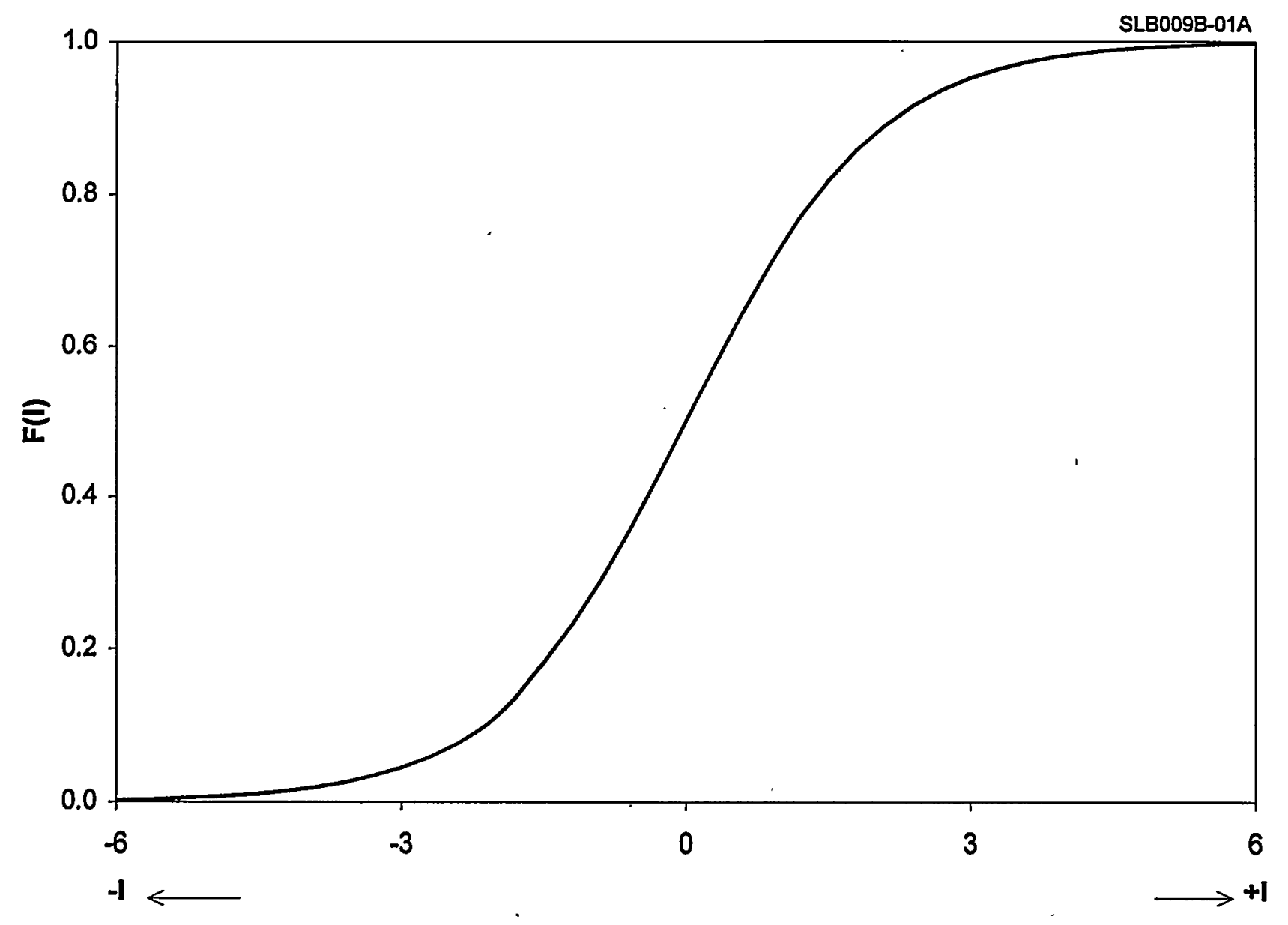

Figure A.3 Sigmoid Transfer Function 
APPENDIX B

EMPIRICAL MODELING THEORY 


\section{APPENDIX B}

\section{EMPIRICAL MODELING THEORY}

\section{Introduction}

In this project, the polynomial form of empirical modeling was used. An empirical model is created by selecting training data, deciding which variables to include as the inputs, and limiting the model order and the number of terms in the model. The modeling algorithm will automatically determine which of the model inputs are most related to the output variable, and also the polynomial form of the relationship. The inputs provided to the model during training with little relationship to the output variable are discarded by the model creation algorithm and are not utilized.

\section{Empirical Modeling Algorithm}

The empirical modeling algorithm creates an optimal nonlinear polynomial model based on a given data set. A polynomial function is determined that best describes the relationship between the provided inputs and outputs in the form given in Equation 1 below:

$$
y=c_{0}+\sum_{l=1}^{m} c_{l} \Phi_{l}(\bar{x})
$$

where $y=$ process variable estimate,

$\overrightarrow{\mathrm{x}}=$ vector of the input signals,

$\mathrm{m}=$ number of terms in the model,

$c_{i}=$ constant coefficients of each term, and

$\Phi_{i}=$ single term nonlinear function of the input signals.

An example of a empirical model of this form is as follows:

$$
y=-5.4+3 x_{1}-2 x_{1} x_{3}+10.4 x_{2}^{2}
$$

The maximum model order and maximum number of terms are specified in advance by the user. The algorithm then proceeds to first find the optimal polynomial-form combination of the provided input variables within the supplied restrictions on model order and number of terms. After 
the optimal polynomial form has been identified, the coefficients of the terms are determined by least-squares linear regression.

The number of possible $\Phi_{i}$ is dependent on the maximum model order and the number of input variables supplied in the training data set. For example, the set of all possible $\Phi_{\mathrm{i}}$ for a second order model with three inputs is $\left\{\mathrm{x}_{1}^{2}, \mathrm{x}_{1} \mathrm{x}_{2}, \mathrm{x}_{1} \mathrm{x}_{3}, \mathrm{x}_{2}^{2}, \mathrm{x}_{2} \mathrm{x}_{3}, \mathrm{x}_{3}^{2}, \mathrm{x}_{1}, \mathrm{x}_{2}, \mathrm{x}_{3}\right\}$. Every $\Phi_{\mathrm{i}}$ is evaluated for each of the (m) input data vectors in the training data set. A vector $\mathrm{v}(\mathrm{i})$ with $\mathrm{m}$ components is formed from the corresponding $\Phi_{i}$ evaluated for each of the $m$ training data points.

$$
\bar{v}(i)=\left[\begin{array}{c}
\Phi_{i}(\bar{x}(1)) \\
\Phi_{i}(\bar{x}(2)) \\
\Phi_{i}(\bar{x}(3)) \\
\vdots \\
\vdots \\
\Phi_{i}(\bar{x}(m))
\end{array}\right]
$$

A projection matrix, $P(i)$ is generated for each $v(i)$ from the following equation:

$$
P(i)=\frac{[\bar{v}(i)][\bar{v}(i)]^{T}}{[\bar{v}(i)]^{T}[\bar{v}(i)]}
$$

The vector composed of the measured output process variable at each measurement is multiplied by each projection matrix, to determine the projection of the output vector in the direction of each v(i).

$$
\bar{y}(i)=P(i) \bar{y}
$$

The scalar length, or norm of each resulting vector $y(i)$ is calculated, giving the magnitudes of the different projections of the output vector. The projection with the largest magnitude 
corresponds to the $\Phi_{\mathrm{i}}$ which best fits the behavior of the output variable over the training data. This $\Phi_{i}$ is selected as the next term for the model.

$$
R(j)=[\bar{y}(i)]^{T}[\bar{y}(i)]
$$

The output vector as well as each of the vectors $v(i)$, are projected into a vector space orthogonal to the vectors $\Phi_{\mathrm{i}}$ previously selected.

$$
\begin{gathered}
M=I-\sum_{k=1}^{J} P(i) \\
\bar{y}=M \bar{y} \\
\bar{v}(i)=M \bar{v}(i)
\end{gathered}
$$

The norm of the new y represents the remaining error not accounted for by the nonlinear terms already selected. If this error is not sufficiently small, as calculated by Equation 5, then the algorithm is repeated from Equation 3 until the error has either been reduced sufficiently or the maximum number of terms have been selected.

Once all of the terms $\Phi_{i}$ have been selected, the coefficients $c_{i}$ are obtained through least-squares linear regression. 


\section{APPENDIX C}

GENERALIZED CONSISTENCY CHECKING THEORY 


\section{APPENDIX C \\ GENERALIZED CONSISTENCY CHECKING THEORY}

Generalized Consistency Checking (GCC), sometimes also called the parity space method, is a technique for inter-comparing signals among a physically redundant sensor group. GCC is performed by comparing each signal pair combination within a redundant sensor group, and incrementing the inconsistency indices of the members of any pair with disagreement of more than a specified limit or threshold.

$$
I_{i}=\sum_{\substack{j=1 \\ j \neq i}}^{n} f\left(m_{i}, m_{j}\right)
$$

where

$\mathrm{m}_{\mathrm{i}}=$ redundant measurement $\mathrm{i}$,

$\mathrm{n}=$ number of redundant measurements,

The inconsistency function ( $f$ ) is given by:

$$
f\left(m_{l}, m_{j}\right)=\left\{\begin{array}{l}
1, \text { for }\left|m_{i}-m_{j}\right|>b_{i}+b_{j} \\
0, \text { for }\left|m_{l}-m_{j}\right| \leq b_{l}+b_{j}
\end{array}\right\}
$$

where $b_{i}$ is the inconsistency threshold corresponding to single signal $i$.

These limits are determined for each pair based on the individual sensor tolerances. After all of the pair comparisons (out of a set of $n$ redundant measurements, there are $n(n-1) / 2$ comparisons) have taken place, those sensors with large inconsistency indices may be excluded. In this manner, anomalous sensors which are present, if any, are isolated. 
The remaining signals are averaged to provide the best estimate of the process. If a signal fails all of its comparisons with the other redundant signals, it will have accumulated an index of $\mathrm{n}-1$. Such totally inconsistent signals are removed, and the consistency check is repeated. If none of the sensors is inconsistent, then all of the signals will have indices of zero. The terms of the average to provide the best estimate are weighted proportionally to the inconsistency index of each signal.

$$
p=\sum_{i=1}^{n} u_{i} m_{i}
$$

where $\mathrm{p}=$ the best estimate, and $u_{i}=$ the weight corresponding to signal $i$ given below:

$$
u_{i}=\frac{w_{i}\left(n-1-l_{i}\right)}{\sum_{i=1}^{n} w_{i}\left(n-1-l_{i}\right)}
$$

where $w_{i}$ reflects the intrinsic accuracy of a measurement (e.g., higher for narrow range sensors, lower for wide range), and the denominator satisfies the normalization criterion that the weights sum to unity.

At least three redundant signals must be available within a group to provide sufficient redundancy for GCC to achieve failure isolation. In the case of a single pair of disagreeing signals, the GCC, though able to detect the inconsistency, is not able to decide which signal is correct. Generally, GCC is only able to detect and identify drift of a sensor (or common mode drift of a group of sensors) when the drifting sensors make up less than half of the number of available redundant sensors. When a sufficient number of physically redundant signals is not available in a group, carefully validated and proven analytically redundant models could be used to provide process estimates for additional inputs. 


\section{APPENDIX D}

SEQUENTIAL PROBABILITY RATIO TEST 


\section{APPENDIX D}

\section{SEQUENTIAL PROBABILITY RATIO TEST}

The Sequential Probability Ratio Test (SPRT) is a technique which is used to monitor sensor degradation. The SPRT does not use analytical or physical redundancy directly to compare with each single measurement to monitor the magnitude of the deviation or discrepancy. Instead, the statistical properties of the deviation between a measured signal and another signal (either a redundant sensor signal or an analytically redundant signal generated by a model) are trended. The SPRT monitors the trend of accumulated information in the measurement history.

The two main features of SPRT are that the user can specify and configure the false-alarm and missed alarm probabilities and that the SPRT is mathematically defined such that it is guaranteed to provide results within the false-alarm and missed alarm probability limits over a shorter sampling period than any other method.

The main limitation of the SPRT method is that it presumes input signals with purely Gaussian independent (white noise) random variable characteristics. This limitation may be alleviated by spectral filtering techniques to remove any serial correlations present between the input signals

The Gaussian probability density function (PDF) of a normally distributed random variable may be expressed as:

$$
p\left(s ; \mu_{0}, \sigma_{0}^{2}\right)=\frac{1}{\sqrt{2 \pi \sigma_{0}^{2}}} \exp \left(-\frac{\left(s-\mu_{0}\right)^{2}}{2 \sigma_{0}^{2}}\right)
$$

where $\mathrm{p}$ is the probability of observing a value $\mathrm{s}$, given that the signal has a mean of $\mu_{0}$ and a variance of $\sigma_{0}^{2}$.

Given the normally distributed measured signal $m(t)$ at time $t$ and the predicted signal $y(t)$ at the same time $t$, define the measurement residual or deviation $s(t)=m(t)-y(t)$. The mean $\mu_{0}$ and the variance $\sigma_{0}{ }^{2}$ of the residual are calculated at every time point. For a pair made up of a measurement and a process estimate, the mean of the residual should tend towards some constant value with a magnitude near 0 , with a small variance corresponding to noise. However, in the case 
of a sensor suffering from a particular defect, the mean and variance of the residual would be expected to tend towards some other values characterizing the nature of the sensor defect.

The SPRT may either be used to test for a particular degradation mode, or simply to monitor for more than one failure criterion. In the latter mode of operation, the SPRT may be used to indicate when a sensor has degraded past separate threshold criteria including noise level and DC bias.

The following function $\lambda_{n}$ is the logarithm of the likelihood ratio (LLR). The likelihood ratio is the ratio of the probability of observing the last $\mathbf{n}$ samples assuming that the error residual is normally distributed with given mean $\mu_{1}$ and variance $\sigma_{1}^{2}$ to the probability of observing the last $\mathbf{n}$ samples assuming a measurement residual with the original "good" mean $\mu_{0}$ and variance $\sigma_{0}^{2}$.

$$
\lambda_{n}=\ln \left(\frac{p\left(s_{1}, s_{2}, \ldots, s_{n} \mid \mu_{1}, \sigma_{1}^{2}\right)}{p\left(s_{1}, s_{2}, \ldots, s_{n} \mid \mu_{0}, \sigma_{0}^{2}\right)}\right)
$$

The LLR can be expressed recursively (in terms of earlier calculations), as each of the previous measurements may be assumed to be independent of one another.

$$
\lambda_{n}=\lambda_{n-1}+\ln \left(\frac{p\left(s_{n} \mid \mu_{1}, \sigma_{1}^{2}\right)}{p\left(s_{n} \mid \mu_{0}, \sigma_{0}^{2}\right)}\right)
$$

The LLR is compared to an upper and a lower threshold. As long as the cumulative result does not reach or exceed either threshold, the cumulative calculation proceeds with the next sample or measurement. The thresholds $\mathrm{A}<0$, and $\mathrm{B}>0$, are given below:

$$
\begin{aligned}
& A=\ln \left(\frac{\beta}{1-\alpha}\right) \\
& B=\ln \left(\frac{1-\beta}{\alpha}\right)
\end{aligned}
$$

where $\alpha$ and $\beta$ are respectively the user-specified probabilities of false and missed alarms. The LLR may be expressed in different forms depending on the characteristic of the residual to be monitored. 
If noise level degradation is disregarded, and only bias degradation is monitored, then the LLR reduces to the following expression:

$$
\lambda_{n}=\lambda_{n-1}+\frac{\mu_{1}}{\sigma^{2}}\left(s_{n}-\frac{\mu_{1}}{2}\right)
$$

Depending on whether absolute bias or relative drift is to be monitored, the residual may be calculated differently. For absolute bias, the residuals $s_{i}$ in the above equation are calculated as defined previously, $s(t)=m(t)-y(t)$, where $m(t)$ is the measured signal at time $t$ and $y(t)$ is the best estimate at the same time $t$. For relative drift monitoring, the residual is calculated similarly, except that the initial bias between signals $m$ and $y$ is subtracted away from the subsequent calculations of the residual.

To monitor exclusively for noise level degradation, the LLR reduces to the following expression:

$$
\lambda_{n}=\lambda_{n-1}+\frac{s_{n}^{2}}{2}\left(\frac{1}{\sigma_{0}^{2}}-\frac{1}{\sigma_{1}^{2}}\right)+\ln \frac{\sigma_{0}}{\sigma_{1}}
$$

This expression of the LIR may be used to detect high noise levels which reach a maximum variance limit or also noise levels less than a minimum limit (perhaps indicating dynamic response degradation). 
APPENDIX E

PATTERN RECOGNITION METHODS 


\section{APPENDIX E}

\section{PATTERN RECOGNITION METHODS}

\section{PROCESS HYPERCUBE}

The Process Hypercube signal validation and processing technique is based on a cartesian interpretation of the plant states. This technique learns the operating states of a system from example data. During signal monitoring, the measurements are compared to previously observed measurements. The states which have been previously observed are declared normal and the signals are declared valid. When a measured state does not coincide with a previously observed state, either one or more signals are in error, or the system state is considered abnormal.

The hypercube technique requires a previous database incorporating and demonstrating all of the possible acceptable states of the plant and the sensor measurements in those states. The data is stored in a hypercube data structure, which gives this signal validation method its name. The hypercube data structure allows large amounts of data to be stored (several hundred sensors stored regularly over months) in only a fraction of the storage space that would be necessary for a conventional database. As the measurements are monitored, they are compared to all of the states previously observed.

The hypercube data structure may be conceived as an $\mathrm{n}$ dimensional histogram of the previous measurements obtained from $\mathrm{n}$ different sensors. Each process signal corresponds geometrically to a direction orthogonal to the all of the other dimensions, and is assigned a discrete value within the maximum and minimum values into a number of user-specified intervals. Each "bin" defined by the intervals in the $\mathrm{n}$ different dimensions represents a state of the system. Only those $\mathrm{n}$-dimensional bins or states which "contain" previously observed measurements need to be stored, affecting a considerable storage reduction over a more conventional data structure. Those bins that contain relatively few observances may be eliminated, affecting another similar storage requirement reduction. In many applications similar to calibration monitoring, around $90 \%$ of the observed states were observed at most once, with the remaining $10 \%$ or so of the bins containing the vast majority of the system state occurrences.

When a newly measured state agrees with none of the previously measured states, the hypercube methodology first tests whether the number of suspected signals is greater than a 
threshold. If so, then the process is declared abnormal. If not, then each suspected variable, and each combination of suspected variables, are varied until the "nearest state" in the hypercube data structure is found.

This "nearest state" is not necessarily the closest in a cartesian sense. If a particular system measurement does not agree with any previously observed system state, and the process is not declared abnormal, then the "nearest state" is the one in which the most individual signals agree with the new measurement. This can result, for example, in the following hypothetical situation illustrated in Figure E.1.

A series of measurements of a system which consists of two different sensors is acquired. The hypercube of such a system would possess only two dimensions, with the axes corresponding to the two different sensors. Out of the entire series of data, only two distinct states (State A and State B) are identified. A new measurement does not happen to agree exactly with either State A or State B. The new measurement seems to agree well in a cartesian manner with State $A$, as it occupies the bin one interval over in each dimension. However, although the new measurement does not agree similarly with State $B$, the system state at the time of the new measurement is identified to be State B. This is because one of the components of the new measurement and State B agree exactly. Since State B differs in only one dimension (or individual signal) from the new measurement, it is nearer than State A, which differs in both dimensions.

If the process is not declared abnormal, then the hypercube is generally able to provide an estimate of the true process state. The hypercube is not always able to supply an estimate, as the newly measured state might be halfway between the two closest neighboring observed states.

\section{SYSTEM STATE ANALYSIS AND UNIVERSAL PROCESS MODELING}

System State Analysis may be viewed as another predictive method relying on a past history of "good" measurements, and incorporating pattern recognition, clustering, and modeling. A new measurement is first compared to past measurements. Those past measurements which are most similar to the current measurement are linearly combined to obtain an estimate of the current true process values. 
AMS-DWG BLK104A

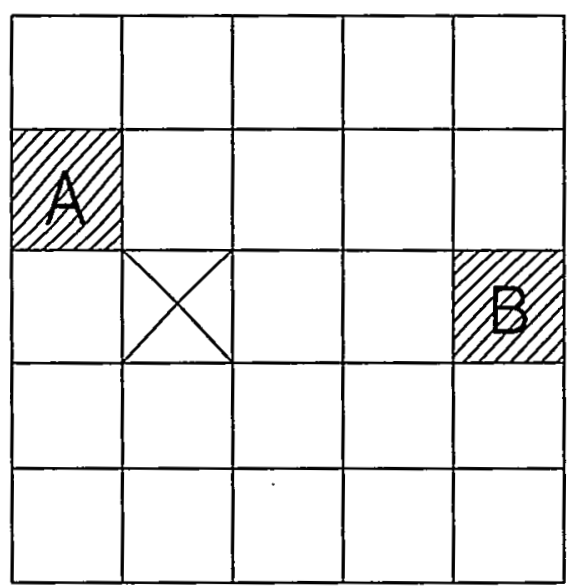

$\triangle$ New Observed Measurement

Previously Observed System States

Figure E.1 Two Dimensional Hypercube Nearest Neighbor Example 


\section{APPENDIX F}

RAW DATA PLOTS FOR CYCLE 1 


\section{APPENDIX F}

\section{RAW DATA PLOTS FOR CYCLE 1}

This Appendix contains raw data plots for the McGuire instrument channels that were monitored during this project. Cycle 1 data collection began in March 1992 after a refueling outage and ended in June 1993 when the plant was shutdown for refueling.

The same data acquisition system and procedure was used in cycle 1 and cycle 2. However, the sampling rates were different. In cycle 1, the output of each instrument was sampled six times per hour while in cycle 2 , the sampling rate was reduced to one sample per hour. The plots that are included in this appendix do not represent every point that was sampled. Rather, points were skipped in plotting the data.

Each figure in this appendix including three plots as follows:

1. The raw data including the spikes and gaps that represent plant trips, shutdowns, and periods where on-line monitoring data could not be collected.

2. Data after the spikes and gaps were removed.

3. Data after it was filtered to remove the extraneous noise. 
Steam Flow SG A

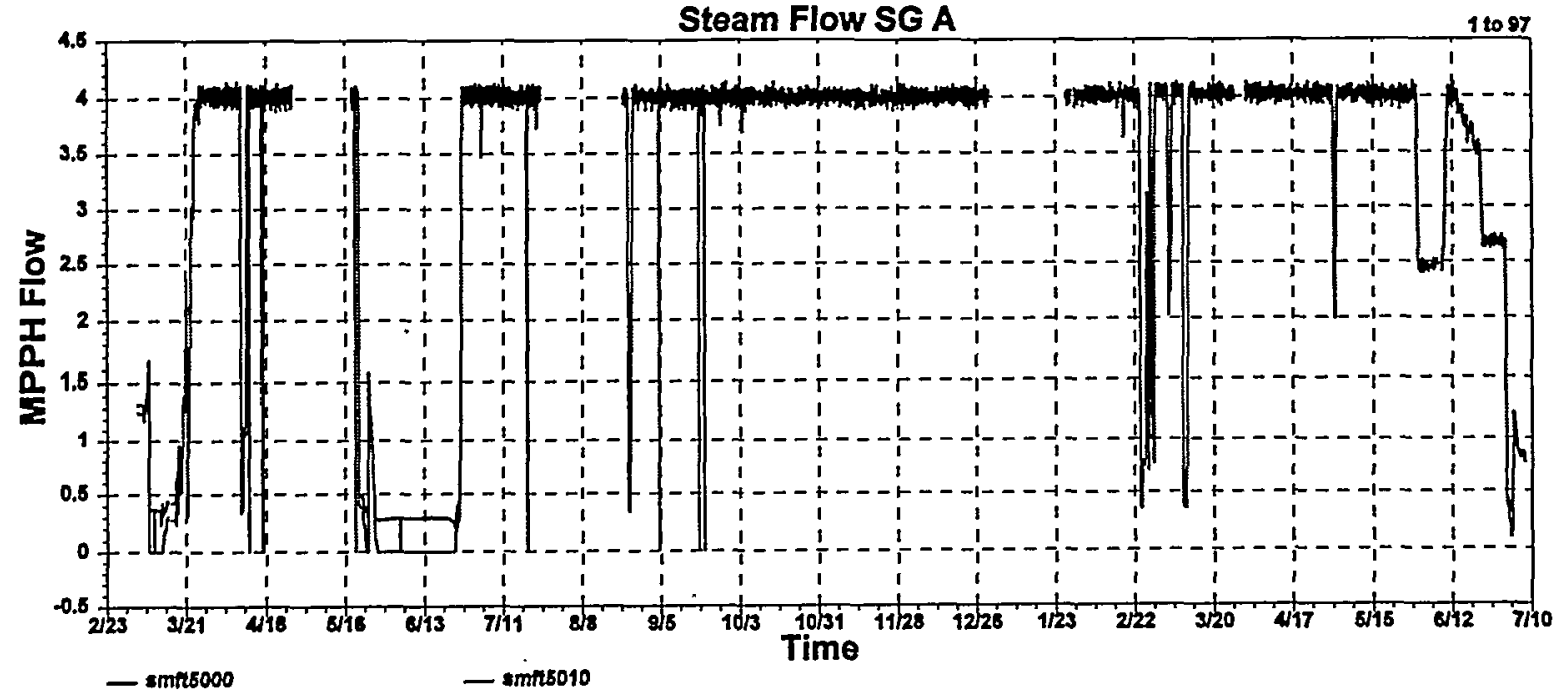

Steam Flow SG A

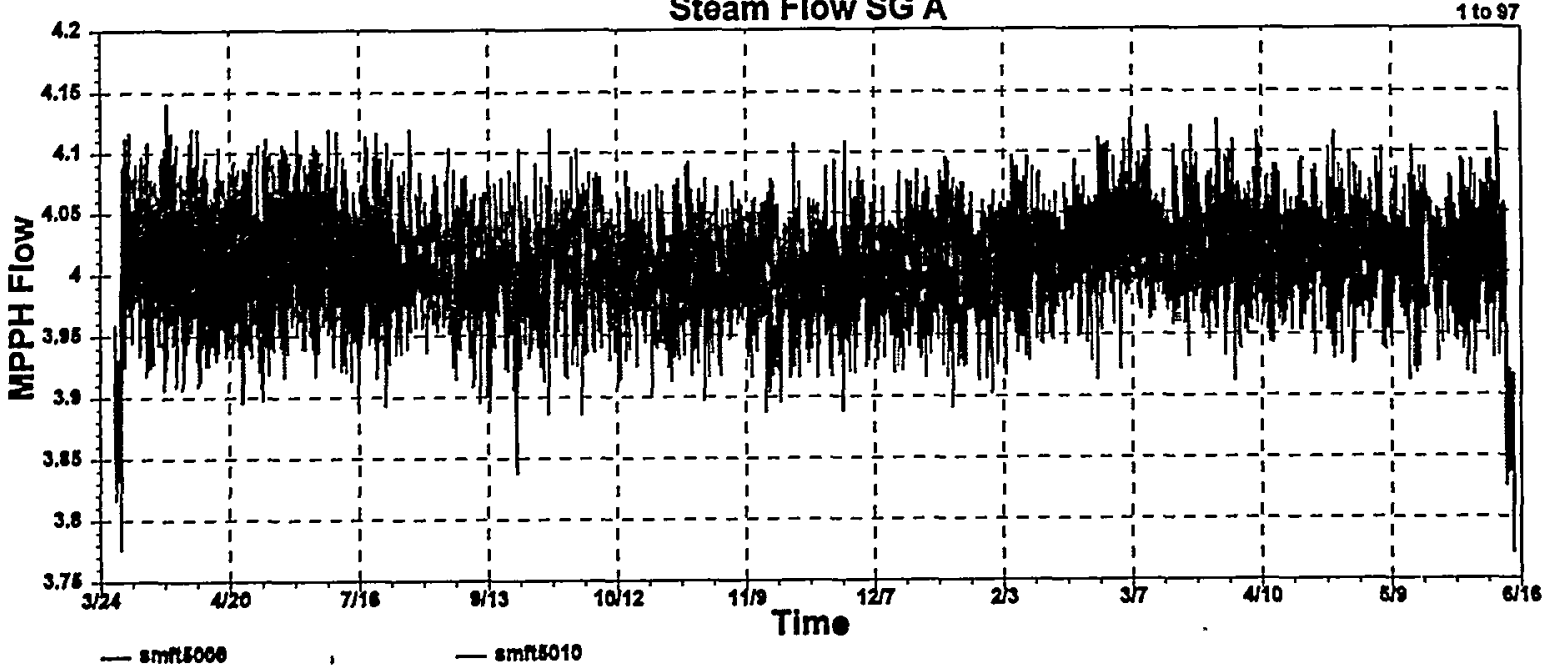

Stoam Flow SG A

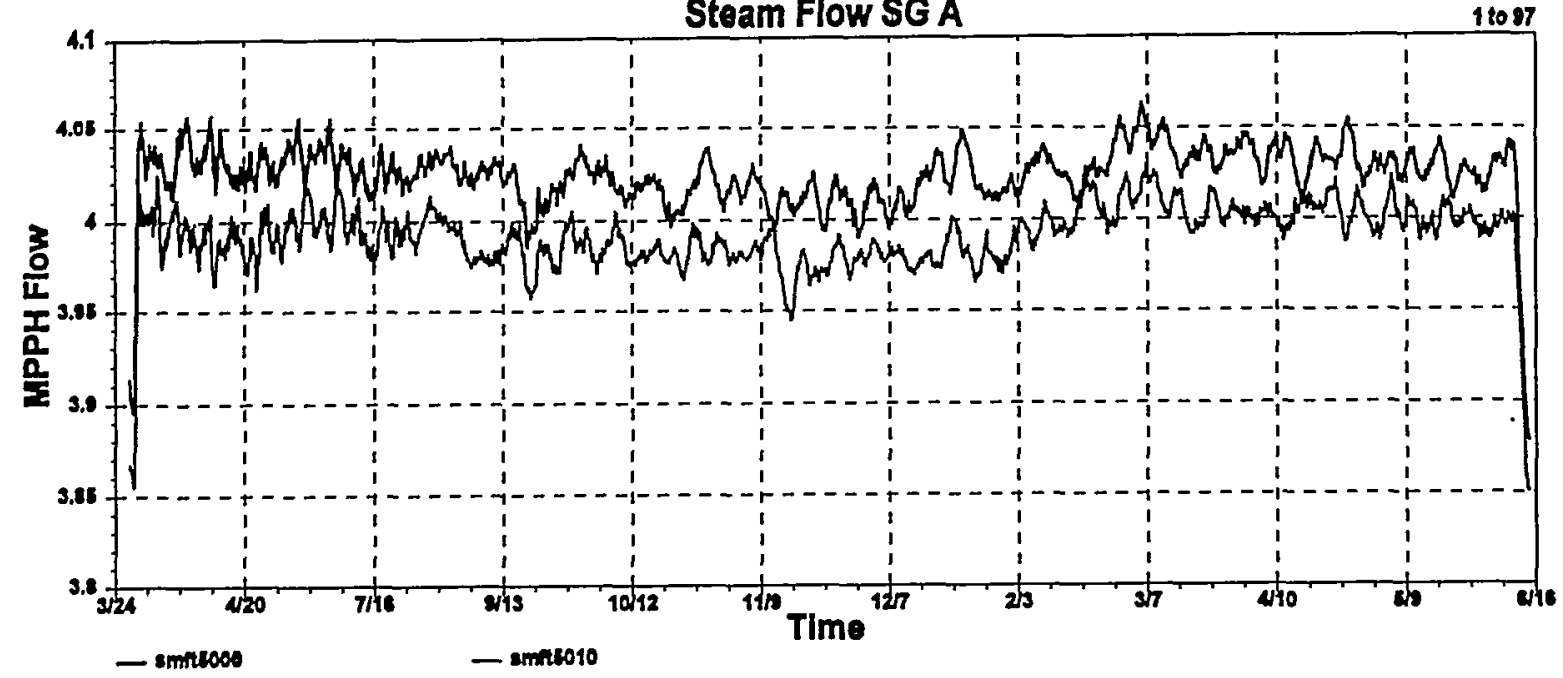

F.1 McGuire Cycle 1 Data for Steam Flow Signals for Loop A 


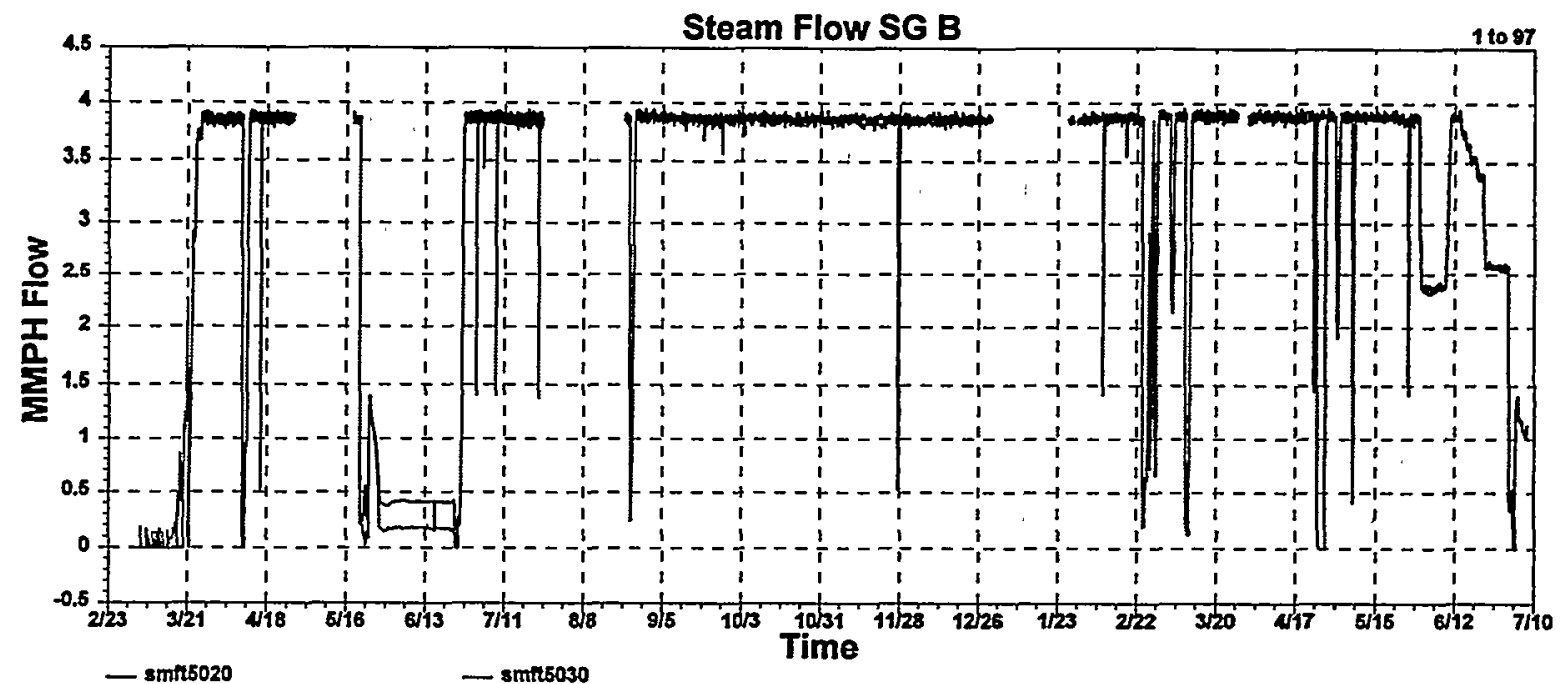

Steam Flow SG B

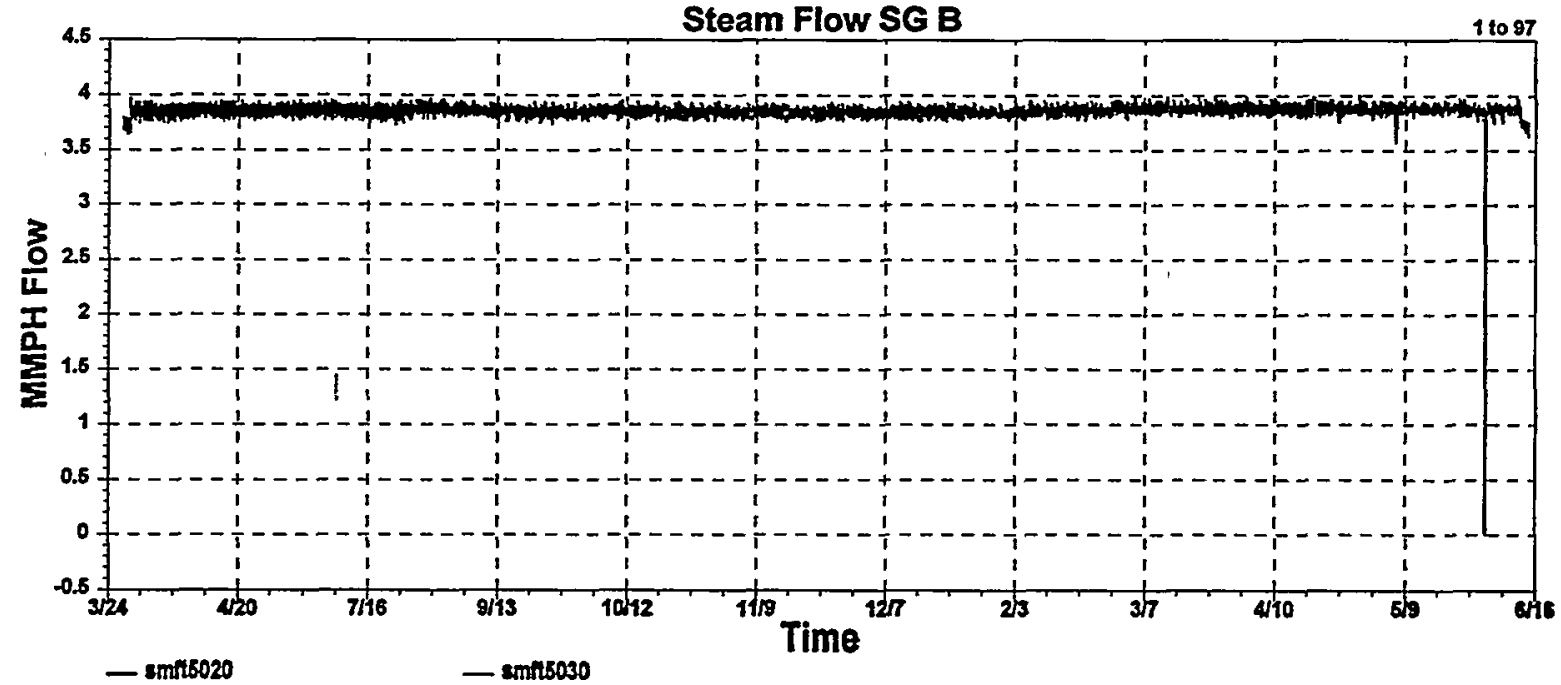

Steam Flow SG B

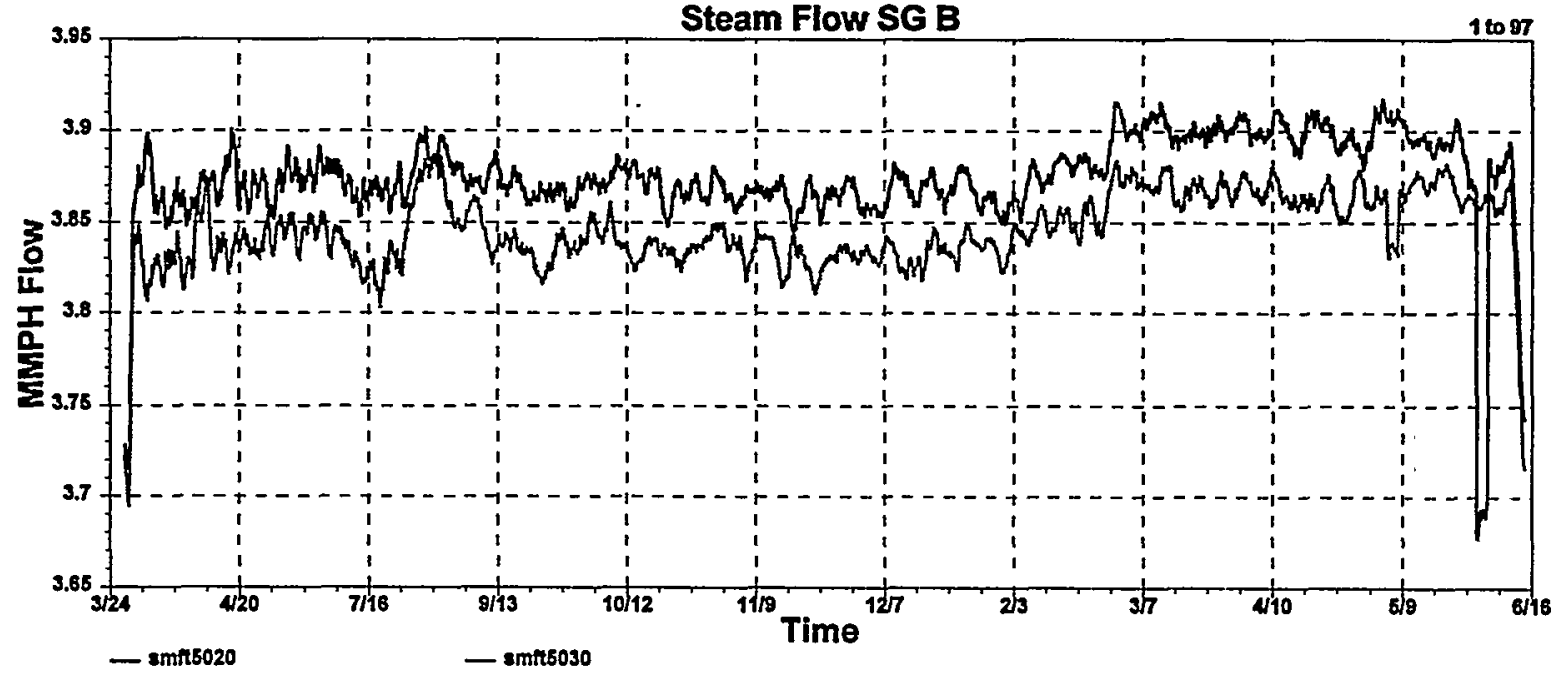

F.2 McGuire Cycle 1 Data for Steam Flow Signals for Loop B 

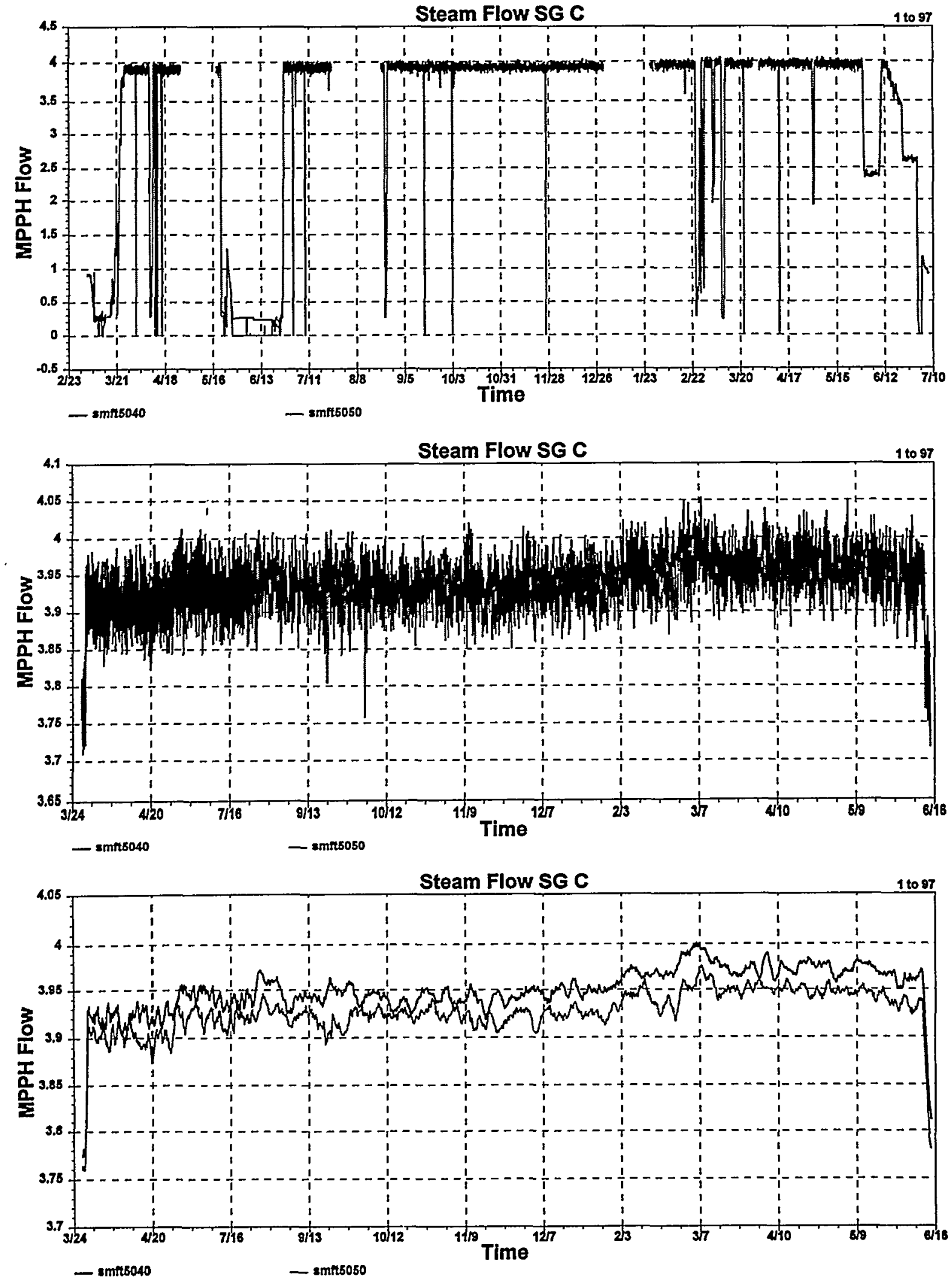

F.3 McGuire Cycle 1 Data for Steam Flow Signals for Loop C 

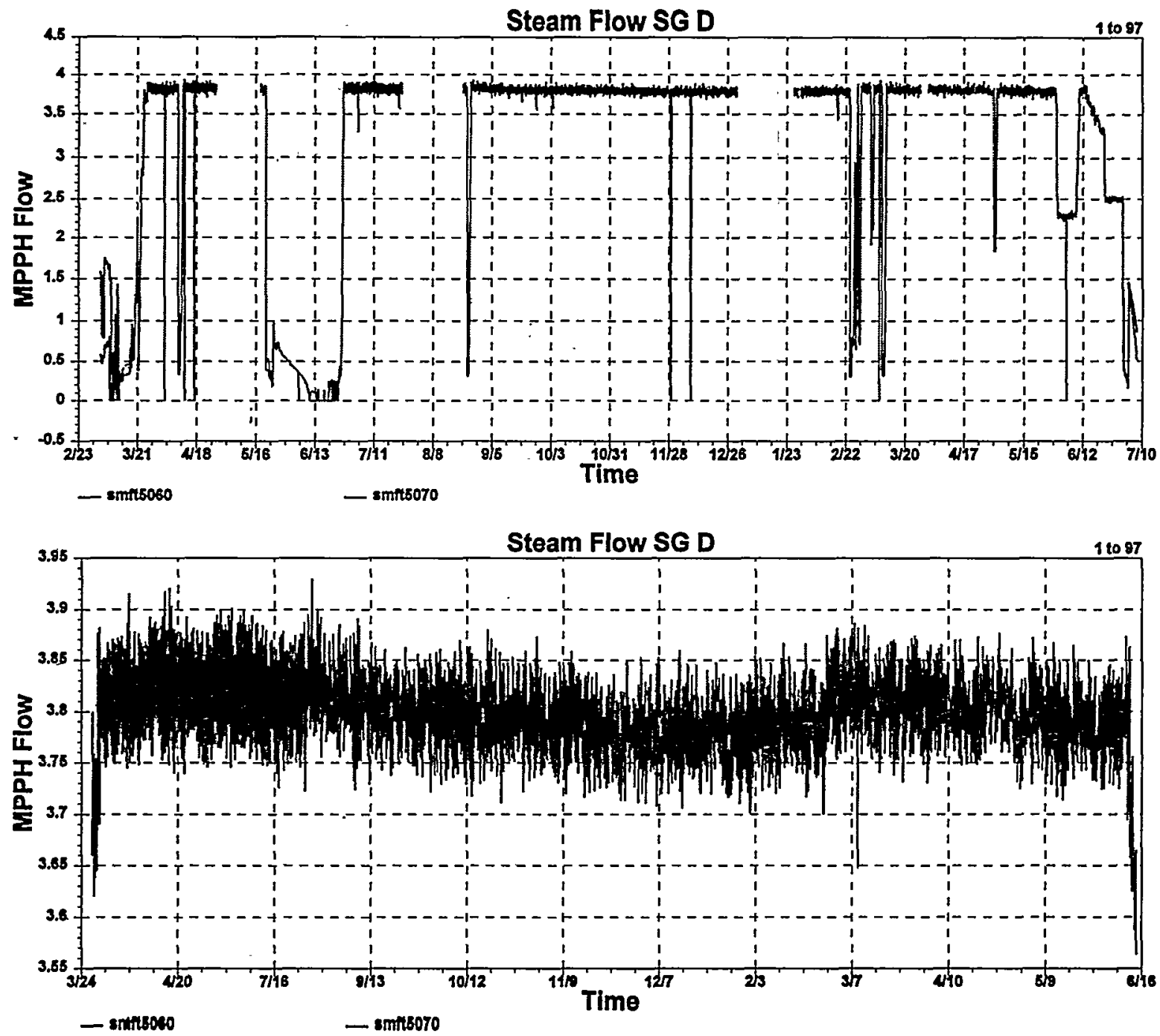

- emis070

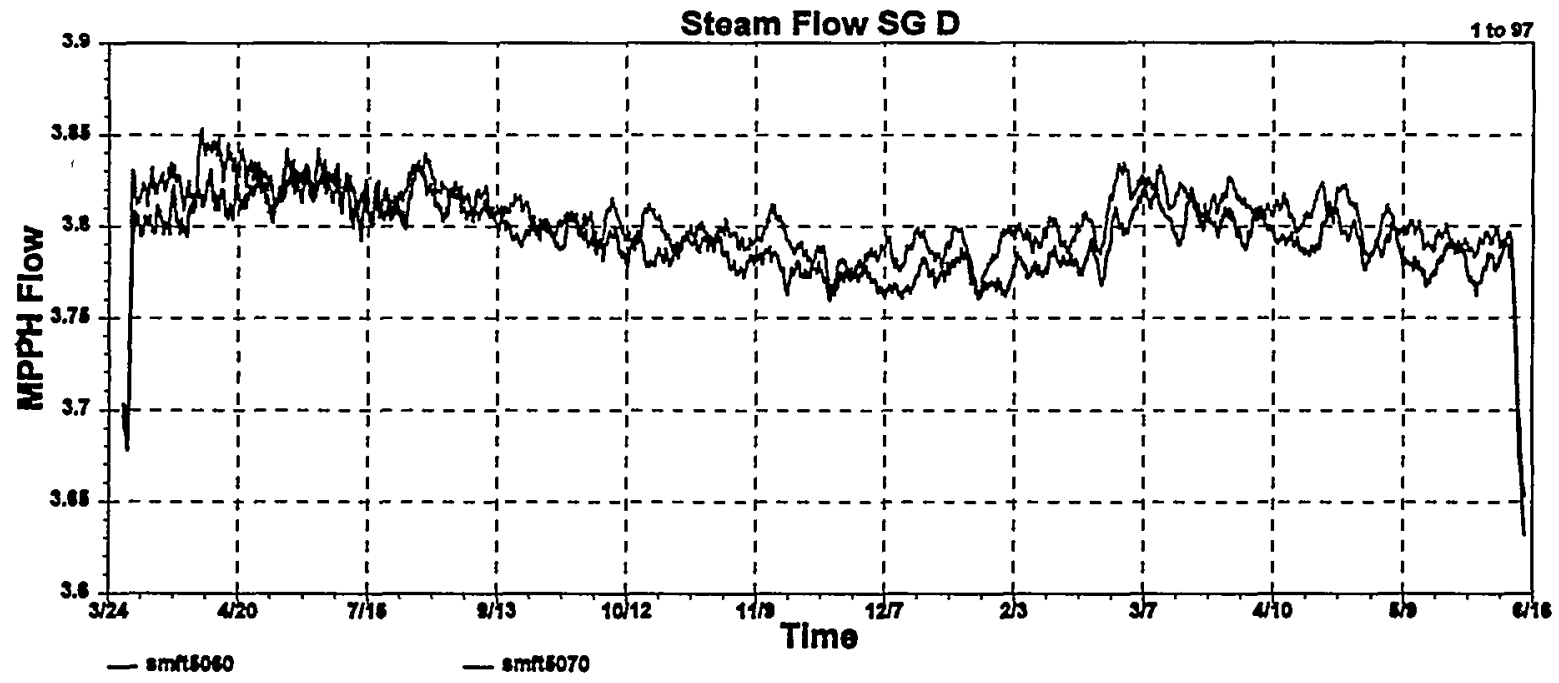

F.4 McGuire Cycle 1 Data for Steam Flow Signals for Loop D 

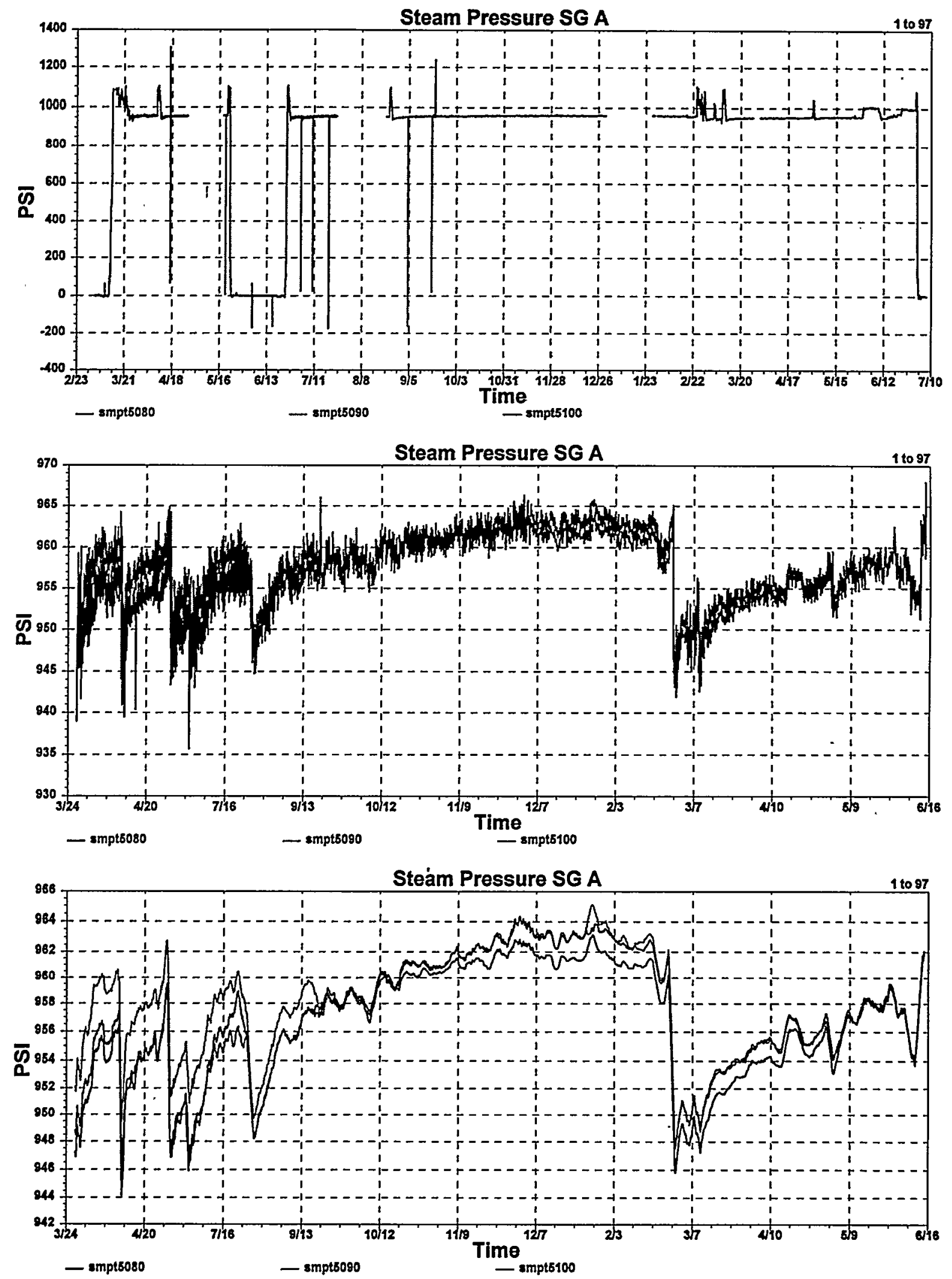

F.5 McGuire Cycle 1 Data for Steam Pressure Signals for Loop A 

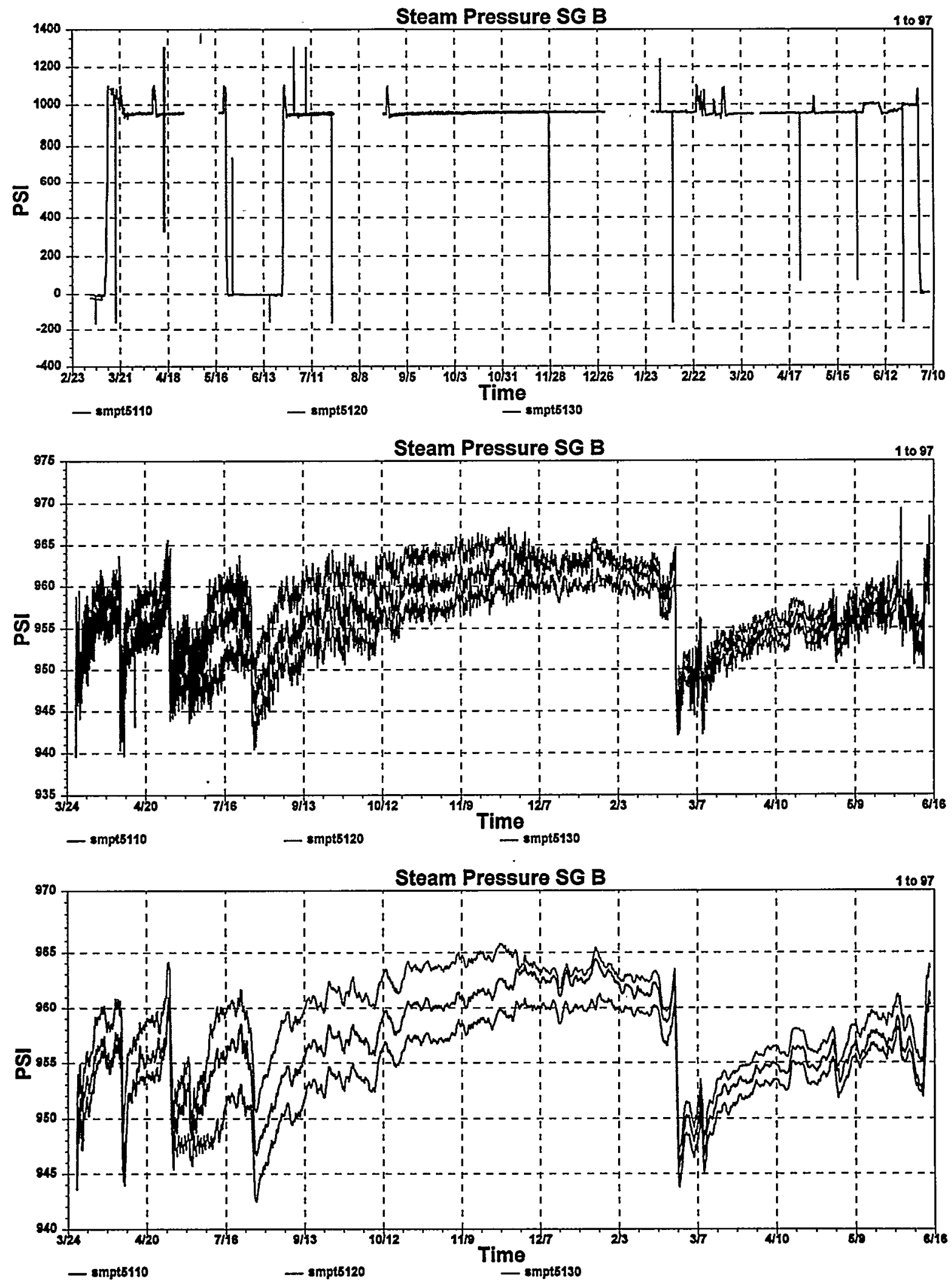

F.6 McGuire Cycle 1 Data for Steam Pressure Signals for Loop B 

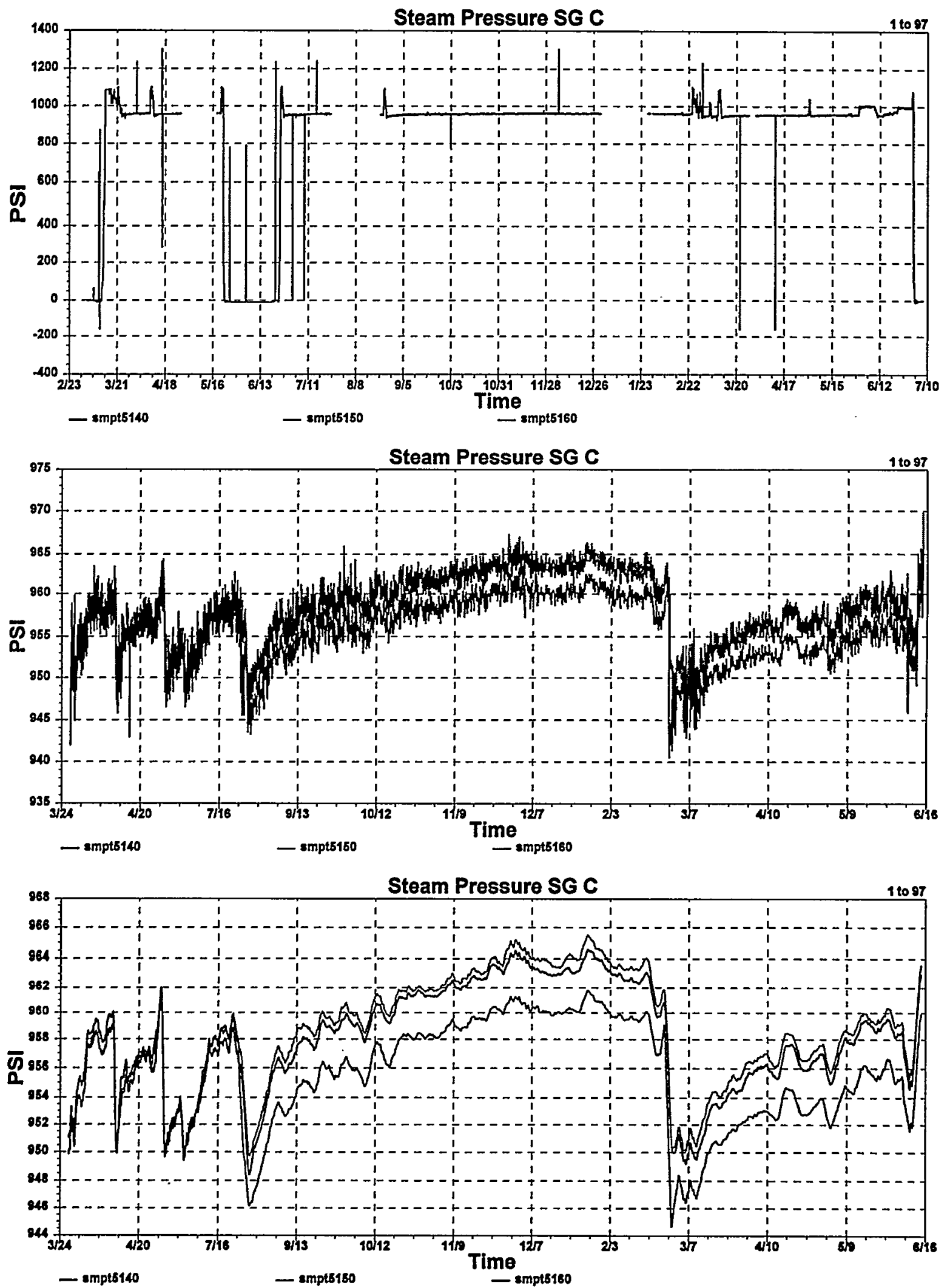

F.7 McGuire Cycle 1 Data for Steam Pressure Signals for Loop C 

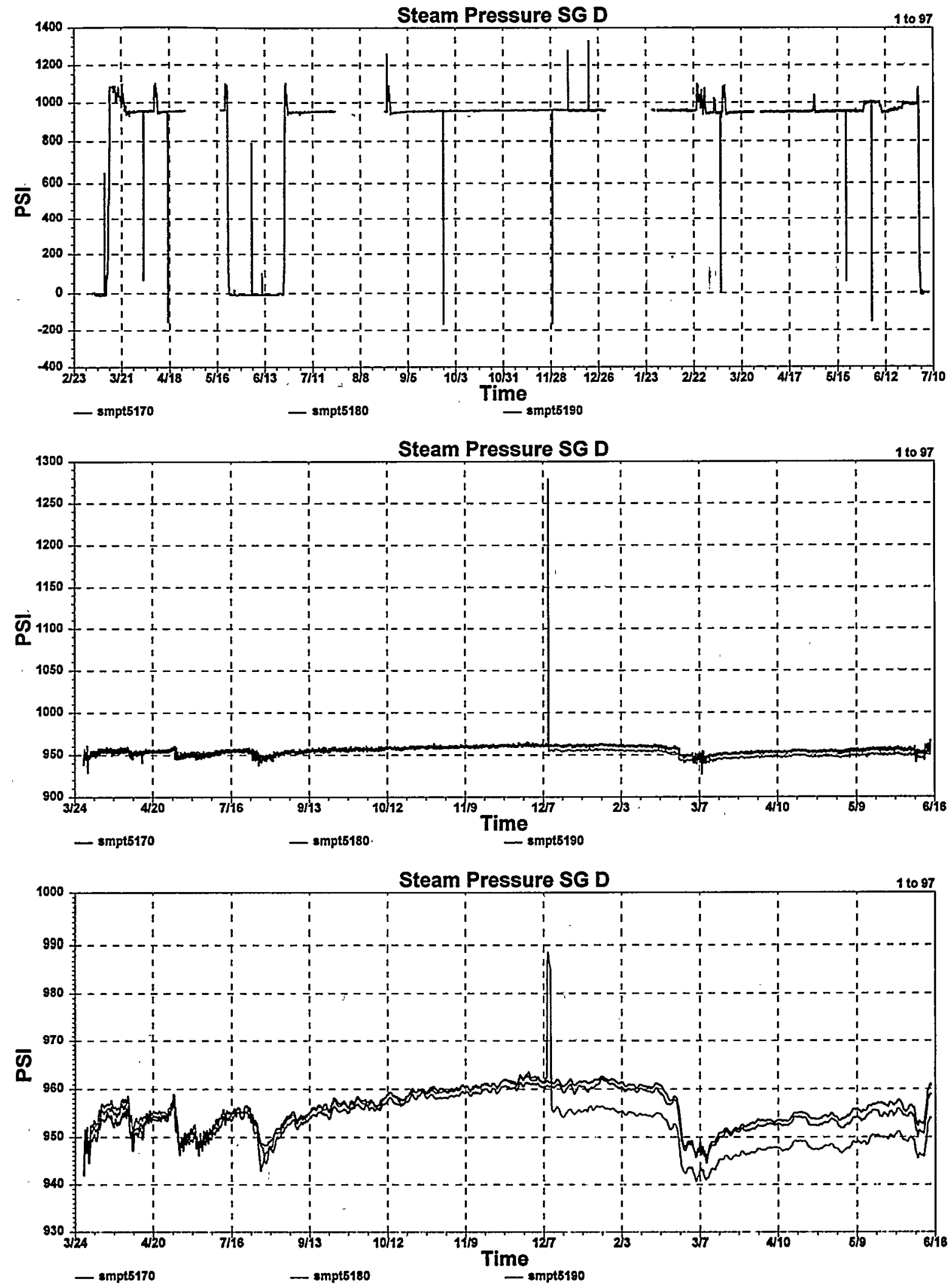

F.8 McGuire Cycle 1 Data for Steam Pressure Signals for Loop D 

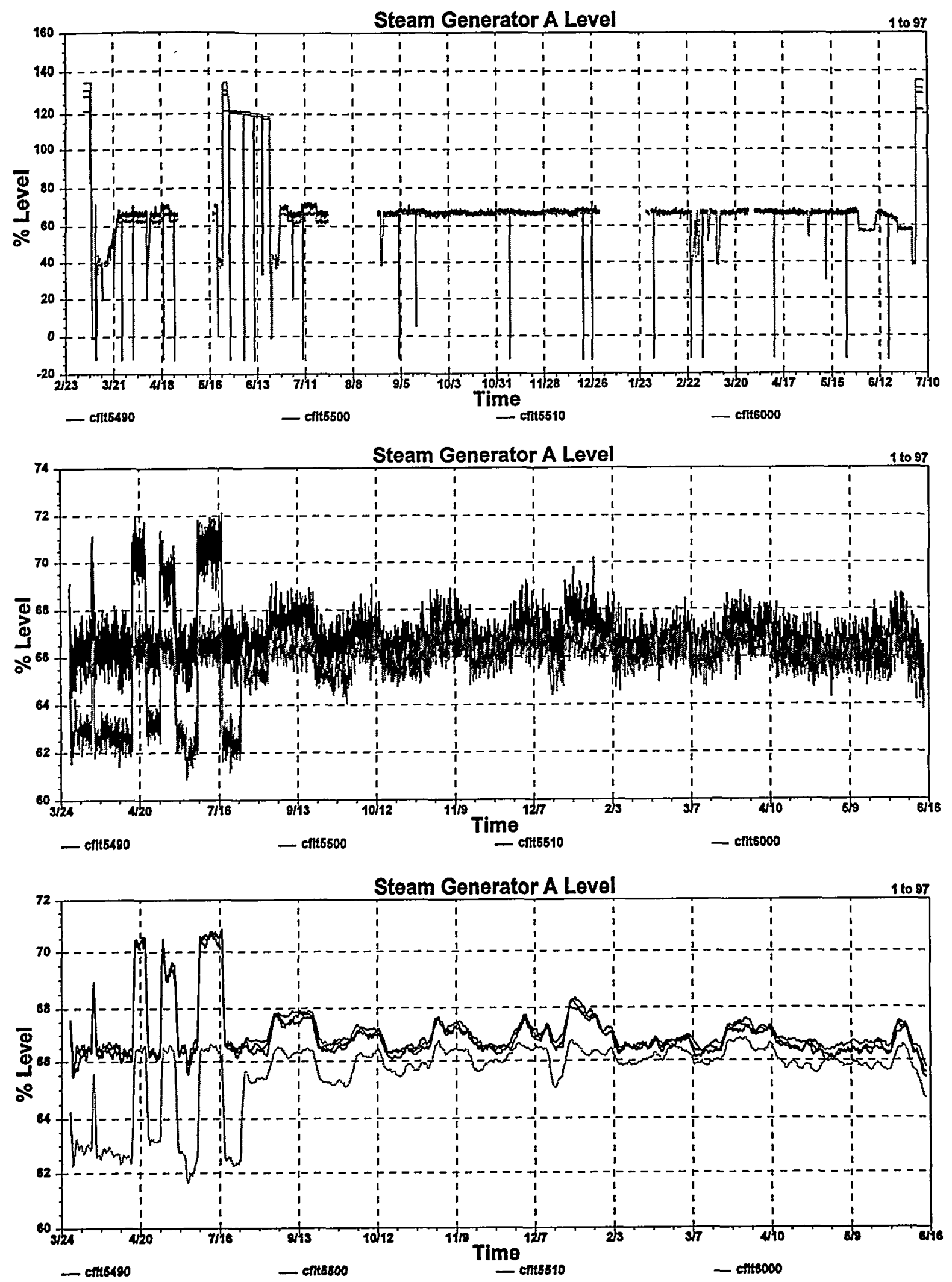

F.9 McGuire Cycle 1 Data for Steam Generator Level Signals for Loop A 

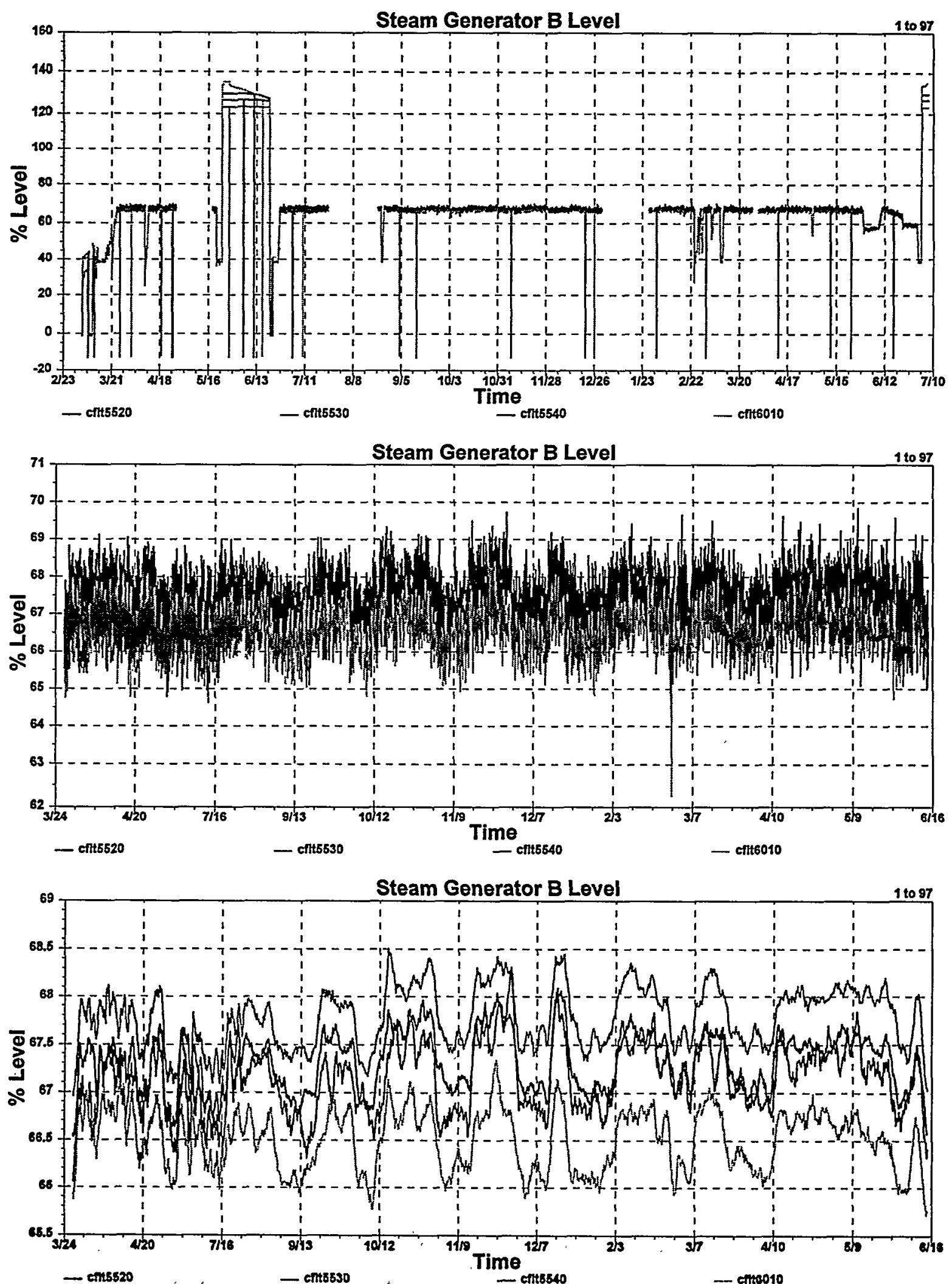

F.10 McGuire Cycle 1 Data for Steam Generator Level Signals for Loop B 

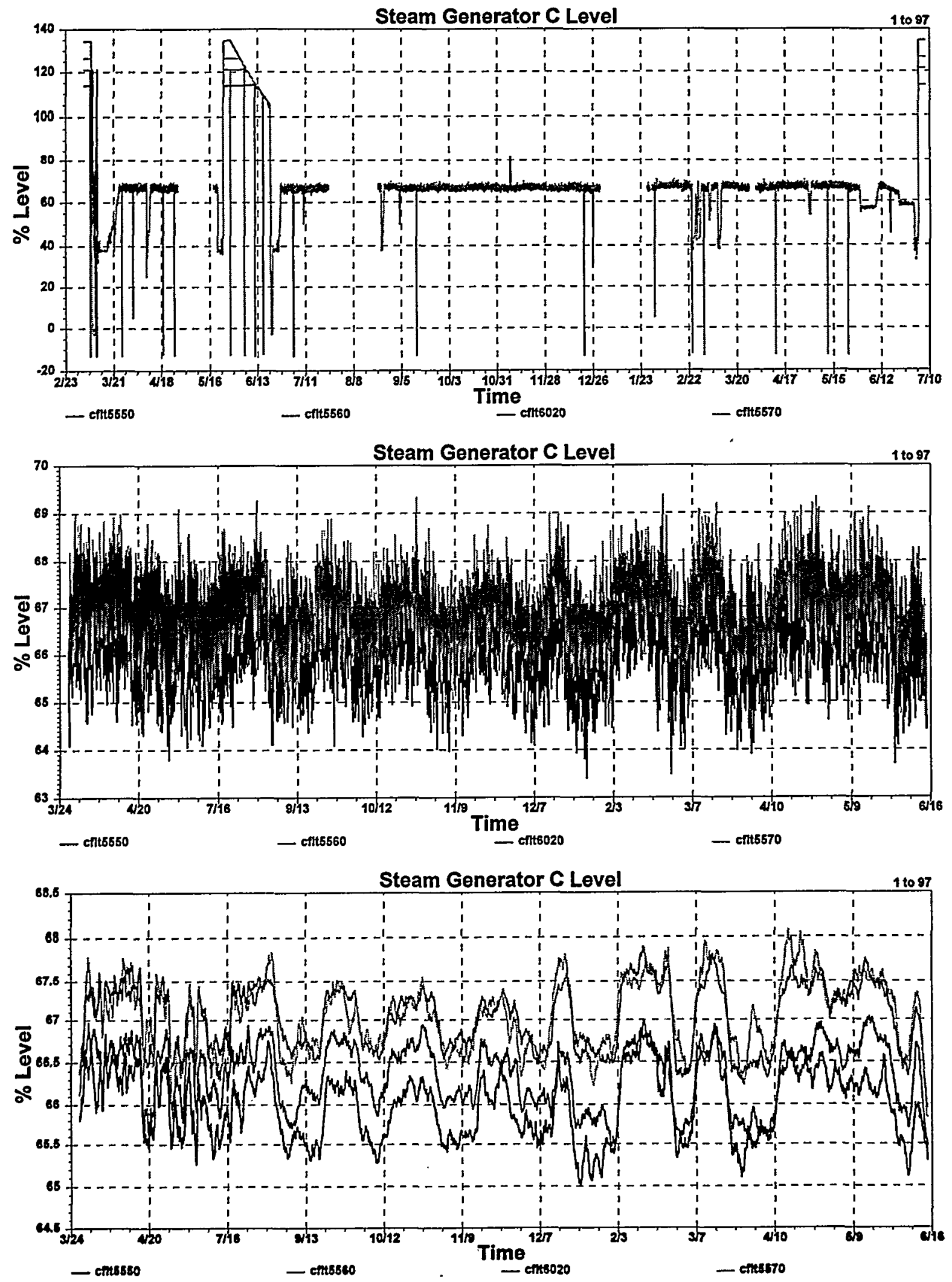

F.11 McGuire Cycle 1 Data for Steam Generator Level Signals for Loop C 

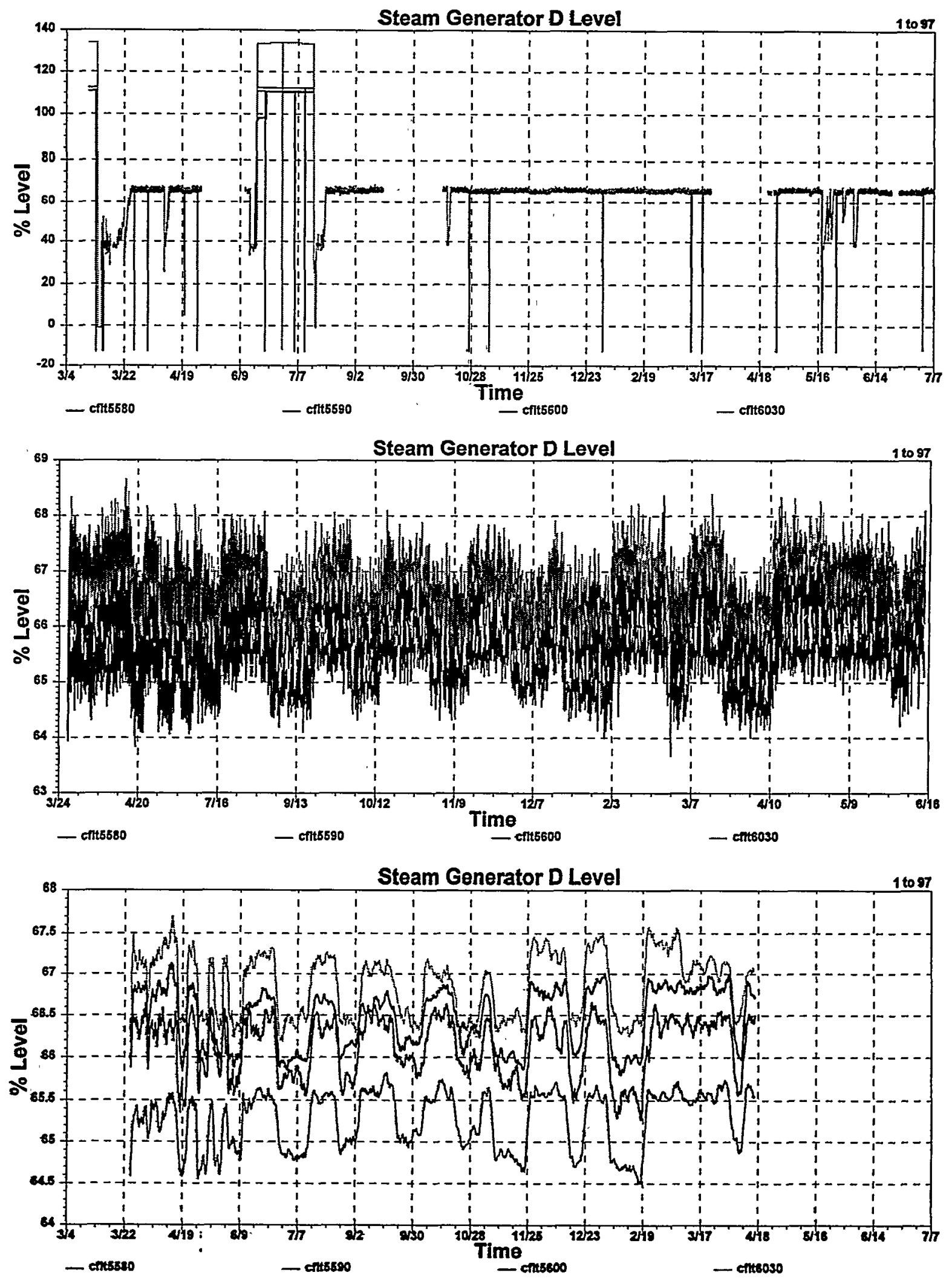

F.12 McGuire Cycle 1 Data for Steam Generator Level Signals for Loop D 
FW Flow SG A

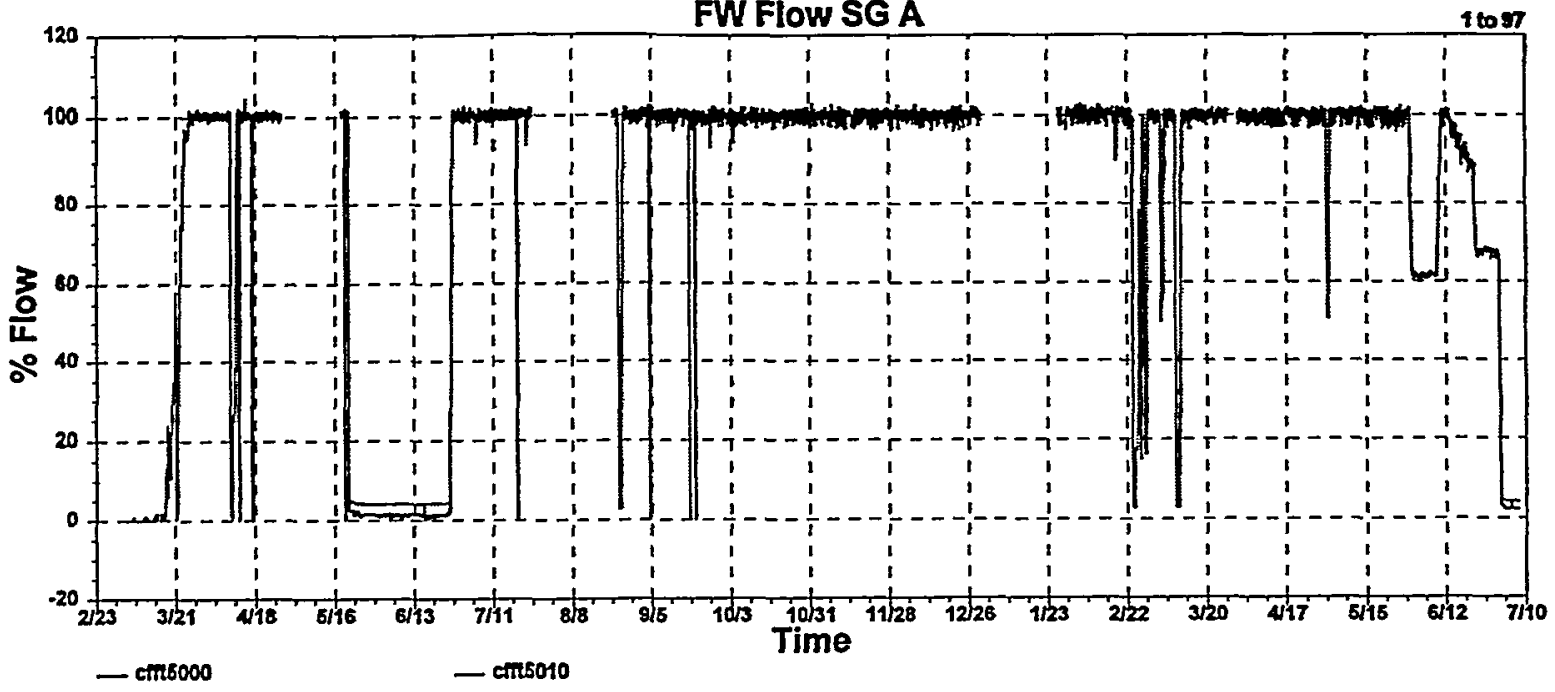

FW Flow SG A

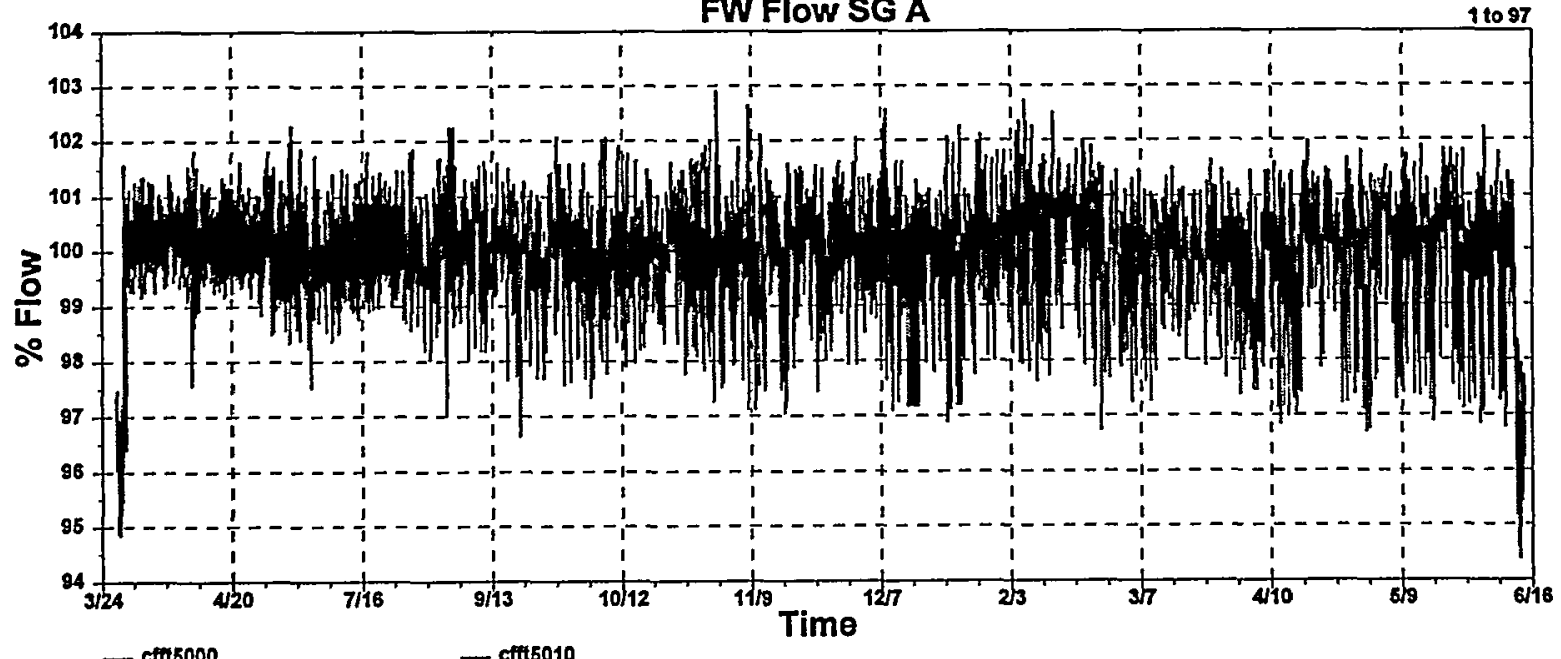

FW Flow SG A

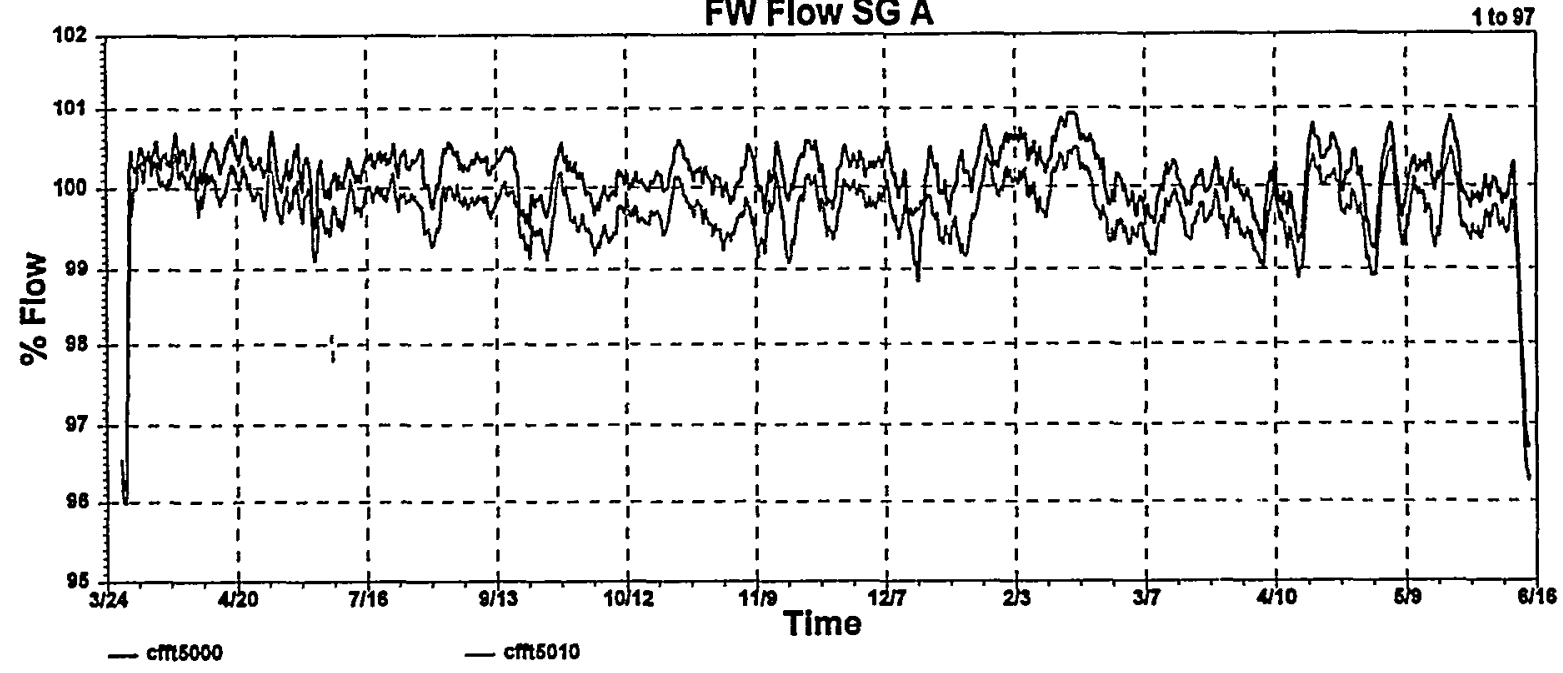

F.13 McGuire Cycle 1 Data for Feedwater Flow Signals for Loop A 

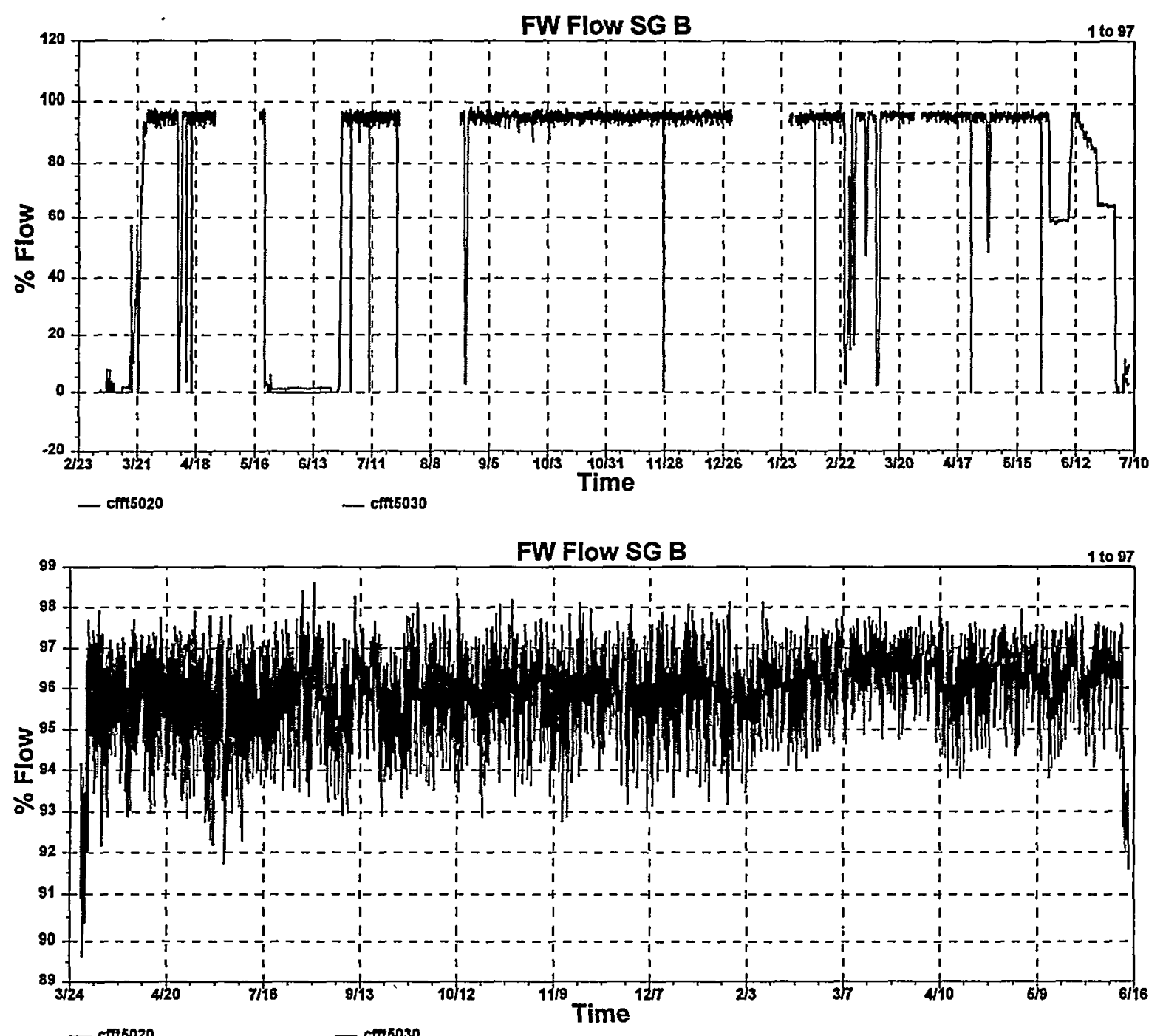

$-\operatorname{cnt} 6020$

FW Flow SG B

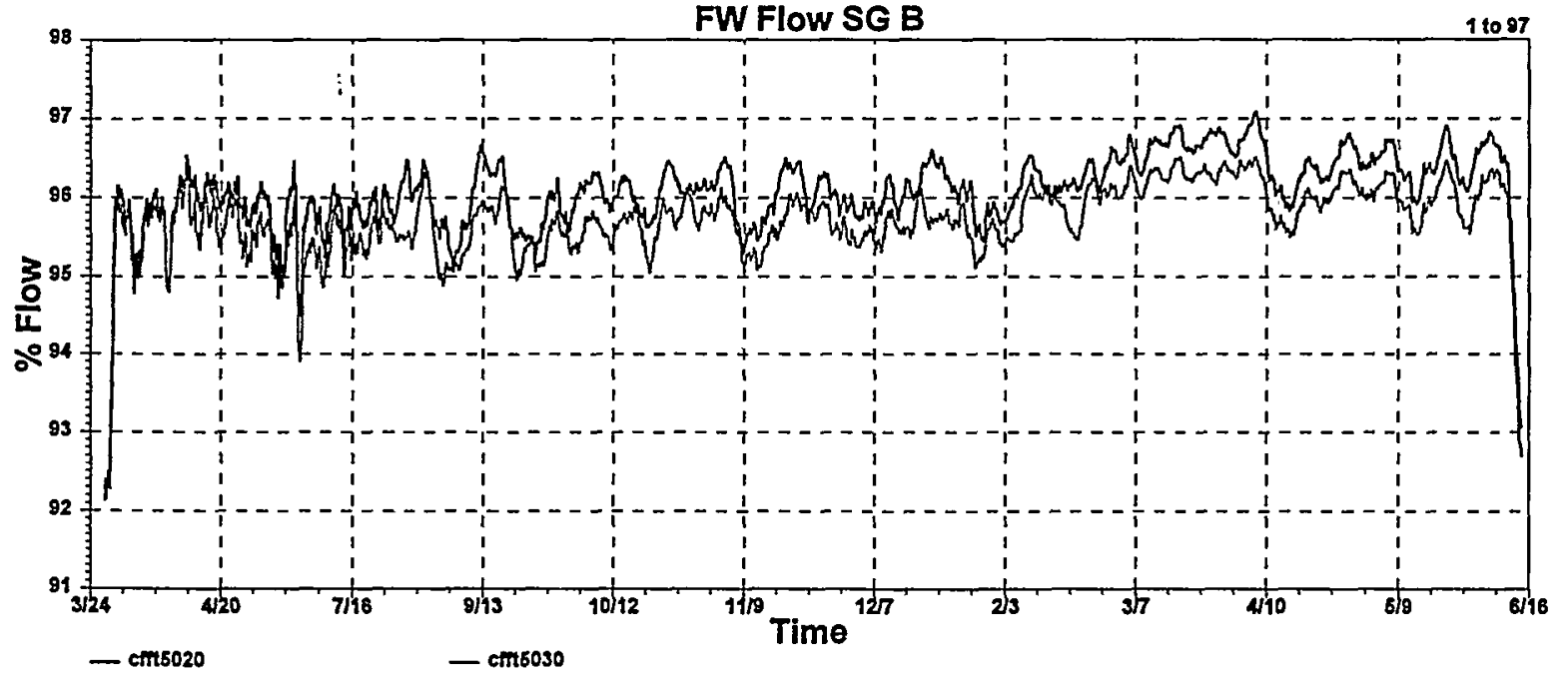

F.14 McGuire Cycle 1 Data for Feedwater Flow Signals for Loop B 

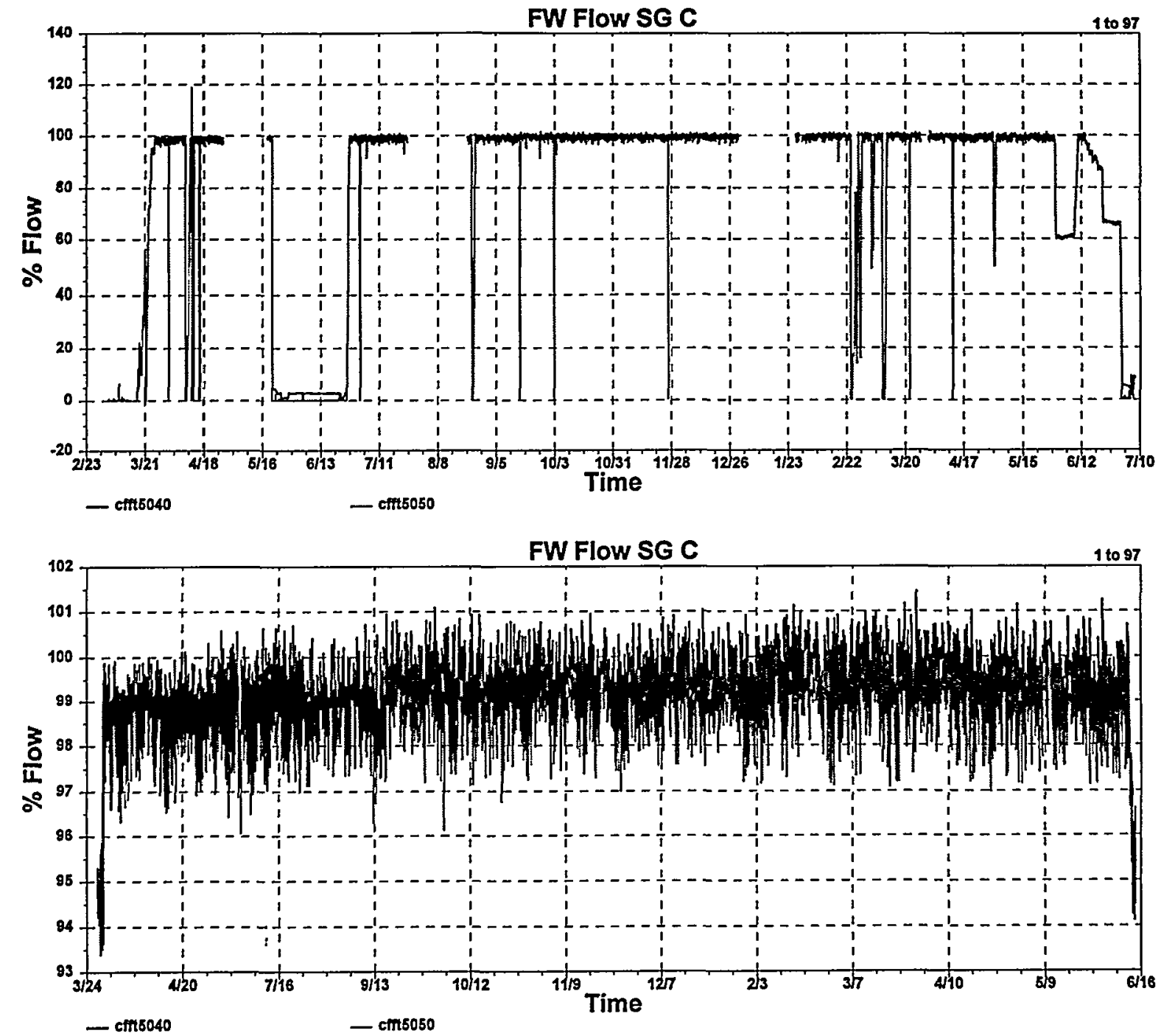

FW Flow SG C

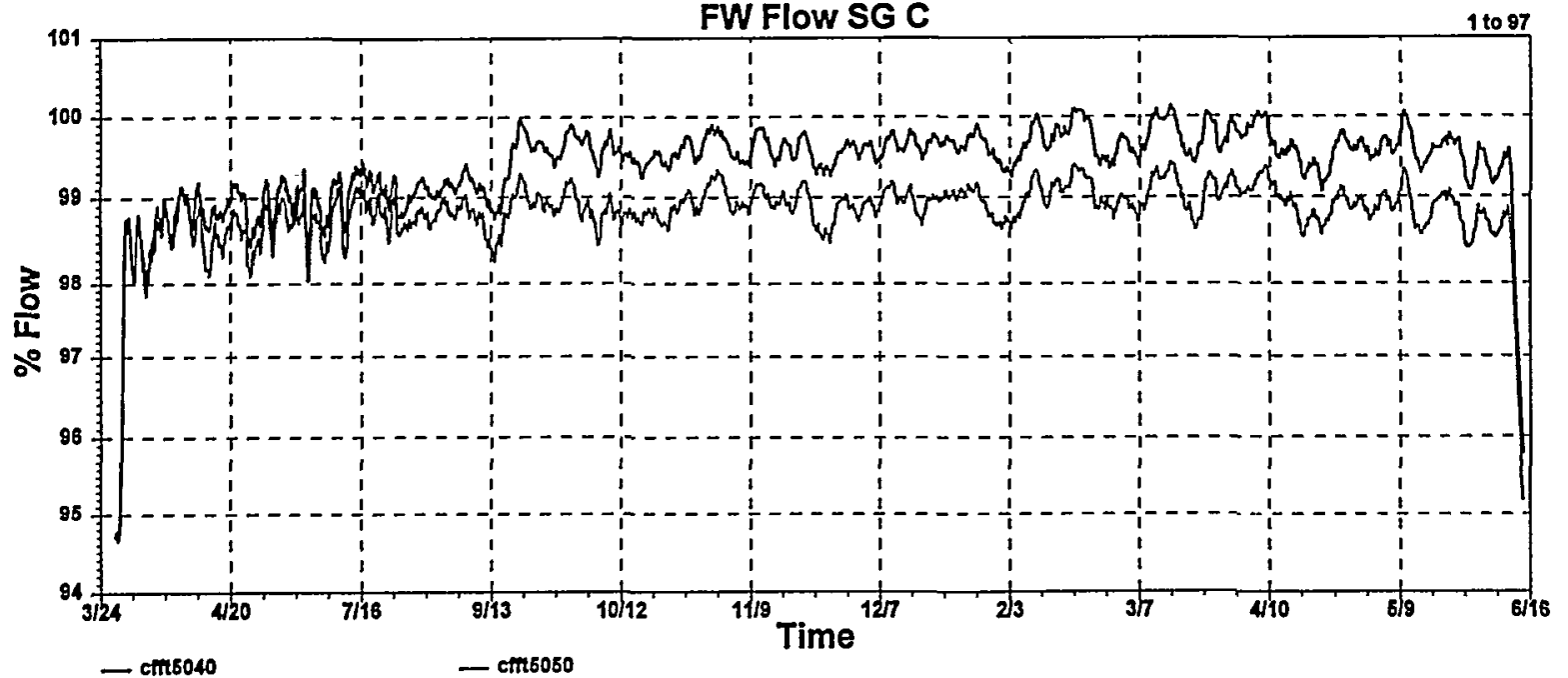

F.15 McGuire Cycle 1 Data for Feedwater Flow Signals for Loop C 


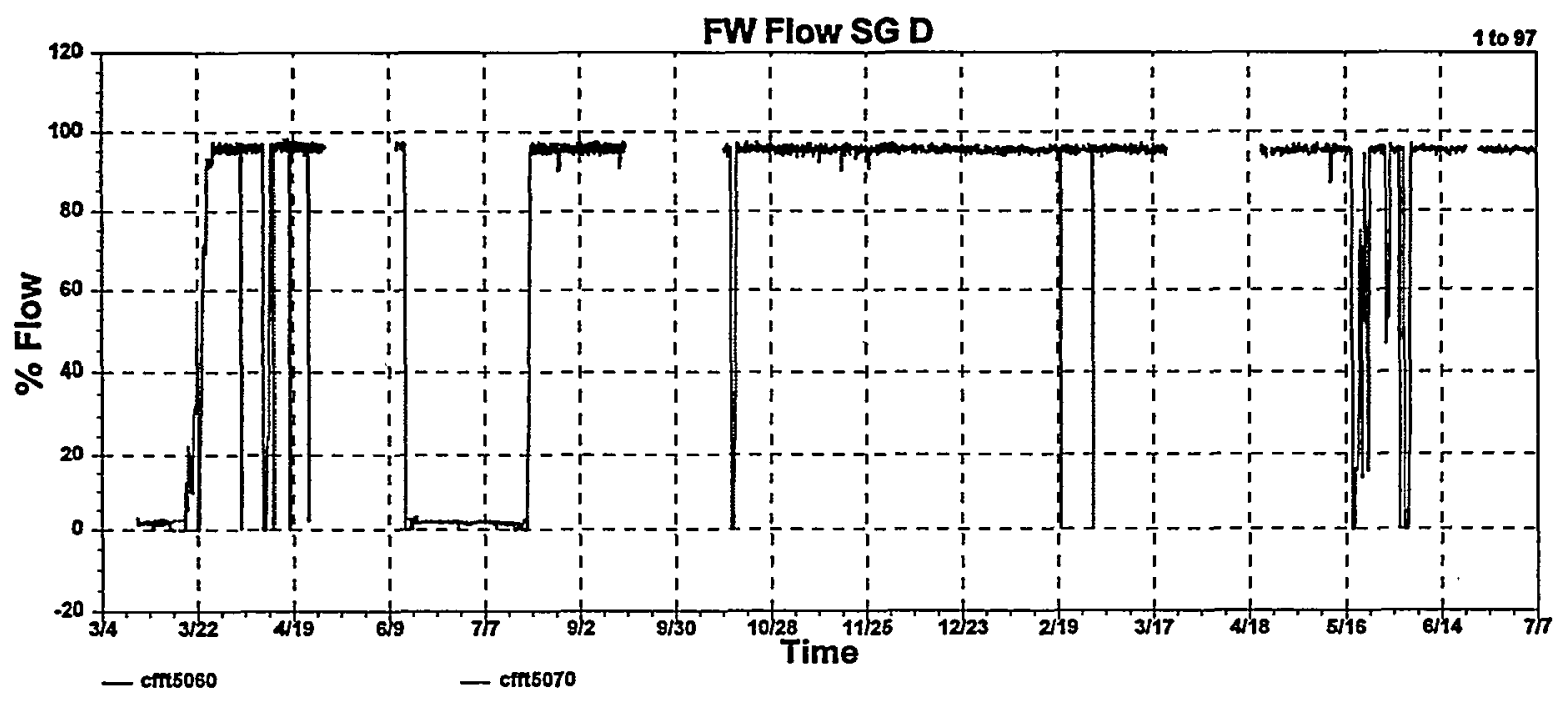

FW Flow SG D

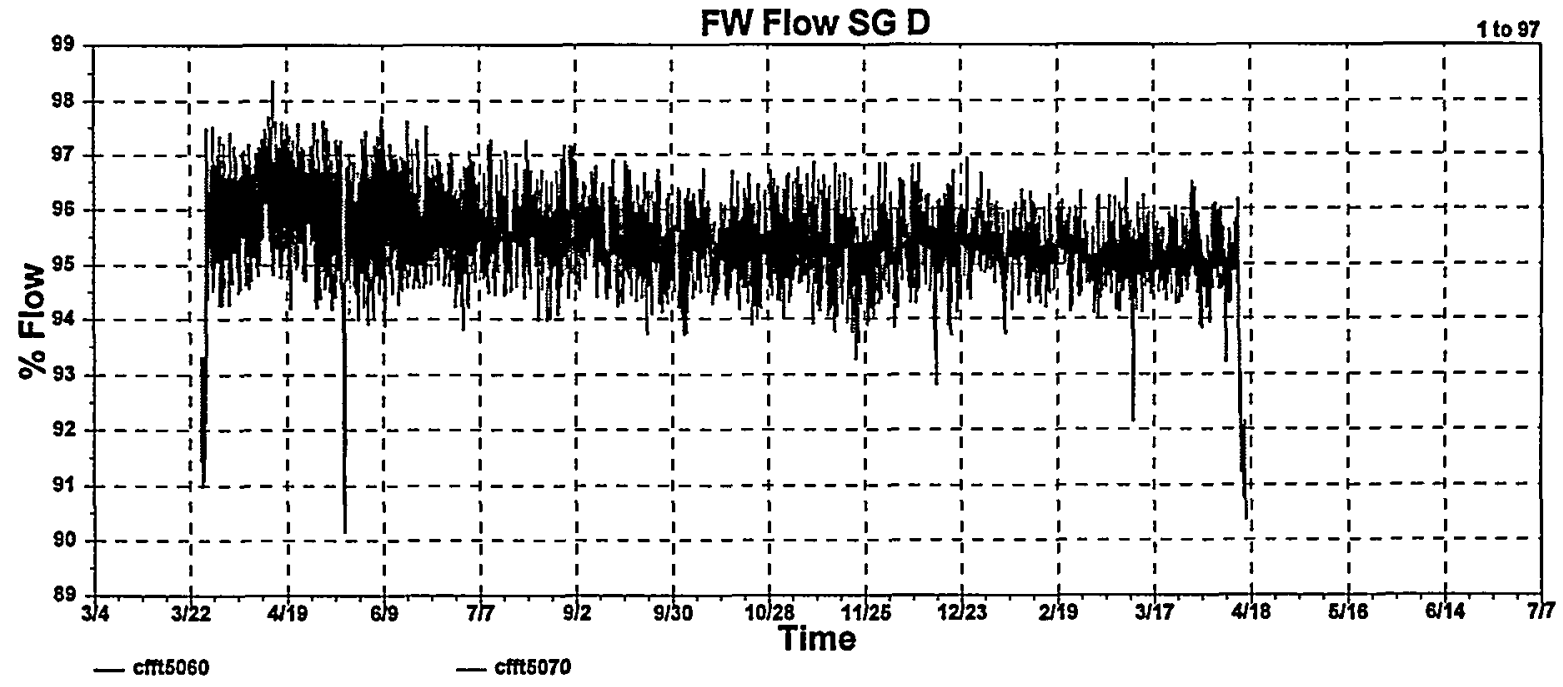

FW Flow SG D

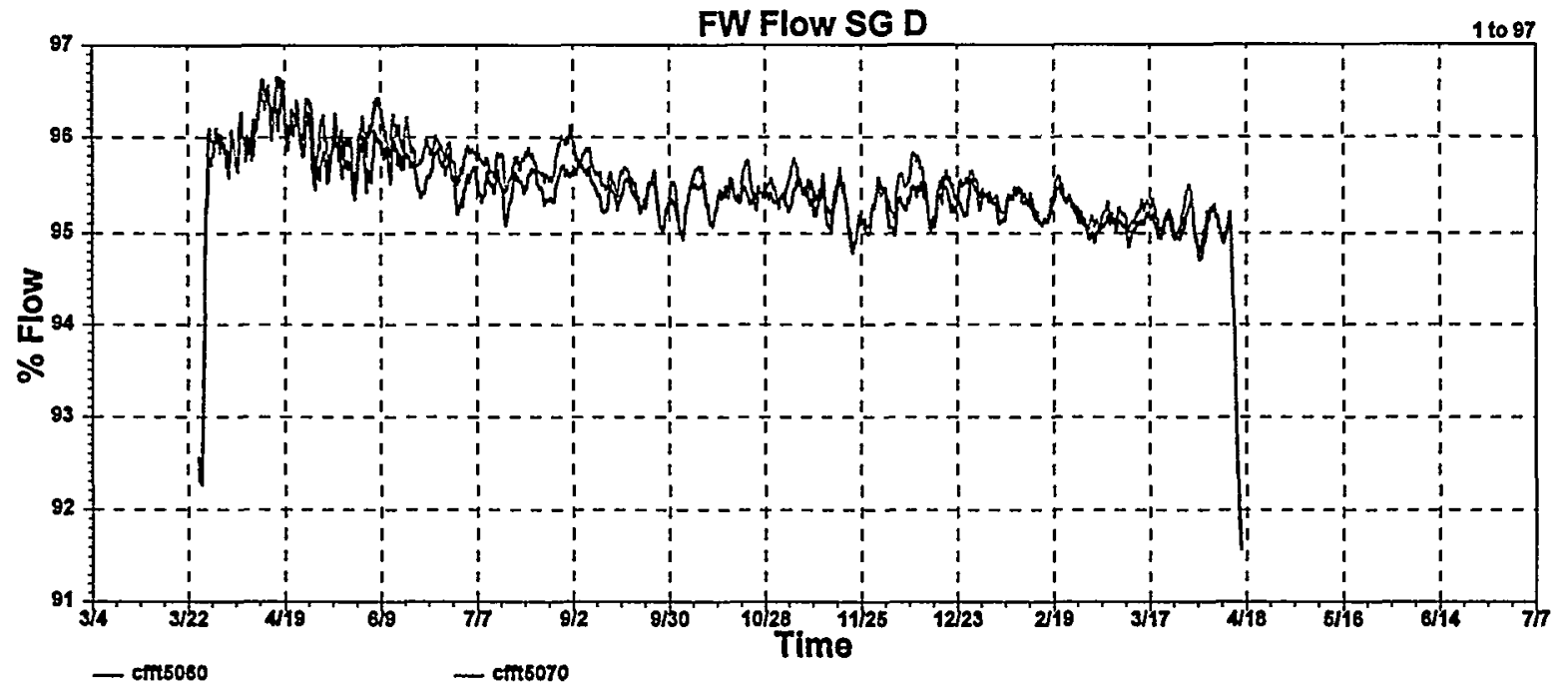

F.16 McGuire Cycle 1 Data for Feedwater Flow Signals for Loop D 

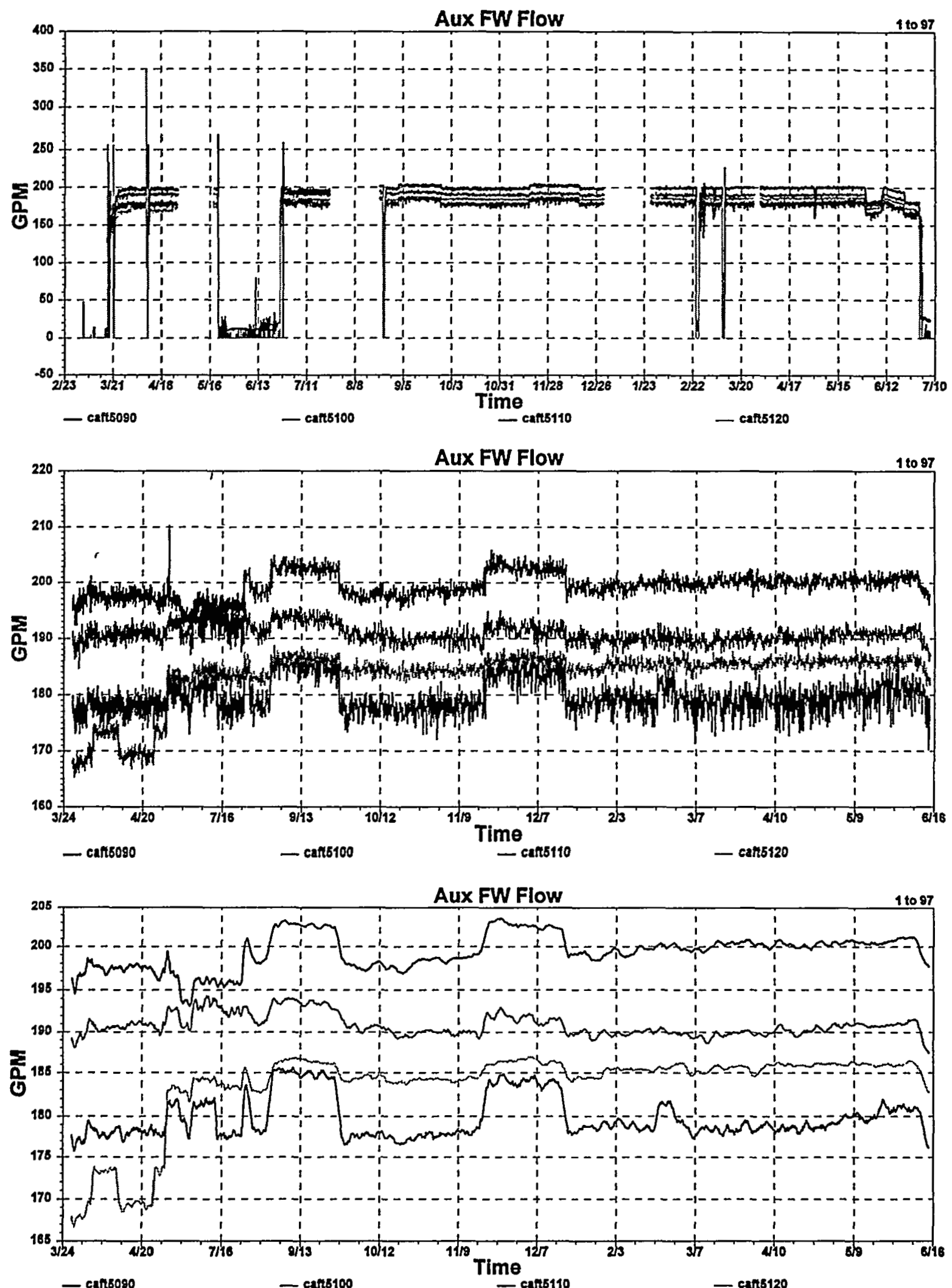

F.17 McGuire Cycle 1 Data for Auxiliary Feedwater Flow Signals 

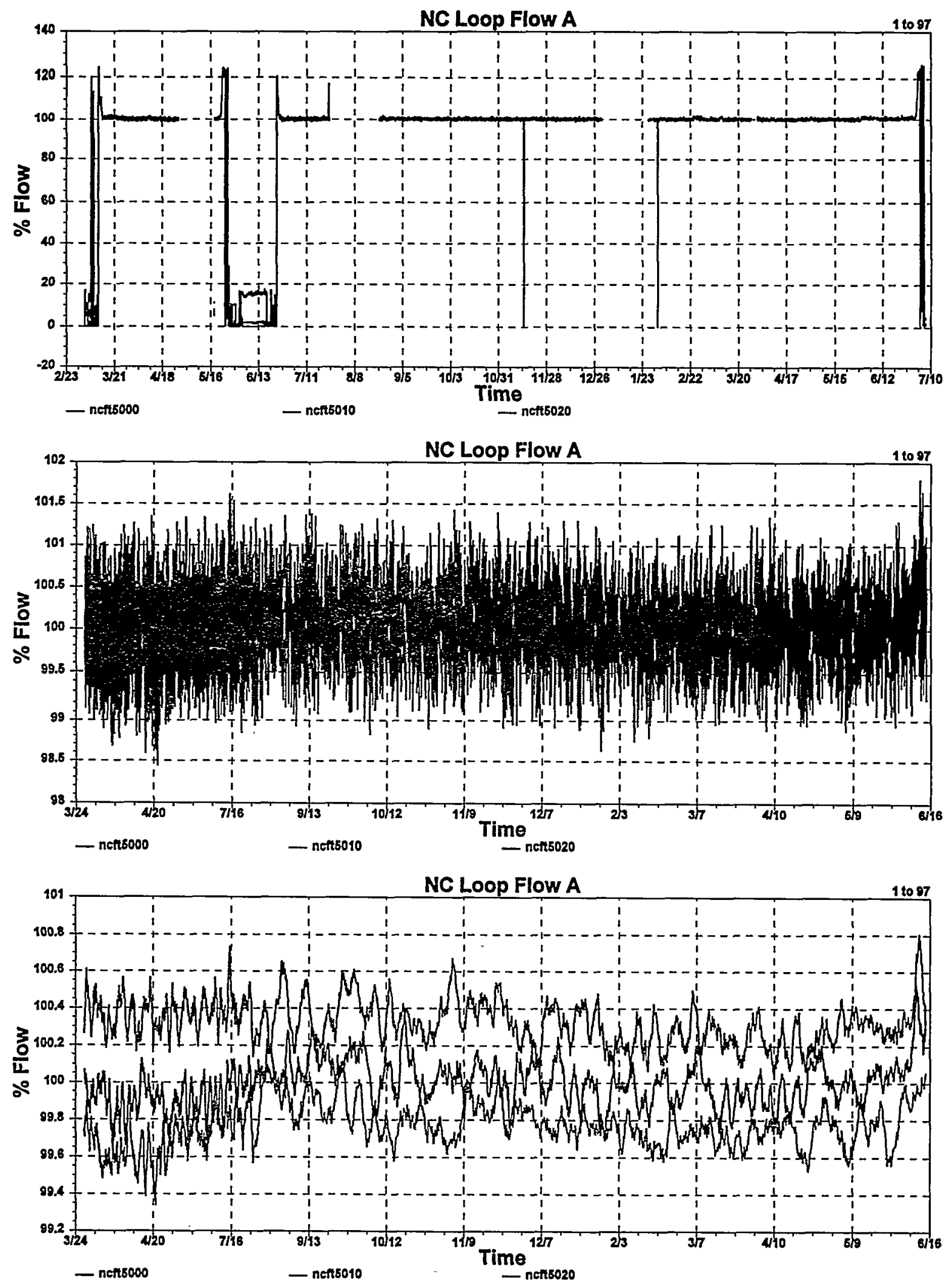

F.18 McGuire Cycle 1 Data for Reactor Coolant Flow Signals for Loop A 

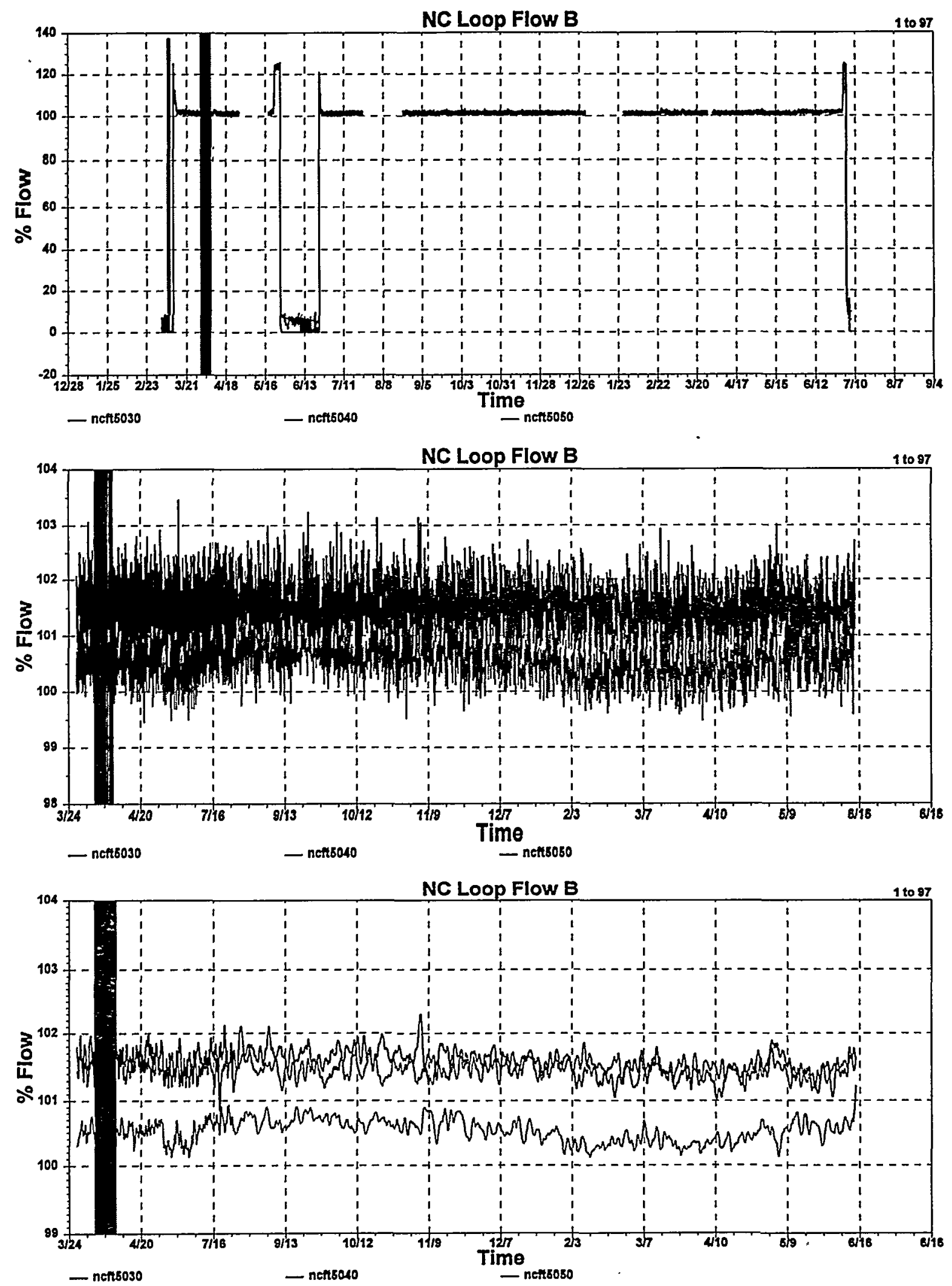

F.19 McGuire Cycle 1 Data for Reactor Coolant Flow Signals for Loop B 


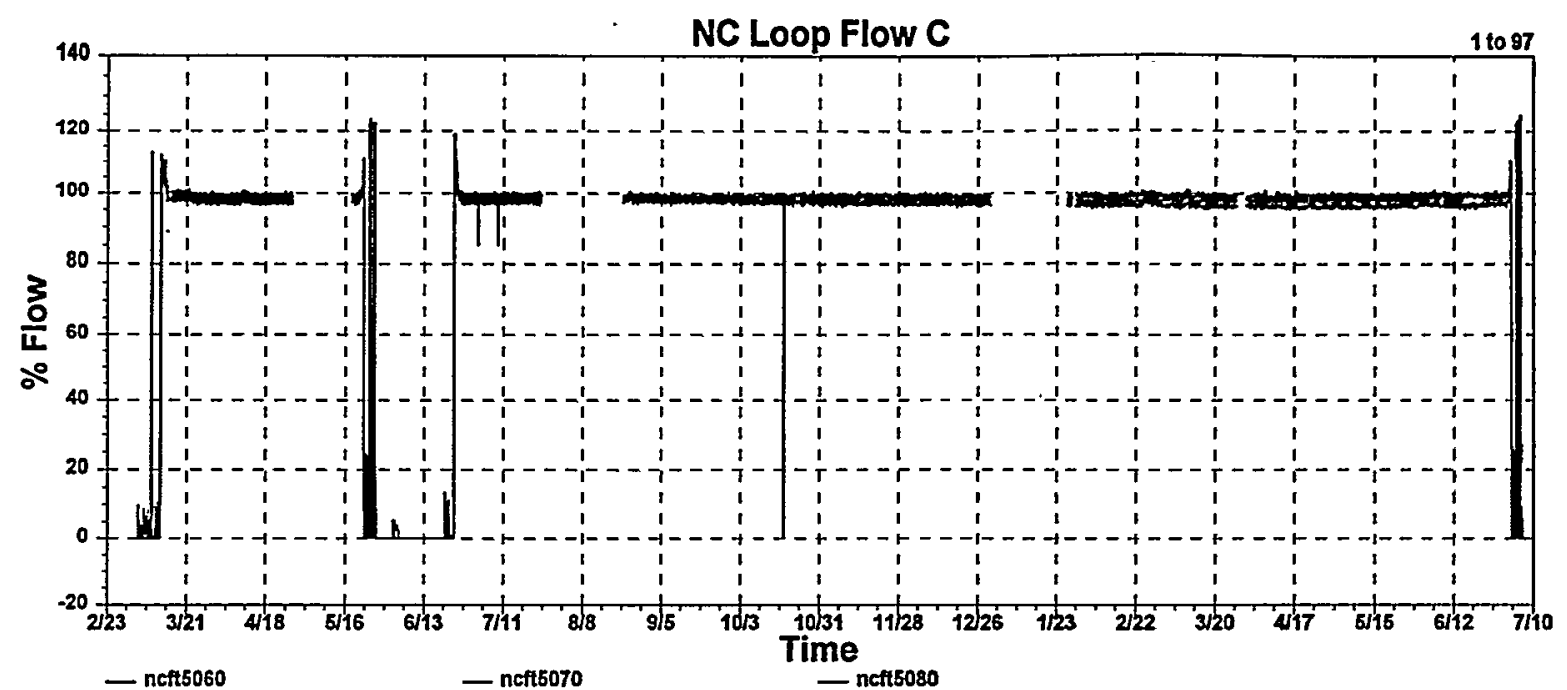

NC Loop Flow C 1 to 97

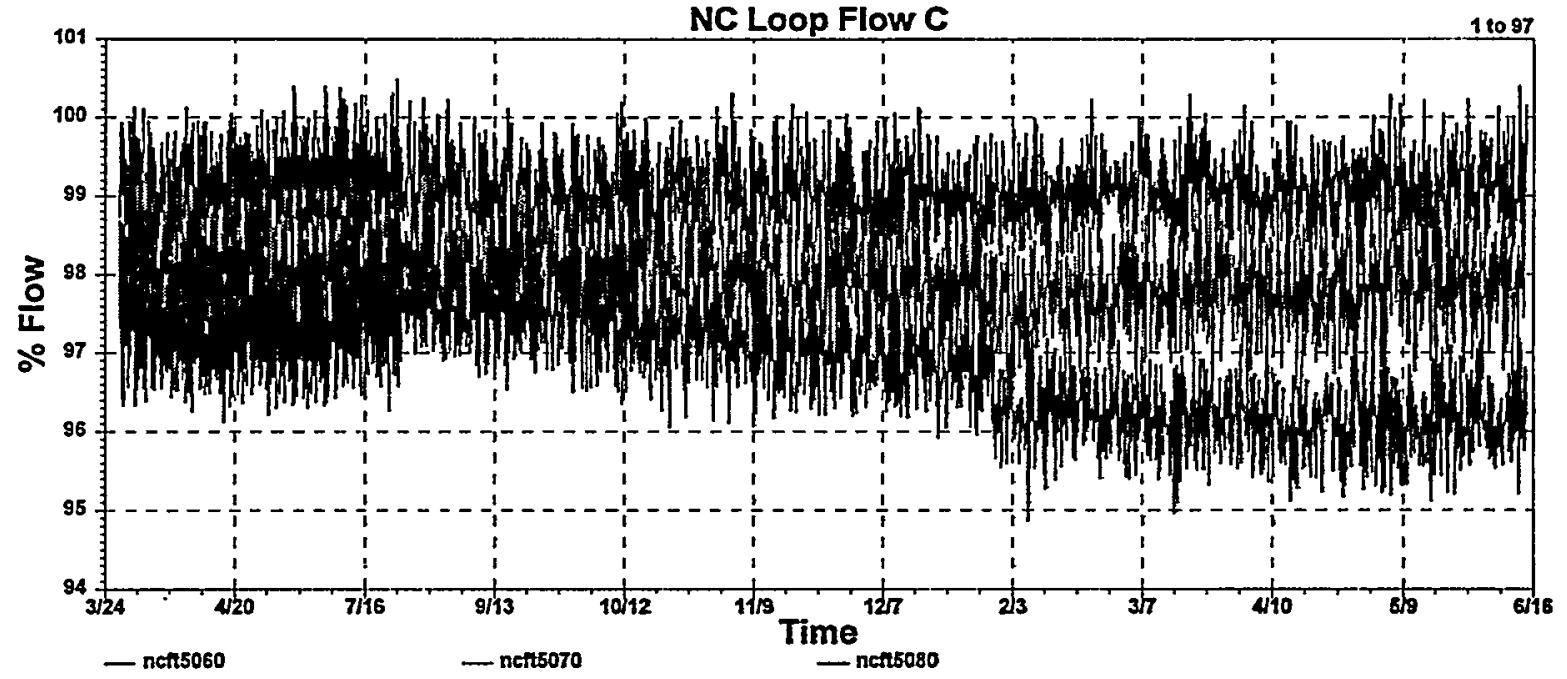

NC Loop Flow C

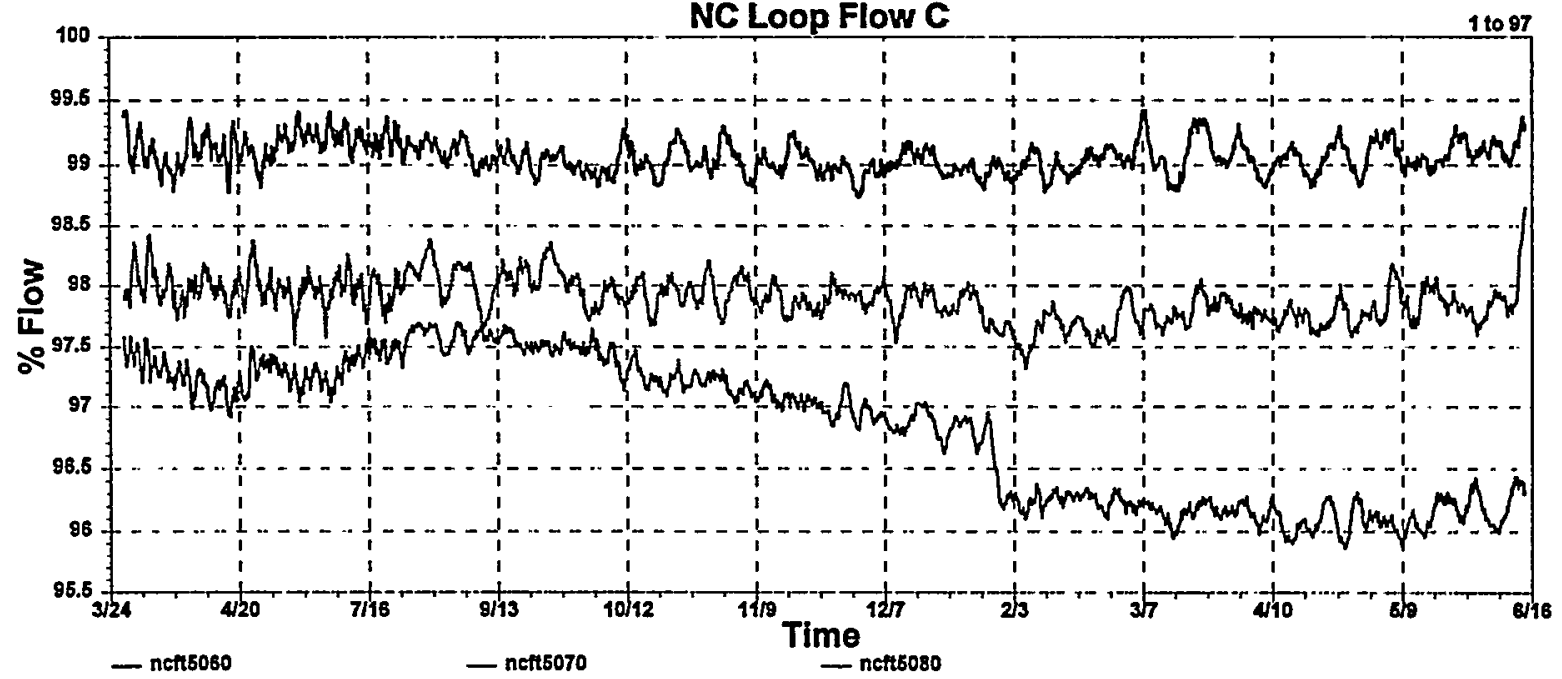

F.20 McGuire Cycle 1 Data for Reactor Coolant Flow Signals for Loop C 

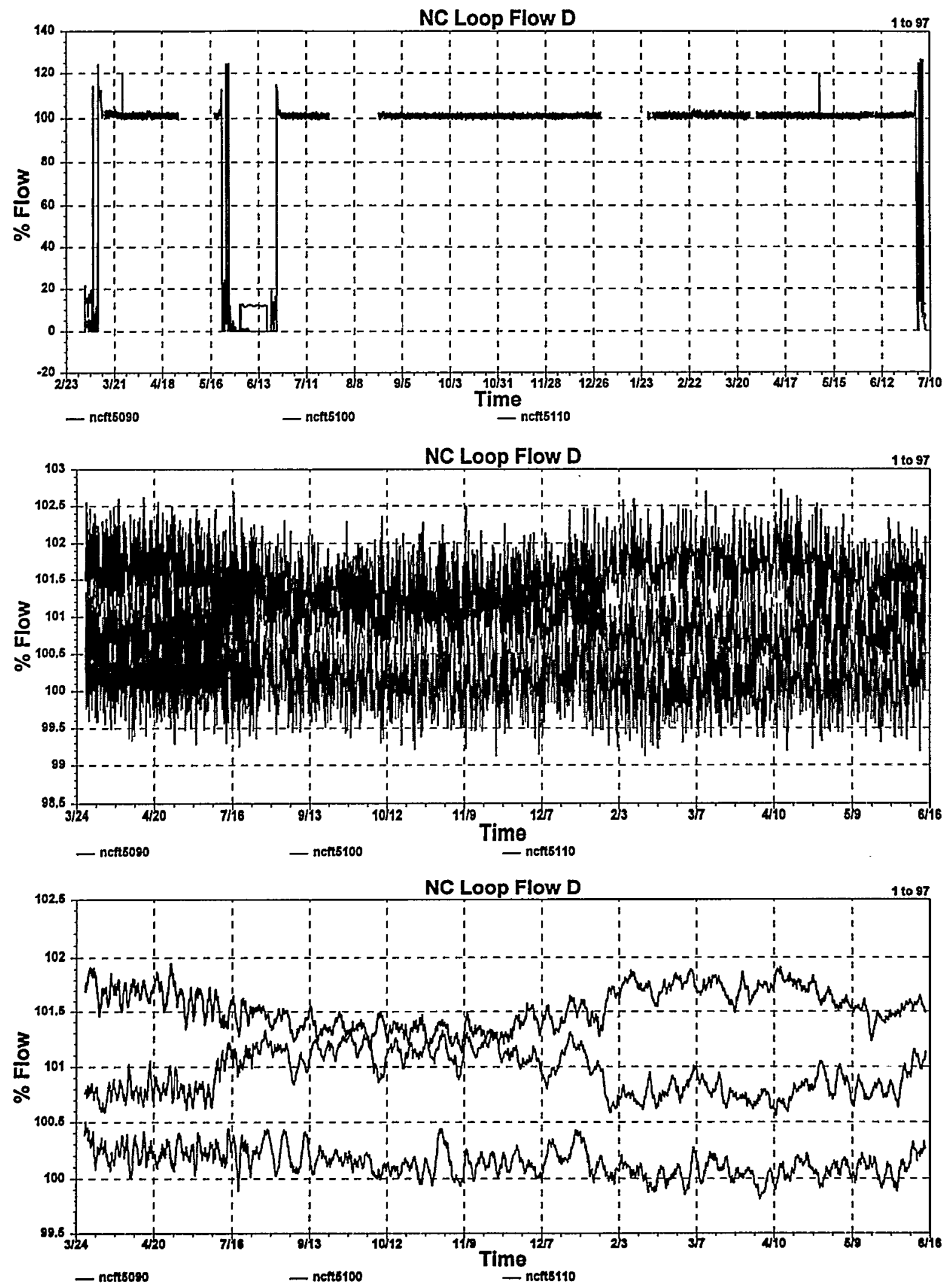

F.21 McGuire Cycle 1 Data for Reactor Coolant Flow Signals for Loop D 

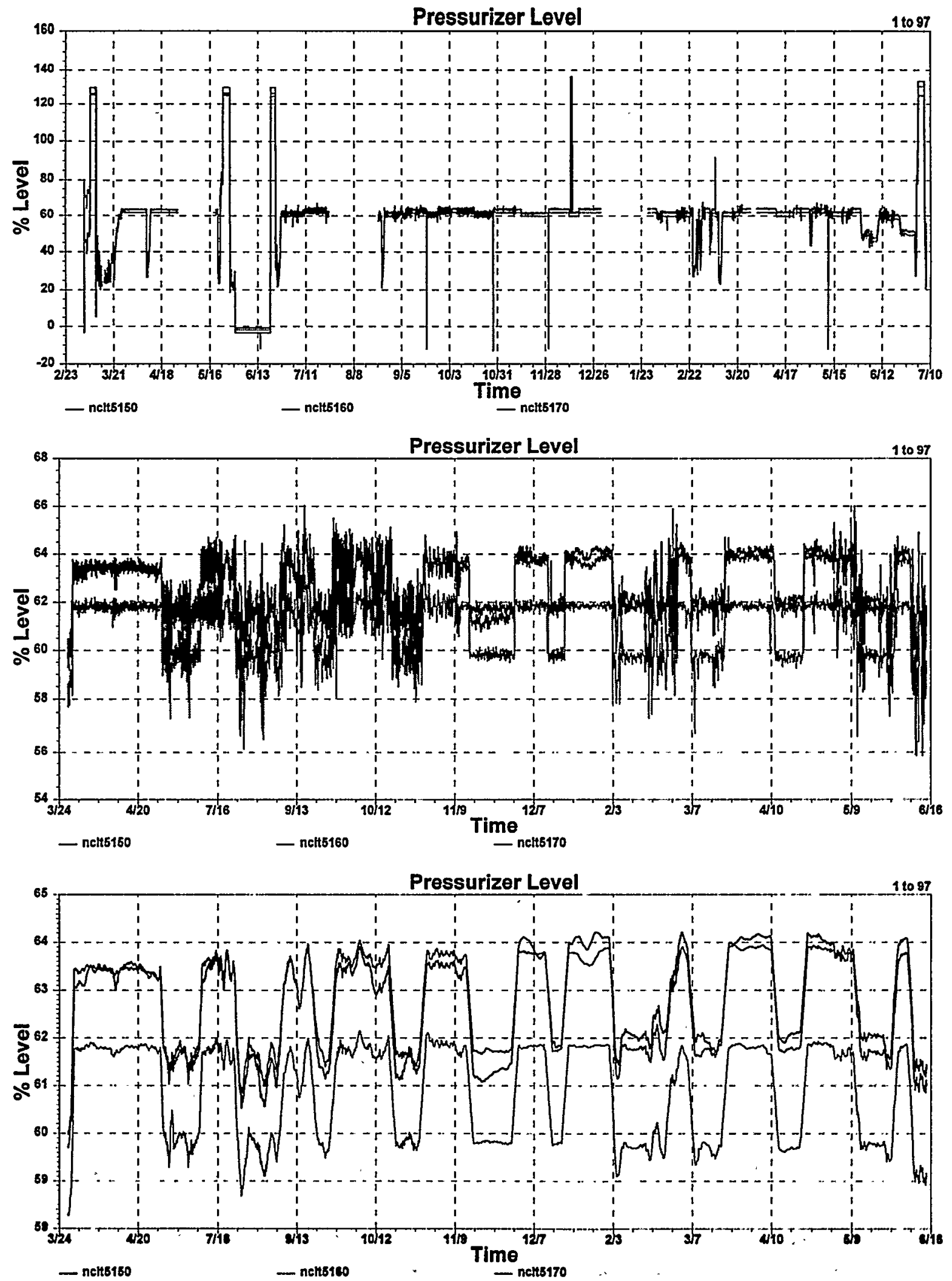

F.22 McGuire Cycle 1 Data for Pressurizer Level Signals 

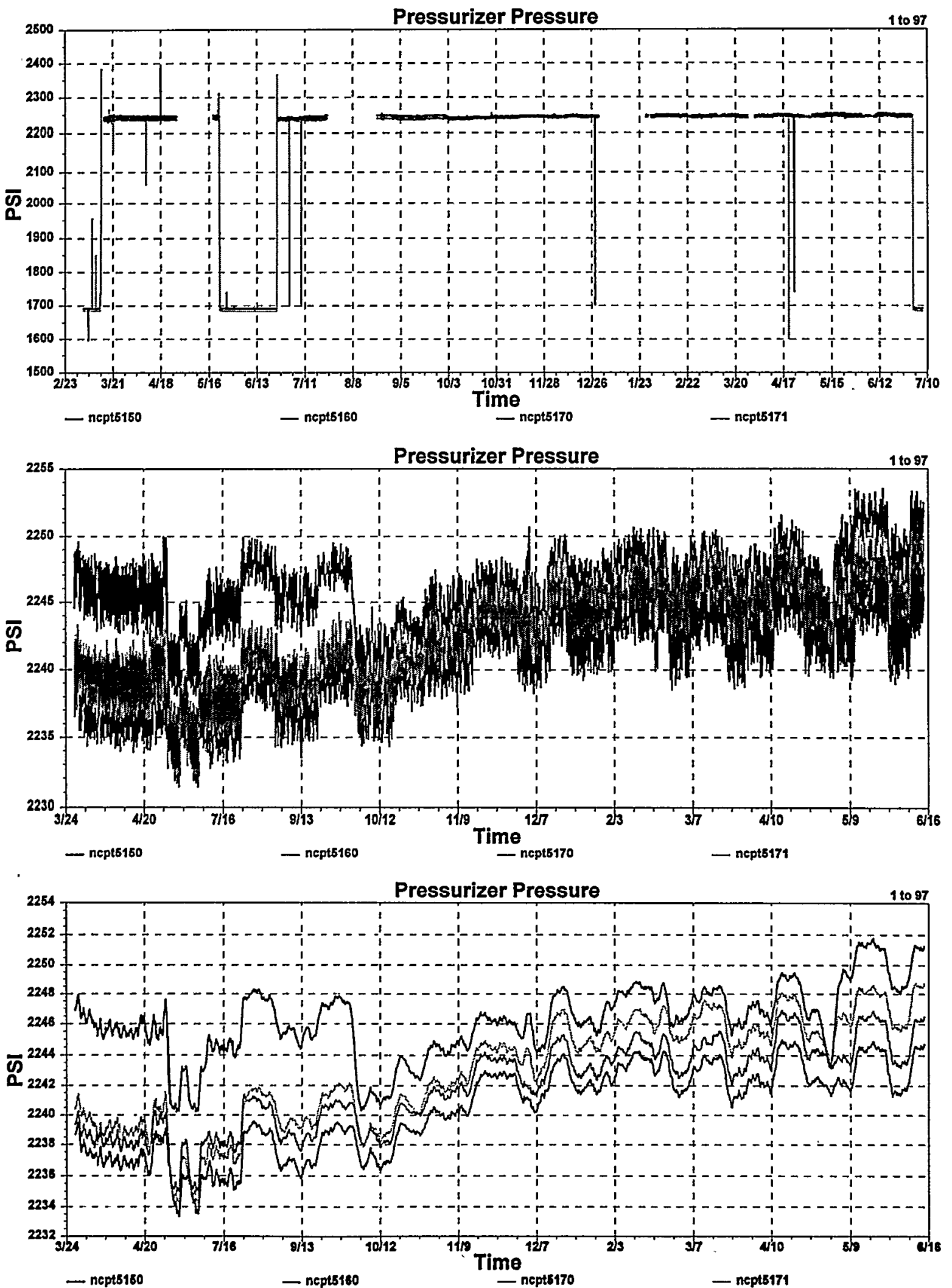

F.23 McGuire Cycle 1 Data for Pressurizer Pressure Signals 

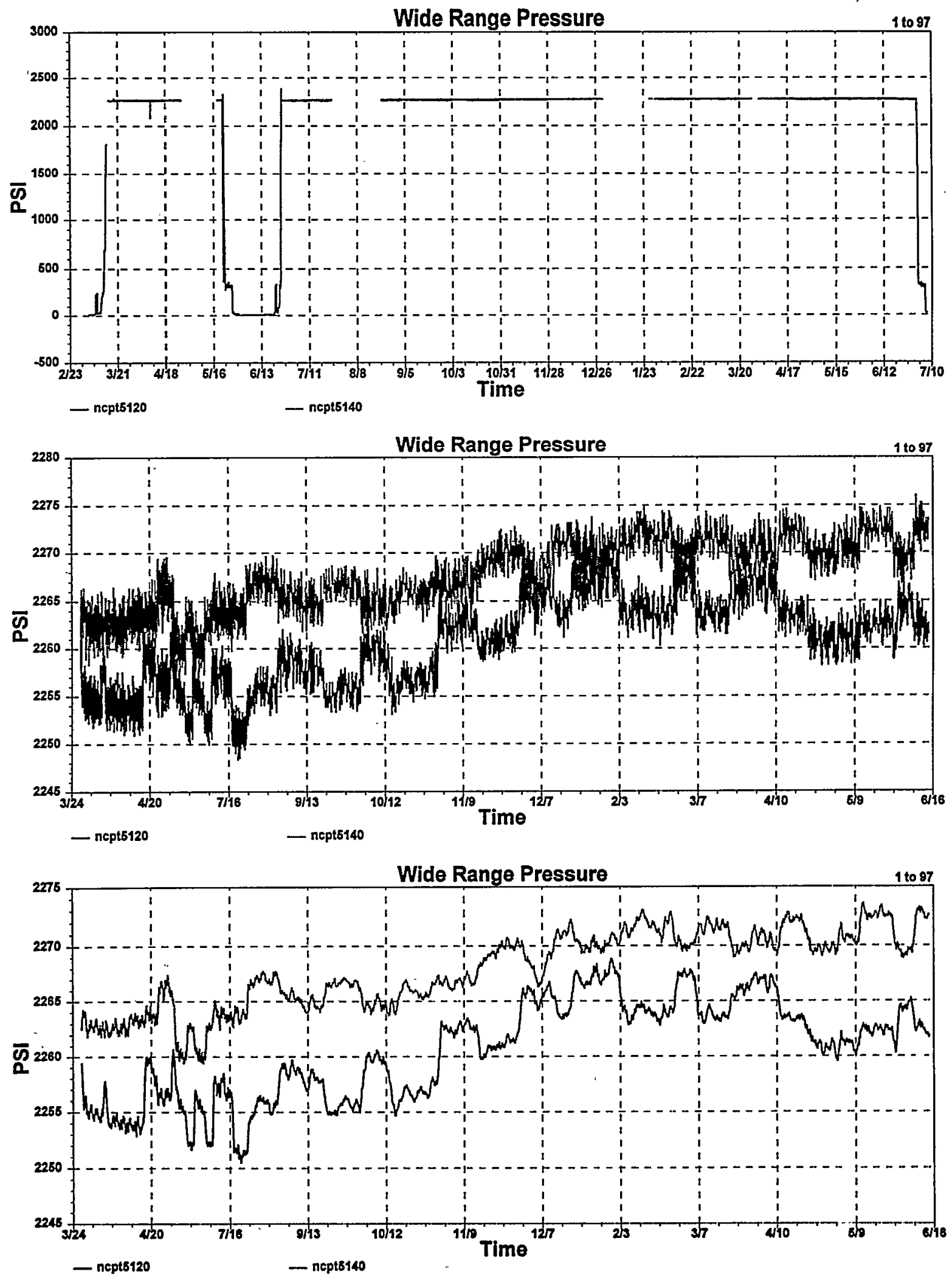

F.24 McGuire Cycle 1 Data for Wide-Range Pressure Signals 

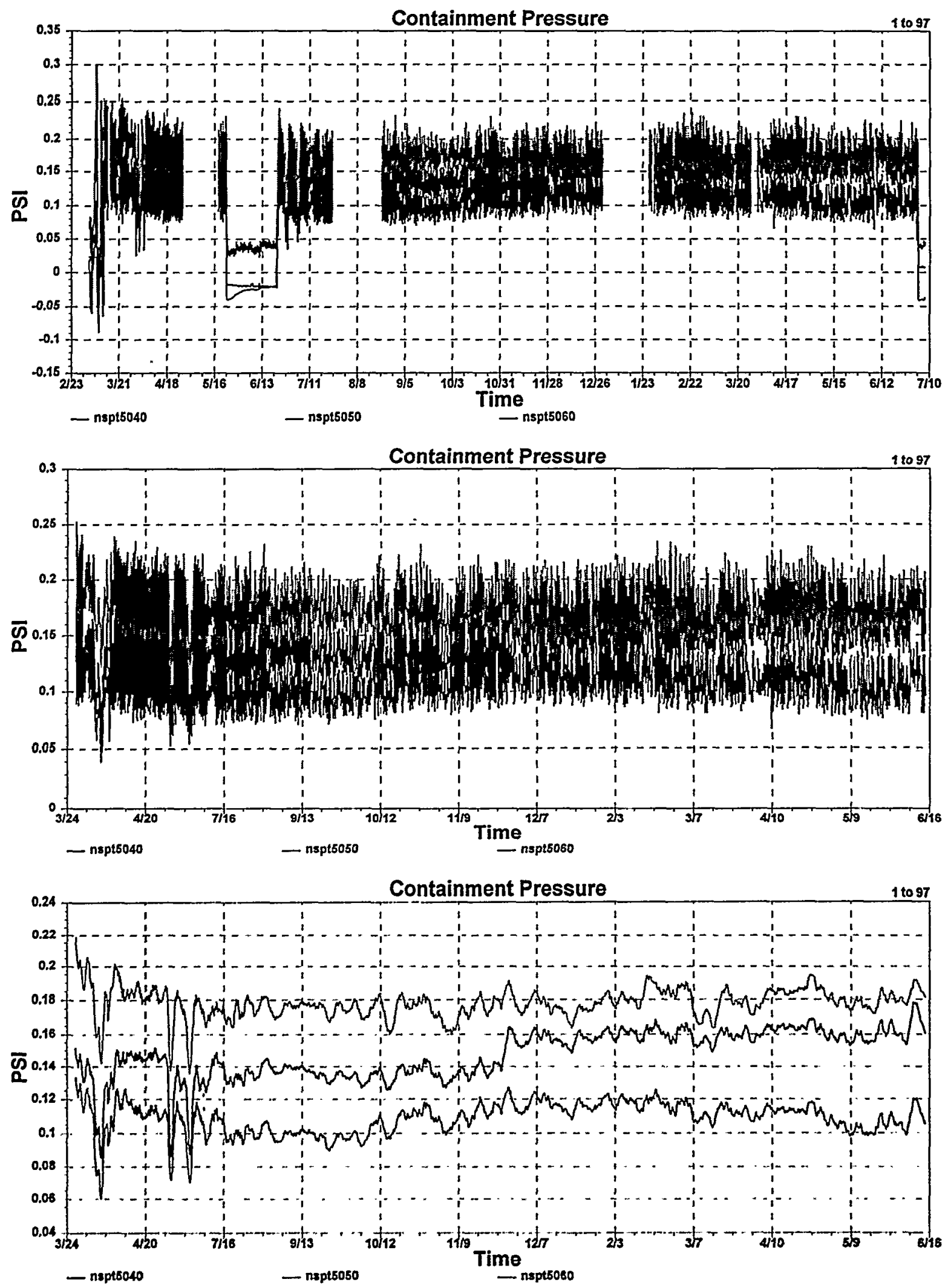

F.25 McGuire Cycle 1 Data for Containment Pressure Signals 

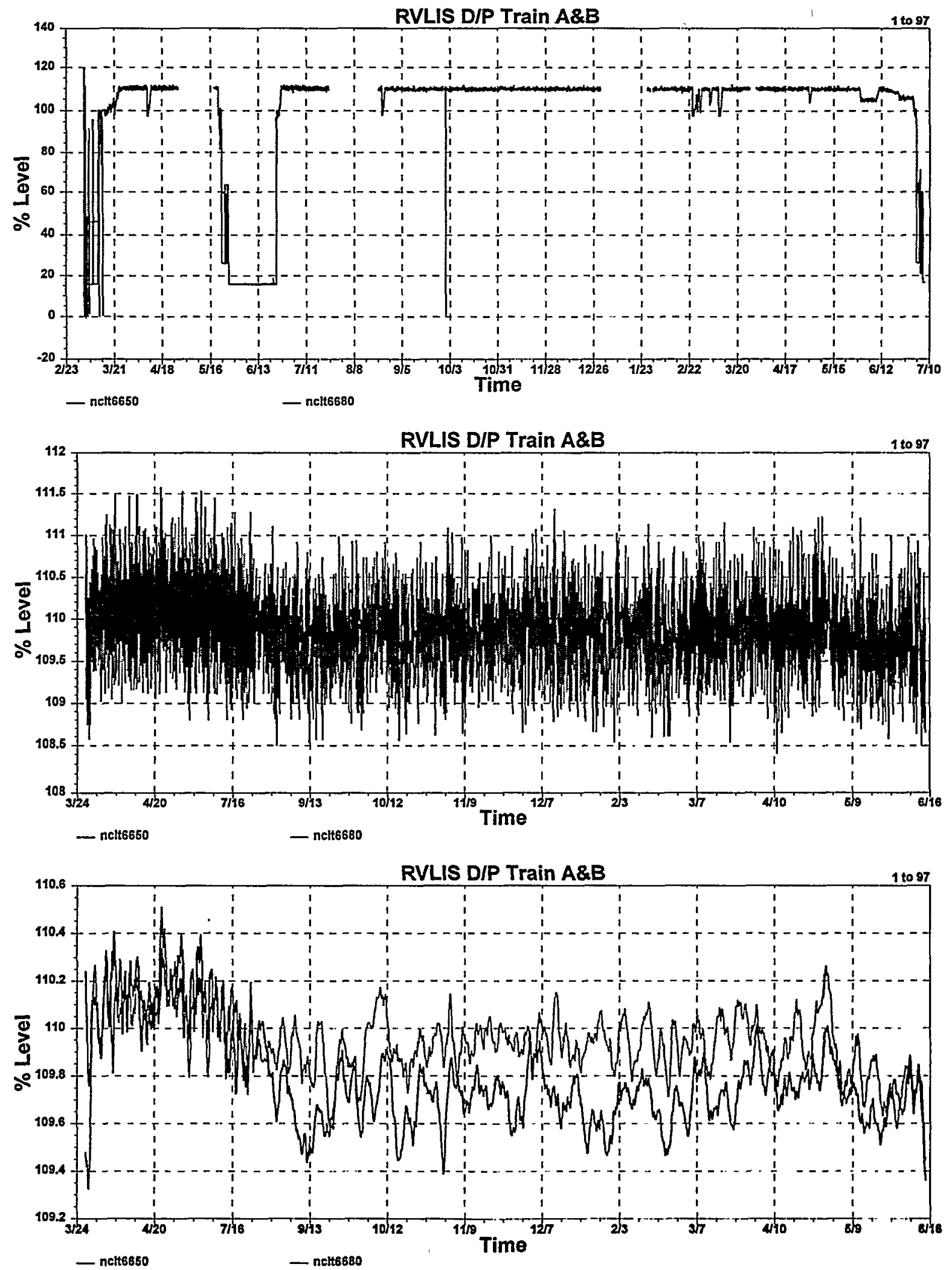

F.26 McGuire Cycle 1 Data for Reactor Vessel Level Indication System Signals 
Turbine Impulse Chamb. Press

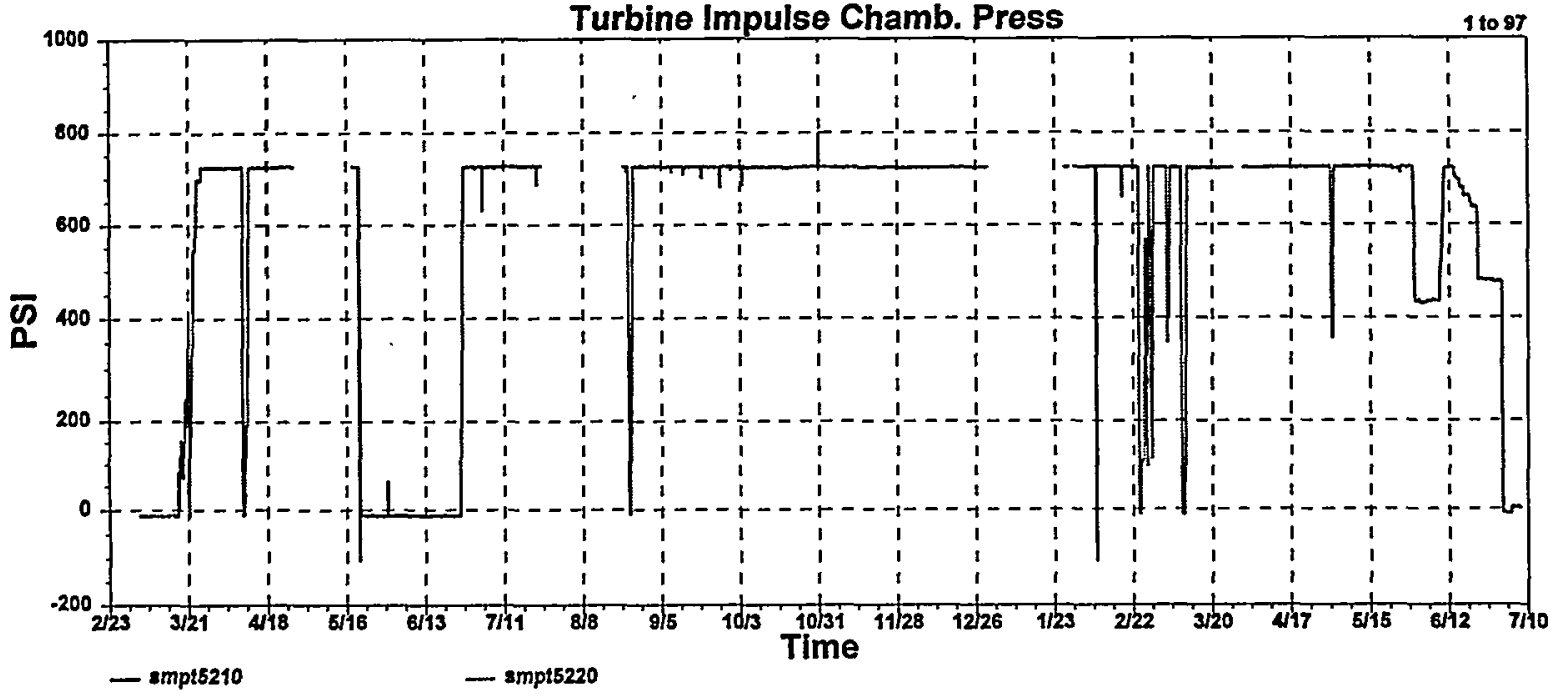

Turbine Impulse Chamb. Press

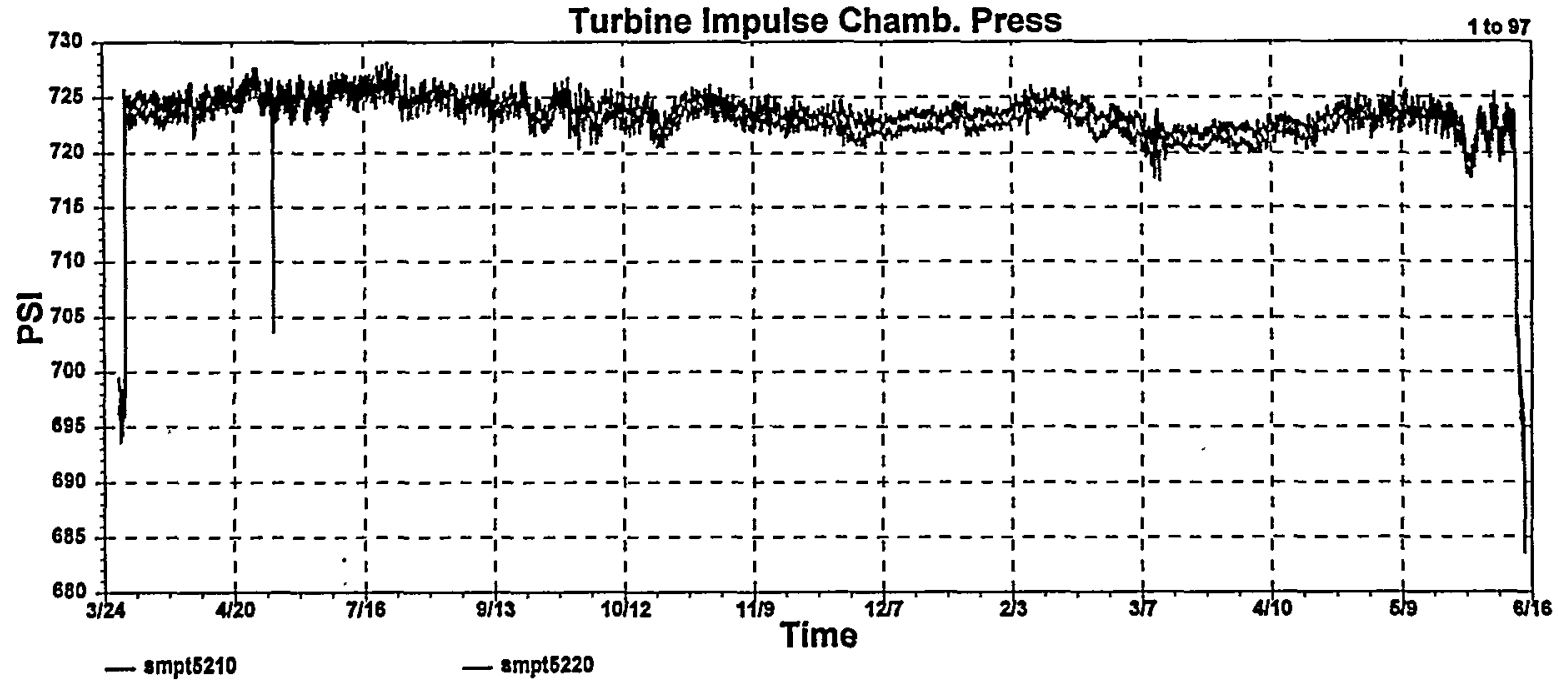

Turbine Impulse Chamb. Press

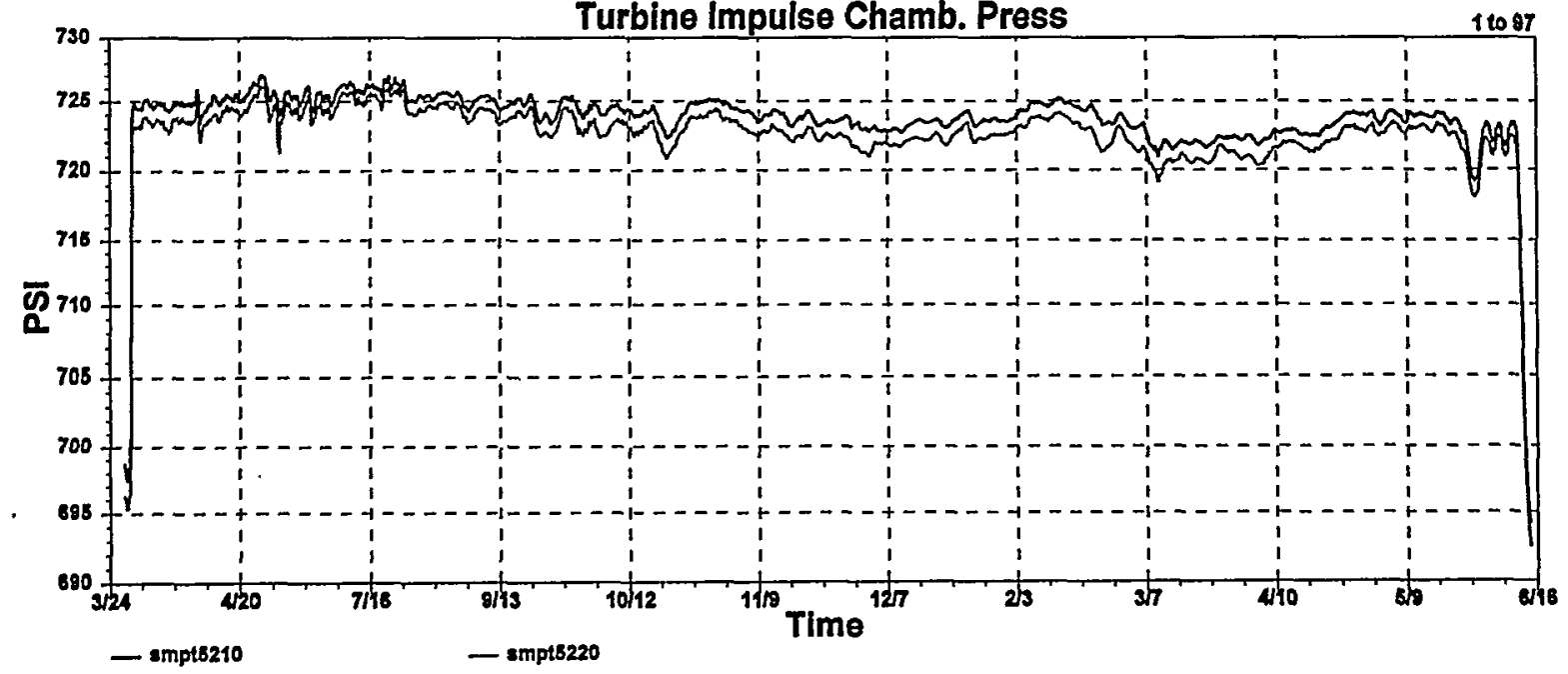

F.27 McGuire Cycle 1 Data for Turbine Impulse Pressure Signals 

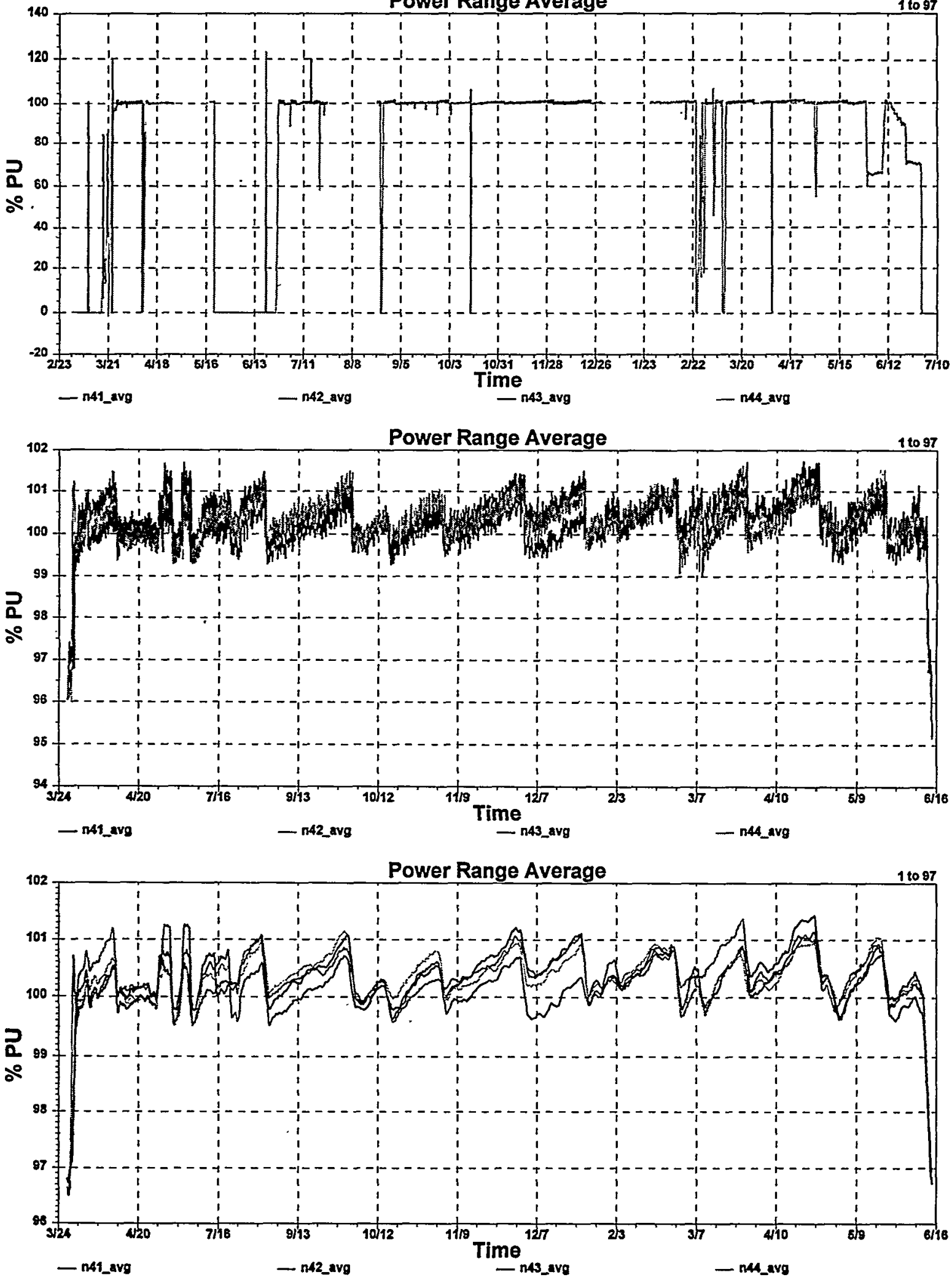

F.28 McGuire Cycle 1 Data for Neutron Sensor Signals 
Hot Leg RTDs Loop A

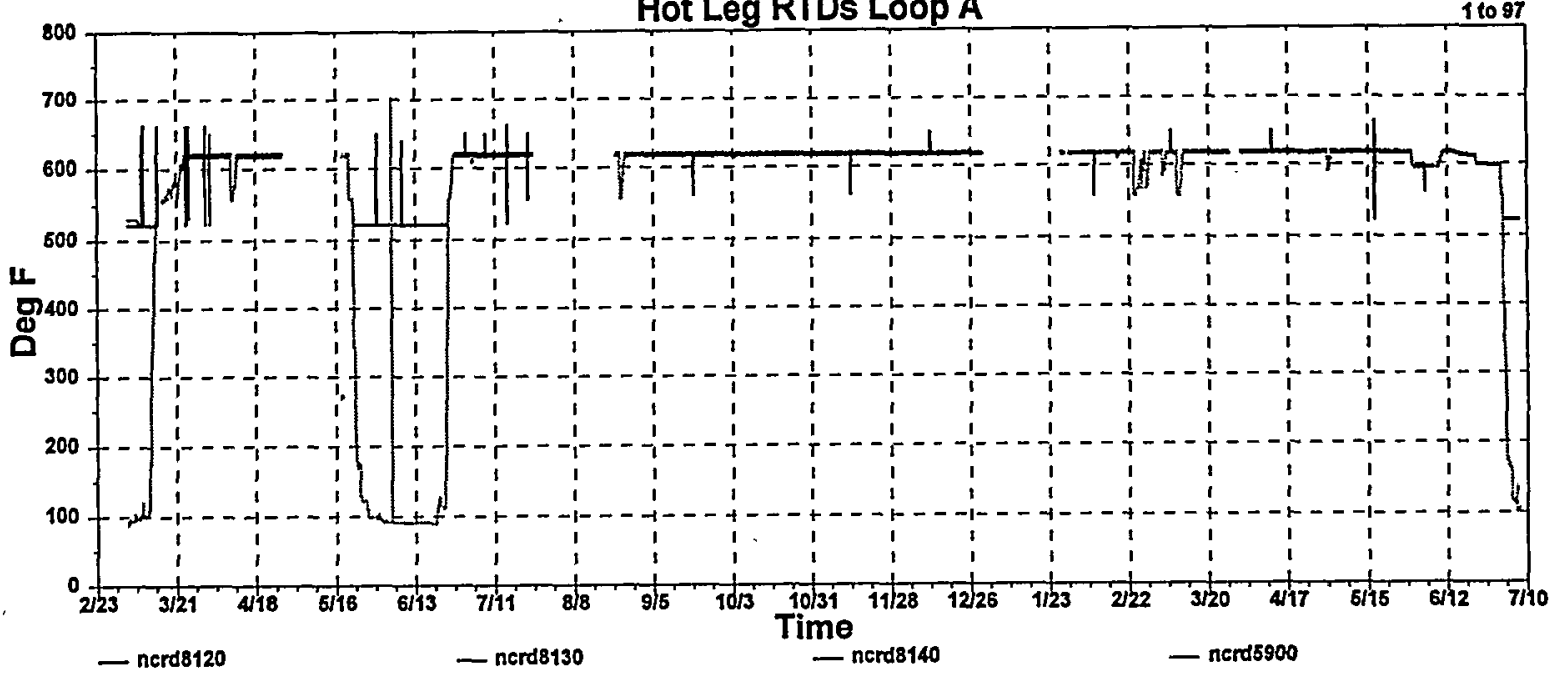

Hot Leg RTDs Loop A

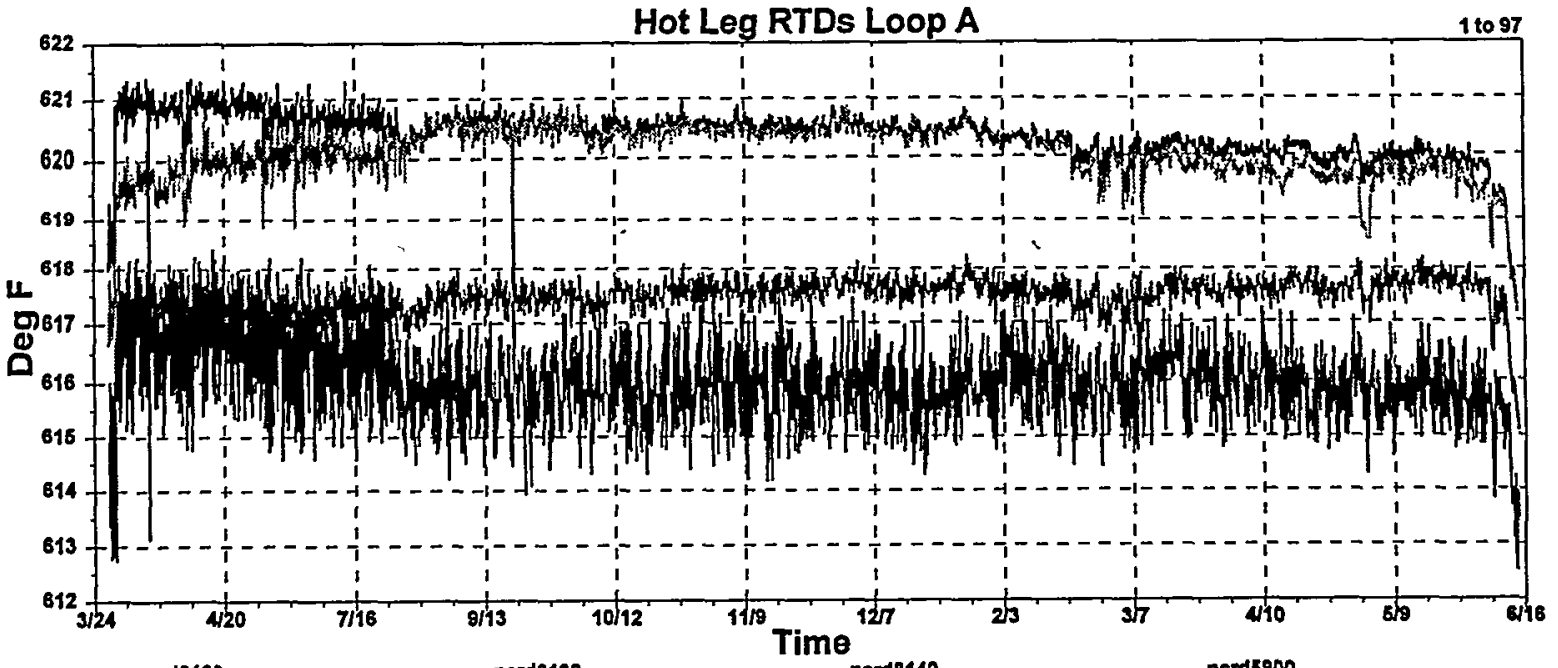

- nerd8120

- ncrd8130

- nerd8140

- nerd5900

Hot Leg RTDs Loop A

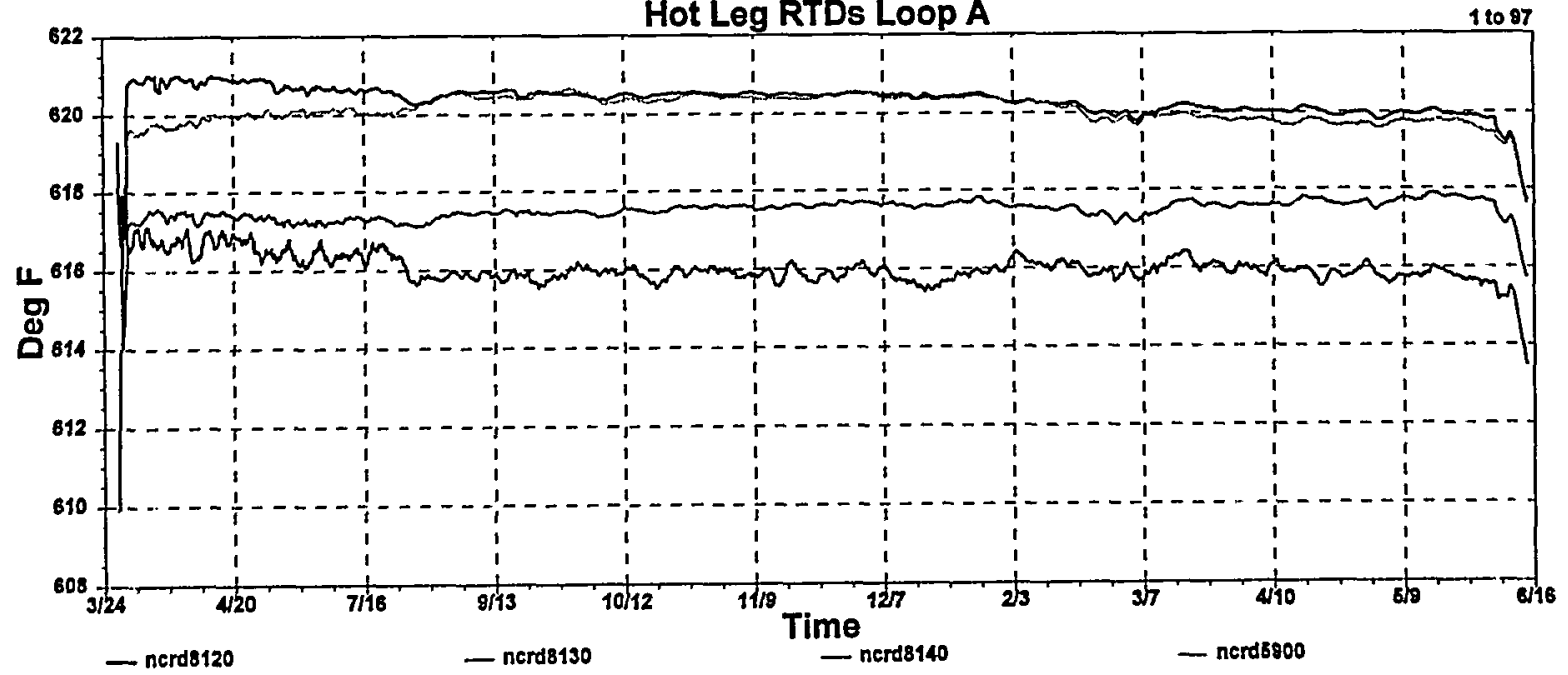

F.29 McGuire Cycle 1 Data for Hot Leg RTD Signals for Loop A 

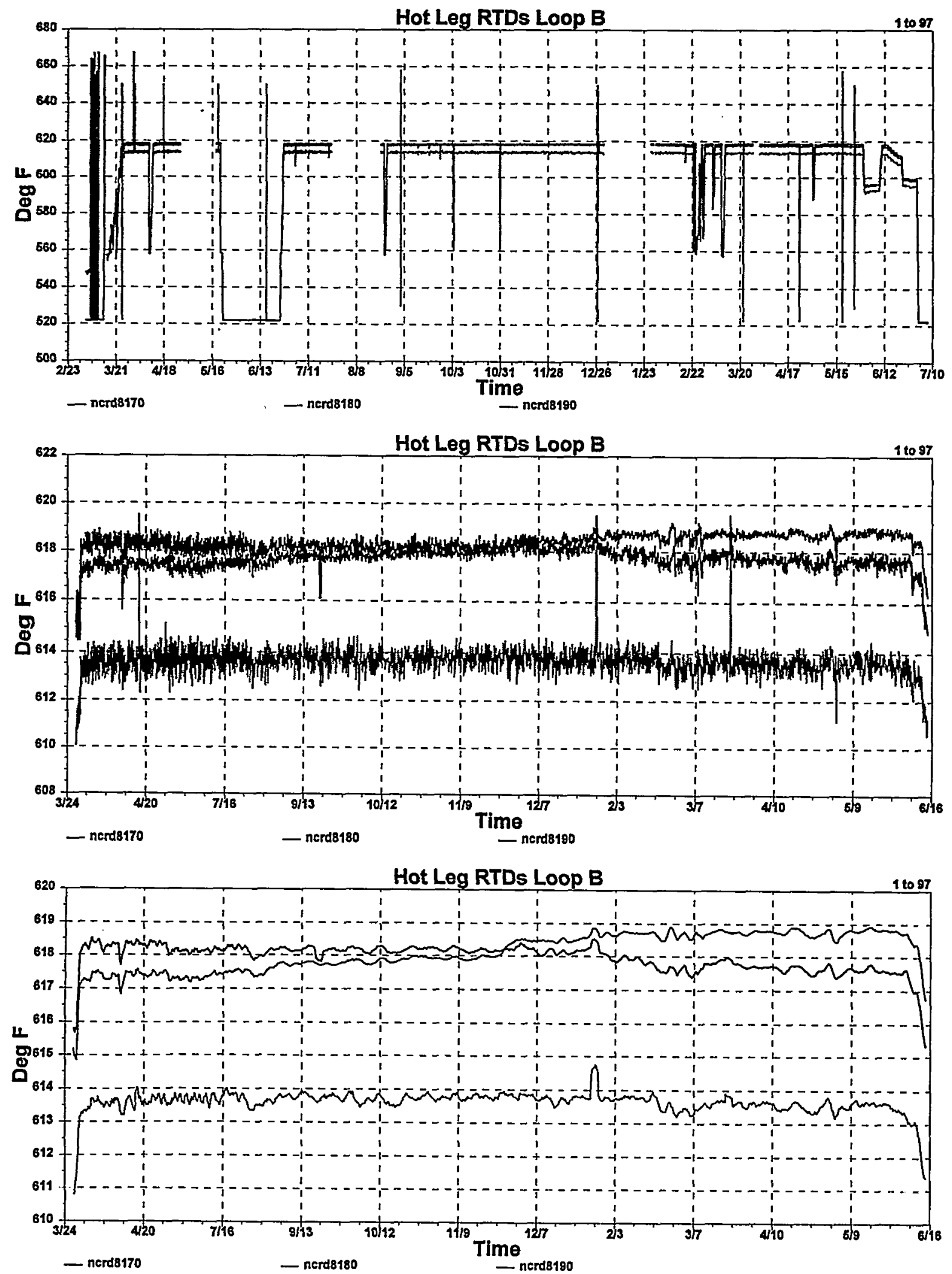

F.30 McGuire Cycle 1 Data for Hot Leg RTD Signals for Loop B 


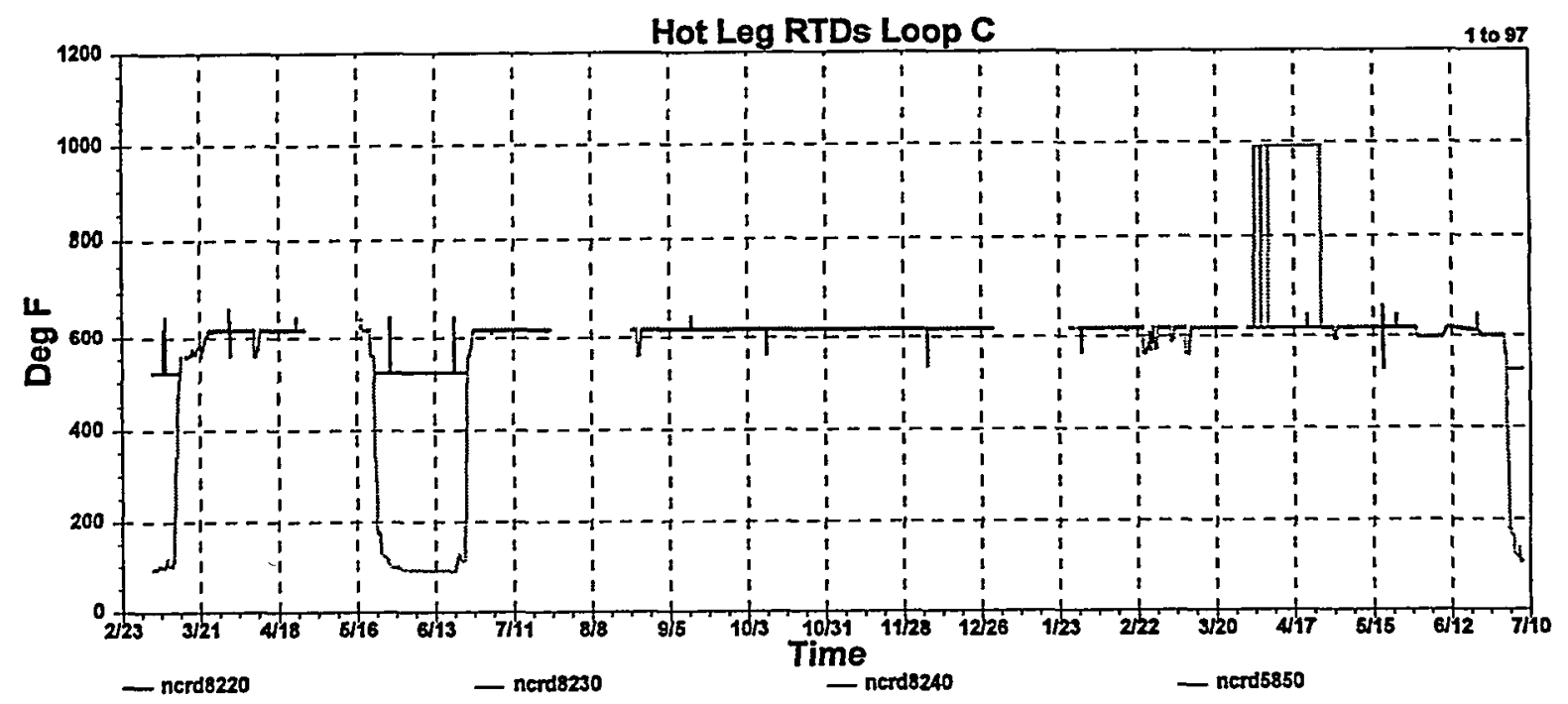

Hot Leg RTDs Loop C

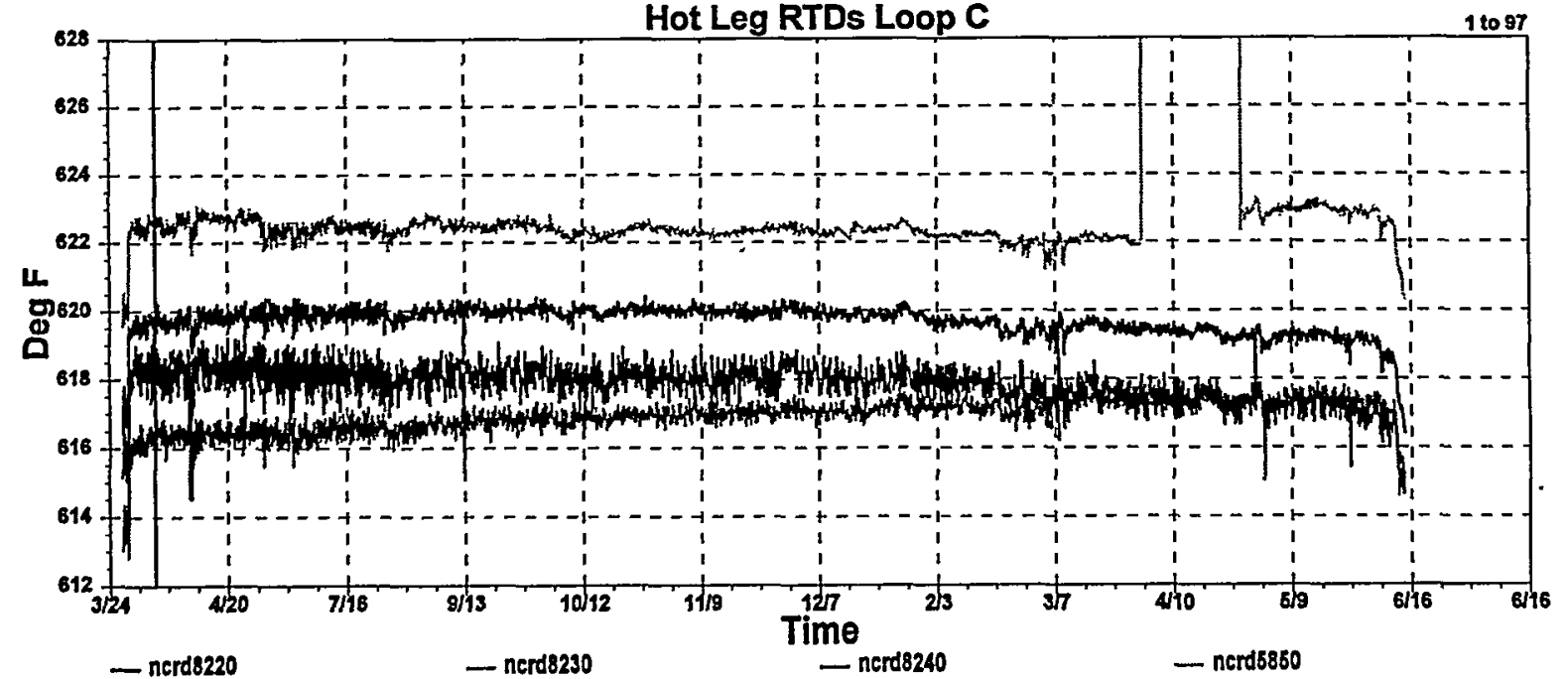

Hot Leg RTDs Loop C

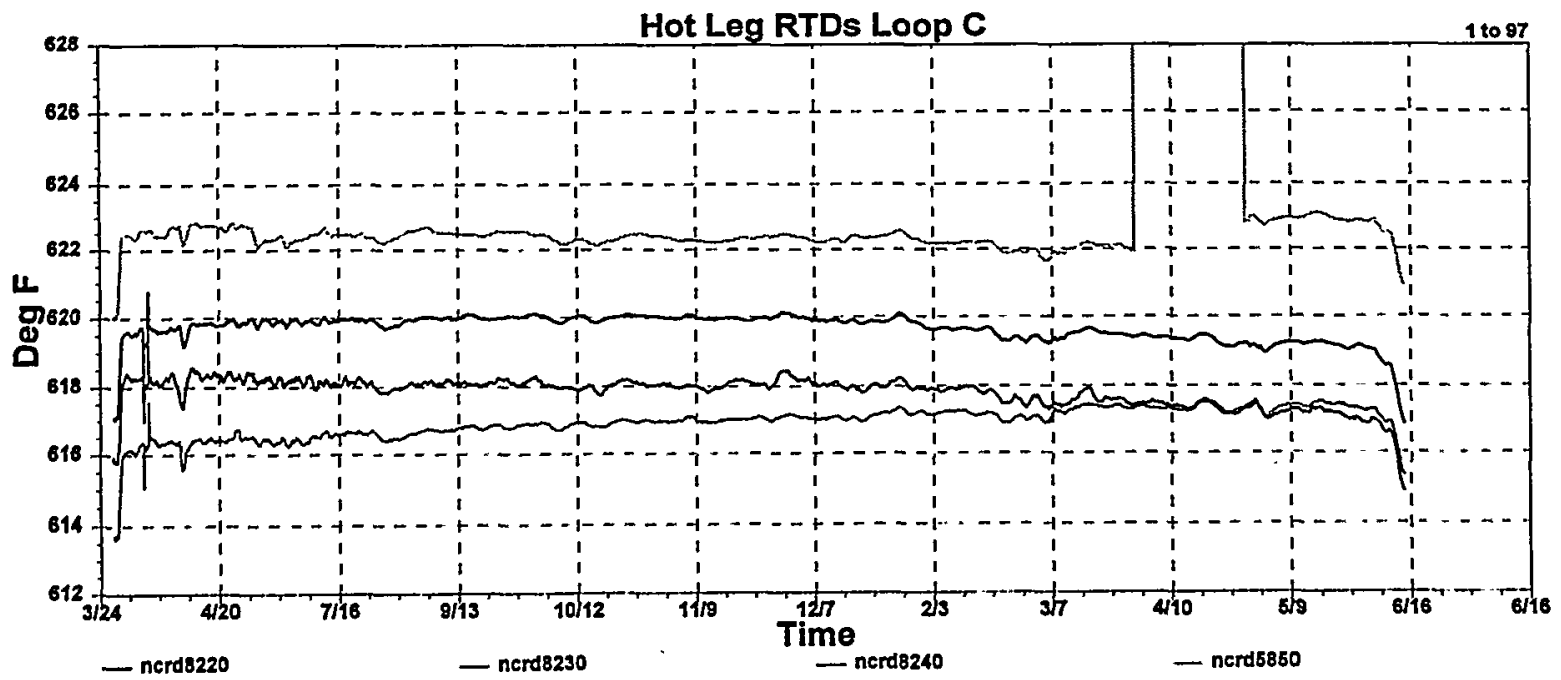

F.31 McGuire Cycle 1 Data for Hot Leg RTD Signals for Loop C 

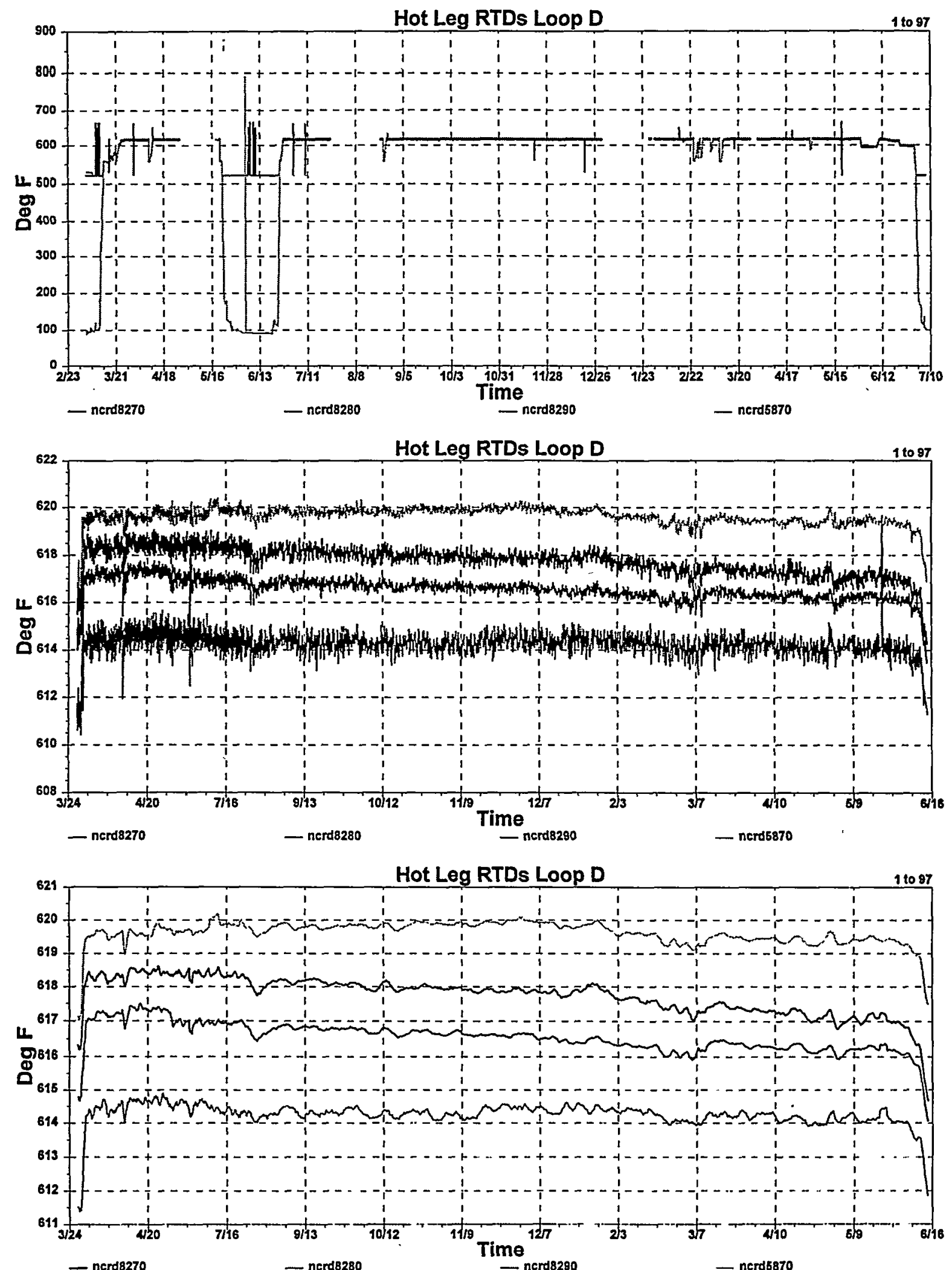

F.32 McGuire Cycle 1 Data for Hot Leg RTD Signals for Loop D 

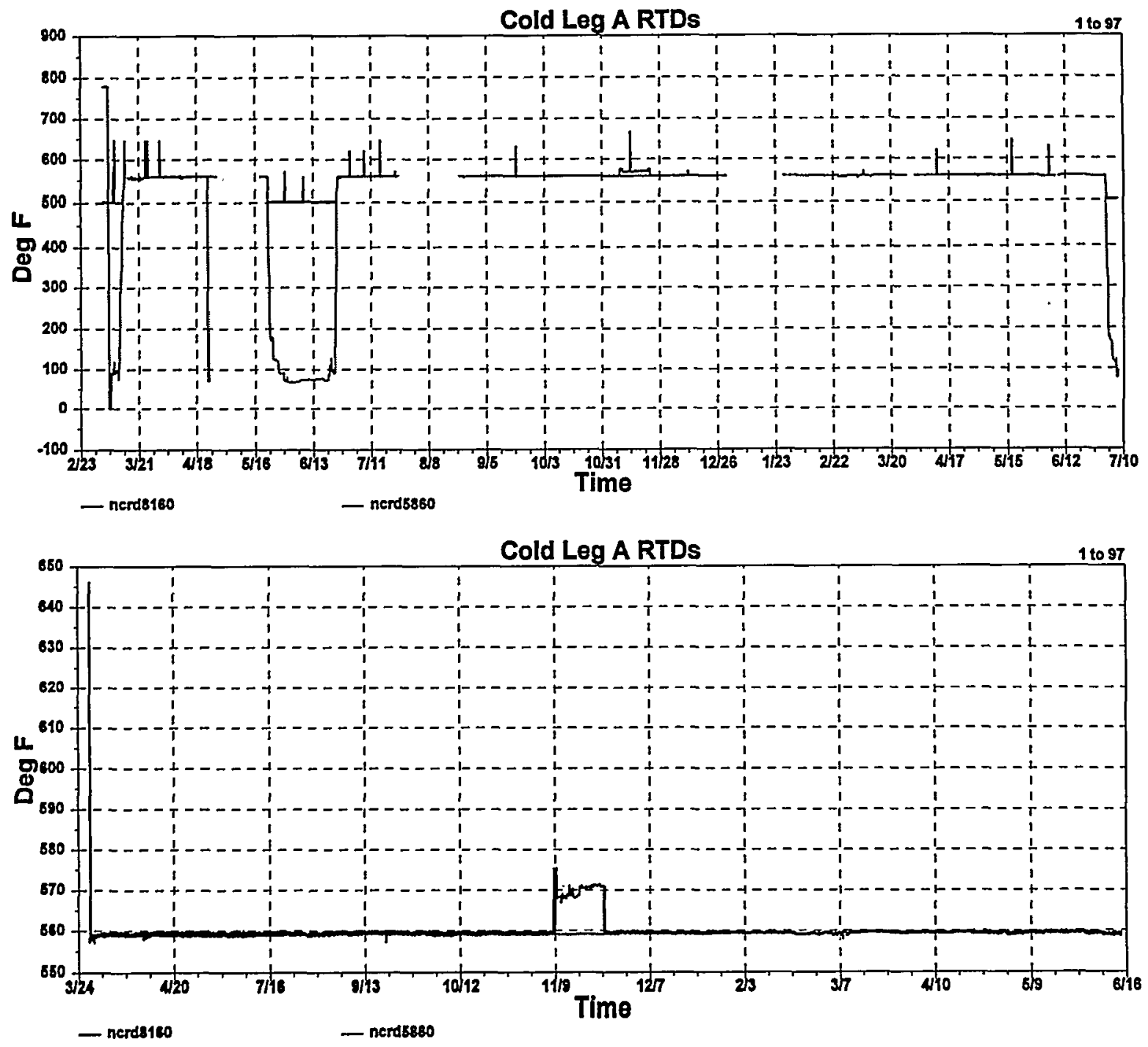

Cold Leg A RTDs

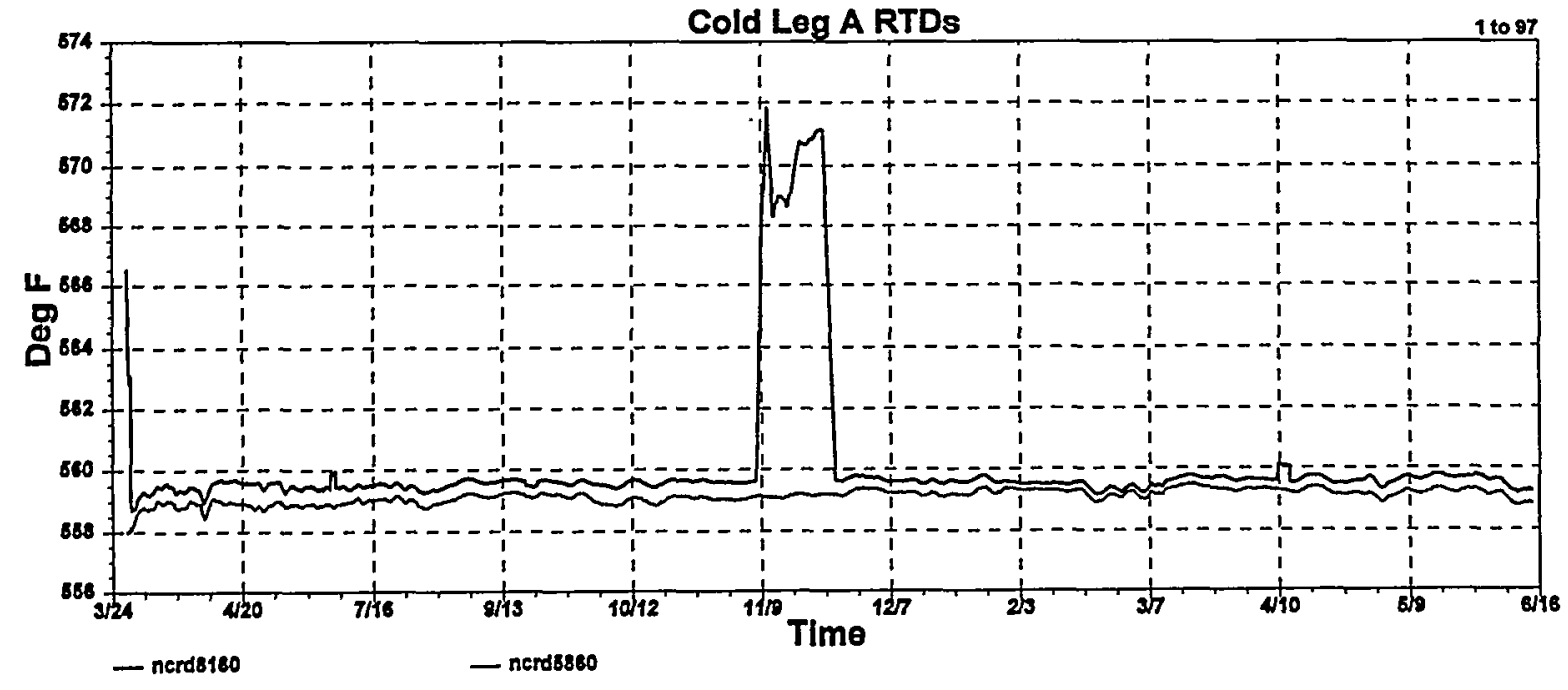

F.33 McGuire Cycle 1 Data for Cold Leg RTD Signals for Loop A 

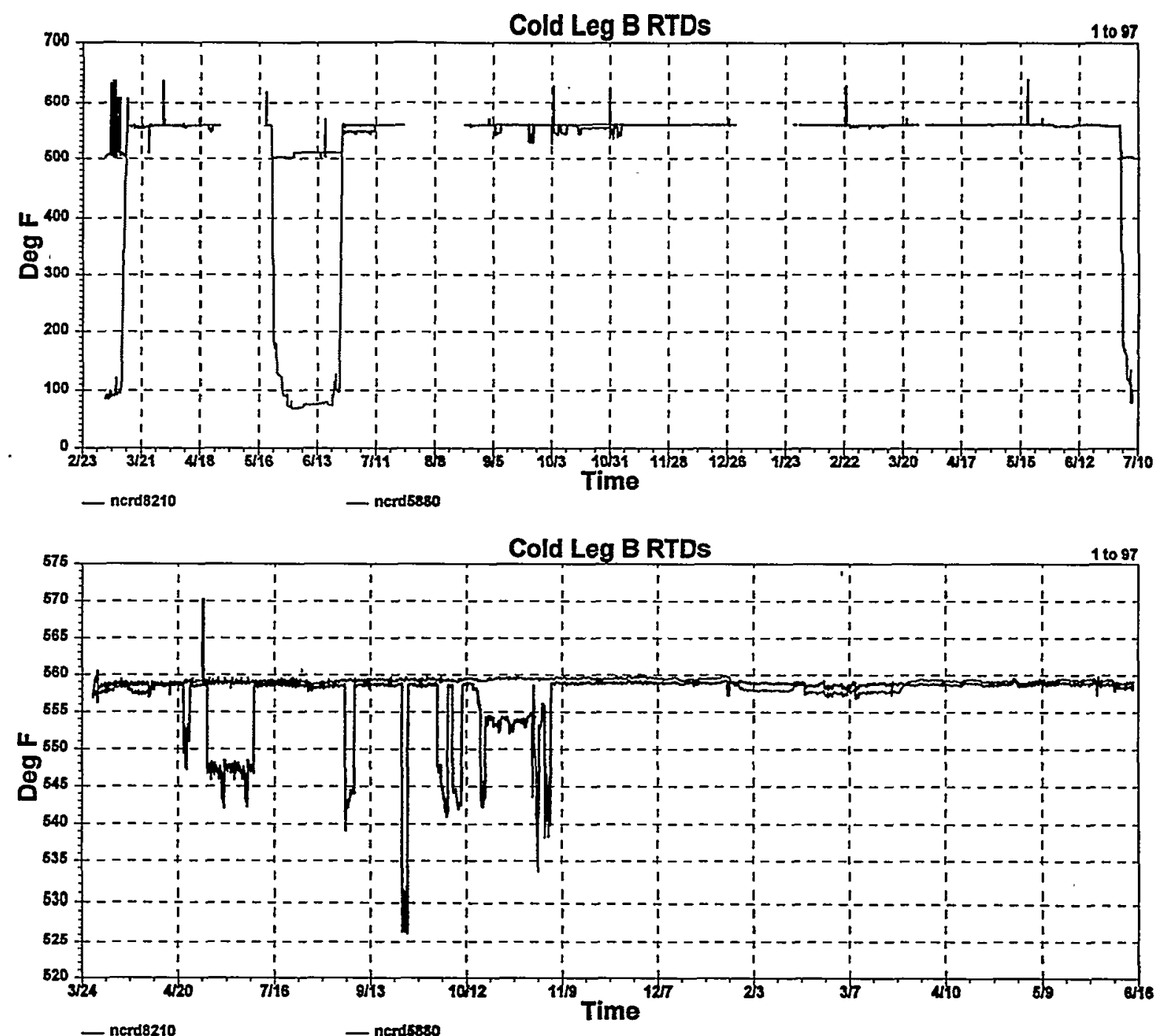

- nerd8210

- nerdsose

Cold Leg B RTDs

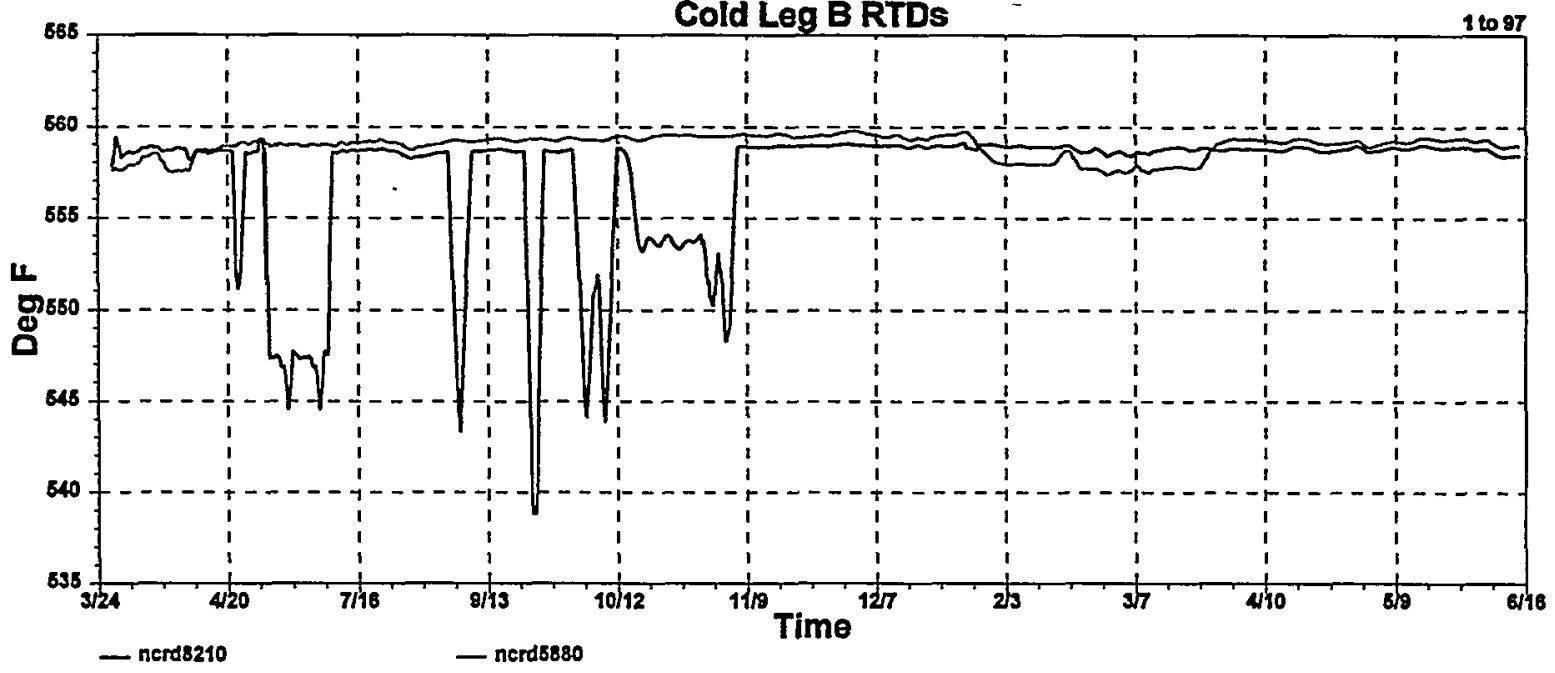

F.34 McGuire Cycle 1 Data for Cold Leg RTD Signals for Loop B 

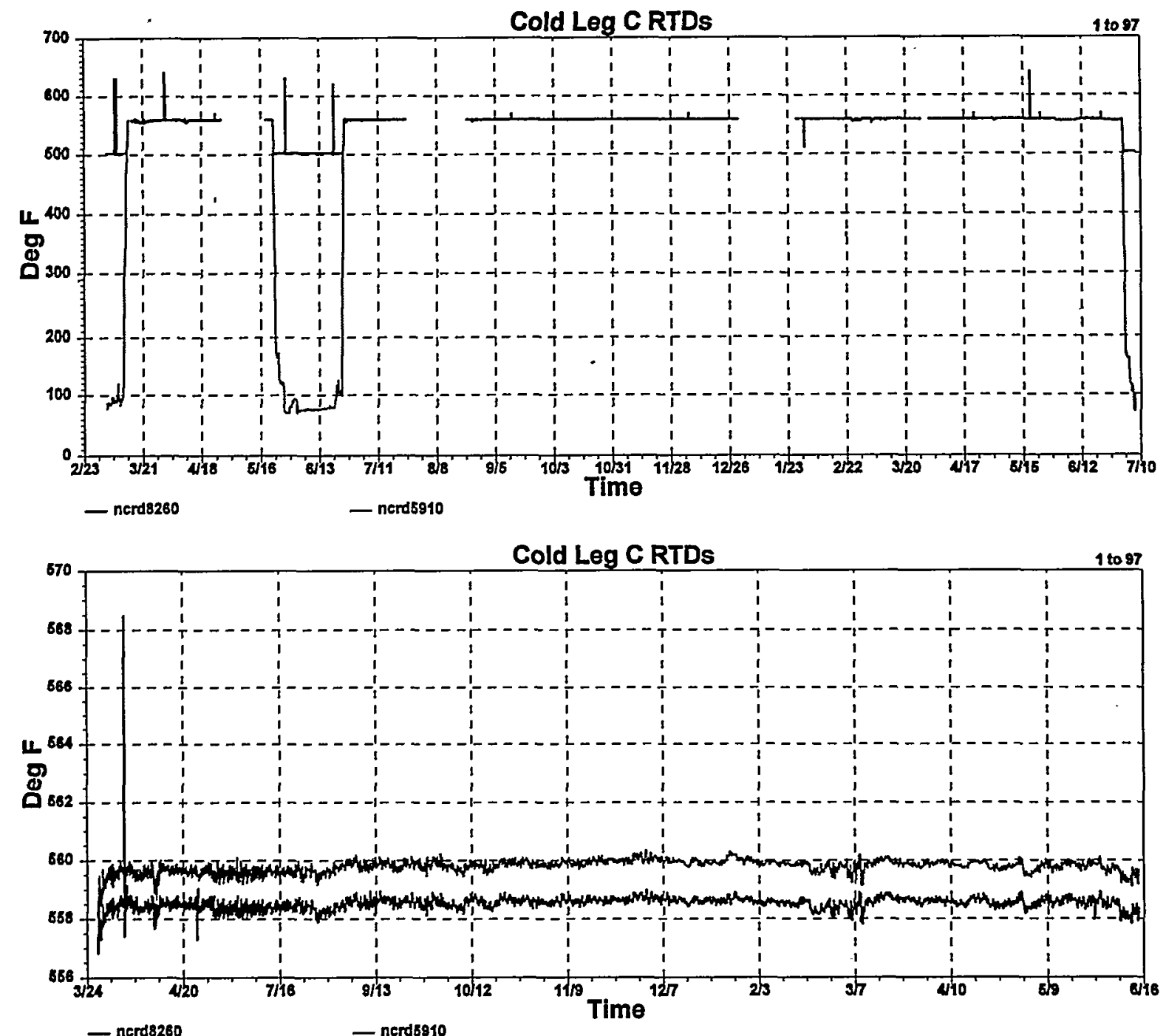

Cold Leg C RTDs

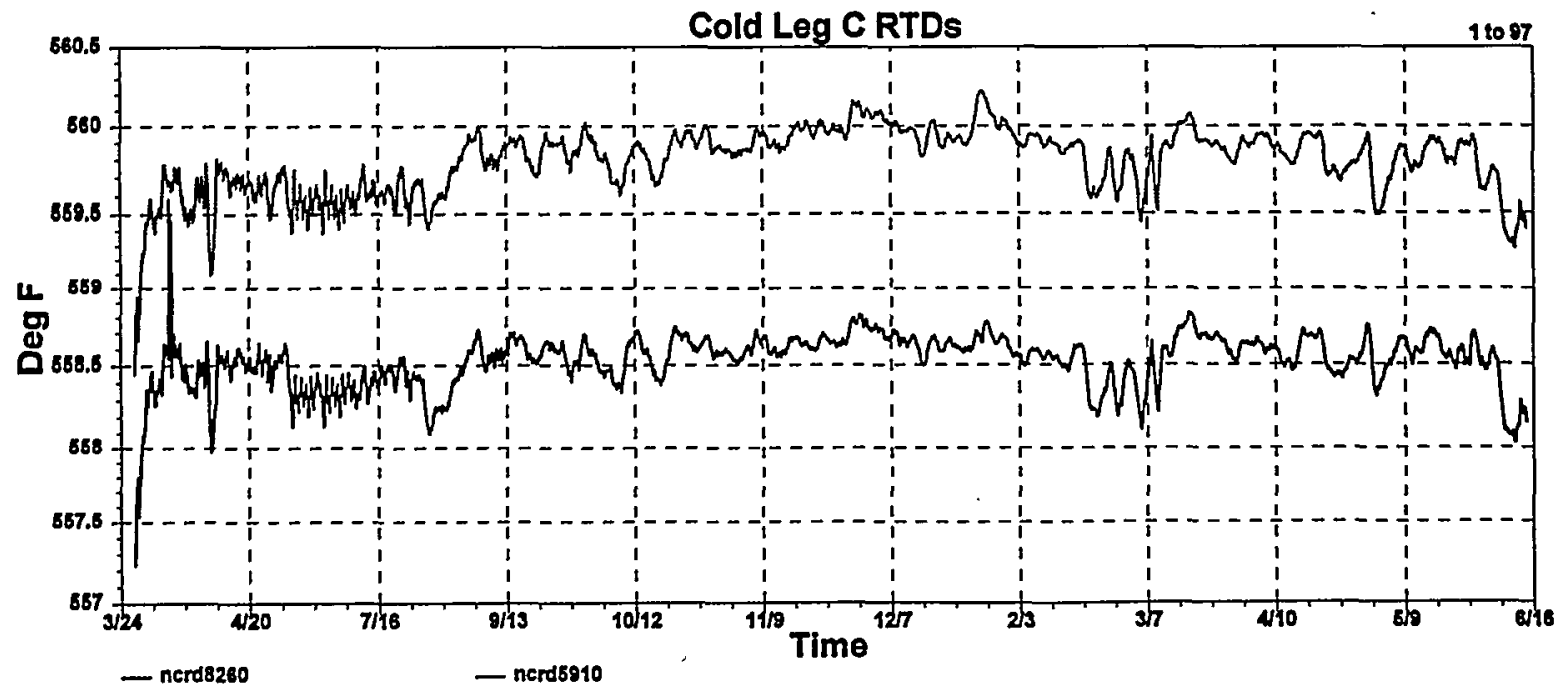

F.35 McGuire Cycle 1 Data for Cold Leg RTD Signals for Loop C 

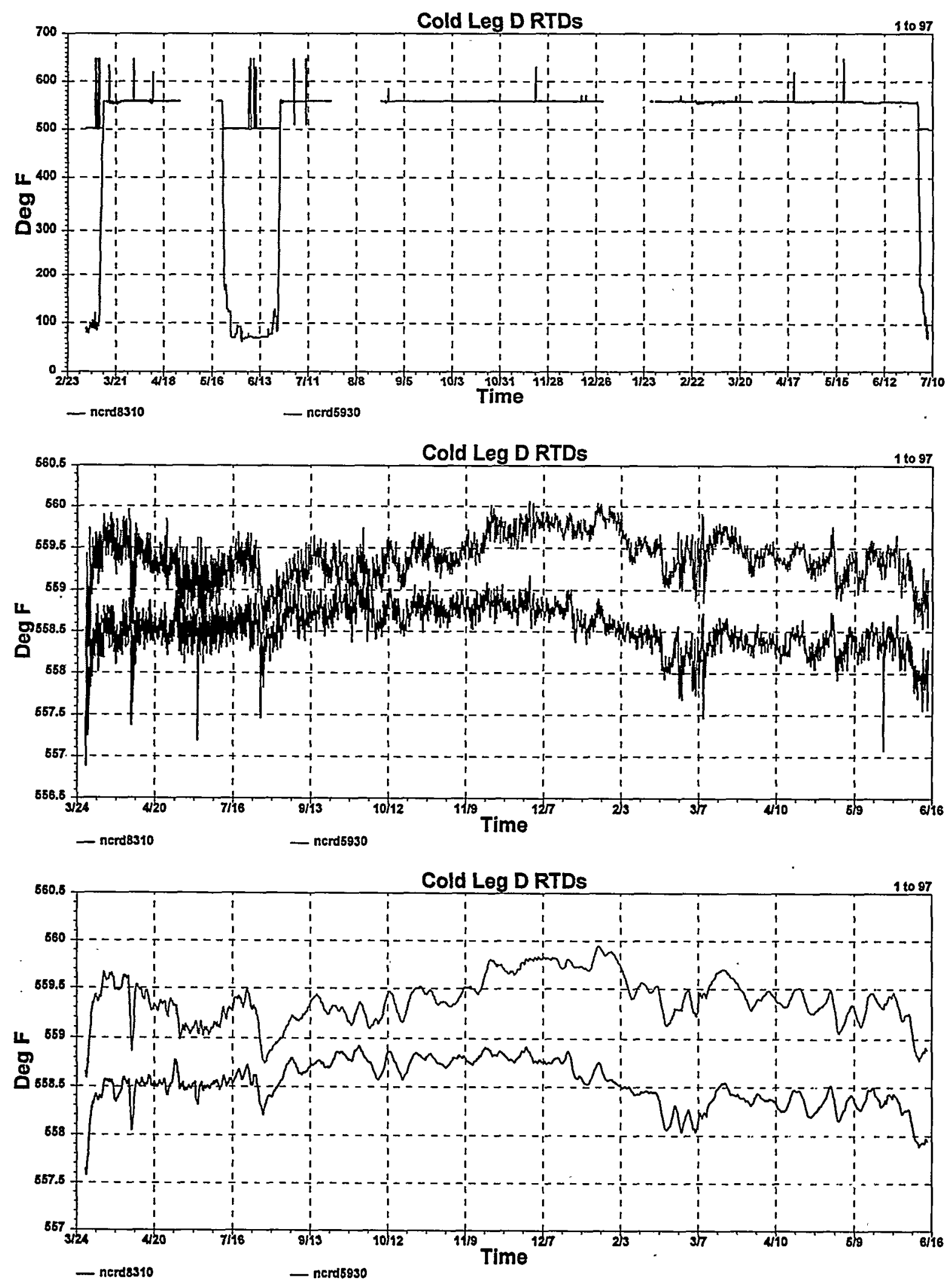

F.36 McGuire Cycle 1 Data for Cold Leg RTD Signals for Loop D 

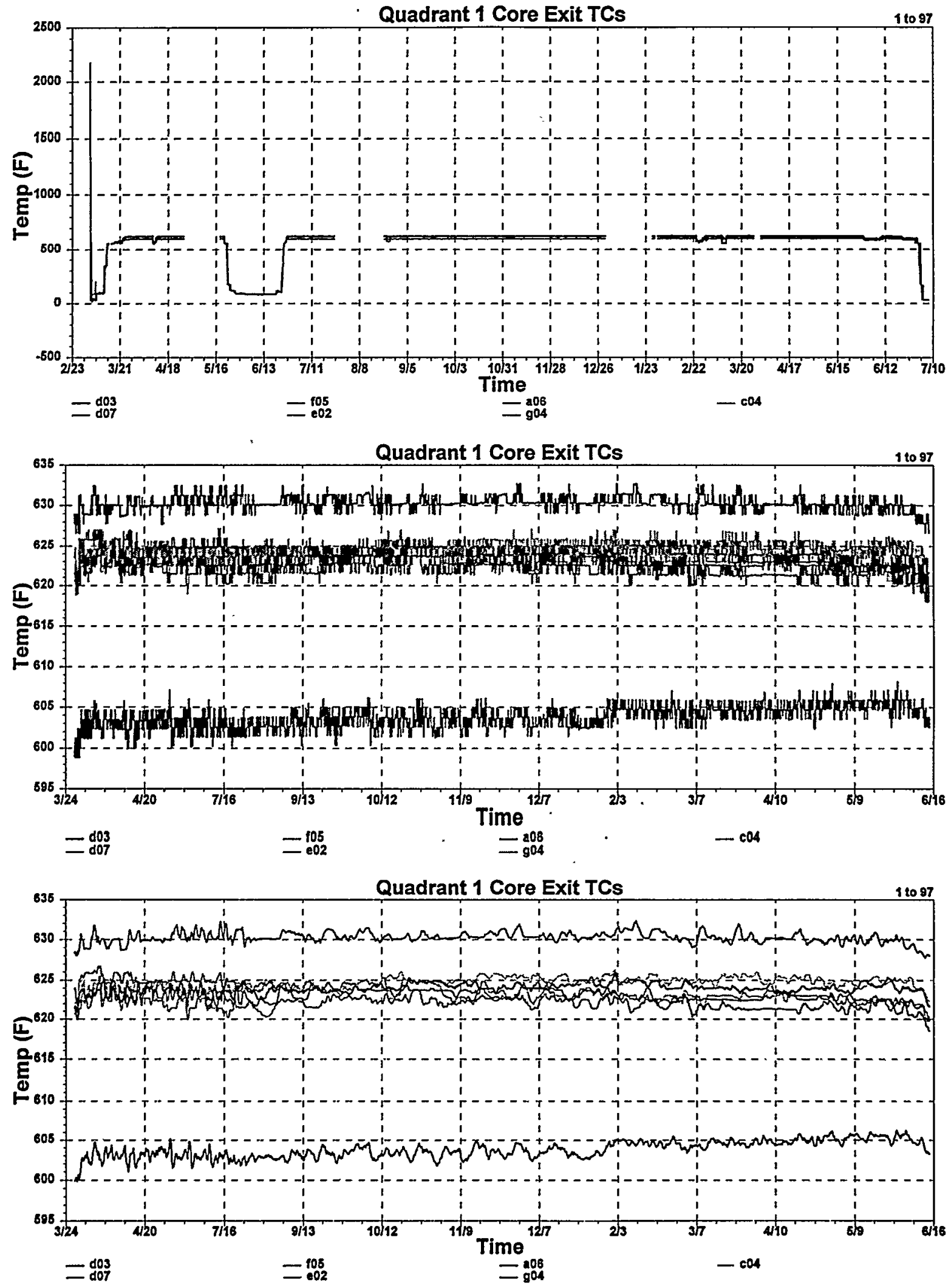

F.37 McGuire Core Exit Thermocouple Signals for Quadrant 1 

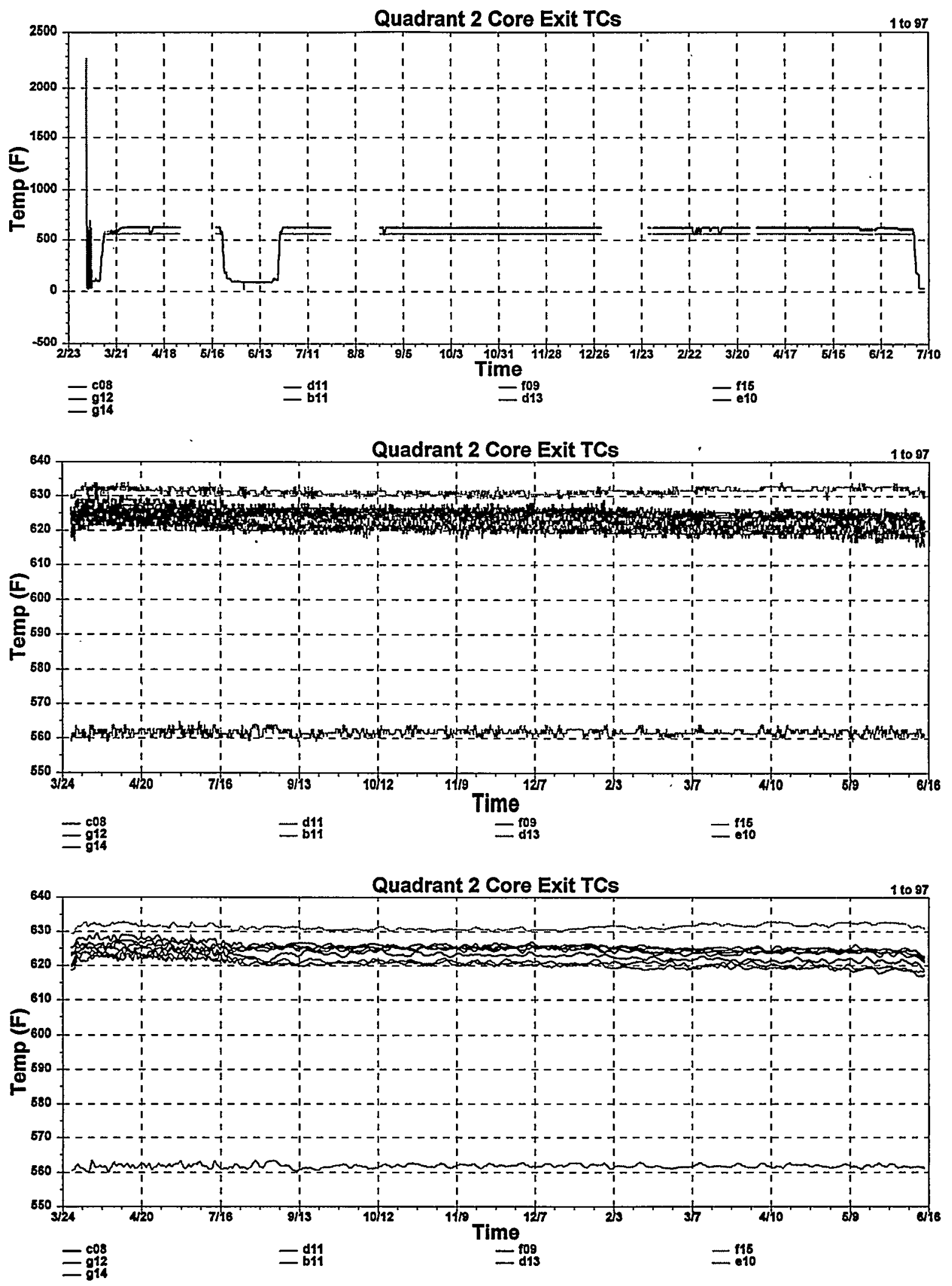

F.38 McGuire Core Exit Thermocouple Signals for Ouadrant 2 

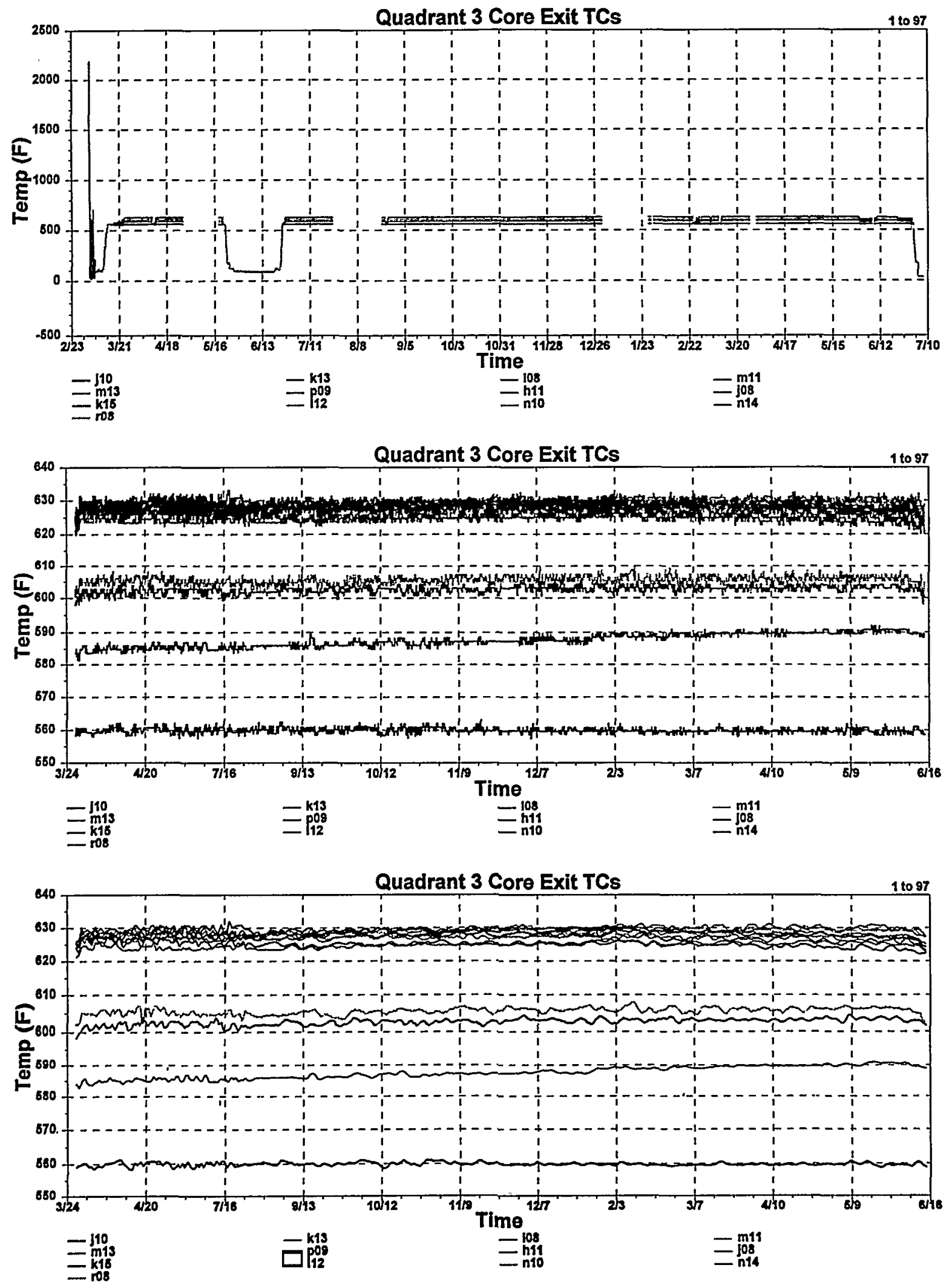

F.39 McGuire Core Exit Thermocouple Signals for Quadrant 3 

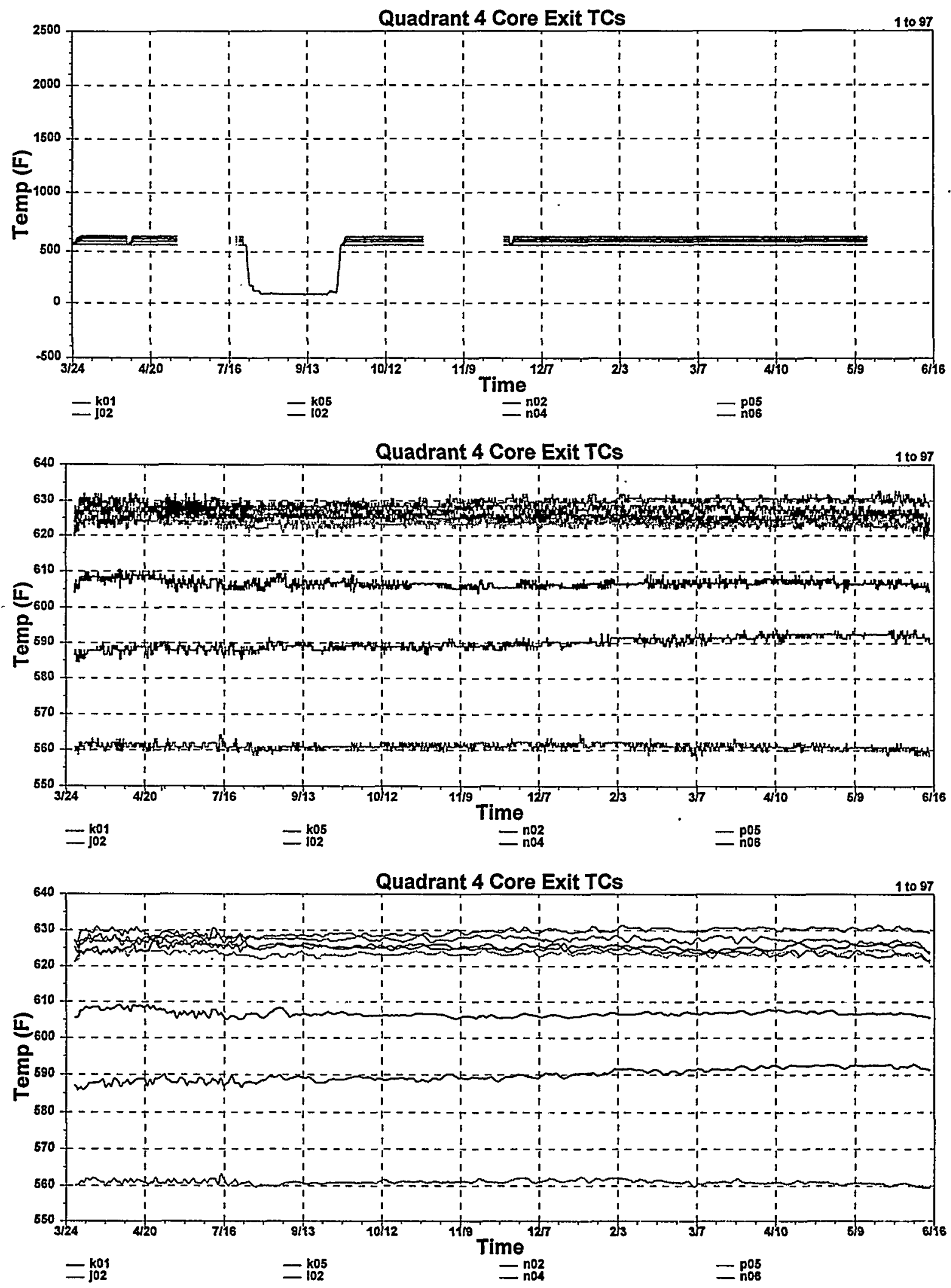

F.40 McGuire Core Exit Thermocouple Signals for Quadrant 4 


\section{APPENDIX G}

RAW DATA PLOTS FOR CYCLE 2 


\section{APPENDIX G}

\section{RAW DATA PLOTS FOR CYCLE 2}

This Appendix contains raw data plots for the McGuire instrument channels that were monitored during this project. Cycle 2 data collection began in October 1993 and ended in October 1994.

The same data acquisition system and procedure was used in cycle 1 and cycle 2. However, the sampling rates were different. In cycle 1, the output of each instrument was sampled six times per hour while in cycle 2 , the sampling rate was reduced to one sample per hour. The plots that are included in this appendix do not represent every point that was sampled. Rather, points were skipped in plotting the data.

Each figure in this appendix including three plots as follows:

1. The raw data including the spikes and gaps that represent plant trips, shutdowns, and periods where on-line monitoring data could not be collected.

2. Data after the spikes and gaps were removed.

3. Data after it was filtered to remove the extraneous noise. 


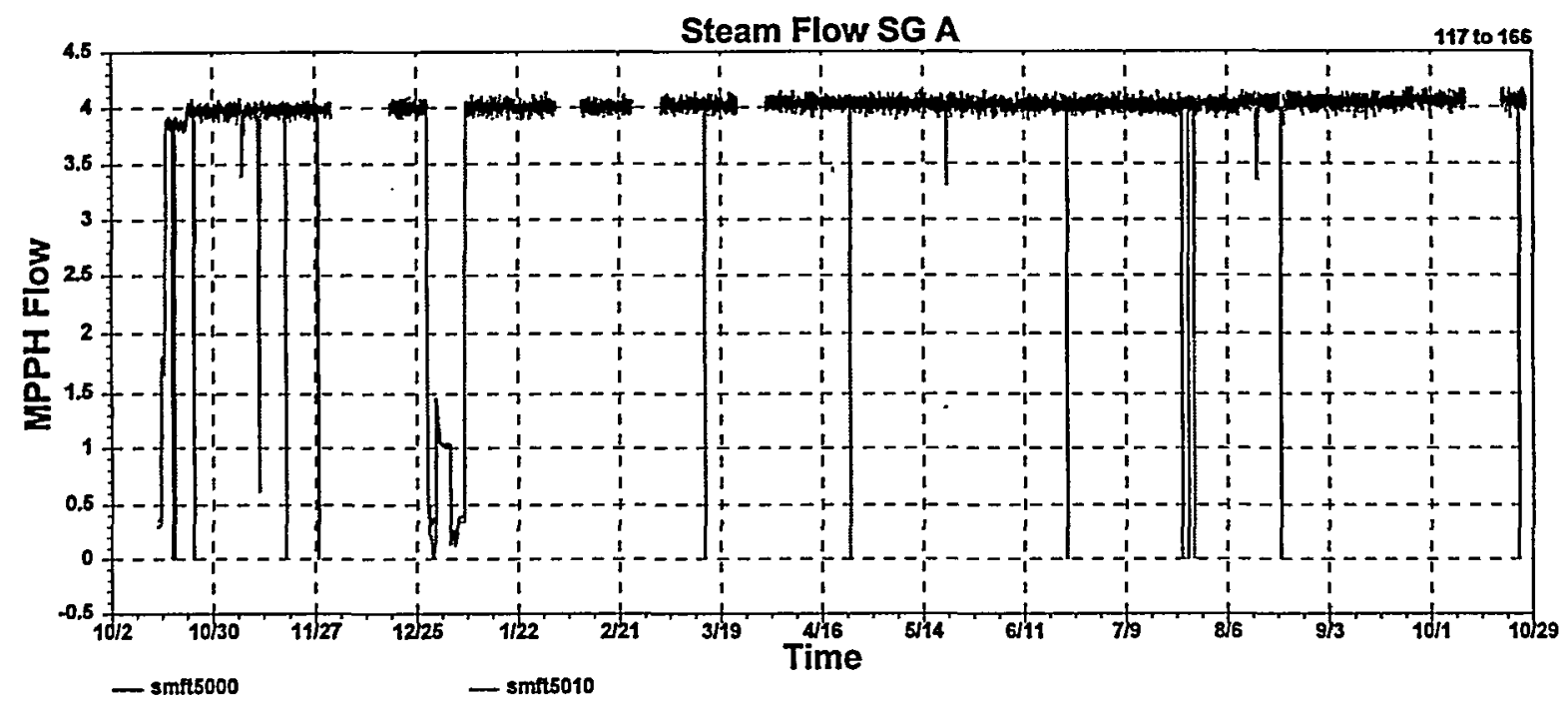

Steam Flow SG A

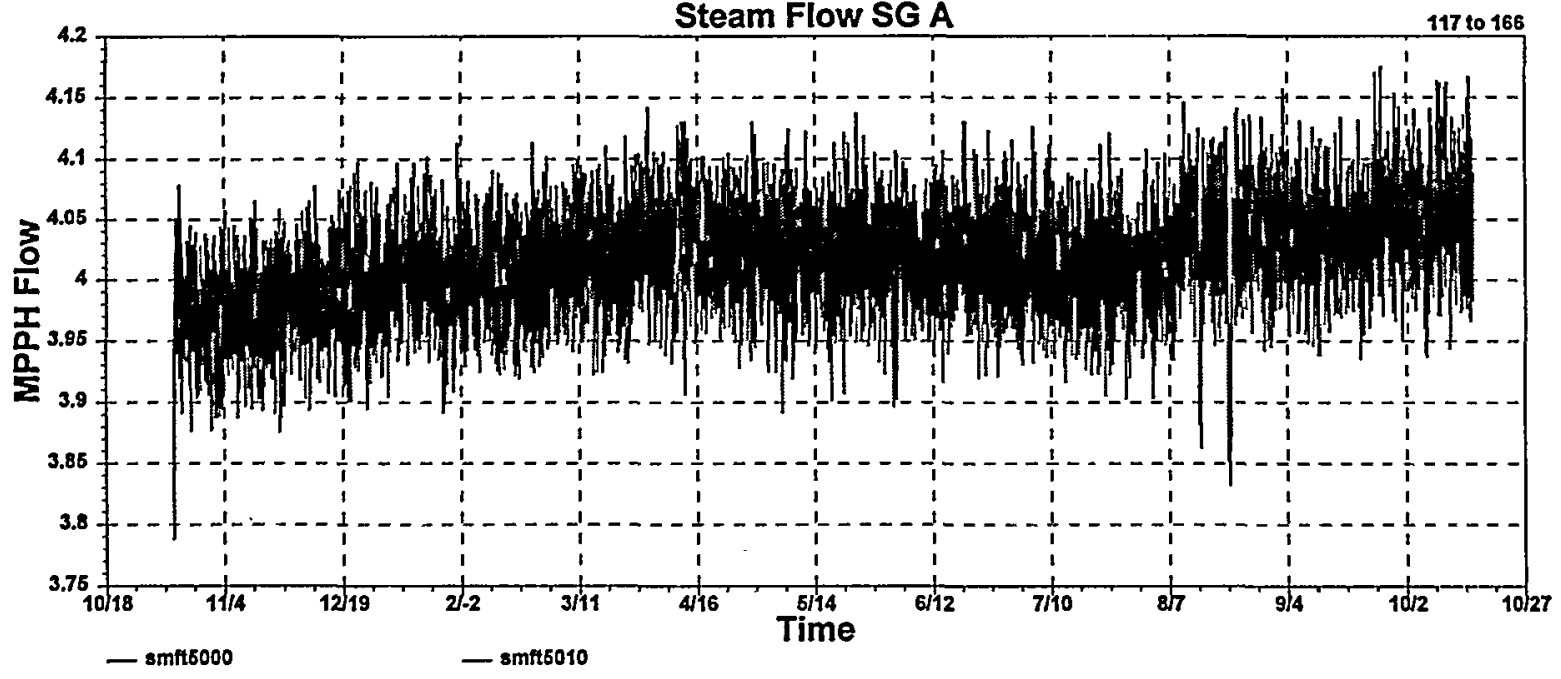

Steam Flow SG A

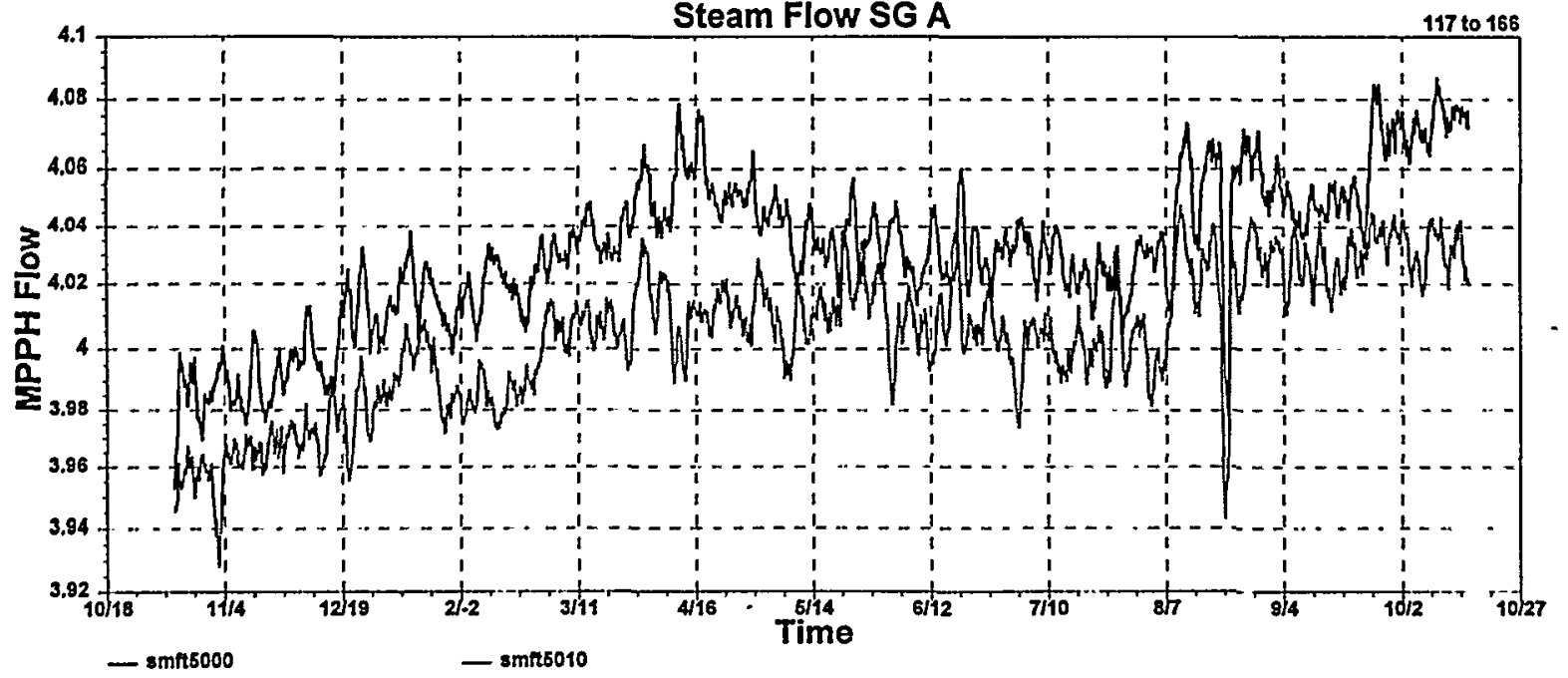

G.1 McGuire Cycle 2 Data for Steam Flow Signals for Loop A 

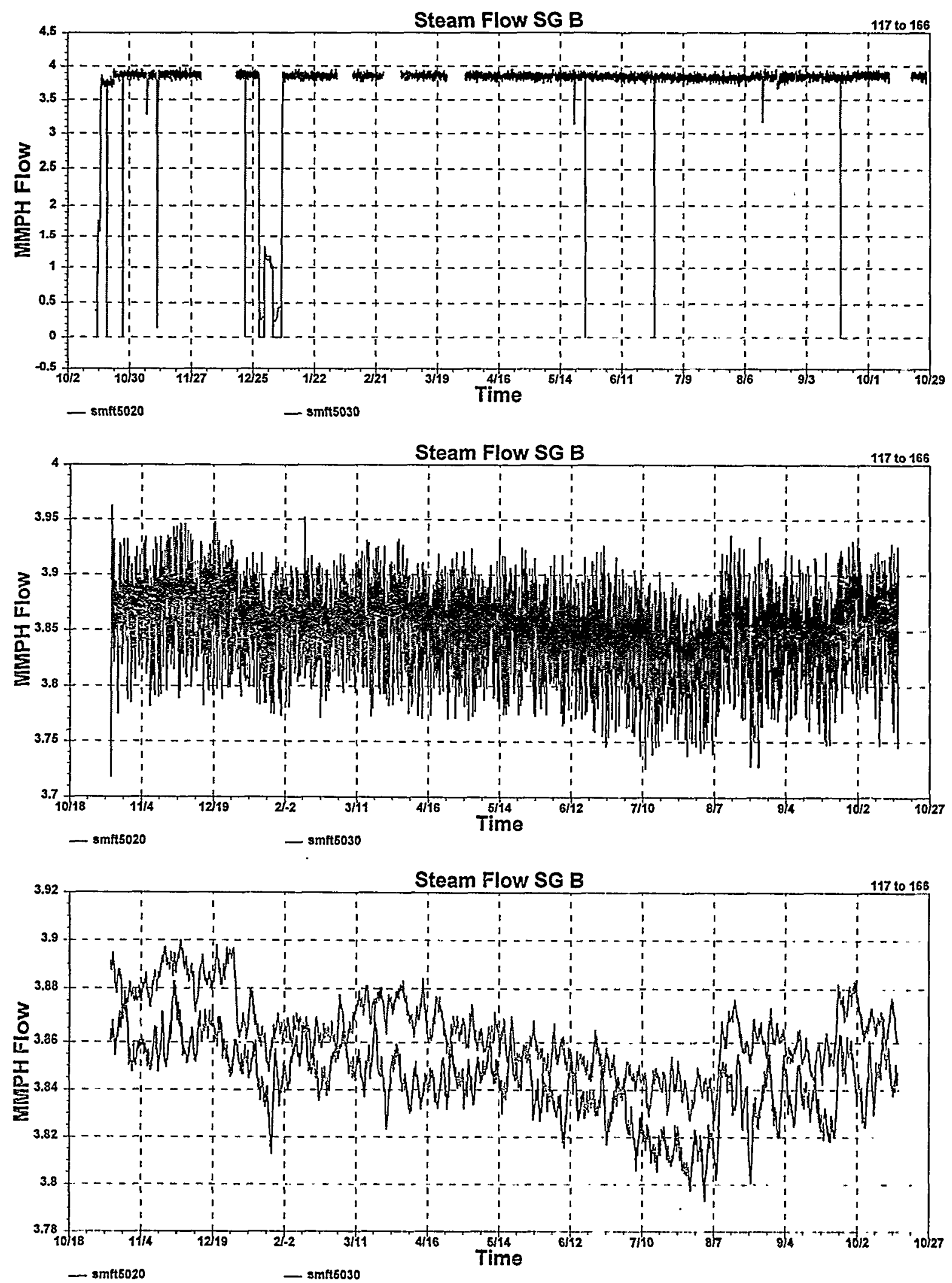

G.2 McGuire Cycle 2 Data for Steam Flow Signals for Loop B 

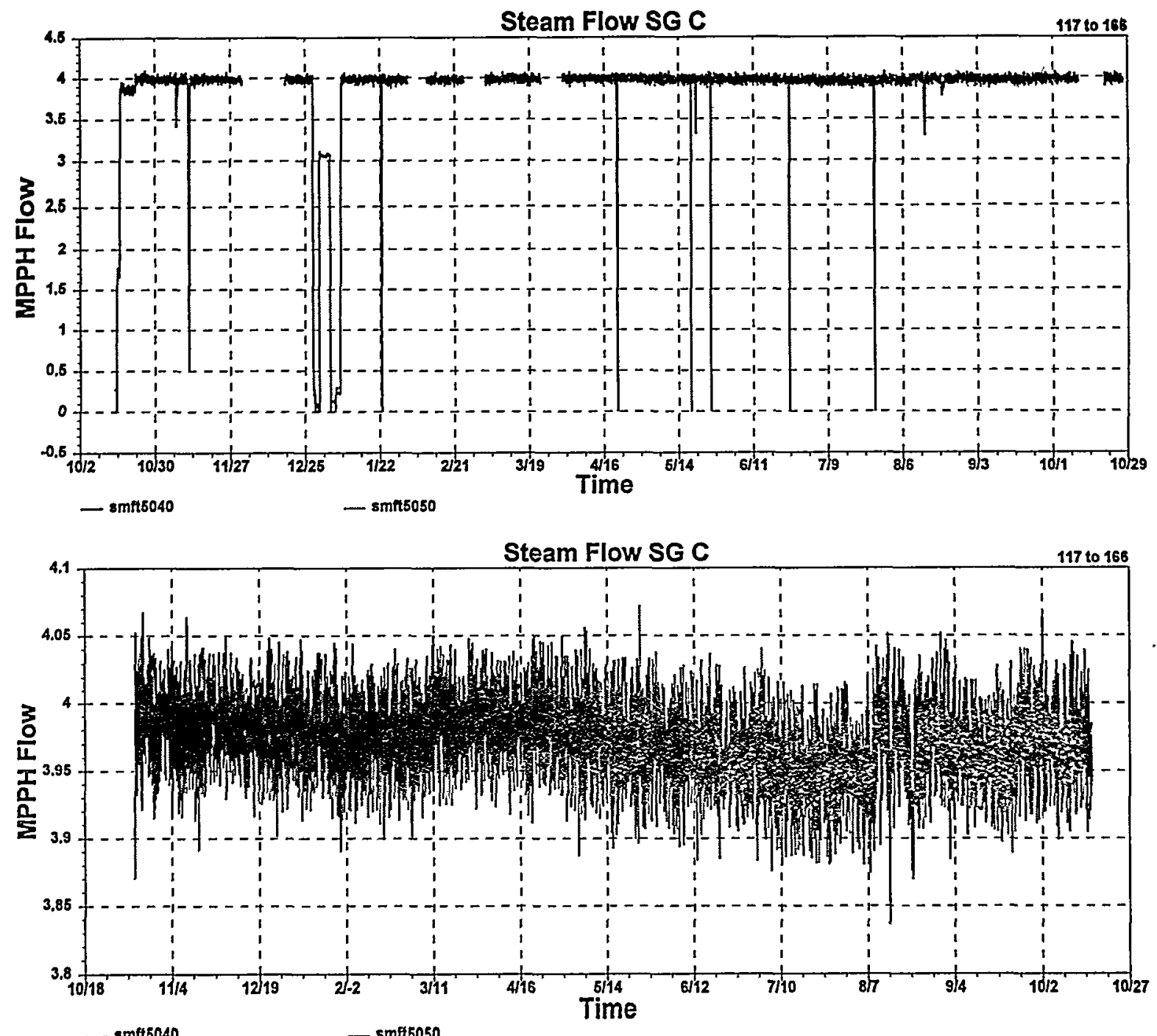

$-\operatorname{sinft5040}$

- smft5050

Steam Flow SG C

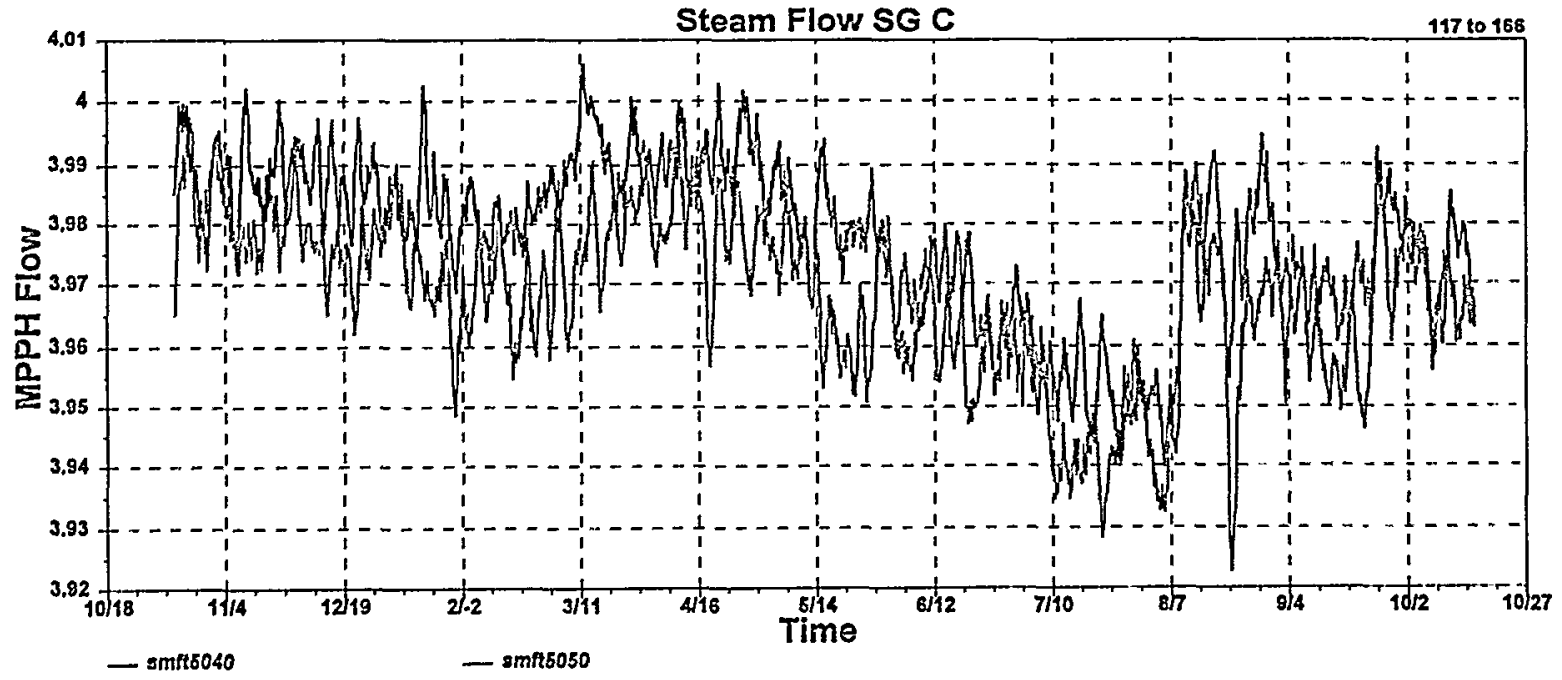

G.3 McGuire Cycle 2 Data for Steam Flow Signals for Loop C 

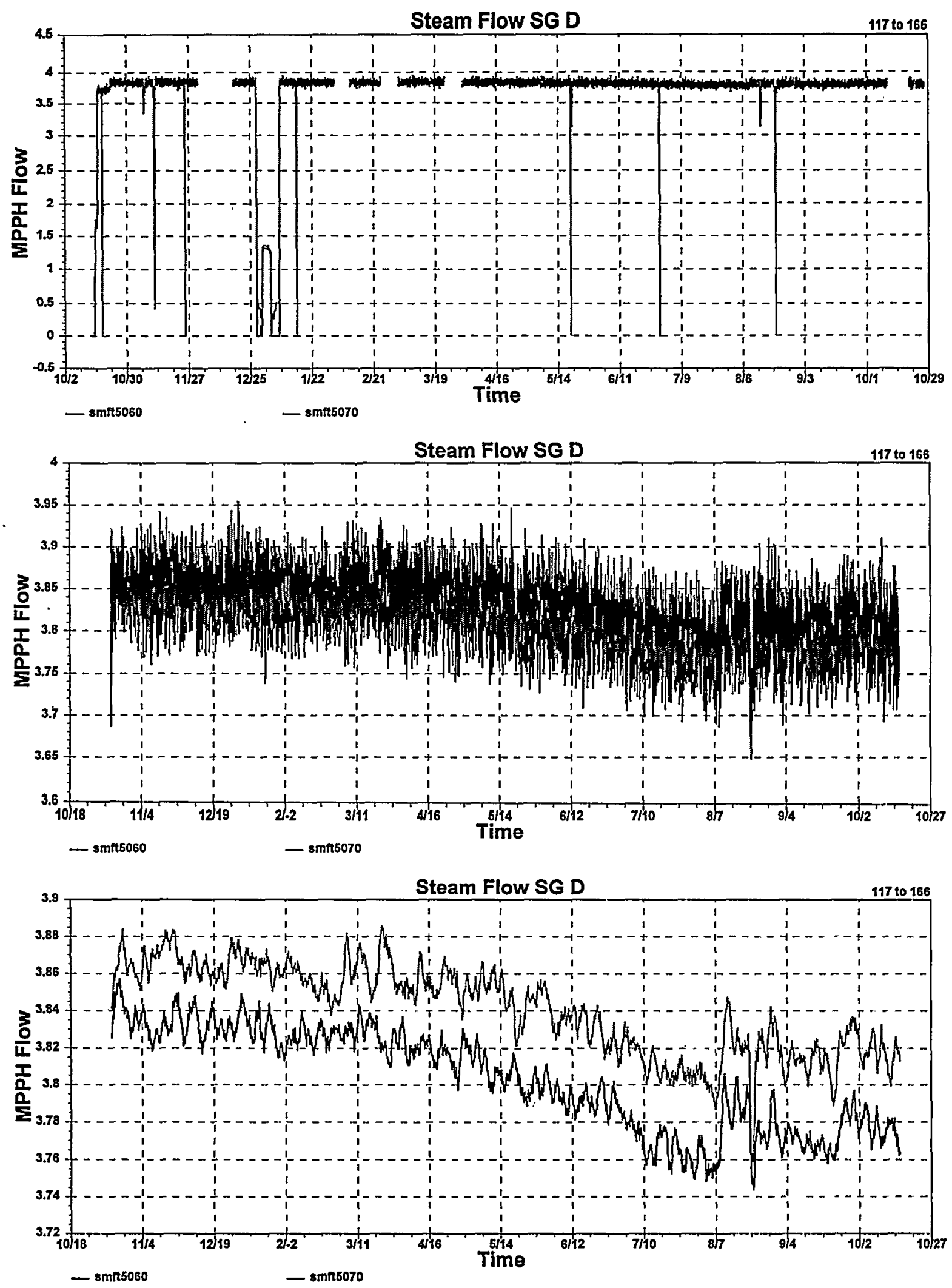

G.4 McGuire Cycle 2 Data for Steam Flow Signals for Loop D 

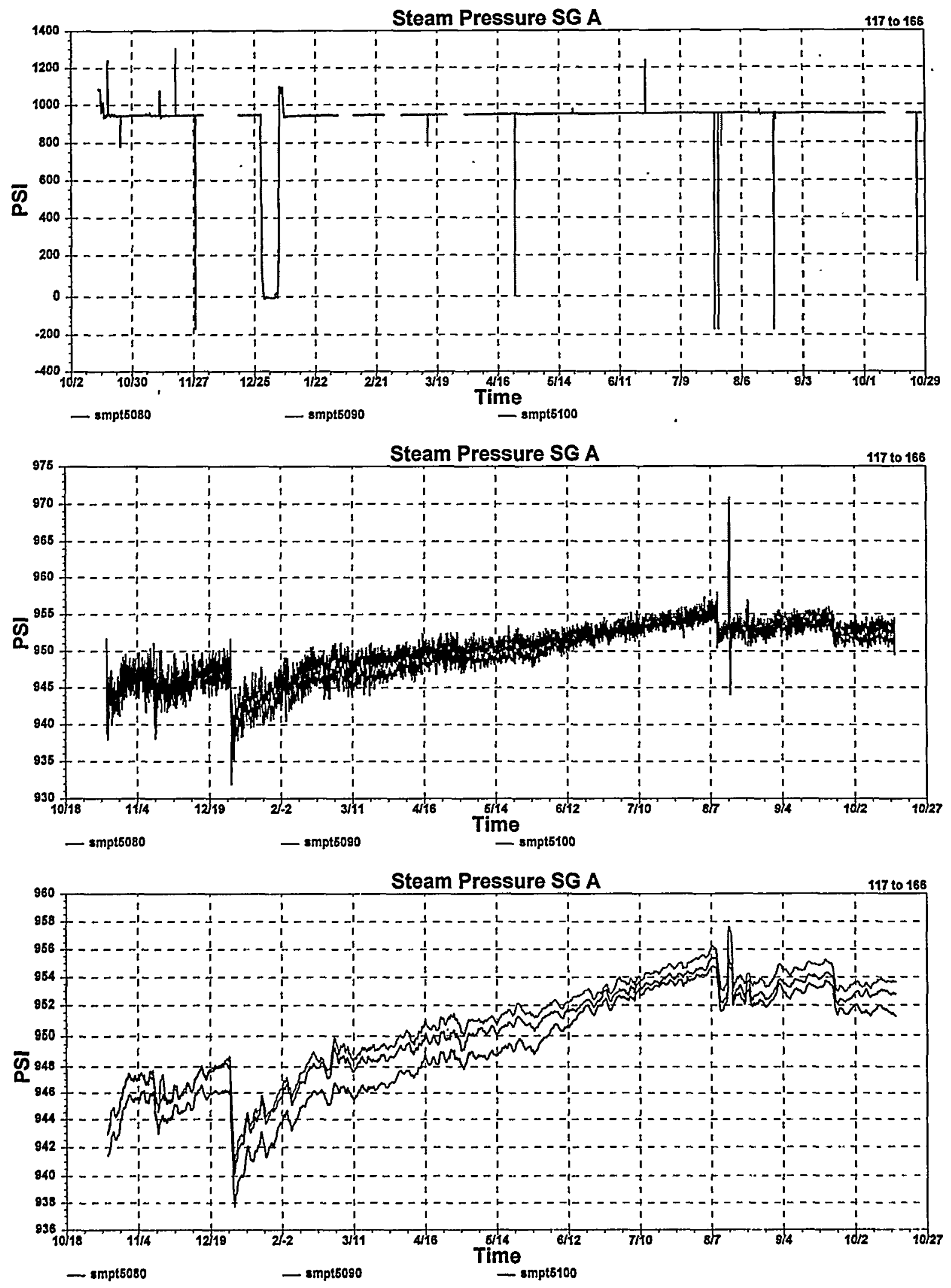

G.5 McGuire Cycle 2 Data for Steam Pressure Signals for Loop A 

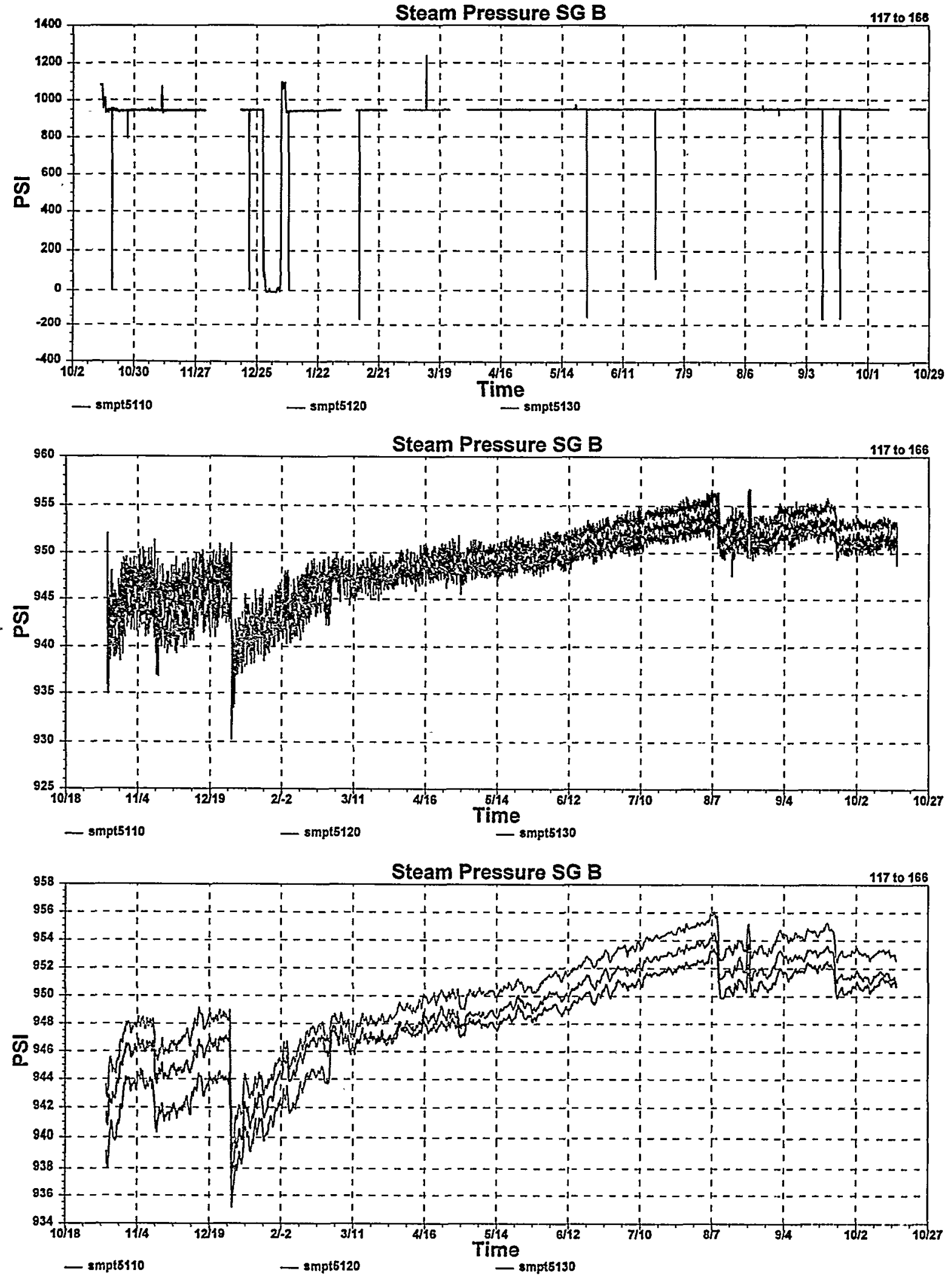

G.6 McGuire Cycle 2 Data for Steam Pressure Signals for Loop B 

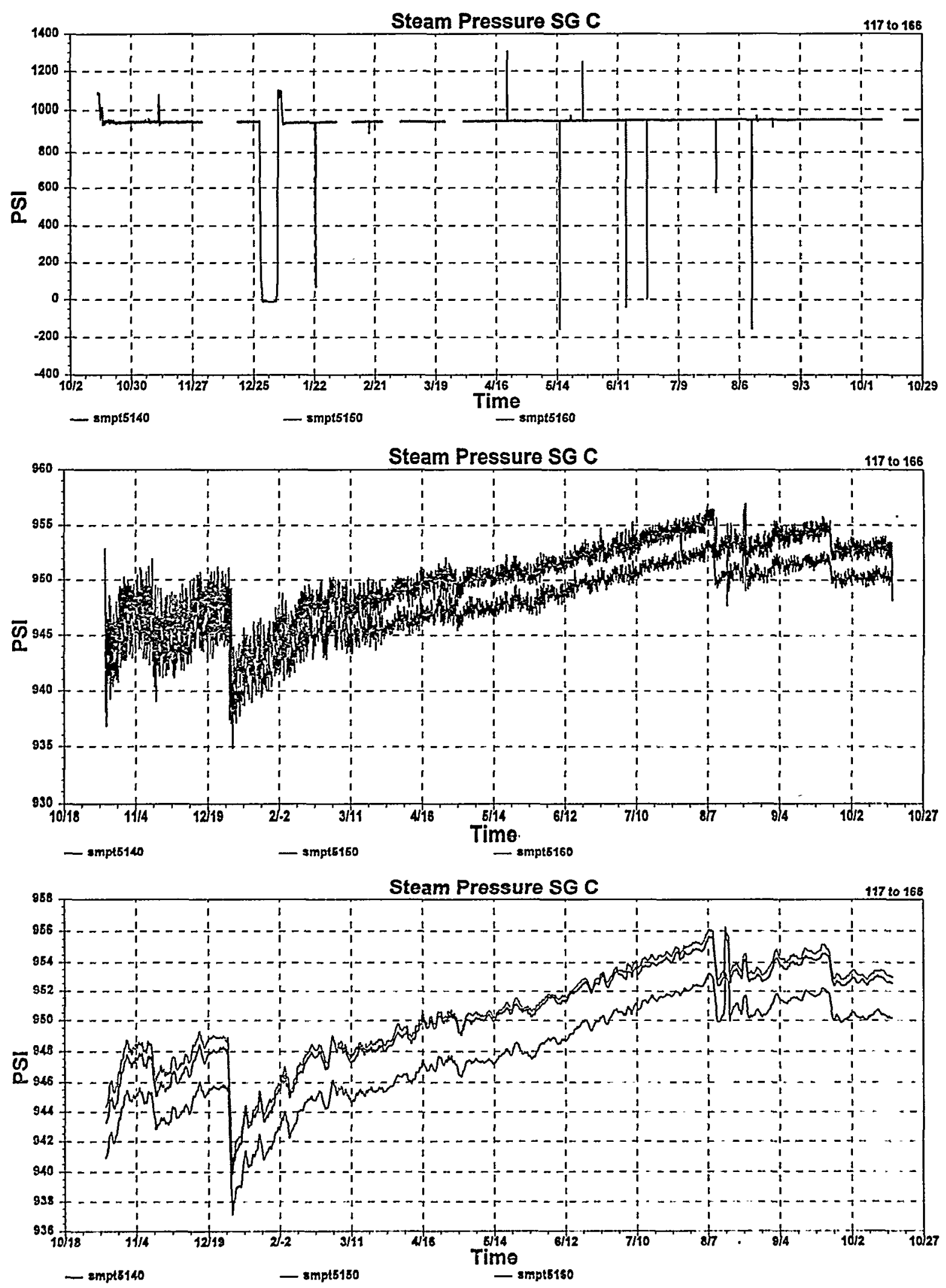

G.7 McGuire Cycle 2 Data for Steam Pressure Signals for Loop C 
Steam Pressure SG D

117 to 166

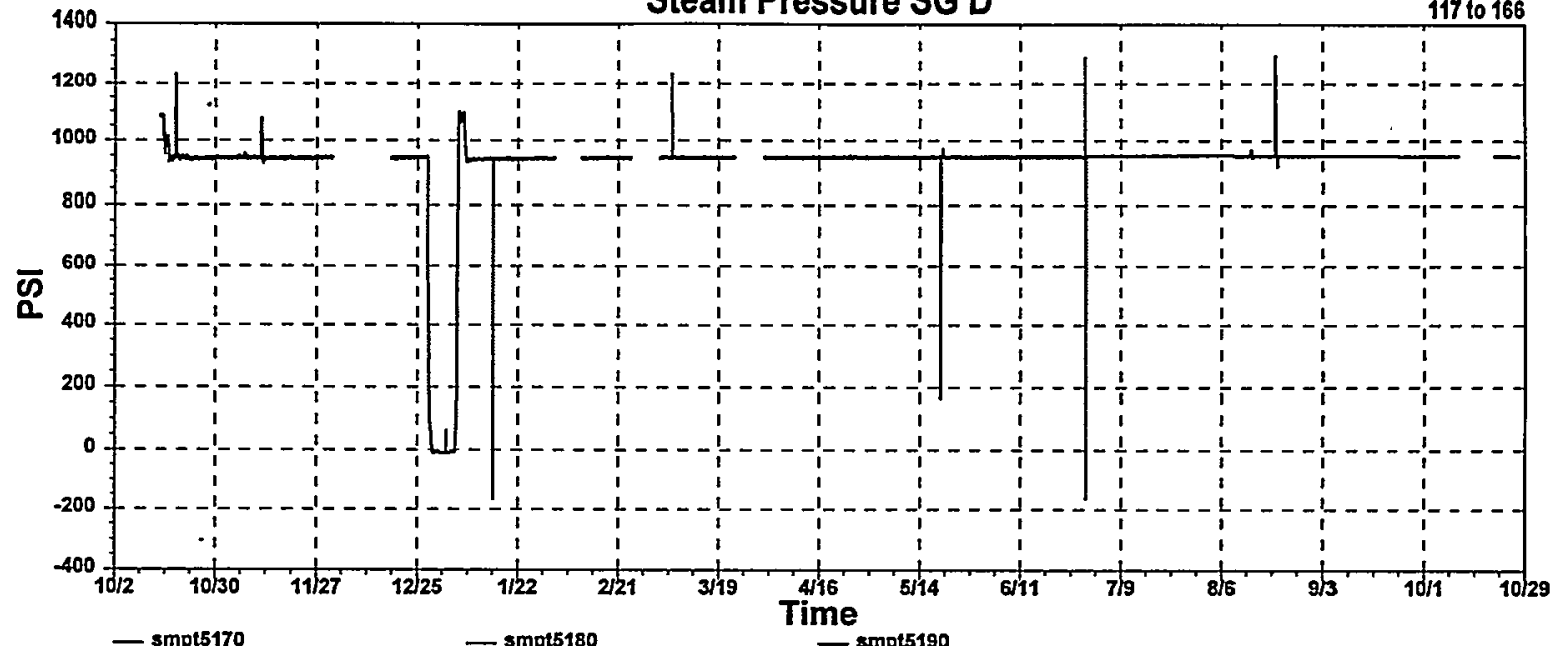

Steam Pressure SG D

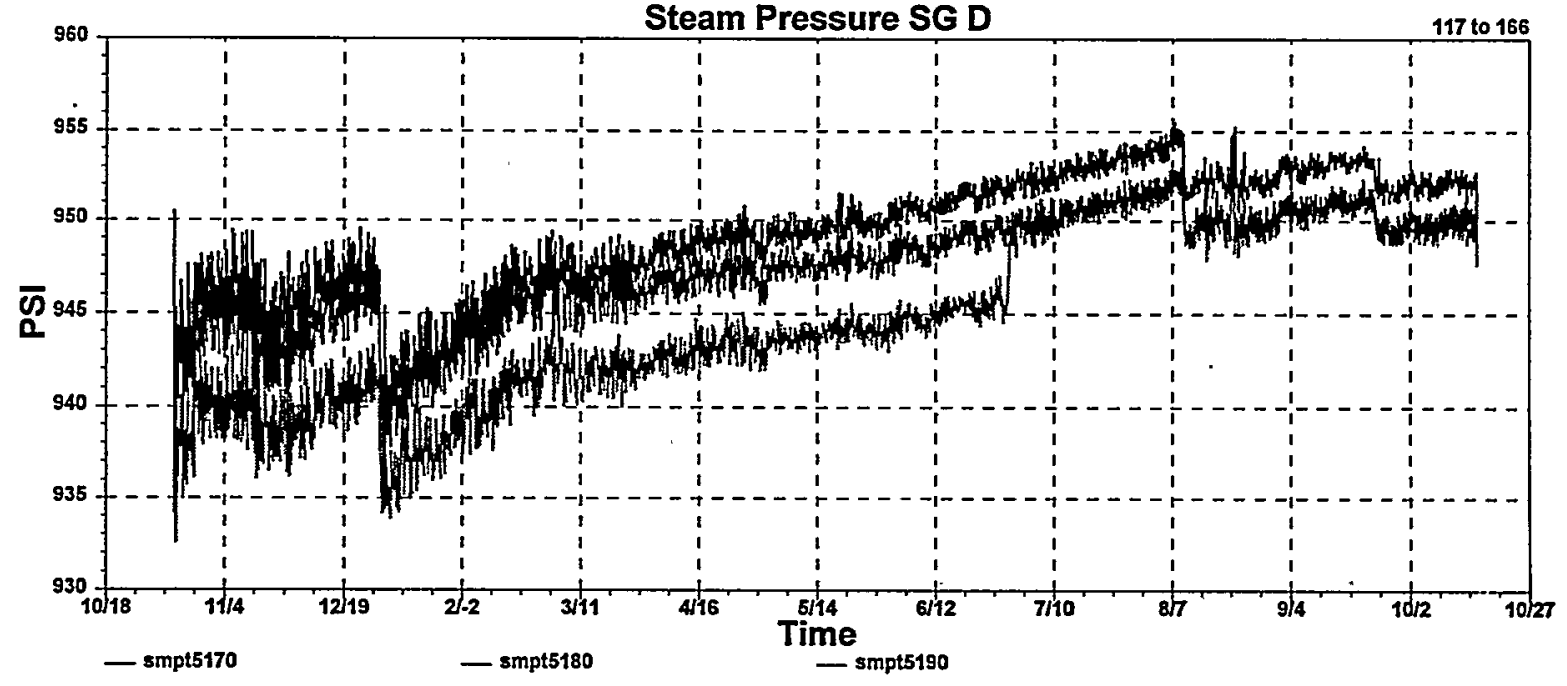

Steam Pressure SG D

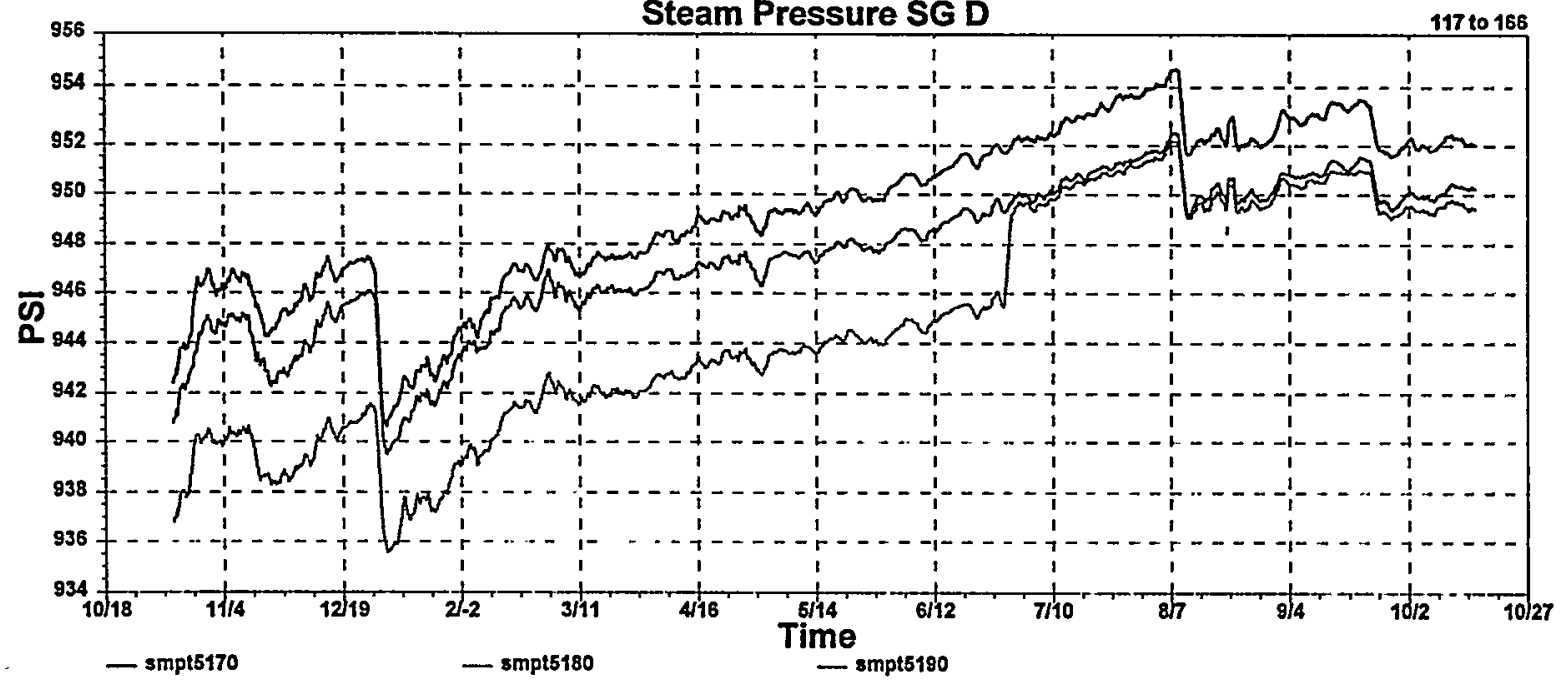

G.8 McGuire Cycle 2 Data for Steam Pressure Signals for Loop D 

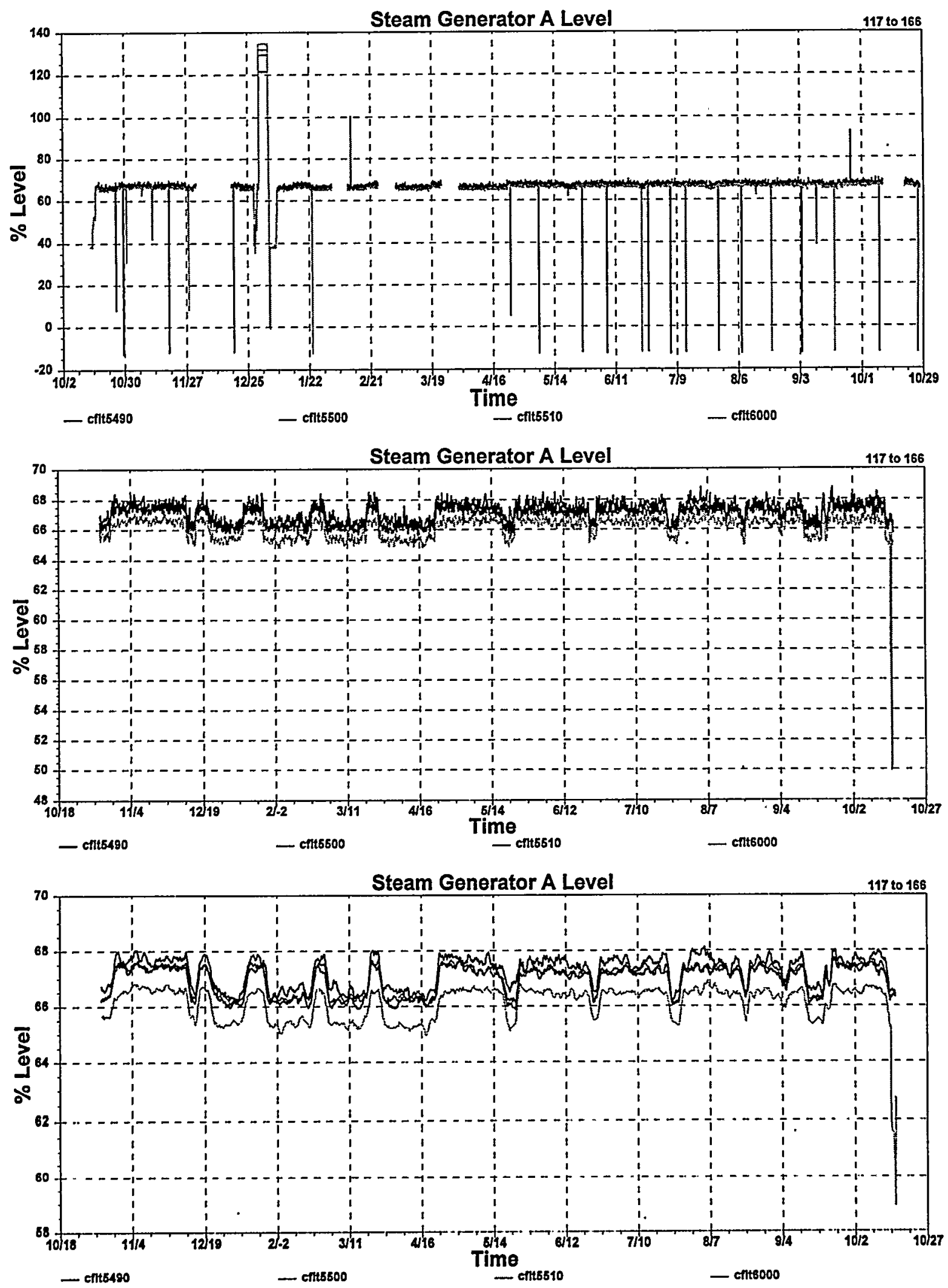

G.9 McGuire Cycle 2 Data for Steam Generator Level Signals for Loop A 

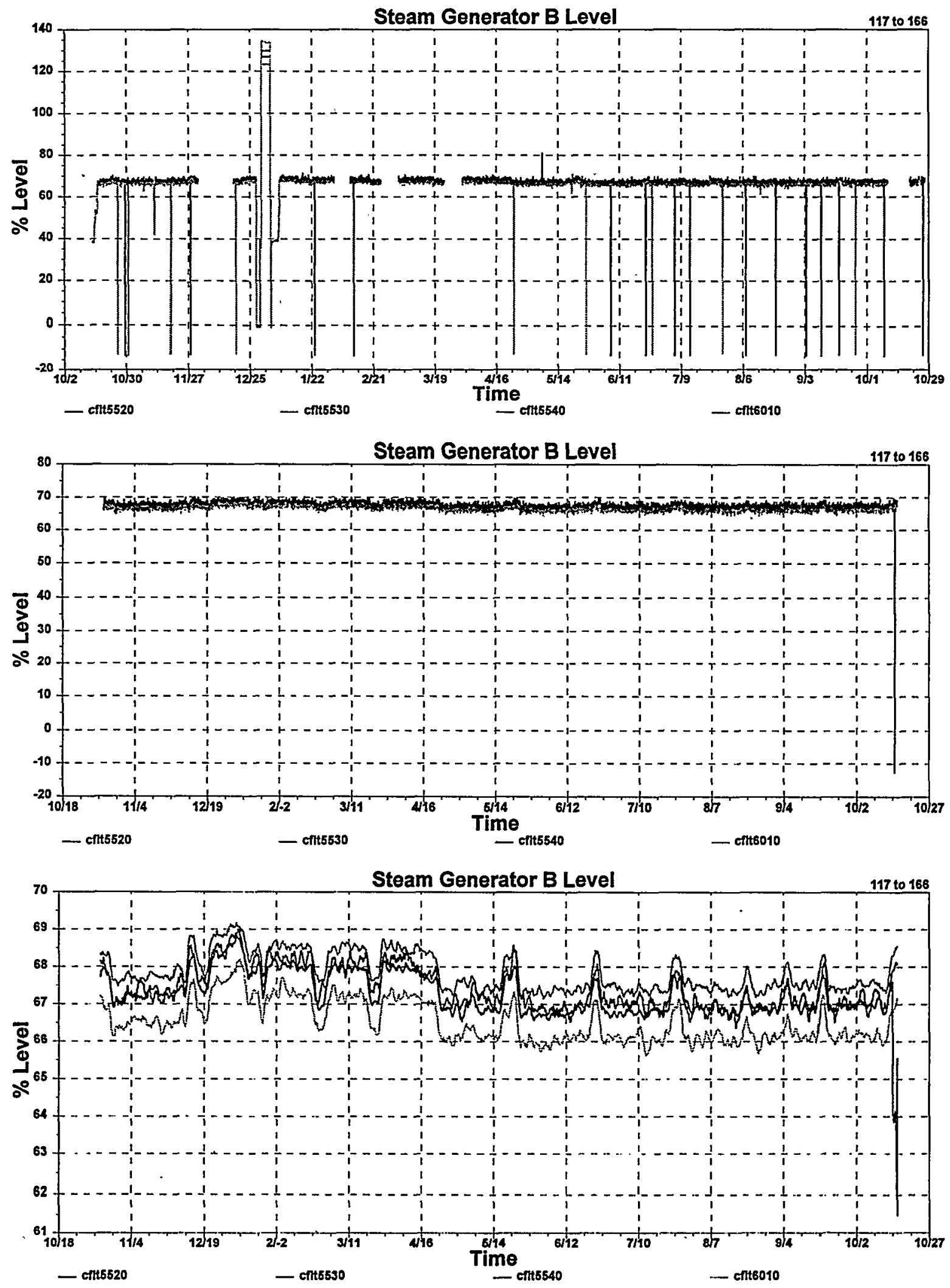

G.10 McGuire Cycle 2 Data for Steam Generator Level Signals for Loop B 

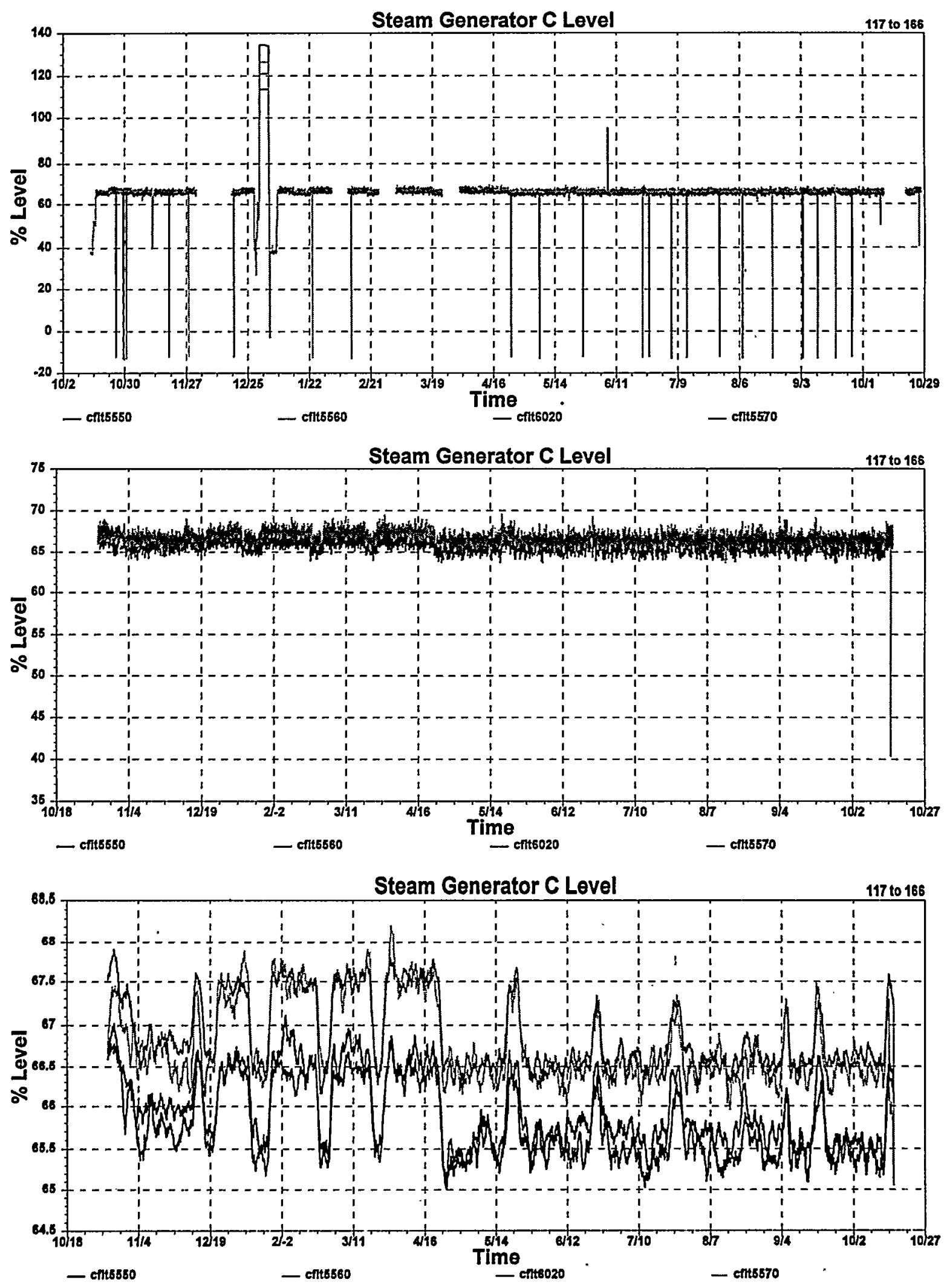

G.11 McGuire Cycle 2 Data for Steam Generator Level Signals for Loop C 

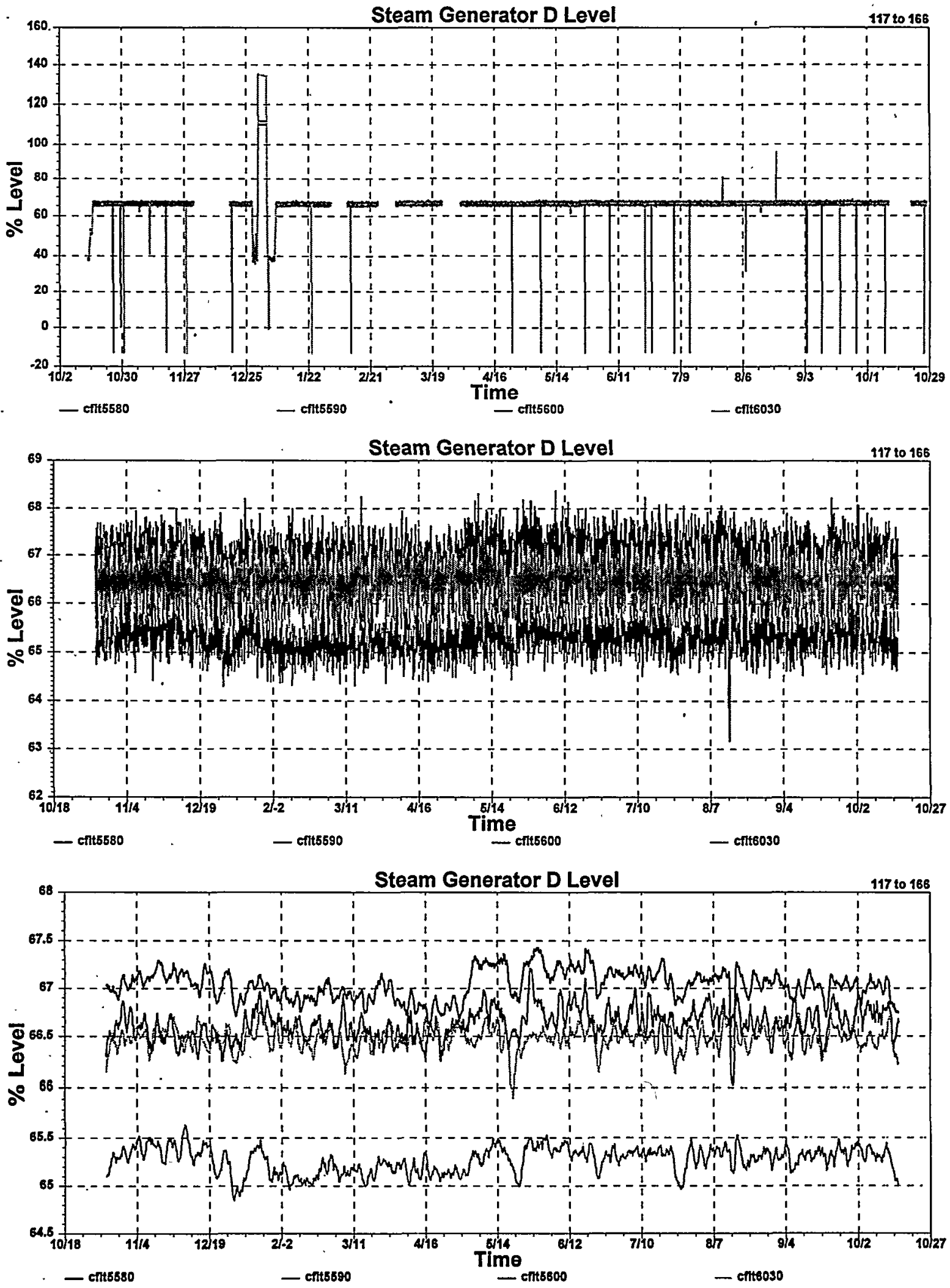

G.12 McGuire Cycle 2 Data for Steam Generator Level Signals for Loop D 

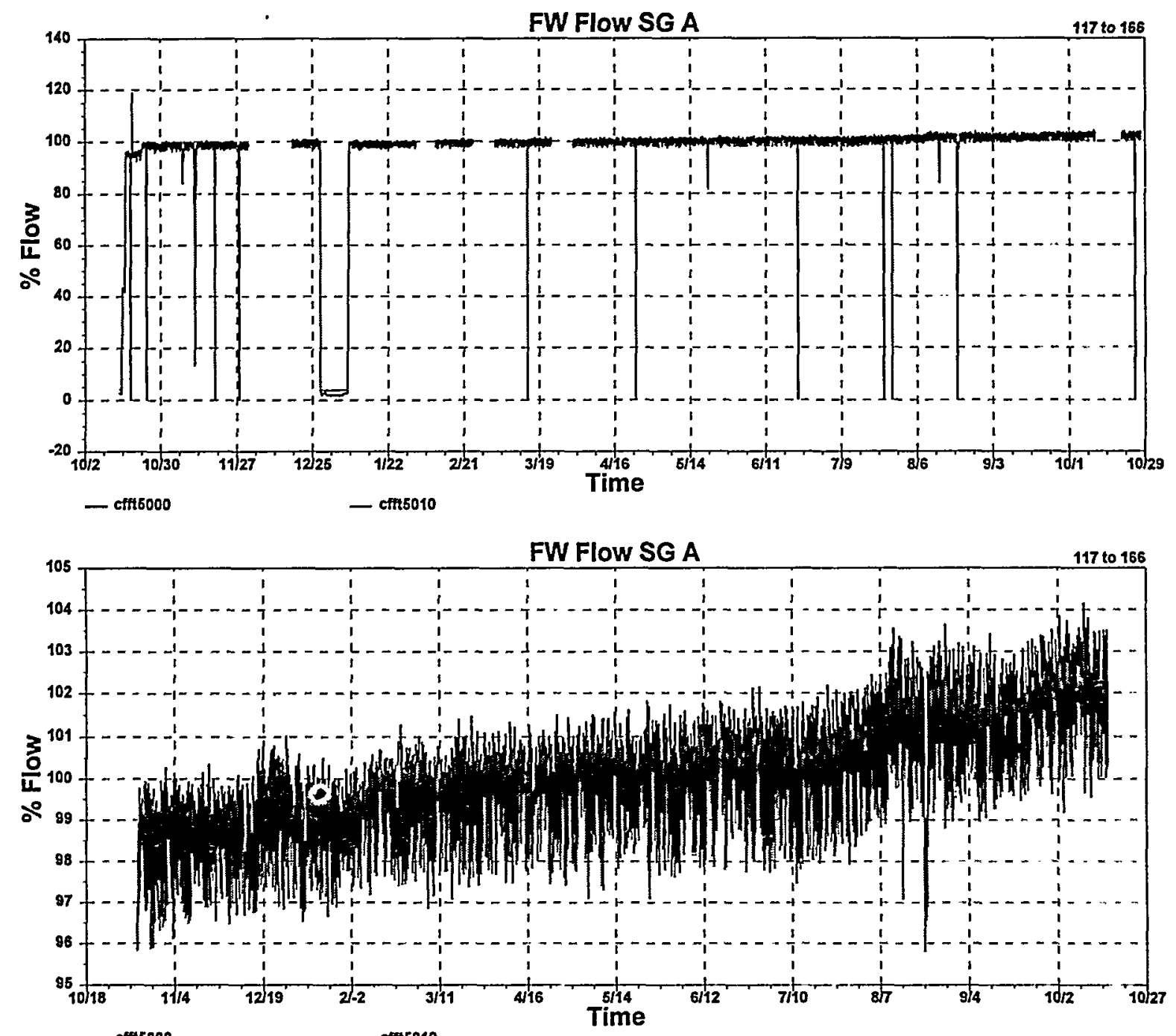

- cftt5000 - cfft5010

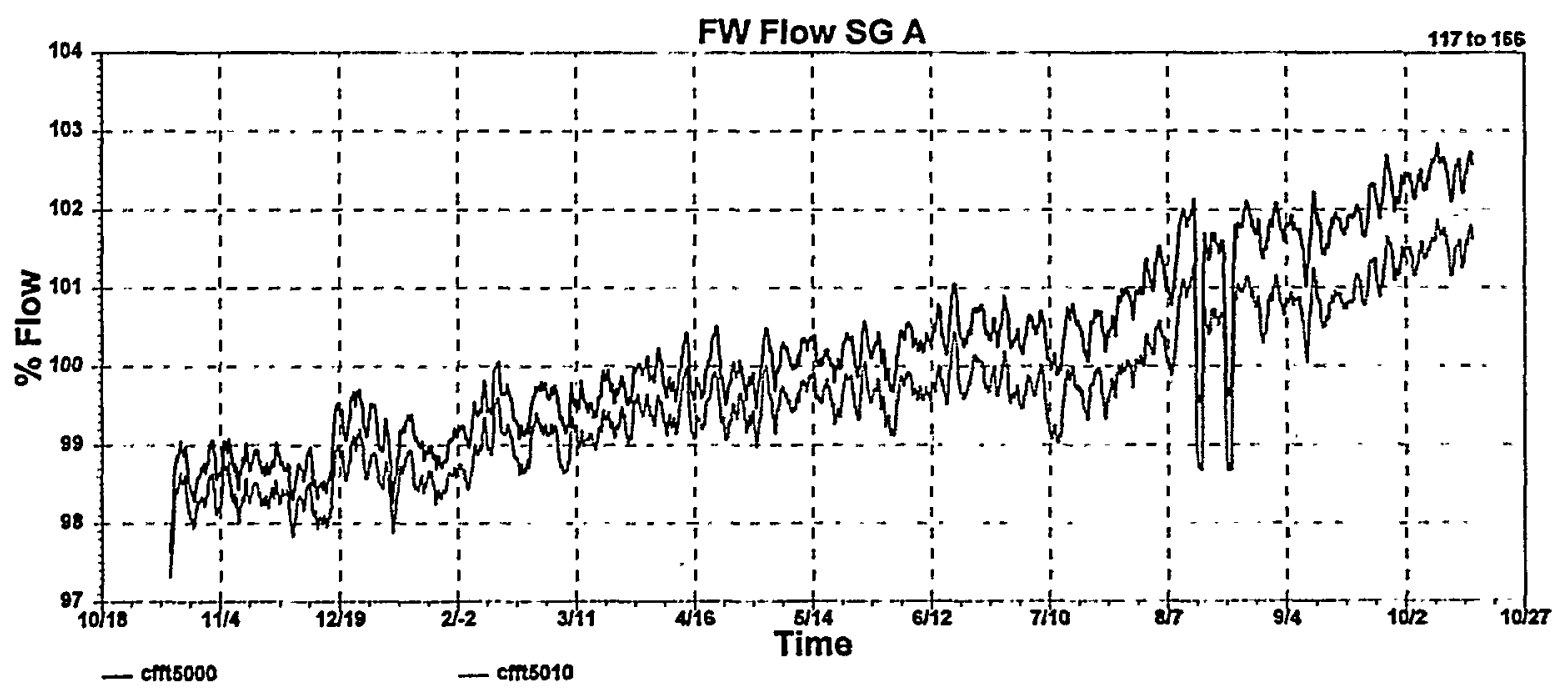

G.13 McGuire Cycle 2 Data for Feedwater Flow Signals for Loop A 

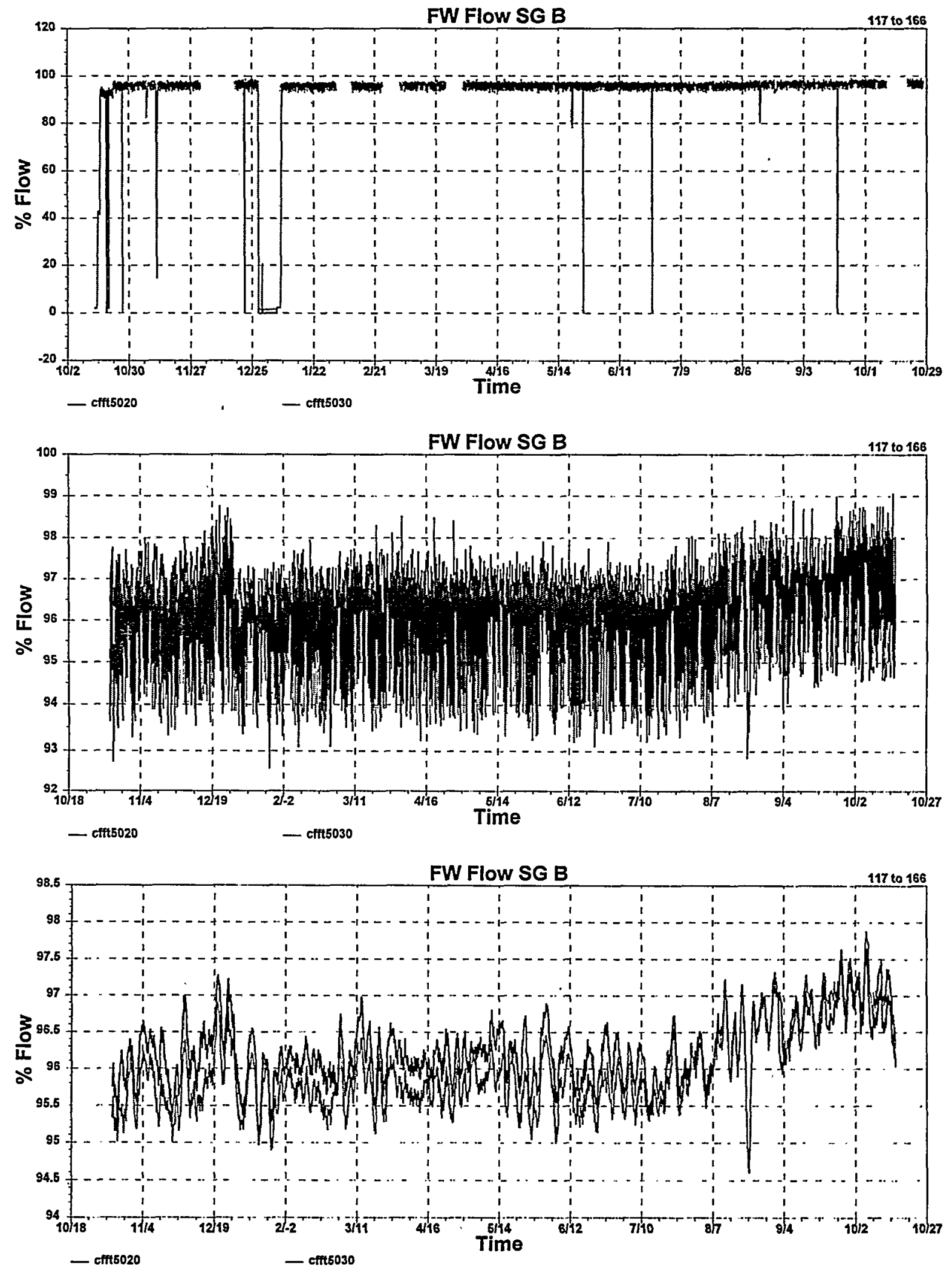

G.14 McGuire Cycle 2 Data for Feedwater Flow Signals for Loop B 

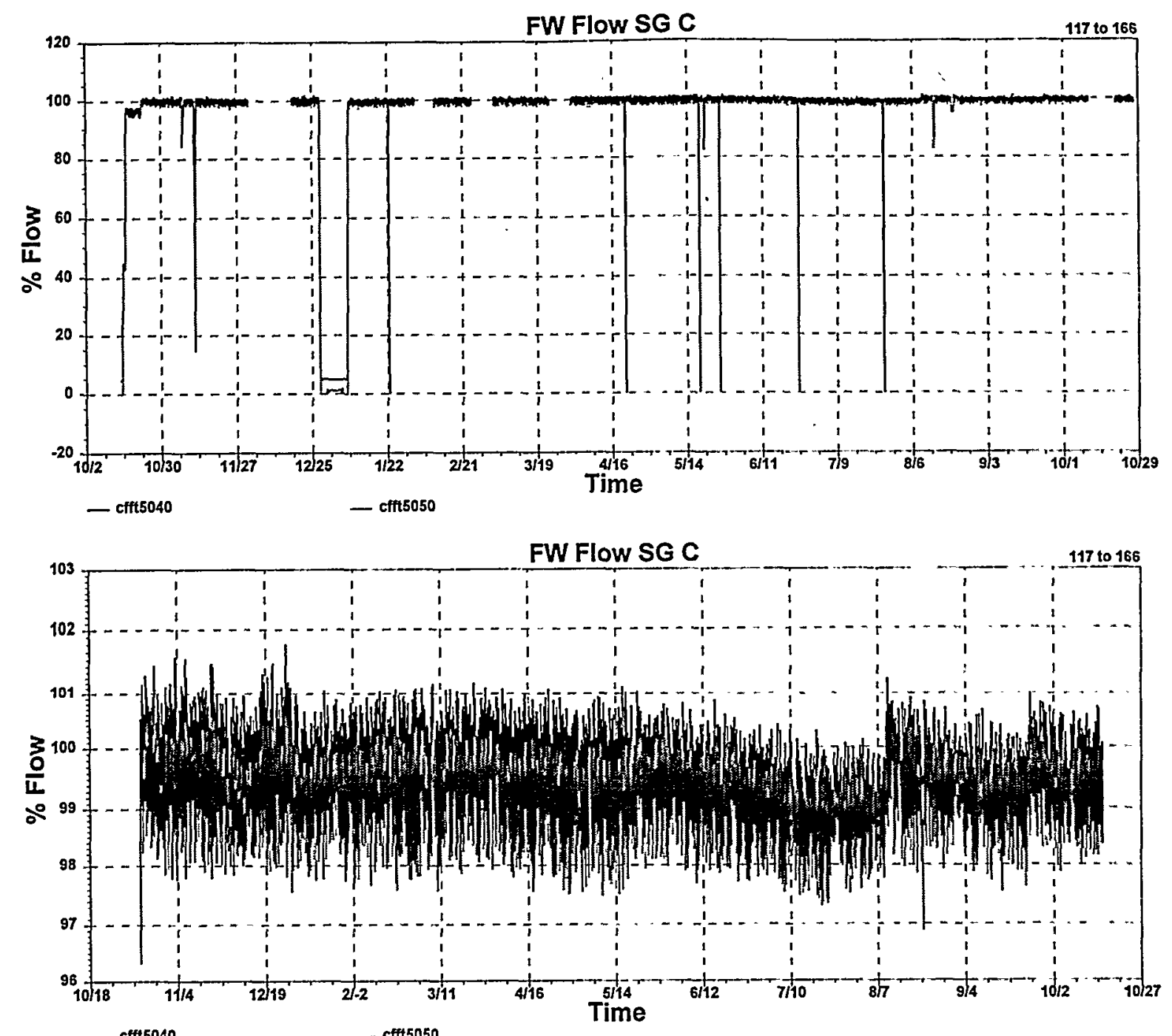

- cfft5040

- efft5050

FW Flow SG C

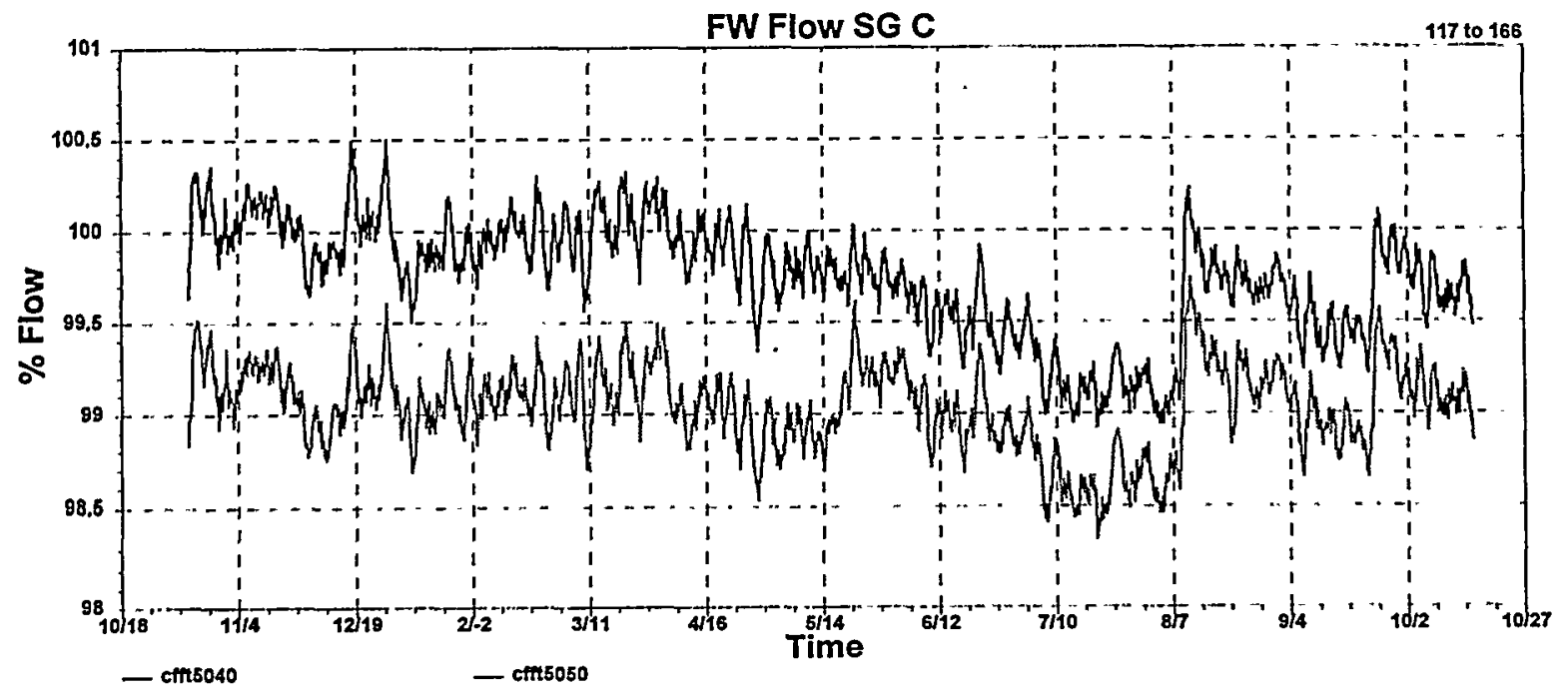

G.15 McGuire Cycle 2 Data for Feedwater Flow Signals for Loop C 

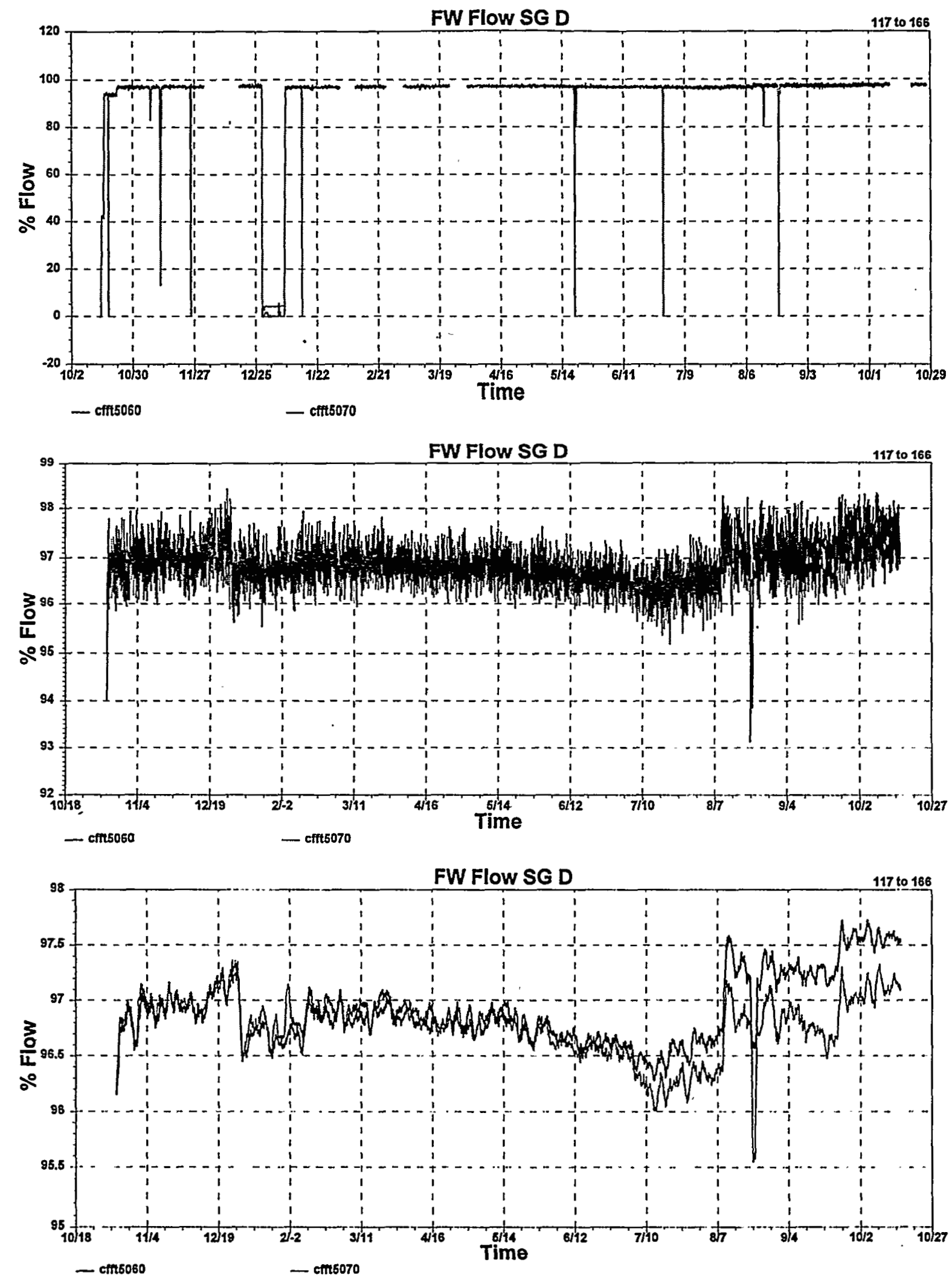

G.16 McGuire Cycle 2 Data for Feedwater Flow Signals for Loop D 


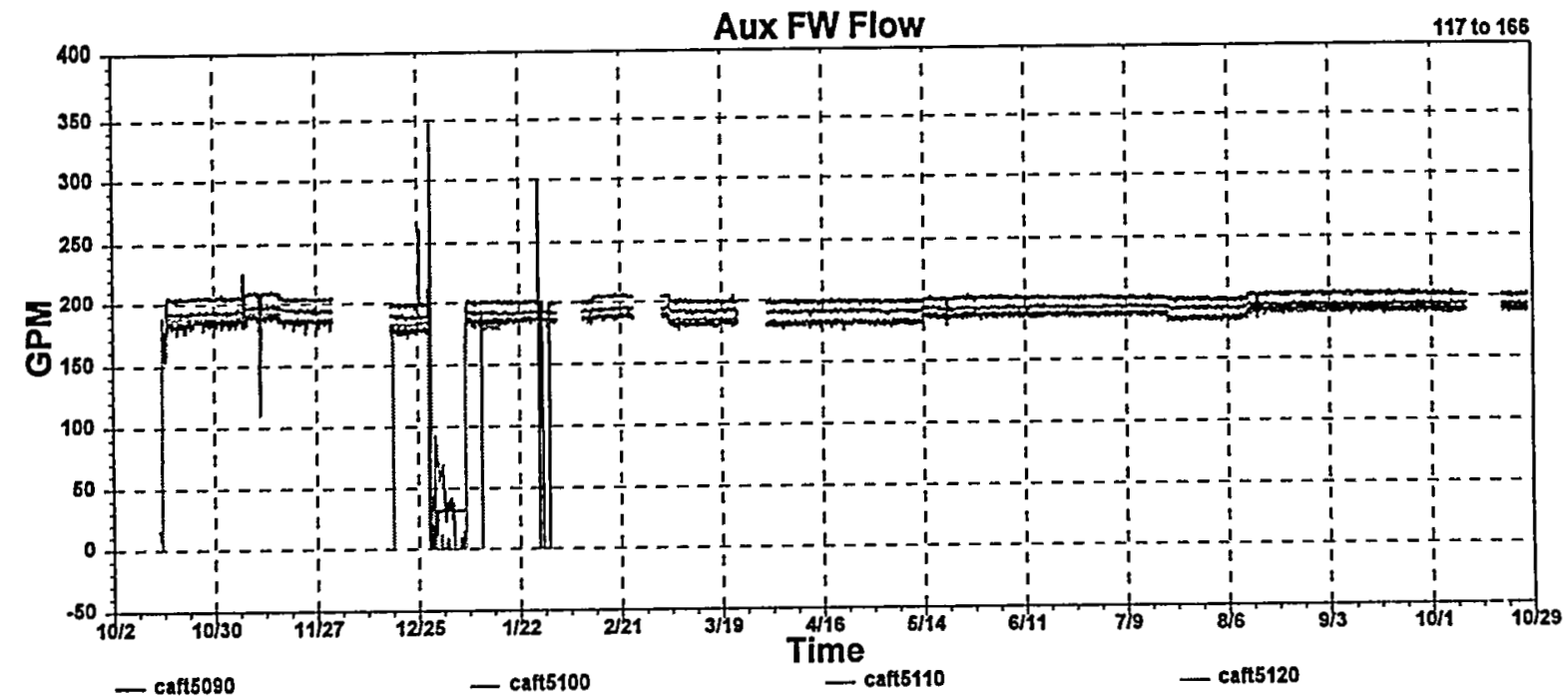

Aux FW Flow 117 to 166
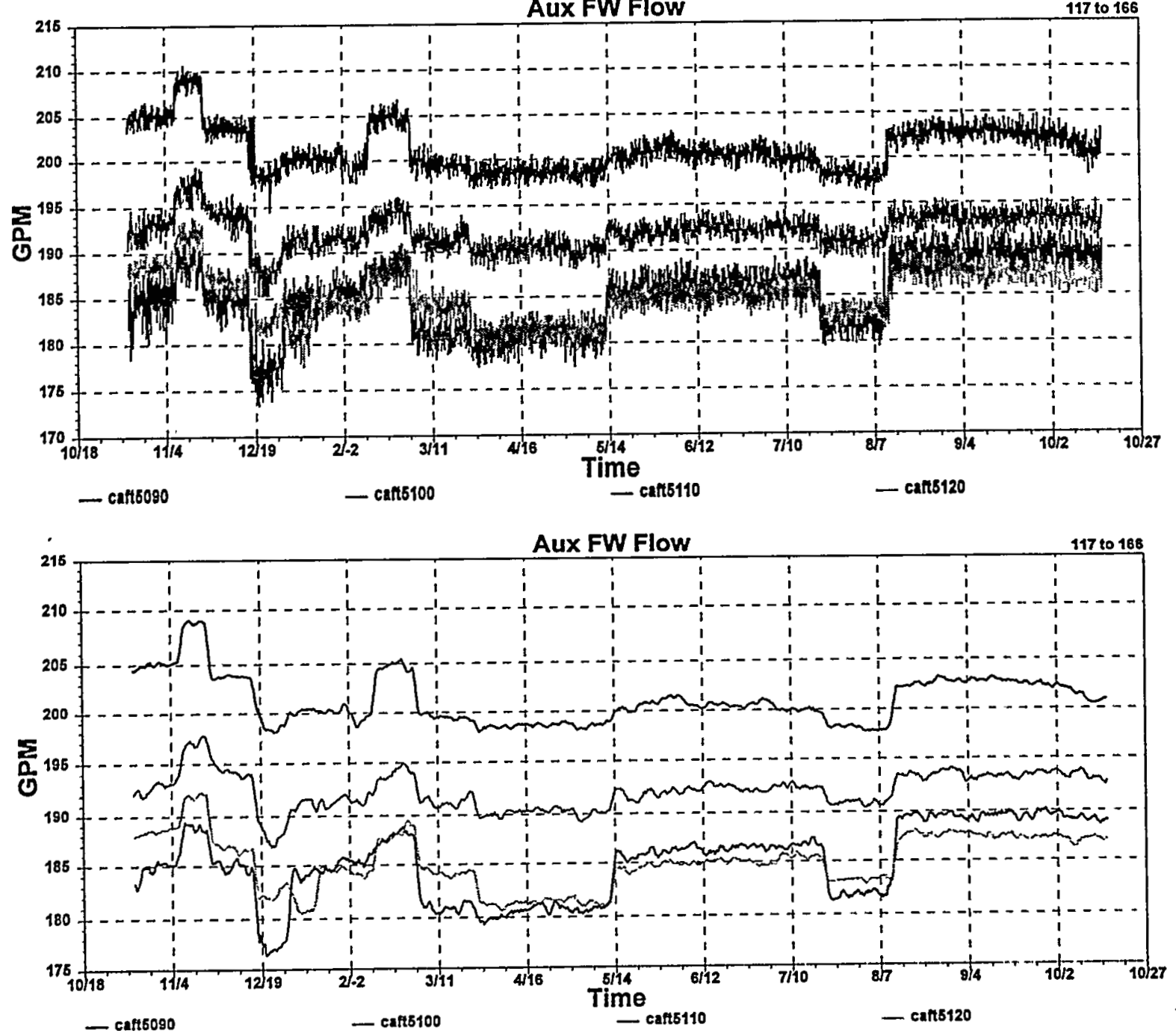

G.17 McGuire Cycle 2 Data for Auxiliary Feedwater Flow Signals 

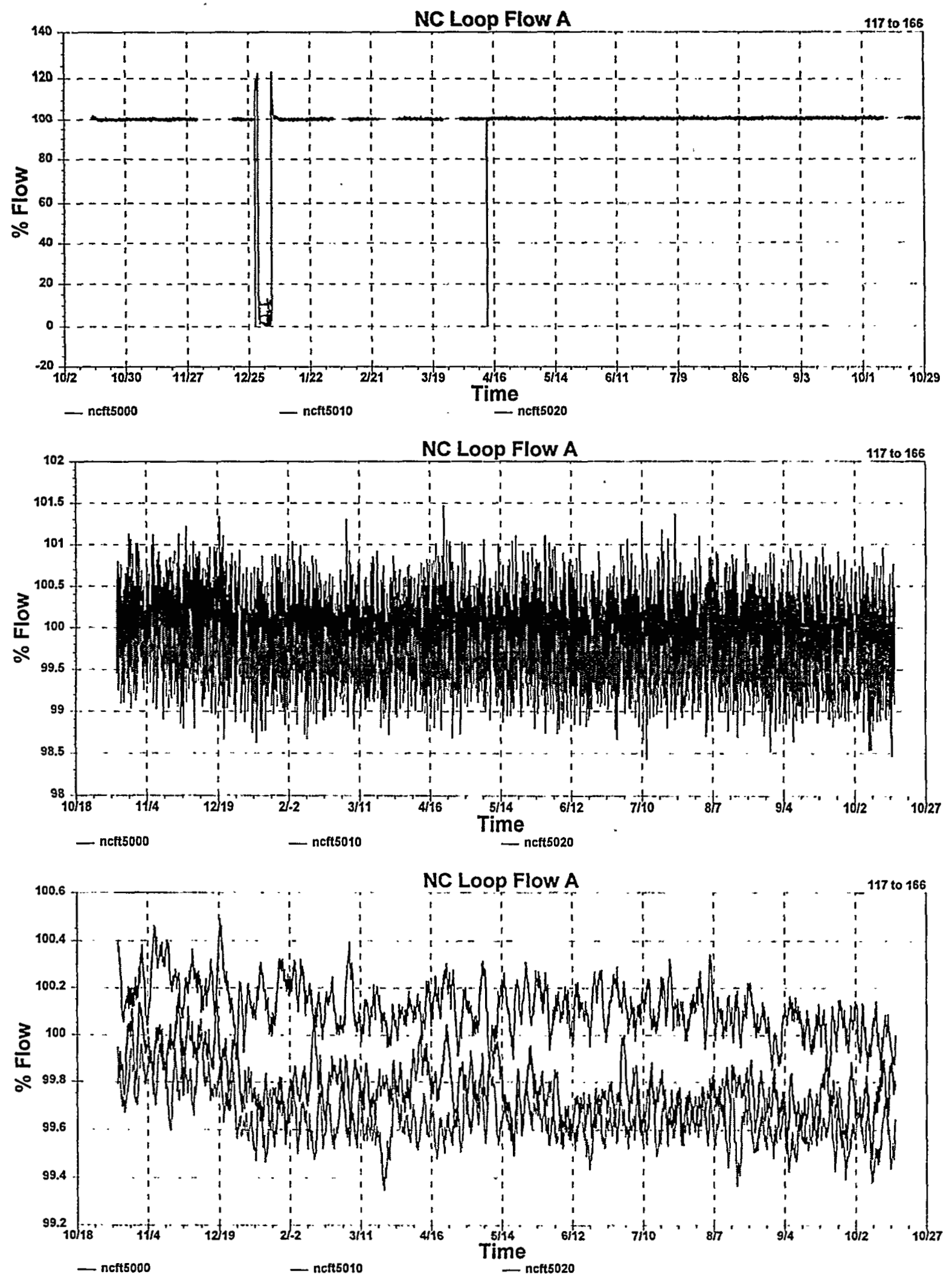

G.18 McGuire Cycle 2 Data for Reactor Coolant Flow Signals for Loop A 

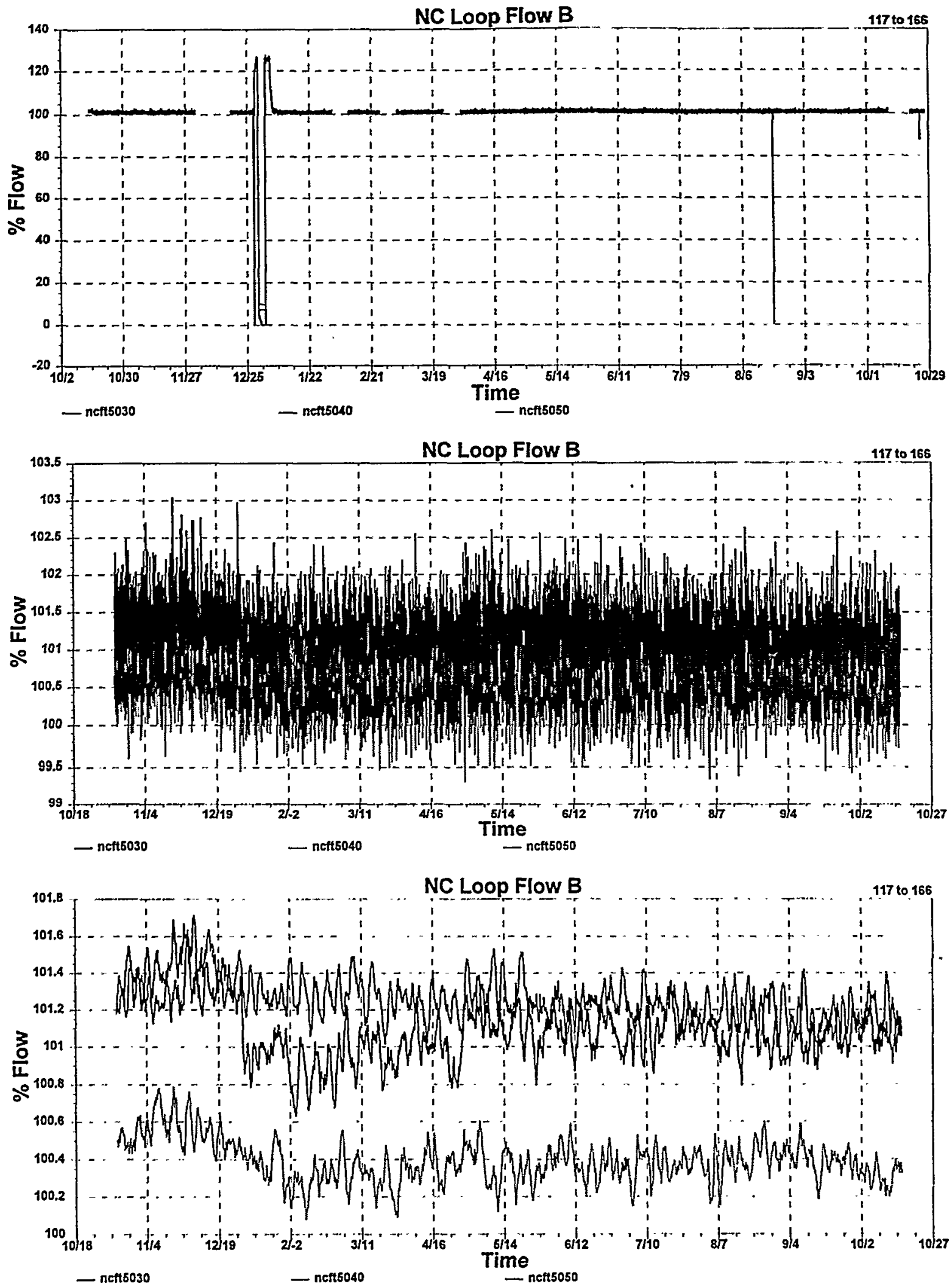

G.19 McGuire Cycle 2 Data for Reactor Coolant Flow Signals for Loop B 

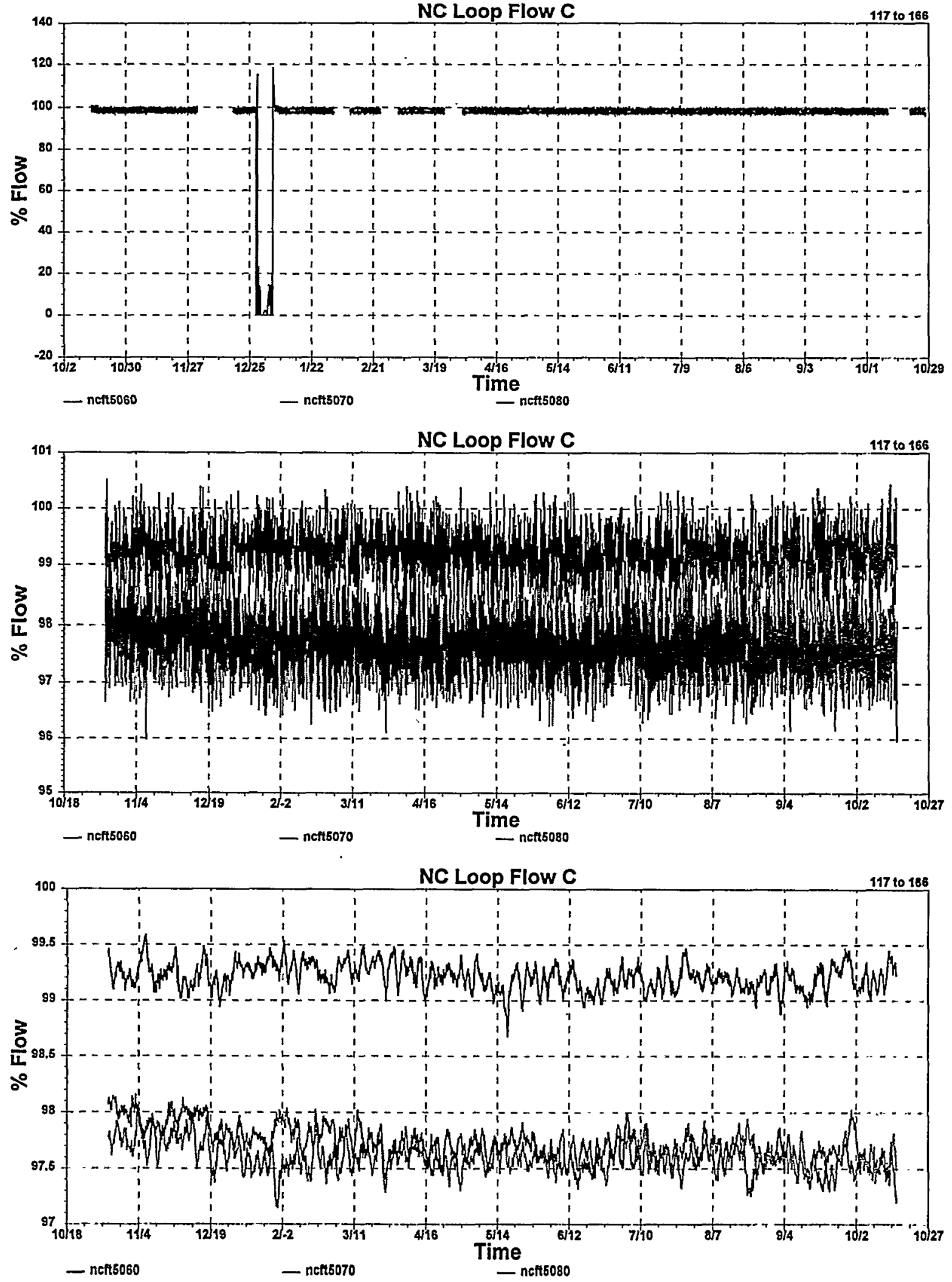

G.20 McGuire Cycle 2 Data for Reactor Coolant Flow Signals for Loop C 


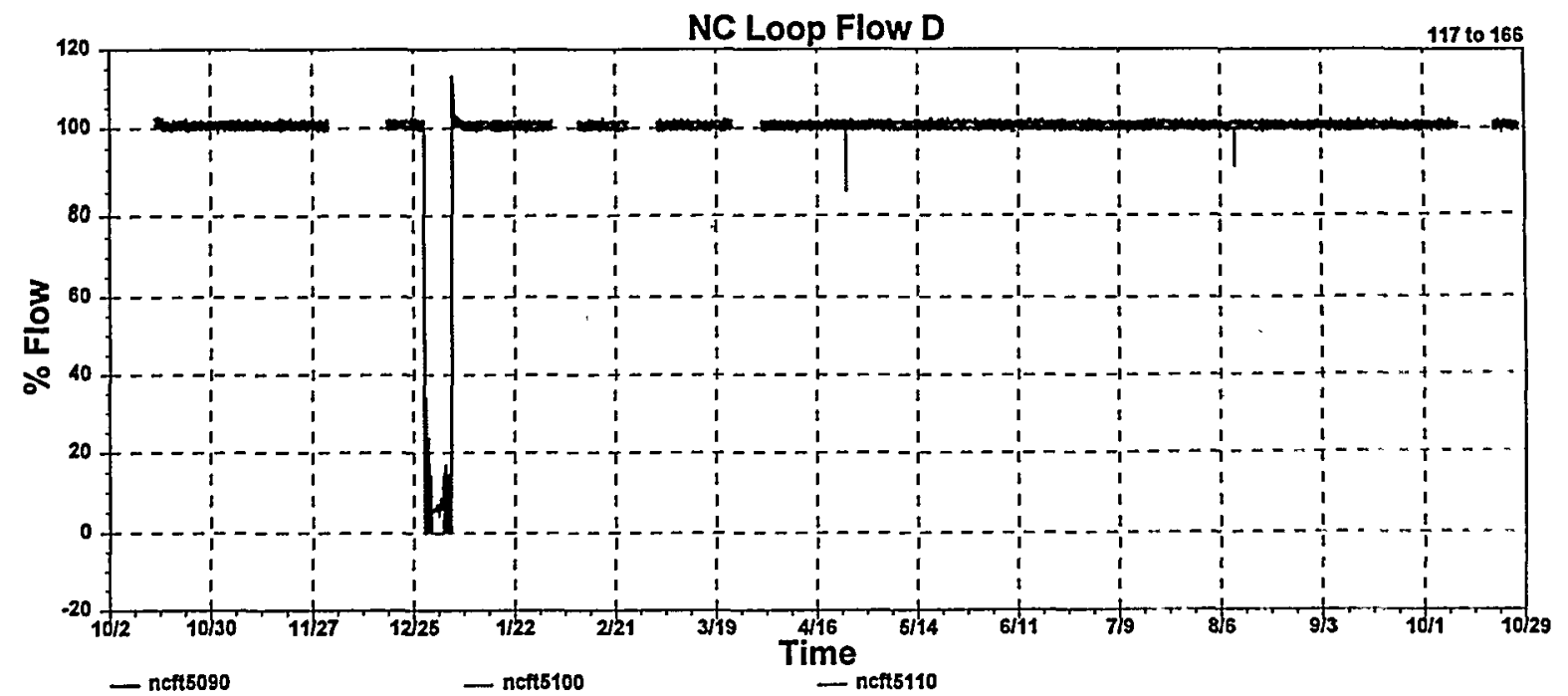

NC Loop Flow D

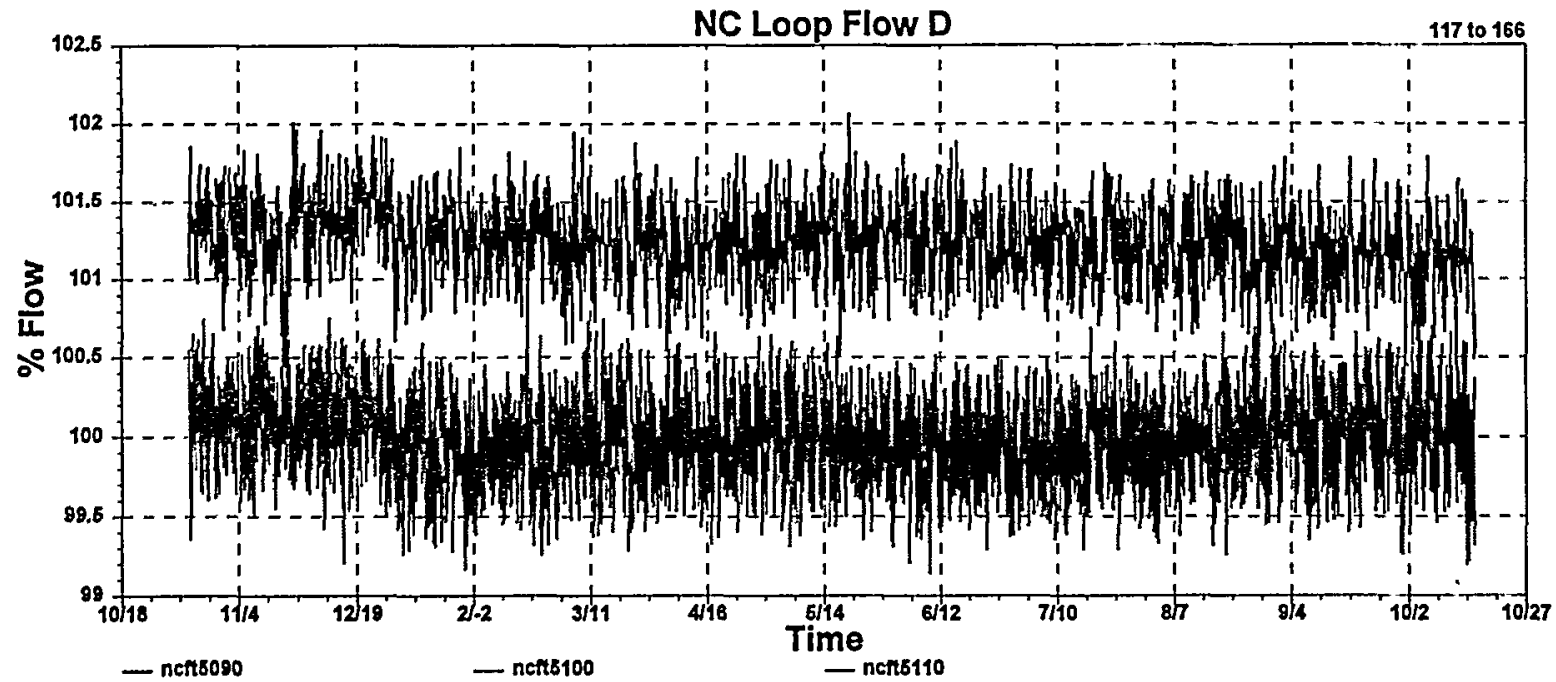

NC Loop Flow D

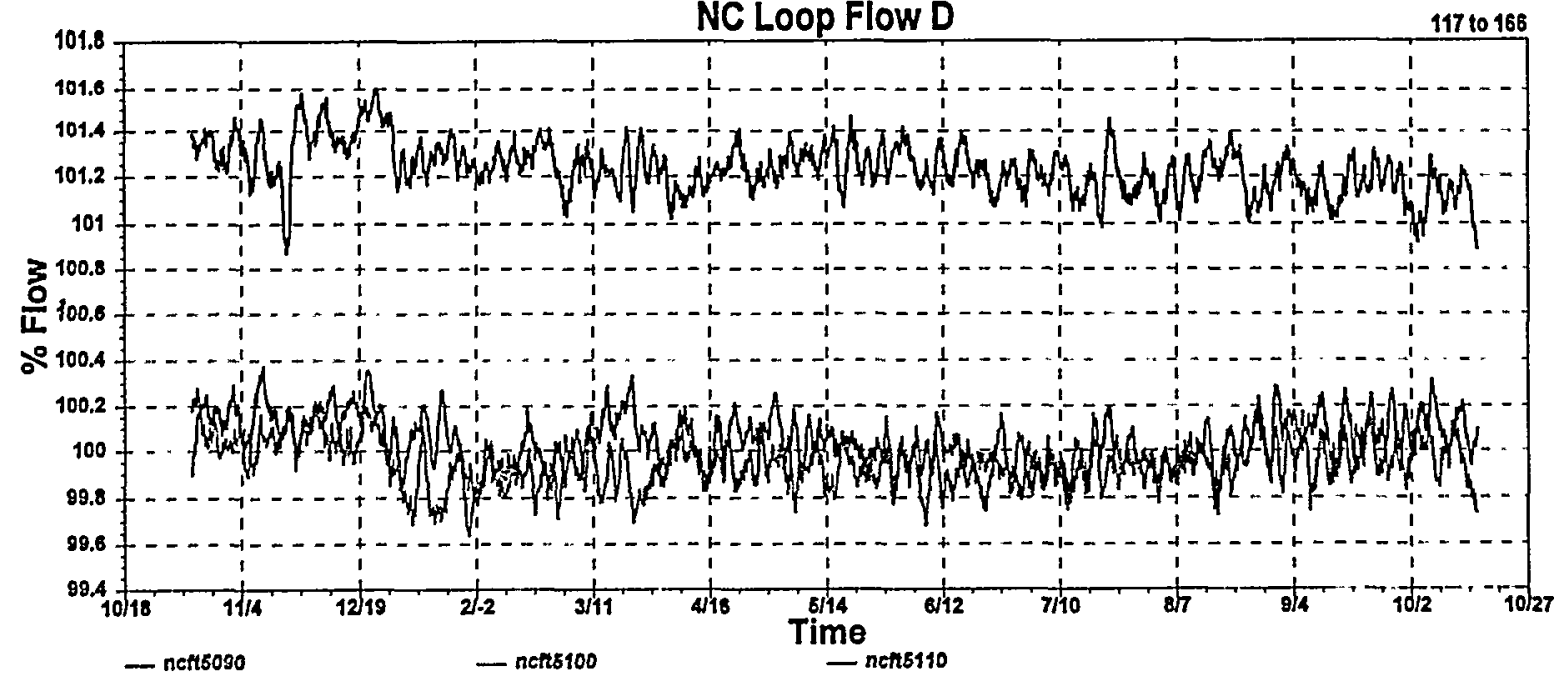

G.21 McGuire Cycle 2 Data for Reactor Coolant Flow Signals for Loop D 

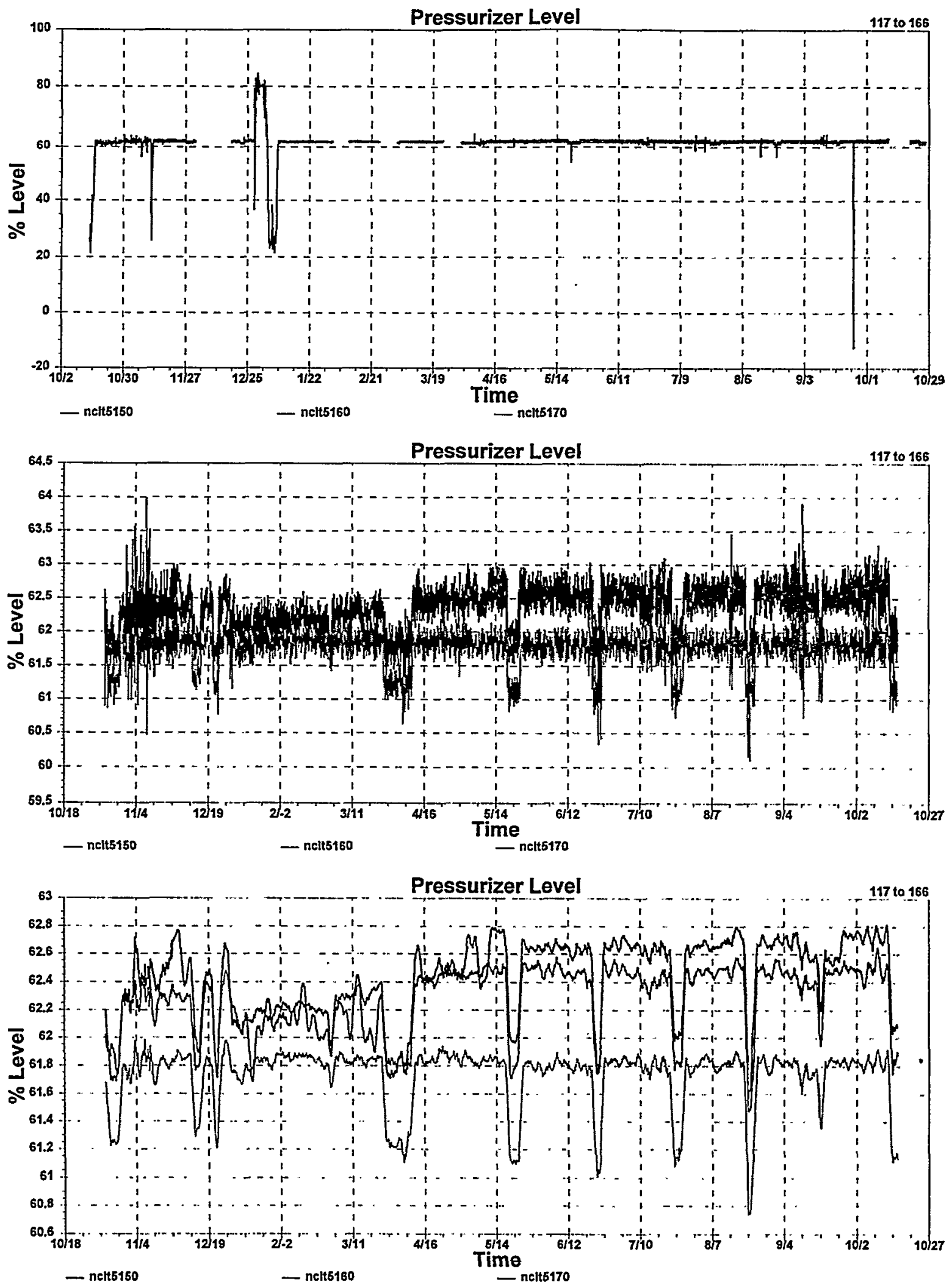

G.22 McGuire Cycle 2 Data for Pressurizer Level Signals 

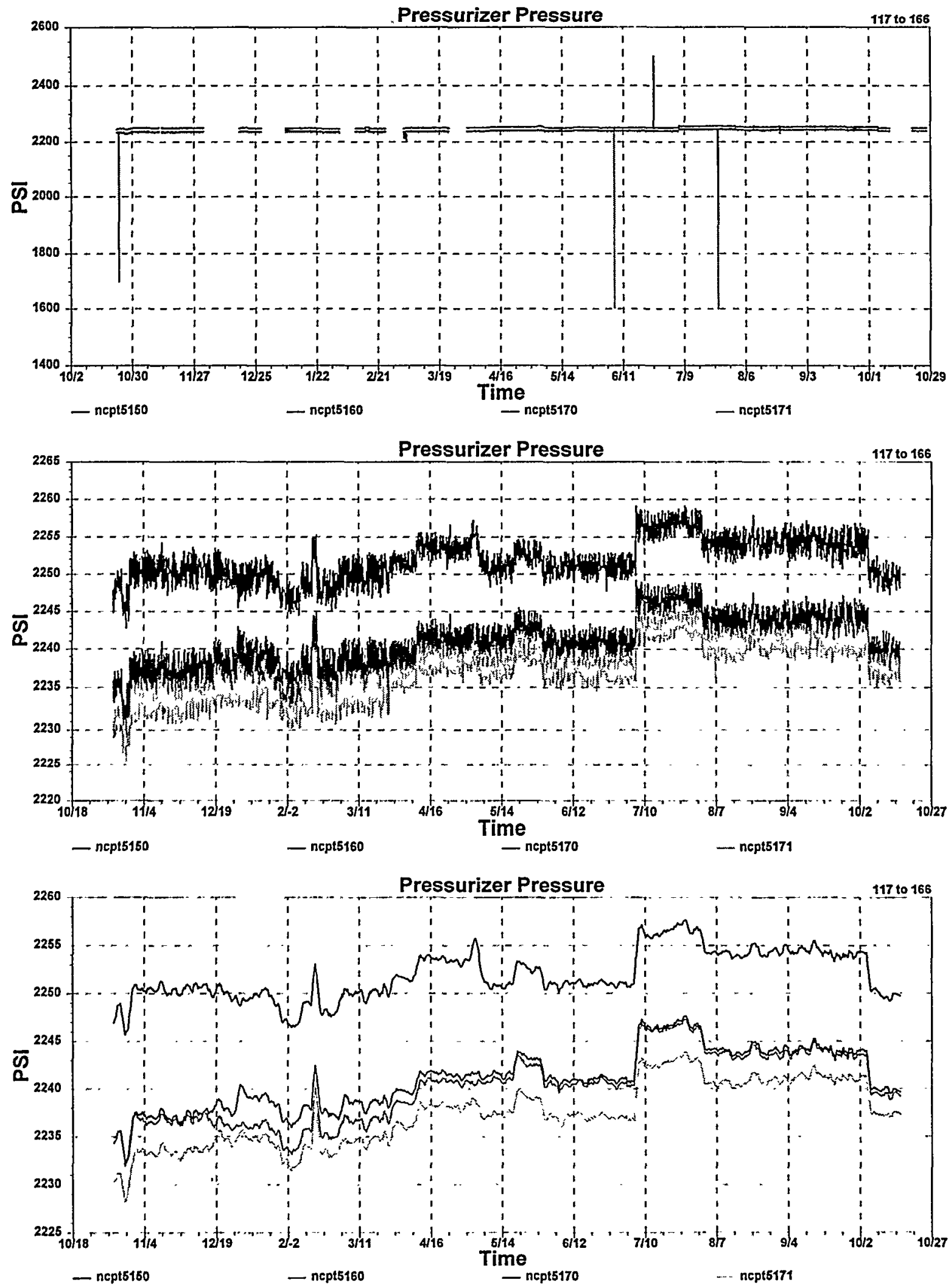

G.23 McGuire Cycle 2 Data for Pressurizer Pressure Signals 

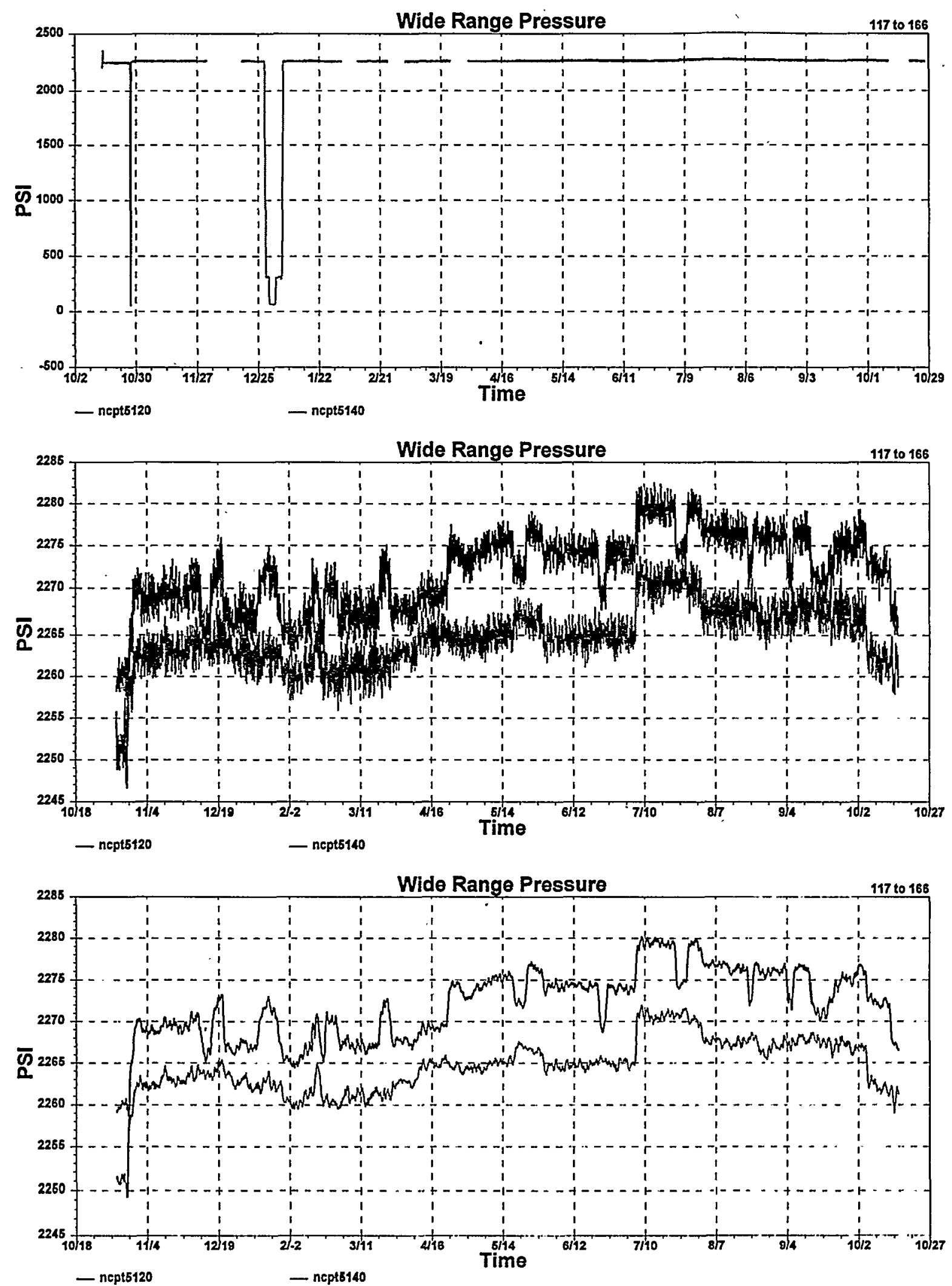

G.24 McGuire Cycle 2 Data for Wide-Range Pressure Signals 

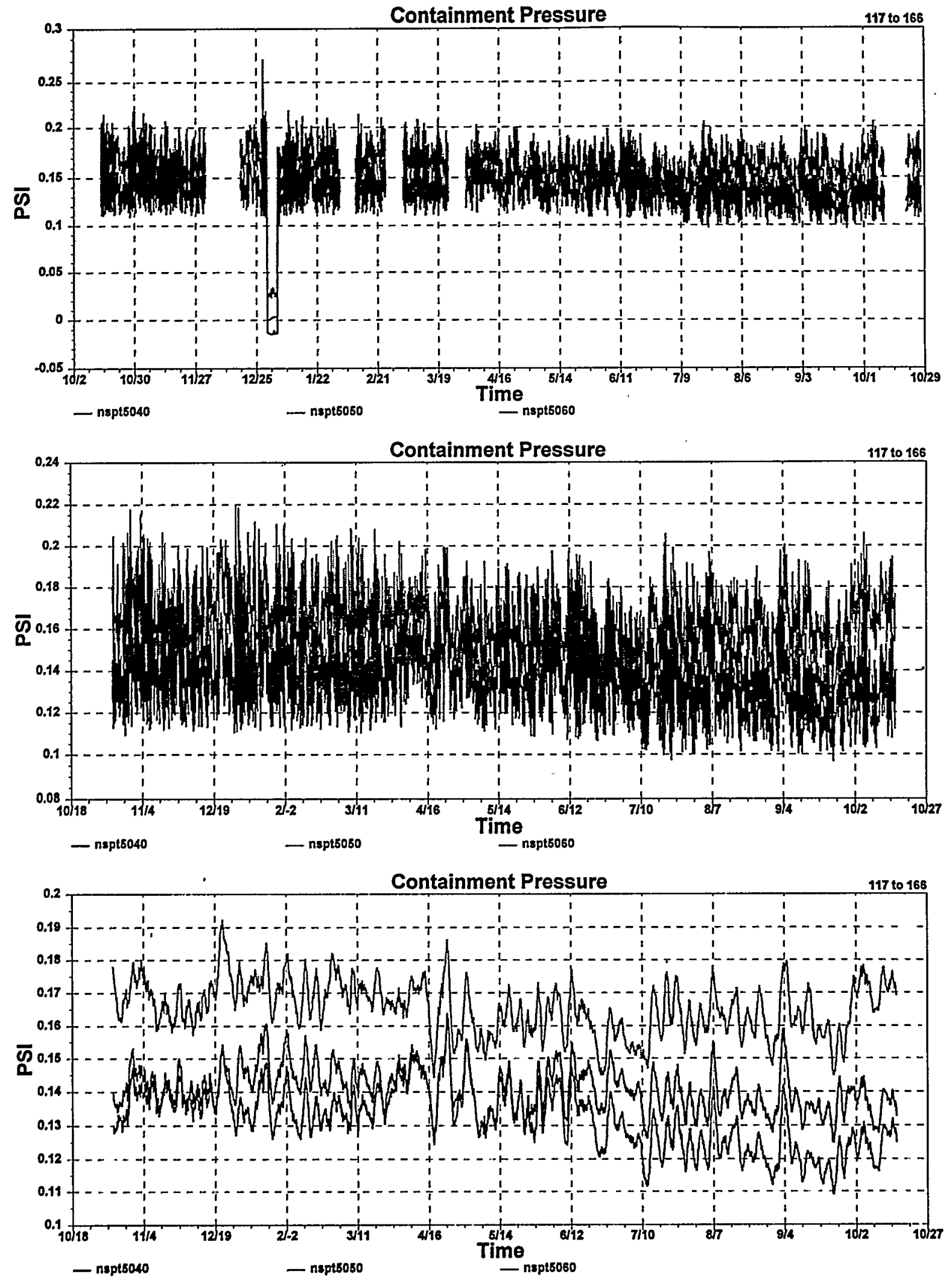

G.25 McGuire Cycle 2 Data for Containment Pressure Signals 

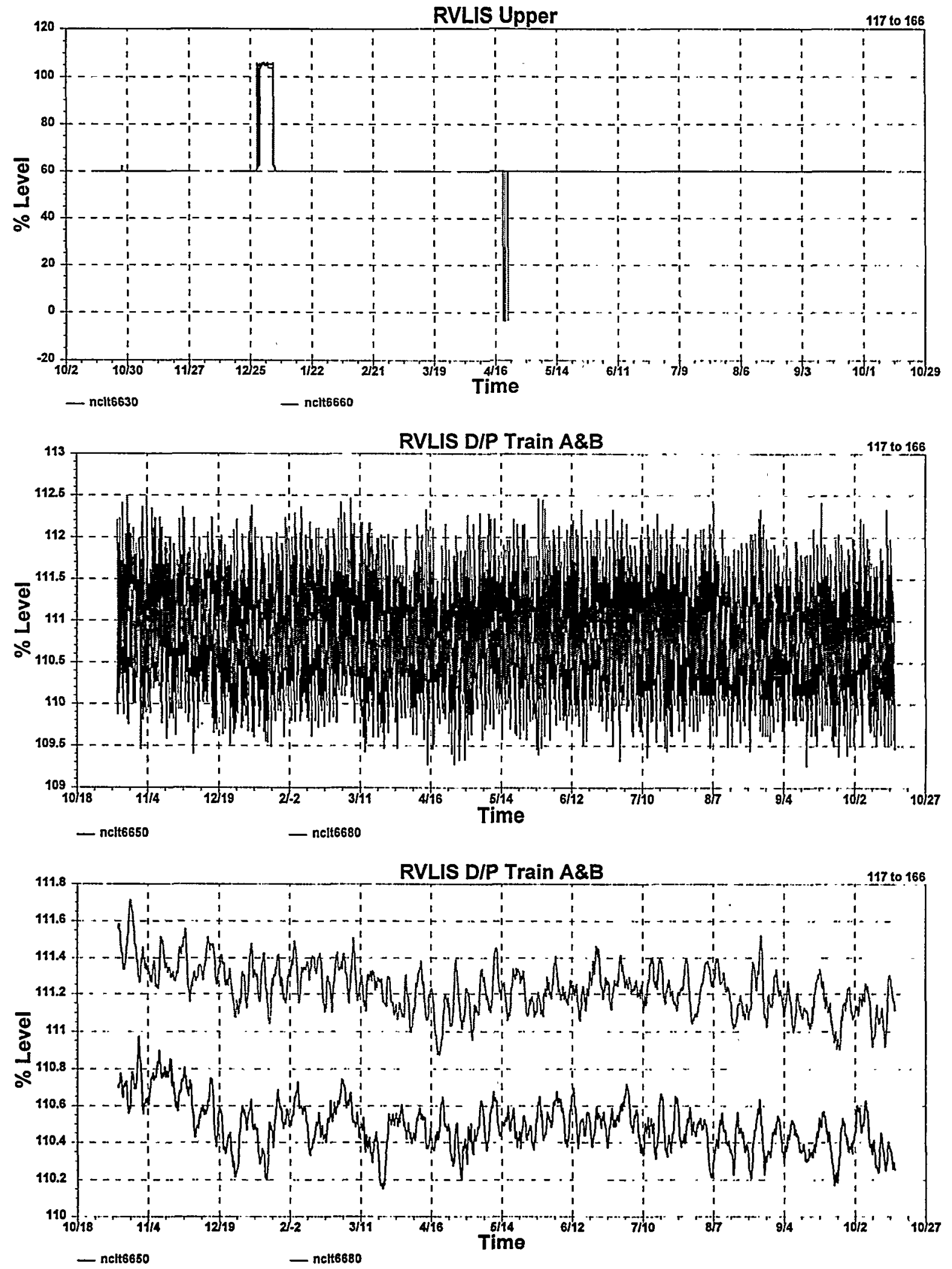

G.26 McGuire Cycle 2 Data for Reactor Vessel Level Indication System Signals 


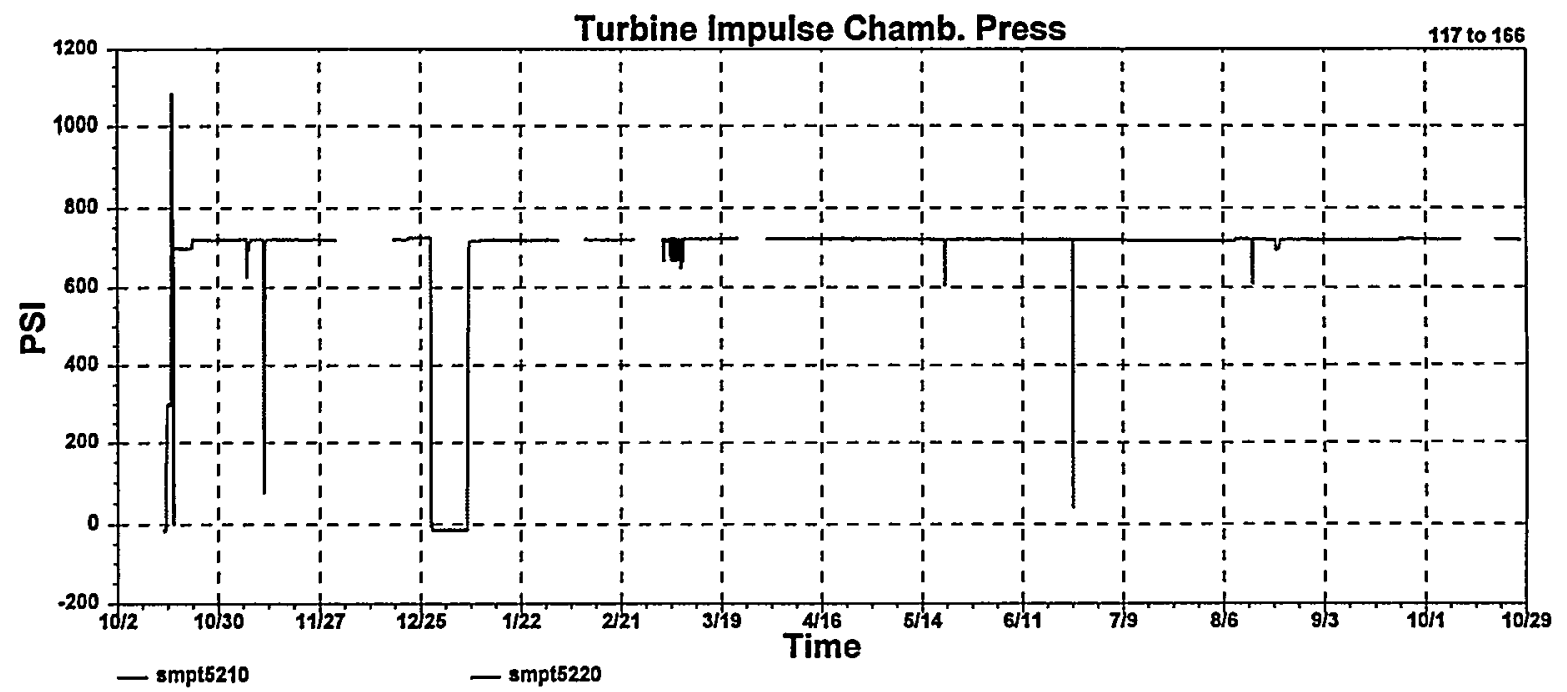

Turbine Impulse Chamb. Press

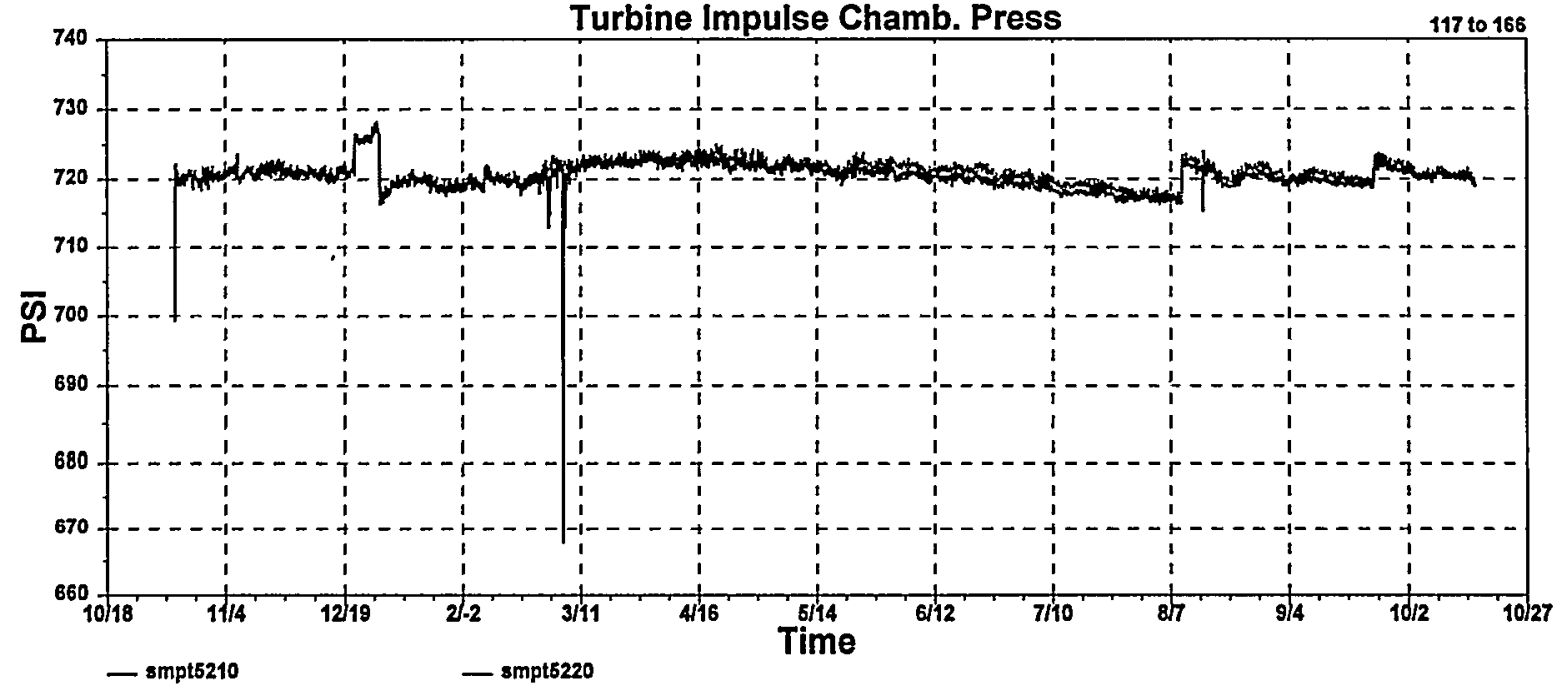

Turbine Impulse Chamb. Press

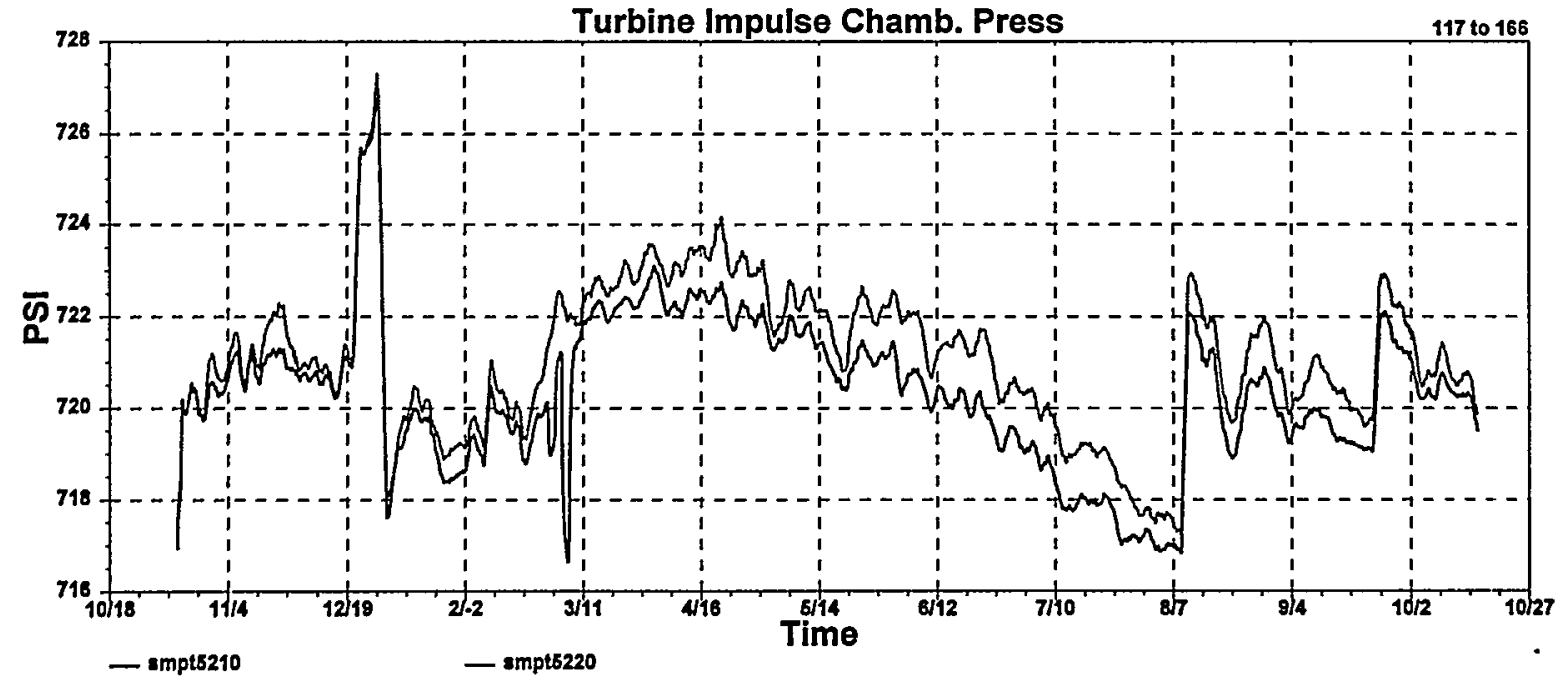

G.27 McGuire Cycle 2 Data for Turbine Impulse Pressure Signals 

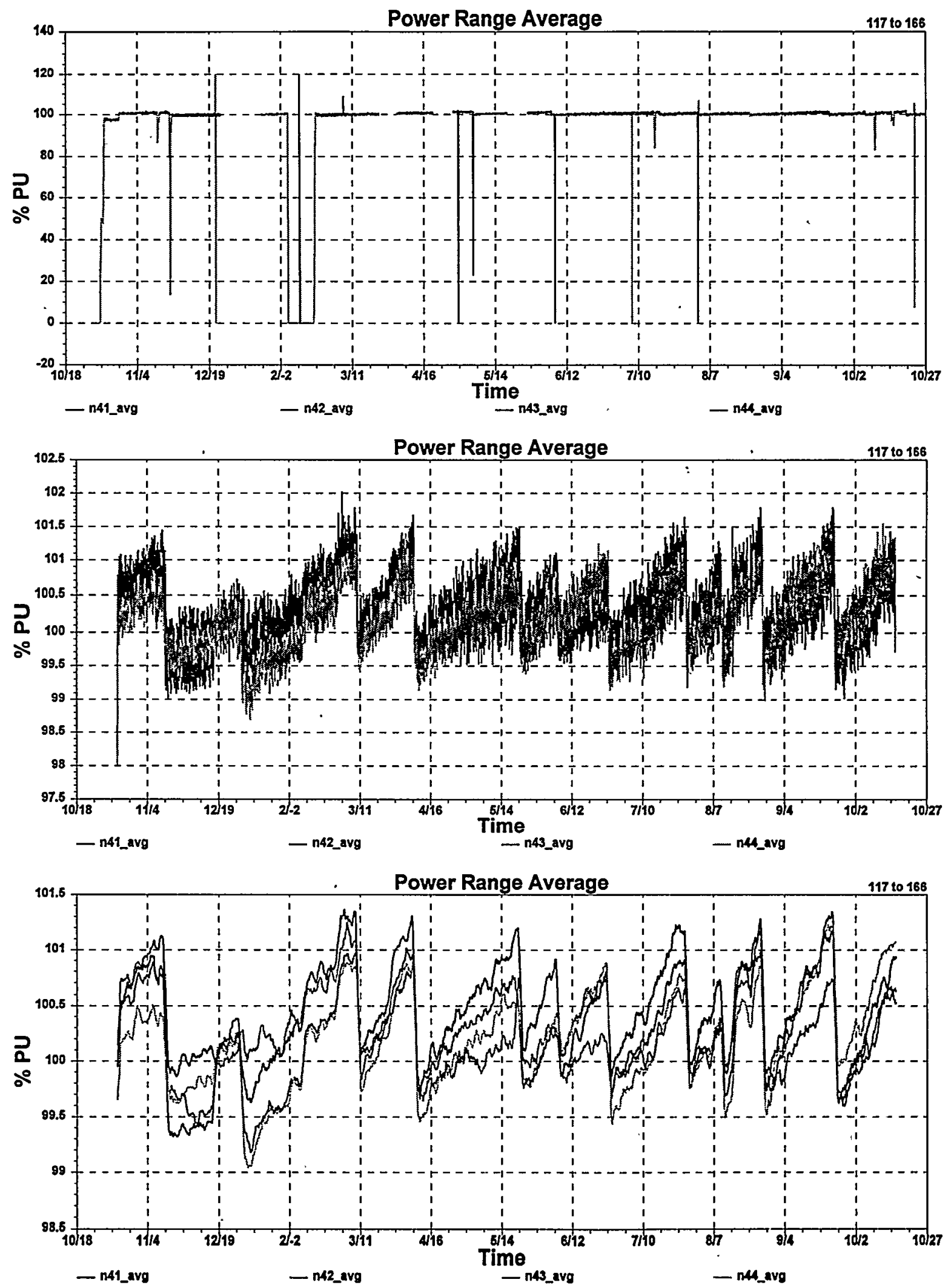

G.28 McGuire Cycle 2 Data for Neutron Sensors 


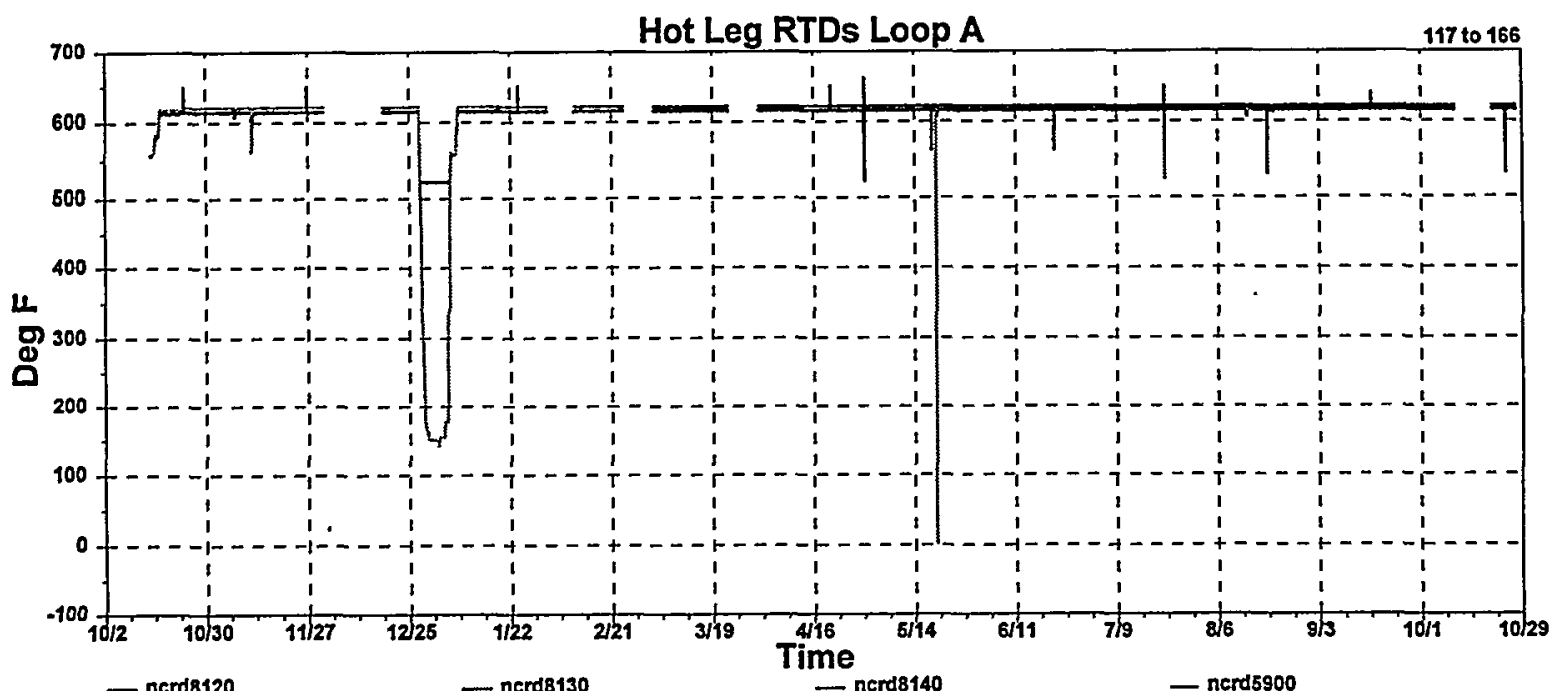

Hot Leg RTDs Loop A

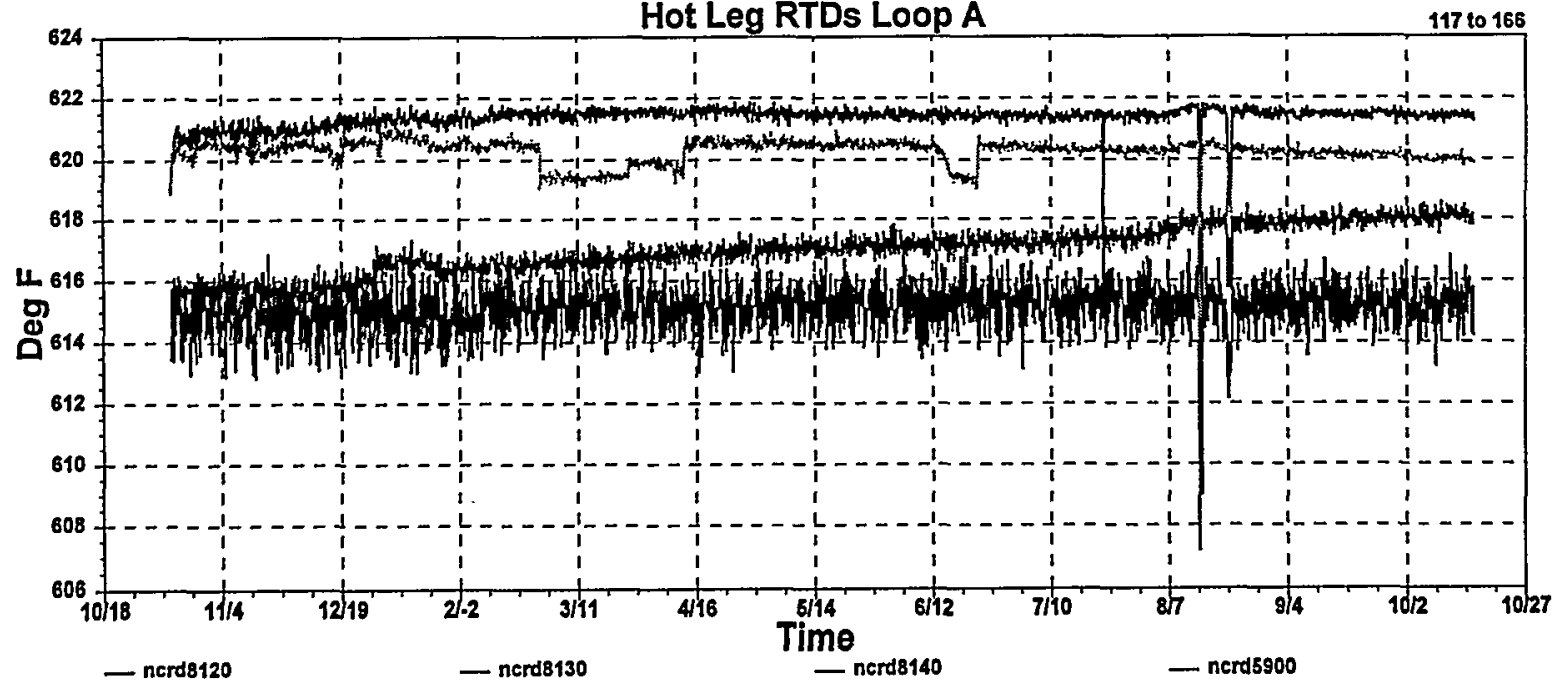

Hot Leg RTDs Loop A

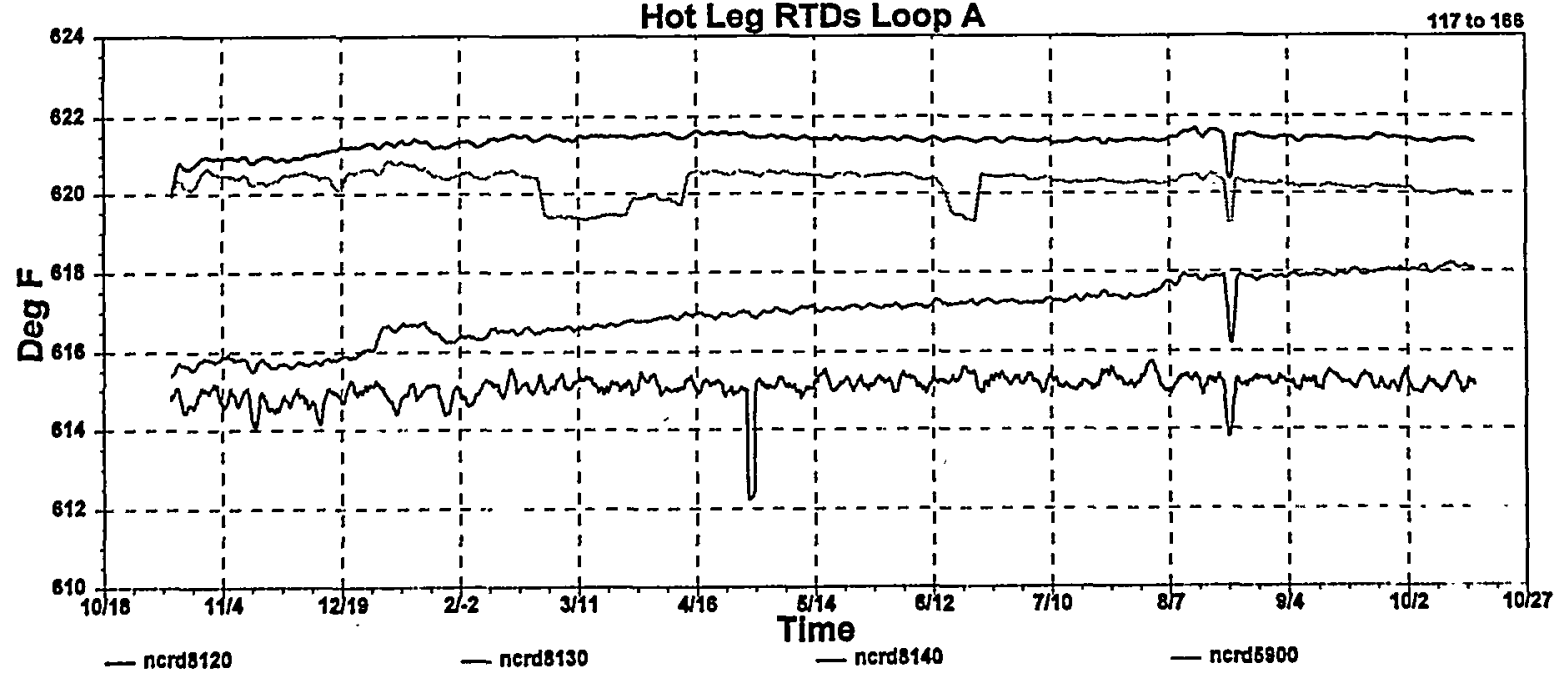

G.29 McGuire Cycle 2 Data for Hot Leg RTD Signals for Loop A 


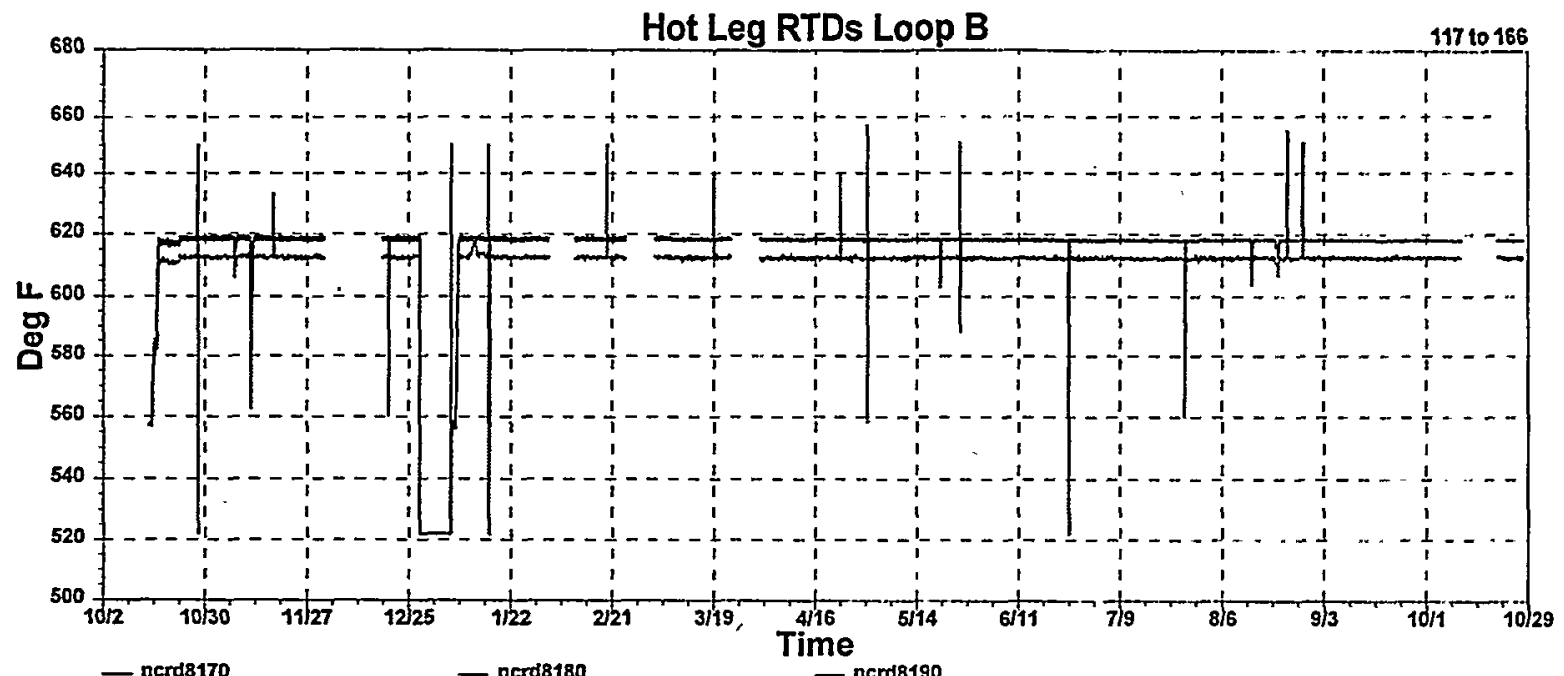

Hot Leg RTDs Loop B
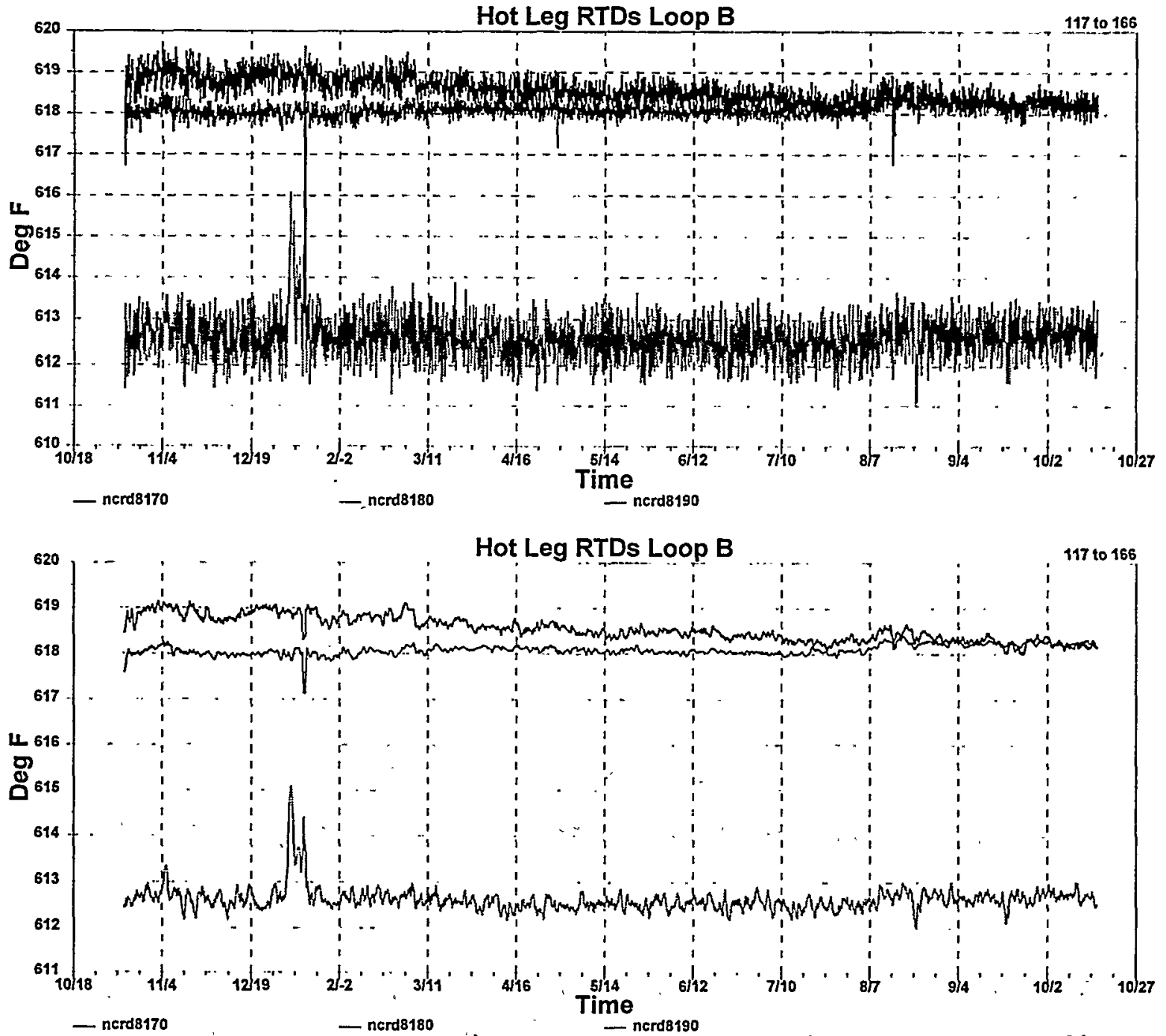

G.30 McGuire Cycle 2 Data for Hot Leg RTD Signals for Loop B 


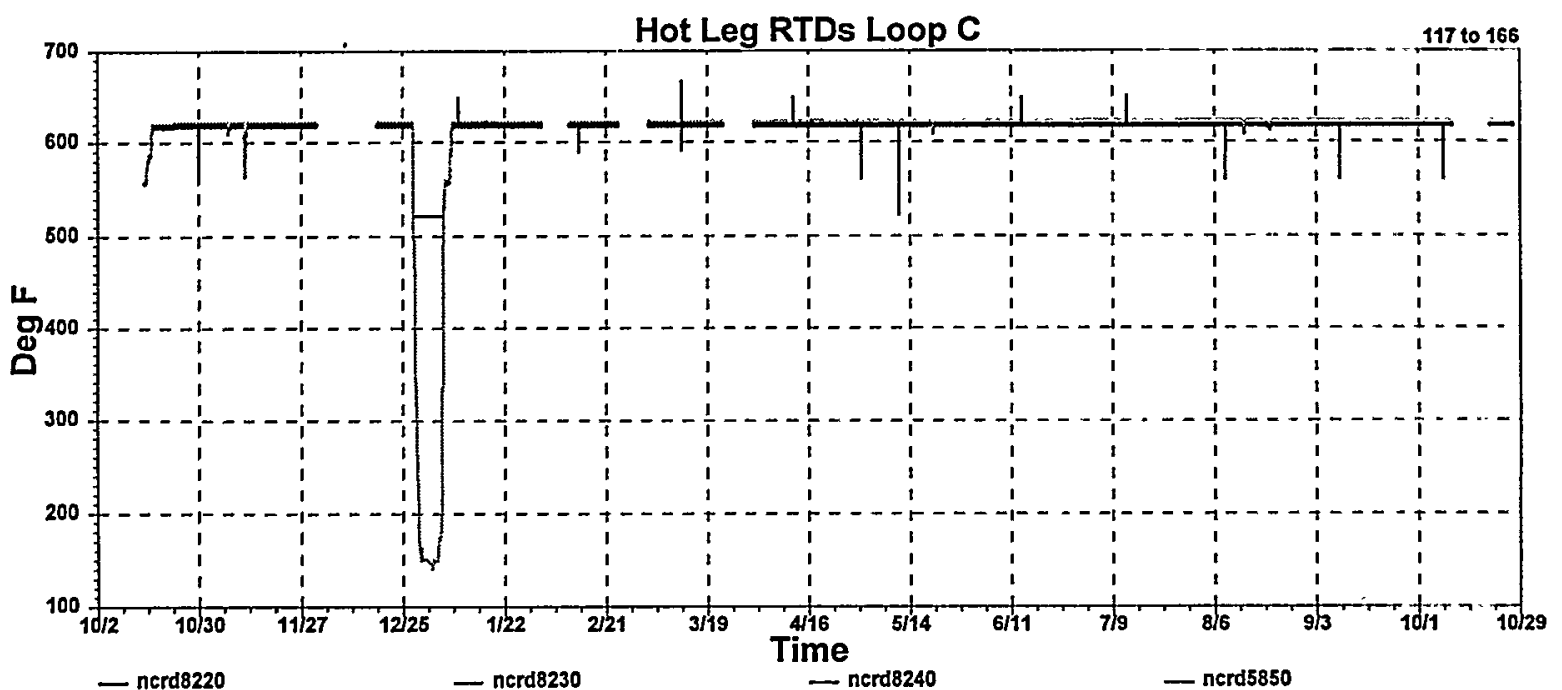

Hot Leg RTDs Loop C

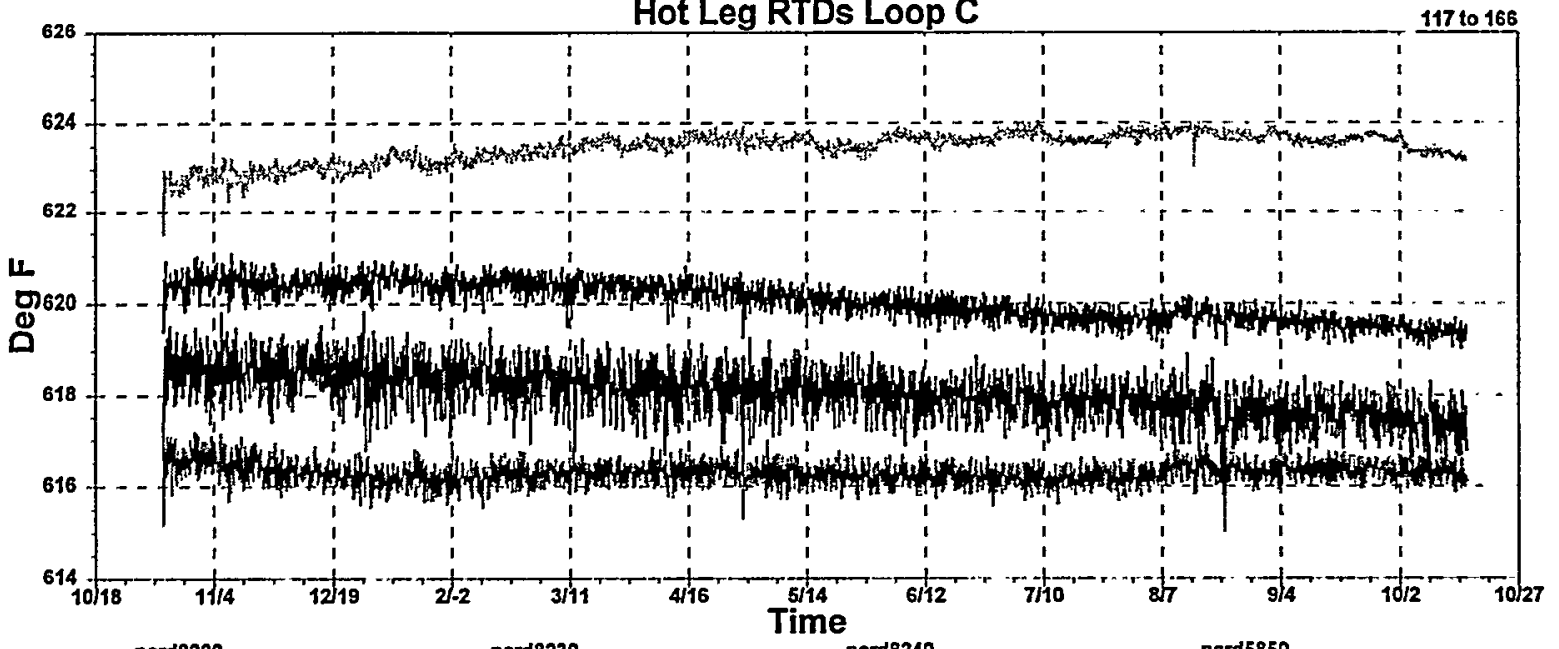

Hot Leg RTDs Loop C

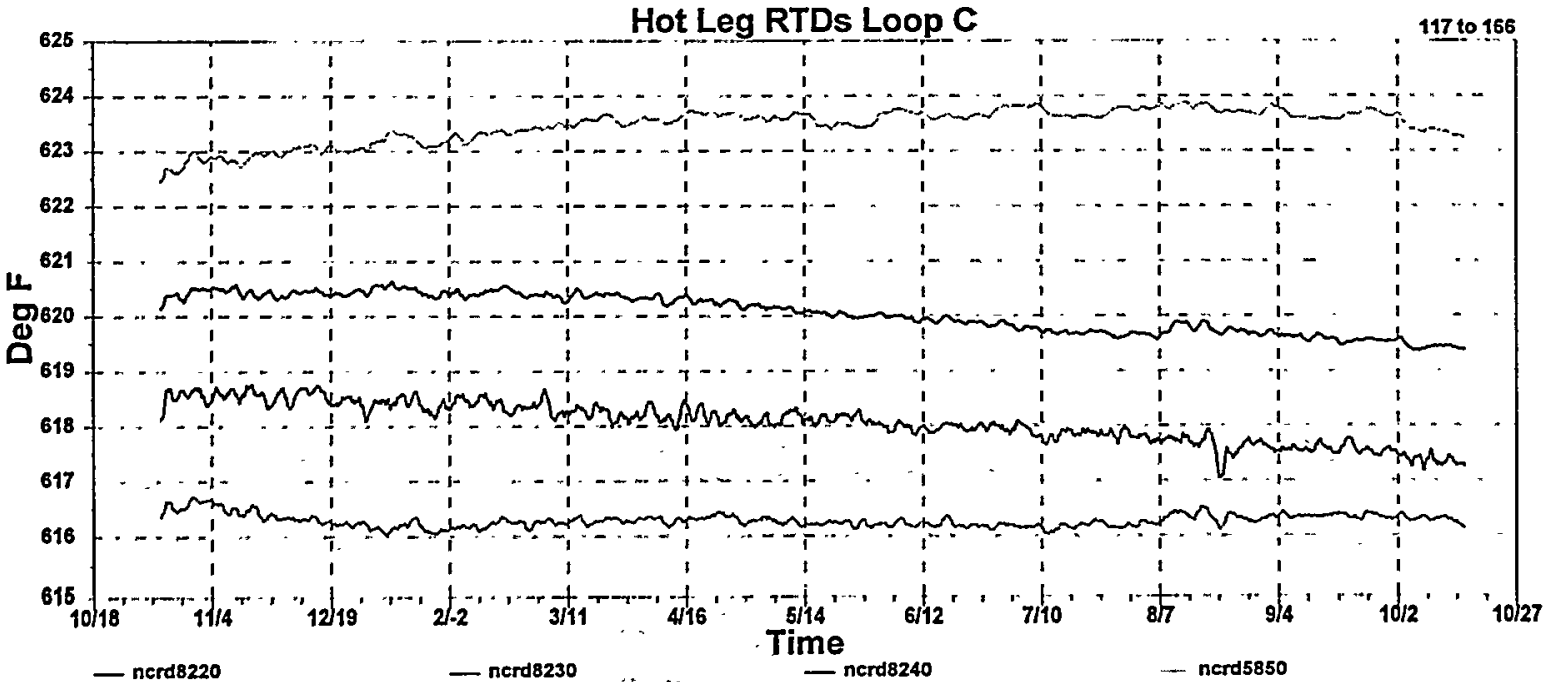

G.31 McGuire Cycle 2 Data for Hot Leg RTD Signals for Loop C, 


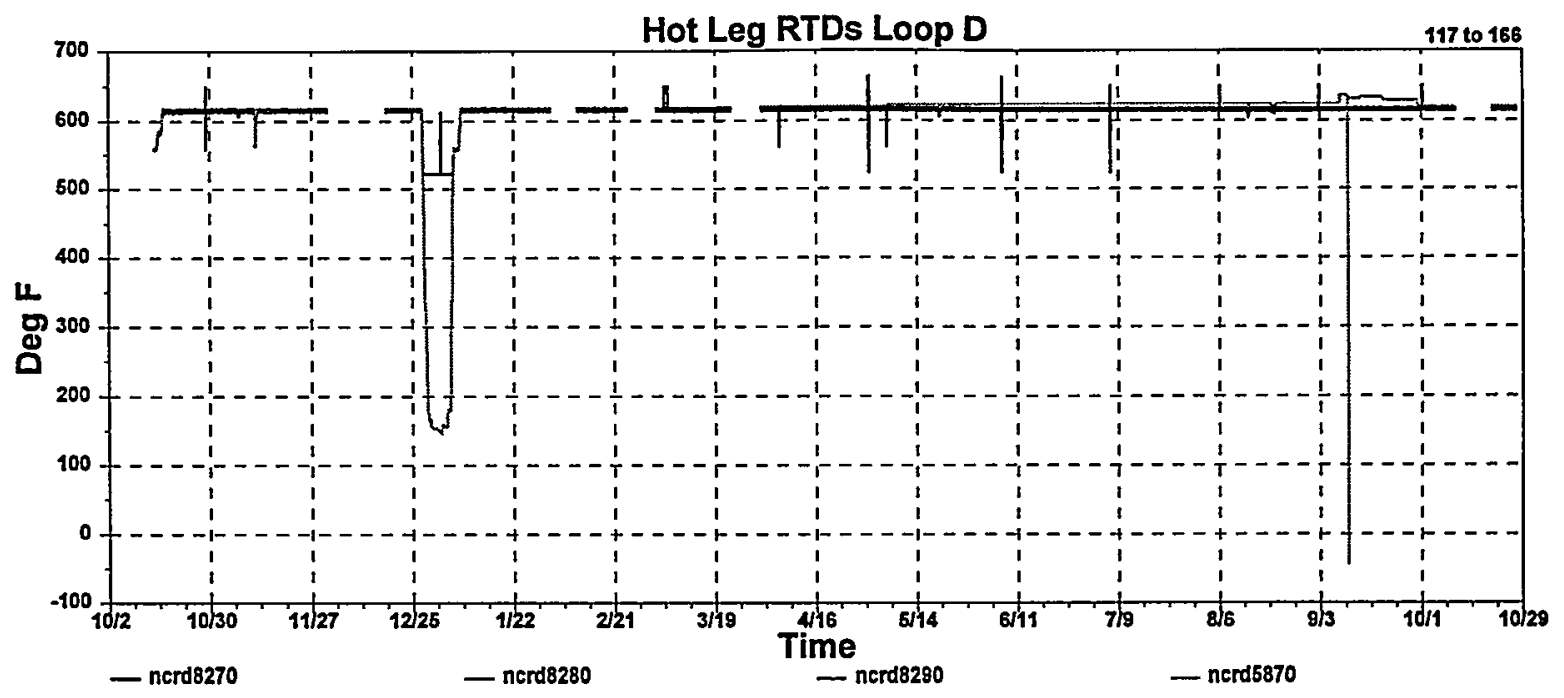

Hot Leg RTDs Loop D

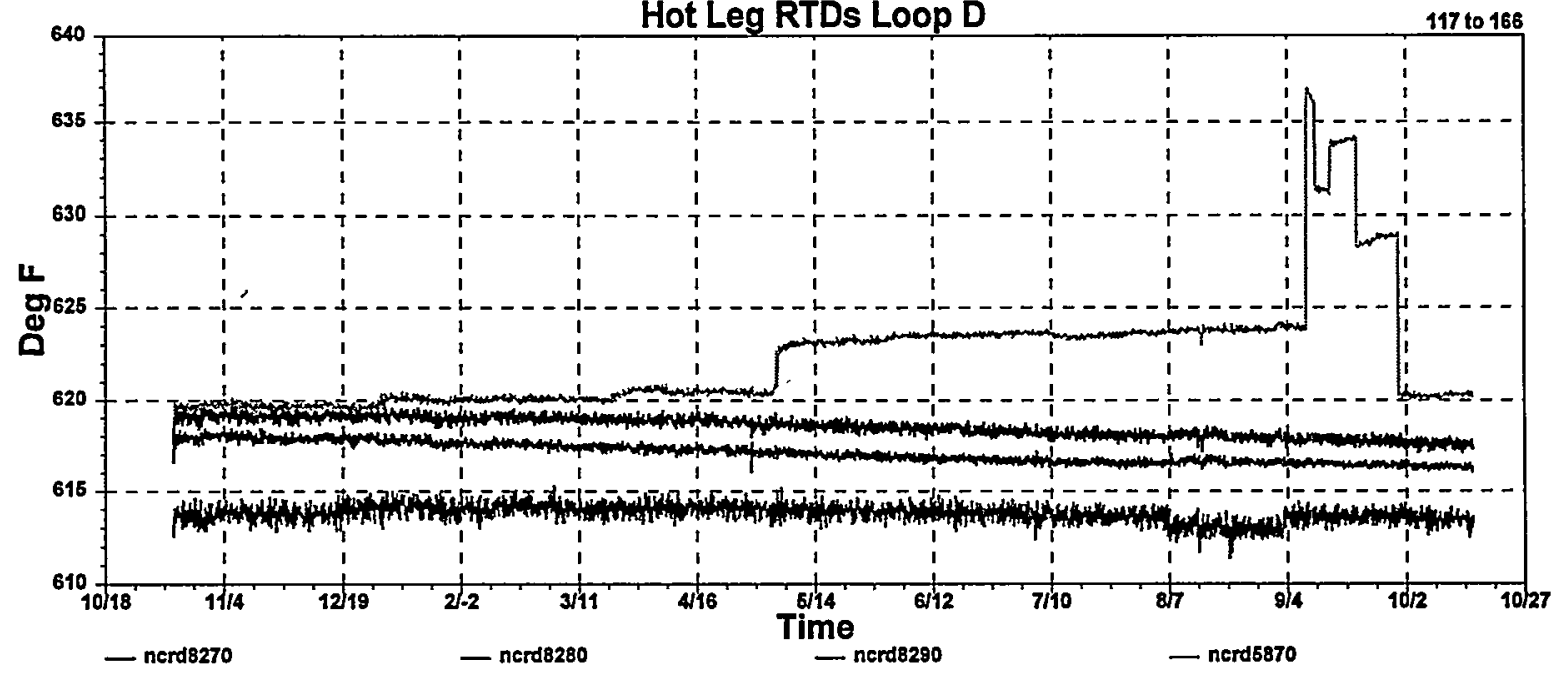

Hot Leg RTDs Loop D

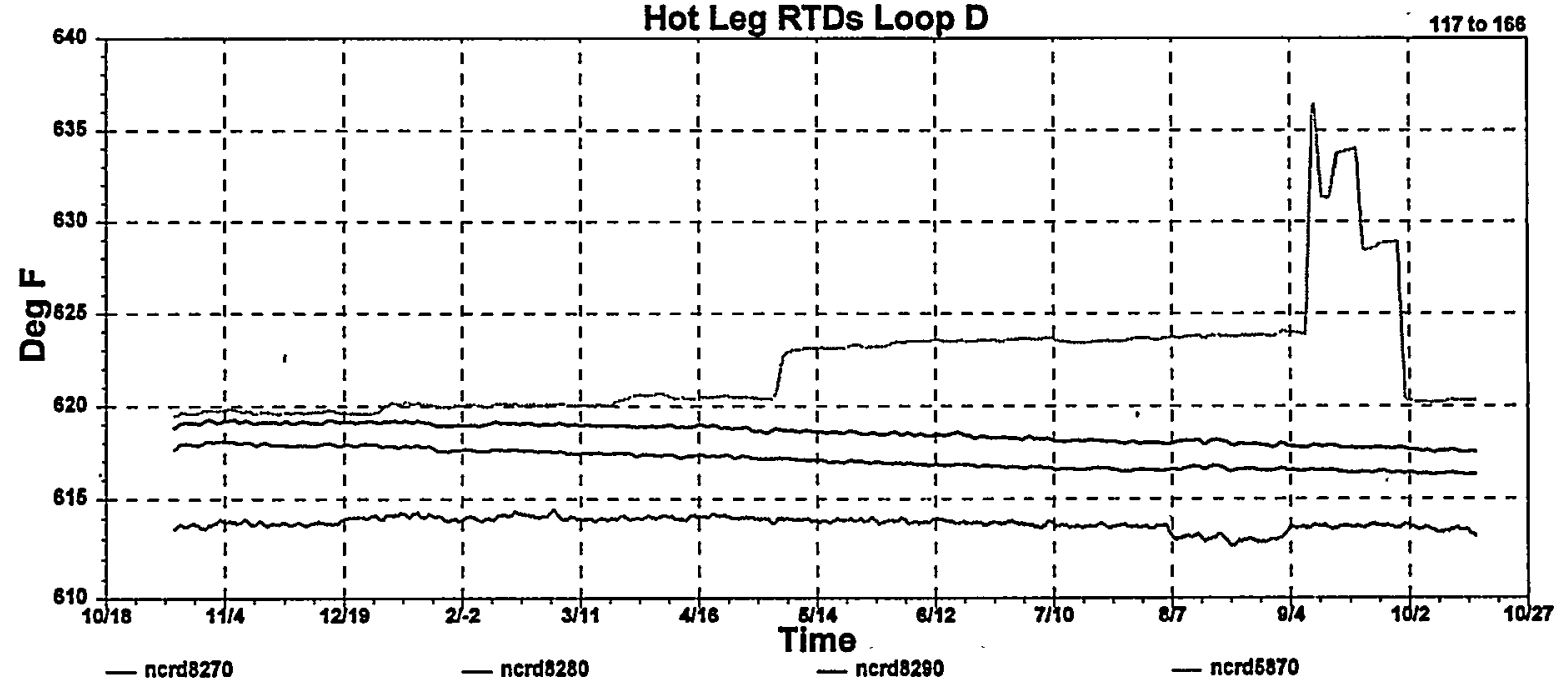

G.32 McGuire Cycle 2 Data for Hot Leg RTD Signals for Loop D 

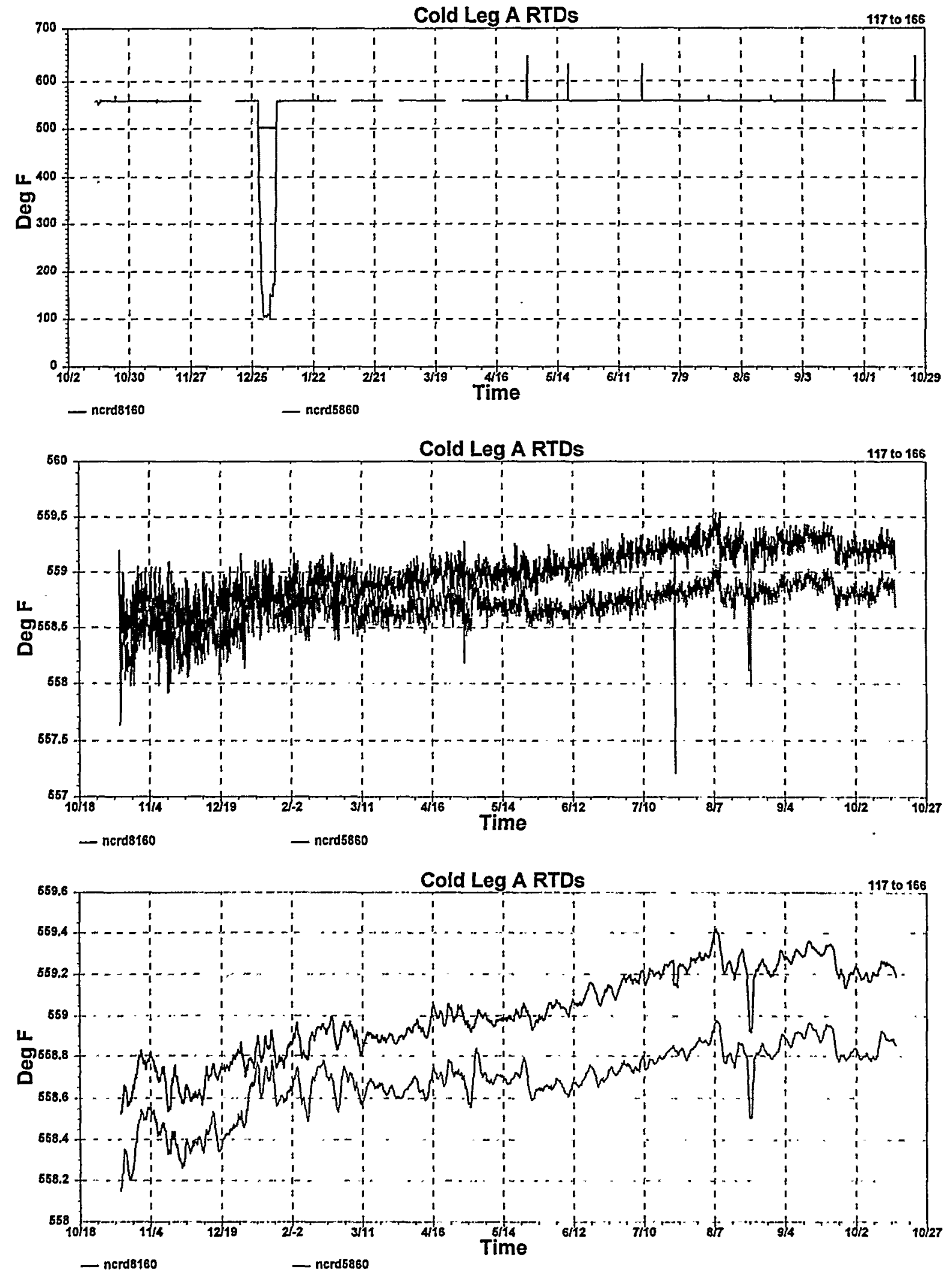

G.33 McGuire Cycle 2 Data for Cold Leg RTD Signals for Loop A 

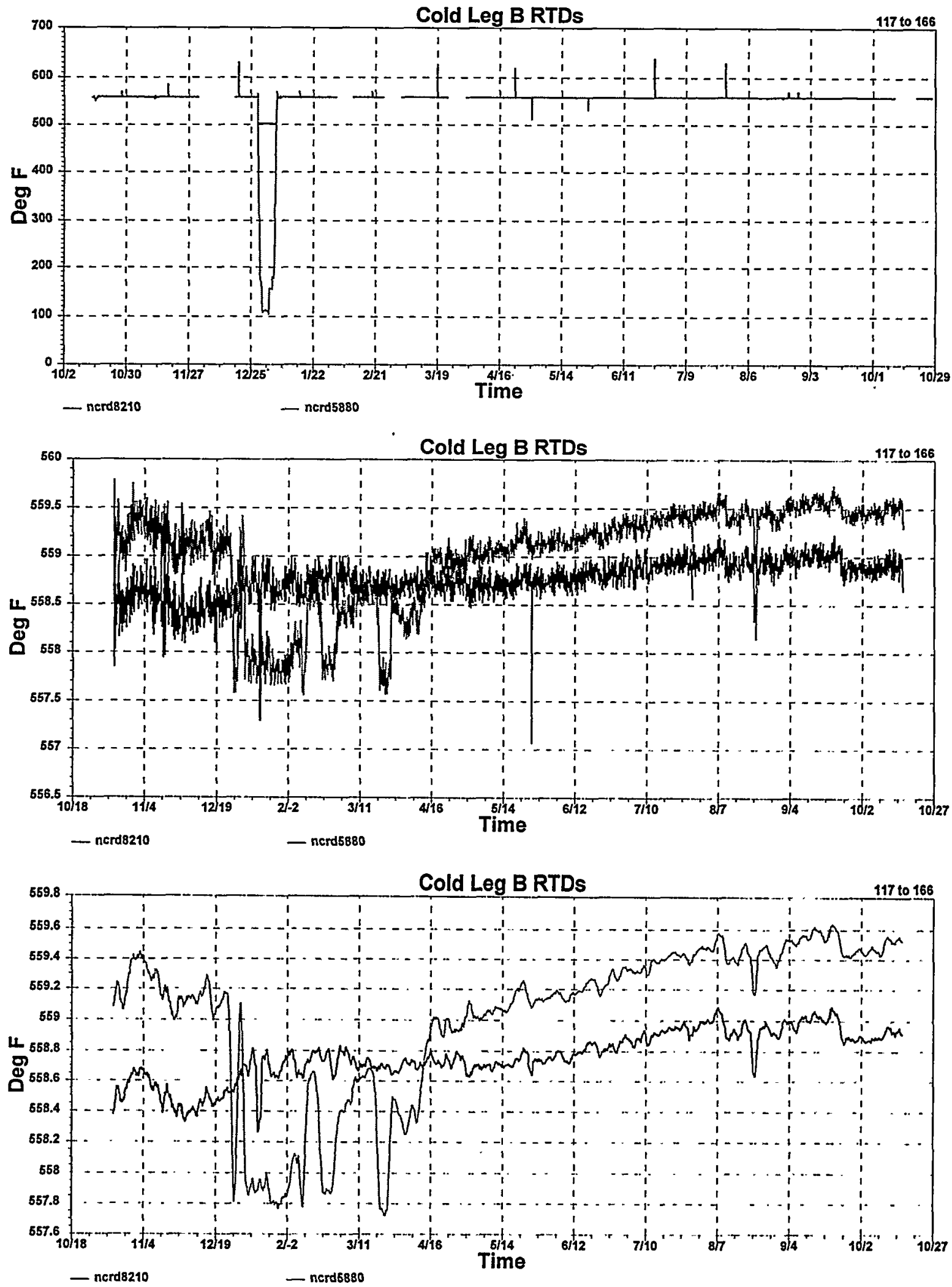

G.34 McGuire Cycle 2 Data for Cold Leg RTD Signals for Loop B 

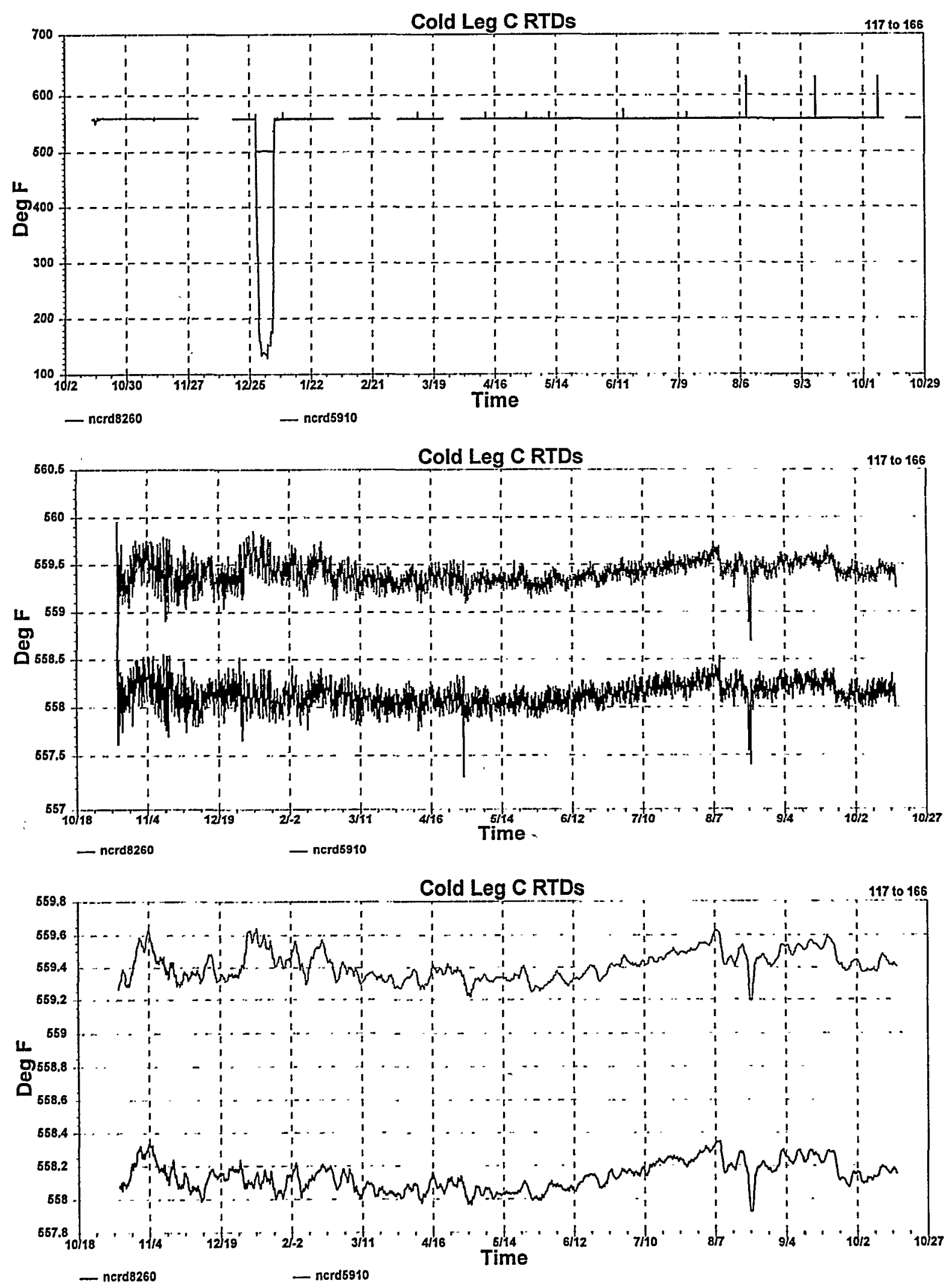

G.35 McGuire Cycle 2 Data for Cold Leg RTD Signals for Loop C 

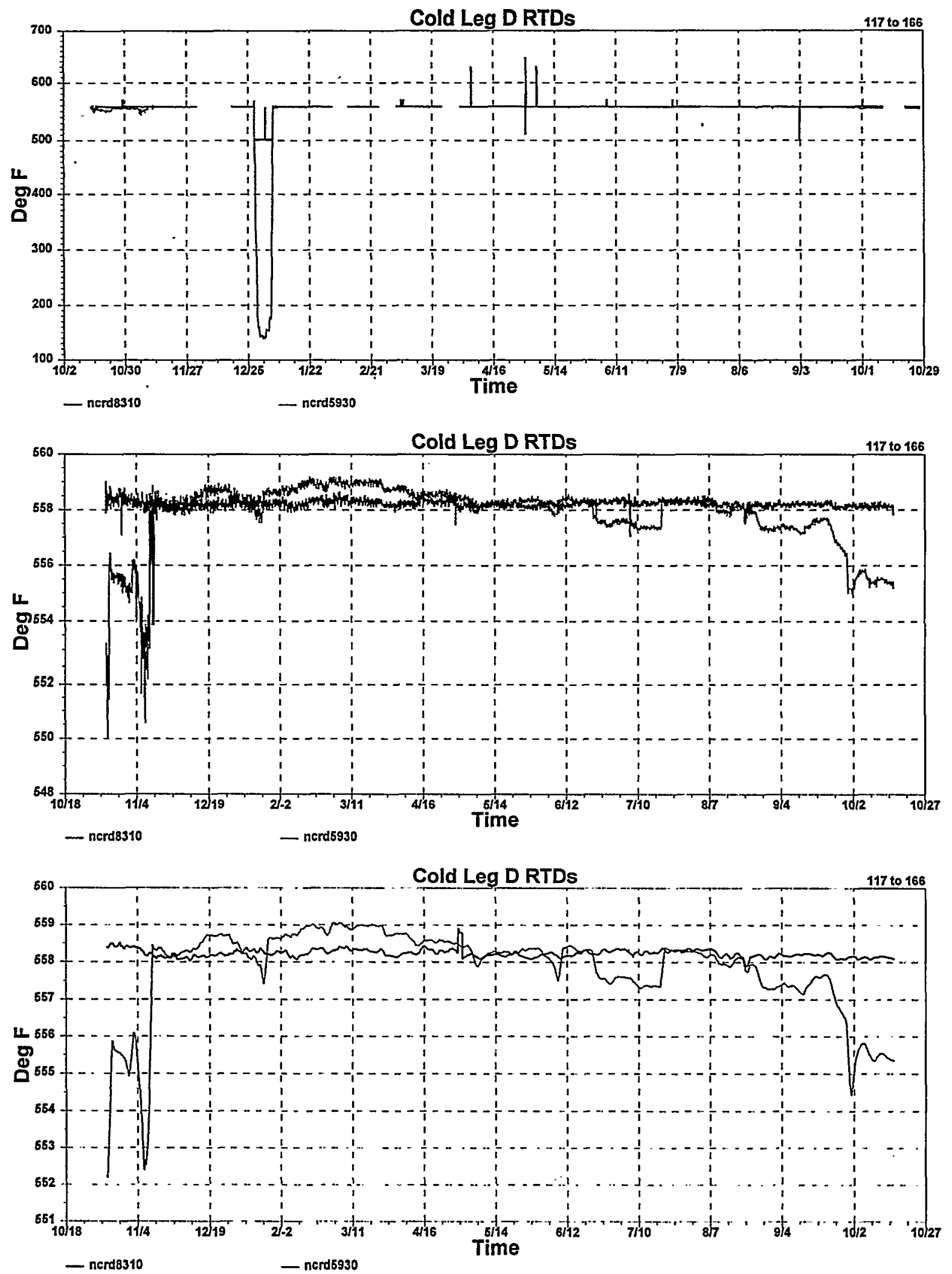

G.36 McGuire Cycle 2 Data for Cold Leg RTD Signals for Loop D 

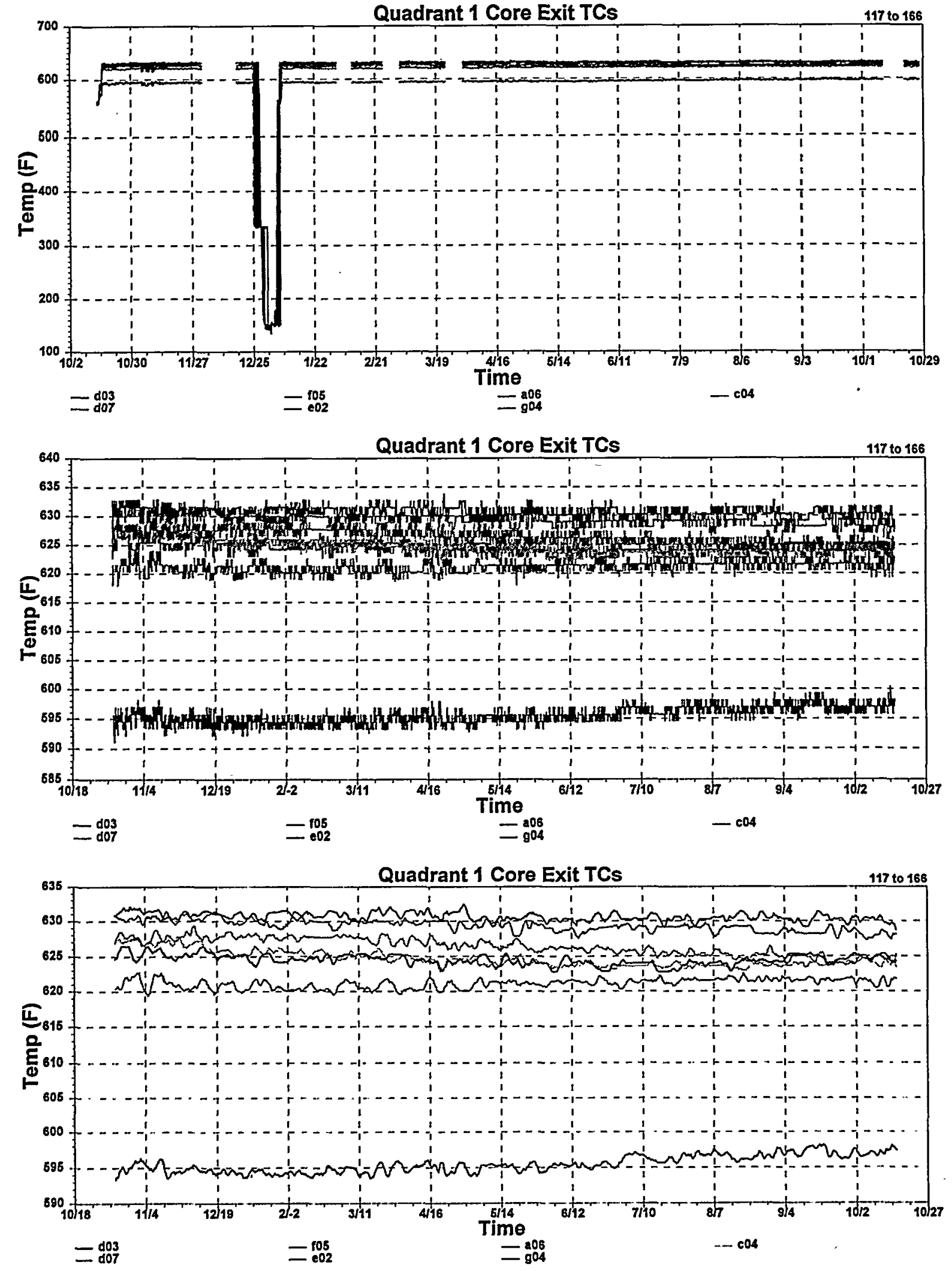

G.37 McGuire Cycle 2 Data for Core Exit Thermocouple Signals for Quadrant 1 

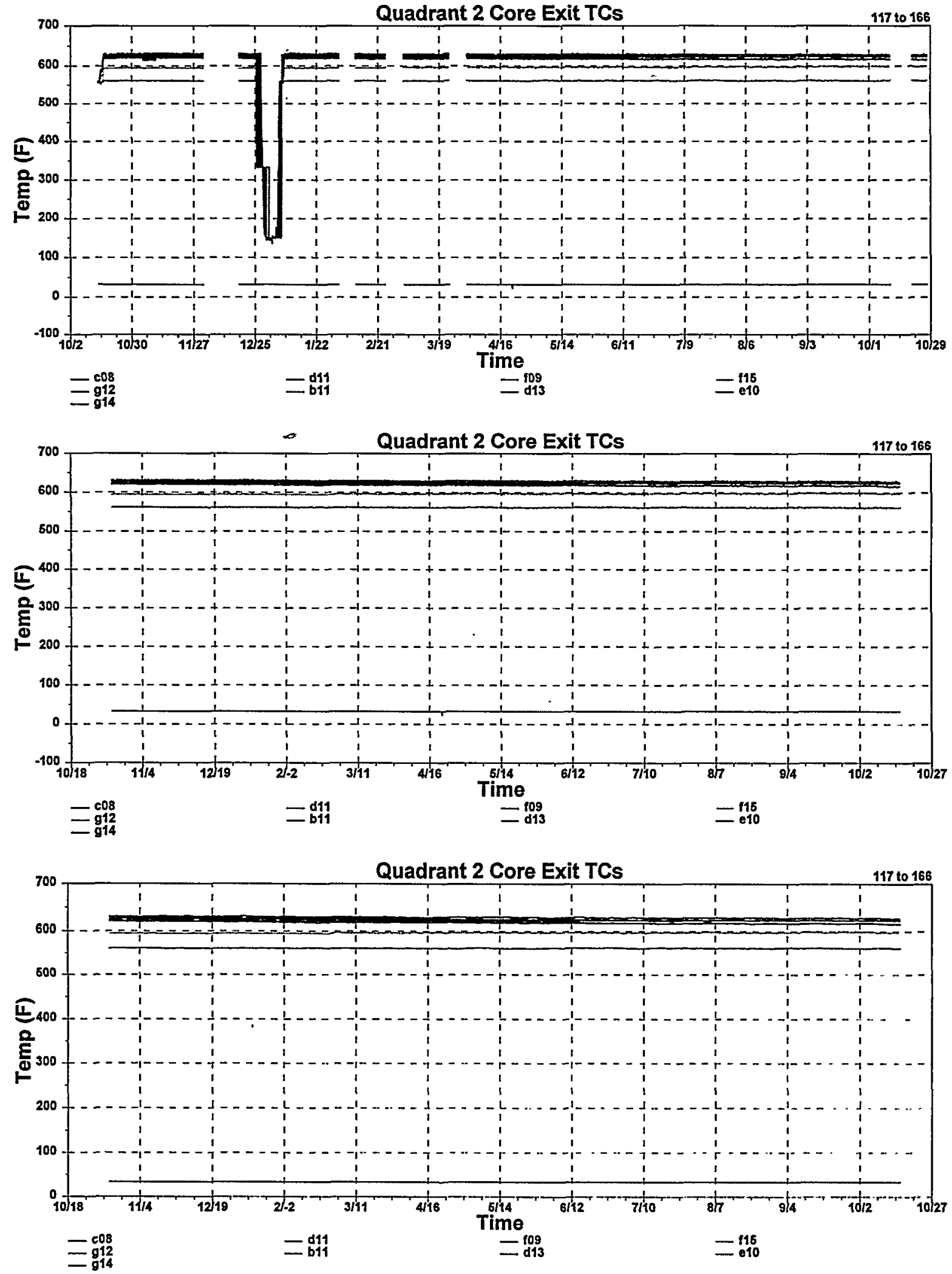

G.38 McGuire Cycle 2 Data for Core Exit Thermocouple Signals for Quadrant 2 

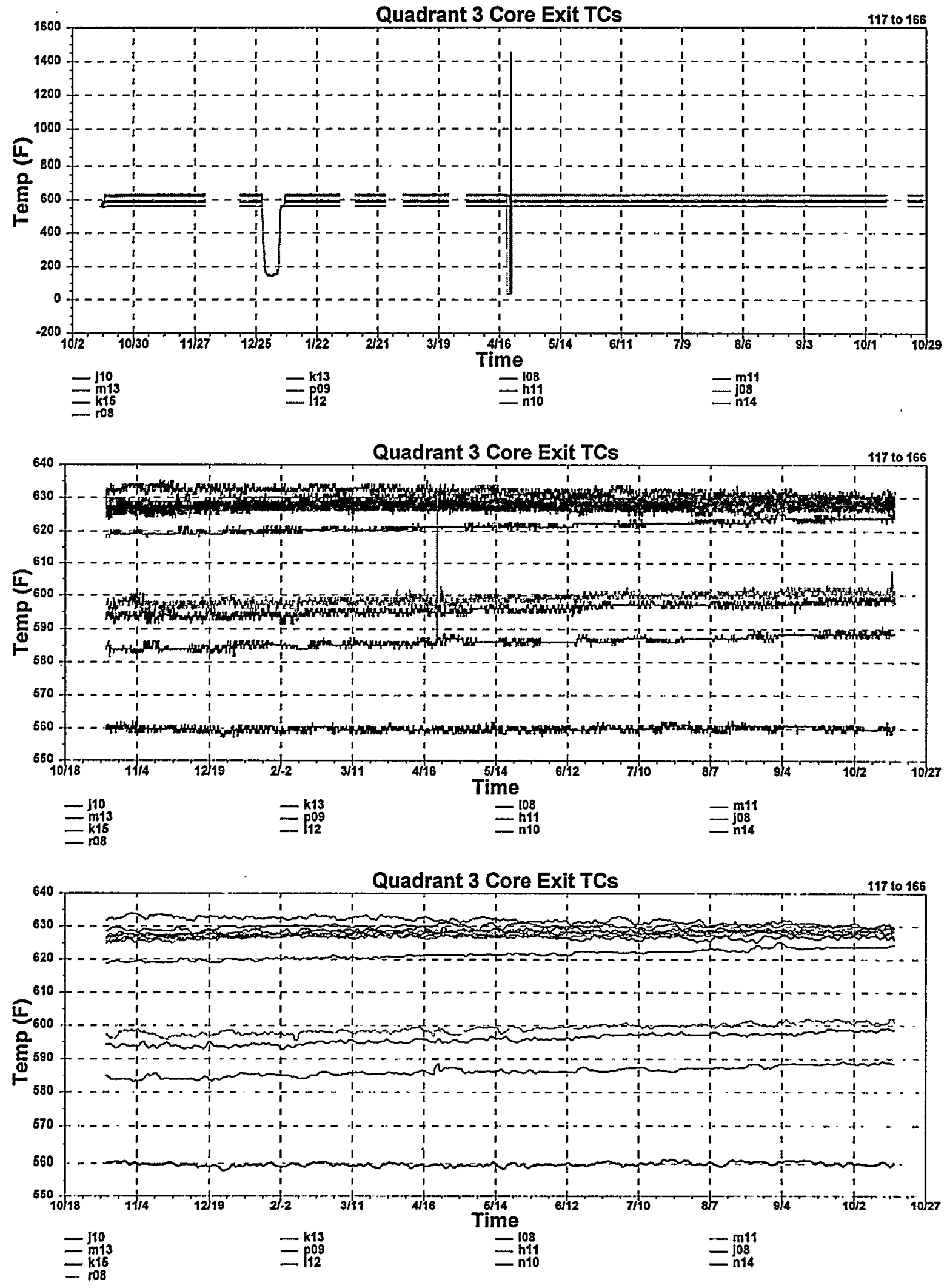

G.39 McGuire Cycle 2 Data for Core Exit Thermocouple Signals for Quadrant 3 

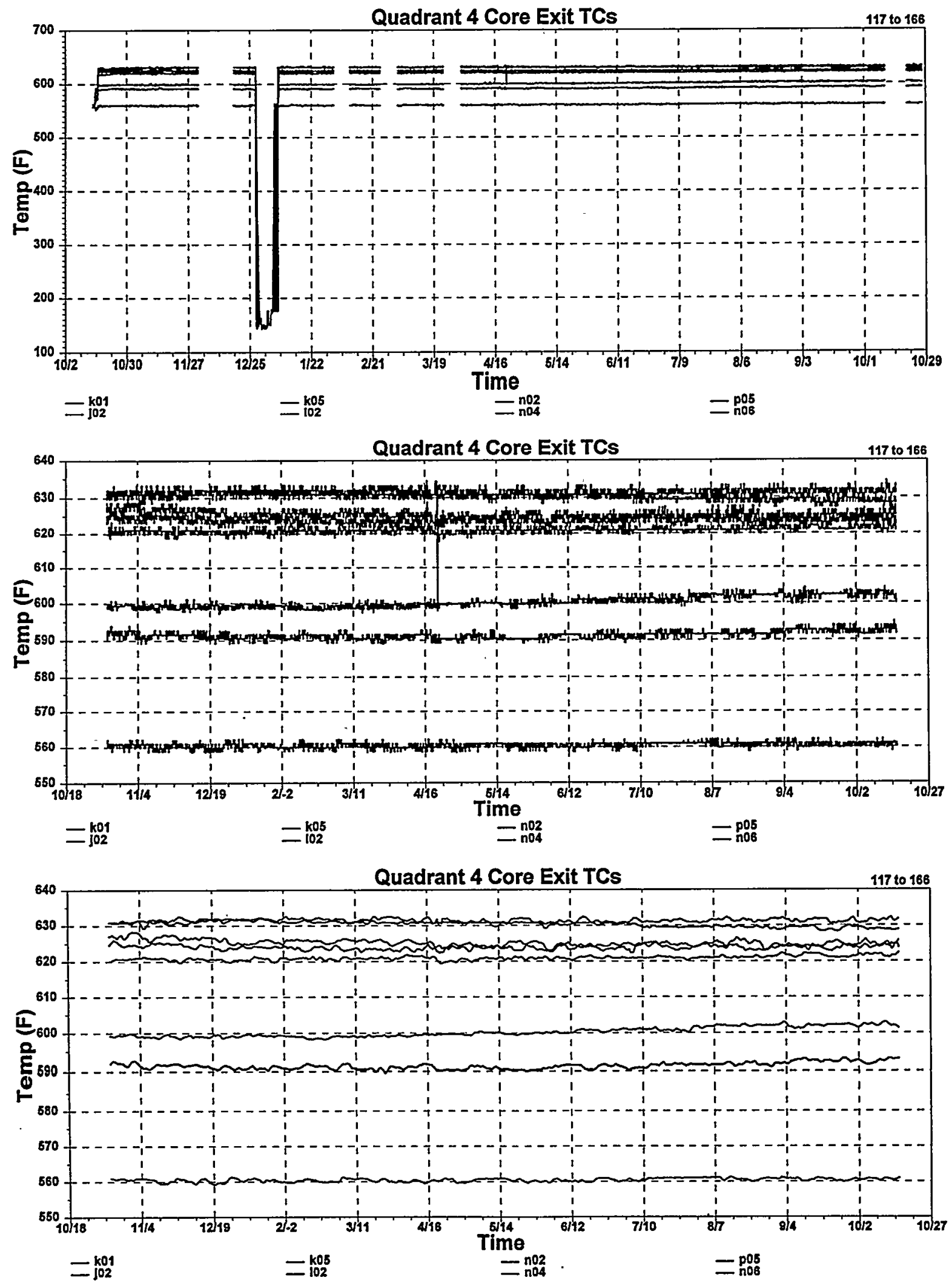

G.40 McGuire Cycle 2 Data for Core Exit Thermocouple Signals for Quadrant 4 


\begin{tabular}{|c|c|}
\hline $\begin{array}{l}\text { U.S. NUCLEAR REGULATORY COMMISSION } \\
\text { BIBLIOGRAPHIC DATA SHEET } \\
\text { (See instructions on the reverse) }\end{array}$ & 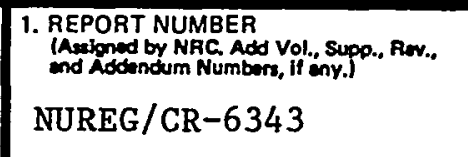 \\
\hline \multirow{4}{*}{$\begin{array}{l}\text { 2. TITLE AND SUBTITLE } \\
\text { On-Line Testing of Calibration of Process Instrumentation } \\
\text { Channels in Nuclear Power Plants }\end{array}$} & \\
\hline & DATE REPORT PUBLISHED \\
\hline & \begin{tabular}{|c|c|} 
MONTH & YEAR \\
November & 1995
\end{tabular} \\
\hline & $\begin{array}{l}\text { 4. FIN OR GRANT NUMBER } \\
\text { L2562 }\end{array}$ \\
\hline \multirow[t]{2}{*}{$\begin{array}{l}\text { 5. AUTHOR(S) } \\
\text { H.M. Hashemian }\end{array}$} & $\begin{array}{l}\text { 6. TYPE OF REPORT } \\
\text { Technical }\end{array}$ \\
\hline & $\begin{array}{l}\text { 7. PERIOD COVERED (Inc/usive Dates) } \\
9 / 30 / 92-6 / 30 / 95\end{array}$ \\
\hline \multicolumn{2}{|c|}{ 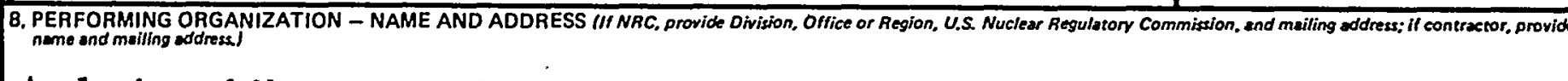 } \\
\hline \multicolumn{2}{|c|}{$\begin{array}{l}\text { Analysis and Measurement Services Corporation } \\
\text { AMS } 9111 \text { Cross Park Drive } \\
\text { Knoxville, TN } 37923\end{array}$} \\
\hline \multicolumn{2}{|c|}{ 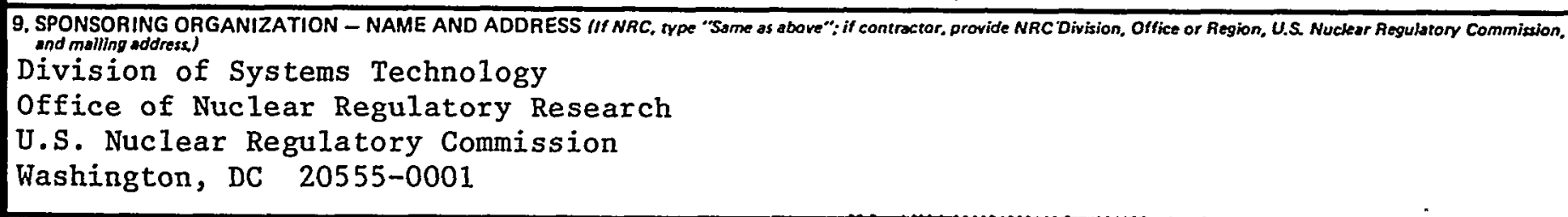 } \\
\hline \multicolumn{2}{|l|}{$\begin{array}{l}\text { 10. SUPPLEMENTARY NOTES Christina Antonescu, NRC Project Manager } \\
\text { Copyrighted by Analysis and Measurement Services Corporation, } 1995\end{array}$} \\
\hline \multicolumn{2}{|c|}{$\begin{array}{l}\text { 11.ABSTACT (200wordsores) } \\
\text { This report presents the results of a comprehensive research and development program } \\
\text { to develop and validate a new technique for remote testing of calibration of process } \\
\text { instrumentation channels in nuclear power plants. This technique can be used to } \\
\text { verify the calibration of instrument channels (including the field sensors) while } \\
\text { the plant is on-line. The method is simple and passive, uses the existing plant } \\
\text { instrumentation, and includes the effects of process operating conditions on the } \\
\text { calibration of the instruments. } \\
\text { The new technique is based on monitoring the steady-state output of process } \\
\text { instrumentation channels on a periodic or continuous basis during each fuel cycle } \\
\text { to identify any significant drift. If the drift is more than an allowable value, } \\
\text { then the channel is calibrated. otherwise, the channel is not calibrated. To } \\
\text { separate process drift from instrument drift, analytical models or a calibrated } \\
\text { reference channel may be used to track the process independently and determine if } \\
\text { it is drifting. On-1ine monitoring is performed not only during normal operation } \\
\text { but also during plant startup and shutdown periods to obtain data to verify } \\
\text { instrument calibrations throughout their operating range. }\end{array}$} \\
\hline 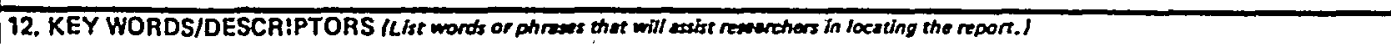 & $\begin{array}{l}\text { 13. AVAILABILITY STATEMENT } \\
\text { Unlimited }\end{array}$ \\
\hline \multirow[t]{3}{*}{$\begin{array}{ll}\text { On-Line Monitoring } & \text { Analytical Models } \\
\text { Calibration Reduction } & \text { Neural Networks } \\
\text { Process Instrumentation } & \text { Expert Systems } \\
\text { Nuclear Power Plants } & \text { Performance Monitoring } \\
\text { Automated Calibration } & \text { Predictive Maintenance }\end{array}$} & $\begin{array}{l}\text { 14. SECUAIYY CLASSIFICATION } \\
\text { Unhls Pest } \\
\text { Unclassified Report } \\
\text { Unclassified }\end{array}$ \\
\hline & $\begin{array}{l}\text { 15. NUMBER OF PAGES } \\
\text { Unclassified } \\
\end{array}$ \\
\hline & \\
\hline
\end{tabular}

